\title{
Zn-Catalyzed Nicotinate-Directed Transamidations in Peptide Synthesis
}

\author{
Charlie Hollanders, ${ }^{\dagger, t}$ Evelien Renders, ${ }^{*}$ Charlène Gadais, ${ }^{\dagger, t}$ Dario Masullo, ${ }^{*}$ Laurent Van \\ Raemdonck, ${ }^{*}$ Clarence C. D. Wybon, ${ }^{*}$ Charlotte Martin,$^{\dagger}$ Wouter A. Herrebout,${ }^{\square}$ Bert U. W. Maes, ${ }^{*}$ \\ and Steven Ballet ${ }^{+}{ }^{\dagger}$ \\ ${ }^{\dagger}$ Organic Chemistry, Departments of Chemistry and Bioengineering Sciences, Vrije Universiteit \\ Brussel, Pleinlaan 2, B-1050 Brussels, Belgium \\ ${ }^{\ddagger}$ Organic Synthesis, Department of Chemistry, University of Antwerp, Groenenborgerlaan 171, B-2020 \\ Antwerp, Belgium \\ $\square$ Molecular Spectroscopy, Department of Chemistry, University of Antwerp, Groenenborgerlaan 171, \\ B-2020 Antwerp, Belgium
}

Corresponding Author

*E-mail: $\quad$ steven.ballet@vub.be

bert.maes@uantwerpen.be 


\section{Table of Contents}

0 General Considerations $\quad$ S3

1 Optimization Data $\quad$ S5

1.1 General Procedure for the Optimization of the Transamidation $\quad$ S5

1.2 Evaluation of the Reaction Parameters of the Transamidation $\quad$ S5

1.3 Optimization of the Synthesis of L-4o: tert-Butyl 2-Chloronicotinate Introduction on the Side Chain $\begin{array}{ll}\text { of Boc-L-Gln-OH } & \text { S17 }\end{array}$

2 NMR Characterization of Compound L-4o $\quad$ S19

$\begin{array}{ll}\text { 2.1 COSY NMR Correlations in L-4o } & \text { S19 }\end{array}$

$2.2{ }^{15} \mathrm{~N}$ and ${ }^{13} \mathrm{C}$ HSQC NMR Correlations in L-4o $\quad$ S21

2.3 NOESY NMR Correlations in L-4o $\quad$ S23

$\begin{array}{lc}2.4 \text { HMBC NMR Correlations in } \mathbf{L - 4 0} & \text { S24 }\end{array}$

3 Comparison of Transamidation with Alcoholysis $\quad$ S32

4 General Procedures $\quad$ S33

5 Synthetic Procedures $\quad$ S36

5.1 Synthesis of the $N$-Boc Protected $t$ Bu Nicotinate Functionalized Amino Acid / peptidic Amides 4

5.2 Effect of tert-Butyl 2-Chloronicotinate Introduction on the Chiral Purity of Boc-L-Gln-OH S48

5.3 Dipeptide Synthesis using Transamidation of $N$-Boc Protected $t$ Bu Nicotinate Functionalized Amino Acid Amides 4

S53

5.4 Segment Coupling using Transamidation $\quad$ S60

$\begin{array}{lc}\text { 5.5 Transamidation on Solid Support } & \text { S68 }\end{array}$

5.6 Cyclization using Transamidation of $t \mathrm{Bu}$ Nicotinate Linear Heptapeptide: Synthesis and Cyclization

5.7 Synthesis of the Chemoselectivity Building Block L,L-15 and Transamidation S81

6 Amide Resonance Energy Calculations $\quad$ S86

$\begin{array}{ll}7 \text { Copies of the NMR spectra } & \text { S167 }\end{array}$

8 References $\quad$ S290 


\section{General Considerations}

Unless stated otherwise, all commercial chemicals were used without further purification. Anhydrous 2methyltetrahydrofuran was obtained by distillation over activated $3 \AA$ molecular sieves. Anhydrous tetrahydrofuran was obtained using a PureSolv Innovative Technology system. Anhydrous 1,4-dioxane was obtained by drying over activated $4 \AA$ molecular sieves for $24 \mathrm{~h}$ under argon atmosphere prior to use. Non-commercial starting materials were prepared based on literature procedures and are described below. ${ }^{1} \mathrm{H}$ and ${ }^{13} \mathrm{C}$ NMR spectra were recorded using different spectrometers. A Bruker Avance II 500 spectrometer was used at $500 \mathrm{MHz}\left({ }^{1} \mathrm{H} \mathrm{NMR}\right)$ and $126 \mathrm{MHz}\left({ }^{13} \mathrm{C} \mathrm{NMR}\right)$ at ambient temperature. Alternatively, A Bruker Avance 400 was used at $400 \mathrm{MHz}\left({ }^{1} \mathrm{H} \mathrm{NMR}\right)$ and $101 \mathrm{MHz}\left({ }^{13} \mathrm{C} \mathrm{NMR}\right)$ at $30^{\circ} \mathrm{C}$. To obtain spectra at $250 \mathrm{MHz}$ and $63 \mathrm{MHz}$, a Bruker Avance DRX 250 was used. The chemical shifts were reported in delta $(\delta)$ units in parts per million $(\mathrm{ppm})$ relative to the signal of the deuterated solvent. For $\mathrm{CDCl}_{3}$, the singlet in ${ }^{1} \mathrm{H}$ NMR was calibrated at $7.26 \mathrm{ppm}$ and the central line of the triplet in ${ }^{13} \mathrm{C}$ NMR at $77.0 \mathrm{ppm}$. When recording in MeOD or DMSO- $d_{6}$, the calibration was performed at 3.31 ppm and $2.50 \mathrm{ppm}$ for ${ }^{1} \mathrm{H}$ NMR and $49.0 \mathrm{ppm}$ and $39.5 \mathrm{ppm}$ for ${ }^{13} \mathrm{C}$ NMR, respectively. Assignments were made using one dimensional (1D) ${ }^{1} \mathrm{H}$ and ${ }^{13} \mathrm{C}$ spectra and two-dimensional (2D) HSQC, HMBC, and COSY spectra. Multiplicities were described as singlet (s), doublet (d), triplet (t), quartet (q), multiplet $(\mathrm{m})$, broad (br), or a combination thereof. The corresponding coupling constants ( $J$ values) were reported in Hertz (Hz). Analytical RP-HPLC was performed on a VWR-Hitachi Chromaster HPLC with a Chromolith HighResolution RP-18C column from Merck (150 mm X $4.6 \mathrm{~mm}, 1.1 \mu \mathrm{m}$, $150 \AA$ A). The flow rate was $3 \mathrm{ml} / \mathrm{min}$ and UV detection was done at $214 \mathrm{~nm}$. The solvent system used consisted of $0.1 \%$ TFA in ultrapure water (A) and $0.1 \%$ TFA in acetonitrile (B) with a gradient from $3 \%$ B to $100 \%$ B over a 6 minutes runtime. For LC-MS analysis, the HPLC unit used was a Waters 600 system combined with a Waters 2487 UV detector at $215 \mathrm{~nm}$ and as stationary phase a Vydac MS RP C18-column (150 mm x $2.1 \mathrm{~mm}, 3 \mu \mathrm{m}, 300 \AA$ ). The solvent system used was $0.1 \%$ formic acid in water (A) and $0.1 \%$ formic acid in acetonitrile (B) with a gradient going from $3 \%$ to $100 \%$ B over 20 minutes with a flow rate of $0.3 \mathrm{ml} / \mathrm{min}$. The MS unit, coupled to the HPLC system, was a Micromass QTOF-micro system. For the high resolution mass spectroscopy, the same MS system was used with reserpine $\left(2.10^{-3} \mathrm{mg} / \mathrm{ml}\right)$ solution in $\mathrm{H}_{2} \mathrm{O}: \mathrm{CH}_{3} \mathrm{CN}(1: 1)$ as reference. Chiral HPLC was recored using a Kontron HPLC autosampler 465, Biotek system pump 522, a Jasco X-LC 3195 CD detector, Water Acquity PDA detector, and a Diacel Chiralpak IA column. Optical rotations were recorded with a Schmidt Haensch Polartronic $\mathrm{M}$ at a wavelength of $589.3 \mathrm{~nm}$ at a concentration between 5 and 15 $\mathrm{mg} / \mathrm{ml}$ in chloroform and a cell of $5 \mathrm{~cm}$. LC-MS samples to check for chiral purity were prepared by dissolving 2-4 mg of the compound in $n$-hexane/EtOH (80/20) and further diluted to a concentration of $10^{-4}$ M. From these samples $20 \mu \mathrm{L}$ was injected and analyzed via a Kontron HPLC autosampler 465 
equipped with a Kontron HPLC pump 522, a Chiralpak IA column, a Waters Photo Diode Array detector and a Jasco X-LC 3195CD system. Automated flash chromatography was performed using Grace ${ }^{\circledR}$ Reveleris X2 system equipped with an ELSD and UV detector (254 nm or $280 \mathrm{~nm}$ ) or Biotage ${ }^{\circledR}$ Isolera One Normal Phase Silicagel Flash Chromatography associated with UV detection. The used normal phase columns for the systems were Grace ${ }^{\circledR}$ Silica Flash Cartridges of $40 \mathrm{~g}$ with a flow rate of $40 \mathrm{ml} / \mathrm{min}$ unless stated otherwise. Semi-preparative RP-HPLC-purifications were done using a Gilson HPLC system with Gilson 322 pump equipped with a Grace ${ }^{\circledR}$ Vydac 150HC C18 (250 mm x 22 mm, 10 $\mu \mathrm{m})$ column and Waters UV/VIS-156 detector at $215 \mathrm{~nm}$. The same solvent system was used as applied for the analytical RP-HPLC with a flow rate of $20 \mathrm{ml} / \mathrm{min}$. The transamidations were performed in sealed microwave vials for a reaction volume of $0.5 \mathrm{ml}-2 \mathrm{ml}$ (Figure S1) equiped with a triangle stirring bar.

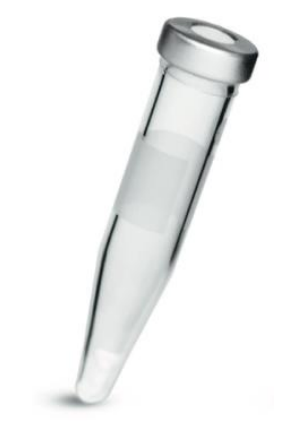

Figure S1. Reaction vial $(0.5 \mathrm{ml}-2 \mathrm{ml})$ used for the transamidation reactions 


\section{Optimization Data}

\subsection{General Procedure for the Optimization of the Transamidation}

To a microwave vial was added Boc-L-Phe-NH- $t$ Bu-nic L-4a (1 equiv, 110 mg, 0.25 mmol), H-L-PheOMe L-5a, additives, and solvent. The vial was sealed with a crimp cap, and stirred at the specified temperature and time. The mixture was then allowed to cool down to room temperature, decapped, and concentrated in vacuo. A known amount of 1,3,5-trimethoxybenzene, between 10 - $15 \mathrm{mg}$, was added to the crude mixture, which was then dissolved in MeOD or DMSO- $d_{6}$. Potential precipitating salts were removed by centrifugation. A ${ }^{1} \mathrm{H}$ NMR of the crude was recorded and signals were integrated in comparison to the internal standard. The mass balance was calculated based on the remaining starting material L-4a and tert-butyl 2-aminonicotinate $\mathbf{S 2}$ by-product of the transamidation. Considering experimental and instrumental errors, a total measured mass balance in the $95-105 \%$ range was considered as a successful experiment. Reported values were subsequently recalculated to $100 \%$. HPLC and LC-MS of the sample were subsequently recorded to confirm the formation of the desired peptide compound.

\section{Procedure for the Desalting of H-L-Phe-OMe Hydrochloric Acid Salt L-5a.HCl}

The desalting of the commercially available H-L-Phe-OMe hydrochloric acid salt L-5a.HCl was performed by adding DIPEA (1 equiv) to a stirring mixture of L-5a.HCl (1.1 equiv) in diethyl ether. The mixture was stirred for 30 minutes at room temperature, filtered and concentrated in vacuo. The purity of the H-L-Phe-OMe L-5a was confirmed by ${ }^{1} \mathrm{H}$ NMR.

\subsection{Evaluation of the Reaction Parameters of the Transamidation}

The transamidation on amino acid and peptide substrates was optimized using the synthesis of the dipeptide, Boc-L-Phe-L-Phe-OMe L,L-6a from Boc-L-Phe-NH- $t$ Bu-nic L-4a and H-L-Phe-OMe hydrochloric acid salt $\mathbf{L}-\mathbf{5 a} \mathbf{a} . \mathbf{H C l}$ or $\mathbf{L - 5 a}$ according to the general procedure.

As a starting point, the reaction conditions of the alcoholysis previously developed in our laboratories were evaluated using L-5a $\left(\mathrm{Zn}(\mathrm{OAc})_{2}\right.$ cat., 3 equiv. $\mathrm{ROH}$ in $t \mathrm{BuOAc}$ at $\left.40^{\circ} \mathrm{C}\right) .{ }^{[1]}$ The solvent screening was evaluated at a concentration of $1 \mathrm{M}$ (Table S1). In $t$ BuOAc a solubilty problem of the starting materials was observed (Table S1, entry 1). The solvents were chosen based on their frequent use for transformations on peptides and amino acids. A good solubility of both starting materials was observed in DMSO, DMF, THF, and 1,4-dioxane (Table S1, Entry 2, 3, 5, 6). The use of THF (Table S1, Entry 5) as solvent resulted in the best conversion and yield for the transamidation. 
Table S1. Evaluation of the Solvent.

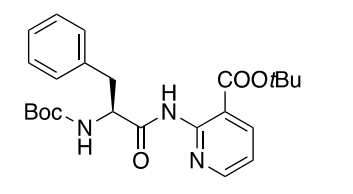

L-4a

(1 equiv)

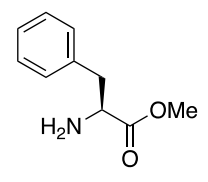

L-5a

(3 equiv)
$\mathrm{Zn}(\mathrm{OAc})_{2}$ (4 mol\%)

Solvent $(C 1 \mathrm{M})$
$40^{\circ} \mathrm{C}$

$48 \mathrm{~h}$

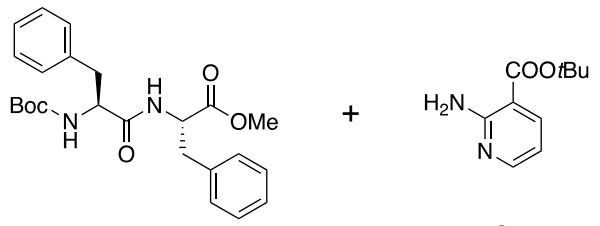

$\mathrm{L}, \mathrm{L}-6 \mathrm{a}$

S2

\begin{tabular}{cccc}
\hline Entry & Solvent & Solubility of & \\
$($ ELN-code $)$ & starting materials $^{[\mathrm{a}]}$ & ${\text { Yield L-4a }(\%)^{[\mathrm{b}]}}$ & ${\text { Yield L,L-6a }(\%)^{[\mathrm{b}]}}$
\end{tabular}

1

$t \mathrm{BuOAc}$

(KH_98.1)

2

DMSO

78

22

(KH_98.3)

3

DMF

23

77

(KH_98.5)

$4 \quad \mathrm{CH}_{2} \mathrm{Cl}_{2}$

(KH_131.1)

5

THF

12

88

(KH_131.2)

6

1,4-dioxane

24

76

(KH_131.3)

[a] $\boldsymbol{*}=$ No clear solution could be obtained when stirring the reaction mixture at $40^{\circ} \mathrm{C}, \boldsymbol{\checkmark}=\mathrm{A}$ clear solution could be obtained when stirring the reaction mixture at $40^{\circ} \mathrm{C}$. [b] ${ }^{1} \mathrm{H}$ NMR yield using 1,3,5trimethoxybenzene as internal standard.

To anticipate possible solubility issues with amino acids and peptides, the concentration of the reaction mixture was subsequently evaluated (Table S2, Entry 1-3), showing no significant change in reaction by decreasing the concentration from $1 \mathrm{M}$ (Table S2, Entry 1) to $0.5 \mathrm{M}$ (Table S2, Entry 2). A further reduction in concentration however affected the conversion negatively (Table S2, Entry 3). To achieve full conversion within $24 \mathrm{~h}$, rather than the currently used $48 \mathrm{~h}$, a catalyst loading of $10 \mathrm{~mol} \%$ at $60^{\circ} \mathrm{C}$ was required (Table S2, Entries 5-6). 
Table S2. Effect of Catalyst Loading, Temperature, Reaction Time and Concentration.
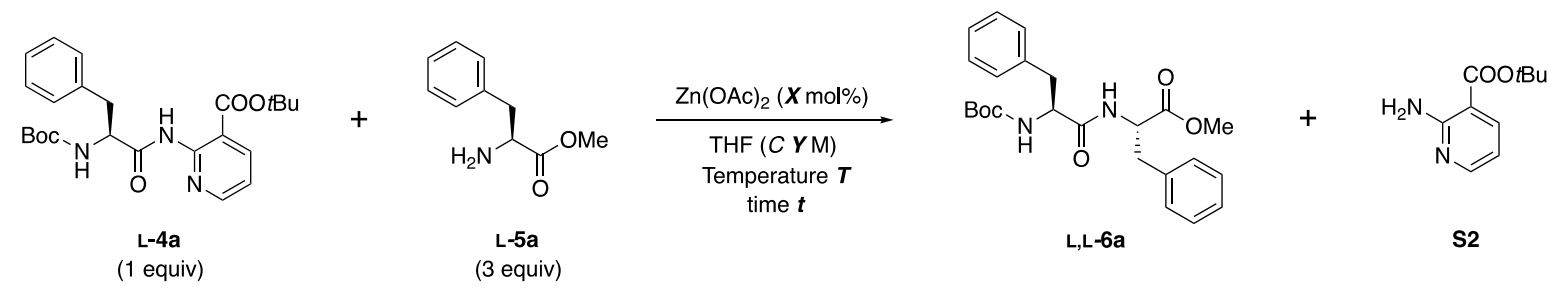

\begin{tabular}{|c|c|c|c|c|c|c|}
\hline $\begin{array}{c}\text { Entry } \\
(\text { ELN-code })\end{array}$ & $\begin{array}{c}\mathrm{Zn}(\mathrm{OAc})_{2}(X \\
\mathrm{mol} \%)\end{array}$ & $\begin{array}{l}\text { Concentration } \\
\qquad Y(\mathrm{M})^{[\mathrm{a}]}\end{array}$ & $\begin{array}{c}\text { Temperature } \\
\boldsymbol{T}\left({ }^{\circ} \mathrm{C}\right)\end{array}$ & $\begin{array}{l}\text { Reaction } \\
\text { Time } \boldsymbol{t}(\mathrm{h})\end{array}$ & $\begin{array}{l}\text { Yield L-4a } \\
\qquad(\%)^{[\mathrm{b}]}\end{array}$ & $\begin{array}{c}\text { Yield } \mathbf{L}, \mathbf{L}- \\
\mathbf{6 a}(\%)^{[\mathrm{b}]}\end{array}$ \\
\hline $\begin{array}{c}1 \\
\left(\mathrm{KH} \_131.2\right)\end{array}$ & 4 & 1 & 40 & 48 & 12 & 88 \\
\hline $\begin{array}{c}2 \\
\left(\mathrm{KH} \_143.3\right)\end{array}$ & 4 & 0.5 & 40 & 48 & 10 & 90 \\
\hline $\begin{array}{c}3 \\
\left(\mathrm{KH} \_144.3\right)\end{array}$ & 4 & 0.2 & 40 & 48 & 29 & 71 \\
\hline $4\left(\mathrm{KH} \_466\right)$ & 4 & 0.5 & 40 & 24 & 28 & 72 \\
\hline $\begin{array}{c}5 \\
\left(\mathrm{KH} \_157.1\right)\end{array}$ & 10 & 0.5 & 40 & 24 & 23 & 77 \\
\hline $\begin{array}{c}6 \\
\left(\mathrm{KH} \_182.1\right)\end{array}$ & 10 & 0.5 & 60 & 24 & 0 & 100 \\
\hline
\end{tabular}

[a] Molar concentration of the limiting reactant. [b] ${ }^{1} \mathrm{H}$ NMR yield using 1,3,5-trimethoxybenzene as internal standard.

The commercial HCl-salt form of the amine L-5a, L-5a.HCl, required the liberation of the amine prior to transamidation. The in situ deprotonation of the amino acid using DIPEA was therefore tested. Both with L-5a and L-5a.HCl, full conversion was obtained at $60^{\circ} \mathrm{C}$ within $24 \mathrm{~h}$ using $10 \mathrm{~mol} \% \mathrm{Zn}(\mathrm{OAc})_{2}$ in THF (Table S3, Entry 3, 6). 
Table S3. Evaluation of Amine Form, L-5a versus L-5a.HCl and Temperature.

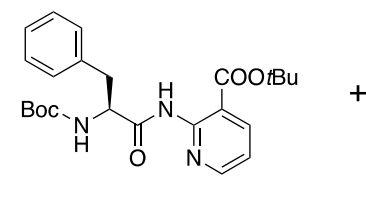

L-4a

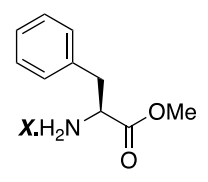

ᄂ-5a.X

(3 equiv)

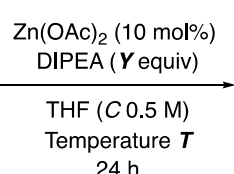

$24 \mathrm{~h}$

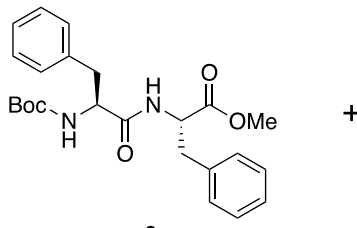

L,L-6a<smiles>CCCCOC(=O)c1cccnc1N</smiles>

S2

\begin{tabular}{|c|c|c|c|c|c|}
\hline $\begin{array}{c}\text { Entry (ELN- } \\
\text { code) }\end{array}$ & $\begin{array}{l}\text { Amine form } \\
\qquad \boldsymbol{X}\end{array}$ & $\begin{array}{c}\text { DIPEA ( } \boldsymbol{Y} \\
\text { equiv) }\end{array}$ & $\begin{array}{c}\text { Temperature } \boldsymbol{T} \\
\left({ }^{\circ} \mathrm{C}\right)\end{array}$ & $\begin{array}{l}\text { Yield L-4a } \\
\qquad(\%)^{[\mathrm{a}]}\end{array}$ & $\begin{array}{c}\text { Yield } \mathbf{L}, \mathbf{L}-\mathbf{6 a} \\
(\%)^{[\mathrm{a}]}\end{array}$ \\
\hline 1 (KH_157.1) & $\mathbf{L}-\mathbf{5} \mathbf{a}^{[\mathrm{b}]}$ & 0 & 40 & 23 & 77 \\
\hline $2\left(\mathrm{KH} \_183\right)$ & $\mathbf{L}-\mathbf{5} \mathbf{a}^{[\mathrm{b}]}$ & 0 & 50 & 14 & 86 \\
\hline 3 (KH_182.1) & $\mathbf{L}-\mathbf{5} \mathbf{a}^{[\mathrm{b}]}$ & 0 & 60 & 0 & 100 \\
\hline $4\left(\mathrm{KH} \_157.3\right)$ & L-5a.HCl & 3 & 40 & 33 & 67 \\
\hline $5\left(\mathrm{KH} \_184\right)$ & L-5a.HCl & 3 & 50 & 17 & 83 \\
\hline $6\left(\mathrm{KH} \_182.3\right)$ & L-5a.HCl & 3 & 60 & 0 & 100 \\
\hline
\end{tabular}

[a] ${ }^{1} \mathrm{H}$ NMR yield using 1,3,5-trimethoxybenzene as internal standard. [b] Free amine obtained as described in the general procedure for desalting $\mathbf{L}-5 \mathbf{a} . \mathbf{H C l}$.

Due to the high concentration, the amount of DIPEA added could be considered as a co-solvent. Consequently, a possible dilution effect needed to be evaluated (Table S4). It was observed that no significant difference was found in either the case where DIPEA was considered as a co-solvent (Table S4, Entry 3), or as a reactant (Table S4, Entry 4). 
Table S4. Effect of Dilution due to in situ Deprotonation with DIPEA.

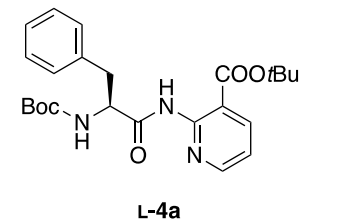

L-4a
(1 equiv)

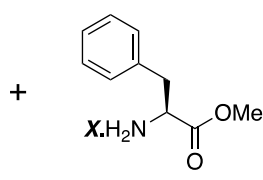

L-5a.X

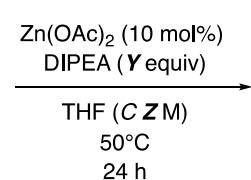

$24 \mathrm{~h}$

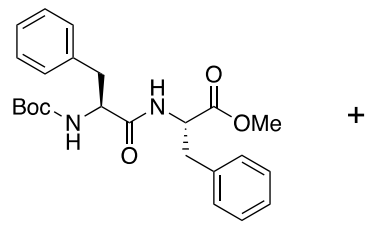

L,L-6a<smiles>CCOC(=O)c1cccnc1N</smiles>

S2

\begin{tabular}{cccccc}
\hline $\begin{array}{c}\text { Entry } \\
(\text { ELN-code })\end{array}$ & Amine form $\boldsymbol{X}$ & $\begin{array}{c}\text { DIPEA }(\boldsymbol{Y} \\
\text { equiv })\end{array}$ & $\begin{array}{c}\text { Concentration } \\
\boldsymbol{Z}(\mathrm{M})\end{array}$ & $\begin{array}{c}\text { Yield L-4a } \\
(\%)^{[\mathrm{a}]}\end{array}$ & $\begin{array}{c}\text { Yield L,L-6a } \\
(\%)^{[\mathrm{a}]}\end{array}$ \\
\hline $1\left(\mathrm{KH} \_183\right)$ & L-5a & 0 & $0.5^{[\mathrm{b}]}$ & 14 & 86 \\
$2\left(\mathrm{KH} \_187\right)$ & L-5a & 0 & $0.39^{[\mathrm{b}]}$ & 10 & 81 \\
$3\left(\mathrm{KH} \_188\right)$ & L-5a.HCl & 3 & $0.5^{[\mathrm{c}]}$ & 19 & 83 \\
$4\left(\mathrm{KH} \_184\right)$ & L-5a.HCl & 3 & $0.39^{[\mathrm{c}]}$ & 17 & 90 \\
\hline
\end{tabular}

[a] ${ }^{1} \mathrm{H}$ NMR yield using 1,3,5-trimethoxybenzene as internal standard. [b] Concentration calculated based on the limiting reactant in the volume THF used. [c] Concentration calculated based on the limiting reactant in the total volume of THF and DIPEA used.

As a combination of catalyst and bases have not been screened in our esterification, ${ }^{[1]}$ a selection of catalysts that performed well in the alcoholysis reaction was tested in the model system with DIPEA. The results showed that, compared to $\mathrm{Zn}(\mathrm{OAc})_{2}$ (Table S5, Entry 1), Zn formate. $2 \mathrm{H}_{2} \mathrm{O}$ (Table S5, Entry 4), $\mathrm{Ni}(\mathrm{OAc})_{2} \cdot \mathrm{H}_{2} \mathrm{O}$ (Table S5, Entry 7), and $\mathrm{Cu}(\mathrm{OAc})_{2} \cdot \mathrm{H}_{2} \mathrm{O}$ (Table S5, Entry 9) also gave good results, though a lower conversion was obtained. 
Table S5. Evaluation of the Catalyst.
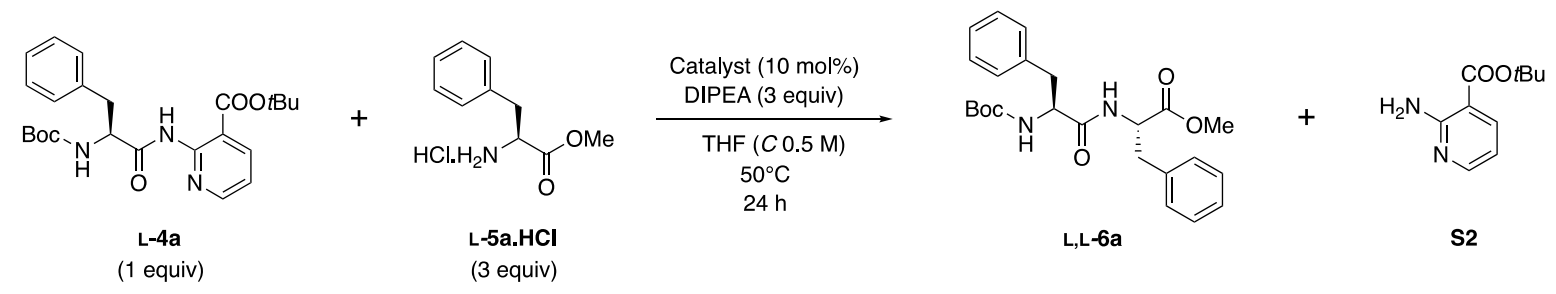

\begin{tabular}{|c|c|c|c|}
\hline $\begin{array}{c}\text { Entry (ELN- } \\
\text { code) }\end{array}$ & Catalyst $(10 \mathrm{~mol} \%)^{[\mathrm{a}]}$ & Yield L-4a $(\%)^{[\mathrm{b}]}$ & Yield $\mathbf{L}, \mathbf{L}-\mathbf{6 a}(\%)^{[\mathrm{b}]}$ \\
\hline 1 (KH_184) & $\mathrm{Zn}(\mathrm{OAc})_{2}$ & 17 & 83 \\
\hline 2 (KH_235) & $\mathrm{Zn}(\mathrm{OTf})_{2} \cdot \mathrm{H}_{2} \mathrm{O}$ & 62 & 38 \\
\hline 3 (KH_1121) & $\mathrm{Zn}(\mathrm{acac})_{2}$ & 56 & 44 \\
\hline 4 (KH_237) & $\mathrm{Zn}$ formate $2 \mathrm{H}_{2} \mathrm{O}$ & 29 & 71 \\
\hline $5\left(\mathrm{KH} \_1282\right)$ & $\mathrm{ZnCl}_{2}$ & 49 & 51 \\
\hline 6 (KH_241) & $\mathrm{Co}(\mathrm{OAc})_{2} \cdot 4 \mathrm{H}_{2} \mathrm{O}$ & 38 & 62 \\
\hline 7 (KH_243) & $\mathrm{Ni}(\mathrm{OAc})_{2} \cdot \mathrm{H}_{2} \mathrm{O}$ & 31 & 69 \\
\hline 8 (KH_246) & $\mathrm{Ni}(\text { acac })_{2}(4 \%$ water $)$ & 39 & 61 \\
\hline 9 (KH_244) & $\mathrm{Cu}(\mathrm{OAc})_{2} \cdot \mathrm{H}_{2} \mathrm{O}$ & 31 & 69 \\
\hline 10 (KH_245) & $\mathrm{Cu}\left(\mathrm{OOCCF}_{3}\right)_{2}$ & 44 & 56 \\
\hline
\end{tabular}

[a] Hydrates determined by TGA analysis. [b] ${ }^{1} \mathrm{H}$ NMR yield using 1,3,5-trimethoxybenzene as internal standard.

The four best catalysts were then evaluated with different tertiary amine bases at both $40^{\circ} \mathrm{C}$ and $50^{\circ} \mathrm{C}$ (Table S6). Despite the fact that at $50^{\circ} \mathrm{C}$ multiple combinations resulted in full conversion (Table S6, Entry $3,5,8,10)$, the corresponding experiments at $40^{\circ} \mathrm{C}$ revealed that $\mathrm{Zn}(\mathrm{OAc})_{2}$ in combination with $\mathrm{Et}_{3} \mathrm{~N}$ is the most promising system. 
Table S6. Evaluation of $\mathrm{Zn}(\mathrm{OAc})_{2}$, $\mathrm{Zn}$ formate. $2 \mathrm{H}_{2} \mathrm{O}, \mathrm{Ni}(\mathrm{OAc})_{2} \cdot \mathrm{H}_{2} \mathrm{O}$ and $\mathrm{Cu}(\mathrm{OAc})_{2} \cdot \mathrm{H}_{2} \mathrm{O}$ with Different Tertiary Amine Bases.
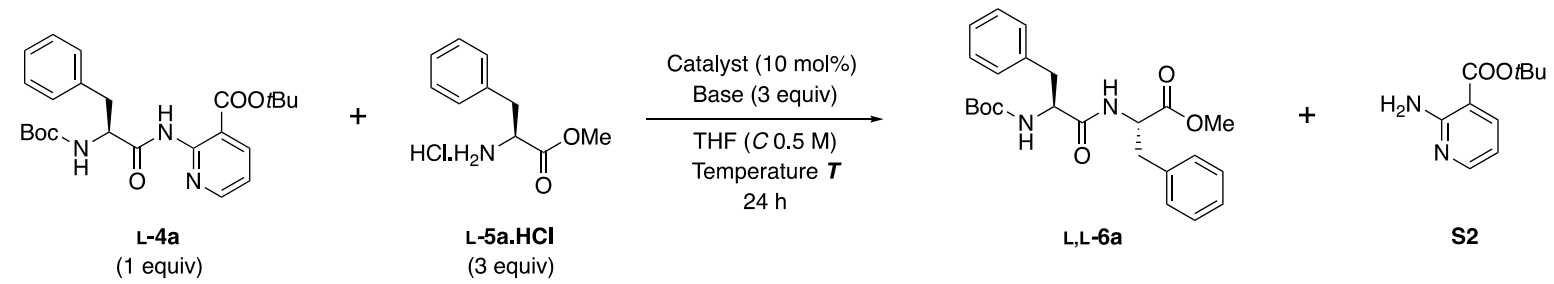

\begin{tabular}{|c|c|c|c|c|c|}
\hline $\begin{array}{c}\text { Entry (ELN- } \\
\text { code) }\end{array}$ & $\begin{array}{c}\text { Catalyst (10 } \\
\text { mol\%) }\end{array}$ & $\begin{array}{l}\text { Base (3 } \\
\text { equiv) }\end{array}$ & $\begin{array}{c}\text { Temperature } \boldsymbol{T} \\
\left({ }^{\circ} \mathrm{C}\right)\end{array}$ & $\begin{array}{l}\text { Yield L-4a } \\
\qquad(\%)^{[\mathrm{b}]}\end{array}$ & $\begin{array}{l}\text { Yield } \mathbf{L}, \mathbf{L}-\mathbf{6 a} \\
\qquad \%)^{[\mathrm{b}]}\end{array}$ \\
\hline $1\left(\mathrm{KH} \_184\right)$ & $\mathrm{Zn}(\mathrm{OAc})_{2}$ & DIPEA & 50 & 17 & 83 \\
\hline 2 (KH_267) & $\mathrm{Zn}(\mathrm{OAc})_{2}$ & $\mathrm{Et}_{3} \mathrm{~N}$ & 40 & 8 & 92 \\
\hline 3 (KH_257) & $\mathrm{Zn}(\mathrm{OAc})_{2}$ & $\mathrm{Et}_{3} \mathrm{~N}$ & 50 & 0 & 100 \\
\hline $4\left(\mathrm{KH} \_270\right)$ & $\mathrm{Zn}(\mathrm{OAc})_{2}$ & $\mathrm{NMM}^{[\mathrm{c}]}$ & 40 & 12 & 88 \\
\hline 5 (KH_258) & $\mathrm{Zn}(\mathrm{OAc})_{2}$ & $\mathrm{NMM}^{[\mathrm{c}]}$ & 50 & 0 & 100 \\
\hline $6\left(\mathrm{KH} \_237\right)$ & $\mathrm{Zn}$ formate $2 \mathrm{H}_{2} \mathrm{O}$ & DIPEA & 50 & 29 & 71 \\
\hline 7 (KH_268) & $\mathrm{Zn}$ formate. $2 \mathrm{H}_{2} \mathrm{O}$ & $\mathrm{Et}_{3} \mathrm{~N}$ & 40 & 18 & 82 \\
\hline 8 (KH_259) & $\mathrm{Zn}$ formate. $2 \mathrm{H}_{2} \mathrm{O}$ & $\mathrm{Et}_{3} \mathrm{~N}$ & 50 & 0 & 100 \\
\hline 9 (KH_271) & $\mathrm{Zn}$ formate $2 \mathrm{H}_{2} \mathrm{O}$ & $\mathrm{NMM}^{[\mathrm{c}]}$ & 40 & 30 & 70 \\
\hline 10 (KH_260) & $\mathrm{Zn}$ formate. $2 \mathrm{H}_{2} \mathrm{O}$ & $\mathrm{NMM}^{[\mathrm{c}]}$ & 50 & 0 & 100 \\
\hline 11 (KH_243) & $\mathrm{Ni}(\mathrm{OAc})_{2} \cdot \mathrm{H}_{2} \mathrm{O}$ & DIPEA & 50 & 31 & 69 \\
\hline 12 (KH_263) & $\mathrm{Ni}(\mathrm{OAc})_{2} \cdot \mathrm{H}_{2} \mathrm{O}$ & $\mathrm{Et}_{3} \mathrm{~N}$ & 50 & 12 & 88 \\
\hline 13 (KH_264) & $\mathrm{Ni}(\mathrm{OAc})_{2} \cdot \mathrm{H}_{2} \mathrm{O}$ & $\mathrm{NMM}^{[\mathrm{c}]}$ & 50 & 18 & 82 \\
\hline 14 (KH_244) & $\mathrm{Cu}(\mathrm{OAc})_{2} \cdot \mathrm{H}_{2} \mathrm{O}$ & DIPEA & 50 & 31 & 69 \\
\hline 15 (KH_265) & $\mathrm{Cu}(\mathrm{OAc})_{2} \cdot \mathrm{H}_{2} \mathrm{O}$ & $\mathrm{Et}_{3} \mathrm{~N}$ & 50 & 9 & 91 \\
\hline 16 (KH_266) & $\mathrm{Cu}(\mathrm{OAc})_{2} \cdot \mathrm{H}_{2} \mathrm{O}$ & $\mathrm{NMM}^{[\mathrm{c}]}$ & 50 & 12 & 88 \\
\hline
\end{tabular}

[a] Hydrates determined by TGA analysis. [b] ${ }^{1} \mathrm{H}$ NMR yield using 1,3,5-trimethoxybenzene as internal standard. [c] NMM = N-methylmorpholine. 
Using the optimal catalyst/tertiary amine base system, the excess of $\mathbf{L - 5 a . H C l}$ and $\mathrm{Et}_{3} \mathrm{~N}$ was simultaneously stepwise reduced to 1.1 equivalents (Table S7, Entry 1-4). This had a significant effect on the conversion; 3 to 1.1 equiv. reduced conversion with $25 \%$. The ratio $\mathbf{L}-\mathbf{5 a} \mathbf{- H C l}$ and $\mathrm{Et}_{3} \mathrm{~N}$ was finally investigated (Table S7, Entries 4-6). With a loading of 1.1 equiv. L-5a.HCl, the use of more base inhibited conversion to a very small extend; 1.1 to 6 equiv. reduced conversion with only $10 \%$.

Table S7. Evaluation of the Excess of $\mathbf{L - 5 a . H C l}$ and $\mathrm{Et}_{3} \mathrm{~N}$
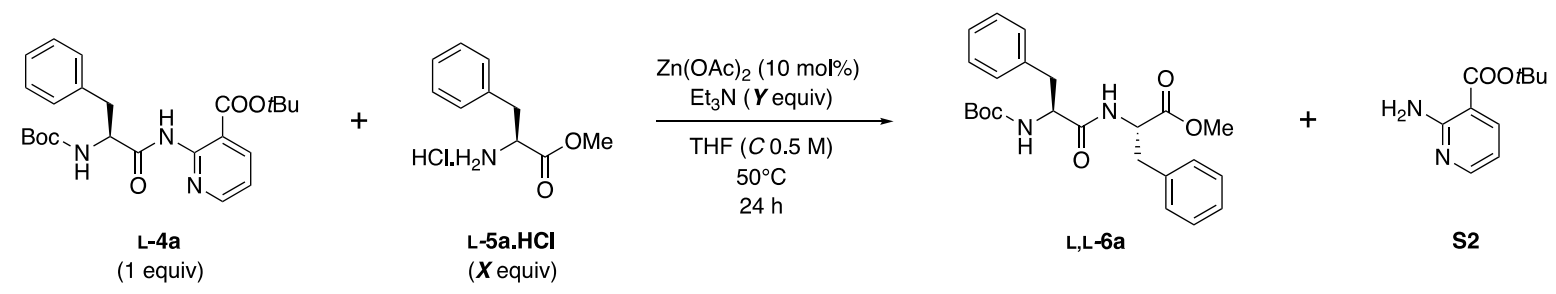

\begin{tabular}{|c|c|c|c|c|}
\hline $\begin{array}{c}\text { Entry } \\
(\text { ELN-code })\end{array}$ & $\begin{array}{c}\text { H-L-Phe-OMe.HCl L- } \\
\text { 5a.HCl ( } \boldsymbol{X} \text { equiv) }\end{array}$ & $\mathrm{Et}_{3} \mathrm{~N}$ ( $\boldsymbol{Y}$ equiv) & Yield L-4a $(\%)^{[\mathrm{a}]}$ & Yield $\mathbf{L}, \mathbf{L}-\mathbf{6 a}(\%)^{[\mathrm{a}]}$ \\
\hline 1 (KH_257) & 3 & 3 & 0 & 100 \\
\hline 2 (KH_274) & 2 & 2 & 7 & 93 \\
\hline 3 (KH_273) & 1.5 & 1.5 & 13 & 87 \\
\hline $4\left(\mathrm{KH} \_355\right)$ & 1.1 & 1.1 & 25 & 75 \\
\hline 5 (KH_349) & 1.1 & 3 & 30 & 70 \\
\hline 6 (KH_352) & 1.1 & 6 & 35 & 65 \\
\hline
\end{tabular}

[a] ${ }^{1} \mathrm{H}$ NMR yield using 1,3,5-trimethoxybenzene as internal standard.

To obtain full conversion using only 1.1 equivalents of $\mathbf{L - 5 a . H C l}$, and 1.1 equivalents of $\mathrm{Et}_{3} \mathrm{~N}$ the catalyst loading was increased alongside with the temperature (Table S8). Here, the use of $20 \mathrm{~mol} \%$ of catalyst at $70^{\circ} \mathrm{C}$ (Table S8, Entry 7), resulted in full conversion. Considering $\mathrm{Zn}(\mathrm{OAc})_{2}$ is cheap, airstable, non-toxic (E650) and aiming a general applicability of the developed transamidation protocol, a higher catalyst loading rather than an excess of L-5a.HCl was preferred as standard optimized conditions. 
Table S8. Evaluation of $\mathrm{Zn}(\mathrm{OAc})_{2}$ Loading and Temperature with 1.1 Equivalents of L-5a.HCl and 1.1 Equivalents of $\mathrm{Et}_{3} \mathrm{~N}$.
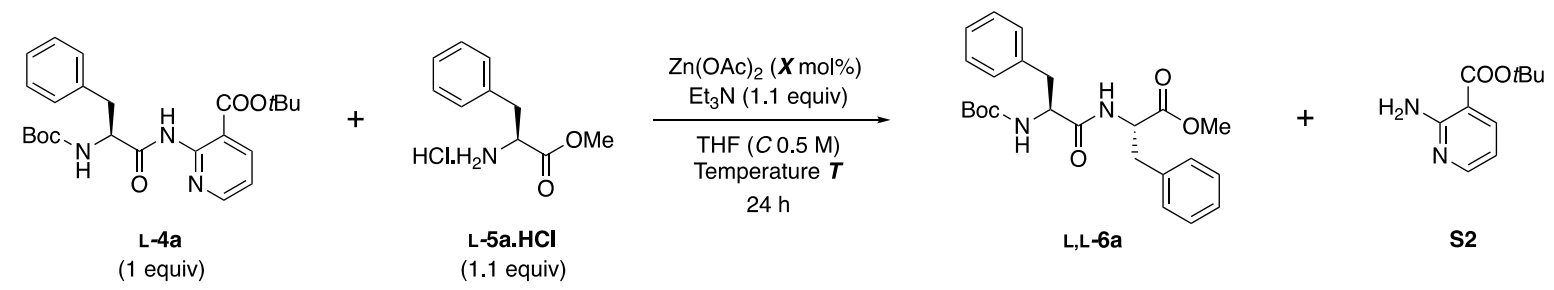

\begin{tabular}{|c|c|c|c|c|}
\hline $\begin{array}{c}\text { Entry } \\
(\text { ELN-code })\end{array}$ & $\begin{array}{c}\mathrm{Zn}(\mathrm{OAc})_{2}(X \\
\mathrm{mol} \%)\end{array}$ & Temperature $\boldsymbol{T}\left({ }^{\circ} \mathrm{C}\right)$ & Yield L-4a $(\%)^{[\mathrm{a}]}$ & Yield L,L-6a $(\%)^{[\mathrm{a}}$ \\
\hline 1 (KH_355) & 10 & 50 & 25 & 75 \\
\hline 2 (KH_356) & 10 & 60 & 21 & 79 \\
\hline 3 (KH_361) & 10 & 70 & 14 & 86 \\
\hline $4\left(\mathrm{KH} \_420\right)$ & 15 & 50 & 19 & 81 \\
\hline 5 (KH_459) & 15 & 70 & 12 & 88 \\
\hline $6\left(\mathrm{KH} \_421\right)$ & 20 & 50 & 12 & 88 \\
\hline $7\left(\mathrm{KH} \_402\right)$ & 20 & 70 & 0 & 100 \\
\hline
\end{tabular}

[a] ${ }^{1} \mathrm{H}$ NMR yield using 1,3,5-trimethoxybenzene as internal standard.

Next, the effect of the transamidation reaction conditions on the chiral purity of both the $t$ Bu-nicotinate activated amide 4a moiety and the nucleophile 5a was evaluated. Consequently, we synthesized the four stereoisomers of Boc-Phe-Phe-OMe $\mathbf{6 a}$ by coupling the enantiomers of Boc-Phe-OH S1 with those of $\mathrm{H}-\mathrm{Phe}-\mathrm{OMe} . \mathrm{HCl}$ 5a.HCl using EDC.HCl and $\mathrm{HOBt} . \mathrm{H}_{2} \mathrm{O}$. These were then analyzed using chiral HPLC (Table S9) and used as references for the evaluation of epimerization under the optimal reaction conditions. 
Table S9. Synthesis and Chiral Analysis of the Four Stereoisomers of Boc-Phe-Phe-OMe 6a
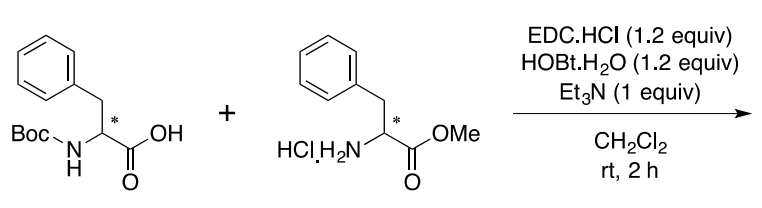

S1

(1 equiv)

5a.HCl

$\begin{array}{lll}\text { Entry (ELN- } & \text { (1 equiv) } & \text { (1 equiv) } \\ \text { code }) & & \text { Isolated yield }(\%)\end{array}$

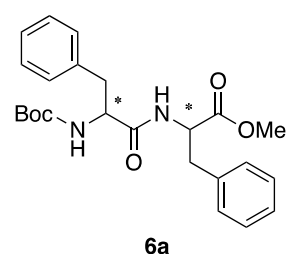

$6 a$

\begin{tabular}{lccc}
\hline $1\left(\mathrm{KH} \_1066\right)$ & L,L & 75 & 3.85 \\
$2\left(\mathrm{KH} \_873\right)$ & $\mathrm{D}, \mathrm{L}$ & 76 & 5.71 \\
$3\left(\mathrm{KH} \_1067\right)$ & L,D & 76 & 3.76 \\
$4\left(\mathrm{KH} \_1068\right)$ & D,D & 84 & 4.26 \\
\hline
\end{tabular}

[a] \%ee measured using HPLC with $n$-hexane: $i$-propanol on a Diacel Chiralpak IA column.

With the ability in hand to distinguish between the different steroisomers, we repeated the transamidation of Boc-L-Phe-NH- $t$ Bu-nic L-4a with H-L-Phe-OMe.HCl L-5a.HCl under the optimized reaction conditions. Dipeptide 6a was isolated with a yield of $67 \%$, however, the chiral analysis showed that a mixture of $\mathbf{L}, \mathbf{L}-\mathbf{6} \mathbf{a}$ and $\mathbf{D , L}-\mathbf{6} \mathbf{a}$ was present in a 88:12 ratio (Scheme $\mathrm{S} 1$ ).
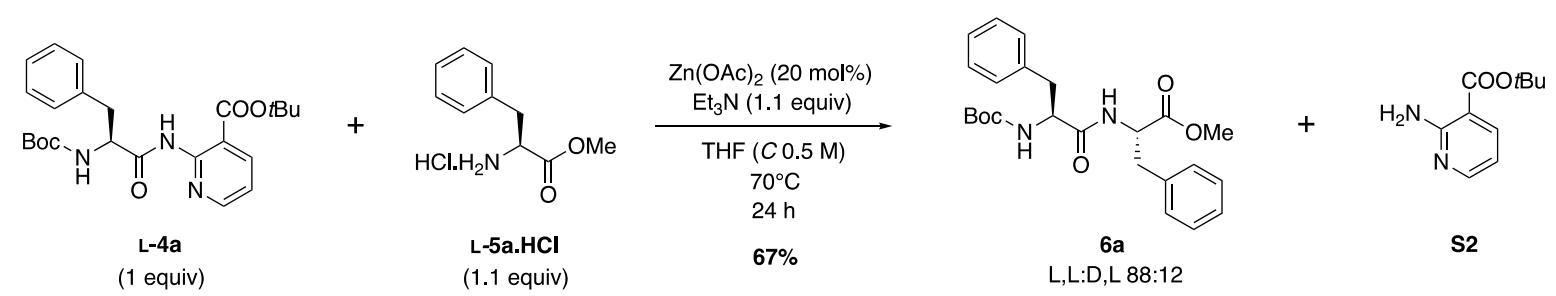

Scheme S1. Transamidation of Boc-L-Phe-NH- $t$ Bu-nic L-4a and H-L-Phe-OMe.HCl L-5a.HCl under optimized conditions.

As the chiral purity of the dipeptide is jeopardized under the optimal reaction conditions, we decided to further investigate the impact of base on the reaction. Besides, full retention of chiral information, full conversion and a high isolated yield were aimed for (Table S10). 
Table S10. Evaluation of the Base on the Chiral Purity of $\mathbf{6 a}$.
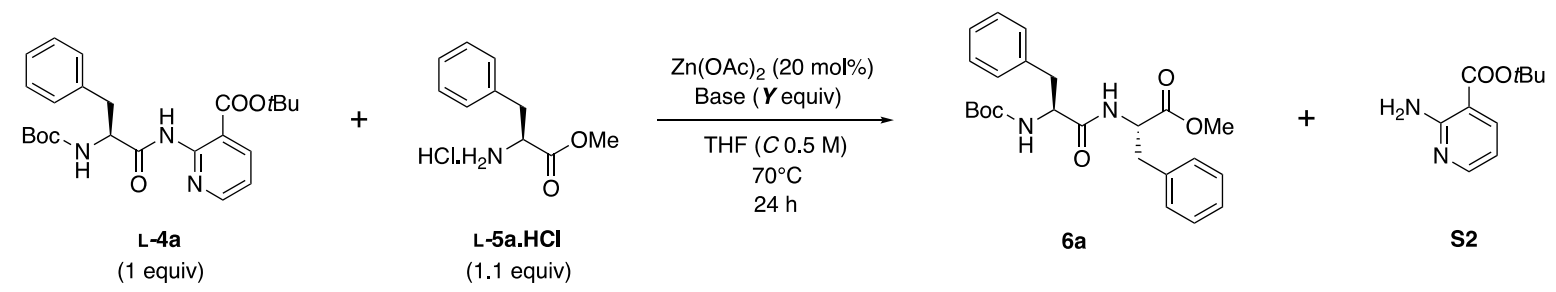

\begin{tabular}{|c|c|c|c|c|c|c|c|}
\hline \multirow{2}{*}{$\begin{array}{c}\text { Entry (ELN- } \\
\text { code) }\end{array}$} & \multirow{2}{*}{ Base } & \multirow{2}{*}{$\begin{array}{c}\text { (Y } \\
\text { equiv) }\end{array}$} & \multirow{2}{*}{$\begin{array}{l}\text { Yield } L \text { - } \\
\mathbf{4 a}(\%)^{[\mathrm{a}]}\end{array}$} & \multirow{2}{*}{$\begin{array}{l}\text { Yield L,L- } \\
\mathbf{6 a}(\%)^{[\mathrm{a}]}\end{array}$} & \multirow{2}{*}{$\begin{array}{c}\text { Isolated yield } \\
\qquad \mathbf{6 a}(\%)\end{array}$} & \multicolumn{2}{|c|}{$\begin{array}{c}\text { Chiral Purity } \\
\text { analysis of } \mathbf{6 a}^{[\mathrm{b}]}\end{array}$} \\
\hline & & & & & & $\% \mathrm{~L}, \mathrm{~L}$ & $\% \mathrm{D}, \mathrm{L}$ \\
\hline 1 (KH_1070) & $\mathrm{Et}_{3} \mathrm{~N}$ & 1.1 & 0 & 100 & 67 & 88 & 12 \\
\hline 2 (KH_1080) & Pyridine & 1.1 & 44 & 56 & 26 & 100 & 0 \\
\hline 3 (KH_1081) & NMM & 1.1 & 9 & 91 & 69 & 100 & 0 \\
\hline 4 (KH_1082) & $\mathrm{K}_{2} \mathrm{CO}_{3}$ & 0.55 & 0 & 100 & 54 & 94 & 6 \\
\hline 5 (KH_1092) & NaOAc & 1.1 & $\mathbf{0}$ & 100 & 79 & 100 & $\mathbf{0}$ \\
\hline $6\left(\mathrm{KH} \_1093\right)$ & $\begin{array}{c}N, N- \\
\text { dimethylanili } \\
\text { ne }\end{array}$ & 1.1 & 47 & 53 & 28 & 100 & 0 \\
\hline 7 (KH_1096) & $\mathrm{Bu}_{3} \mathrm{~N}$ & 1.1 & 0 & 100 & 38 & 91 & 9 \\
\hline
\end{tabular}

[a] ${ }^{1} \mathrm{H}$ NMR yield using 1,3,5-trimethoxybenzene as internal standard. [b] \%ee measured using HPLC with $n$-hexane: $i$-propanol on a Diacel Chiralpak IA column, corrected for chiral purity of the starting material.

Similar to $\mathrm{Et}_{3} \mathrm{~N}$ as base (Table S10, Entry 1), full conversion was obtained for $\mathrm{K}_{2} \mathrm{CO}_{3}$ (Table S10, Entry 4), NaOAc (Table S10, Entry 5) and $\mathrm{Bu}_{3} \mathrm{~N}$ (Table S10, Entry 7). However, in the chiral purity analysis, from these only NaOAc showed no epimerization during the transamidation. Therefore NaOAc was selected as the optimal base (Table S10, Entry 5).

With the optimized conditions in hand, the need for the catalyst was evaluated (Table S11). Table S11, Entry 1 shows the reaction does occur without catalyst revealing 82\% conversion. Full conversion without catalyst can be achieved within $48 \mathrm{~h}$. Chiral purity analysis, however, shows that the chiral purity is jeopardized under these conditions. The addition of $\mathrm{Zn}(\mathrm{OAc})_{2}$ has a significant effect as it allows to achieve full conversion within $24 \mathrm{~h}$ and retains the enantiomeric purity of the product. 
Table S11. Effect of $\mathrm{Zn}(\mathrm{OAc})_{2}$.

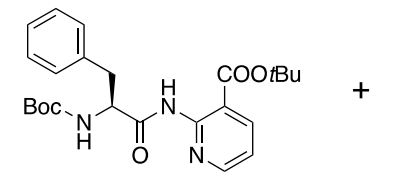

L-4a (1 equiv)

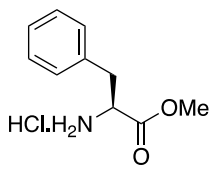

L-5a.HCl

(1.1 equiv)
$\mathrm{Zn}(\mathrm{OAc})_{2}(\boldsymbol{X} \mathrm{mol} \%)$

$\mathrm{NaOAc}$ (1.1 equiv)

$\operatorname{THF}(C 0.5 \mathrm{M})$ $70^{\circ} \mathrm{C}$
time $t$

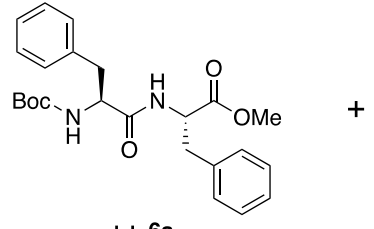

L,L-6a<smiles>CCOC(=O)c1cccnc1N</smiles>

S2

\begin{tabular}{|c|c|c|c|c|c|c|}
\hline \multirow{2}{*}{$\begin{array}{l}\text { Entry (ELN- } \\
\text { code) }\end{array}$} & \multirow{2}{*}{$\begin{array}{l}\mathrm{Zn}(\mathrm{OAc})_{2} \\
(X \mathrm{~mol} \%)\end{array}$} & \multirow{2}{*}{$\begin{array}{l}\text { Reaction } \\
\text { Time } t(h)\end{array}$} & \multirow{2}{*}{$\begin{array}{l}\text { Yield L-4a } \\
\qquad(\%)^{[\mathrm{a}]}\end{array}$} & \multirow{2}{*}{$\begin{array}{l}\text { Yield } \mathbf{L}, \mathbf{L}-\mathbf{6 a} \\
(\%)^{[\mathrm{a}]}\end{array}$} & \multicolumn{2}{|c|}{$\begin{array}{c}\text { Chiral Purity analysis } \\
\text { of } \mathbf{6 a}^{[\mathrm{b}]}\end{array}$} \\
\hline & & & & & $\% \mathrm{~L}, \mathrm{~L}$ & $\% \mathrm{D}, \mathrm{L}$ \\
\hline 1 (KH_1195) & 0 & 24 & 18 & 82 & 95 & 5 \\
\hline 2 (KH_1195) & 0 & 48 & 0 & 100 & 88 & 12 \\
\hline 3 (KH_1092) & 20 & 24 & 0 & 100 & 100 & 0 \\
\hline
\end{tabular}

[a] ${ }^{1}$ H NMR yield using 1,3,5-trimethoxybenzene as internal standard. [b] \%ee measured using HPLC with $n$-hexane:i-propanol on a Diacel Chiralpak IA column, corrected for chiral purity of the starting material. 


\subsection{Optimization of the Synthesis of L-4o: tert-Butyl 2-Chloronicotinate Introduction On The Side Chain of Boc-L-GIn-OH}

The introduction of tert-butyl 2-chloronicotinate 2 in the side chain of Boc-L-Gln-OH was evaluated. As starting point, two different reaction conditions previously reported in our lab were tested (Table S12, entries 1-2). ${ }^{[1]}$ Since the combination of $\mathrm{Pd}(\mathrm{OAc})_{2}$ and Xantphos (Table S12, Entry 2) afforded the formation of the desired product, a further optimization of that method was required to obtain higher yields. The best results were obtained in entry 6 , where $5 \mathrm{~mol} \%$ of $\mathrm{Pd}(\mathrm{OAc})_{2}, 10 \mathrm{~mol} \%$ of Xantphos and 1.2 equiv. of $\mathrm{Cs}_{2} \mathrm{CO}_{3}$ in 1,4-dioxane at $90{ }^{\circ} \mathrm{C}$ were used. The addition of $1 \%$ of formic acid in water during the purification revealed to be a crucial factor to obtain good yields. Details about the chiral purity of the obtained compound will be discussed in a separate section.

Table S12. Optimization of DG introduction on the Gln side chain.

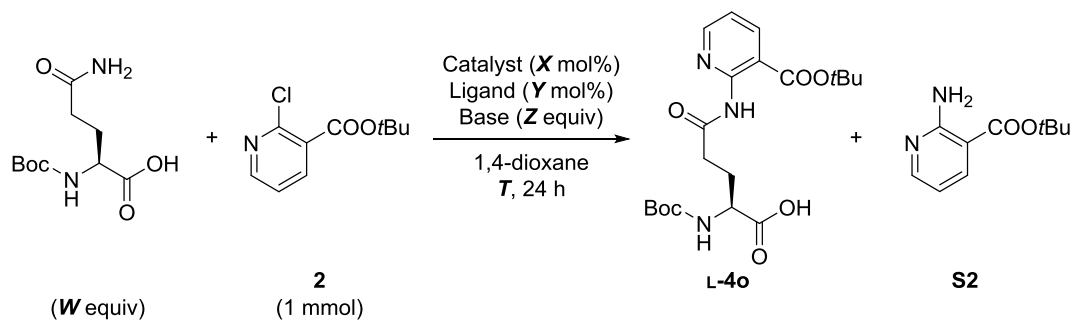

\begin{tabular}{|c|c|c|c|c|c|c|c|c|c|}
\hline $\begin{array}{l}\text { Entry } \\
\text { (ELN } \\
\text { code) }\end{array}$ & $\begin{array}{c}\text { Catalyst ( } \boldsymbol{X} \\
\text { mol\%) }\end{array}$ & $\begin{array}{c}\text { Ligand ( } \boldsymbol{Y} \\
\text { mol\%) }\end{array}$ & $\begin{array}{l}\text { Base }(\boldsymbol{Z} \\
\text { equiv) }\end{array}$ & $\begin{array}{c}\boldsymbol{T} \\
\left({ }^{\circ} \mathrm{C}\right)\end{array}$ & $\begin{array}{c}(\boldsymbol{W} \\
\text { equiv) }\end{array}$ & $\begin{array}{c}2 \\
(\%)^{\mathrm{a}, \mathrm{b}}\end{array}$ & $\begin{array}{l}\mathbf{L}-40 \\
(\%)^{\mathrm{a}}\end{array}$ & $\begin{array}{c}\mathbf{S 2} \\
(\%)^{\mathrm{a}}\end{array}$ & $\begin{array}{l}\mathrm{MB} \\
(\%)^{\mathrm{a}, \mathrm{c}}\end{array}$ \\
\hline $\begin{array}{c}1 \\
(\mathrm{ER} 1310)\end{array}$ & $\begin{array}{l}\text { Pd-G3-dcpf } \\
(10 \mathrm{~mol} \%)\end{array}$ & - & $\begin{array}{c}\mathrm{Cs}_{2} \mathrm{CO}_{3} \\
\text { (7.5 equiv) }\end{array}$ & 40 & 1.1 & 93 & 0 & 0 & 93 \\
\hline $\begin{array}{c}2 \\
(\mathrm{DM} 614)\end{array}$ & $\begin{array}{l}\mathrm{Pd}(\mathrm{OAc})_{2} \\
(5 \mathrm{~mol} \%)\end{array}$ & $\begin{array}{l}\text { Xantphos } \\
(10 \mathrm{~mol} \%)\end{array}$ & $\begin{array}{c}\mathrm{K}_{2} \mathrm{CO}_{3} \\
\text { (7.5 equiv) }\end{array}$ & 90 & 1.1 & 38 & 10 & 46 & 94 \\
\hline $\begin{array}{c}3 \\
(\mathrm{DM} 607)\end{array}$ & $\begin{array}{l}\mathrm{Pd}(\mathrm{OAc})_{2} \\
(5 \mathrm{~mol} \%)\end{array}$ & $\begin{array}{l}\text { Xantphos } \\
(10 \mathrm{~mol} \%)\end{array}$ & $\begin{array}{c}\mathrm{K}_{2} \mathrm{CO}_{3} \\
\text { (1.2 equiv) }\end{array}$ & 90 & 1.5 & 32 & 0 & 58 & 90 \\
\hline $\begin{array}{c}4 \\
(\mathrm{DM} 600)\end{array}$ & $\begin{array}{l}\mathrm{Pd}(\mathrm{OAc})_{2} \\
(5 \mathrm{~mol} \%)\end{array}$ & $\begin{array}{l}\text { Xantphos } \\
(10 \mathrm{~mol} \%)\end{array}$ & $\begin{array}{c}\mathrm{Rb}_{2} \mathrm{CO}_{3} \\
\text { (1.2 equiv) }\end{array}$ & 90 & 1.5 & 26 & 0 & 24 & 50 \\
\hline $\begin{array}{c}5 \\
(\mathrm{DM} 596)\end{array}$ & $\begin{array}{l}\mathrm{Pd}(\mathrm{OAc})_{2} \\
(5 \mathrm{~mol} \%)\end{array}$ & $\begin{array}{l}\text { Xantphos } \\
(10 \mathrm{~mol} \%)\end{array}$ & $\begin{array}{c}\mathrm{Cs}_{2} \mathrm{CO}_{3} \\
\text { (1.2 equiv) }\end{array}$ & 90 & 1.5 & 0 & 50 & 5 & 55 \\
\hline 6 & $\mathrm{Pd}(\mathrm{OAc})_{2}$ & Xantphos & $\mathrm{Cs}_{2} \mathrm{CO}_{3}$ & 90 & 1.5 & 0 & $60^{\mathrm{d}}$ & 9 & 69 \\
\hline
\end{tabular}


(DM613) $\quad(5 \mathrm{~mol} \%) \quad(10 \mathrm{~mol} \%) \quad(1.2$ equiv $)$

$\begin{array}{cccccccccc}7 & \mathrm{Pd}(\mathrm{OAc})_{2} & \text { Xantphos } & \mathrm{Cs}_{2} \mathrm{CO}_{3} & 90 & 1.5 & 0 & 40 & 3 & 43 \\ (\mathrm{DM} 598) & (5 \mathrm{~mol} \%) & (10 \mathrm{~mol} \%) & (1.0 \text { equiv }) & & & & & & \end{array}$

$\begin{array}{cccccccccc}8 & \mathrm{Pd}(\mathrm{OAc})_{2} & \text { Xantphos } & \mathrm{Cs}_{2} \mathrm{CO}_{3} & 90 & 1.5 & 30 & 0 & 40 & 70 \\ (\mathrm{DM} 601) & (5 \mathrm{~mol} \%) & (10 \mathrm{~mol} \%) & (3 \text { equiv }) & & & & & \end{array}$

$\begin{array}{cccccccccc}9 & \mathrm{Pd}(\mathrm{OAc})_{2} & \text { Xantphos } & \mathrm{Cs}_{2} \mathrm{CO}_{3} & 90 & 1.1 & 0 & 30 & 7 & 37 \\ (\mathrm{DM} 511) & (5 \mathrm{~mol} \%) & (10 \mathrm{~mol} \%) & (1.2 \text { equiv }) & & & & & & \end{array}$

$\begin{array}{crcccccccc}10 & \mathrm{Pd}(\mathrm{OAc})_{2} & \text { Xantphos } & \mathrm{Cs}_{2} \mathrm{CO}_{3} & 90 & 2.0 & 0 & 50 & 8 & 58 \\ (\mathrm{DM} 599) & (5 \mathrm{~mol} \%) & (10 \mathrm{~mol} \%) & (1.2 \text { equiv }) & & & & & & \end{array}$

[a] Isolated yields after column chromatography. General work up: the mixture was acidified to $\mathrm{pH}=5$ with $1 \mathrm{M} \mathrm{HCl}$ [b] Recovered 2 [c] Mass balance. [d] No acidification in the work up. $\mathrm{H}_{2} \mathrm{O}+1 \%$ formic acid was used in the purification. 


\section{NMR Characterization of Compound L-4o}

To demonstrate that the $t \mathrm{Bu}$-nic directing group was selectively installed on the side chain primary amide of Boc-L-Gln-OH, full 2D NMR analysis of compound L-4o was performed. The ${ }^{1} \mathrm{H}-\mathrm{NMR}$ of $\mathbf{L}-$ 40 is given in Figure $\mathbf{S} 2$.

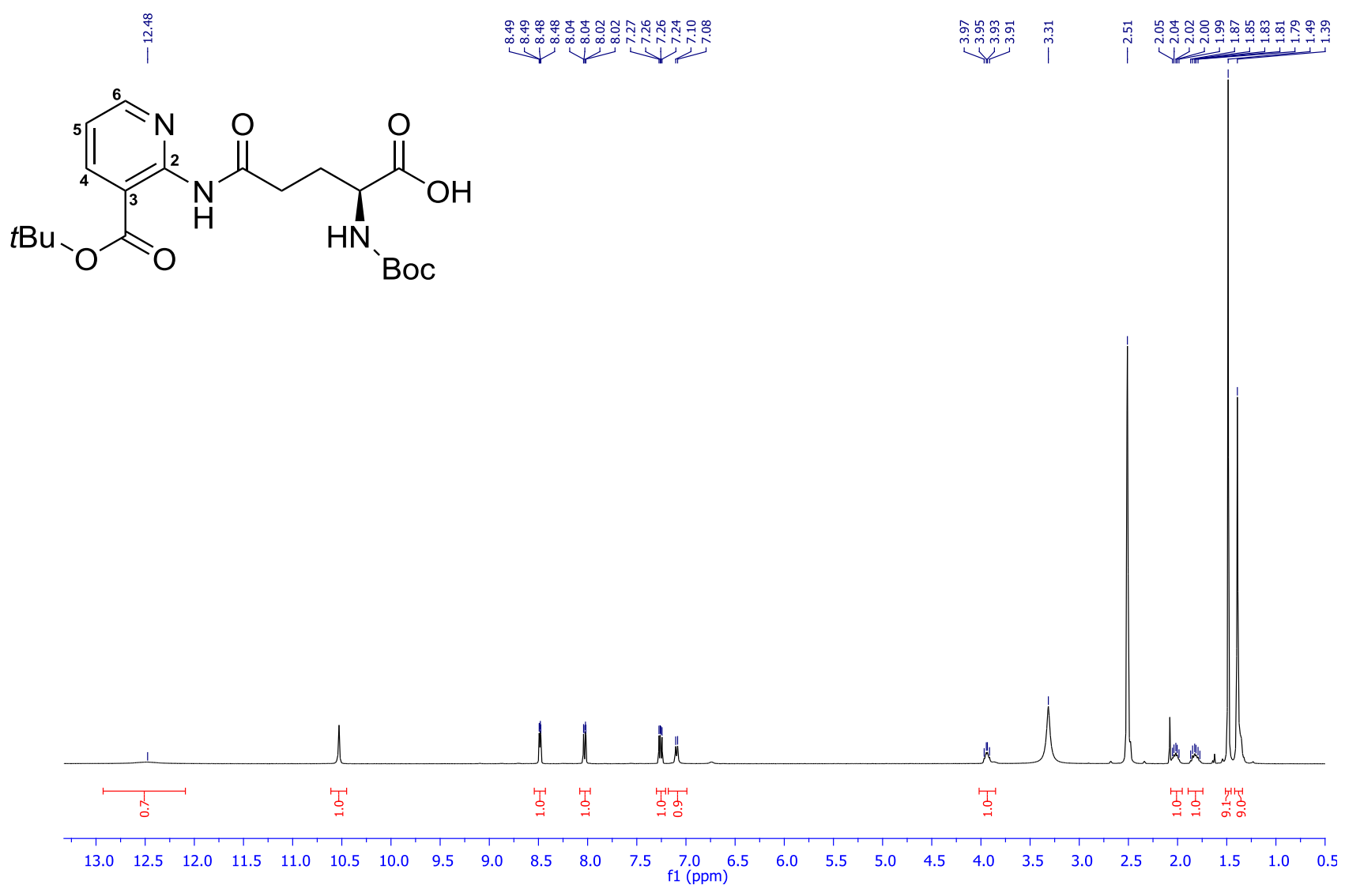

Figure S2. Full ${ }^{1} \mathrm{H}$ NMR spectrum (in DMSO- $d_{6}$ ) of $\mathbf{L}-\mathbf{4 o}$.

\subsection{COSY NMR Correlations in L-4o}

The COSY spectrum showed the presence of two different spin systems, the first in the aromatic area of the proton NMR (spin system A, Figure S3) and the second in the aliphatic area (spin system B, Figure S4). The analysis of the spin system A (Figure S2) allowed to place the proton at $7.26 \mathrm{ppm}(\mathrm{dd}, J=7.7$, $4.8 \mathrm{~Hz})$ in position 5 on the pyridine ring, since it both couples with the protons at $8.03 \mathrm{ppm}(\mathrm{dd}, J=$ 7.7, $1.7 \mathrm{~Hz})$ and $8.48 \mathrm{ppm}(\mathrm{dd}, J=4.7,1.7 \mathrm{~Hz})$. The latter proton could be placed in position 6 according to its higher chemical shift and, consequently, the proton at $8.03 \mathrm{ppm}$ could be attributed to position 4. 


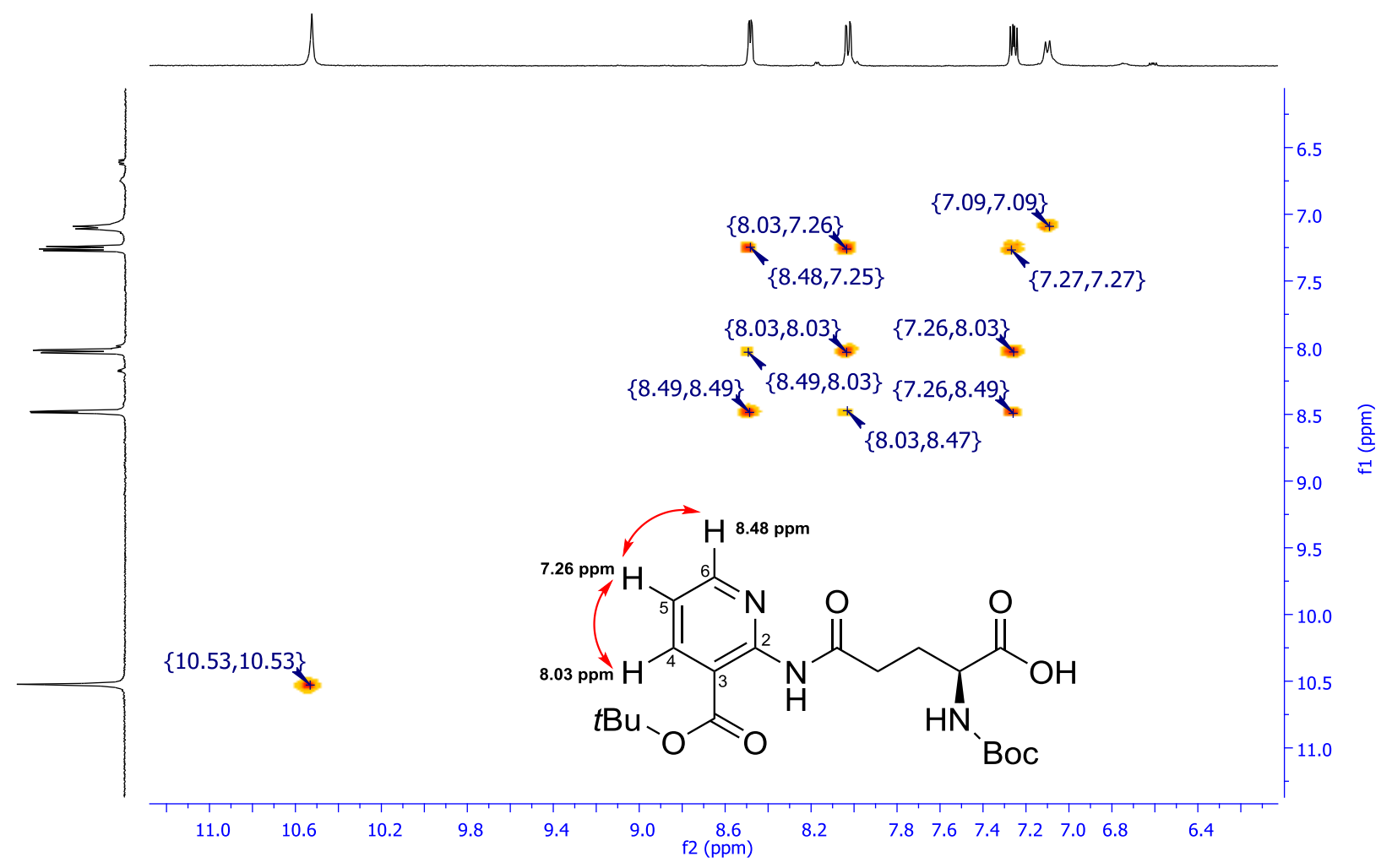

Figure S3. COSY correlations (in DMSO- $d_{6}$ ) in the aromatic spin system A of $\mathbf{L}-\mathbf{4 o}$.

Regarding the spin system B, the analysis started from the proton at $7.09 \mathrm{ppm}(\mathrm{d}, J=7.9 \mathrm{~Hz})$, which corresponds to the amine group from the amino acid (see also section 2.2). This proton couples with the proton at $3.94 \mathrm{ppm}(\mathrm{dd}, J=13.1,8.7 \mathrm{~Hz})$ (Figure S4, pink arrow) that in turn couples with the protons at $2.03(\mathrm{~m})$ and $1.83 \mathrm{ppm}(\mathrm{td}, J=16.2,7.8 \mathrm{~Hz}$ ) (Figure S4, red arrows). Finally, a COSY correlation between the protons at $2.51 \mathrm{ppm}$ (overlapping with DMSO signals) and the protons at 2.03 and 1.83 ppm was found (Figure S4, blue arrows). 


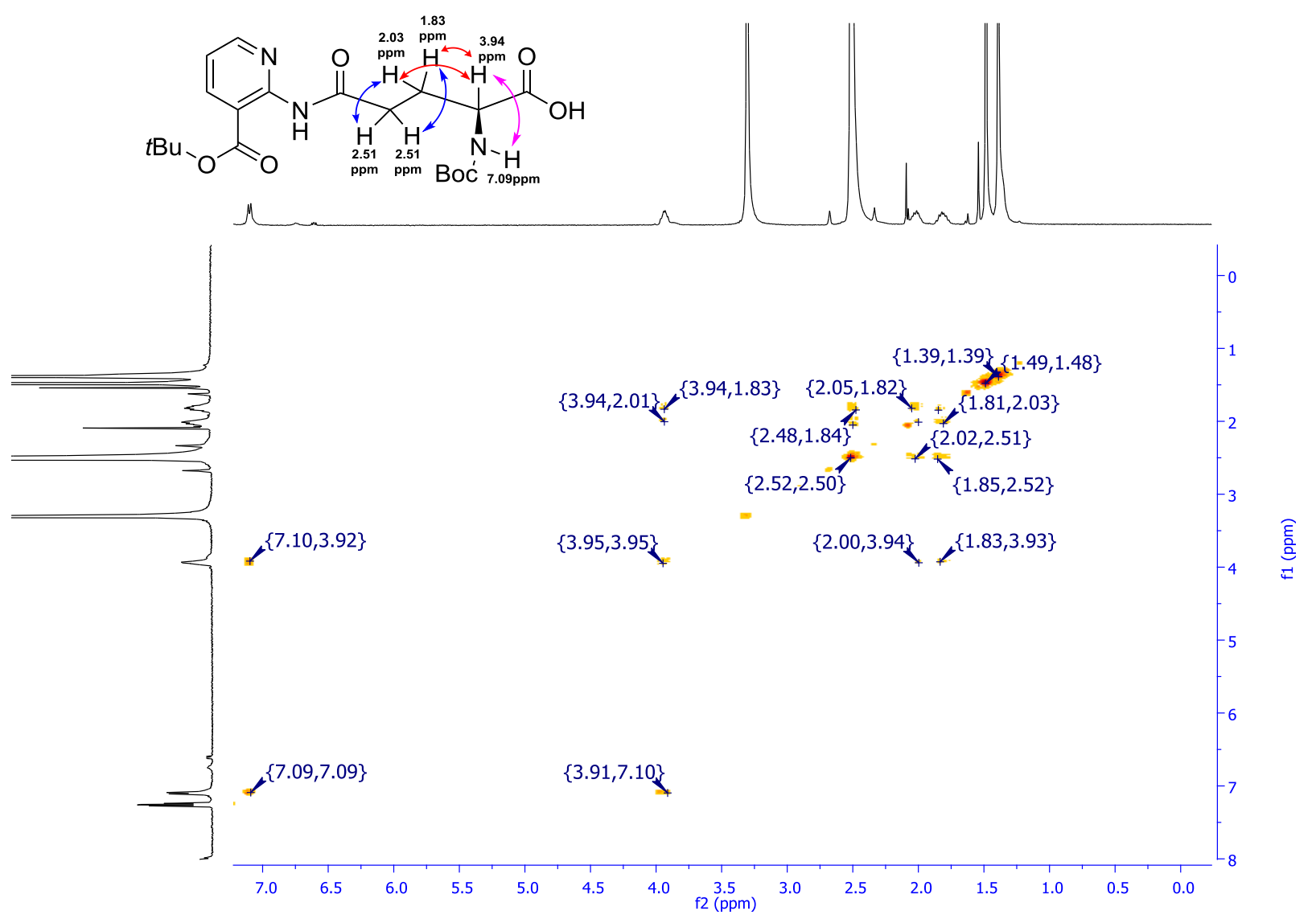

Figure S4. COSY correlations (in DMSO- $d_{6}$ ) in aliphatic spin system B of $\mathbf{L}-\mathbf{4 o}$.

\section{$2.2{ }^{15} \mathrm{~N}$ and ${ }^{13} \mathrm{C}$ HSQC NMR Correlations in L-4o}

${ }^{15} \mathrm{~N}$ HSQC revealed that the proton at $7.09 \mathrm{ppm}$ is directly bound to a nitrogen atom (Figure S5). In combination with the COSY correlation $(\mathrm{d}, J=7.9 \mathrm{~Hz})$ to the aliphatic carbon chain of glutamine (Figure S4), allowed us to identify this proton as the Boc-NH. The proton at $10.53 \mathrm{ppm}$ (s) also belongs to a nitrogen atom, however no proton coupling to the aliphatic carbon chain is observed, identifying it as the $t \mathrm{Bu}-\mathrm{nic}-\mathrm{NH}$. 


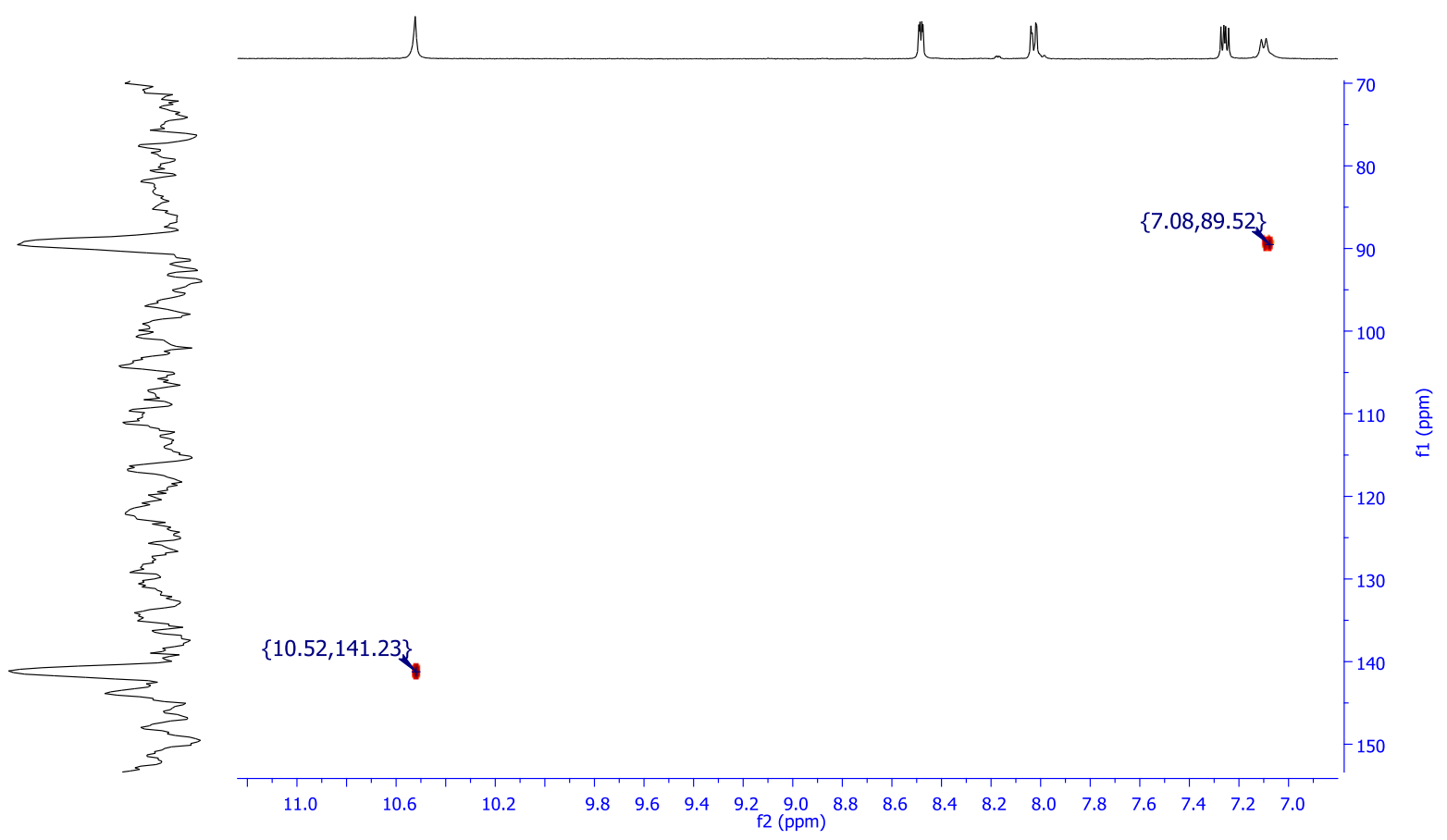

Figure S5. ${ }^{15} \mathrm{~N}$ HSQC correlations in DMSO- $d_{6}$ of $\mathbf{L}-\mathbf{4 o}$.

The analysis of the ${ }^{13} \mathrm{C}$ HSQC allowed us to assign the values of the carbons directly bound to protons (Figure S6). The proton at $3.94 \mathrm{ppm}$ coupled with the carbon at $53.9 \mathrm{ppm}$. APT NMR revealed that this is the only aliphatic $\mathrm{C}-\mathrm{H}$, consequently, it could be unambiguously assigned as the chiral carbon of glutamine. The protons at 2.03 and $1.83 \mathrm{ppm}$ both couple with the same carbon $\left(27.0 \mathrm{ppm}\right.$ in $\left.{ }^{13} \mathrm{C} \mathrm{NMR}\right)$, indicating it belongs to a $\mathrm{CH}_{2}$. Finally, the two protons at $2.51 \mathrm{ppm}$ couple both with the carbon at 33.2 ppm, which also point to a $\mathrm{CH}_{2}$. APT NMR confirmed the signals at 27.0 and $33.2 \mathrm{ppm}$ are $\mathrm{CH}_{2}$ 's. Regarding the HSQC NMR correlation of the pyridine ring, the protons at $8.48 \mathrm{ppm}, 8.03 \mathrm{ppm}$ and 7.26 ppm correlate with the carbons at 151.4 ppm, $139.6 \mathrm{ppm}$ and $120.7 \mathrm{ppm}$ respectively. APT NMR confirmed that the signals at $151.4 \mathrm{ppm}, 139.6 \mathrm{ppm}$ and $120.7 \mathrm{ppm}$ are $\mathrm{CH}$ 's. 


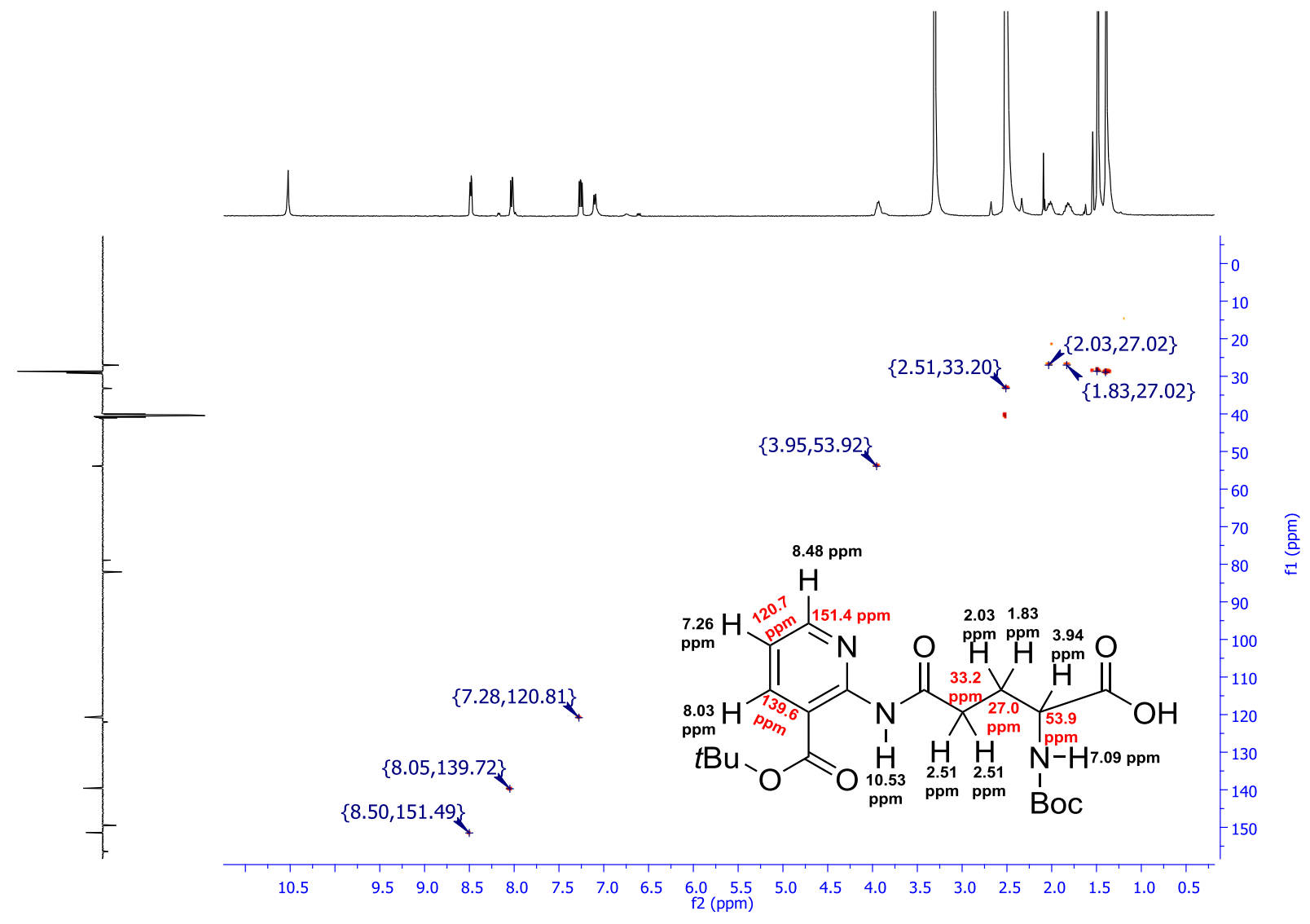

Figure S6. ${ }^{13} \mathrm{C}$ HSQC correlations in DMSO- $d_{6}$ of $\mathbf{L}-\mathbf{4 o}$.

\subsection{NOESY NMR Correlations in L-4o}

The key NOESY correlations of the two N-H protons are reported in Figure S7. The N-H at 7.09 ppm couples with the proton at $3.94 \mathrm{ppm}$ (the proton attached to the chiral carbon), the proton at $1.82 \mathrm{ppm}$ and the two protons at $2.51 \mathrm{ppm}$ (Figure S7, blue arrows). Furthermore, the proton at $7.09 \mathrm{ppm}$ correlates with the signal at $1.39 \mathrm{ppm}$ (s, 9H, see Figure 1) (Figure S7 purple arrow). The N-H proton at $10.53 \mathrm{ppm}$ correlates with the two protons at $2.51 \mathrm{ppm}$ on the side chain of the glutamine (Figure S7, red arrows) and with the protons at $1.49 \mathrm{ppm}$ (s, 9H, see Figure 1) (Figure S7, green arrow). The signal at $1.49 \mathrm{ppm}$ couples also with the proton at $8.03 \mathrm{ppm}$ in position 4 on the pyridine ring, indicating that the $t \mathrm{Bu}$ group at $1.49 \mathrm{ppm}$ can be linked to the pyridine ring, whereas the signal at $1.39 \mathrm{ppm}$ correspond to the $t \mathrm{Bu}$ group on the amine nitrogen. Consequently, it can be stated that the $\mathrm{N}-\mathrm{H}$ at $10.53 \mathrm{ppm}$ is located in between the pyridine ring and the side chain of the glutamine. 


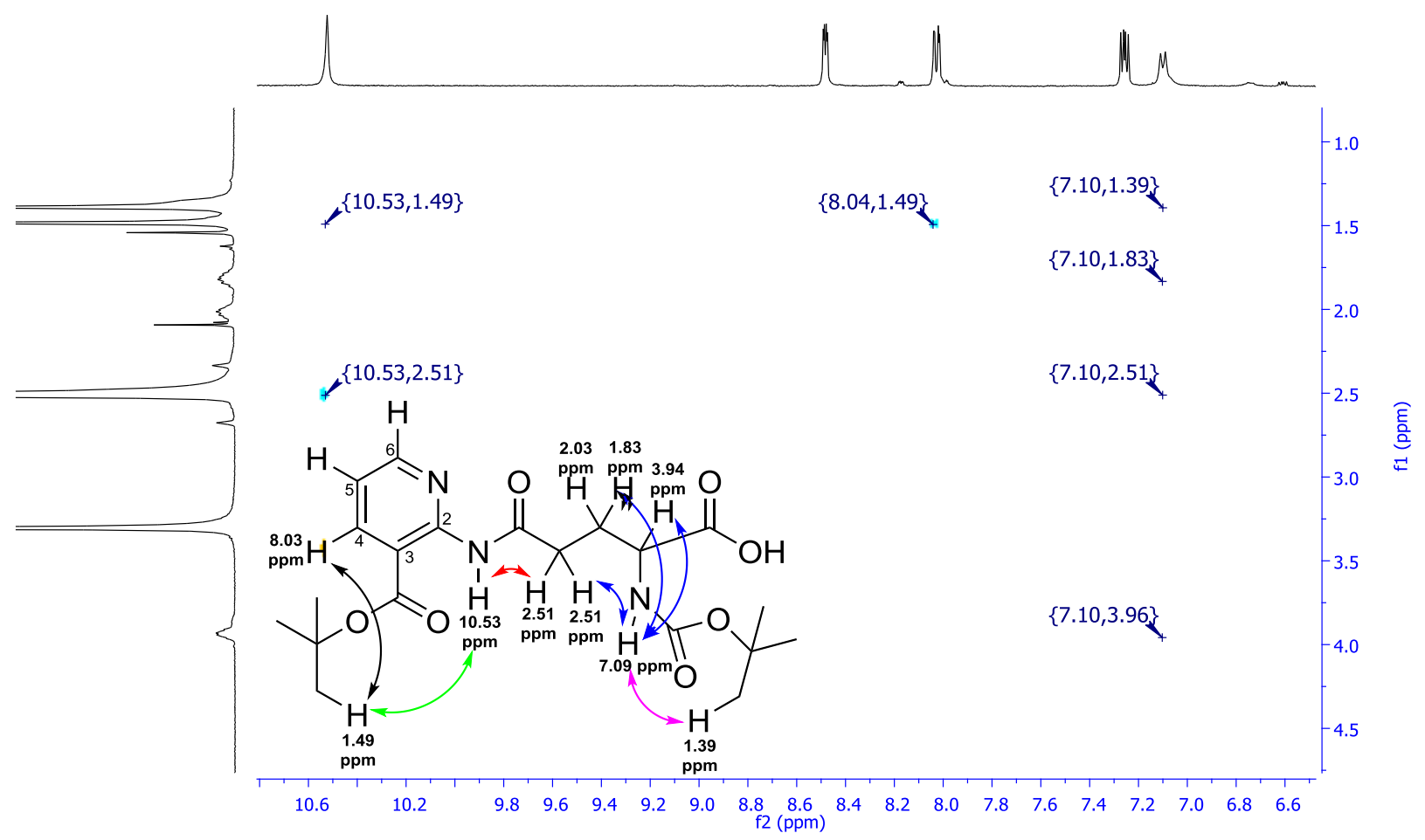

Figure S7. NOESY correlations in DMSO- $d_{6}$ of the N-H protons of L-4o.

\subsection{HMBC NMR Correlations in L-4o}

In order to assign the quaternary carbons of the pyridine ring and the carbonyls, the HMBC spectrum was analyzed. The protons at 8.48 and $8.03 \mathrm{ppm}$ both coupled with the carbon at $149.5 \mathrm{ppm}$, identifying it as the carbon in position 2 of the pyridine ring (Figure S8 blue arrows). The correlation between the proton at $8.03 \mathrm{ppm}$ and the carbon at $165.9 \mathrm{ppm}$ allowed to assign that carbon as the carbonyl of the $t \mathrm{Bu}$ ester on the pyridine ring (Figure S8, pink arrow). The HMBC correlation between the proton at 7.26 ppm and the carbon at 121.9 ppm established the placement of the latter carbon in position 3 of the pyridine ring (Figure S8, red arrow). 


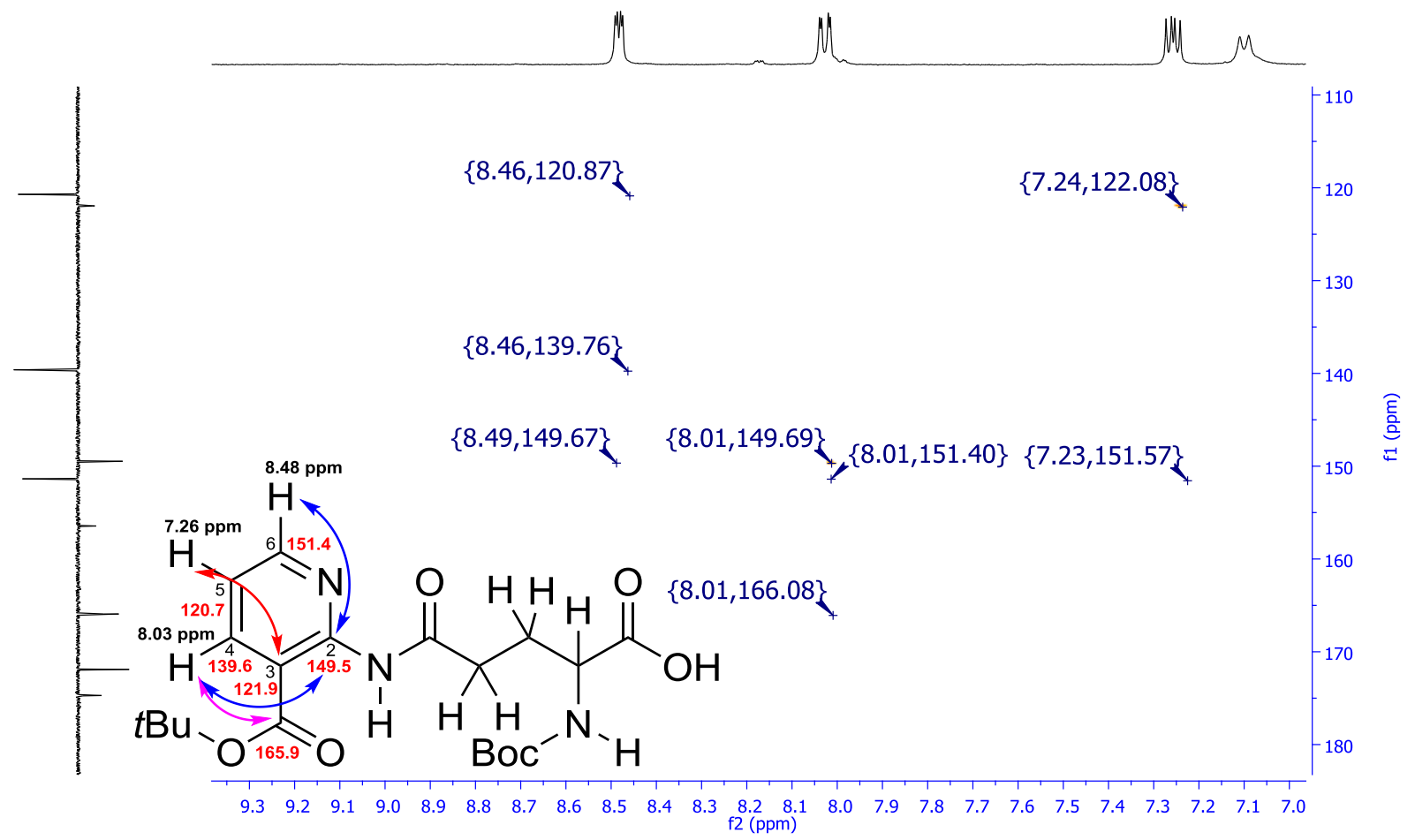

Figure S8: HMBC correlations in DMSO- $\mathrm{d}_{6}$ of the pyridine ring in $\mathbf{L}-\mathbf{4 o}$.

Regarding the HMBC correlations in the aliphatic chain, the two protons at $2.51 \mathrm{ppm}$ couple with the carbonyl at $171.9 \mathrm{ppm}$ and with the carbons at $27.0 \mathrm{ppm}$ and $53.9 \mathrm{ppm}$, while the protons at $2.03 \mathrm{ppm}$ and $1.83 \mathrm{ppm}$ couple with the carbons at $33.2 \mathrm{ppm}$ and $53.9 \mathrm{ppm}$, also confirming the HSQC of the side chain (Figure S9)

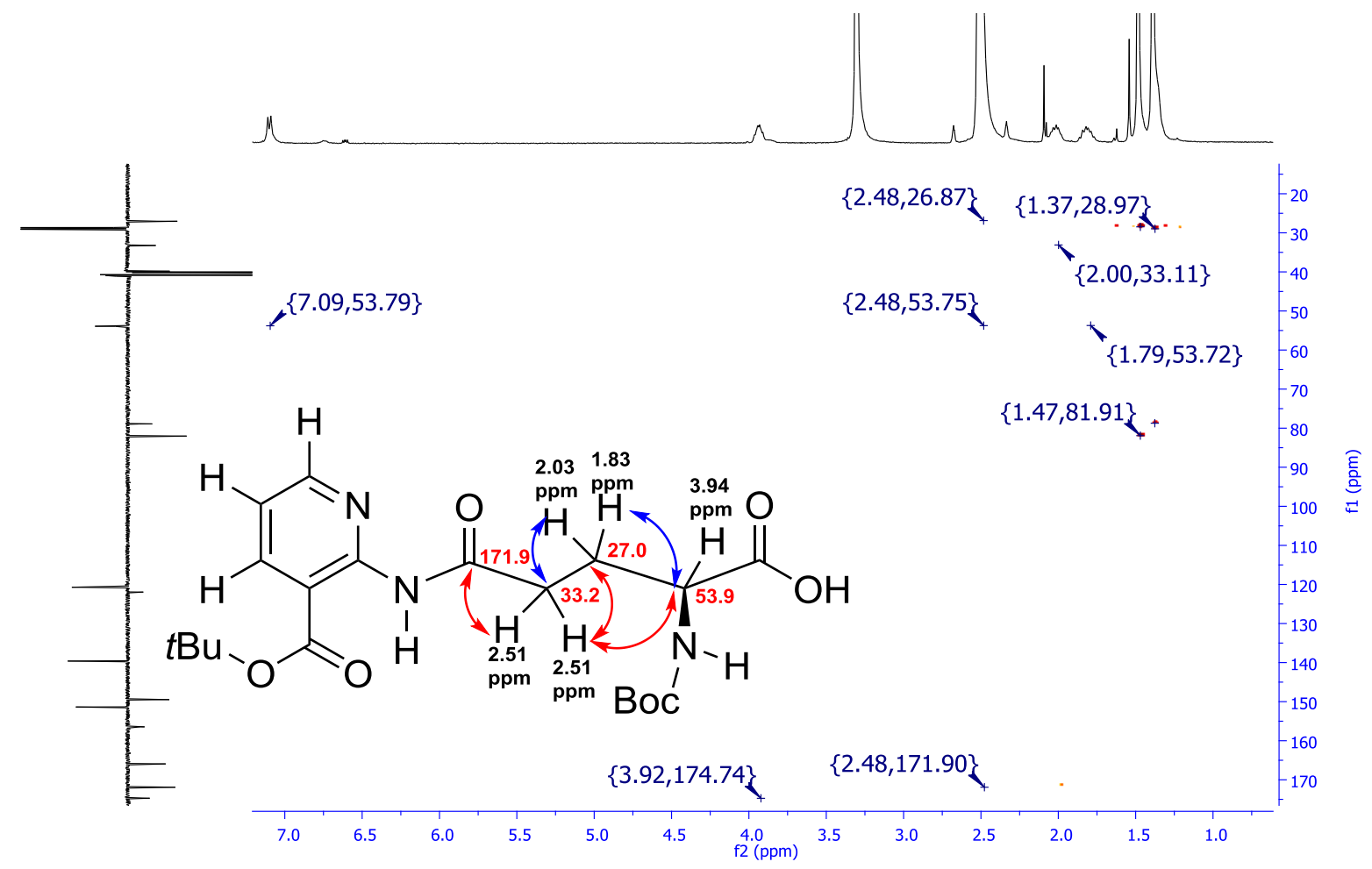

Figure S9: HMBC correlations in DMSO- $\mathrm{d}_{6}$ of the aliphatic chain in $\mathbf{L}-\mathbf{4 o}$. 
The proton at $3.94 \mathrm{ppm}$ showed an HMBC correlation with the carbonyl at $174.7 \mathrm{ppm}$, hereby identified as the carboxylic acid. Furthermore, a correlation between the $\mathrm{N}-\mathrm{H}$ proton at $7.09 \mathrm{ppm}$ and the carbon at $53.9 \mathrm{ppm}$ was observed, further confirming it as the chiral carbon (Figure S10). The N-H proton at $10.56 \mathrm{ppm}$ couples both with the carbon at $121.9 \mathrm{ppm}$ (the C-3 of the pyridine ring) and the remaining carbonyl at $171.9 \mathrm{ppm}$. Interestingly, no HMBC correlation between the proton at $10.53 \mathrm{ppm}$ and the carbon at $149.5 \mathrm{ppm}$ were found, even when the relaxation time of carbon NMR was increased. Furthermore, the carbonyl at $171.9 \mathrm{ppm}$ coupled also with the protons at $2.51 \mathrm{ppm}$. Those data, together with the information described in the section 2.3, unambiguously demonstrate that the $t$ Bu-nic directing group was selectively installed on the side chain primary amide.

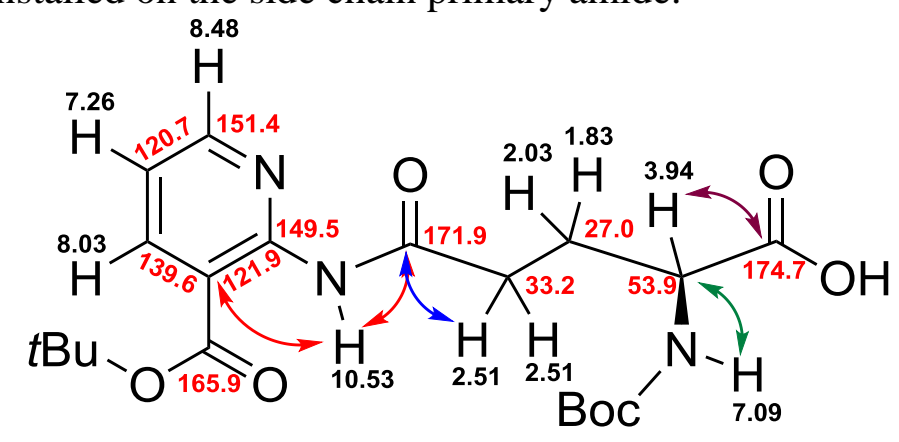

Figure S10. HMBC correlations in DMSO- $d_{6}$ distinguishing the position of the $t$ Bu-nic group in L-4o. 
$N^{2}$-(tert-Butoxycarbonyl)- $N^{5}$-(3-(tert-butoxycarbonyl)pyridin-2-yl)-L-glutamine $\quad$ (Boc-L-Gln(NH- $t \mathrm{Bu}-$ nic)-OH, DM613) L-4o - COSY NMR (400 MHz, DMSO- $d_{6}$ )

(udd) $\mathrm{T}$

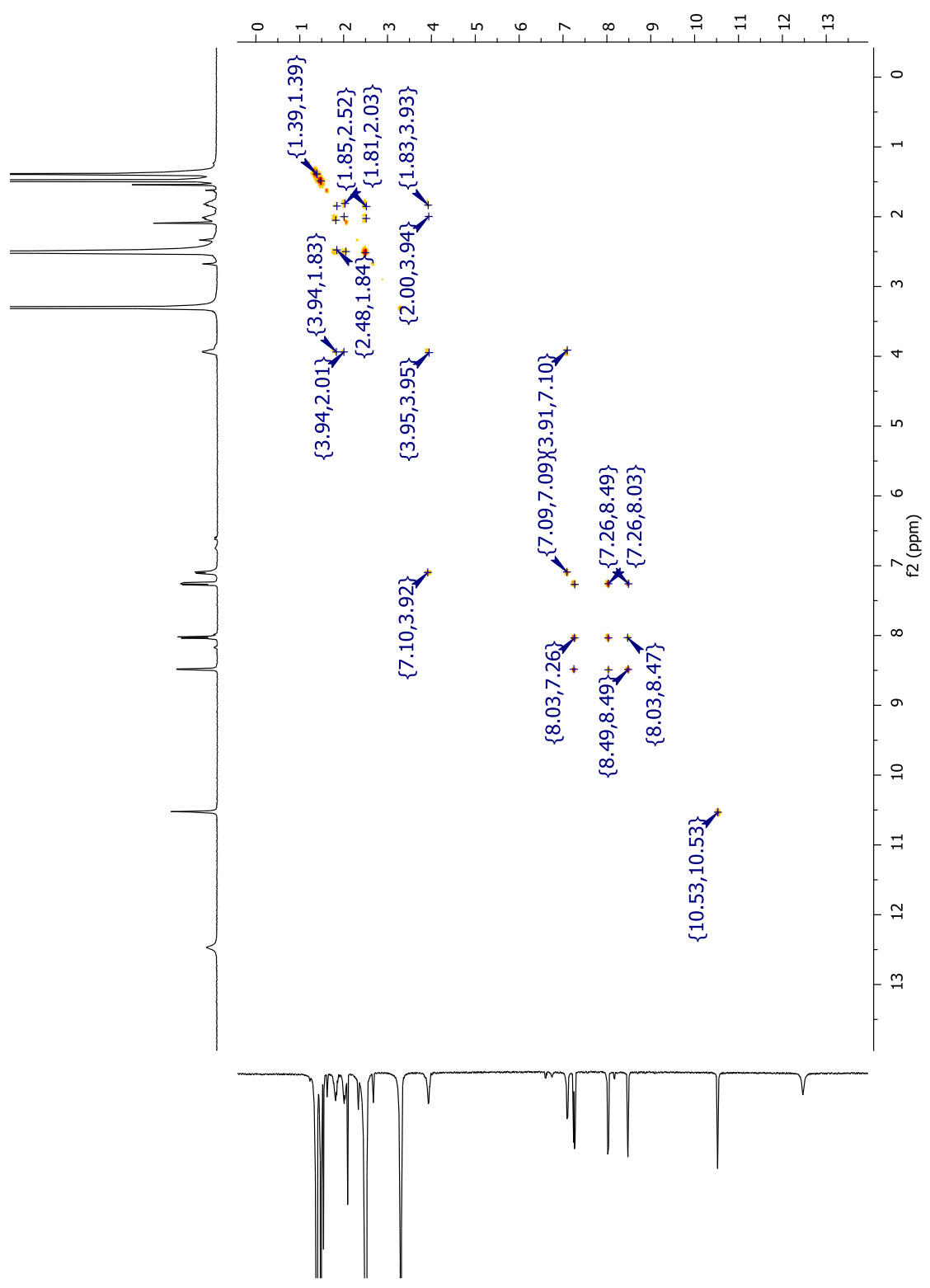


$N^{2}$-(tert-Butoxycarbonyl)- $N^{5}$-(3-(tert-butoxycarbonyl)pyridin-2-yl)-L-glutamine $\quad$ (Boc-L-Gln(NH- $t \mathrm{Bu}-$ nic)-OH, DM613) L-4o - ${ }^{15}$ N HSQC NMR (400 MHz, DMSO-d $)_{6}$

(udd) It

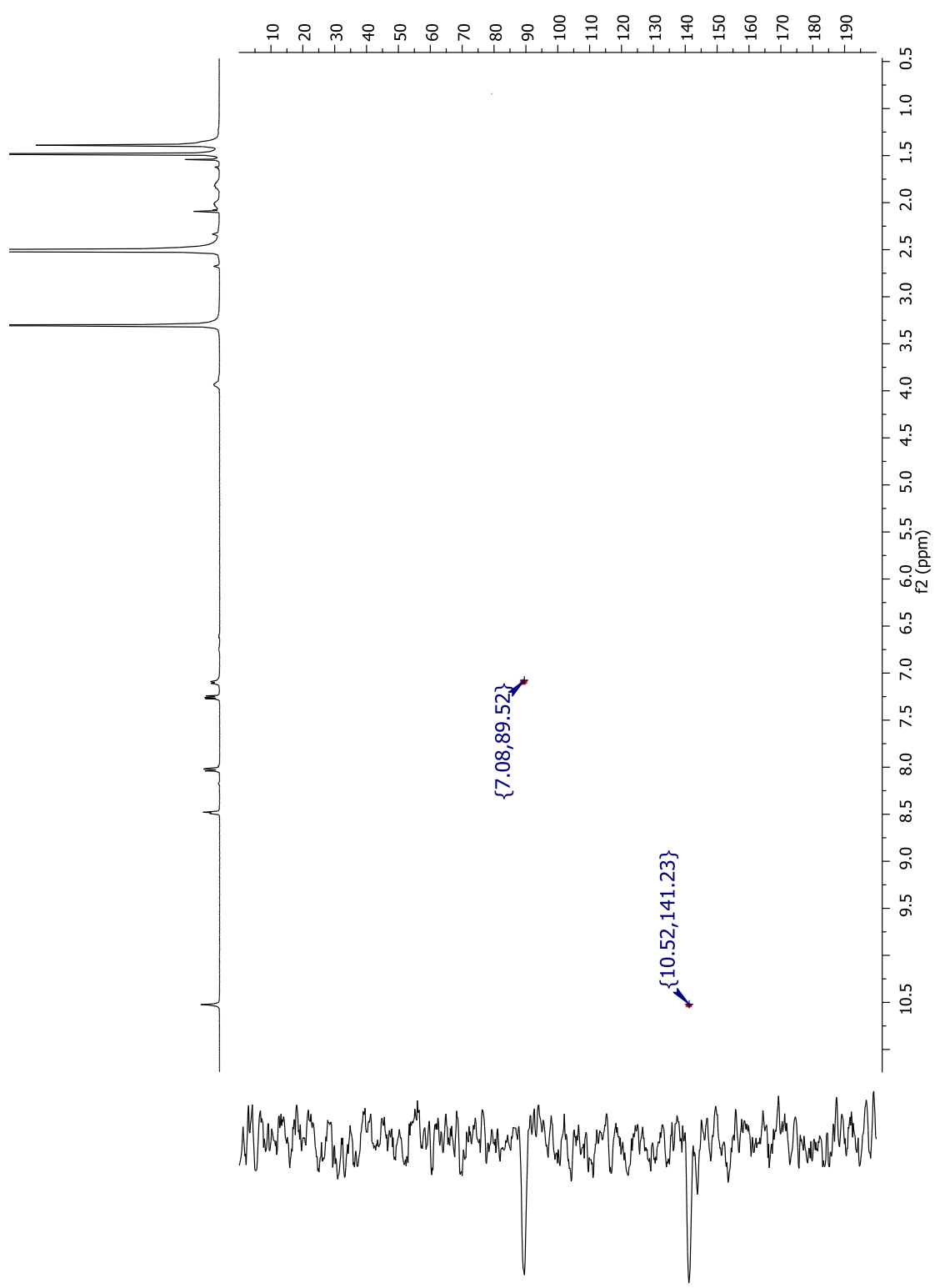


$N^{2}$-(tert-Butoxycarbonyl)- $N^{5}$-(3-(tert-butoxycarbonyl)pyridin-2-yl)-L-glutamine $\quad$ (Boc-L-Gln(NH- $t \mathrm{Bu}-$ nic)-OH, DM613) L-4o - HSQC NMR (400 MHz, DMSO- $d_{6}$ )

(udd) $\mathrm{i}$

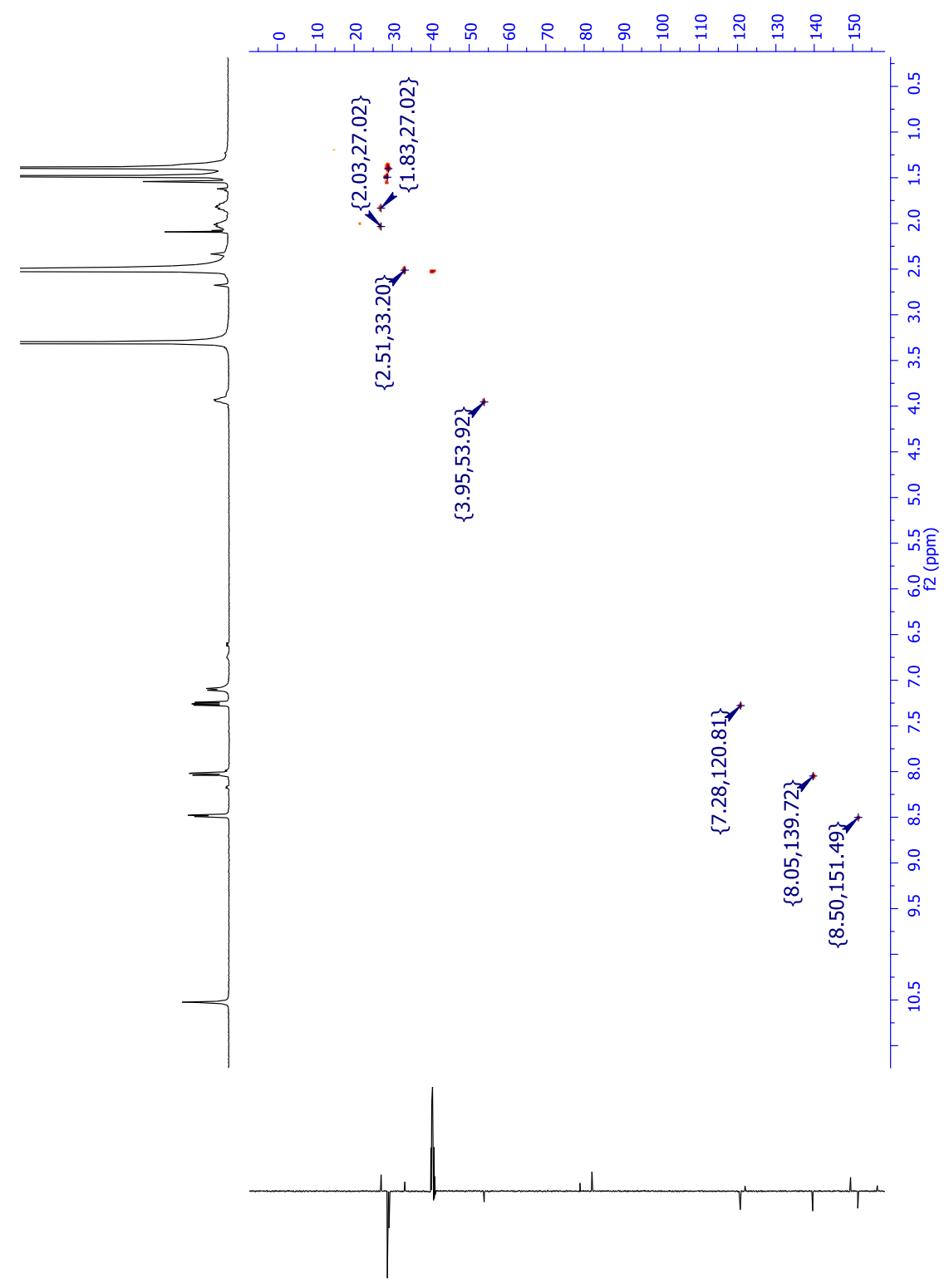


$N^{2}$-(tert-Butoxycarbonyl)- $N^{5}$-(3-(tert-butoxycarbonyl)pyridin-2-yl)-L-glutamine $\quad$ (Boc-L-Gln(NH- $t \mathrm{Bu}-$ nic)-OH, DM613) L-4o - NOESY NMR (400 MHz, DMSO-d $\left.\boldsymbol{d}_{\boldsymbol{\sigma}}\right)$

(mdd) If

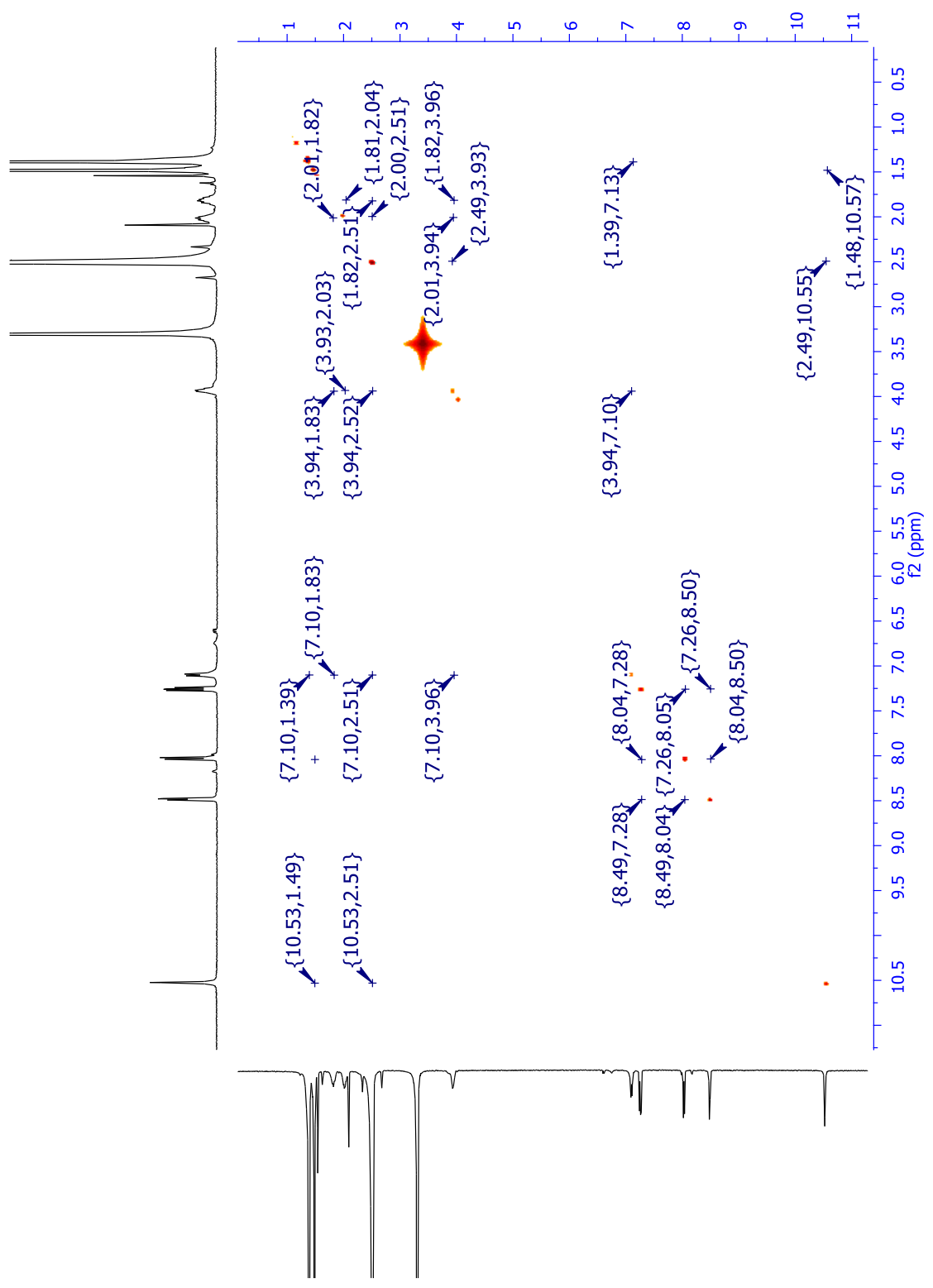


$N^{2}$-(tert-Butoxycarbonyl)- $N^{5}$-(3-(tert-butoxycarbonyl)pyridin-2-yl)-L-glutamine $\quad$ (Boc-L-Gln(NH- $t \mathrm{Bu}-$ nic)-OH, DM613) L-4o - HMBC NMR (400 MHz, DMSO-d $\left.{ }_{6}\right)$

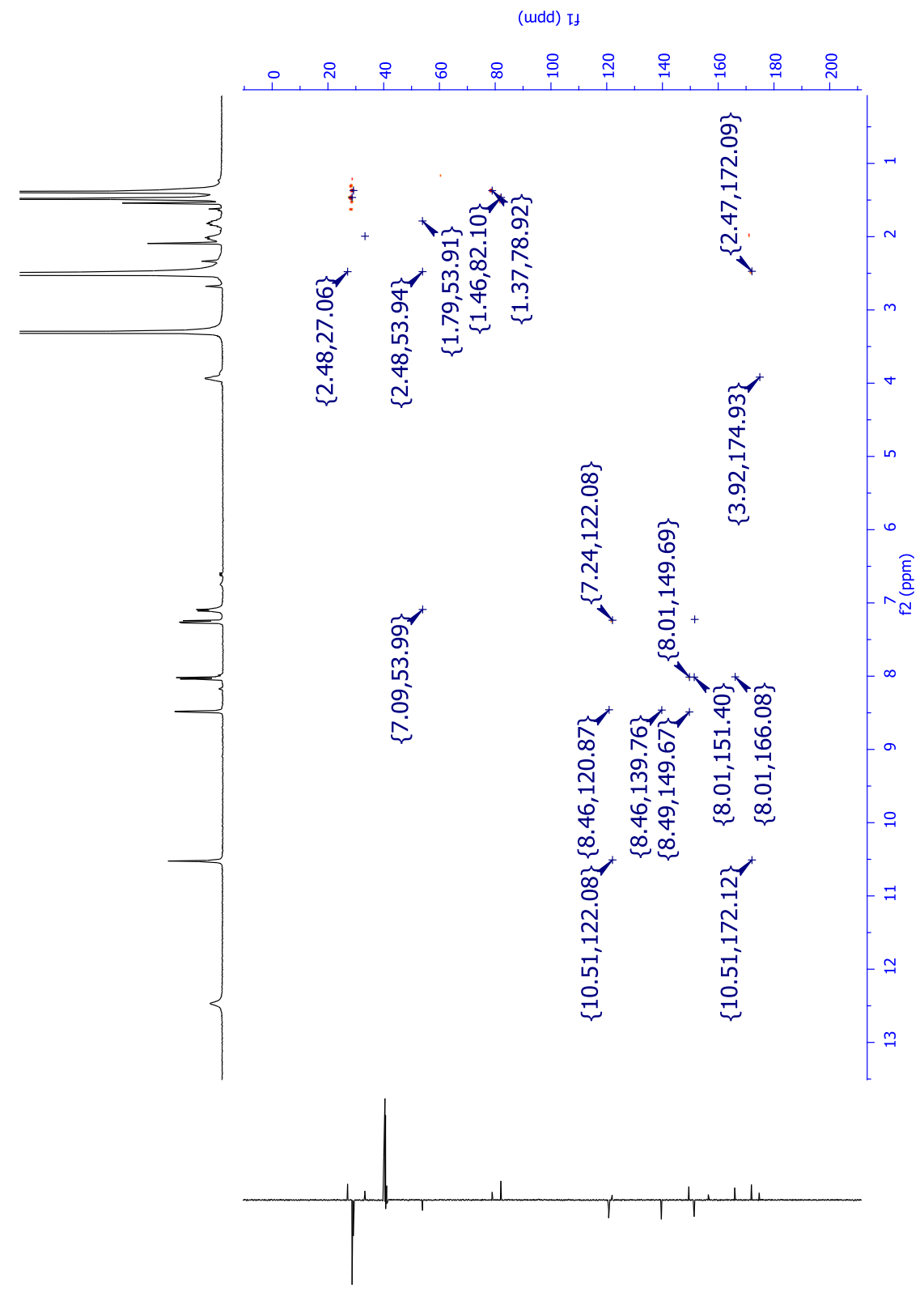




\section{Comparison of Transamidation and Alcoholysis}

In the model system, the synthesis of Boc-L-Phe-L-Phe-OMe (L,L-6a) via transamidation of Boc-L-Phe$\mathrm{NH}-t \mathrm{Bu}-\mathrm{nic}(\mathbf{L}-\mathbf{4 a})$ with H-L-Phe-OMe.HCl (L-5a.HCI), optimized conditions involved 1.1 equivalents L-5a.HCl and 1.1 equivalents $\mathrm{NaOAc}$ with $20 \mathrm{~mol} \% \mathrm{Zn}(\mathrm{OAc})_{2}$ at $70{ }^{\circ} \mathrm{C}$ (see section 1.2). This reaction can also be performed at lower temperature, i.e. $50{ }^{\circ} \mathrm{C}$, as used in the previously reported alcoholysis. The standard amount of alcohol used in the alcoholysis is 3 equivalents (Scheme S2 - B). When the same excess of amine. $\mathrm{HCl}$ (3 equivalents L-5a.HCl requiring 3 equivalents $\mathrm{NaOAc}$ to in situ deprotonate) was used in the amide cleavage of the model substrate $\mathbf{L}-\mathbf{4 a}$ at $50{ }^{\circ} \mathrm{C}$, full conversion and an excellent isolated yield was also obtained (Scheme S2 - A). Reactions can therefore be performed at a lower temperature provided a larger amount of reactant is used.

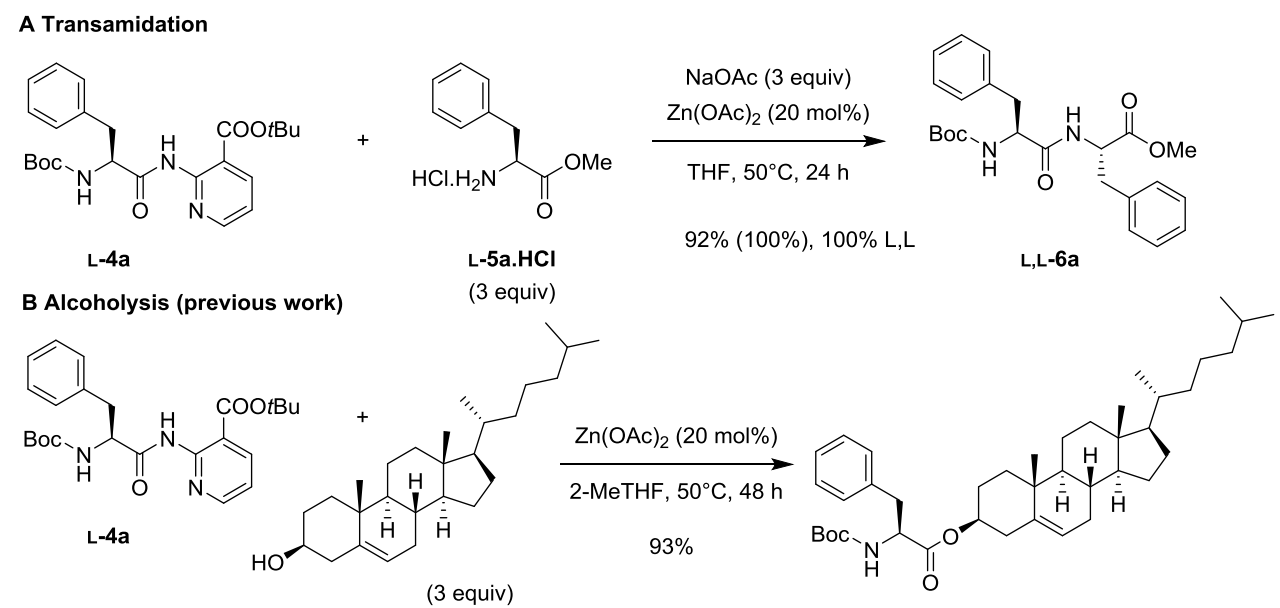

Scheme S2. A) Transamidation of Boc-L-Phe-NH- $t$ Bu-nic L-4a with 3 equiv of H-L-Phe-OMe.HCl L-5a.HCl and 3 equiv NaOAc. ${ }^{1} \mathrm{H}$ NMR yield between brackets using 1,3,5-trimethoxybenzene as internal standard. B) Alcoholysis of Boc-L-Phe-NH- $t$ Bu-nic L-4a with 3 equiv of cholesterol. 


\section{General procedures}

\section{General procedure A: Synthesis of $N$-Boc Protected $t$ Bu-nicotinate Functionalized Amino Acid /}

Peptidic Amides 4

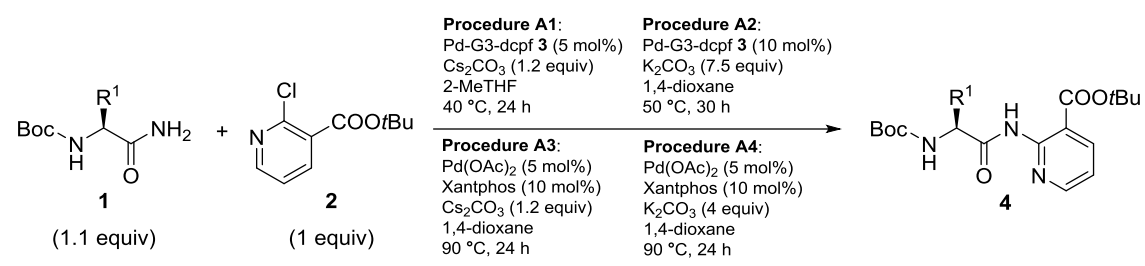

General procedure A1: A flame dried round bottomed flask was charged with $\mathrm{Cs}_{2} \mathrm{CO}_{3}(1.2$ equiv), $\mathrm{N}$-Boc protected amino acid amide 1 (1.1 equiv), Pd-G3-dcpf 3 (5 mol\%) and tert-butyl 2chloronicotinate 2 (1 equiv), dissolved in anhydrous 2-MeTHF $(0.167 \mathrm{M})$. The vial was flushed with argon and then capped. The mixture was vigorously stirred at $40{ }^{\circ} \mathrm{C}$ for $24 \mathrm{~h}$. After cooling down to room temperature, the mixture was filtered over celite ${ }^{\circledR}$ and concentrated in vacuo. The mixture was purified using automated normal phase flash chromatography and the chiral purity was confirmed using chiral HPLC. Following this procedure, compounds L-4d, L-4f $-\mathbf{4 k}, \mathbf{L}-\mathbf{4 m}$ and $\mathbf{L}, \mathbf{L}-\mathbf{4 n}$ were obtained.

General procedure A2: A flame dried round bottomed flask was charged with $\mathrm{K}_{2} \mathrm{CO}_{3}$ (7.5 equiv), $N$-Boc protected amino acid amide 1 (1.1 equiv), Pd-G3-dcpf 3 (10 mol\%) and tert-butyl 2chloronicotinate 2 ( 1 equiv), dissolved in anhydrous 1,4-dioxane $(0.167 \mathrm{M})$. The vial was flushed with argon and then capped. The mixture was vigorously stirred at $50^{\circ} \mathrm{C}$ for $30 \mathrm{~h}$. After cooling down to room temperature, the mixture was filtered over celite ${ }^{\circledR}$ and concentrated in vacuo. The mixture was purified using automated normal phase flash chromatography and the chiral purity was confirmed using chiral HPLC. Following this procedure, compounds L-4a-4c, $\mathbf{L - 4 e}$, and $\mathbf{L}-\mathbf{4 l}$ were obtained.

General procedure A3: An oven-dried microwave vial was charged with Boc-L-Gln-OH (1.5 equiv), $\mathrm{Pd}(\mathrm{OAc})_{2}(5 \mathrm{~mol} \%)$, Xantphos $(10 \mathrm{~mol} \%)$, tert-butyl 2-chloronicotinate 2 (1 equiv) and anhydrous 1,4-dioxane $(0.167 \mathrm{M})$. While vigorous stirring, $\mathrm{Cs}_{2} \mathrm{CO}_{3}$ (1.2 equiv) was added and the resulting mixture was flushed with argon and then the vial was capped. The mixture was vigorously stirred at $90^{\circ} \mathrm{C}$ for $24 \mathrm{~h}$. After cooling down to room temperature, the mixture was purified using automated reversed phase flash chromatography. Following this procedure, compound $N^{2}$-(tertButoxycarbonyl)- $N^{5}$-(3-(tert-butoxycarbonyl)pyridin-2-yl)-L-glutamine (Boc-L-Gln(NH-tBu-nic)-OH, DM613) L-4o was obtained.

General procedure A4: An oven-dried microwave vial was charged with $\mathrm{K}_{2} \mathrm{CO}_{3}$ (4 equiv), $N$-Boc protected amino acid amide 1 (1.1 equiv), $\mathrm{Pd}(\mathrm{OAc})_{2}$ (5 mol\%), Xantphos (10 mol\%), tert-butyl 2chloronicotinate 2 ( 1 equiv) and anhydrous 1,4-dioxane $(0.167 \mathrm{M})$. The vial was flushed with argon and 
then capped. The mixture was vigorously stirred at $90^{\circ} \mathrm{C}$ for $24 \mathrm{~h}$. After cooling down to room temperature, the mixture was filtered over celite ${ }^{\circledR}$ and concentrated in vacuo. The mixture was purified using automated normal phase flash chromatography and the chiral purity was confirmed using chiral HPLC. Following this procedure, compounds $\mathbf{L}-\mathbf{4 a}-\mathbf{L}-\mathbf{4} \mathbf{k}, \mathbf{L}-\mathbf{4} \mathbf{m}$ and $\mathbf{L}, \mathbf{L}-\mathbf{4 n}$ were obtained.

\section{General procedure B: Transamidation of $N$-Boc Protected $t$ Bu Nicotinate Functionalized Amino} Acid / Peptidic Amides 4

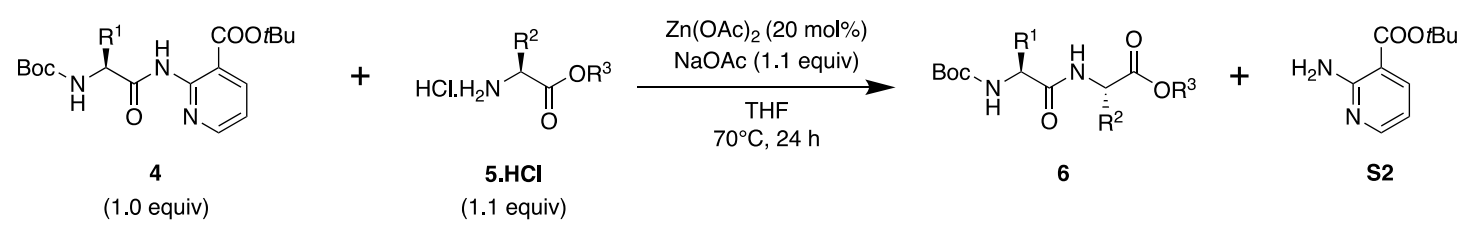

Unless stated otherwise, a microwave vial was charged with $\mathrm{Zn}(\mathrm{OAc})_{2}(20 \mathrm{~mol} \%, 0.1 \mathrm{mmol}, 18 \mathrm{mg}), N$ Boc protected $t \mathrm{Bu}$ nicotinate functionalized amino acid amide 4 ( 1 equiv, $0.5 \mathrm{mmol}$ ), 5.HCl (1.1 equiv, $0.55 \mathrm{mmol})$, NaOAc (1.1 equiv, $0.55 \mathrm{mmol}, 46 \mathrm{mg})$ and dissolved in THF $(1 \mathrm{ml})$. The mixture was sealed and stirred at $70^{\circ} \mathrm{C}$ for $24 \mathrm{~h}$. Following, the mixture was concentrated, coated on silica and purified using automated normal phase flash chromatography.

\section{General procedure C: Boc Deprotection of the $N$-Boc Protected $t$ Bu Nicotinate Functionalized}

\section{Amino Acid / Peptidic Amides 4}

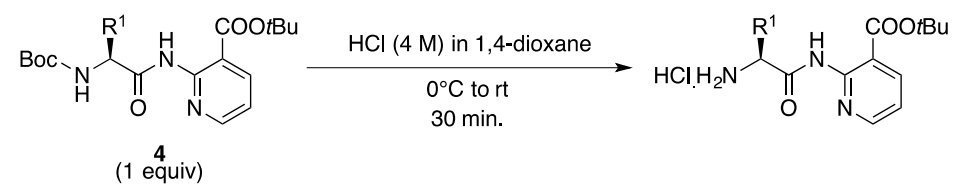

For the deprotection of the $N$-Boc protected $t \mathrm{Bu}$ nicotinate functionalized amino acids, a literature procedure was used allowing a Boc deprotection in the presence of $t$ Bu ester. ${ }^{[2]}$ In a round-bottom flask equipped with stirring bar, a solution of $\mathrm{HCl}$ in dioxane $(4 \mathrm{ml}, 4 \mathrm{M})$ was added and cooled down to $0^{\circ} \mathrm{C}$. The $N$-Boc protected $t \mathrm{Bu}$ nicotinate functionalized amino acid $4(0.2 \mathrm{mmol})$ was added at once. The mixture was allowed to warm up to room temperature and the mixture was stirred for 30 minutes. The solvent was removed in vacuo and the crude was triturated with diethyl ether.

\section{General procedure D: Solution Peptide Synthesis}

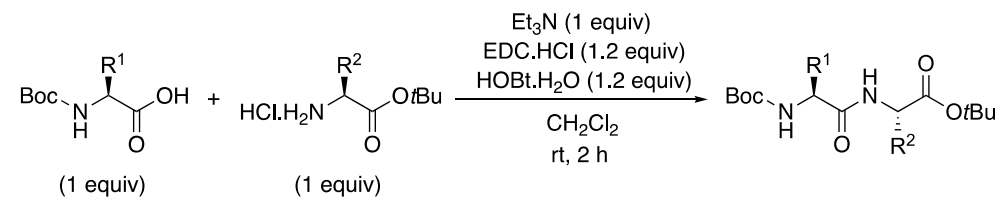

Unless stated otherwise, a round-bottom flask was charged with $N$-Boc protected amino acid (1 equiv), EDC. $\mathrm{HCl}$ (1.2 equiv) HOBt. $\mathrm{H}_{2} \mathrm{O}$ (1.2 equiv) in $\mathrm{CH}_{2} \mathrm{Cl}_{2}$. The solution was stirred at room temperature for 15 minutes. Alongside, $t \mathrm{Bu}$ amino acid ester hydrochloric acid salt (1 equiv) in $\mathrm{CH}_{2} \mathrm{Cl}_{2}$ was brought 
up to $\mathrm{pH} 9$ with $\mathrm{Et}_{3} \mathrm{~N}$ (1 equiv). This solution was subsequently added to the pre-activated $N$-Boc protected amino acid mixture and the reaction was stirred for $2 \mathrm{~h}$ at room temperature. The solvent was removed in vacuo and the residue was redissolved in EtOAc. The organic phase was extracted with $\mathrm{HCl}$ $(3 \mathrm{x}, 1 \mathrm{M})$ and $\mathrm{NaHCO}_{3}\left(3 \mathrm{x}\right.$, sat.), washed with brine, dried over $\mathrm{MgSO}_{4}$, filtered and concentrated in vacuo. 


\section{Synthetic Procedures}

\subsection{Synthesis of the $N$-Boc Protected $t$ Bu Nicotinate Functionalized Amino Acid / Peptidic Amides}

4

The synthesis and characterisation of the $N$-Boc $t \mathrm{Bu}$ nicotinate functionalized amino acid / peptide amides L-4a, L-4d, L-4e, L-4f, L-4g, L-4h, L-4i, L-4j, L-4k, L,L-4n has previously been reported by our laboratories. ${ }^{[1]} \mathbf{L}-\mathbf{4 b}, \mathbf{L}-\mathbf{4 c}, \mathbf{L}-\mathbf{4 l}, \mathbf{L}-\mathbf{4 m}$, and $\mathbf{L}-\mathbf{4 o}$ have not been reported before. They are all accessed from the $N$-Boc amino acid / peptide amides through $\mathrm{Pd}$-catalyzed coupling with $t \mathrm{Bu} 2$-chloronicotinate 2 (see section 4). To increase the yield of Boc-L-Gln(NH-tBu-nic)-OH (L-4o), featuring side chain DG introduction, a small optimization was performed (see section 1.3). There are generally two set of conditions, depending on the catalyst used [Pd-G3-dcpf 3 (A1 and A2) or $\mathrm{Pd}(\mathrm{OAc})_{2} / \mathrm{Xantphos}(\mathrm{A} 3$ and A4)]. Both catalysts were used with either $\mathrm{Cs}_{2} \mathrm{CO}_{3}$ (A1 and A3) or $\mathrm{K}_{2} \mathrm{CO}_{3}$ (A2 and A4). Generally, with some expections ( $\mathbf{L}-\mathbf{4 d}$ and $\mathbf{L}, \mathbf{L}-\mathbf{4 n})$, conditions A4 based on $\mathrm{Pd}(\mathrm{OAc})_{2} / \mathrm{Xantphos}$ gave epimerisation. All reaction conditions have been summarized in Table S13, also for those $N$-Boc $t$ Bu-nicotinate functionalized amino acid / peptide amides $\mathbf{4}$ previously prepared. The $N$-Boc amino acid amides $\mathbf{1}$, not commercially available, were synthesized based on the commercially available $N$-Boc amino acid precursors, using a literature protocol. ${ }^{[5]}$ The spectroanalytical data and purity were checked and compared with literature characterisations. 
Table S13. Synthesis of the $N$-Boc Protected $t$ Bu Nicotinate Functionalized Amino Acid / Peptidic Amides 4

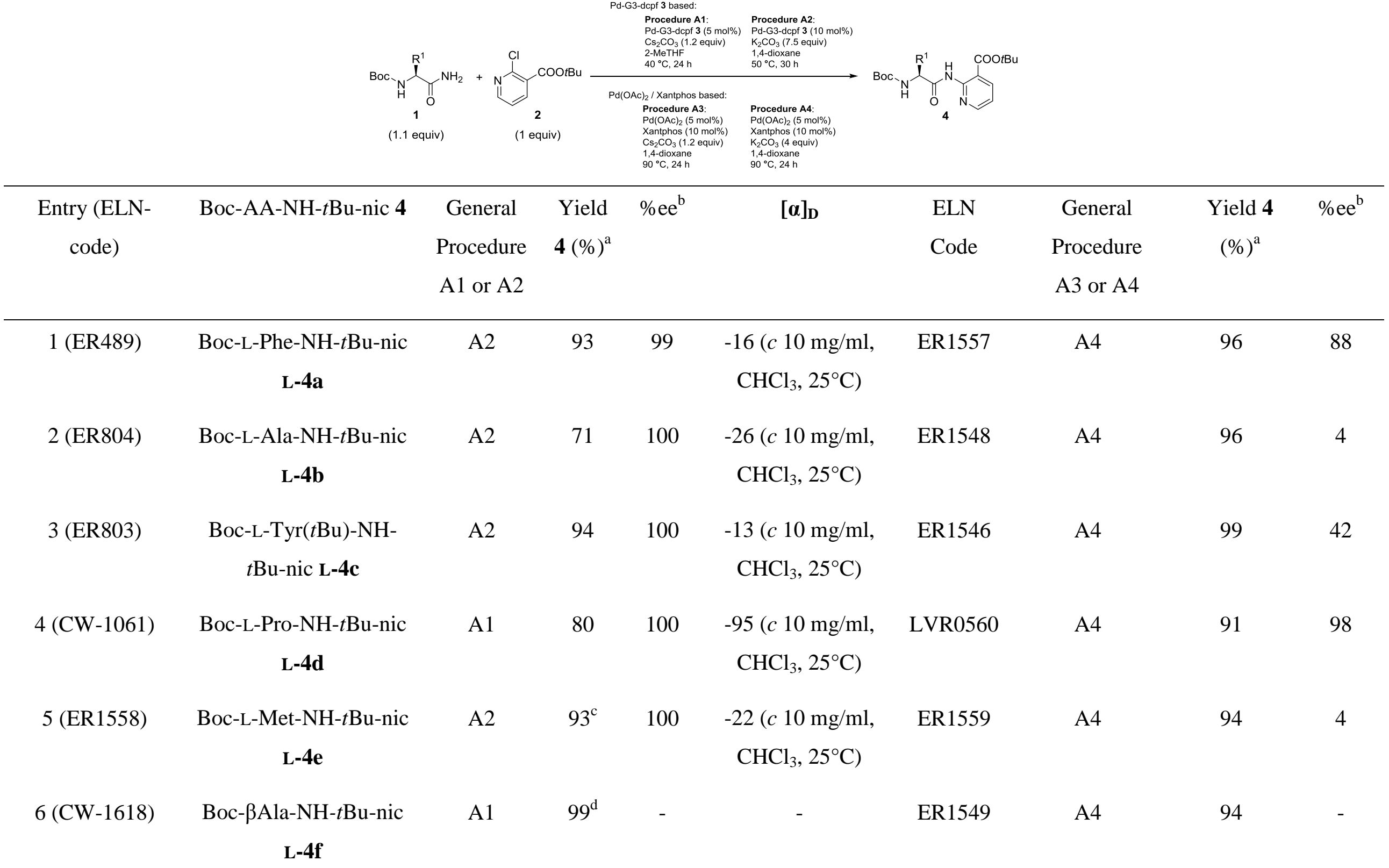




\begin{tabular}{|c|c|c|c|c|c|c|c|c|c|}
\hline $7(\mathrm{CW}-1124)$ & $\begin{array}{c}\text { Boc-Gly-NH- } t \text { Bu-nic } \\
\text { L-4g }\end{array}$ & A1 & 83 & - & - & ER1559 & A4 & 91 & - \\
\hline 8 (ER1538) & $\begin{array}{c}\text { Boc-L-Val-NH- } t \text { Bu-nic } \\
\mathbf{L}-\mathbf{4 h}\end{array}$ & A1 & $96^{\mathrm{d}}$ & 96 & $\begin{array}{c}-14(c 10 \mathrm{mg} / \mathrm{ml}, \\
\left.\mathrm{CHCl}_{3}, 25^{\circ} \mathrm{C}\right)\end{array}$ & ER499 & A4 & 94 & 93 \\
\hline 9 (DM679) & $\begin{array}{c}\text { Boc-L-Ile-NH- } t \text { Bu-nic } \\
\qquad \mathbf{L}-\mathbf{4 i}\end{array}$ & A1 & $82^{\mathrm{d}}$ & $100^{\mathrm{e}}$ & $\begin{array}{l}-18(c 10 \mathrm{mg} / \mathrm{ml}, \\
\left.\mathrm{CHCl}_{3}, 27^{\circ} \mathrm{C}\right)\end{array}$ & DM682 & A4 & 79 & $84^{\mathrm{e}}$ \\
\hline 10 (LVR0603) & $\begin{array}{c}\text { Boc-L-Ser }(t \mathrm{Bu})-\mathrm{NH}- \\
t \mathrm{Bu}-\operatorname{nic} \mathbf{L}-\mathbf{4} \mathbf{j}\end{array}$ & A1 & $72^{f}$ & 99 & $\begin{array}{c}-24(c 10 \mathrm{mg} / \mathrm{ml}, \\
\left.\mathrm{CHCl}_{3}, 27^{\circ} \mathrm{C}\right)\end{array}$ & ER1561 & A4 & 91 & 40 \\
\hline 11 (ER1718) & $\begin{array}{c}\text { Boc-L-Lys(Boc)-NH- } \\
t \text { Bu-nic } \mathbf{L}-\mathbf{4 k}\end{array}$ & A1 & 79 & 100 & $\begin{array}{c}-15(c 10 \mathrm{mg} / \mathrm{ml}, \\
\left.\mathrm{CHCl}_{3}, 20^{\circ} \mathrm{C}\right)\end{array}$ & ER1565 & A4 & 89 & 48 \\
\hline 12 (ER1676) & $\begin{array}{c}\text { Boc-L-Cys }(t \mathrm{Bu})-\mathrm{NH}- \\
t \text { Bu-nic } \mathbf{L}-\mathbf{4 l}\end{array}$ & $\mathrm{A} 2$ & 89 & 100 & $\begin{array}{c}-33(c 10 \mathrm{mg} / \mathrm{ml}, \\
\left.\mathrm{CHCl}_{3}, 21^{\circ} \mathrm{C}\right)\end{array}$ & ER1621 & A4 & Trace & - \\
\hline 13 (ER1738) & $\begin{array}{c}\text { Boc-L-His(trt)-NH- } \\
\quad t \text { Bu-nic } \mathbf{L}-\mathbf{4 m}\end{array}$ & A1 & $56^{\mathrm{g}}$ & 98 & $\begin{array}{c}-26(c 10 \mathrm{mg} / \mathrm{ml}, \\
\left.\mathrm{CHCl}_{3}, 19^{\circ} \mathrm{C}\right)\end{array}$ & ER1703 & A4 & 73 & 56 \\
\hline $14(\mathrm{CW}-1843)$ & $\begin{array}{c}\text { Boc-L-Pro-L-Leu-Gly- } \\
\text { NH- } t \text { Bu-nic L,L-4n }\end{array}$ & A1 & $99^{\mathrm{d}}$ & 100 & $\begin{array}{c}-102(c 10 \mathrm{mg} / \mathrm{ml}, \\
\left.\mathrm{CHCl}_{3}, 21^{\circ} \mathrm{C}\right)\end{array}$ & DM868 & A4 & 25 & 100 \\
\hline 15 & $\begin{array}{c}\text { Boc-L-Gln(NH-tBu- } \\
\text { nic)-OH } \quad \mathbf{L - 4 o}\end{array}$ & - & - & - & $\begin{array}{c}+17(c 10 \mathrm{mg} / \mathrm{ml}, \\
\left.\mathrm{CHCl}_{3}, 25^{\circ} \mathrm{C}\right)\end{array}$ & DM613 & A3 & 60 & 100 \\
\hline
\end{tabular}

[a] Isolated yield after column chromatography. [b] \%ee measured using LC-CD analysis on a Chiralpak IA column, $250 \mathrm{~nm}, 1 \mathrm{ml} / \mathrm{min} . \quad[\mathrm{c}] \mathrm{T}=60^{\circ} \mathrm{C}$.

[d] $\mathrm{Cs}_{2} \mathrm{CO}_{3}$ (2 equiv). [e] \%de. [f] $\mathrm{K}_{2} \mathrm{CO}_{3}$ (6 equiv). [g] 10 mol\% Pd-G3-dcpf, 16h, 50v/v\% DMF. 
tert-Butyl 2-\{[N-(tert-butoxycarbonyl)-L-phenylalanyl]amino $\}$ nicotinate (Boc-L-Phe-NH- $t$ Bu-nic, ER489) L-4a

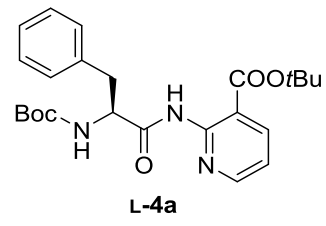

The title compound was prepared using general procedure A2, from Boc-L-Phe$\mathrm{NH}_{2}$ (145 mg, $0.55 \mathrm{mmol}, 1.1$ equiv), $\mathrm{K}_{2} \mathrm{CO}_{3}$ (0.518 g, $3.75 \mathrm{mmol}, 7.5$ equiv), $\mathrm{Pd}-$ G3-dcpf 3 (52 mg, $0.05 \mathrm{mmol}, 10 \mathrm{~mol} \%$ ) and tert-butyl 2-chloronicotinate 2 (107 $\mathrm{mg}, 0.5 \mathrm{mmol}, 1$ equiv) in anhydrous 1,4-dioxane $(3 \mathrm{ml})$. This yielded, using Biotage ${ }^{\circledR}$ Isolera One Normal phase silicagel flash chromatography ( $n$-Heptane:EtOAc, 100:0 to 50:50 over $1 \mathrm{~h}$ ), the desired compound as a white solid in $93 \%$ (207 mg, $0.469 \mathrm{mmol})$. M.p. $159{ }^{\circ} \mathrm{C}{ }^{1} \mathbf{H}$ NMR (400 MHz, $\left.\mathbf{C D C l}_{3}\right) \delta 11.28(\mathrm{br} \mathrm{s}, 1 \mathrm{H}), 8.60(\mathrm{~d}, J=3.2 \mathrm{~Hz}, 1 \mathrm{H}), 8.22(\mathrm{dd}, J=7.7,1.1 \mathrm{~Hz}, 1 \mathrm{H}), 7.26(\mathrm{~d}$, $J=4.3 \mathrm{~Hz}, 4 \mathrm{H}) 7.22-7.15(\mathrm{~m}, 1 \mathrm{H}), 7.04$ (dd, $J=7.7,4.8 \mathrm{~Hz}, 1 \mathrm{H}), 5.18$ (br d, $J=6.8 \mathrm{~Hz}, 1 \mathrm{H}), 4.98$ (br s, 1H), $3.28(\mathrm{dd}, J=13.9,5.8 \mathrm{~Hz}, 1 \mathrm{H}), 3.09(\mathrm{dd}, J=12.5,6.7 \mathrm{~Hz}, 1 \mathrm{H}), 1.58(\mathrm{~s}, 9 \mathrm{H}), 1.40(\mathrm{~s}, 9 \mathrm{H}) \mathrm{ppm} .{ }^{13} \mathrm{C}$

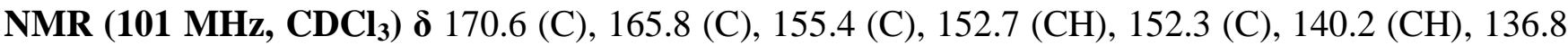
$(\mathrm{C}), 129.6(\mathrm{CH}), 128.6(\mathrm{CH}), 126.9(\mathrm{CH}), 118.4(\mathrm{CH}), 112.8(\mathrm{C}), 83.4(\mathrm{C}), 80.0(\mathrm{C}), 56.9(\mathrm{CH}), 38.9$ $\left(\mathrm{CH}_{2}\right), 28.4\left(\mathrm{CH}_{3}\right), 28.2\left(\mathrm{CH}_{3}\right)$ ppm. HRMS (ESI+) m/z calcd. for $\mathrm{C}_{24} \mathrm{H}_{32} \mathrm{~N}_{3} \mathrm{O}_{5}[\mathrm{M}+\mathrm{H}]^{+}: 442.2336$, found 442.2322. Spectroscopic data are reported in literature. ${ }^{1}[\alpha]_{\mathbf{D}}=-16\left(c 10 \mathrm{mg} / \mathrm{ml}, \mathrm{CHCl}_{3}, 25^{\circ} \mathrm{C}\right)$. LC-CD analysis on a Chiralpak IA column (retention time $=3.94 \mathrm{~min}, 250 \mathrm{~nm}, 1.0 \mathrm{ml} / \mathrm{min}$ ) revealed $99 \%$ ee.

tert-Butyl (S)-2-(2-((tert-butoxycarbonyl)amino)propanamido)nicotinate (Boc-L-Ala-NH-tBu-nic, ER804) L-4b

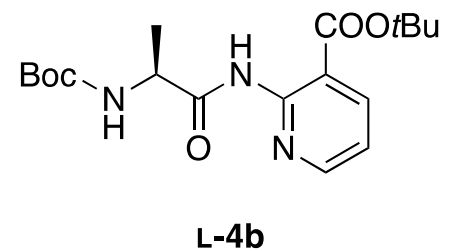

The title compound was prepared using general procedure A2, from Boc-LAla- $\mathrm{NH}_{2}$ (214 mg, $1.1 \mathrm{mmol}, 1.1$ equiv), $\mathrm{K}_{2} \mathrm{CO}_{3}(1.04 \mathrm{~g}, 7.5 \mathrm{mmol}, 7.5$ equiv), Pd-G3-dcpf 3 (104 mg, $0.1 \mathrm{mmol}, 10 \mathrm{~mol} \%$ ) and tert-butyl 2chloronicotinate 2 (214 mg, $1 \mathrm{mmol}, 1$ equiv) in anhydrous 1,4-dioxane (6 ml). This yielded, using Biotage ${ }^{\circledR}$ Isolera One Normal phase silicagel flash chromatography ( $n$ Heptane:EtOAc, 100:0 to 0:100 over $1 \mathrm{~h}$ ), the desired compound as a white solid in 71\% (260 $\mathrm{mg}, 0.7$ mmol). ${ }^{1} \mathbf{H}$ NMR (500 MHz, $\left.\mathbf{C D C l}_{3}\right) \boldsymbol{\delta} 11.34$ (br s, 1H), 8.56 (br s, 1H), 8.22 (d, J=6.6 Hz, 1H), 7.02 (br s, 1H), 5.30-5.22 (m, 1H), 4.70-4.62 (m, 1H), 1.57 (s, 9H), $1.47(\mathrm{~d}, J=5 \mathrm{~Hz}, 3 \mathrm{H}), 1.43(\mathrm{~s}, 9 \mathrm{H}) \mathrm{ppm}$. ${ }^{13}$ C NMR (126 MHz, CDCl $)$ ) $171.5(\mathrm{C}), 165.8(\mathrm{C}), 155.1(\mathrm{C}), 152.5(\mathrm{CH}), 152.2(\mathrm{C}), 140.0(\mathrm{CH})$, $118.2(\mathrm{CH}), 112.7(\mathrm{C}), 83.3(\mathrm{C}), 79.6(\mathrm{C}), 51.4(\mathrm{CH}), 28.3\left(\mathrm{CH}_{3}\right), 28.0\left(\mathrm{CH}_{3}\right), 19.0\left(\mathrm{CH}_{3}\right)$ ppm. HRMS (ESI+) $\mathrm{m} / \mathrm{z}$ calcd. for $\mathrm{C}_{18} \mathrm{H}_{28} \mathrm{~N}_{3} \mathrm{O}_{5}[\mathrm{M}+\mathrm{H}]^{+}:$366.2024, found 366.2013. No spectroscopic data are reported in literature. $[\boldsymbol{\alpha}]_{\mathbf{D}}=-26\left(c 10 \mathrm{mg} / \mathrm{ml}, \mathrm{CHCl}_{3}, 25^{\circ} \mathrm{C}\right)$.

LC-CD analysis on a Chiralpak IA column (retention time $=3.35 \mathrm{~min}, 250 \mathrm{~nm}, 1.0 \mathrm{ml} / \mathrm{min}$ ) revealed $100 \%$ ee. 
tert-Butyl (S)-2-(3-(4-(tert-butoxy)phenyl)-2-((tert-butoxycarbonyl)amino)propanamido)nicotinate (Boc-L-Tyr( $t \mathrm{Bu})-\mathrm{NH}-t \mathrm{Bu}-\mathrm{nic}$, ER803) L-4c

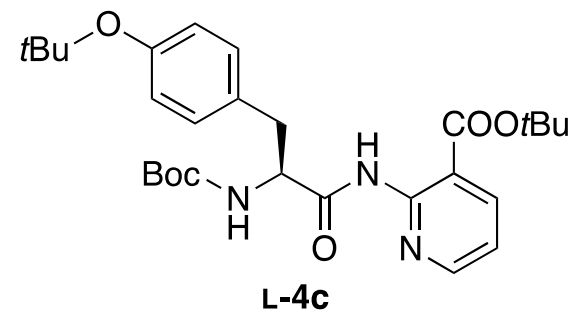

The title compound was isolated using general procedure $\mathbf{A 2}$, from Boc-L-Tyr $(t \mathrm{Bu})-\mathrm{NH}_{2}\left(370 \mathrm{mg}, 1.1 \mathrm{mmol}, 1.1\right.$ equiv), $\mathrm{K}_{2} \mathrm{CO}_{3}(1.03$ g, 7.5 mmol, 7.5 equiv), Pd-G3-dcpf 3 (103 mg, 0.1 mmol, 10 mol\%) and tert-butyl 2-chloronicotinate 2 (214 mg, $1 \mathrm{mmol}, 1$ equiv) in anhydrous 1,4-dioxane $(6 \mathrm{ml})$. The reaction mixture was

purified using the Biotage ${ }^{\circledR}$ Isolera One Normal phase silicagel flash chromatography $(n-$ Heptane:EtOAc, 100:0 to 0:100 over $1 \mathrm{~h}$ ) to obtain a white solid in 94\% (481 mg, $0.93 \mathrm{mmol}$ ) yield. ${ }^{1} \mathbf{H}$

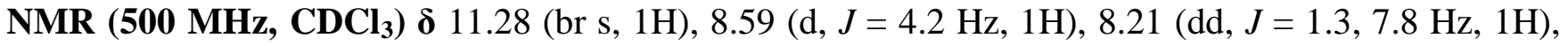
$7.14(\mathrm{~d}, J=8.5 \mathrm{~Hz}, 2 \mathrm{H}), 7.03(\mathrm{dd}, J=4.8,7.7 \mathrm{~Hz}, 1 \mathrm{H}), 6.87(\mathrm{~d}, J=8.3 \mathrm{~Hz}, 2 \mathrm{H}), 5.23-5.10(\mathrm{~m}, 1 \mathrm{H})$, 4.93 (br s, 1H), 3.24 (dd, $J=5.6,14.2 \mathrm{~Hz}, 1 \mathrm{H}), 3.02$ (dd, $J=7.1,12.7 \mathrm{~Hz}, 1 \mathrm{H}), 1.58$ (s, 9H), 1.40 (s, 9H), 1.28 (s, 9H) ppm. ${ }^{13} \mathbf{C}$ NMR (126 MHz, CDCl $\mathbf{3}$ ) $\delta 170.6$ (C), 165.8 (C), 155.4 (C), 154.3 (C), $152.6(\mathrm{CH}), 152.2$, (C) 140.1 (CH) , $131.5(\mathrm{C}), 129.9(\mathrm{CH}), 124.2(\mathrm{CH}), 118.4(\mathrm{CH}), 112.9(\mathrm{C}), 83.4$ (C), $79.8(\mathrm{C}), 78.3(\mathrm{C}), 57.0(\mathrm{CH}), 38.3\left(\mathrm{CH}_{2}\right), 28.9\left(\mathrm{CH}_{3}\right), 28.4\left(\mathrm{CH}_{3}\right), 28.2\left(\mathrm{CH}_{3}\right)$ ppm. HRMS (ESI+) $\mathrm{m} / \mathrm{z}$ calcd. for $\mathrm{C}_{28} \mathrm{H}_{40} \mathrm{~N}_{3} \mathrm{O}_{6}[\mathrm{M}+\mathrm{H}]^{+}: 514.2911$ found 514.2933. $[\alpha]_{\mathbf{D}}=-13\left(c 10 \mathrm{mg} / \mathrm{ml}, \mathrm{CHCl}_{3}, 25^{\circ} \mathrm{C}\right)$. No spectroscopic data are reported in literature.

LC-CD analysis on a Chiralpak IA column (retention time $=3.87 \mathrm{~min}, 250 \mathrm{~nm}, n$-hexane:EtOH 80:20, $1.0 \mathrm{ml} / \mathrm{min}$ ) revealed $100 \%$ ee.

tert-Butyl 2-\{[1-(tert-butoxycarbonyl)-L-prolyl]amino\}nicotinate (Boc-L-Pro-NH-tBu-nic, CW-1061) L-4d

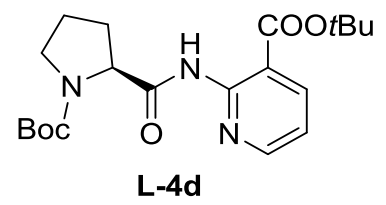

The title compound was isolated using general procedure A1, from Boc-LPro- $\mathrm{NH}_{2}$ (1.178 g, $5.5 \mathrm{mmol}, 1.1$ equiv), $\mathrm{Cs}_{2} \mathrm{CO}_{3}(1.955 \mathrm{~g}, 6.0 \mathrm{mmol}, 1.2$ equiv), Pd-G3-dcpf 3 (257 mg, $0.250 \mathrm{mmol}, 5 \mathrm{~mol} \%$ ), and tert-butyl 2chloronicotinate 2 (1.068 mg, $5.0 \mathrm{mmol}, 1$ equiv) in anhydrous 2-MeTHF $(30.0 \mathrm{ml})$. The reaction mixture was purified using the Biotage ${ }^{\circledR}$ Isolera One Normal phase silicagel flash chromatography ( $n$-Heptane:EtOAc, 100:0 to 40:60 over $1 \mathrm{~h}$ ) to obtain a brown solid in $80 \%$ (1.566 g, $4.00 \mathrm{mmol}$ ) yield. M.p. $98^{\circ} \mathrm{C} .{ }^{1} \mathbf{H}$ NMR (400 $\mathbf{~ M H z , ~} \mathbf{C D C l}_{3}$, mixture of rotamers) $\delta 11.54$ (br s, $0.6 \mathrm{H}), 11.46$ (br s, 0.4H), 8.58 (br s, $1 \mathrm{H}), 8.22$ (br s, 0.4H), 8.20 (br s, 0.6H), 7.01 (d, J=4.8 Hz, 1H), 4.56 (br s, 0.3H), 4.36 (br s, 0.6H), 3.74-3.33 (m, 2H), 2.36-2.09 (m, 2H), 2.01-1.81 (m, 2H), 1.54 (s, 9H), 1.45 (s, 3H), 1.32 (s, 6H) ppm. ${ }^{13} \mathbf{C}$ NMR (101 $\mathbf{M H z}, \mathbf{C D C l}_{3}$, mixture of rotamers) $\delta$ [171.6, $171.1(\mathrm{C})], 166.0(\mathrm{C}),[155.1,154.2(\mathrm{C})]$, [152.9, $152.7(\mathrm{CH})], 152.2(\mathrm{C}), 140.0(\mathrm{CH})$, [118.6, 118.1 $(\mathrm{CH})],[113.0,112.8(\mathrm{C})],[83.5,83.0(\mathrm{C})],[80.3,80.0(\mathrm{C})],[62.9,62.2(\mathrm{CH})],\left[47.3,46.9\left(\mathrm{CH}_{2}\right)\right],[31.6$, 
$30.5\left(\mathrm{CH}_{2}\right)$ ], [28.6, $\left.28.3\left(\mathrm{CH}_{3}\right)\right], 28.1\left(\mathrm{CH}_{3}\right)$, [24.3, $\left.23.8\left(\mathrm{CH}_{2}\right)\right]$ ppm. Spectroscopic data are reported in literature. ${ }^{1}$ HRMS (ESI+) m/z calcd. for $\mathrm{C}_{20} \mathrm{H}_{30} \mathrm{~N}_{3} \mathrm{O}_{5}[\mathrm{M}+\mathrm{H}]^{+}: 392.2180$ found $392.2179[\boldsymbol{\alpha}]_{\mathbf{D}}=-95(c$ $10 \mathrm{mg} / \mathrm{ml}, \mathrm{CHCl}_{3}, 25^{\circ} \mathrm{C}$ ).

LC-CD analysis on a Chiralpak IA column (retention time $=3.33 \mathrm{~min}, 250 \mathrm{~nm}, 1.0 \mathrm{ml} / \mathrm{min}$ ) revealed $100 \%$ ee.

tert-Butyl 2-\{[N-(tert-butoxycarbonyl)-L-methionyl]amino $\}$ nicotinate

(Boc-L-Met-NH- $t$ Bu-nic, ER1558) L-4e<smiles>CCOC(=O)c1cccnc1NC(=O)C(CCSC)NC(=O)OCc1ccccc1</smiles>

The title compound was prepared using general procedure A2, from Boc-LMet- $\mathrm{NH}_{2}$ (273 mg, $1.1 \mathrm{mmol}, 1.1$ equiv), $\mathrm{K}_{2} \mathrm{CO}_{3}$ (1.037 g, $7.5 \mathrm{mmol}, 7.5$ equiv), Pd-G3-dcpf 3 (104 mg, $0.1 \mathrm{mmol}, 10 \mathrm{~mol} \%$ ) and tert-butyl 2chloronicotinate 2 (214 mg, $1.0 \mathrm{mmol}, 1$ equiv) in anhydrous 1,4-dioxane (6 $\mathrm{ml})$. This yielded, using Biotage ${ }^{\circledR}$ Isolera One Normal phase silicagel flash chromatography ( $n$-Heptane:EtOAc, 100:0 to 50:50 over $1 \mathrm{~h}$ ), the desired compound as a white solid in 93\% (396 mg, 0.932 mmol). M.p. $117{ }^{\circ} \mathrm{C}^{\mathbf{1}} \mathbf{H}$ NMR (400 MHz, $\mathbf{C D C l}_{3}$ ) $\boldsymbol{\delta} 11.34$ (br s, 1H), 8.56 (dd, $J=$ 7.0, 1.6 Hz, 1H), 8.23 (dd, $J=7.9,1.9 \mathrm{~Hz}, 1 \mathrm{H}), 7.04$ (dd, $J=7.8,4.8 \mathrm{~Hz}, 1 \mathrm{H}), 5.38$ (br d, $J=7.3 \mathrm{~Hz}$, $1 \mathrm{H}), 4.86$ (br s, 1H), 2.68-2.56 (m, 2H), 2.33-2.22 (m, 1H), 2.09 (s, 3H), 2.00-1.89 (m, 1H), 1.59 (s,

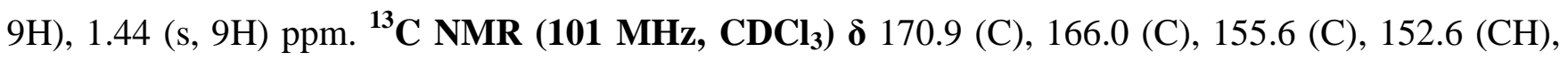
$152.2(\mathrm{C}), 140.2(\mathrm{CH}), 118.5(\mathrm{CH}), 113.0(\mathrm{C}), 83.6(\mathrm{C}), 80.0(\mathrm{C}), 55.1(\mathrm{CH}), 32.8\left(\mathrm{CH}_{2}\right), 30.4\left(\mathrm{CH}_{2}\right)$, $28.4\left(\mathrm{CH}_{3}\right), 28.2\left(\mathrm{CH}_{3}\right), 15.4\left(\mathrm{CH}_{3}\right)$ ppm. HRMS (ESI+) m/z calcd. for $\mathrm{C}_{26} \mathrm{H}_{43} \mathrm{~N}_{4} \mathrm{O}_{7}[\mathrm{M}+\mathrm{H}]^{+}:$523.3126, found 523.3126. Spectroscopic data are reported in literature. ${ }^{1}[\alpha]_{\mathbf{D}}=-22\left(c 10 \mathrm{mg} / \mathrm{ml}, \mathrm{CHCl}_{3}, 25^{\circ} \mathrm{C}\right)$. LC-CD analysis on a Chiralpak IA column (retention time $=7.0 \mathrm{~min}, 250 \mathrm{~nm}, 1.0 \mathrm{ml} / \mathrm{min}$ ) revealed $100 \%$ ee.

tert-Butyl 2-\{3-[(tert-butoxycarbonyl)amino]propanamido\}nicotinate (Boc- $\beta$ Ala-NH-tBu-nic, CW1618) $4 \mathbf{f}$

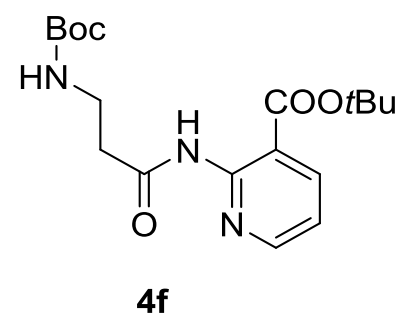

The title compound was isolated using general procedure A1, from , Boc$\beta$ Ala- $\mathrm{NH}_{2}$ (1.035 g, $5.5 \mathrm{mmol}, 1.1$ equiv), $\mathrm{Cs}_{2} \mathrm{CO}_{3}$ (3.26 g, $10.0 \mathrm{mmol}, 2.0$ equiv), Pd-G3-dcpf 3 (257 mg, $0.250 \mathrm{mmol}, 5 \mathrm{~mol} \%$ ), and tert-butyl 2chloronicotinate 2 (1.068 mg, $5.0 \mathrm{mmol}, 1$ equiv) in anhydrous 2-MeTHF $(30.0 \mathrm{ml})$. The reaction mixture was purified using the Biotage ${ }^{\circledR}$ Isolera One Normal phase silicagel flash chromatography ( $n$-Heptane:EtOAc, 100:0 to 25:75 over $1 \mathrm{~h}$ ) to obtain a white solid in 99\% (1.820 g, 4.99 mmol) yield. M.p. $99^{\circ} \mathrm{C}^{\mathbf{1}} \mathbf{H}$ NMR (400 $\mathbf{~ M H z , ~} \mathbf{C D C l}_{3}$ ) $\delta 10.93$ (br s, 
$1 \mathrm{H}), 8.53(\mathrm{dd}, J=4.8,1.9 \mathrm{~Hz}, 1 \mathrm{H}), 8.23(\mathrm{dd}, J=7.9,1.9 \mathrm{~Hz}, 1 \mathrm{H}), 7.02(\mathrm{dd}, J=7.9,4.8 \mathrm{~Hz}, 1 \mathrm{H}), 5.35$ (br s, 1H), 3.50 (q, $J=5.8 \mathrm{~Hz}, 2 \mathrm{H}), 2.89$ (t, $J=5.6 \mathrm{~Hz}, 2 \mathrm{H}), 1.59$ (s, 9H), $1.41(\mathrm{~s}, 9 \mathrm{H}) \mathrm{ppm} .{ }^{\mathbf{1 3}} \mathbf{C}$ NMR

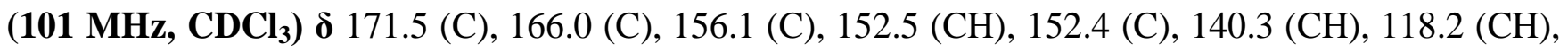
$112.6(\mathrm{C}), 83.6(\mathrm{C}), 79.1(\mathrm{C}), 38.4\left(\mathrm{CH}_{2}\right), 36.2\left(\mathrm{CH}_{2}\right), 28.5\left(\mathrm{CH}_{3}\right), 28.2\left(\mathrm{CH}_{3}\right)$ ppm. Spectroscopic data are reported in literature. ${ }^{1}$ HRMS (ESI+) m/z calcd. for $\mathrm{C}_{18} \mathrm{H}_{28} \mathrm{~N}_{3} \mathrm{O}_{5}[\mathrm{M}+\mathrm{H}]^{+}: 366.2023$ found 366.2022 .

tert-Butyl 2-\{[N-(tert-butoxycarbonyl)glycyl]amino $\}$ nicotinate (Boc-Gly-NH-tBu-nic, CW-1124) 4g

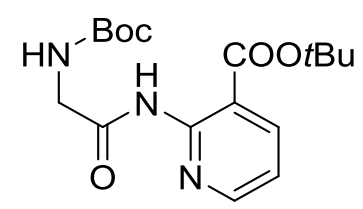

$4 g$

The title compound was isolated using general procedure A1, from , Boc-Gly$\mathrm{NH}_{2}$ (0.958 g, 5.5 mmol, 1.1 equiv), $\mathrm{Cs}_{2} \mathrm{CO}_{3}$ (1.955 g, 6.0 mmol, 1.2 equiv), Pd-G3-dcpf 3 (257 mg, $0.250 \mathrm{mmol}, 5 \mathrm{~mol} \%)$, and tert-butyl 2chloronicotinate 2 (1.068 g, $5.0 \mathrm{mmol}, 1$ equiv) in anhydrous 2-MeTHF (30.0 $\mathrm{ml}$ ). The reaction mixture was purified using the Biotage ${ }^{\circledR}$ Isolera One Normal phase silicagel flash chromatography ( $n$-Heptane:EtOAc, 100:0 to 30:70 over $1 \mathrm{~h}$ ) to obtain a white solid in 83\% (1.49 g, $4.25 \mathrm{mmol}$ ) yield. M.p. $129^{\circ} \mathrm{C}{ }^{1} \mathbf{H}$ NMR (400 MHz, $\left.\mathbf{C D C l}_{3}\right) \boldsymbol{\delta} 11.19$ (br s, 1H), $8.51(\mathrm{dd}, J=4.8,1.4 \mathrm{~Hz}, 1 \mathrm{H}), 8.24$ (dd, $J=7.9,1.9 \mathrm{~Hz}, 1 \mathrm{H}), 7.02$ (dd, $J=7.8,4.8 \mathrm{~Hz}, 1 \mathrm{H}), 5.43$ (br s,

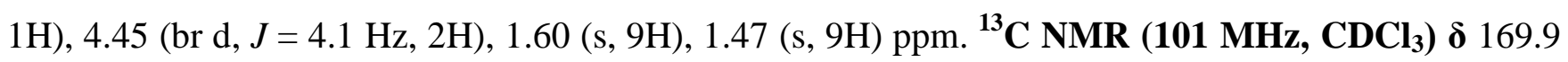
(C), $165.8(\mathrm{C}), 156.0(\mathrm{C}), 152.4(\mathrm{CH}), 152.2(\mathrm{C}), 140.4(\mathrm{CH}), 118.2(\mathrm{CH}), 112.3(\mathrm{C}), 83.6(\mathrm{C}), 79.9(\mathrm{C})$, $46.8\left(\mathrm{CH}_{2}\right), 28.5\left(\mathrm{CH}_{3}\right), 28.2\left(\mathrm{CH}_{3}\right)$ ppm. Spectroscopic data are reported in literature. ${ }^{1}$ HRMS (ESI+) $\mathrm{m} / \mathrm{z}$ calcd. for $\mathrm{C}_{17} \mathrm{H}_{26} \mathrm{~N}_{3} \mathrm{O}_{5}[\mathrm{M}+\mathrm{H}]^{+}: 352.1867$ found 352.1859 .

tert-Butyl 2-\{[N-(tert-butoxycarbonyl)-L-valyl]amino\}nicotinate (Boc-L-Val-NH-tBu-nic, ER1538) L4h<smiles>CCCCOC(=O)c1cccnc1NC(=O)C(NC(=O)OC(C)(C)C)C(C)C</smiles>

$(30.0 \mathrm{ml})$. The reaction mixture was purified using the Biotage ${ }^{\circledR}$ Isolera One Normal phase silicagel flash chromatography ( $n$-Heptane:EtOAc, 100:0 to 40:60 over 1 h) to obtain a white solid in $96 \%$ (1.896 g, 4.82 mmol) yield. M.p. $132^{\circ} \mathrm{C} .{ }^{1} \mathbf{H}$ NMR (400 MHz, $\mathbf{C D C l}_{3}$ ) $\boldsymbol{\delta} 11.30$ (br s, 1H), 8.57 (dd, $J=$ 4.4, $1.3 \mathrm{~Hz}, 1 \mathrm{H}), 8.22(\mathrm{dd}, J=7.9,1.9 \mathrm{~Hz}, 1 \mathrm{H}), 7.02$ (dd, $J=7.8,4.8 \mathrm{~Hz}, 1 \mathrm{H}), 5.25$ (br d, $J=8.5 \mathrm{~Hz}$, 1H), 4.53 (br s, 1H), $2.37-2.24(\mathrm{~m}, 1 \mathrm{H}), 1.58$ (s, 9H), 1.44 (s, 9H), 1.04 (d, J=6.8 Hz, 3H), 0.94 (d, J $=6.9 \mathrm{~Hz}, 3 \mathrm{H}) \mathrm{ppm} .{ }^{13} \mathbf{C}$ NMR (101 MHz, $\left.\mathbf{C D C l}_{3}\right) \boldsymbol{\delta} 170.6(\mathrm{C}), 166.0(\mathrm{C}), 156.0(\mathrm{C}), 152.7(\mathrm{CH}), 152.3$ (C), $140.1(\mathrm{CH}), 118.4(\mathrm{CH}), 112.9(\mathrm{C}), 83.5(\mathrm{C}), 79.7(\mathrm{C}), 60.7(\mathrm{CH}), 31.1(\mathrm{CH}), 28.4\left(\mathrm{CH}_{3}\right), 28.2$ 
$\left(\mathrm{CH}_{3}\right), 19.6\left(\mathrm{CH}_{3}\right), 17.2\left(\mathrm{CH}_{3}\right)$ ppm. Spectroscopic data are reported in literature. ${ }^{1} \mathbf{H R M S}(\mathrm{ESI}+) \mathrm{m} / \mathrm{z}$ calcd. for $\mathrm{C}_{20} \mathrm{H}_{32} \mathrm{~N}_{3} \mathrm{O}_{5}[\mathrm{M}+\mathrm{H}]^{+}: 394.2336$ found $394.2339[\boldsymbol{\alpha}]_{\mathbf{D}}=-14\left(c 10 \mathrm{mg} / \mathrm{ml}, \mathrm{CHCl}_{3}, 25^{\circ} \mathrm{C}\right)$.

LC-CD analysis on a Chiralpak IA column (retention time $=8.6 \mathrm{~min}, 250 \mathrm{~nm}, 1.0 \mathrm{ml} / \mathrm{min}$ ) revealed $96 \%$ ee.

tert-Butyl 2-\{[N-(tert-butoxycarbonyl)-L-isoleucyl]amino $\}$ nicotinate (Boc-L-Ile-NH-tBu-nic, DM679) $\mathbf{L}-\mathbf{4} \mathbf{i}$

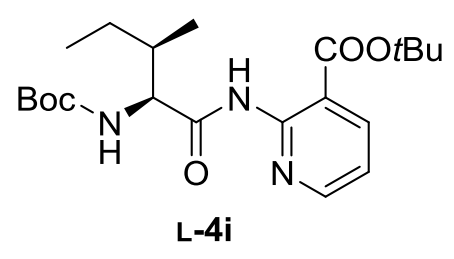

The title compound was isolated using general procedure A1, from Boc-LIle- $\mathrm{NH}_{2}$ (0.760 g, $3.3 \mathrm{mmol}, 1.1$ equiv), $\mathrm{Cs}_{2} \mathrm{CO}_{3}(1.955 \mathrm{~g}, 6.0 \mathrm{mmol}, 2.0$ equiv), Pd-G3-dcpf 3 (143 mg, $0.150 \mathrm{mmol}, 5 \mathrm{~mol} \%$ ), and tert-butyl 2chloronicotinate 2 (0.641 g, $3.0 \mathrm{mmol}, 1$ equiv) in anhydrous 2-MeTHF $(19.0 \mathrm{ml})$. The reaction mixture was purified using the Biotage ${ }^{\circledR}$ Isolera One Normal phase silicagel flash chromatography ( $n$-Heptane:EtOAc, 100:0 to 0:100 over $1 \mathrm{~h}$ ) to obtain a colorless oil in 82\% (1.00 g, 2.45 mmol) yield. ${ }^{1} \mathbf{H}$ NMR (400 MHz, $\left.\mathbf{C D C l}_{3}\right) \boldsymbol{\delta} 11.31$ (br s, 1H), 8.56 (dd, $\left.J=4.5,1.4 \mathrm{~Hz}, 1 \mathrm{H}\right)$, $8.20(\mathrm{dd}, J=7.9 \mathrm{~Hz}, 1.8 \mathrm{~Hz}, 1 \mathrm{H}), 7.01$ (dd, $J=7.8,4.8 \mathrm{~Hz}), 5.22$ (br d, $J=8.6 \mathrm{~Hz}, 1 \mathrm{H}), 4.48$ (br s, 1H), $2.09-1.95(\mathrm{~m}, 1 \mathrm{H}), 1.56(\mathrm{~s}, 9 \mathrm{H}), 1.54-1.47(\mathrm{~m}, 1 \mathrm{H}) 1.42(\mathrm{~s}, 9 \mathrm{H}), 1.19-1.07(\mathrm{~m}, 1 \mathrm{H}), 1.00$ ( d, $J=6.8$ $\mathrm{Hz}, 3 \mathrm{H}), 0.89$ (t, $J=7.4 \mathrm{~Hz}, 3 \mathrm{H})$ ppm. ${ }^{13} \mathbf{C}$ NMR (101 MHz, CDCl $\left.\mathbf{3}\right) \boldsymbol{\delta} 170.4$ (C), 166.0 (C), 155.9 (C), $152.7(\mathrm{CH}), 152.2(\mathrm{C}), 140.0(\mathrm{CH}), 118.4(\mathrm{CH}), 113.0(\mathrm{C}), 83.4(\mathrm{C}), 79.7(\mathrm{C}), 60.5(\mathrm{CH}), 37.8(\mathrm{CH})$, $28.4\left(\mathrm{CH}_{3}\right), 28.1\left(\mathrm{CH}_{3}\right), 24.4\left(\mathrm{CH}_{2}\right), 15.8\left(\mathrm{CH}_{3}\right), 11.8\left(\mathrm{CH}_{3}\right)$ ppm. Spectroscopic data are reported in literature. ${ }^{1}$ HRMS (ESI+) m/z calcd. for $\mathrm{C}_{21} \mathrm{H}_{34} \mathrm{~N}_{3} \mathrm{O}_{5}[\mathrm{M}+\mathrm{H}]^{+}: 408.2493$ found 408.2506. [ $\left.\boldsymbol{\alpha}\right]_{\mathbf{D}}=-18(c$ $\left.10 \mathrm{mg} / \mathrm{ml}, \mathrm{CHCl}_{3}, 27^{\circ} \mathrm{C}\right)$.

tert-Butyl 2-\{[N-(tert-butoxycarbonyl)-O-tert-butyl-L-seryl]amino $\}$ nicotinate (Boc-L-Ser $(t \mathrm{Bu})-\mathrm{NH}$ $t$ Bu-nic, LVR0603) L-4j<smiles>CCCCCCCCC(C)(C)C</smiles>

The title compound was isolated using general procedure A2, from Boc-L$\operatorname{Ser}(t \mathrm{Bu})-\mathrm{NH}_{2}(0.141 \mathrm{~g}, 0.55 \mathrm{mmol}, 1.1$ equiv $), \mathrm{K}_{2} \mathrm{CO}_{3}(0.416 \mathrm{~g}, 3.0 \mathrm{mmol}$, 6.0 equiv), Pd-G3-dcpf 3 (51 mg, $0.05 \mathrm{mmol}, 10 \mathrm{~mol} \%$ ), and tert-butyl 2chloronicotinate 2 ( $0.107 \mathrm{~g}, 0.5 \mathrm{mmol}, 1$ equiv) in anhydrous 1,4-dioxane $(3 \mathrm{ml})$. The reaction mixture was purified using the Biotage $®$ Isolera One Normal phase silicagel flash chromatography ( $n$-Heptane:EtOAc, 100:0 to 60:40 over $1 \mathrm{~h}$ ) to obtain a brown oil in 72\% (0.158 g, 0.36 mmol) yield. ${ }^{1} \mathbf{H}$ NMR (400 MHz, $\mathbf{C D C l}_{3}$ ) $\boldsymbol{\delta} 11.44$ (br s, 1H), 8.54 (dd, $J=3.0 \mathrm{~Hz}, 1 \mathrm{H}), 8.18(\mathrm{~d}, J=7.0 \mathrm{~Hz}, 1 \mathrm{H}), 6.98(\mathrm{dd}, J=7.4,4.9 \mathrm{~Hz}, 1 \mathrm{H}), 5.53(\mathrm{br} \mathrm{d}, J=5.2 \mathrm{~Hz}, 1 \mathrm{H})$, 4.48 (br s, 1H), 3.87 (br s, 1H), 3.52 (br s, 1H), 1.54 (s, 9H), 1.42 (s, 9H), 1.10 (s, 9H) ppm. ${ }^{13} \mathbf{C}$ NMR

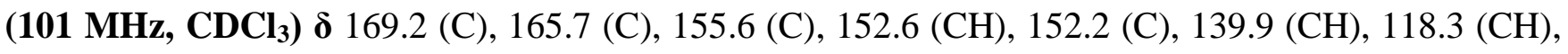


$113.2(\mathrm{C}), 83.0(\mathrm{C}), 79.8(\mathrm{C}), 73.6(\mathrm{C}), 62.0\left(\mathrm{CH}_{2}\right), 56.2(\mathrm{CH}), 28.4\left(\mathrm{CH}_{3}\right), 28.1\left(\mathrm{CH}_{3}\right), 27.3\left(\mathrm{CH}_{3}\right) \mathrm{ppm}$. Spectroscopic data are reported in literature. ${ }^{1}$ HRMS (ESI+) $\mathrm{m} / \mathrm{z}$ calcd. for $\mathrm{C}_{22} \mathrm{H}_{36} \mathrm{~N}_{3} \mathrm{O}_{6}[\mathrm{M}+\mathrm{H}]^{+}$: 438.2599 found 438.2588. [ $\alpha]_{\mathbf{D}}=-24\left(c 10 \mathrm{mg} / \mathrm{ml}, \mathrm{CHCl}_{3}, 27^{\circ} \mathrm{C}\right)$.

LC-CD analysis on a Chiralpak IA column (retention time $=3.02 \mathrm{~min}, 250 \mathrm{~nm}, 1.0 \mathrm{~mL} / \mathrm{min}$ ) revealed $99 \%$ ee.

tert-Butyl 2-\{[ $N^{2}, N^{6}$-bis(tert-butoxycarbonyl)-L-lysyl]amino $\}$ nicotinate (Boc-L-Lys(Boc)-NH-tBu-nic, ER1718) L-4k

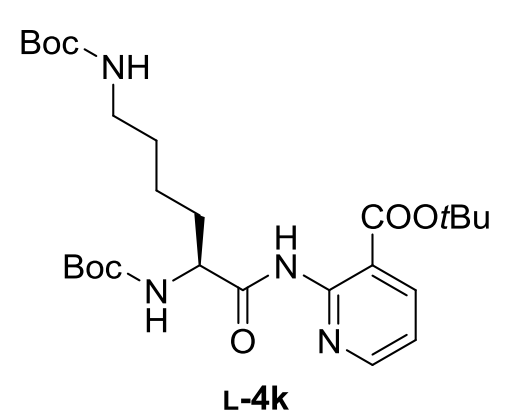

The title compound was isolated using general procedure A1, from BocL-Lys(Boc)- $\mathrm{NH}_{2}$ (0.380 g, $1.1 \mathrm{mmol}, 1.1$ equiv), $\mathrm{Cs}_{2} \mathrm{CO}_{3}(0.391 \mathrm{~g}, 1.2$ mmol, 1.2 equiv), Pd-G3-dcpf 3 (52 mg, $0.050 \mathrm{mmol}, 5 \mathrm{~mol} \%$ ), and and tert-butyl 2-chloronicotinate 2 (214 $\mathrm{mg}, 1.0 \mathrm{mmol}, 1$ equiv) in anhydrous 2-MeTHF $(3.0 \mathrm{ml})$. The reaction mixture was purified using the Biotage ${ }^{\circledR}$ Isolera One Normal phase silicagel flash chromatography ( $n$-Heptane:EtOAc, 100:0 to 50:50 over $1 \mathrm{~h}$ ) to obtain a white solid in $78 \%$ (0.406 g, $0.778 \mathrm{mmol}$ ) yield. M.p. $162^{\circ} \mathrm{C}^{1}{ }^{1} \mathbf{H}$ NMR (400 MHz, $\left.\mathbf{C D C l}_{3}\right) \boldsymbol{\delta} 11.31$ (br s, $\left.1 \mathrm{H}\right), 8.56(\mathrm{~d}, J=3.2 \mathrm{~Hz}, 1 \mathrm{H}), 8.23$ (dd, $J=$ 7.9, $1.9 \mathrm{~Hz}, 1 \mathrm{H}), 7.03$ (dd, J= 7.8, $4.8 \mathrm{~Hz}, 1 \mathrm{H}), 5.33$ (br d, $J=4.7 \mathrm{~Hz}, 1 \mathrm{H}), 4.70$ (br s, 1H), 4.62 (br s, 1H), 3.18-3.02 (m, 2H), 2.04-1.90 (m, 1H), 1.77 - $1.63(\mathrm{~m}, 2 \mathrm{H}), 1.59$ (s, 9H), 1.45 (s, 9H), 1.55 - 1.47

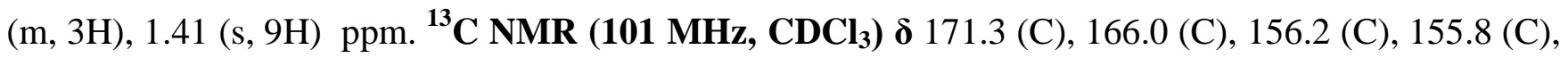
$152.7(\mathrm{CH}), 152.4(\mathrm{C}), 140.2(\mathrm{CH}), 118.4(\mathrm{CH}), 112.9(\mathrm{C}), 83.6(\mathrm{C}), 79.9(\mathrm{C}), 79.1(\mathrm{C}), 55.6(\mathrm{CH}), 40.3$ $\left(\mathrm{CH}_{2}\right), 32.8\left(\mathrm{CH}_{2}\right), 29.6\left(\mathrm{CH}_{2}\right), 28.6\left(\mathrm{CH}_{3}\right), 28.5\left(\mathrm{CH}_{3}\right), 28.2\left(\mathrm{CH}_{3}\right), 22.6\left(\mathrm{CH}_{2}\right) \mathrm{ppm}$. Spectroscopic data are reported in literature. ${ }^{1}$ HRMS (ESI+) m/z calcd. for $\mathrm{C}_{26} \mathrm{H}_{43} \mathrm{~N}_{4} \mathrm{O}_{7}[\mathrm{M}+\mathrm{H}]^{+}: 523.3126$ found 523.3126 $[\boldsymbol{\alpha}]_{\mathbf{D}}=-15\left(\right.$ c $\left.10 \mathrm{mg} / \mathrm{ml}, \mathrm{CHCl}_{3}, 20^{\circ} \mathrm{C}\right)$.

LC-CD analysis on a Chiralpak IA column (retention time $=4.25 \mathrm{~min}, 250 \mathrm{~nm}, 1.0 \mathrm{~mL} / \mathrm{min}$ ) revealed $100 \%$ ee.

tert-Butyl (R)-2-(2-((tert-butoxycarbonyl)amino)-3-(tert-butylthio)propanamido)nicotinate (Boc-LCys( $t \mathrm{Bu})-\mathrm{NH}-t \mathrm{Bu}-\mathrm{nic}$, ER1625/ER1676) L-4l

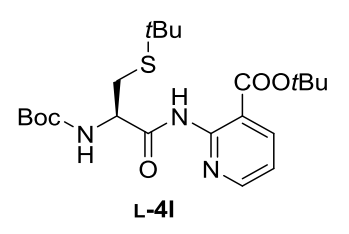

The title compound was isolated using general procedure A2, from Boc-L$\mathrm{Cys}(t \mathrm{Bu})-\mathrm{NH}_{2}\left(0.304 \mathrm{mg}, 1.1 \mathrm{mmol}, 1.1\right.$ equiv), $\mathrm{K}_{2} \mathrm{CO}_{3}(1.03 \mathrm{~g}, 7.5 \mathrm{mmol}, 7.5$ equiv), Pd-G3-dcpf 3 (103 mg, $0.1 \mathrm{mmol}, 10 \mathrm{~mol} \%$ ) and tert-butyl 2chloronicotinate 2 (214 mg, $1 \mathrm{mmol}, 1$ equiv) in anhydrous 1,4-dioxane (6 ml). The reaction mixture was purified using the Biotage ${ }^{\circledR}$ Isolera One Normal phase silicagel flash chromatography ( $n$-Heptane:EtOAc, 100:0 to 50:50 over $1 \mathrm{~h}$ ) to obtain a brown oil in 89\% (405 $\mathrm{mg}$, 0.89 mmol) yield. ${ }^{1} \mathbf{H}$ NMR (400 MHz, $\mathbf{C D C l}_{3}$ ) $\boldsymbol{\delta} 11.49$ (br s, $\left.1 \mathrm{H}\right), 8.59$ (dd, $\left.J=4.7,1.8 \mathrm{~Hz}, 1 \mathrm{H}\right), 8.24$ 
(dd, $J=7.9,1.9 \mathrm{~Hz}, 1 \mathrm{H}), 7.04$ (dd, $J=7.8,4.8 \mathrm{~Hz}, 1 \mathrm{H}), 5.48$ (br s, 1H), 4.79 (br s, 1H), 3.07 (d, $J=5.3$ $\mathrm{Hz}, 2 \mathrm{H}), 1.60$ (s, 9H), 1.4 (s, 9H), 1.33 (s, 9H) ppm. ${ }^{13} \mathbf{C}$ NMR (101 MHz, CDCl$)_{3} \delta 169.5$ (C), 165.9 (C), $152.8(\mathrm{CH}), 152.3(\mathrm{C}), 140.1(\mathrm{CH}), 118.5(\mathrm{CH}), 113.2(\mathrm{C}), 83.4(\mathrm{C}), 80.1(\mathrm{C}), 55.8(\mathrm{CH}), 42.9(\mathrm{C})$, $31.3\left(\mathrm{CH}_{2}\right), 31.0\left(\mathrm{CH}_{3}\right), 28.5\left(\mathrm{CH}_{3}\right), 28.2\left(\mathrm{CH}_{3}\right)$ ppm. HRMS (ESI+) m/z calcd. for $\mathrm{C}_{28} \mathrm{H}_{40} \mathrm{~N}_{3} \mathrm{O}_{6}$ $[\mathrm{M}+\mathrm{H}]^{+}: 454.2371$ found $454.2376 .[\alpha]_{\mathbf{D}}=-33\left(c 10 \mathrm{mg} / \mathrm{ml}, \mathrm{CHCl}_{3}, 21^{\circ} \mathrm{C}\right)$.

LC-CD analysis on a Chiralpak IA column (retention time $=3.87 \mathrm{~min}, 250 \mathrm{~nm}, n$-hexane:EtOH 80:20, $1.0 \mathrm{ml} / \mathrm{min}$ ) revealed $100 \%$ ee.

tert-Butyl 2-\{[N-(tert-butoxycarbonyl)-1-(triphenylmethyl)-L-histidyl $]$ amino $\}$ pyridine-3-carboxylate (Boc-L-His(Trt)-NH-tBu-nic, ER1738) L-4m

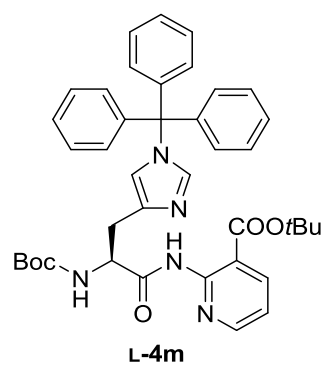

The title compound was isolated using a slightly modified general procedure A1, from Boc- L-His(Trt)- $\mathrm{NH}_{2}\left(0.273 \mathrm{~g}, 0.55 \mathrm{mmol}, 1.1\right.$ equiv), $\mathrm{Cs}_{2} \mathrm{CO}_{3}(0.195 \mathrm{~g}, 0.6$ mmol, 1.2 equiv), Pd-G3-dcpf 3 (52 mg, $0.05 \mathrm{mmol}, 10 \mathrm{~mol} \%)$, and tert-butyl 2chloronicotinate 2 (107 $\mathrm{mg}, 0.5 \mathrm{mmol}, 1$ equiv) in a mixture of anhydrous 2MeTHF and DMF $(1: 1 \mathrm{v} / \mathrm{v}, 3.13 \mathrm{ml})$. The reaction mixture was stirred at $40{ }^{\circ} \mathrm{C}$ for $16 \mathrm{~h}$. The reaction mixture was purified using the Biotage ${ }^{\circledR}$ Isolera One Normal phase silicagel flash chromatography ( $n$-Heptane:EtOAc, 100:0 to 0:100 over $1 \mathrm{~h}$ ) to obtain a brown oil in 56\% (188 mg, $0.28 \mathrm{mmol})$ yield. ${ }^{\mathbf{1}} \mathbf{H} \mathbf{N M R}\left(\mathbf{4 0 0} \mathbf{M H z}, \mathbf{C D C l}_{3}\right) \boldsymbol{\delta} 11.48(\mathrm{~s}, 1 \mathrm{H}), 8.56(\mathrm{~d}, J=$ $2.8 \mathrm{~Hz}, 1 \mathrm{H}), 8.23(\mathrm{dd}, J=7.8,1.7 \mathrm{~Hz}, 1 \mathrm{H}), 7.36(\mathrm{~s}, 1 \mathrm{H}), 7.30-7.18(\mathrm{~m}, 9 \mathrm{H}), 7.05(\mathrm{~d}, J=7.0 \mathrm{~Hz}, 7 \mathrm{H})$, $6.68(\mathrm{~s}, 1 \mathrm{H}), 6.47(\mathrm{~d}, J=5.9 \mathrm{~Hz}, 1 \mathrm{H}), 4.73(\mathrm{~s}, 1 \mathrm{H}), 3.22(\mathrm{dd}, J=9.4,8.3 \mathrm{~Hz}, 1 \mathrm{H}), 3.10$ (dd, $J=14.1,4.0$ Hz, 1H), 1.56 (s, 9H), 1.45 (s, 9H) ppm. ${ }^{13} \mathbf{C}$ NMR (101 MHz, CDCl $\mathbf{3}$ ) $\boldsymbol{\delta} 170.3$ (C), 165.5 (C), 155.8 (C), $152.5(\mathrm{C}), 152.2(\mathrm{CH}), 142.3(\mathrm{C}), 139.8(\mathrm{CH}), 138.5(\mathrm{CH}), 136.6(\mathrm{C}), 129.7(\mathrm{CH}), 127.9(\mathrm{CH})$, $127.9(\mathrm{CH}), 119.7(\mathrm{CH}), 118.1(\mathrm{CH}), 113.2(\mathrm{C}), 82.9(\mathrm{C}), 79.5(\mathrm{C}), 75.2(\mathrm{C}), 56.3(\mathrm{CH}), 30.2\left(\mathrm{CH}_{2}\right)$, $28.4\left(\mathrm{CH}_{3}\right), 28.0\left(\mathrm{CH}_{3}\right)$ ppm. No spectroscopic data are reported in literature. HRMS (ESI+) m/z calcd. for $\mathrm{C}_{40} \mathrm{H}_{44} \mathrm{~N}_{5} \mathrm{O}_{5}[\mathrm{M}+\mathrm{H}]^{+}: 674.3337$ found $674.3347[\alpha]_{\mathbf{D}}=-26\left(c 10 \mathrm{mg} / \mathrm{ml}, \mathrm{CHCl}_{3}, 19^{\circ} \mathrm{C}\right)$.

LC-CD analysis on a Chiralpak IA column (retention time $=8.58 \mathrm{~min}, 250 \mathrm{~nm}, n$-hexane: $i \operatorname{PrOH}$ 65:35, $1.0 \mathrm{ml} / \mathrm{min}$ ) revealed $98 \%$ ee.

1-(tert-Butoxycarbonyl)-L-prolyl-L-leucyl- $N$-[3-tert-butyoxycarbonyl)pyridin-2-yl)]glycinamide (BocL-Pro-L-Leu-Gly-NH-tBu-nic, CW-1843) L,L-4n

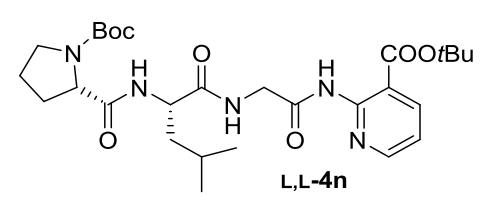

The title compound was isolated using general procedure A1, from BocL-Pro-L-Leu-Gly-NH 2 (1.230 g, 3.20 mmol, 1.1 equiv), $\mathrm{Cs}_{2} \mathrm{CO}_{3}(7.11 \mathrm{~g}$, 21.81 mmol, 7.5 equiv), Pd-G3-dcpf 3 (299 mg, $0.291 \mathrm{mmol}, 10 \mathrm{~mol} \%$ ), and tert-butyl 2-chloronicotinate 2 (621 mg, 2.91mmol, 1 equiv) in anhydrous 2-MeTHF (20.0 ml). The 
reaction mixture was purified using the Biotage ${ }^{\circledR}$ Isolera One Normal phase silicagel flash chromatography ( $n$-Heptane:EtOAc, 100:0 to 0:100 over $1 \mathrm{~h}$ ) to obtain a white solid in 99\% (1.625 g, $2.90 \mathrm{mmol}$ ) yield. M.p. $182^{\circ} \mathrm{C} .{ }^{1} \mathbf{H}$ NMR (400 $\mathbf{~ M H z , ~} \mathbf{C D C l}_{3}$, mixture of rotamers) $\delta 11.16$ (br s, $1 \mathrm{H}$ ), 8.54 (dd, $J=4.6,1.0 \mathrm{~Hz}, 1 \mathrm{H}), 8.24$ (dd, $J=7.9,1.9 \mathrm{~Hz}, 1 \mathrm{H}), 7.33$ (br s, 0.6H), 7.08 (br s, 0.4H), 7.03 (dd, $J=7.8,4.8 \mathrm{~Hz}, 1 \mathrm{H}), 6.86$ (br s, $0.4 \mathrm{H}), 6.47$ (br s, 0.5H), 4.59 (br, 1H), 4.49 (dd, $J=18.5,5.1 \mathrm{~Hz}$, 1H), 4.31 (br s, 2H), 3.42 (br s, 2H), 2.26 (br, 1H), 2.07 (br, 1H), 1.90 (br s, 3H), 1.69-1.63 (m, 1H), $1.61(\mathrm{~s}, 9 \mathrm{H}), 1.43(\mathrm{~s}, 9 \mathrm{H}), 0.93(\mathrm{t}, J=6.1 \mathrm{~Hz}, 6 \mathrm{H}) \mathrm{ppm} .{ }^{13} \mathbf{C} \mathbf{N M R}\left(\mathbf{1 0 1} \mathbf{M H z}, \mathbf{C D C l}_{3}\right) \boldsymbol{\delta} 172.7(\mathrm{C})$, $172.3(\mathrm{C}), 168.6(\mathrm{C}), 165.8(\mathrm{C}), 156.3(\mathrm{C}), 152.6(\mathrm{CH}), 152.1(\mathrm{C}), 140.4(\mathrm{CH}), 118.3(\mathrm{CH}), 112.2(\mathrm{C})$, $83.5(\mathrm{C}), 80.9(\mathrm{C}), 60.7(\mathrm{CH}), 51.7(\mathrm{CH}), 47.4\left(\mathrm{CH}_{2}\right), 45.4\left(\mathrm{CH}_{2}\right), 40.4\left(\mathrm{CH}_{2}\right), 32.0\left(\mathrm{CH}_{2}\right), 29.8\left(\mathrm{CH}_{2}\right)$, $29.4\left(\mathrm{CH}_{2}\right), 28.4\left(\mathrm{CH}_{3}\right), 28.2\left(\mathrm{CH}_{3}\right), 25.0\left(\mathrm{CH}_{3}\right), 21.5(\mathrm{CH})$ ppm. Spectroscopic data are reported in

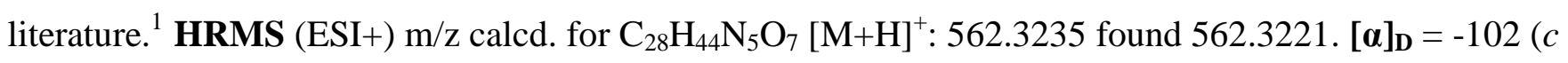
$\left.10 \mathrm{mg} / \mathrm{ml}, \mathrm{CHCl}_{3}, 21^{\circ} \mathrm{C}\right)$. 
$N^{2}$-(tert-Butoxycarbonyl)- $N^{5}$-(3-(tert-butoxycarbonyl)pyridin-2-yl)-L-glutamine $\quad$ (Boc-L-Gln(NH- $t \mathrm{Bu}-$ nic)-OH, DM613) L-4o

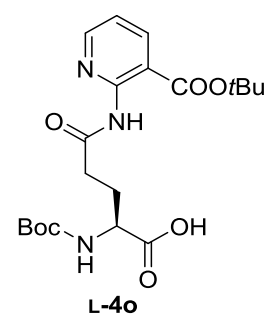

The title compound was isolated using general procedure A3, from Boc-L-Gln-OH (369 mg, $1.5 \mathrm{mmol}), \mathrm{Pd}(\mathrm{OAc})_{2}(11 \mathrm{mg}, 0.05 \mathrm{mmol}, 5 \mathrm{~mol} \%)$, 9,9-dimethyl-4,5bis(diphenylphosphino)xanthene (58 $\mathrm{mg}, 0.1 \mathrm{mmol}, 10 \mathrm{~mol} \%$ ), tert-butyl 2chloronicotinate $2(214 \mathrm{mg}, 1 \mathrm{mmol})$ and anhydrous 1,4-dioxane (1.3 ml). While vigorous stirring, $\mathrm{Cs}_{2} \mathrm{CO}_{3}(391 \mathrm{mg}, 1.2 \mathrm{mmol})$ was added and the resulting mixture was flushed with argon. The mixture was vigorously stirred at $90^{\circ} \mathrm{C}$ for $24 \mathrm{~h}$. After cooling down to room temperature, EtOAc was added and the mixture was purified with Combiflash ${ }^{\circledR}$ RF200 reverse phase silica gel flash chromatography (Water $+1 \%$ formic acid:Acetonitrile, 100:0 to 0:100 in $80 \mathrm{~min}$ ) (Chromabond®Flash RS25 C18 Cartridges, $25 \mathrm{~g}, 25 / 67 \mathrm{~mL}$; flow rate $18 \mathrm{~mL} / \mathrm{min}$, max. operating pressure 150 psi - 10 bar.), yielding the desired compound as a pale yellow solid in $60 \%$ isolated yield (214 mg, 0.5 mmol). ${ }^{1} \mathbf{H}$ NMR (400 MHz, DMSO-d $\left.\boldsymbol{d}_{\boldsymbol{6}}\right) \boldsymbol{\delta} 12.48$ (s, 1H), 10.53 (s, 1H), 8.48 (dd, $J=4.7$, $1.7 \mathrm{~Hz}, 1 \mathrm{H}), 8.03(\mathrm{dd}, J=7.7,1.7 \mathrm{~Hz}, 1 \mathrm{H}), 7.26(\mathrm{dd}, J=7.7,4.8 \mathrm{~Hz}, 1 \mathrm{H}), 7.09$ (d, $J=7.9 \mathrm{~Hz}, 1 \mathrm{H}), 3.94$ (dd, $J=13.1,8.7 \mathrm{~Hz}, 1 \mathrm{H}), 2.12-1.95(\mathrm{~m}, 1 \mathrm{H}), 1.82$ (td, $J=16.2,7.8 \mathrm{~Hz}, 1 \mathrm{H}), 1.49$ (s, 9H), 1.39 (s,

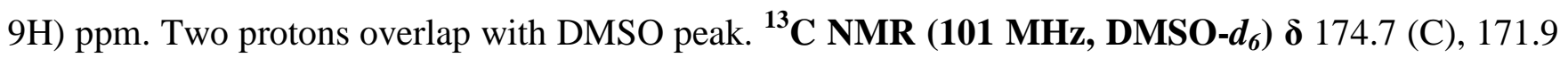
(C), $165.9(\mathrm{C}), 156.4(\mathrm{C}), 151.4(\mathrm{CH}), 149.5(\mathrm{C}), 139.6(\mathrm{CH}), 121.9(\mathrm{C}), 120.7(\mathrm{CH}), 82.0\left(\mathrm{CH}_{2}\right), 78.9$ $\left(\mathrm{CH}_{2}\right), 53.9(\mathrm{CH}), 33.2(\mathrm{C}), 29.1\left(\mathrm{CH}_{3}\right), 28.7\left(\mathrm{CH}_{3}\right), 27.0(\mathrm{C}) \mathrm{ppm}$. HRMS (ESI+) m/z calcd. for

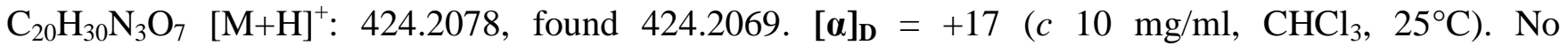
spectroscopic data are reported in literature. The chiral purity was checked by transformation into a dipeptide (see section 5.2).

$N$-[3-(tert-butoxycarbonyl)pyridin-2-yl]- $N$-2-\{[(9H-fluoren-9-yl)methoxy]carbonyl $\}$-L-glutamine (Fmoc-L-Gln(NH-tBu-nic)-OH, DM683) L-4p
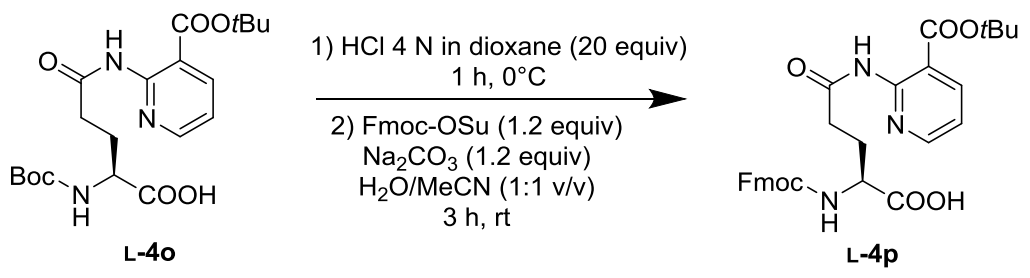

Step 1: $\quad N^{2}$-(tert-butoxycarbonyl)- $N$-[3-(tert-butoxycarbonyl)pyridyn-2-yl]-L-glutamine [Boc-L$\mathrm{Gln}(t \mathrm{Bu}-\mathrm{nic})-\mathrm{OH}] \mathbf{L}-4 \mathbf{0}(1.12 \mathrm{~g}, 2.64 \mathrm{mmol})$ was cooled to $0^{\circ} \mathrm{C}$ and $4 \mathrm{M}$ Hydrochloric acid in dioxane 
(13.22 $\mathrm{ml}, 52.9 \mathrm{mmol})$ was added dropwise. After $1 \mathrm{~h}$, the solvents were removed in vacuo to yield the corresponding ammonium chloride that was used directly in the subsequent reaction step.

Step 2: The amino acid coming from the previous step was dissolved in water $(26.4 \mathrm{ml})$ and acetonitrile $(26.4 \mathrm{ml})$. Then, Fmoc-OSu $(1.071 \mathrm{~g}, 3.17 \mathrm{mmol})$ and sodium carbonate $(0.336 \mathrm{~g}, 3.17$ mmol) were added until a $\mathrm{pH}$ in the range between 8-10 was reached. After $3 \mathrm{~h}$, the solvent was removed and the crude was dissolved in EtOAc $(100 \mathrm{ml})$, washed with $1 \mathrm{M} \mathrm{HCl}(50 \mathrm{ml})$. The organic phase was then dried over $\mathrm{MgSO}_{4}$ and evaporated. The mixture was then purified by flash column chromatography using Combiflash ${ }^{\circledR}$ RF200 reverse phase silica gel flash chromatography (Water $+1 \%$ formic acid:Acetonitrile, 100:0 to 0:100 in $80 \mathrm{~min}$ ), yielding $N$-[3-(tert-butoxycarbonyl)pyridin-2-yl]- $N$ 2-\{[(9H-fluoren-9-yl)methoxy]carbonyl $\}$ - L-glutamine [Fmoc-L-Gln( $t \mathrm{Bu}$-nic)-OH] as a white crystals in $78 \%$ isolated yield (1.13 g, $2.01 \mathrm{mmol}) .{ }^{1} \mathbf{H}$ NMR (400 MHz, DMSO-d 6 ) $\delta 10.56(\mathrm{~s}, 1 \mathrm{H}), 8.47(\mathrm{dd}, J=$ 4.8, $1.8 \mathrm{~Hz}, 1 \mathrm{H}), 8.03(\mathrm{dd}, \mathrm{J}=7.7,1.7 \mathrm{~Hz}, 1 \mathrm{H}), 7.90(\mathrm{~d}, J=7.5 \mathrm{~Hz}, 2 \mathrm{H}), 7.73(\mathrm{~d}, J=7.4 \mathrm{~Hz}, 2 \mathrm{H}), 7.65$ $(\mathrm{d}, J=7.8 \mathrm{~Hz}, 1 \mathrm{H}), 7.42(\mathrm{t}, J=7.4 \mathrm{~Hz}, 2 \mathrm{H}), 7.34(\mathrm{t}, J=7.4 \mathrm{~Hz}, 2 \mathrm{H}), 7.25(\mathrm{dd}, J=7.7,4.8 \mathrm{~Hz}, 1 \mathrm{H}), 4.33$ $-4.18(\mathrm{~m}, 3 \mathrm{H}), 4.02(\mathrm{dd}, J=13.2,8.8 \mathrm{~Hz}, 1 \mathrm{H}), 2.16-2.03(\mathrm{~m}, 1 \mathrm{H}), 1.88(\mathrm{td}, J=16.4,7.9 \mathrm{~Hz}, 1 \mathrm{H})$, $1.48(\mathrm{~s}, 9 \mathrm{H}) \mathrm{pm}$. Two protons overlap with DMSO peak. ${ }^{13} \mathbf{C}$ NMR (100 MHz, DMSO-d $\left.\boldsymbol{\sigma}\right) \boldsymbol{\delta} 174.0(\mathrm{C})$, $171.5(\mathrm{C}), 165.5(\mathrm{C}), 156.6(\mathrm{C}), 151.0(\mathrm{CH}), 149.1(\mathrm{C}), 144.3(\mathrm{C}), 141.2(\mathrm{C}), 139.2(\mathrm{CH}), 128.1(\mathrm{CH})$, $127.5(\mathrm{CH}), 125.7(\mathrm{CH}), 121.5(\mathrm{C}), 120.6(\mathrm{CH}), 120.3(\mathrm{CH}), 81.6(\mathrm{C}), 66.1\left(\mathrm{CH}_{2}\right), 53.9(\mathrm{CH}), 47.1$ $(\mathrm{CH}), 32.8\left(\mathrm{CH}_{2}\right), 28.2\left(\mathrm{CH}_{3}\right), 26.7\left(\mathrm{CH}_{2}\right)$ ppm. HRMS (ESI+) m/z calcd. for $\mathrm{C}_{30} \mathrm{H}_{31} \mathrm{~N}_{3} \mathrm{O}_{7}[\mathrm{M}+\mathrm{Na}]^{+}$: 568.2054, found 568.2060. $[\alpha]_{\mathbf{D}}=+17\left(c 10 \mathrm{mg} / \mathrm{ml}, \mathrm{CHCl}_{3}, 21^{\circ} \mathrm{C}\right)$. No spectroscopic data are reported in literature.

Remark: Compounds L-4e, L-4h, L-4i, L-4k were synthesized starting from enantiopure peptidic amide starting material. In our previous publication, ${ }^{[1]}$ the substrates were partially epimerized which explains the difference observed in comparison to the current data.

\subsection{Effect of tert-butyl 2-chloronicotinate introduction on the chiral purity of Boc-L-Gln-OH}

The evaluation of the tert-butyl 2-chloronicotinate introduction on the chiral purity of the Boc-L-Gln$\mathrm{OH}$ side chain was performed by means of coupling Boc-L-Gln(tBu-nic)-OH L-4o with H-L-Val-OMe. The diastereoisomer of Boc-L-Gln( $t$ Bu-nic)-L-Val-OMe L,L-21, i.e. Boc-D-Gln( $t$ Bu-nic)-L-Val-OMe D,L-22, was synthesized as reference. Boc-D-Gln( $t$ Bu-nic)-OH D-4o was obtained by introducing the $t \mathrm{Bu}-n i c$ directing group on the side chain of Boc-D-Gln-OH and then coupled with H-L-Val-OMe. The two diasteromeric dipeptides, Boc-L-Gln( $t$ Bu-nic)-L-Val-OMe L,L-21 and Boc-D-Gln( $t$ Bu-nic)-L-ValOMe D,L-21 obtained, were subsequently analysed by LC. 
$N^{2}$-(tert-Butoxycarbonyl)- $N^{5}$-(3-(tert-butoxycarbonyl)pyridin-2-yl)-D-glutamine $\quad$ (Boc-D-Gln( $t$ Bu-nic)$\mathrm{OH}, \mathrm{DM} 602) \mathbf{D}-\mathbf{4 0}$

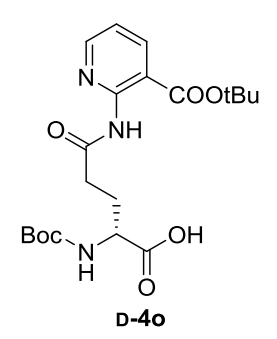

The title compound was isolated using general procedure A4. An oven-dried microwave vial was charged with Boc-D-Gln-OH (369 mg, $1.5 \mathrm{mmol}), \mathrm{Pd}(\mathrm{OAc})_{2}(11$ $\mathrm{mg}, 0.05 \mathrm{mmol}$ ), 9,9-dimethyl-4,5-bis(diphenylphosphino)xanthene (58 mg, 0.1 mmol), tert-butyl 2-chloronicotinate $2(214 \mathrm{mg}, 1 \mathrm{mmol})$ and anhydrous 1,4-dioxane $(1.3 \mathrm{ml})$. While vigorous stirring, $\mathrm{Cs}_{2} \mathrm{CO}_{3}(391 \mathrm{mg}, 1.2 \mathrm{mmol})$ was added and the resulting mixture was flushed with argon. The mixture was vigorously stirred at $90^{\circ} \mathrm{C}$ for 24 hours. After cooling down to room temperature, EtOAc was added and the mixture was purified with Combiflash ${ }^{\circledR}$ RF200 reverse phase silica gel flash chromatography (Water $+1 \%$ formic acid:Acetonitrile, 100:0 to 0:100 in $80 \mathrm{~min}$ ) (Chromabond®Flash RS25 C18 Cartridges, $25 \mathrm{~g}, 25 / 67 \mathrm{~mL}$; flow rate $18 \mathrm{~mL} / \mathrm{min}$, max. operating pressure $150 \mathrm{psi}$ - 10 bar.), yielding the desired compound as a pale yellow solid in 60\% isolated yield (211 mg, $0.5 \mathrm{mmol}){ }^{\mathbf{1}} \mathbf{H}$ NMR (400 MHz, DMSO- $\boldsymbol{d}_{\boldsymbol{\sigma}}$ ) $\boldsymbol{\delta} 12.48$ (s, 1H), $10.53(\mathrm{~s}, 1 \mathrm{H}), 8.48(\mathrm{dd}, J=4.7,1.7 \mathrm{~Hz}, 1 \mathrm{H}), 8.03(\mathrm{dd}, J=7.7,1.7 \mathrm{~Hz}, 1 \mathrm{H}), 7.26(\mathrm{dd}, J=7.7$, $4.8 \mathrm{~Hz}, 1 \mathrm{H}), 7.09(\mathrm{~d}, J=7.9 \mathrm{~Hz}, 1 \mathrm{H}), 3.94(\mathrm{dd}, J=13.1,8.7 \mathrm{~Hz}, 1 \mathrm{H}), 2.12-1.95(\mathrm{~m}, 1 \mathrm{H}), 1.82(\mathrm{td}, J=$ 16.2, $7.8 \mathrm{~Hz}, 1 \mathrm{H}), 1.49$ (s, 9H), 1.39 (s, 9H) ppm. Two protons overlap with DMSO peak. ${ }^{\mathbf{1 3}} \mathbf{C}$ NMR (101 MHz, DMSO-d d $_{\text {) }} \boldsymbol{\delta} 174.7$ (C), 171.9 (C), 165.9 (C), 156.4 (C), $151.4(\mathrm{CH}), 149.5$ (C), 139.6 (CH), $121.9(\mathrm{C}), 120.7(\mathrm{CH}), 82.0\left(\mathrm{CH}_{2}\right), 78.9\left(\mathrm{CH}_{2}\right), 53.9(\mathrm{CH}), 33.2(\mathrm{C}), 29.1\left(\mathrm{CH}_{3}\right), 28.7\left(\mathrm{CH}_{3}\right), 27.0(\mathrm{C})$ ppm. HRMS (ESI+) m/z calcd. for $\mathrm{C}_{20} \mathrm{H}_{30} \mathrm{~N}_{3} \mathrm{O}_{7}[\mathrm{M}+\mathrm{H}]^{+}:$424.2078, found 424.2069. [ $\left.\boldsymbol{\alpha}\right]_{\mathbf{D}}=-14$ (c 10 $\mathrm{mg} / \mathrm{ml}, \mathrm{CHCl}_{3}, 20^{\circ} \mathrm{C}$ ). No spectroscopic data are reported in literature.

Methyl $N^{2}$-(tert-butoxycarbonyl)- $N$-[3-(tert-butoxycarbonyl)pyridin-2-yl]-L-glutaminyl-L-valinate (BocL-Gln( $t$ Bu-nic)-L-Val-OMe, DM584) L,L-21

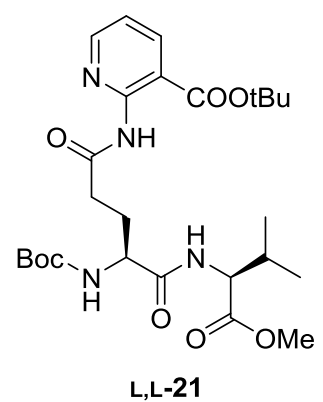

The title compound was obtained following general procedure D. A roundbottom flask was charged with Boc-L-Gln(tBu-nic)-OH L-4o $(190 \mathrm{mg}, 0.45$ mmol), EDC.HCl (103 mg, $0.54 \mathrm{mmol})$, HOBt. $\mathrm{H}_{2} \mathrm{O}$ (82 mg, $\left.0.54 \mathrm{mmol}\right)$ in $\mathrm{CH}_{2} \mathrm{Cl}_{2}(10 \mathrm{ml})$. The solution was stirred at room temperature for 15 minutes. Alongside, H-L-Val-OMe hydrochloric acid salt (75 mg, $0.45 \mathrm{mmol}$ ) in $\mathrm{CH}_{2} \mathrm{Cl}_{2}$ $(10 \mathrm{ml})$ was brought up to $\mathrm{pH} 9$ with $\mathrm{Et}_{3} \mathrm{~N}(33 \mu \mathrm{l}, 0.45 \mathrm{mmol})$. This solution was subsequently added to the pre-activated $\mathrm{N}$-Boc protected amino acid mixture and the reaction was stirred for 2 hours at room temperature. The solvent was removed in vacuo and the residue was redissolved in EtOAc. The organic phase was extracted with $\mathrm{HCl}(3 \mathrm{x}, 1 \mathrm{M})$ and $\mathrm{NaHCO}_{3}(3 \mathrm{x}$, sat.), 
washed with brine, dried over $\mathrm{MgSO}_{4}$, filtered and concentrated in vacuo. The crude product was purified via Biotage ${ }^{\circledR I s o l e r a}$ one automatic flash chromatography with Heptane:EtOAc ( $0 \%$ EtOAc to 100\% EtOAc in $60 \mathrm{~min}, 20.0 \mathrm{ml} / \mathrm{min}$ ) (Grace®Silica Flash Cartridges $12 \mathrm{~g}$ : GraceResolv Silica 12/20ml; flow rate $20 \mathrm{ml} / \mathrm{min}$, max. operating pressure $150 \mathrm{psi}$ - $10 \mathrm{bar}$.) affording the desired product as a pale yellow solid in 95\% isolated yield (40 mg, $0.04 \mathrm{mmol}) .{ }^{\mathbf{1}} \mathbf{H} \mathbf{N M R}\left(\mathbf{4 0 0} \mathbf{M H z}, \mathbf{C D C l}_{3}\right) \boldsymbol{\delta} 10.99$ $(\mathrm{s}, 1 \mathrm{H}), 8.56(\mathrm{~d}, J=3.1 \mathrm{~Hz}, 1 \mathrm{H}), 8.24(\mathrm{~d}, J=6.4 \mathrm{~Hz}, 1 \mathrm{H}), 7.05(\mathrm{dd}, J=7.8,4.8 \mathrm{~Hz}, 1 \mathrm{H}), 6.86(\mathrm{~s}, 1 \mathrm{H})$, $5.83(\mathrm{~s}, 1 \mathrm{H}), 4.53(\mathrm{dd}, J=8.7,5.0 \mathrm{~Hz}, 1 \mathrm{H}), 4.28(\mathrm{~s}, 1 \mathrm{H}), 3.72(\mathrm{~s}, 3 \mathrm{H}), 3.00-2.88(\mathrm{~m}, 1 \mathrm{H}), 2.88-2.76$ $(\mathrm{m}, 1 \mathrm{H}), 2.35-2.06(\mathrm{~m}, 3 \mathrm{H}), 1.61(\mathrm{~s}, 9 \mathrm{H}), 1.44(\mathrm{~s}, 9 \mathrm{H}), 0.94(\mathrm{dd}, J=16.7,6.8 \mathrm{~Hz}, 6 \mathrm{H}) \mathrm{ppm} .{ }^{\mathbf{1 3}} \mathbf{C} \mathbf{N M R}$ $\left(\mathbf{1 0 0} \mathbf{M H z}, \mathbf{C D C l}_{3}\right) \boldsymbol{\delta} 172.3(\mathrm{C}), 172.0(\mathrm{C}), 171.8(\mathrm{C}), 165.8(\mathrm{C}), 152.2(\mathrm{CH}), 152.1(\mathrm{C}), 140.1(\mathrm{CH})$, $118.1(\mathrm{CH}), 83.4(\mathrm{C}), 77.2(\mathrm{C}), 57.2(\mathrm{CH}), 52.0(\mathrm{CH}), 34.5\left(\mathrm{CH}_{2}\right), 31.1\left(\mathrm{CH}_{2}\right), 28.3\left(\mathrm{CH}_{3}\right), 28.1\left(\mathrm{CH}_{3}\right)$, $19.0\left(\mathrm{CH}_{3}\right), 17.7(\mathrm{CH})$ ppm. HRMS (ESI+) m/z calcd for $\mathrm{C}_{26} \mathrm{H}_{41} \mathrm{~N}_{4} \mathrm{O}_{8}[\mathrm{M}+\mathrm{H}]^{+}:$537.2919, found 537.2928. $[\alpha]_{\mathbf{D}}=+10\left(c 10 \mathrm{mg} / \mathrm{ml}, \mathrm{CHCl}_{3}, 20^{\circ} \mathrm{C}\right)$. No spectroscopic data are reported in literature. LC$\mathrm{CD}$ analysis on a Chiralpak IA column (retention time $=6.43 \mathrm{~min}, 250 \mathrm{~nm}, n$-hexane:EtOH 80:20, 1.0 $\mathrm{ml} / \mathrm{min}$ ) revealed $100 \%$ de and ee and indirectly $100 \%$ ee for $\mathbf{L}-\mathbf{4 o}$.

Methyl $N^{2}$-(tert-butoxycarbonyl)- $N$-[3-(tert-butoxycarbonyl)pyridin-2-yl]-D-glutaminyl-L-valinate (Boc-D-Gln( $t$ Bu-nic)-L-Val-OMe, DM593) D,L-21

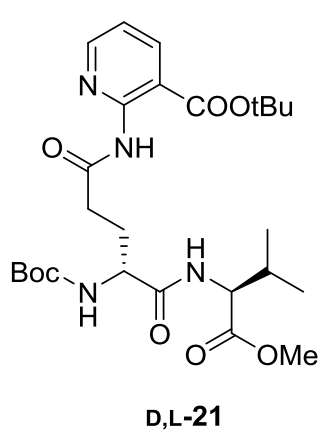

The title compound was obtained following general procedure $\mathbf{D}$. A round-bottom flask was charged with Boc-D-Gln(tBu-nic)-OH D-4o (100 mg, $0.24 \mathrm{mmol})$, EDC.HCl (54 mg, $0.28 \mathrm{mmol})$, HOBt. $\mathrm{H}_{2} \mathrm{O}$ (43 mg, $\left.0.28 \mathrm{mmol}\right)$ in $\mathrm{CH}_{2} \mathrm{Cl}_{2}(5 \mathrm{ml})$. The solution was stirred at room temperature for 15 minutes. Alongside, H-L-ValOMe hydrochloric acid salt (40 mg, $0.24 \mathrm{mmol})$ in $\mathrm{CH}_{2} \mathrm{Cl}_{2}(5 \mathrm{ml})$ was brought up to $\mathrm{pH} 9$ with $\mathrm{Et}_{3} \mathrm{~N}(33 \mu \mathrm{l}, 0.24 \mathrm{mmol})$. This solution was subsequently added to the pre-activated $N$-Boc protected amino acid mixture and the reaction was stirred for 2 hours at room temperature. The solvent was removed in vacuo and the residue was redissolved in EtOAc. The organic phase was extracted with $\mathrm{HCl}(3 \mathrm{x}, 1 \mathrm{M})$ and $\mathrm{NaHCO}_{3}$ (3 x, sat.), washed with brine, dried over $\mathrm{MgSO}_{4}$, filtered and concentrated in vacuo. The crude product was purified via Biotage $囚$ Isolera one automatic flash chromatography with Heptane:EtOAc (0\% EtOAc to 100\% EtOAc in $60 \mathrm{~min}, 20.0 \mathrm{ml} / \mathrm{min}$ ) (Grace®Silica Flash Cartridges $12 \mathrm{~g}$ : GraceResolv Silica 12/20ml; flow rate 20 $\mathrm{ml} / \mathrm{min}$, max. operating pressure $150 \mathrm{psi}$ - $10 \mathrm{bar}$.) affording the desired product as a pale yellow solid in $31 \%$ isolated yield (40 mg, $0.075 \mathrm{mmol}){ }^{\mathbf{1}} \mathbf{H}$ NMR (400 MHz, $\mathbf{C D C l}_{3}$ ) $\boldsymbol{\delta} 10.94(\mathrm{~s}, 1 \mathrm{H}), 8.54$ (dd, $J=$ $4.7,1.8 \mathrm{~Hz}, 1 \mathrm{H}), 8.23(\mathrm{dd}, J=7.8,1.9 \mathrm{~Hz}, 1 \mathrm{H}), 7.10(\mathrm{~d}, J=8.1 \mathrm{~Hz}, 1 \mathrm{H}), 7.04(\mathrm{dd}, J=7.8,4.8 \mathrm{~Hz}, 1 \mathrm{H})$, $5.61(\mathrm{~s}, 1 \mathrm{H}), 4.51(\mathrm{dd}, J=8.7,5.1 \mathrm{~Hz}, 1 \mathrm{H}), 4.30(\mathrm{~d}, J=6.0 \mathrm{~Hz}, 1 \mathrm{H}), 3.72(\mathrm{~s}, 3 \mathrm{H}), 2.90(\mathrm{qd}, J=15.7,8.1$ $\mathrm{Hz}, 2 \mathrm{H}), 2.27-2.06(\mathrm{~m}, 3 \mathrm{H}), 1.61(\mathrm{~s}, 9 \mathrm{H}), 1.42(\mathrm{~s}, 9 \mathrm{H}), 0.93(\mathrm{dd}, J=8.8,7.0 \mathrm{~Hz}, 6 \mathrm{H}) \mathrm{ppm} .{ }^{\mathbf{1 3}} \mathbf{C}$ NMR 
(100 MHz, $\left.\mathbf{C D C l}_{3}\right) \boldsymbol{\delta} 172.4(\mathrm{C}), 172.1(\mathrm{C}), 171.9(\mathrm{C}), 165.8(\mathrm{C}), 152.2(\mathrm{CH}), 152.0(\mathrm{C}), 140.0(\mathrm{CH})$, $118.2(\mathrm{CH}), 83.4(\mathrm{C}), 77.2(\mathrm{C}), 57.4(\mathrm{CH}), 52.0(\mathrm{CH}), 34.4\left(\mathrm{CH}_{2}\right), 31.0\left(\mathrm{CH}_{2}\right), 28.3\left(\mathrm{CH}_{3}\right), 28.1\left(\mathrm{CH}_{3}\right)$, $19.0\left(\mathrm{CH}_{3}\right), 17.8(\mathrm{CH})$ ppm. HRMS (ESI+) $\mathrm{m} / \mathrm{z}$ calcd for $\mathrm{C}_{26} \mathrm{H}_{41} \mathrm{~N}_{4} \mathrm{O}_{8}[\mathrm{M}+\mathrm{H}]^{+}:$537.2919, found 537.2928. $[\boldsymbol{\alpha}]_{\mathbf{D}}=-14\left(\right.$ c $\left.5 \mathrm{mg} / \mathrm{ml}, \mathrm{CHCl}_{3}, 20^{\circ} \mathrm{C}\right)$. No spectroscopic data are reported in literature. LC$\mathrm{CD}$ analysis on a Chiralpak IA column (retention time $=7.48 \mathrm{~min}, 250 \mathrm{~nm}, 1.0 \mathrm{ml} / \mathrm{min}$ ) revealed $100 \%$ de and ee and indirectly $100 \%$ ee for D-4o

As for L-4o, the chiral purity of L-4p was assessed by coupling with H-L-Val-OMe to obtain the dipeptide Fmoc-L-Gln(tBu-nic)-L-Val-OMe L,L-23. The dipeptide L,L-23 was converted into the dipeptide L,L-21 by the interchanging of the Fmoc protecting group with Boc. Finally, the chiral purity of the so obtained dipeptide $\mathbf{L , L - 2 1}$ was assessed by means of both NMR and LC analysis.

Methyl $\quad N$-[3-(tert-butoxycarbonyl)pyridin-2-yl]- $N^{2} \sim-\{[(9 H$-fluoren-9-yl)methoxy $]$ carbonyl $\}$-Lglutaminyl-L-valinate (Fmoc-L-Gln(tBu-nic)-L-Val-OMe L, L-23

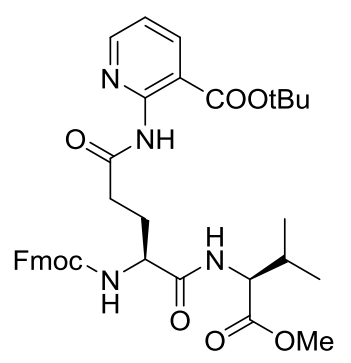

L,L-23

To a solution of Fmoc-L-Gln( $t$ Bu-nic)-OH L-4p $(0.136 \mathrm{~g}, 0.25 \mathrm{mmol})$ in DCM $(4.17 \mathrm{ml}), \mathrm{EDC}(0.058 \mathrm{~g}, 0.300 \mathrm{mmol})$ and 1-hydroxybenzotriazolehydrate $(0.046$ $\mathrm{g}, 0.300 \mathrm{mmol}$ ) were added and the solution was stirred for $20 \mathrm{~min}$. In parallel, to a flask containing H-L-Val-OMe $(0.042 \mathrm{~g}, 0.250 \mathrm{mmol})$ dissolved in DCM (4.17 $\mathrm{ml}), N, N$-di-isopropylethylamine $(0.044 \mathrm{ml}, 0.250 \mathrm{mmol})$ was added. This solution was then added to the activated carboxylic acid mixture and the reaction was stirred for $1 \mathrm{~h}$ at room temperature. Then, the solvent was removed under vacuum and the crude was redissolved in EtOAc $(50 \mathrm{ml})$. The organic layer was washed with $10 \%$ citric acid in water $(3 \times 30 \mathrm{ml})$, saturated $\mathrm{NaHCO}_{3}(3 \times 30 \mathrm{ml})$ and brine, dried over $\mathrm{MgSO}_{4}$ and concentrated in vacuo. The brown oil

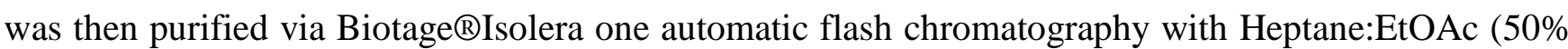
EtOAc to $100 \%$ EtOAc in $60 \mathrm{~min}, 12.0 \mathrm{ml} / \mathrm{min}$ ) (Grace®Silica Flash Cartridges $12 \mathrm{~g}$ : GraceResolv Silica 12/20 ml; flow rate $20 \mathrm{ml} / \mathrm{min}$, max. operating pressure $150 \mathrm{psi}-10 \mathrm{bar}$.) affording the desired product as a pale yellow solid in 58\% isolated yield (96 mg, $0.146 \mathrm{mmol}) .{ }^{\mathbf{1}} \mathbf{H} \mathbf{~ N M R}\left(400 \mathbf{~ M H z}, \mathbf{C D C l}_{3}\right)$ $\boldsymbol{\delta} 10.98(\mathrm{~s}, 1 \mathrm{H}), 8.51(\mathrm{dd}, J=4.8,1.9 \mathrm{~Hz}, 1 \mathrm{H}), 8.22(\mathrm{dd}, J=7.8,1.9 \mathrm{~Hz}, 1 \mathrm{H}), 7.74(\mathrm{~d}, J=7.7 \mathrm{~Hz}, 2 \mathrm{H})$, $7.59(\mathrm{~d}, J=5.8 \mathrm{~Hz}, 2 \mathrm{H}), 7.38(\mathrm{t}, J=7.8 \mathrm{~Hz}, 2 \mathrm{H}), 7.30(\mathrm{~d}, J=7.6 \mathrm{~Hz}, 2 \mathrm{H}), 7.03(\mathrm{dd}, J=7.8,4.8 \mathrm{~Hz}$, $1 \mathrm{H}), 5.98(\mathrm{~d}, J=6.2 \mathrm{~Hz}, 1 \mathrm{H}), 4.51(\mathrm{dd}, J=8.5,5.0 \mathrm{~Hz}, 1 \mathrm{H}), 4.43(\mathrm{dd}, J=13.0,6.8 \mathrm{~Hz}, 1 \mathrm{H}), 4.36(\mathrm{~d}, J$ $=7.1 \mathrm{~Hz}, 2 \mathrm{H}), 4.20(\mathrm{t}, J=7.2 \mathrm{~Hz}, 1 \mathrm{H}), 3.72(\mathrm{~s}, 3 \mathrm{H}), 3.04-2.86(\mathrm{~m}, 2 \mathrm{H}), 2.28-2.11(\mathrm{~m}, 3 \mathrm{H}), 1.56(\mathrm{~s}$, 9H), $0.94(\mathrm{dd}, J=9.0,7.0 \mathrm{~Hz}, 6 \mathrm{H}) \mathrm{ppm} .{ }^{13} \mathbf{C}$ NMR (101 MHz, $\left.\mathbf{C D C l}_{3}\right) \boldsymbol{\delta} 172.6(\mathrm{C}), 172.1(\mathrm{C}), 171.7$ (C), $165.8(\mathrm{C}), 152.2(\mathrm{C}), 151.9$ (C), 143.8 (C), 141.8 (C), 141.3 (C), $140.0(\mathrm{CH}), 127.7$ (CH), 127.1 $(\mathrm{CH}), 125.2(\mathrm{CH}), 119.9(\mathrm{CH}), 118.3(\mathrm{CH}), 83.5(\mathrm{C}), 57.6(\mathrm{CH}), 54.0(\mathrm{CH}), 52.1(\mathrm{CH}), 47.1\left(\mathrm{CH}_{2}\right)$, 
$34.3(\mathrm{CH}), 30.8\left(\mathrm{CH}_{2}\right), 29.7\left(\mathrm{CH}_{2}\right), 28.1\left(\mathrm{CH}_{3}\right), 19.0\left(\mathrm{CH}_{3}\right), 17.9\left(\mathrm{CH}_{3}\right) \mathrm{ppm}$. HRMS (ESI+) m/z calcd for $\mathrm{C}_{36} \mathrm{H}_{42} \mathrm{~N}_{4} \mathrm{O}_{8}[\mathrm{M}+\mathrm{H}]^{+}:$658.3003, found 658.3012. $[\alpha]_{\mathbf{D}}=+34$ (c $\left.1 \mathrm{mg} / \mathrm{ml}, \mathrm{CHCl}_{3}, 20^{\circ} \mathrm{C}\right)$. No spectroscopic data are reported in literature.

Methyl $N^{2}$-(tert-butoxycarbonyl)- $N$-[3-(tert-butoxycarbonyl)pyridin-2-yl]-D-glutaminyl-L-valinate (Boc-L-Gln( $t$ Bu-nic)-L-Val-OMe, DM758) L,L-21 from L,L-23

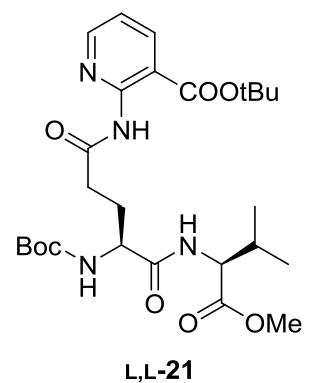

Fmoc-L-GIn( $t$ Bu-nic)-L-Val-OMe L,L-23 (0.096 g, $0.146 \mathrm{mmol})$ was dissolved in $20 \mathrm{~mL}$ of piperidine/DMF solution (1:4). Reaction was stirred for $30 \mathrm{~min}$ at room temperature. The solvent was evaporated and $25 \mathrm{ml}$ of cold ether was added to precipitate the amino acid. The suspended mixture was filtrated over filter paper and rinsed with cold ether. The solid was dried at lyophilisator and used in the next step without purification.

The amino acid coming from the previous step was dissolved in acetonitrile $(2.91 \mathrm{ml})$ and 4dimethylaminopyridine $(3.56 \mathrm{mg}, 0.029 \mathrm{mmol})$ and di-tert-butyldicarbonate $(0.038 \mathrm{~g}, 0.175 \mathrm{mmol})$ were added. After the reaction was completed $(16 \mathrm{~h})$, EtOAc $(50 \mathrm{ml})$ was added and the mixture was washed first with $1 \mathrm{~N} \mathrm{HCl}(20 \mathrm{ml})$ and then with brine $(20 \mathrm{ml})$. The organic phase was then dried over $\mathrm{MgSO}_{4}$ and evaporated. The crude product was purified via Biotage®Isolera one automatic flash chromatography with Heptane:EtOAc (0\% EtOAc to $100 \%$ EtOAc in $60 \mathrm{~min}, 20.0 \mathrm{ml} / \mathrm{min}$ ) (Grace®Silica Flash Cartridges $12 \mathrm{~g}$ : GraceResolv Silica 12/20ml; flow rate $20 \mathrm{ml} / \mathrm{min}$, max. operating pressure 150 psi - 10 bar.) affording the desired product as a pale yellow solid in $83 \%$ isolated yield (65 mg, 0.12 mmol). ${ }^{1} \mathbf{H}$ NMR (400 MHz, $\left.\mathbf{C D C l}_{3}\right) \boldsymbol{\delta} 10.99$ (s, 1H), 8.56 (d, $\left.J=3.1 \mathrm{~Hz}, 1 \mathrm{H}\right), 8.24$ (d, $J=6.4$ $\mathrm{Hz}, 1 \mathrm{H}), 7.05(\mathrm{dd}, J=7.8,4.8 \mathrm{~Hz}, 1 \mathrm{H}), 6.86(\mathrm{~s}, 1 \mathrm{H}), 5.83(\mathrm{~s}, 1 \mathrm{H}), 4.53(\mathrm{dd}, J=8.7,5.0 \mathrm{~Hz}, 1 \mathrm{H}), 4.28$ $(\mathrm{s}, 1 \mathrm{H}), 3.72(\mathrm{~s}, 3 \mathrm{H}), 3.00-2.88(\mathrm{~m}, 1 \mathrm{H}), 2.88-2.76(\mathrm{~m}, 1 \mathrm{H}), 2.35-2.06(\mathrm{~m}, 3 \mathrm{H}), 1.61(\mathrm{~s}, 9 \mathrm{H}), 1.44$ (s, 9H), $0.94(\mathrm{dd}, J=16.7,6.8 \mathrm{~Hz}, 6 \mathrm{H}) \mathrm{ppm} .{ }^{13} \mathbf{C}$ NMR (100 MHz, $\left.\mathbf{C D C l}_{3}\right) \delta 172.3(\mathrm{C}), 172.0$ (C), $171.8(\mathrm{C}), 165.8(\mathrm{C}), 152.2(\mathrm{CH}), 152.1(\mathrm{C}), 140.1(\mathrm{CH}), 118.1(\mathrm{CH}), 83.4(\mathrm{C}), 77.2(\mathrm{C}), 57.2(\mathrm{CH})$, $52.0(\mathrm{CH}), 34.5\left(\mathrm{CH}_{2}\right), 31.1\left(\mathrm{CH}_{2}\right), 28.3\left(\mathrm{CH}_{3}\right), 28.1\left(\mathrm{CH}_{3}\right), 19.0\left(\mathrm{CH}_{3}\right), 17.7(\mathrm{CH})$ ppm. HRMS (ESI+)

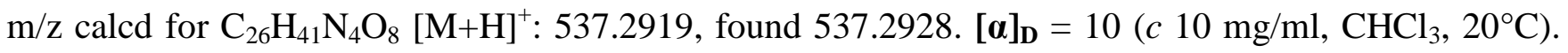
No spectroscopic data are reported in literature. LC-CD analysis on a Chiralpak IA column (retention time $=6.43 \mathrm{~min}, 250 \mathrm{~nm}, n$-hexane:EtOH 80:20, $1.0 \mathrm{ml} / \mathrm{min}$ ) revealed 100\%de and ee and indirectly $100 \%$ ee for $\mathbf{L}-\mathbf{4} \mathbf{p}$. 


\subsection{Dipeptide Synthesis using Transamidation of $N$-Boc Protected $t$ Bu Nicotinate Functionalized}

Amino Acid Amides 4

Methyl (tert-butoxycarbonyl)-L-phenylalanyl-L-phenylalaninate (Boc-L-Phe-L-Phe-OMe, KH_1160) L,L-6a

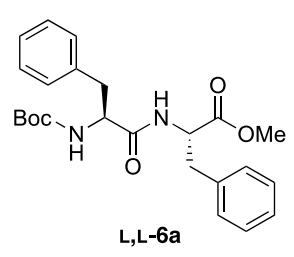

The title compound was prepared using general procedure $\mathbf{B}$ from Boc-L-Phe-NH$t$ Bu-nic L-4a (221 mg, $0.5 \mathrm{mmol}, 1$ equiv), H-L-Phe-OMe hydrochloric acid salt L5a.HCl (119 mg, $0.55 \mathrm{mmol}, 1.1$ equiv), $\mathrm{NaOAc}$ (46 mg, $0.55 \mathrm{mmol}, 1.1$ equiv) and $\mathrm{Zn}(\mathrm{OAc})_{2}(18 \mathrm{mg}, 0.1 \mathrm{mmol}, 20 \mathrm{~mol} \%)$ in THF $(1 \mathrm{ml})$. This yielded, after Grace Reveleris® X2 Normal Phase silicagel flash chromatography $\left(\mathrm{CH}_{2} \mathrm{Cl}_{2}\right.$ :EtOAc, 100:0 to 70:30 over 10 $\mathrm{CV}$ ), the desired compound as a white powder in 95\% (203 mg, $0.477 \mathrm{mmol})$ yield. ${ }^{1} \mathbf{H}$ NMR (500 MHz, $\left.\mathbf{C D C l}_{3}\right) \boldsymbol{\delta}$ 7.21-7.09 (m, 8H), 6.91-6.90 (m, 2H), 6.29 (br s, 1H), 4.94 (br s, 1H), $4.72(\mathrm{dd}, J=$ 5.4, $11.5 \mathrm{~Hz}, 1 \mathrm{H}), 4.28$ (br s, 1H), $3.58(\mathrm{~s}, 3 \mathrm{H}), 3.01-2.92(\mathrm{~m}, 4 \mathrm{H}), 1.32$ (s, 9H) ppm. ${ }^{\mathbf{1 3}} \mathbf{C}$ NMR (126 MHz, $\left.\mathbf{C D C l}_{3}\right) \boldsymbol{\delta} 171.5$ (C), 170.9 (C), 155.4 (C), 136.6 (C), 135.7 (C), $129.4(\mathrm{CH}), 129.3(\mathrm{CH}), 128.7$ $(\mathrm{CH}), 128.6(\mathrm{CH}), 127.2(\mathrm{CH}), 127.0(\mathrm{CH}), 80.2(\mathrm{C}), 55.7(\mathrm{CH}), 53.4(\mathrm{CH}), 52.3\left(\mathrm{CH}_{3}\right), 38.3\left(\mathrm{CH}_{2}\right)$. $38.0\left(\mathrm{CH}_{2}\right), 28.3\left(\mathrm{CH}_{3}\right)$ ppm. HRMS (ESI+) m/z: calcd. for $\mathrm{C}_{24} \mathrm{H}_{31} \mathrm{~N}_{2} \mathrm{O}_{5} \mathrm{Na}[\mathrm{M}+\mathrm{Na}]^{+}: 449.2047$ found 449.2057. $[\alpha]_{\mathbf{D}}=+26\left(c 10 \mathrm{mg} / \mathrm{ml}, \mathrm{CHCl}_{3}, 25^{\circ} \mathrm{C}\right)$. The spectroscopic data were in accordance with those previously reported. ${ }^{[6]}$

Prop-1-en-1-yl (tert-butoxycarbonyl)-L-alanyl-L-leucinate (Boc-L-Ala-L-Leu-OAll, KH_1197) L,L-6b

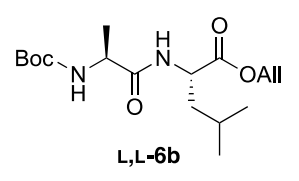

The title compound was prepared using general procedure B from Boc-L-Ala-NHtBu-nic L-4b (183 mg, 0.5 mmol, 1 equiv), H-L-Leu-OAll hydrochloric acid salt L-

5b.HCl (114 mg, $0.55 \mathrm{mmol}, 1.1$ equiv), NaOAc (46 mg, $0.55 \mathrm{mmol}, 1.1$ equiv) and $\mathrm{Zn}(\mathrm{OAc})_{2}(18 \mathrm{mg}, 0.1 \mathrm{mmol}, 20 \mathrm{~mol} \%)$ in THF $(1 \mathrm{ml})$. This yielded, after Grace Reveleris® X2 Normal Phase silica gel flash chromatography $\left(\mathrm{CH}_{2} \mathrm{Cl}_{2}\right.$ :EtOAc, 100:0 to 0:100 over $\left.20 \mathrm{CV}\right)$, the desired compound as a colorless oil in 99\% (170 mg, $0.496 \mathrm{mmol})$ yield. ${ }^{1} \mathbf{H}$ NMR (500 MHz, $\left.\mathbf{C D C l}_{3}\right) \boldsymbol{\delta} 6.52$ (br s, 1H), 5.91 (ddt, $J=10.5,17.1,5.2 \mathrm{~Hz}, 1 \mathrm{H}), 5.33$ (dd, $J=17.1,1.0 \mathrm{~Hz}, 1 \mathrm{H}), 5.26$ (dd, $J=10.5,1.0$ $\mathrm{Hz}, 1 \mathrm{H}), 5.00$ (br s, 1H), 4.65-4.60 (m, 2H), 4.22-4.14 (m, 1H), 1.70-1.64 (m, 3H), 1.61-1.56 (m, 1H), $1.45(\mathrm{~s}, 9 \mathrm{H}), 1.36(\mathrm{~d}, J=7.1 \mathrm{~Hz}, 3 \mathrm{H}), 0.94(\mathrm{~d}, J=6.1 \mathrm{~Hz}, 6 \mathrm{H}) \mathrm{ppm} .{ }^{\mathbf{1 3}} \mathbf{C} \mathbf{N M R}\left(\mathbf{1 2 6} \mathbf{M H z}, \mathbf{C D C l}_{3}\right) \boldsymbol{\delta}$ $172.5(\mathrm{C}), 172.5(\mathrm{C}), 155.7(\mathrm{C}), 131.7(\mathrm{CH}), 118.9\left(\mathrm{CH}_{2}\right), 80.3(\mathrm{C}), 66.0\left(\mathrm{CH}_{2}\right), 50.9(\mathrm{CH}), 50.0(\mathrm{CH})$, $41.7\left(\mathrm{CH}_{2}\right), 28.4\left(\mathrm{CH}_{3}\right), 24.9(\mathrm{CH}), 23.0\left(\mathrm{CH}_{3}\right), 21.9\left(\mathrm{CH}_{3}\right), 18.0\left(\mathrm{CH}_{3}\right) \mathrm{ppm}$. HRMS (ESI+) m/z: calcd.

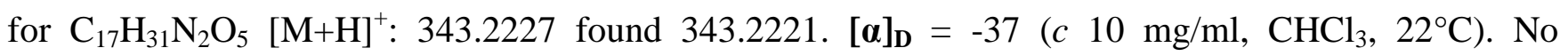
spectroscopic data are reported in literature. 
tert-Butyl ((S)-3-(4-(tert-butoxy)phenyl)-2-((tert-butoxycarbonyl)amino)propanoyl)-L-valinate (Boc-LTyr(tBu)-L-Val-OtBu, KH_1245) L,L-6c

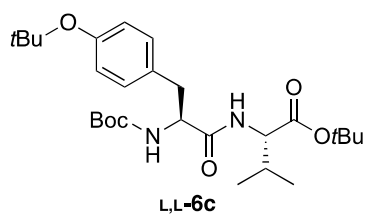

The title compound was prepared using general procedure B, from Boc-L$\operatorname{Tyr}(t \mathrm{Bu})-\mathrm{NH}-t \mathrm{Bu}-\mathrm{nic} \mathbf{L}-4 \mathrm{c}$ (256 mg, $0.5 \mathrm{mmol}, 1$ equiv), H-L-Val-O $t \mathrm{Bu}$ hydrochloric acid salt L-5c.HCl (116 mg, $0.55 \mathrm{mmol}, 1.1$ equiv), NaOAc (46 $\mathrm{mg}, 0.55 \mathrm{mmol}, 1.1$ equiv) and $\mathrm{Zn}(\mathrm{OAc})_{2}(18 \mathrm{mg}, 0.1 \mathrm{mmol}, 20 \mathrm{~mol} \%)$ in THF $(1 \mathrm{ml})$. This yielded, after Grace Reveleris ${ }^{\circledR}$ X2 Normal Phase silicagel flash chromatography (Petroleum Ether:EtOAc, 100:0 to 0:100 over $20 \mathrm{CV}$ ), the desired compound as a colorless oil in 85\% (210 mg, $0.426 \mathrm{mmol}){ }^{\mathbf{1}} \mathbf{H}\left(\mathbf{5 0 0} \mathbf{~ M H z}\right.$, DMSO-d $\boldsymbol{d}_{6}$, mixture of rotamers) $\boldsymbol{\delta}$ 8.00-7.92 (m, 0.2H), 7.88 $(\mathrm{d}, J=8.3 \mathrm{~Hz}, 0.8 \mathrm{H}), 7.21(\mathrm{~d}, J=7.2 \mathrm{~Hz}, 0.2 \mathrm{H}), 7.17(\mathrm{~d}, J=8.3 \mathrm{~Hz}, 1.8 \mathrm{H}), 6.92-6.82(\mathrm{~m}, 2.9 \mathrm{H}), 6.4(\mathrm{br}$ $\mathrm{s}, 0.1 \mathrm{H}), 4.33-4.19(\mathrm{~m}, 1 \mathrm{H}), 4.12-4.02(\mathrm{~m}, 1 \mathrm{H}), 2.96-2.83(\mathrm{~m}, 1 \mathrm{H}), 2.73-2.60(\mathrm{~m}, 1 \mathrm{H}), 2.10-1.97(\mathrm{~m}$, 1H), 1.41 (s, 9H), 1.29 (s, 7.5H), 1.26 (s, 10.5H), 0.89 (dd, $J=6.8,6.8 \mathrm{~Hz}, 5.6 \mathrm{H}), 0.82$ (dd, $J=6.3,6.3$ $\mathrm{Hz}, 0.4 \mathrm{H}) \mathrm{ppm} .{ }^{13} \mathrm{C}$ (126 MHz, DMSO-d $\boldsymbol{d}_{\boldsymbol{\sigma}}$, mixture of rotamers) $\boldsymbol{\delta} 171.9(\mathrm{C}), 170.4(\mathrm{C}), 155.1$ (C), $153.3(\mathrm{C}), 132.7(\mathrm{C}), 129.6(\mathrm{CH}), 123.1(\mathrm{CH}), 80.6(\mathrm{C}), 77.9(\mathrm{C}), 77.4(\mathrm{C}), 57.7(\mathrm{CH}), 55.4(\mathrm{CH}), 36.7$ $\left(\mathrm{CH}_{2}\right), 30.1(\mathrm{CH}), 28.5\left(\mathrm{CH}_{3}\right)$, [28.0, 27.8] $\left(\mathrm{CH}_{3}\right), 27.6\left(\mathrm{CH}_{3}\right), 18.8\left(\mathrm{CH}_{3}\right), 17.9\left(\mathrm{CH}_{3}\right)$ ppm. NMR-data was acquired at elevated temperature so that peaks arising from hindered rotation coalesced. ${ }^{1} \mathbf{H}$ NMR (500 MHz, DMSO-d 6 , 60 ${ }^{\circ}$ C) $67.72(\mathrm{~d}, J=7.2 \mathrm{~Hz}, 1 \mathrm{H}), 7.15(\mathrm{~d}, J=8.2 \mathrm{~Hz}, 2 \mathrm{H}), 6.84(\mathrm{~d}, J=8.2 \mathrm{~Hz}$, 2H), 6.76 (br s, 1H), 4.26 (ddd, $J=9.3,9.3,4.0 \mathrm{~Hz}, 1 \mathrm{H}), 4.10(\mathrm{dd}, J=5.9,8.2 \mathrm{~Hz}, 1 \mathrm{H}), 2.94(\mathrm{dd}, J=$ 14.0, $4.0 \mathrm{~Hz}, 1 \mathrm{H}), 2.71(\mathrm{dd}, J=10.7,13.1 \mathrm{~Hz}, 1 \mathrm{H}), 2.10-2.00(\mathrm{~m}, 1 \mathrm{H}), 1.42(\mathrm{~s}, 9 \mathrm{H}), 1.31(\mathrm{~s}, 9 \mathrm{H}), 1.28$ (s, 9H), 0.94-0.88 (m, 6H) ppm. ${ }^{13} \mathbf{C}\left(126\right.$ MHz, DMSO-d 6 , 60$\left.^{\circ} \mathbf{C}\right) \boldsymbol{\delta} 171.5(\mathrm{C}), 170.0(\mathrm{C}), 154.8(\mathrm{C})$, $153.3(\mathrm{C}), 132.3(\mathrm{C}), 129.4(\mathrm{CH}), 122.8(\mathrm{CH}), 80.4(\mathrm{C}), 77.8(\mathrm{C}), 77.2(\mathrm{C}), 57.6(\mathrm{CH}), 55.3(\mathrm{CH}), 36.6$ $\left(\mathrm{CH}_{2}\right), 30.0(\mathrm{CH}), 28.3\left(\mathrm{CH}_{3}\right), 27.8\left(\mathrm{CH}_{3}\right), 27.4\left(\mathrm{CH}_{3}\right), 18.5\left(\mathrm{CH}_{3}\right), 17.7\left(\mathrm{CH}_{3}\right)$ ppm. HRMS (ESI+) $\mathrm{m} / \mathrm{z}$ : calcd. for $\mathrm{C}_{27} \mathrm{H}_{44} \mathrm{~N}_{2} \mathrm{O}_{6} \mathrm{Na}[\mathrm{M}+\mathrm{Na}]^{+}: 515.3091$ found 515.3090 . [ $\left.\boldsymbol{\alpha}\right]_{\mathbf{D}}=+5$ (c $10 \mathrm{mg} / \mathrm{ml}, \mathrm{CHCl}_{3}$, $25^{\circ} \mathrm{C}$ ). No spectroscopic data are reported in literature.

tert-Butyl $\quad(S)$-2-(((S)-1-methoxy-4-methyl-1-oxopentan-2-yl)carbamoyl)pyrrolidine-1-carboxylate (Boc-L-Pro-L-Leu-OMe, KH_1196) L,L-6d

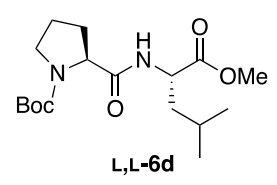

The title compound was prepared using general procedure B, from Boc-L-Pro-NH$t$ Bu-nic L-4d (196 mg, 0.5 mmol, 1 equiv), H-L-Leu-OMe hydrochloric acid salt L5d.HCl (100 mg, $0.55 \mathrm{mmol}, 1.1$ equiv), $\mathrm{NaOAc}$ (46 mg, $0.55 \mathrm{mmol}, 1.1$ equiv) and $\mathrm{Zn}(\mathrm{OAc})_{2}(18 \mathrm{mg}, 0.1 \mathrm{mmol}, 20 \mathrm{~mol} \%)$ in THF $(1 \mathrm{ml})$. This yielded, after Grace Reveleris ${ }^{\circ}$ X2 Normal Phase silicagel flash chromatography (Petroleum Ether:EtOAc, 100:0 to 0:100 over $20 \mathrm{CV}$ ), the desired compound as a colorless oil in 95\% (162 mg, $0.474 \mathrm{mmol})$ yield. ${ }^{\mathbf{1}} \mathbf{H}$ (500 MHz, DMSO-d $\boldsymbol{d}_{6}$, mixture of rotamers) $\delta 8.20(\mathrm{~d}, J=7.8 \mathrm{~Hz}, 0.7 \mathrm{H}), 8.14(\mathrm{~d}, J=7.6 \mathrm{~Hz}, 0.3 \mathrm{H}), 4.32-4.24(\mathrm{~m}, 1 \mathrm{H}), 4.17-4.10$ (m, 
1H), $3.61(\mathrm{~s}, 2.1 \mathrm{H}), 3.60(\mathrm{~s}, 0.9 \mathrm{H}), 3.40-3.32(\mathrm{~m}, 1 \mathrm{H}), 3.29-3.20(\mathrm{~m}, 1 \mathrm{H}), 2.17-2.07(\mathrm{~m}, 0.7 \mathrm{H}), 2.05-$ $1.97(\mathrm{~m}, 0.3 \mathrm{H}), 1.82-1.70(\mathrm{~m}, 3 \mathrm{H}), 1.69-1.64(\mathrm{~m}, 1 \mathrm{H}), 1.60$ (ddt, $J=5.0,9.2,8.9 \mathrm{~Hz}, 1 \mathrm{H}), 1.47$ (ddd, $J$ $=4.4,9.3,13.5 \mathrm{~Hz}, 1 \mathrm{H}), 1.38(\mathrm{~s}, 2.6 \mathrm{H}), 1.32(\mathrm{~s}, 6.4 \mathrm{H}), 0.88(\mathrm{~d}, J=6.6 \mathrm{~Hz}, 3 \mathrm{H}), 0.84$ (pseudo t, $J=5.4$ $\mathrm{Hz}, 3 \mathrm{H}) \mathrm{ppm} .{ }^{13} \mathbf{C}$ (126 MHz, DMSO-d $\boldsymbol{d}_{\boldsymbol{\sigma}}$, mixture of rotamers) $\boldsymbol{\delta} 172.9$ (C), [172.7, 172.3] (C), [153.5, 153.3] (C), [78.5, 78.3] (C), [59.2, 58.9] (CH), $51.7\left(\mathrm{CH}_{3}\right)$, [50.2, 50.0] (CH), [46.6, 46.4] $\left(\mathrm{CH}_{2}\right), 30.9$ $\left(\mathrm{CH}_{2}\right), 29.6\left(\mathrm{CH}_{2}\right),[28.1,27.9]\left(\mathrm{CH}_{3}\right)$, [24.1, 23.8] $(\mathrm{CH}), 22.9\left(\mathrm{CH}_{2}\right),[22.8,22.8]\left(\mathrm{CH}_{3}\right)$, [21.3, 21.0] $\left(\mathrm{CH}_{3}\right)$ ppm. NMR-data was acquired at elevated temperature so that peaks arising from hindered rotation coalesced. ${ }^{1} \mathbf{H}$ NMR $\left(\mathbf{5 0 0} \mathbf{M H z}\right.$, DMSO-d $\left._{\boldsymbol{6}}, \mathbf{6 0}^{\circ} \mathbf{C}\right) \boldsymbol{\delta} 8.00$ (br s , 1H), 4.31 (ddd, $J=5.1,8.0$, $9.6 \mathrm{~Hz}, 1 \mathrm{H}), 4.15(\mathrm{dd}, J=1.9,8.7 \mathrm{~Hz}, 1 \mathrm{H}), 3.62(\mathrm{~s}, 3 \mathrm{H}), 3.41-3.34(\mathrm{~m}, 1 \mathrm{H}), 3.31-3.25(\mathrm{~m}, 1 \mathrm{H}), 2.17-$ $2.07(\mathrm{~m}, 1 \mathrm{H}), 1.85-1.72(\mathrm{~m}, 3 \mathrm{H}), 1.71-1.62(\mathrm{~m}, 1 \mathrm{H}), 1.60(\mathrm{ddd}, J=4.7,9.7,13.8 \mathrm{~Hz}, 1 \mathrm{H}) 1.50(\mathrm{ddd}, J=$ 4.9, 8.9, $13.7 \mathrm{~Hz}, 1 \mathrm{H}), 1.35$ (s, 9H), $0.90(\mathrm{~d}, J=6.7 \mathrm{~Hz}, 3 \mathrm{H}), 0.86(\mathrm{~d}, J=6.4 \mathrm{~Hz}, 3 \mathrm{H}) \mathrm{ppm} .{ }^{\mathbf{1 3}} \mathbf{C}(\mathbf{1 2 6}$ MHz, DMSO-d $\left.\boldsymbol{~}^{\circ} \mathbf{6 0}^{\circ} \mathrm{C}\right) \boldsymbol{\delta} 172.5(\mathrm{C}), 172.3(\mathrm{C}), 153.2(\mathrm{C}), 78.1(\mathrm{C}), 59.0(\mathrm{CH}), 51.4\left(\mathrm{CH}_{3}\right), 50.0(\mathrm{CH})$, $46.3\left(\mathrm{CH}_{2}\right), 30.6\left(\mathrm{CH}_{2}\right), 29.3\left(\mathrm{CH}_{2}\right), 27.7\left(\mathrm{CH}_{3}\right), 23.9(\mathrm{CH}), 22.7\left(\mathrm{CH}_{2}\right), 22.4\left(\mathrm{CH}_{3}\right), 21.0\left(\mathrm{CH}_{3}\right) \mathrm{ppm}$.

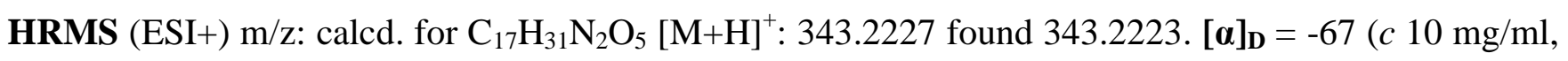
$\left.\mathrm{CHCl}_{3}, 25^{\circ} \mathrm{C}\right)$. The spectroscopic data were in accordance with those previously reported. ${ }^{[7]}$

Benzyl (tert-butoxycarbonyl)-L-methionyl-L-tryptophanate (Boc-L-Met-L-Trp-OBn, DM769) L,L-6e

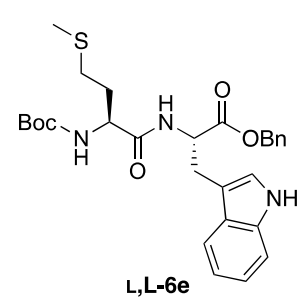

The title compound was prepared using general procedure $\mathbf{B}$, from Boc-L-Met-NH$t$ Bu-nic L-4e (213 mg, $0.5 \mathrm{mmol}, 1$ equiv), H-L-Trp-OBn hydrochloric acid salt L5e.HCl (182 mg, $0.55 \mathrm{mmol}, 1.1$ equiv), $\mathrm{NaOAc}$ (46 mg, $0.55 \mathrm{mmol}, 1.1$ equiv) and $\mathrm{Zn}(\mathrm{OAc})_{2}(18 \mathrm{mg}, 0.1 \mathrm{mmol}, 20 \mathrm{~mol} \%)$ in THF $(1 \mathrm{ml})$. This yielded, after Grace Reveleris ${ }^{\circledR}$ X2 Normal Phase silicagel flash chromatography (Petroleum Ether:EtOAc, 100:0 to 0:100 over $20 \mathrm{CV})$, the desired compound as a white powder in $80 \%(210 \mathrm{mg}$, 0.399 mmol) yield. ${ }^{1} \mathbf{H}$ NMR (400 MHz, DMSO-d $\left.\boldsymbol{d}_{\boldsymbol{6}}\right) \boldsymbol{\delta} 10.90$ (s, 1H), 8.29 (d, $\left.J=7.2 \mathrm{~Hz}, 1 \mathrm{H}\right), 7.52$ (d, $J$ $=7.9 \mathrm{~Hz}, 1 \mathrm{H}), 7.39(\mathrm{~d}, J=8.1 \mathrm{~Hz}, 1 \mathrm{H}), 7.35-7.30(\mathrm{~m}, 3 \mathrm{H}), 7.23-7.16(\mathrm{~m}, 3 \mathrm{H}), 7.13-7.07(\mathrm{~m}, 1 \mathrm{H})$, $7.01(\mathrm{dd}, J=11.0,3.9 \mathrm{~Hz}, 1 \mathrm{H}), 6.94(\mathrm{~d}, J=8.2 \mathrm{~Hz}, 1 \mathrm{H}), 5.12-4.97(\mathrm{~m}, 2 \mathrm{H}), 4.65(\mathrm{dd}, J=13.6,6.8 \mathrm{~Hz}$, $1 \mathrm{H}), 4.13(\mathrm{dd}, J=12.7,7.9 \mathrm{~Hz}, 1 \mathrm{H}), 3.20(\mathrm{qd}, J=14.7,7.0 \mathrm{~Hz}, 2 \mathrm{H}), 2.45(\mathrm{t}, J=7.0 \mathrm{~Hz}, 2 \mathrm{H}), 2.02(\mathrm{~s}$,

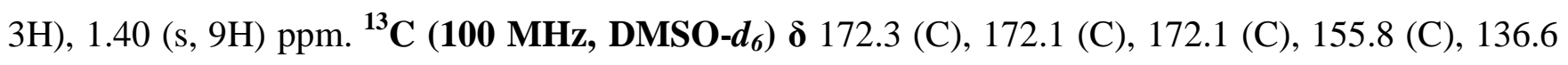
(C), $136.1(\mathrm{C}), 128.8(\mathrm{CH}), 128.8(\mathrm{CH}), 128.4(\mathrm{CH}), 128.2(\mathrm{CH}), 127.6(\mathrm{CH}), 127.5(\mathrm{C}), 124.2(\mathrm{CH})$, $121.5(\mathrm{CH}), 118.9(\mathrm{CH}), 118.5(\mathrm{CH}), 111.9(\mathrm{CH}), 109.7(\mathrm{C}), 78.6(\mathrm{C}), 66.5\left(\mathrm{CH}_{2}\right), 53.8(\mathrm{CH}), 53.7$ $(\mathrm{CH}), 32.4\left(\mathrm{CH}_{2}\right), 30.14\left(\mathrm{CH}_{2}\right), 28.65\left(\mathrm{CH}_{2}\right), 27.55\left(\mathrm{CH}_{3}\right), 15.10\left(\mathrm{CH}_{3}\right)$ ppm. HRMS (ESI+) m/z: calcd.

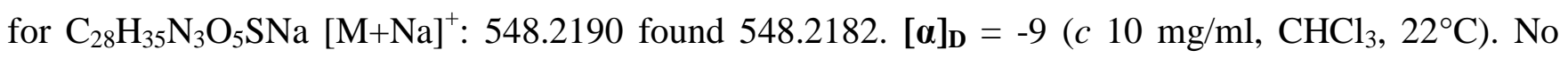
spectroscopic data are reported in literature. 
tert-Butyl (S)-(3-((1-amino-1-oxo-3-phenylpropan-2-yl)amino)-3-oxopropyl)carbamate (Boc- $\beta$ Ala-LPhe-NH 2 , DM332) L-6f

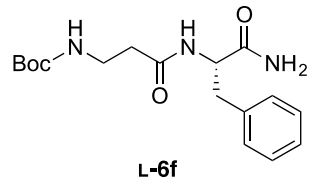

The title compound was prepared using general procedure $\mathbf{B}$, from Boc- $\beta$ Ala- $\mathrm{NH}-$ $t$ Bu-nic $4 \mathbf{f}$ (183 mg, 0.42 mmol, 1 equiv), $\mathrm{H}-\mathrm{L}-\mathrm{Phe}-\mathrm{NH}_{2}$ hydrochloric acid salt L5f.HCl (110 mg, $0.55 \mathrm{mmol}, 1.1$ equiv), NaOAc (45 mg, $0.55 \mathrm{mmol}, 1.1$ equiv) and $\mathrm{Zn}(\mathrm{OAc})_{2}(18 \mathrm{mg}, 0.1 \mathrm{mmol}, 20 \mathrm{~mol} \%)$ in $\mathrm{THF}(1 \mathrm{ml})$, by stirring at $70^{\circ} \mathrm{C}$ for $48 \mathrm{~h}$. This yielded, after Biotage ${ }^{\circledR}$ Isolera One Normal phase silicagel flash chromatography (n-Heptane:EtOAc, 100:0 to 0:100 for $40 \mathrm{~min}$ at a flow rate of $22 \mathrm{ml} / \mathrm{min})$, the desired compound as a white powder in $72 \%(120$ mg, 0.358 mmol) yield. ${ }^{1} \mathbf{H}$ NMR (400 MHz, DMSO-d d $) \boldsymbol{\delta} 8.01(\mathrm{~d}, J=8.27 \mathrm{~Hz}, 1 \mathrm{H}), 7.40$ (br s, 1H), 7.30-7.17 (m, 5H), 7.03 (br s, 1H), 6.57 (br s, 1H), 4.43 (dt, $J=4.5,4.3 \mathrm{~Hz}, 1 \mathrm{H}), 3.07-2.97$ (m, 3H), $2.74(\mathrm{dd}, J=10.0,3.9 \mathrm{~Hz}, 1 \mathrm{H}), 2.28-2.12(\mathrm{~m}, 2 \mathrm{H}), 1.37$ (s, 9H) ppm. ${ }^{13} \mathbf{C}$ NMR (100 MHz, DMSO-d $\left.)\right)$ $\delta 173.1(\mathrm{C}), 170.1(\mathrm{C}), 155.3(\mathrm{C}), 138.1(\mathrm{C}), 129.0(\mathrm{CH}), 127.9(\mathrm{CH}), 126.1(\mathrm{CH}), 77.5(\mathrm{C}), 53.6(\mathrm{CH})$, $37.5\left(\mathrm{CH}_{2}\right), 36.5\left(\mathrm{CH}_{2}\right), 35.5\left(\mathrm{CH}_{2}\right), 28.2\left(\mathrm{CH}_{3}\right)$ ppm. HRMS (ESI+) m/z: calcd. for $\mathrm{C}_{17} \mathrm{H}_{26} \mathrm{~N}_{3} \mathrm{O}_{4}$ $[\mathrm{M}+\mathrm{H}]^{+}: 336.1918$ found 336.1923. $[\alpha]_{\mathbf{D}}=-11\left(\right.$ c $\left.5 \mathrm{mg} / \mathrm{ml}, \mathrm{CHCl}_{3}, 25^{\circ} \mathrm{C}\right)$. No spectroscopic data are reported in literature.

tert-Butyl ( $\quad(S)-1-(((S)-1-a m i n o-1-o x o-3-p h e n y l p r o p a n-2-y l) a m i n o)-4-(m e t h y l t h i o)-1-o x o b u t a n-2-$ yl)carbamate (Boc-L-Met-L-Phe-NH2, DM770) L,L-6g

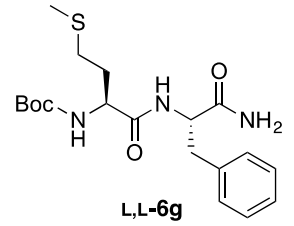

The title compound was prepared using general procedure B, from Boc-L-Met-NH$t$ Bu-nic L-4e (213 mg, 0.5 mmol, 1 equiv), H-L-Phe- $\mathrm{NH}_{2}$ hydrochloric acid salt Lff.HCl (301 mg, $1.5 \mathrm{mmol}, 3$ equiv), NaOAc (123 mg, $1.5 \mathrm{mmol}, 3$ equiv) and $\mathrm{Zn}(\mathrm{OAc})_{2}(18 \mathrm{mg}, 0.1 \mathrm{mmol}, 20 \mathrm{~mol} \%)$ in THF $(1 \mathrm{ml})$. This yielded, after Grace Reveleris ${ }^{\circledR}$ X2 Normal Phase silicagel flash chromatography (Petroleum Ether:EtOAc, 100:0 to 0:100 over $20 \mathrm{CV})$, the desired compound as a white powder in $89 \%(175 \mathrm{mg}, 0.443 \mathrm{mmol})$ yield. ${ }^{1} \mathbf{H}$ NMR (400 MHz, DMSO-d $\boldsymbol{d}_{\boldsymbol{6}} \boldsymbol{\delta} 7.77(\mathrm{~d}, J=8.3 \mathrm{~Hz}, 1 \mathrm{H}), 7.40(\mathrm{~s}, 1 \mathrm{H}), 7.29-7.16(\mathrm{~m}, 5 \mathrm{H}), 7.13(\mathrm{~s}, 1 \mathrm{H}), 7.03$ $(\mathrm{d}, J=7.4 \mathrm{~Hz}, 1 \mathrm{H}), 4.49(\mathrm{dd}, J=12.8,8.1 \mathrm{~Hz}, 1 \mathrm{H}), 3.93(\mathrm{dd}, J=13.4,6.6 \mathrm{~Hz}, 1 \mathrm{H}), 3.04(\mathrm{dd}, J=13.6$, $4.5 \mathrm{~Hz}, 1 \mathrm{H}), 2.85(\mathrm{dd}, J=13.7,8.9 \mathrm{~Hz}, 1 \mathrm{H}), 2.33(\mathrm{t}, J=6.5 \mathrm{~Hz}, 2 \mathrm{H}), 1.99(\mathrm{~s}, 3 \mathrm{H}), 1.72(\mathrm{dd}, J=13.9$, $7.0 \mathrm{~Hz}, 2 \mathrm{H}), 1.38$ (s, 9H), 1.29 (s, 1H) ppm. ${ }^{13} \mathbf{C}$ NMR (100 MHz, DMSO-d $)^{\text {) } \delta} 173.2$ (C), 171.7 (C), $155.87(\mathrm{C}), 138.2(\mathrm{C}), 129.7(\mathrm{CH}), 128.4(\mathrm{CH}), 126.7(\mathrm{CH}), 78.8(\mathrm{C}), 54.5(\mathrm{CH}), 53.8(\mathrm{CH}), 38.0$ $\left(\mathrm{CH}_{2}\right), 32.0\left(\mathrm{CH}_{2}\right), 30.0\left(\mathrm{CH}_{2}\right), 28.6\left(\mathrm{CH}_{3}\right), 15.0\left(\mathrm{CH}_{3}\right)$ ppm. HRMS (ESI+) m/z: calcd. for $\mathrm{C}_{19} \mathrm{H}_{29} \mathrm{~N}_{3} \mathrm{O}_{4} \mathrm{SNa}[\mathrm{M}+\mathrm{Na}]^{+}: 418.1771$ found $418.1754 .[\alpha]_{\mathbf{D}}=-33\left(\right.$ c $\left.5 \mathrm{mg} / \mathrm{ml}, \mathrm{CHCl}_{3}, 25^{\circ} \mathrm{C}\right)$. No spectroscopic data are reported in literature. 
Methyl (tert-butoxycarbonyl)-L-alanyl-L-prolinate (Boc-L-Ala-L-Pro-OMe, KH_1204) L,L-6h

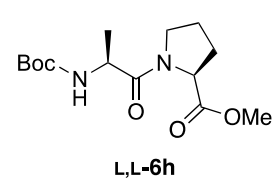

The title compound was prepared using general procedure $\mathbf{B}$, from Boc-L-Ala-NH$t$ Bu-nic L-4b (183 mg, 0.5 mmol, 1 equiv), H-L-Pro-OMe hydrochloric acid salt L5g.HCl ( $\mathrm{mg}, 1.5 \mathrm{mmol}, 3$ equiv), NaOAc (123 mg, $1.5 \mathrm{mmol}, 3$ equiv) and $\mathrm{Zn}(\mathrm{OAc})_{2}(18 \mathrm{mg}, 0.1 \mathrm{mmol}, 20 \mathrm{~mol} \%)$ in $\mathrm{THF}(1 \mathrm{ml})$, by stirring at $100^{\circ} \mathrm{C}$ for $24 \mathrm{~h}$. This yielded, after Grace Reveleris ${ }^{\circledR}$ X2 Normal Phase silicagel flash chromatography (Petroleum Ether:EtOAc, 100:0 to 0:100 over $20 \mathrm{CV}$ ), the desired compound as a white powder in $68 \%(102 \mathrm{mg}, 0.338 \mathrm{mmol})$ yield. ${ }^{\mathbf{1}} \mathbf{H}$ NMR (500 MHz, CDCl $\left.{ }_{3}\right) \delta 5.34(\mathrm{~d}, J=7.8 \mathrm{~Hz}, 1 \mathrm{H}), 4.53(\mathrm{dd}, J=4.4,7.0 \mathrm{~Hz}, 1 \mathrm{H}), 4.49-4.42$ (m, 1H), $3.72(\mathrm{~s}, 3 \mathrm{H}), 3.72-3.67(\mathrm{~m}, 1 \mathrm{H}), 3.64-3.57(\mathrm{~m}, 1 \mathrm{H}), 2.26-2.16(\mathrm{~m}, 1 \mathrm{H}), 2.10-1.94(\mathrm{~m}, 3 \mathrm{H}), 1.42(\mathrm{~s}, 9 \mathrm{H})$, $1.35(\mathrm{~d}, J=6.9 \mathrm{~Hz}, 3 \mathrm{H}) \mathrm{ppm} .{ }^{\mathbf{1 3}} \mathbf{C}$ NMR (126 MHz, $\left.\mathbf{C D C l}_{3}\right) \boldsymbol{\delta} 172.5(\mathrm{C}), 171.8(\mathrm{C}), 155.4(\mathrm{C}), 79.7$ (C), $58.8(\mathrm{CH}), 52.3\left(\mathrm{CH}_{3}\right), 47.9(\mathrm{CH}), 46.9\left(\mathrm{CH}_{2}\right), 29.0\left(\mathrm{CH}_{2}\right), 28.5\left(\mathrm{CH}_{3}\right), 25.0\left(\mathrm{CH}_{2}\right), 18.4\left(\mathrm{CH}_{3}\right)$

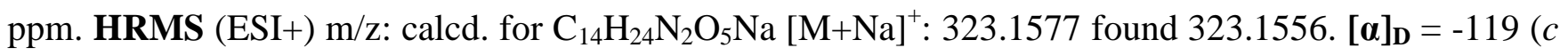
$5 \mathrm{mg} / \mathrm{ml}, \mathrm{CHCl}_{3}, 22^{\circ} \mathrm{C}$ ). No spectroscopic data are reported in literature.

Methyl $\quad N^{6}$-((benzyloxy)carbonyl)- $N^{2}-(N$-(tert-butoxycarbonyl)-S-(tert-butyl)-L-cysteinyl)-L-lysinate (Boc-L-Cys $\left.(t \mathrm{Bu})-\mathrm{L}-\mathrm{Lys}(\mathrm{Cbz})-\mathrm{OMe}, \mathrm{KH} \_1315\right)$ L,L-6i

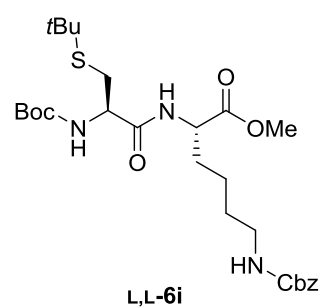

The title compound was prepared using general procedure B from Boc-LCys( $t \mathrm{Bu})-\mathrm{NH}-t \mathrm{Bu}-\mathrm{nic}$ L-4l (226 mg, $0.5 \mathrm{mmol}, 1$ equiv), H-L-Lys(Cbz)-OMe hydrochloric acid salt L-5h.HCl (496 mg, 1.65 mmol, 3 equiv), NaOAc (124 mg, $1.65 \mathrm{mmol}, 3$ equiv) and $\mathrm{Zn}(\mathrm{OAc})_{2}(18 \mathrm{mg}, 0.1 \mathrm{mmol}, 20 \mathrm{~mol} \%)$ in THF (1 ml). The reaction mixture was concentrated in vacuo and redisolved in EtOAc. The organic phase was washed with $\mathrm{HCl}(3 \mathrm{x}, 1 \mathrm{M}), \mathrm{NaHCO}_{3}(3 \mathrm{x}$, sat.) and brine. The organic phase was dried over $\mathrm{MgSO}_{4}$, filtrated and concentrated in vacuo. After Grace Reveleris ${ }^{\circledR}$ X2 Normal Phase silicagel flash chromatography $\left(\mathrm{CH}_{2} \mathrm{Cl}_{2}\right.$ :EtOAc, 100:0 to 70:30 over $\left.10 \mathrm{CV}\right)$, this yielded the desired compound as a white powder in $57 \%(157 \mathrm{mg}, 0.285 \mathrm{mmol})$ yield. ${ }^{1} \mathbf{H}$ NMR (500 MHz, CDCl $) \boldsymbol{\delta}$ 7.38-7.27 (m, 4H), 6.99-6.90 (m, 1H), 6.07 (br s, 1H), 5.43 (d, J= 7.0 Hz, 1H), 5.14-5.07 (m, 2H), 4.96 (br s, 1H), 4.59 (dd, $J=16.1,7.5 \mathrm{~Hz}, 1 \mathrm{H}), 4.32-4.25$ (m, 1H), 3.73 (s, 3H), 3.22-3.13 (m, 2H), 2.99 (dd, $J=12.9,5.58 \mathrm{~Hz}, 1 \mathrm{H}), 2.82(\mathrm{dd}, J=12.7,6.3 \mathrm{~Hz}, 1 \mathrm{H}), 1.91-1.83(\mathrm{~m}, 1 \mathrm{H}), 1.74-1.65(\mathrm{~m}, 1 \mathrm{H}), 1.57-1.49$

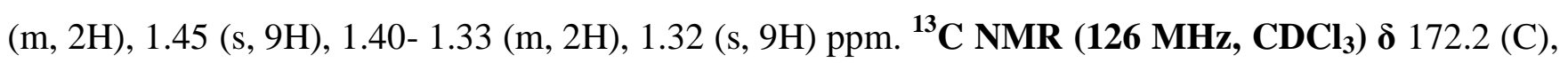
$170.8(\mathrm{C}), 156.6(\mathrm{C}), 155.5(\mathrm{C}), 136.5(\mathrm{C}), 128.5(\mathrm{CH}), 128.1(\mathrm{CH}), 80.5(\mathrm{C}), 66.7(\mathrm{CH}), 54.2(\mathrm{CH})$, $52.4\left(\mathrm{CH}_{3}\right), 52.0(\mathrm{CH}), 42.9(\mathrm{C}), 40.6\left(\mathrm{CH}_{2}\right), 31.9\left(\mathrm{CH}_{2}\right), 30.8\left(\mathrm{CH}_{3}\right), 30.4\left(\mathrm{CH}_{2}\right), 29.1\left(\mathrm{CH}_{2}\right), 28.2$ $\left(\mathrm{CH}_{3}\right), 22.1\left(\mathrm{CH}_{2}\right)$ ppm. HRMS (ESI+) m/z: calcd. for $\mathrm{C}_{27} \mathrm{H}_{43} \mathrm{~N}_{3} \mathrm{O}_{7} \mathrm{SNa}[\mathrm{M}+\mathrm{Na}]^{+}: 576.2720$ found 576.2776. $[\alpha]_{\mathbf{D}}=-6\left(c 10 \mathrm{mg} / \mathrm{ml}, \mathrm{CHCl}_{3}, 20^{\circ} \mathrm{C}\right)$. No spectroscopic data are reported in literature. 
Methyl $N^{6}$-((benzyloxy)carbonyl)- $N^{2}$-( $N^{\prime}$-(tert-butoxycarbonyl)- $N^{\tau}$-trityl-L-histidyl)-L-lysinate (Boc-LHis(Trt)-L-Lys(Cbz)-OMe, KH_1308) L,L-6j

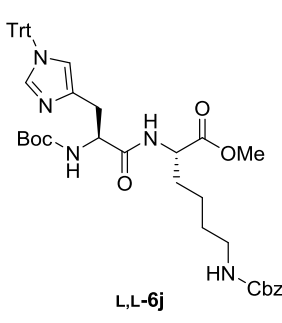

The title compound was prepared using general procedure B from Boc-L-His(Trt)NH- $t$ Bu-nic L,L-4m (336 mg, 0.5 mmol, 1 equiv), H-L-Lys(Cbz)-OMe hydrochloric acid salt L-5h.HCl (496 mg, 1.65 mmol, 3 equiv), NaOAc (124 mg, 1.65 mmol, 3 equiv) and $\mathrm{Zn}(\mathrm{OAc})_{2}(18 \mathrm{mg}, 0.1 \mathrm{mmol}, 20 \mathrm{~mol} \%)$ in THF $(1 \mathrm{ml})$. This yielded, after Grace Reveleris® X2 Normal Phase silicagel flash chromatography $\left(\mathrm{CH}_{2} \mathrm{Cl}_{2}\right.$ :EtOAc, 100:0 to 70:30 over $10 \mathrm{CV}$ ), the desired compound as a white powder in $72 \%$ (276 $\mathrm{mg}$, $0.36 \mathrm{mmol}$ ) yield. ${ }^{1} \mathbf{H}$ NMR (500 MHz, MeOD) $\delta 8.68$ (br s, 1H), 7.51-7.40 (m, 10H), 7.38-7.30 (m, 3H), 7.29-723 (m, 3H), 7.22-7.17 (m, 6H), 5.05 (s, 2H), 4.43 (dd, $J=\mathrm{Hz}, 1 \mathrm{H}), 4.38$ (dd, $J=\mathrm{Hz}, 1 \mathrm{H})$, $3.57(\mathrm{~s}, 3 \mathrm{H}), 3.16(\mathrm{dd}, J=15.0,4.3 \mathrm{~Hz}, 1 \mathrm{H}), 3.12-3.08(\mathrm{~m}, 2 \mathrm{H}), 3.00(\mathrm{dd}, J=\mathrm{Hz}, 1 \mathrm{H}), 1.90-1.80(\mathrm{~m}$, 1H), 1.73-1.65 (m, 1H), 1.56-1.45 (m, 4H), 1.40 (s, 9H) ppm. ${ }^{13}$ C NMR (126 MHz, MeOD) $\delta 174.2$ (C), $173.8(\mathrm{C}), 158.9(\mathrm{C}), 157.5(\mathrm{C}), 143.6(\mathrm{C}), 138.4$ (C), $137.6(\mathrm{C}), 130.9(\mathrm{CH}), 129.4(\mathrm{CH}), 129.3$ $(\mathrm{CH}), 129.3(\mathrm{CH}), 128.9(\mathrm{CH}), 128.8(\mathrm{CH}), 121.6(\mathrm{CH}), 80.6(\mathrm{C}), 67.3(\mathrm{C}), 55.8(\mathrm{CH}), 53.5(\mathrm{CH}), 52.7$ $\left(\mathrm{CH}_{3}\right), 41.4\left(\mathrm{CH}_{2}\right), 32.3\left(\mathrm{CH}_{2}\right), 31.6\left(\mathrm{CH}_{2}\right), 30.3\left(\mathrm{CH}_{2}\right), 28.8\left(\mathrm{CH}_{3}\right), 23.8\left(\mathrm{CH}_{2}\right)$ ppm. HRMS (ESI+) $\mathrm{m} / \mathrm{z}$ : calcd. for $\mathrm{C}_{45} \mathrm{H}_{52} \mathrm{~N}_{5} \mathrm{O} 7[\mathrm{M}+\mathrm{H}]^{+}: 774.3867$ found 774.3804 . $[\boldsymbol{\alpha}]_{\mathbf{D}}=-5$ (c $\left.10 \mathrm{mg} / \mathrm{ml}, \mathrm{CHCl}_{3}, 20^{\circ} \mathrm{C}\right)$. No spectroscopic data are reported in literature.

tert-Butyl $\quad(S)-2-(((S)-1-((2-(((S)-1-m e t h o x y-1-o x o-3-p h e n y l p r o p a n-2-y l) a m i n o)-2-o x o e t h y l) a m i n o)-4-$ methyl-1-oxopentan-2-yl)carbamoyl)pyrrolidine-1-carboxylate (Boc-L-Pro-L-Leu-Gly-L-Phe-OMe, KH_1265) L,L,L-6k

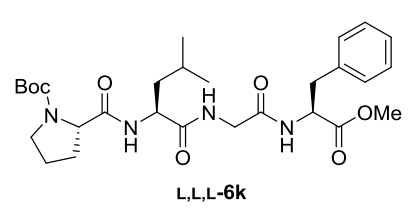

The title compound was prepared using general procedure B, from Boc-L-ProL-Leu-Gly-NH- $t$ Bu-nic L-4n (281 mg, 0.5 mmol, 1 equiv), H-L-Phe-OMe hydrochloric acid salt L-5a.HCl (119 mg, 0.55 mmol, 1.1 equiv), NaOAc (45 $\mathrm{mg}, 0.55 \mathrm{mmol}, 1.1$ equiv) and $\mathrm{Zn}(\mathrm{OAc})_{2}(18 \mathrm{mg}, 0.1 \mathrm{mmol}, 20 \mathrm{~mol} \%)$ in THF (1 ml). This yielded, after Grace Reveleris ${ }^{\circledR}$ X2 Normal Phase silicagel flash chromatography (Petroleum Ether:EtOAc, 100:0 to 0:100 over $20 \mathrm{CV}$ ), the desired compound as a yellow oil in $88 \%$ (240 mg, $0.439 \mathrm{mmol})$ yield. ${ }^{1} \mathbf{H}\left(500 \mathrm{MHz}\right.$, DMSO- $\boldsymbol{d}_{\boldsymbol{6}}$, mixture of rotamers) $\boldsymbol{\delta} 8.26(\mathrm{~d}, J=7.6 \mathrm{~Hz}$, $0.6 \mathrm{H}), 8.20(\mathrm{~d}, J=8.0 \mathrm{~Hz}, 0.4 \mathrm{H}), 8.09(\mathrm{t}, J=5.0 \mathrm{~Hz}, 0.6 \mathrm{H}), 8.04(\mathrm{t}, J=5.2 \mathrm{~Hz}, 0.4 \mathrm{H}), 7.98-7.92(\mathrm{~m}$, $1 \mathrm{H}), 7.30-7.24(\mathrm{~m}, 2 \mathrm{H}), 7.23-7.16(\mathrm{~m}, 3 \mathrm{H}), 4.50-4.43(\mathrm{~m}, 1 \mathrm{H}), 4.31-4.19(\mathrm{~m}, 1 \mathrm{H}), 4.13(\mathrm{dd}, J=3.1,8.6$ $\mathrm{Hz}, 1 \mathrm{H}), 3.73(\mathrm{dd}, J=6.1,16.8 \mathrm{~Hz}, 1 \mathrm{H}), 3.68-3.60$ (m, $1 \mathrm{H}), 3.57$ (s, 3H), 3.34-3.29 (m, 1H), 3.28-3.22 $(\mathrm{m}, 1 \mathrm{H}), 3.00(\mathrm{dd}, J=6.1,13.8 \mathrm{~Hz}, 1 \mathrm{H}), 2.91(\mathrm{dd}, J=8.9,13.9 \mathrm{~Hz}, 1 \mathrm{H}), 2.12-1.96(\mathrm{~m}, 1 \mathrm{H}), 1.81-1.68$ 
(m, 3H), 1.68-1.58 (m, 1H), 1.53-1.41 (m, 2H), $1.36(\mathrm{~s}, 3 \mathrm{H}), 1.31(\mathrm{~s}, 6 \mathrm{H}), 0.91-0.80(\mathrm{~m}, 6 \mathrm{H})$ ppm. ${ }^{\mathbf{1 3}} \mathbf{C}$ (126 MHz, DMSO-d $\boldsymbol{d}_{\boldsymbol{6}}$, mixture of rotamers) $\boldsymbol{\delta} 172.4$ (C), 172.2 (C), 171.7 (C), 168.7 (C), 153.3 (C), $136.9(\mathrm{C}), 129.0(\mathrm{CH}), 128.2(\mathrm{CH}), 126.5(\mathrm{CH}),[78.7,78.3](\mathrm{C})$, [59.3, 59.2] $(\mathrm{CH}), 53.6(\mathrm{CH}), 51.8$ $\left(\mathrm{CH}_{3}\right)$, [51.2, 51.0] $(\mathrm{CH})$, [46.6, 46.4] $\left(\mathrm{CH}_{2}\right), 41.5\left(\mathrm{CH}_{2}\right), 40.8(\mathrm{CH}), 36.8\left(\mathrm{CH}_{2}\right), 30.9\left(\mathrm{CH}_{2}\right), 29.5$ $\left(\mathrm{CH}_{2}\right)$, [28.0, 27.9] $\left(\mathrm{CH}_{3}\right), 24.0\left(\mathrm{CH}_{2}\right)$, [23.0, 22.9] $\left(\mathrm{CH}_{3}\right), 21.5\left(\mathrm{CH}_{3}\right)$ ppm. NMR-data was acquired at elevated temperature so that peaks arising from hindered rotation coalesced. ${ }^{1} \mathbf{H}\left(\mathbf{5 0 0} \mathbf{M H z}\right.$, DMSO- $\boldsymbol{d}_{\boldsymbol{6}}$, $\mathbf{8 0}^{\circ}$ C) $\boldsymbol{\delta} 7.94(\mathrm{~d}, J=6.5 \mathrm{~Hz}, 1 \mathrm{H}), 7.79(\mathrm{t}, J=5.3 \mathrm{~Hz}, 1 \mathrm{H}), 7.67(\mathrm{~d}, J=7.4 \mathrm{~Hz}, 1 \mathrm{H}), 7.30-7.25(\mathrm{~m}, 2 \mathrm{H})$, 7.23-7.18 (m, 3H), 4.57-4.51 (m, 1H), 4.33-4.26 (m, 1H), $4.13(\mathrm{dd}, J=2.8,8.1 \mathrm{~Hz}, 1 \mathrm{H}), 3.74(\mathrm{dd}, J=$ 5.7, $16.7 \mathrm{~Hz}, 1 \mathrm{H}$, overlap with HDO), 3.67 (dd, $J=5.7,16.7 \mathrm{~Hz}, 1 \mathrm{H}$ overlap with HDO), 3.60 (s, 3H overlap with HDO), 3.38-2.27 (m, 2H), $3.04(\mathrm{dd}, J=13.8,6.0 \mathrm{~Hz} 1 \mathrm{H}), 2.92$ (dd, $J=8.0,13.8 \mathrm{~Hz}, 1 \mathrm{H})$, 2.10-2.03 (m, 1H), 1.91-1.84 (m, 1H), 1.83-1.73 (m, 2H), 1.72-1.63 (m, 1H), 1.51 (dd, J = 6.7, $6.7 \mathrm{~Hz}$, 2H), $1.38(\mathrm{~s}, 9 \mathrm{H}), 0.89(\mathrm{~d}, J=6.7 \mathrm{~Hz}, 3 \mathrm{H}), 0.87(\mathrm{~d}, J=6.7 \mathrm{~Hz}, 3 \mathrm{H}) \mathrm{ppm} .{ }^{13} \mathbf{C}(126 \mathrm{MHz}$, DMSO-d $\boldsymbol{6}$, $\left.\mathbf{8 0}^{\circ} \mathbf{C}\right) \boldsymbol{\delta} 171.9(\mathrm{C}), 171.8(\mathrm{C}), 171.1(\mathrm{C}), 168.2(\mathrm{C}), 153.4(\mathrm{C}), 136.6(\mathrm{C}), 128.6(\mathrm{CH}), 127.8(\mathrm{CH})$, 126.1 (CH), $78.3(\mathrm{C}), 59.2(\mathrm{CH}), 53.2(\mathrm{CH}), 51.2\left(\mathrm{CH}_{3}\right), 51.1(\mathrm{CH}), 46.2\left(\mathrm{CH}_{2}\right), 41.6\left(\mathrm{CH}_{2}\right), 40.6(\mathrm{CH})$, $36.7\left(\mathrm{CH}_{2}\right), 29.0\left(\mathrm{CH}_{2}\right), 27.7\left(\mathrm{CH}_{3}\right), 23.8\left(\mathrm{CH}_{2}\right), 23.0\left(\mathrm{CH}_{2}\right), 22.5\left(\mathrm{CH}_{3}\right), 21.2\left(\mathrm{CH}_{3}\right)$ ppm. HRMS (ESI+) $\mathrm{m} / \mathrm{z}$ : calcd. for $\mathrm{C}_{27} \mathrm{H}_{42} \mathrm{~N}_{2} \mathrm{O}_{6} \mathrm{Na}[\mathrm{M}+\mathrm{Na}]^{+}: 515.3091$ found $515.3090 .[\alpha]_{\mathbf{D}}=-31(c 5 \mathrm{mg} / \mathrm{ml}$, $\mathrm{CHCl}_{3}, 22^{\circ} \mathrm{C}$ ). No spectroscopic data are reported in literature.

Remark: Diastereomerical purity was checked on the target compounds. The diastereomerical excess (\%de) was evaluated with HPLC (UV detector) on a Chiralpak IA column and/or ${ }^{1} \mathrm{H}$ NMR analysis. 


\subsection{Segment Coupling using Transamidation}

Synthesis of the Directing Group Functionalized Peptide Segment

tert-Butyl 2-((S)-2-((S)-2-((tert-butoxycarbonyl)amino)-4-methylpentanamido)-3-

phenylpropanamido)nicotinate (Boc-L-Leu-L-Phe-NH-tBu-nic, KH_549/ER1730) L,L-7

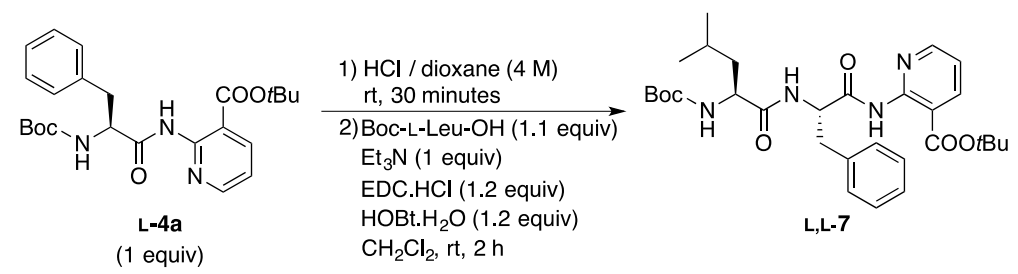

KH_549: The synthesis of Boc-L-Leu-L-Phe-NH- $t$ Bu-nic L,L-7 was started from Boc-L-Phe-NH- $t$ Bu-nic L-4a (221 mg, $0.5 \mathrm{mmol}, 1$ equiv), initially deprotected following general procedure $\mathbf{C}$ with $\mathrm{HCl} /$ dioxane $(10 \mathrm{ml}, 4 \mathrm{M})$. After removal of the solvent under vacuum, the resulting crude solid material (L-Phe-NH- $t \mathrm{Bu}-\mathrm{nic} . \mathrm{HCl}$ ) was used in the subsequent step without further purification.

The next step was performed following general procedure D. The crude was dissolved in $\mathrm{CH}_{2} \mathrm{Cl}_{2}(20$ $\mathrm{ml}$ ) and $\mathrm{Et}_{3} \mathrm{~N}$ was added until the mixture reached $\mathrm{pH}$ 9. This was added to the pre-activated mixture of Boc-L-Leu-OH (137 mg, $0.55 \mathrm{mmol}, 1.1$ equiv), EDC.HCl (125 mg, $0.65 \mathrm{mmol}, 1.2$ equiv) and HOBt. $\mathrm{H}_{2} \mathrm{O}$ (100 mg, $0.65 \mathrm{mmol}, 1.2$ equiv). The reaction mixture was stirred for $2 \mathrm{~h}$. After extraction, the title compound L,L-7 could be obtained as a white powder with $96 \%$ (266 mg, $0.48 \mathrm{mmol}$ ) over the two steps.

${ }^{1}$ H NMR (500 MHz, DMSO-d 6 ) $\boldsymbol{\delta} 10.82$ (br s, 1H), 8.53 (br s, 1H), 8.06 (d, $J=7.7$ Hz, 1H), 7.94 (d, $J$ $=8.8 \mathrm{~Hz}, 1 \mathrm{H}), 7.32-7.28(\mathrm{~m}, 3 \mathrm{H}), 7.26-7.20(\mathrm{~m}, 2 \mathrm{H}), 7.20-7.15(\mathrm{~m}, 1 \mathrm{H}), 6.83(\mathrm{~d}, J=8.9 \mathrm{~Hz}, 1 \mathrm{H}), 4.94-$ $4.86(\mathrm{~m}, 1 \mathrm{H}), 3.98-3.91(\mathrm{~m}, 1 \mathrm{H}), 3.13(\mathrm{~d}, J=13.8 \mathrm{~Hz}, 1 \mathrm{H}), 2.83(\mathrm{dd}, J=11.8,11.8 \mathrm{~Hz}, 1 \mathrm{H}), 1.47(\mathrm{~s}$, 9H), 1.36 (s, 9H), 1.28-1.22 (m, 3H), 0.84-0.76 (m, 6H) ppm. ${ }^{13}$ C NMR (126 MHz, DMSO-d $)^{\text {) } \delta} 172.2$ (C), $170.1(\mathrm{C}), 164.8(\mathrm{C}), 155.0(\mathrm{C}), 150.6(\mathrm{CH}), 148.3(\mathrm{C}), 138.8(\mathrm{CH}), 137.3(\mathrm{C}), 129.3(\mathrm{CH}), 127.9$ $(\mathrm{CH}), 126.2(\mathrm{CH}), 120.8(\mathrm{C}), 120.1(\mathrm{CH}), 81.3(\mathrm{C}), 78.0(\mathrm{C}), 53.8(\mathrm{CH}), 52.9(\mathrm{CH}), 41.1\left(\mathrm{CH}_{2}\right), 37.4$ $\left(\mathrm{CH}_{2}\right), 28.1\left(\mathrm{CH}_{3}\right), 27.7\left(\mathrm{CH}_{3}\right), 24.1(\mathrm{CH}), 22.8\left(\mathrm{CH}_{3}\right), 21.5\left(\mathrm{CH}_{3}\right) \mathrm{ppm}$. HRMS (ESI+) m/z calcd. for

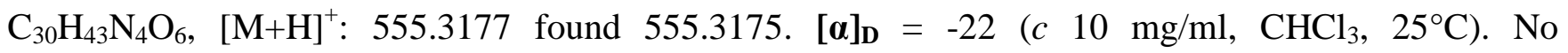
spectroscopic data are reported in literature. 


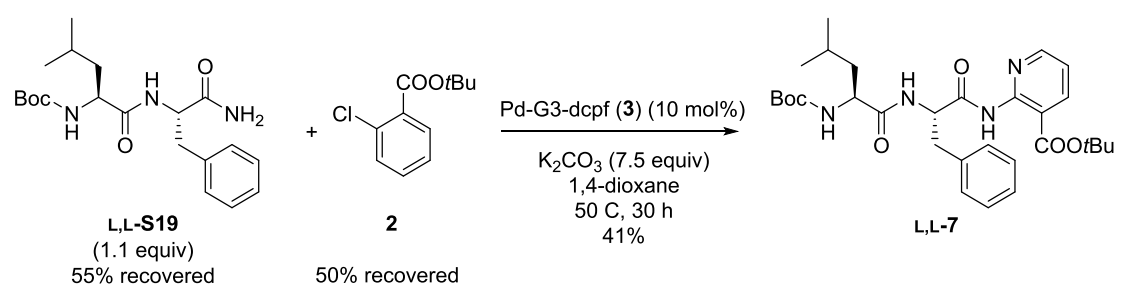

ER1730: The title compound was synthesized using general procedure A2, from Boc-L-Leu-L-Phe- $\mathrm{NH}_{2}$ L,L-S19 (0.208 mg, 0.55 mmol, 1.1 equiv), $\mathrm{K}_{2} \mathrm{CO}_{3}$ (0.518 g, 3.75 mmol, 7.5 equiv), Pd-G3-dcpf 3 (52 $\mathrm{mg}, 0.05 \mathrm{mmol}, 10 \mathrm{~mol} \%)$ and tert-butyl 2-chloronicotinate 2 (107 $\mathrm{mg}, 0.5 \mathrm{mmol}, 1 \mathrm{equiv})$ in anhydrous 1,4-dioxane $(3.13 \mathrm{~mL})$. The reaction mixture was purified using the Biotage ${ }^{\circ}$ Isolera One Normal phase silicagel flash chromatography ( $n$-Heptane:EtOAc, 100:0 to 50:50 over $45 \mathrm{~min}$ ) to obtain white powder in $41 \%(114 \mathrm{mg}, 0.2 \mathrm{mmol})$ yield. Boc-L-Leu-L-Phe- $\mathrm{NH}_{2}$ and tert-butyl 2chloronicotinate 2 were recoverd in $55 \%$ (113 mg, $0.3 \mathrm{mmol})$ and $50 \%$ (54 mg, $0.25 \mathrm{mmol})$ yield. ${ }^{1} \mathbf{H}$ NMR (500 MHz, DMSO-d d $_{\text {) }} \boldsymbol{\delta} 10.82$ (br s, 1H), 8.53 (br s, 1H), 8.06 (d, $\left.J=7.7 \mathrm{~Hz}, 1 \mathrm{H}\right), 7.94$ (d, $J=$ $8.8 \mathrm{~Hz}, 1 \mathrm{H}), 7.32-7.28(\mathrm{~m}, 3 \mathrm{H}), 7.26-7.20(\mathrm{~m}, 2 \mathrm{H}), 7.20-7.15(\mathrm{~m}, 1 \mathrm{H}), 6.83(\mathrm{~d}, J=8.9 \mathrm{~Hz}, 1 \mathrm{H}), 4.94-$ $4.86(\mathrm{~m}, 1 \mathrm{H}), 3.98-3.91(\mathrm{~m}, 1 \mathrm{H}), 3.13(\mathrm{~d}, J=13.8 \mathrm{~Hz}, 1 \mathrm{H}), 2.83(\mathrm{dd}, J=11.8,11.8 \mathrm{~Hz}, 1 \mathrm{H}), 1.47$ (s, 9H), 1.36 (s, 9H), 1.28-1.22 (m, 3H), 0.84-0.76 (m, 6H) ppm. ${ }^{13}$ C NMR (126 MHz, DMSO-d $)$ 反 172.2 (C), $170.1(\mathrm{C}), 164.8(\mathrm{C}), 155.0(\mathrm{C}), 150.6(\mathrm{CH}), 148.3(\mathrm{C}), 138.8(\mathrm{CH}), 137.3(\mathrm{C}), 129.3(\mathrm{CH}), 127.9$ $(\mathrm{CH}), 126.2(\mathrm{CH}), 120.8(\mathrm{C}), 120.1(\mathrm{CH}), 81.3(\mathrm{C}), 78.0(\mathrm{C}), 53.8(\mathrm{CH}), 52.9(\mathrm{CH}), 41.1\left(\mathrm{CH}_{2}\right), 37.4$ $\left(\mathrm{CH}_{2}\right), 28.1\left(\mathrm{CH}_{3}\right), 27.7\left(\mathrm{CH}_{3}\right), 24.1(\mathrm{CH}), 22.8\left(\mathrm{CH}_{3}\right), 21.5\left(\mathrm{CH}_{3}\right) \mathrm{ppm}$. HRMS (ESI+) m/z calcd. for

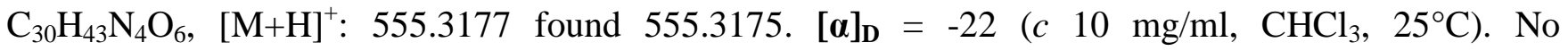
spectroscopic data are reported in literature.

tert-Butyl 2-((S)-2-((S)-2-((tert-butoxycarbonyl)amino)-4-methylpentanamido)-3phenylpropanamido)nicotinate (Boc-L-Leu-L-Phe- $\mathrm{NH}_{2}, \mathrm{KH}_{-}$1281) L,L-S19

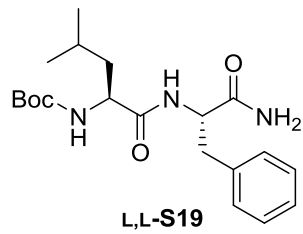

The synthesis of Boc-L-Leu-L-Phe- $\mathrm{NH}_{2} \mathbf{L}, \mathbf{L}-\mathbf{S 1 9}$ was performed following general procedure $\mathbf{D}$ from Boc-L-Leu-OH. $\mathrm{H}_{2} \mathrm{O} 499 \mathrm{mg}, 2 \mathrm{mmol}, 1$ equiv), $\mathrm{H}-\mathrm{Phe}-\mathrm{NH}_{2} \cdot \mathrm{HCl}$ (328 mg, $2 \mathrm{mmol}, 1$ equiv), EDC.HCl (460 mg, $2.4 \mathrm{mmol}, 1.2$ equiv) and HOBt. $\mathrm{H}_{2} \mathrm{O}$ (368 mg, $2.4 \mathrm{mmol}, 1.2$ equiv), $\mathrm{Et}_{3} \mathrm{~N}$ (0.279 ml, 2 mmol, 1 equiv) in $\mathrm{CH}_{2} \mathrm{Cl}_{2}(50 \mathrm{ml})$. This yielded, after extraction, the title compound as a colourless oil with $94 \%$ (706 mg, $1.87 \mathrm{mmol})$.

${ }^{1}$ H NMR (500 MHz, DMSO-d $)^{\text {) }} \boldsymbol{\delta} 7.68$ (d, $\left.J=8.3 \mathrm{~Hz}, 1 \mathrm{H}\right), 7.39$ (br s , 1H), 7.25-7.15 (m, 5H), 7.10 (br s, $1 \mathrm{H}), 6.96(\mathrm{~d}, J=8.05 \mathrm{~Hz}, 1 \mathrm{H}), 4.50-4.42(\mathrm{~m}, 1 \mathrm{H}), 3.91-3.77(\mathrm{~m}, 1 \mathrm{H}), 3.00$ (dd, $J=14.1,4.8 \mathrm{~Hz}$, $1 \mathrm{H}), 2.82(\mathrm{dd}, 14.2,8.9 \mathrm{~Hz}, 1 \mathrm{H}), 1.52-1.41(\mathrm{~m}, 1 \mathrm{H}), 1.37(\mathrm{~s}, 9 \mathrm{H}), 1.33-1.26(\mathrm{~m}, 1 \mathrm{H}), 1.26-1.20(\mathrm{~m}, 1 \mathrm{H})$, $0.81(\mathrm{~d}, J=6.7 \mathrm{~Hz}, 3 \mathrm{H}), 0.77(\mathrm{~d}, J=6.5 \mathrm{~Hz}, 3 \mathrm{H}) \mathrm{ppm} .{ }^{13} \mathrm{C}$ NMR (126 MHz, DMSO-d $)^{\boldsymbol{\delta}} 172.7(\mathrm{C})$, $172.0(\mathrm{C}), 155.3(\mathrm{C}), 137.7(\mathrm{C}), 129.2(\mathrm{CH}), 127.9(\mathrm{CH}), 126.1(\mathrm{CH}), 78.2(\mathrm{C}), 53.3(\mathrm{CH}), 53.1(\mathrm{CH})$, 
$40.7\left(\mathrm{CH}_{2}\right), 37.6\left(\mathrm{CH}_{2}\right), 28.2\left(\mathrm{CH}_{3}\right), 24.1(\mathrm{CH}), 22.8\left(\mathrm{CH}_{3}\right), 21.6\left(\mathrm{CH}_{3}\right)$ ppm. HRMS (ESI+) m/z calcd. $\mathrm{C}_{20} \mathrm{H}_{32} \mathrm{~N}_{3} \mathrm{O}_{4}$ for $[\mathrm{M}+\mathrm{H}]^{+}: 378.2393$ found 378.2347. No spectroscopic data are reported in literature.

tert-Butyl 2-((S)-2-((S)-3-(tert-butoxy)-2-((tert butoxycarbonyl)amino)propanamido)propanamido) nicotinate (Boc-L-Ser( $t$ Bu)-L-Ala-NH-tBu-nic, KH_635) L,L-8

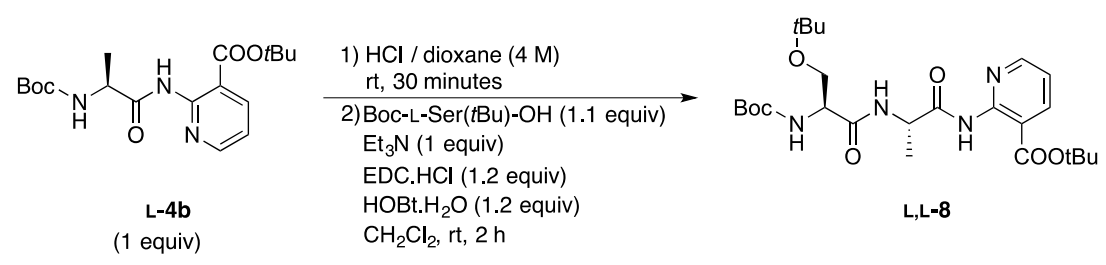

For the synthesis of Boc-L-Ser $(t \mathrm{Bu})$-L-Ala-NH- $t \mathrm{Bu}-n i c$ L,L-8 was started from Boc-L-Ala-NH- $t \mathrm{Bu}-n i c$ L-4b (512 mg, $1.4 \mathrm{mmol}$, 1 equiv) that was deprotected following general procedure $\mathbf{C}$ with $\mathrm{HCl} /$ dioxane $(20 \mathrm{ml}, 4 \mathrm{M})$. After removal of the solvent under vacuum, the resulting crude solid material (L-Ala-NH-tBu-nic.HCl) was used in the subsequent step without further purification.

The next step was performed following general procedure $\mathbf{D}$. The crude was dissolved in $\mathrm{CH}_{2} \mathrm{Cl}_{2}(30$ $\mathrm{ml}$ ) and $\mathrm{Et}_{3} \mathrm{~N}$ was added until the mixture reached $\mathrm{pH}$ 9. This was added to the pre-activated mixture of Boc-L-Ser( $t \mathrm{Bu})-\mathrm{OH}$ (366 mg, $1.4 \mathrm{mmol}, 1$ equiv), EDC.HCl (322 mg, $1.68 \mathrm{mmol}, 1.2$ equiv) and HOBt. $\mathrm{H}_{2} \mathrm{O}$ (257 mg, $1.68 \mathrm{mmol}, 1.2$ equiv). The reaction mixture was stirred for $2 \mathrm{~h}$. After extraction the title compound $\mathbf{L}, \mathbf{L}-\mathbf{8}$ could be obtained as a white powder with quantitive yield (748 mg, $1.4 \mathrm{mmol}$ ) over the two steps.

${ }^{1}$ H NMR (500 MHz, $\left.\mathbf{C D C l}_{3}\right) \boldsymbol{\delta} 11.22(\mathrm{~s}, 1 \mathrm{H}), 8.58(\mathrm{dd}, J=2.1,4.8 \mathrm{~Hz}, 1 \mathrm{H}), 8.23(\mathrm{dd}, J=2.1,7.9 \mathrm{~Hz}$, 1H), 7.67 (br s, 1H), 7.04 (dd, $J=4.8,7.9 \mathrm{~Hz}, 1 \mathrm{H}), 5.47-5.39(\mathrm{~m}, 1 \mathrm{H}), 4.93$ (br s, 1H), 4.27-4.21 (m, $1 \mathrm{H}), 3.80(\mathrm{~d}, J=6.0 \mathrm{~Hz}, 1 \mathrm{H}), 3.48(\mathrm{dd}, J=7.4,8.6 \mathrm{~Hz}, 1 \mathrm{H}), 1.59(\mathrm{~s}, 9 \mathrm{H}), 1.51(\mathrm{~d}, J=7.0 \mathrm{~Hz}, 3 \mathrm{H}), 1.44$ (s, 9H), 1.20 (s, 9H) ppm. ${ }^{13} \mathbf{C}$ NMR (126 MHz, CDCl 3 ) $\delta 171.0(\mathrm{C}), 170.6(\mathrm{C}), 166.1(\mathrm{C}), 155.8(\mathrm{C})$, $152.7(\mathrm{CH}), 152.3(\mathrm{C}), 140.2(\mathrm{CH}), 118.5(\mathrm{CH}), 113.0(\mathrm{C}), 83.7(\mathrm{C}), 80.0(\mathrm{C}), 74.2(\mathrm{C}), 61.8\left(\mathrm{CH}_{2}\right)$, $54.4(\mathrm{CH}), 50.7(\mathrm{CH}), 28.5\left(\mathrm{CH}_{3}\right), 28.2\left(\mathrm{CH}_{3}\right), 27.6\left(\mathrm{CH}_{3}\right), 19.0\left(\mathrm{CH}_{3}\right) \mathrm{ppm}$. HRMS (ESI+) m/z calcd.

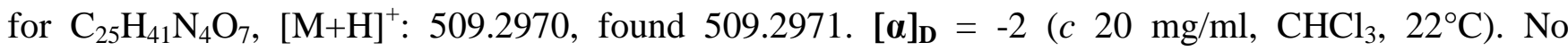
spectroscopic data are reported in literature. 
tert-Butyl L-leucyl-L-valinate hydrochloric acid salt (H-L-Leu-L-Val-OtBu.HCl, KH_562) L,L-9.HCl

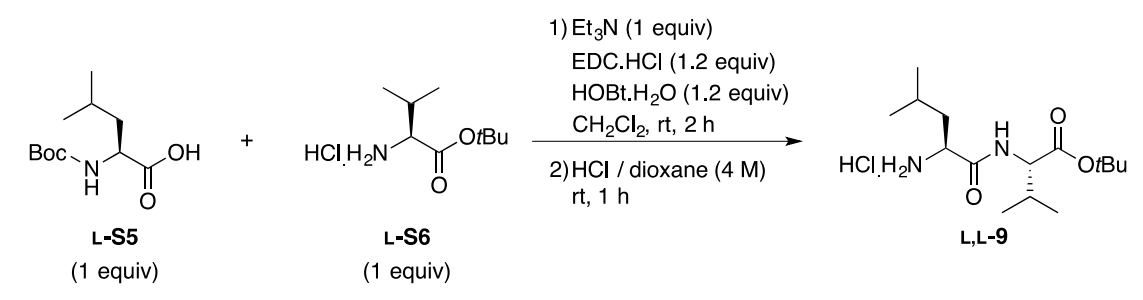

The title compound was obtained by means of a two-step synthesis. The amide coupling was performed following general procedure D from Boc-L-Leu-OH L-S5 (578 mg, 2.5 mmol, 1 equiv), H-L-Val-OtBu hydrochloric acid salt L-S6 (524 mg, $2.5 \mathrm{mmol}, 1$ equiv), EDC.HCl (575 mg, 3 mmol, 1.2 equiv), HOBt. $\mathrm{H}_{2} \mathrm{O}$ (459 mg, $3 \mathrm{mmol}, 1.2$ equiv) and $\mathrm{Et}_{3} \mathrm{~N}(0.35 \mathrm{ml}, 2.5 \mathrm{mmol}, 1$ equiv). After extraction, the $N-$ Boc protected dipeptide was obtained in a 97\% (936 mg, $2.4 \mathrm{mmol})$ yield. Without intermediate purification, the product was deprotected following general procedure $\mathbf{C}$ from Boc-L-Leu-L-Val-OtBu (387 mg, $1 \mathrm{mmol}, 1$ equiv) in $\mathrm{HCl}$ in dioxane $(20 \mathrm{ml}, 4 \mathrm{M}$ ) by stirring $30 \mathrm{~min}$ at room temperature. After removal of solvent and lyophilisation, the desired compound L,L-9.HCl was obtained in nearly quantitative yield (320 mg, $0.99 \mathrm{mmol}$ ) as a white powder. ${ }^{1} \mathbf{H}$ NMR (500 MHz, DMSO-d 6 ) $\delta$ 8.66 (d, $J$ $=7.8 \mathrm{~Hz}, 1 \mathrm{H}), 8.32(\mathrm{br} \mathrm{s}, 2 \mathrm{H}), 4.04(\mathrm{dd}, J=6.2,7.9 \mathrm{~Hz}, 1 \mathrm{H}), 3.90(\mathrm{dd}, J=7.2,7.2 \mathrm{~Hz}, 1 \mathrm{H}), 2.04$ (qdd, $J$ $=6.6,13.1,13.1 \mathrm{~Hz}, 1 \mathrm{H}), 1.68(\mathrm{qdt}, J=6.6,6.6,6.6 \mathrm{~Hz}, 1 \mathrm{H}), 1.56(\mathrm{ddd}, J=7.2,7,2,2.1 \mathrm{~Hz}, 2 \mathrm{H}), 1.40$ (s, 9H), 0.94-0.86 (m, 12H) ppm. ${ }^{13}$ C NMR (126 MHz, DMSO-d $\left.\boldsymbol{d}_{\boldsymbol{6}}\right) \boldsymbol{\delta} 169.9(\mathrm{C}), 169.2(\mathrm{C}), 80.8(\mathrm{C})$, $58.4(\mathrm{CH}), 50.5(\mathrm{CH}), 40.3\left(\mathrm{CH}_{2}\right), 29.8(\mathrm{CH}), 27.6\left(\mathrm{CH}_{3}\right), 23.5\left(\mathrm{CH}_{3}\right), 22.6(\mathrm{CH}), 22.2\left(\mathrm{CH}_{3}\right), 18.9$ $\left(\mathrm{CH}_{3}\right), 18.2\left(\mathrm{CH}_{3}\right)$ ppm. HRMS (ESI+) m/z: calcd. for $\mathrm{C}_{15} \mathrm{H}_{31} \mathrm{~N}_{2} \mathrm{O}_{3},[\mathrm{M}+\mathrm{H}]^{+}:$287.2329 found 287.2311. $[\boldsymbol{\alpha}]_{\mathbf{D}}=8\left(c 10 \mathrm{mg} / \mathrm{ml}, \mathrm{CHCl}_{3}, 19^{\circ} \mathrm{C}\right)$. No spectroscopic data are reported in literature.

tert-Butyl L-alanyl-L-phenylalaninate hydrochloride (H-L-Ala-L-Phe-OtBu.HCl, KH_782) L,L-10.HCl

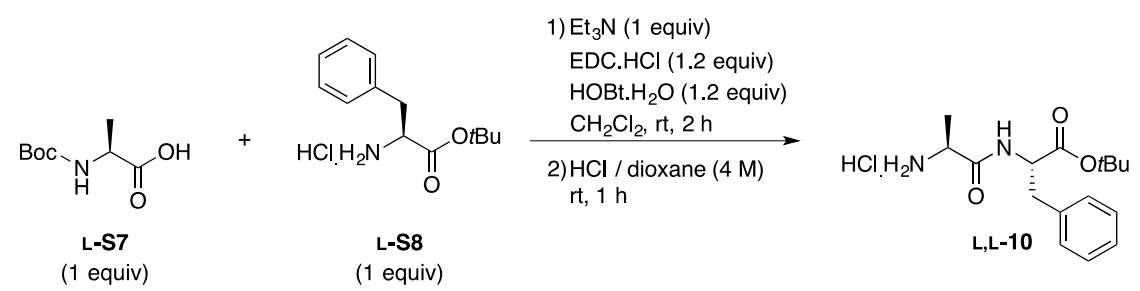

The title compound was obtained by means of a two step synthesis. The peptide synthesis was performed following general procedure D from Boc-L-Ala-OH L-S7 (473 mg, 2.5 mmol, 1 equiv), H-LPhe-O $t$ Bu hydrochloric acid salt L-S8 (644 mg, 2.5 mmol, 1 equiv), EDC.HCl (575 mg, 3 mmol, 1.2 equiv), $\mathrm{HOBt} . \mathrm{H}_{2} \mathrm{O}$ (459 mg, $3 \mathrm{mmol}, 1.2$ equiv) and $\mathrm{Et}_{3} \mathrm{~N}$ (0.35 ml, $2.5 \mathrm{mmol}, 1$ equiv). After extraction, the $N$-Boc protected dipeptide was obtained in a $95 \%$ (930 mg, $2.37 \mathrm{mmol}$ ) yield. Without 
intermediate purification, the product was deprotected following general procedure $\mathbf{C}$, from Boc-L-AlaL-Phe-O $t \mathrm{Bu}$ (903 mg, $2.3 \mathrm{mmol}, 1$ equiv) in $\mathrm{HCl} /$ dioxane $(50 \mathrm{ml}, 4 \mathrm{M})$ by stirring $1 \mathrm{~h}$ at room temperature. After removal of solvent and lyophilisation, the desired compound L,L-10.HCl was obtained in nearly quantitive yield $(749 \mathrm{mg}, 2.27 \mathrm{mmol})$ as a white powder. ${ }^{1} \mathbf{H}$ NMR (500 MHz, $\mathbf{C D C l}_{3}$ ) $\boldsymbol{\delta} 8.27$ (br s, 2H), 7.95 (br s, 1H), 7.21-7.18 (m, 4H), 7.15-7.12 (m, 1H), 4.58 (q, J = 6.2 Hz, $1 \mathrm{H}), 4.45$ (br s, 1H), 3.14 (dd, $J=4.9,13.3 \mathrm{~Hz}, 1 \mathrm{H}), 2.99$ (dd, $J=7.8,13.0 \mathrm{~Hz}, 1 \mathrm{H}), 1.59$ (d, $J=5.1 \mathrm{~Hz}$,

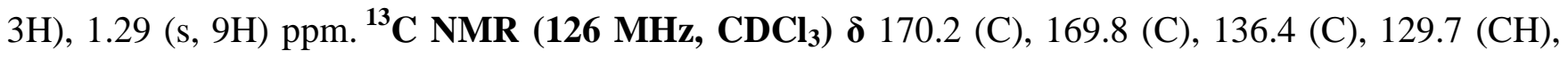
$128.5(\mathrm{CH}), 126.9(\mathrm{CH}), 82.2(\mathrm{C}), 55.2(\mathrm{CH}), 49.7(\mathrm{CH}), 37.6\left(\mathrm{CH}_{2}\right), 28.0\left(\mathrm{CH}_{3}\right), 17.8\left(\mathrm{CH}_{3}\right)$ ppm.

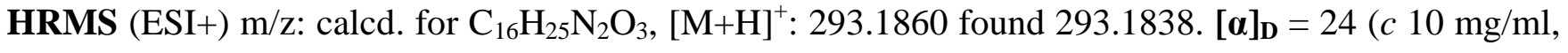
$\left.\mathrm{CHCl}_{3}, 19^{\circ} \mathrm{C}\right)$. No spectroscopic data are reported in literature.

Segment Condensation using Transamidation of N-Boc Protected tert-Butyl Nicotinate Functionalized Dipeptides

tert-Butyl (tert-butoxycarbonyl)-L-leucyl-L-phenylalanyl-L-leucyl-L-valinate (Boc-L-Leu-L-Phe-L-LeuL-Val-OtBu, KH_1224) L,L,L,L-11

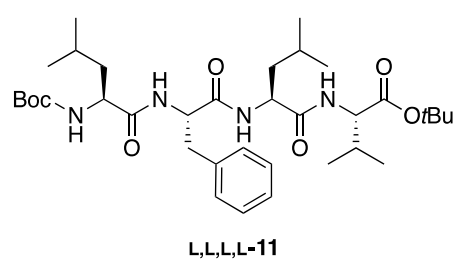

The title compound was prepared using general procedure $\mathbf{B}$, from Boc-LLeu-L-Phe-NH- $t$ Bu-nic L,L-7 (277 mg, 0.5 mmol, 1 equiv), H-L-Leu-L-Val$\mathrm{O} t \mathrm{Bu}$ hydrochloric acid salt L,L-9.HCl (178 mg, $0.55 \mathrm{mmol}, 1.1$ equiv), $\mathrm{NaOAc}$ (46 mg, $0.55 \mathrm{mmol}, 1.1$ equiv) and $\mathrm{Zn}(\mathrm{OAc})_{2}(18 \mathrm{mg}, 0.1 \mathrm{mmol}, 20$ mol\%) in THF (1 ml). This yielded, after Grace Reveleris ${ }^{\circledR}$ X2 Normal Phase silica gel flash chromatography (Petroleum Ether:EtOAc, 100:0 to 50:50 over $25 \mathrm{CV}$ to 0:100 in $2 \mathrm{CV}$ ), the desired compound L,L,L,L-11 as a white powder in $83 \%$ (269 mg, $0.415 \mathrm{mmol}$ ) yield. ${ }^{1} \mathbf{H}$ NMR (500 MHz, $\left.\mathbf{C D C l}_{3}\right) \boldsymbol{\delta}$ 7.29-7.25 (m, 2H), 7.24-7.20 (m, 1H), 7.19-7.16 (m, 2H), 6.87-6.81 (m, 2H), $6.72(\mathrm{~d}, J=6.9$ $\mathrm{Hz}, 1 \mathrm{H}), 4.93(\mathrm{~d}, J=6.0 \mathrm{~Hz}, 1 \mathrm{H}), 4.71(\mathrm{dt}, J=6.7,6.8 \mathrm{~Hz}, 1 \mathrm{H}), 4.57-4.52(\mathrm{~m}, 1 \mathrm{H}), 4.36(\mathrm{dd}, J=5.4$, $8.7 \mathrm{~Hz}, 1 \mathrm{H}), 4.00(\mathrm{td}, J=5.0,9.9 \mathrm{~Hz}, 1 \mathrm{H}), 3.17(\mathrm{dd}, J=5.4,13.1 \mathrm{~Hz}, 1 \mathrm{H}), 3.04(\mathrm{dd}, J=6.0,13.9 \mathrm{~Hz}$, $1 \mathrm{H}), 2.15(\mathrm{dq}, J=6.7,13.1 \mathrm{~Hz}, 1 \mathrm{H}), 1.75-1.68(\mathrm{~m}, 1 \mathrm{H}), 1.66-1.54(\mathrm{~m}, 2 \mathrm{H}), 1.47$ (s, 9H), 1.45-1.40 (m, 1H), 1.36 (s, 9H), 0.94-0.85 (m, 20H) ppm. ${ }^{13} \mathbf{C}$ NMR (126 MHz, CDCl $\left.\mathbf{~}_{3}\right) \boldsymbol{\delta} 172.8(\mathrm{C}), 171.8(\mathrm{C}), 170.8$ (C), $170.7(\mathrm{C}), 156.1(\mathrm{C}), 136.3(\mathrm{C}), 129.4(\mathrm{CH}), 128.9(\mathrm{CH}), 127.2(\mathrm{CH}), 81.8(\mathrm{C}), 80.6(\mathrm{C}), 58.0$ $(\mathrm{CH}), 54.3(\mathrm{CH}), 54.0(\mathrm{CH}) 52.0(\mathrm{CH}), 41.1\left(\mathrm{CH}_{2}\right), 40.9\left(\mathrm{CH}_{2}\right), 37.4\left(\mathrm{CH}_{2}\right), 31.2(\mathrm{CH}), 28.4\left(\mathrm{CH}_{3}\right)$, $28.1\left(\mathrm{CH}_{3}\right), 24.9\left(\mathrm{CH}_{3}\right), 24.6\left(\mathrm{CH}_{3}\right), 23.1\left(\mathrm{CH}_{3}\right), 23.0\left(\mathrm{CH}_{3}\right), 21.9(\mathrm{CH}), 19.1\left(\mathrm{CH}_{3}\right), 18.1\left(\mathrm{CH}_{3}\right) \mathrm{ppm}$.

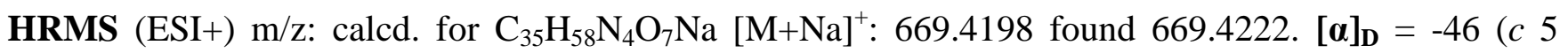
$\mathrm{mg} / \mathrm{ml}, \mathrm{CHCl}_{3}, 25^{\circ} \mathrm{C}$ ).No spectroscopic data are reported in literature. 
tert-Butyl $\mathrm{N}$-(tert-butoxycarbonyl)-O-(tert-butyl)-L-seryl-L-alanyl-L-alanyl-L-phenylalaninate (Boc-L$\operatorname{Ser}(t \mathrm{Bu})$-L-Ala-L-Ala-L-Phe-OtBu, KH_1229), L,L,L,L-12

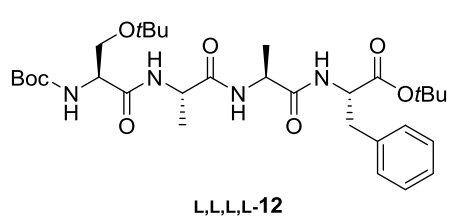

L,L,L,L-12

The title compound was prepared using general procedure $\mathbf{B}$, from Boc-L$\operatorname{Ser}(t \mathrm{Bu})$-L-Ala-NH- $t$ Bu-nic L,L-8 $(254$ mg, 0.5 mmol, 1 equiv), H-L-Ala-LPhe-O $t \mathrm{Bu}$ hydrochloric acid salt L,L-10.HCl $(181 \mathrm{mg}, 0.55 \mathrm{mmol}, 1.1$ equiv), $\mathrm{NaOAc}$ (46 mg, $0.55 \mathrm{mmol}, 1.1$ equiv) and $\mathrm{Zn}(\mathrm{OAc})_{2}(18 \mathrm{mg}, 0.1$ mmol, $20 \mathrm{~mol} \%)$ in THF (1 ml). This yielded, after Grace Reveleris ${ }^{\circledR}$ X2 Normal Phase silicagel flash chromatography (Petroleum Ether:EtOAc, 100:0 to 50:50 over $25 \mathrm{CV}$ to 0:100 in $2 \mathrm{CV}$ ), the desired compound L,L,L,L-12 as a white powder in 74\% (224 mg, $0.37 \mathrm{mmol})$ yield. ${ }^{\mathbf{1}} \mathbf{H}$ NMR (500 MHz, DMSO-d $\left.\boldsymbol{d}_{\boldsymbol{6}}\right) \boldsymbol{\delta} 8.11(\mathrm{~d}, J=7.0 \mathrm{~Hz}, 1 \mathrm{H}), 7.95(\mathrm{~d}, J=7.7 \mathrm{~Hz}, 1 \mathrm{H}), 7.83(\mathrm{~d}, J=7.4 \mathrm{~Hz}, 1 \mathrm{H}), 7.29-7.25(\mathrm{~m}$, 2H), 7.23-7.18 (m, 3H), $6.66(\mathrm{~d}, J=8.3 \mathrm{~Hz}, 1 \mathrm{H}), 4.34-4.27(\mathrm{~m}, 3 \mathrm{H}), 4.02-3.96(\mathrm{~m}, 1 \mathrm{H}), 3.47-3.44(\mathrm{~m}$, $1 \mathrm{H}), 3.42-3.38(\mathrm{~m}, 1 \mathrm{H}), 2.99-2.90(\mathrm{~m}, 2 \mathrm{H}), 1.38(\mathrm{~s}, 9 \mathrm{H}), 1.31(\mathrm{~s}, 9 \mathrm{H}), 1.20-1.14(\mathrm{~m}, 6 \mathrm{H}), 1.10(\mathrm{~s}, 9 \mathrm{H})$

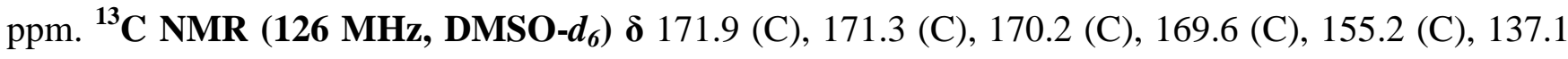
(C), $129.1(\mathrm{CH}), 128.1(\mathrm{CH}), 126.4(\mathrm{CH}), 80.5(\mathrm{C}), 78.2(\mathrm{C}), 72.8(\mathrm{C}), 61.8\left(\mathrm{CH}_{2}\right), 55.0(\mathrm{CH}), 54.1$ $(\mathrm{CH}), 47.9(\mathrm{CH}), 47.6(\mathrm{CH}), 36.7\left(\mathrm{CH}_{2}\right), 28.1\left(\mathrm{CH}_{3}\right), 27.4\left(\mathrm{CH}_{3}\right), 27.1\left(\mathrm{CH}_{3}\right), 18.4\left(\mathrm{CH}_{3}\right), 18.3\left(\mathrm{CH}_{3}\right)$

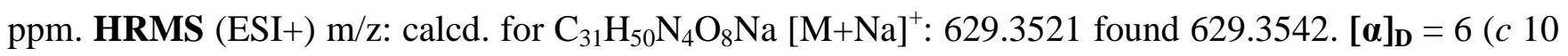
$\mathrm{mg} / \mathrm{ml}, \mathrm{CHCl}_{3}, 25^{\circ} \mathrm{C}$ ). No spectroscopic data are reported in literature.

\section{HPLC Analysis of the Peptide 11 and 12 obtained by transamidation}

To confirm the NMR analysis indicating peptide $\mathbf{L}, \mathbf{L}, \mathbf{L}, \mathbf{L}-\mathbf{1 1}$ and $\mathbf{L}, \mathbf{L}, \mathbf{L}, \mathbf{L}-\mathbf{1 2}$ are diasteriomerically pure tetrapeptides, HPLC analysis was performed. Initially, a mixture of $\mathbf{L}, \mathbf{L}, \mathbf{L}, \mathbf{L}-$ and $\mathbf{L}, \mathbf{D}, \mathbf{L}, \mathbf{L}-$ diastereomers, obtained through classical peptide synthesis, was prepared for both peptides. The mixture was fully resolved on a Diacel Chiralpak IA column with an isocratic gradient of heptane/ethanol (80:20) (cf. Figure S11 for peptide 11 and Figure S13 for peptide 12). Subsequently, the analysis of peptide 11 (KH_1224) and peptide 12 (KH_1229) obtained through transamidation was performed. Herein, peptide 11 (KH_1224; Figure S12) lacked any sign of the L,D,L,L-11. For peptide 12 (KH_1229; Figure S14), the analysis revealed less than $2 \%$ of the $\mathbf{L}, \mathbf{D}, \mathbf{L}, \mathbf{L}-\mathbf{- 1 2}$. 


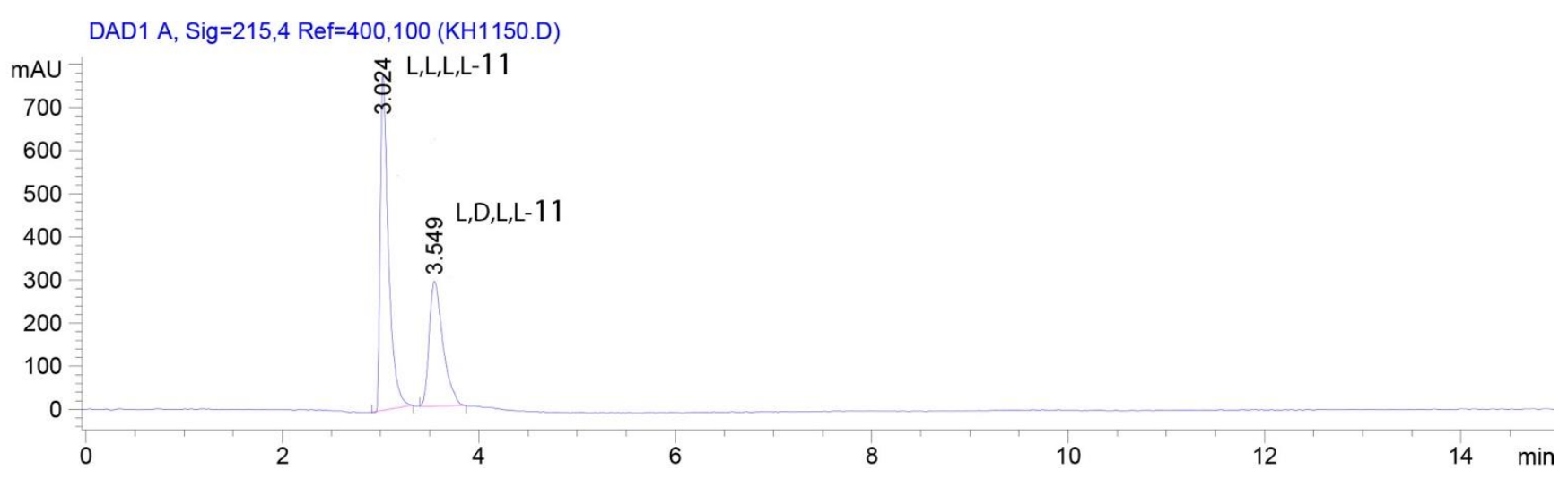

Figure S11. Chromatogram obtained at $215 \mathrm{~nm}$ by HPLC analysis with heptane/ethanol (80:20) of a mixture of $\mathbf{L}, \mathbf{L}, \mathbf{L}, \mathbf{L}-\mathbf{1 1}$ and $\mathbf{L}, \mathbf{D}, \mathbf{L}, \mathbf{L}-\mathbf{1 1}$.

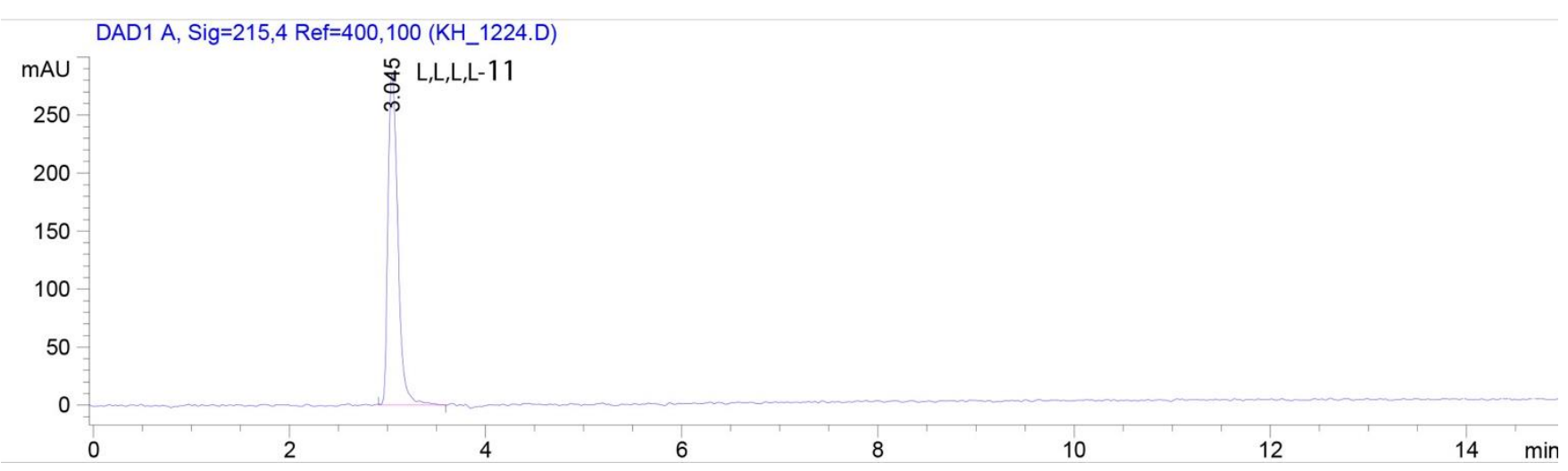

Figure S12. Chromatogram obtained of peptide 11 (KH_1224) synthesized via transamidation at 215 nm by HPLC analysis with heptane/ethanol (80:20) revealing L,L,L,L-11 purity.

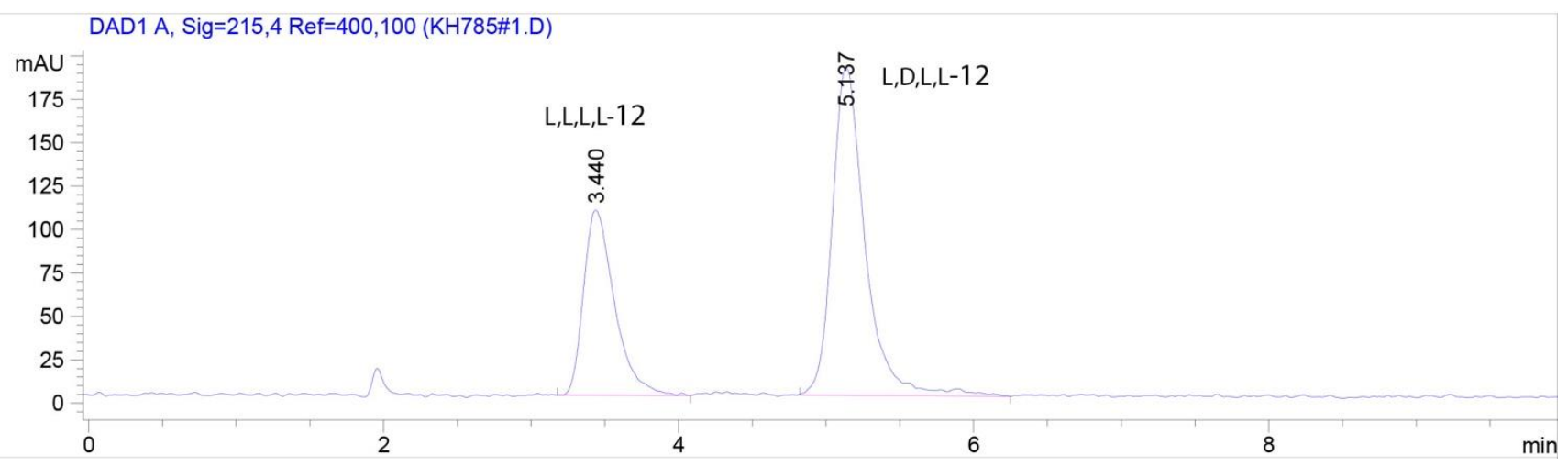

Figure S13. Chromatogram obtained at $215 \mathrm{~nm}$ by HPLC analysis with heptane/ethanol (80:20) of a mixture of $\mathbf{L}, \mathbf{L}, \mathbf{L}, \mathbf{L}-\mathbf{1 2}$ and $\mathbf{L}, \mathbf{D}, \mathbf{L}, \mathbf{L}-\mathbf{1 2}$. 
DAD1 A, Sig=215,4 Ref=400,100 (KH1229\#1.D)

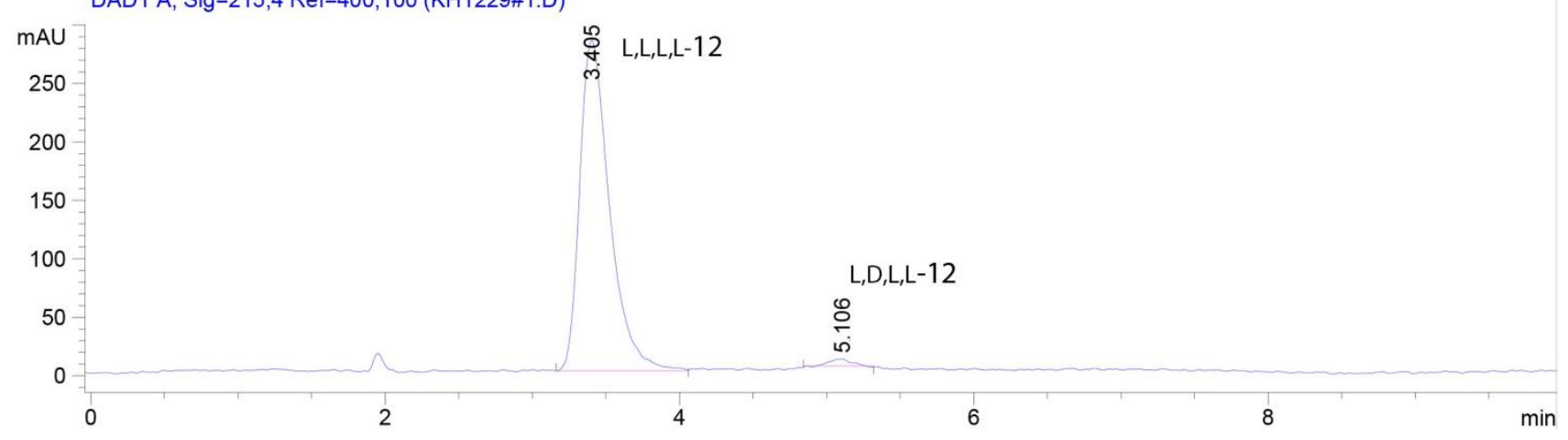

Figure S14. Chromatogram obtained of peptide 12 (KH_1229) synthesized via transamidation at 215 nm by HPLC analysis with heptane/ethanol (80:20) revealing L,L,L,L-12 purity. 


\subsection{Transamidation on Solid Support}

Fmoc rink amide resin $=$

4-(2',4'-Dimethoxyphenyl-Fmoc-aminomethyl)phenoxyacetamidoaminomethyl resin

Assemble of the peptide sequence Boc-Gln(tBu-nic)-Phe-Lys(Boc)-Phe 13 on the solid support

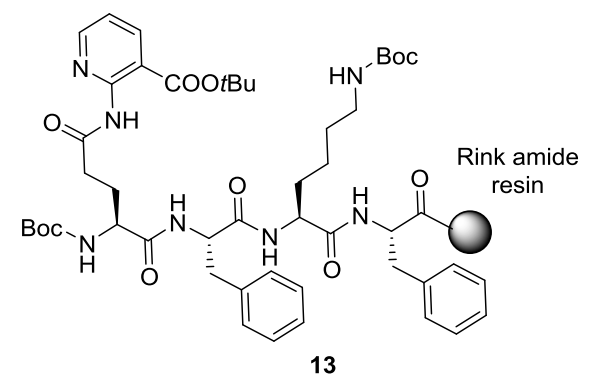

Into an SPPS reactor, Fmoc rink amide resin $(303 \mathrm{mg}, 0.1 \mathrm{mmol}$ $0.33 \mathrm{mmol} / \mathrm{g}, 1$ equiv) was weighted and swollen in $\mathrm{CH}_{2} \mathrm{Cl}_{2}$ for 15 minutes under constant shaking. The solvent was removed by filtration and the deprotection of the Fmoc protection group was achieved by treatment of the resin with a solution of 4methylpiperidine (20\%) in DMF (5 and 15 minutes of shaking). In the meantime, a mixture of $\mathrm{N}$-Fmoc protected amino acid ( 3 equiv), HBTU (114 mg, $0.3 \mathrm{mmol}, 3$ equiv) and DIPEA ( $87 \mu 1,0.5 \mathrm{mmol}, 5$ equiv) was stirred for 15 minutes in DMF ( $1 \mathrm{ml})$. The resin was washed with DMF (3x) and $\mathrm{CH}_{2} \mathrm{Cl}_{2}(3 \mathrm{x})$ before the pre-stirred mixture was added. After 40 minutes of shaking, the excess of reagents was removed by filtration and the resin was washed with DMF (3x) and $\mathrm{CH}_{2} \mathrm{Cl}_{2}(3 \mathrm{x})$. The Kaiser test was applied to determine if the peptide coupling was finished. The same deprotection and coupling strategy was applied for the coupling of the following amino acids. Coupling of the Boc-L-Gln( $t$ Bu-nic)-OH L-4o was achieved overnight.

Kaiser test

For this test, some beats were transferred in a glass tube. Then two droplets of each of the following solutions were added:

1) a solution of ninhydrin $(2.5 \mathrm{~g})$ in $\mathrm{EtOH}(50 \mathrm{ml})$;

2) a solution of phenol $(40 \mathrm{~g})$ in $\mathrm{EtOH}(10 \mathrm{ml})$

3) a solution of $\mathrm{KCN}(1 \mathrm{ml}, 0.001 \mathrm{M}$, aqueous $)$ in pyridine $(49 \mathrm{ml})$.

The glass tube was heated at $110^{\circ} \mathrm{C}$ in a sand bath for approximately $5 \mathrm{~min}$. A pale yellow colored mixture indicates less than $1 \%$ free amine present on the resin, while a dark blue color reveals that the coupling was incomplete. In that case, the coupling was repeated.

$N$-[(1S)-1-carboxyethyl]-L-glutaminyl-L-phenylalanyl-L-lysyl-L-phenylalanine 14.TFA from 13

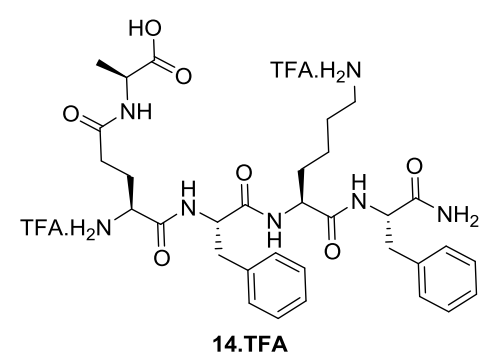

In the SPPS reactor, the resin with the assembled peptide 13 was rinsed with DMF (3x), $\mathrm{CH}_{2} \mathrm{Cl}_{2}(3 \mathrm{x})$ and THF (3x). The resin is swollen in THF for $5 \mathrm{~min}$, then carefully transferred in round-bottom shape microwave vial equipped with a magnet. Remaining beads in the SPPS reactor were recovered using additional THF. Subsequently, the beads were allowed 
to deposit at the bottom of the vial and the excess of THF supernatant was carefully removed by pipette to reduce the solvent to a minimal volume. $\mathrm{Zn}(\mathrm{OAc})_{2}(3.6 \mathrm{mg}, 0.02 \mathrm{mmol}, 20 \mathrm{~mol} \%, 0.02 \mathrm{mmol})$, $\mathrm{NaOAc}$ (41 mg, 0.5 mmol, 5 equiv) and H-L-Ala-OtBu.HCl L-5i.HCl (91 mg, 0.5 mmol, 5 equiv) were added and the vial was sealed with a crimp cap. The reaction was stirred over $48 \mathrm{~h}$ at $90^{\circ} \mathrm{C}$. After it was allowed to cool down to room temperature, the reacting mixture was diluted with THF and $\mathrm{CH}_{2} \mathrm{Cl}_{2}$ and then transferred to the SPPS syringe reactor. The resin is thoroughly washed with THF and $\mathrm{CH}_{2} \mathrm{Cl}_{2}$. The resin is then cleaved using TFA/TIS/ $\mathrm{H}_{2} \mathrm{O}(95: 2.5: 2.5)$ cocktail for 2 hours at room temperature. After filtration, the solvent is removed in vасио and the crude cleaved product is lyophilized and purified using semi-preparative HPLC $\left(\mathrm{H}_{2} \mathrm{O}: \mathrm{CH}_{3} \mathrm{CHN}\right.$ 80:20 to 45:55 over 20 min). This yielded, after lyophilization, the title compound 14.TFA with $59 \%(51 \mathrm{mg}, 0.059 \mathrm{mmol})$ yield. HPLC $\mathrm{rt}=2.69 \mathrm{~min}$. HRMS (ESI+) m/z: 640.3459 found 640.3411. [ $\alpha]_{\mathrm{D}}=-8\left(\mathrm{c} 5 \mathrm{mg} / \mathrm{ml}, \mathrm{CH}_{3} \mathrm{OH}, 21^{\circ} \mathrm{C}\right)$.

Assemble of the peptide sequence Boc-Phe-Gln(tBu-nic)-Phe-Lys(Boc)-Phe $\mathbf{1 5}$ on the solid support

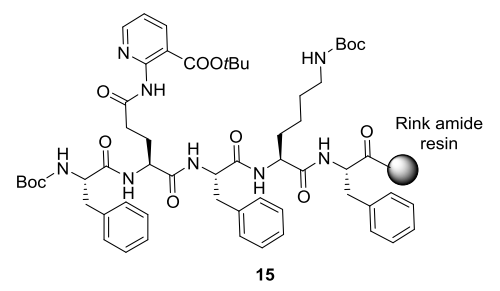

Into an SPPS reactor, Fmoc rink amide resin $(151 \mathrm{mg}, 0.1 \mathrm{mmol} 0.67$ mmol/g, 1 equiv) was weighted and swollen in $\mathrm{CH}_{2} \mathrm{Cl}_{2}$ for 15 minutes under constant shaking. The solvent was removed by filtration and the deprotection of the Fmoc protection group was achieved by treatment of the resin with a solution of 4-methylpiperidine (20\%) in DMF (5 and 15 minutes of shaking). In the meantime, a mixture of $N$-Fmoc protected amino acid ( 3 equiv), HBTU (114 mg, $0.3 \mathrm{mmol}, 3$ equiv) and DIPEA ( $87 \mu \mathrm{l}, 0.5 \mathrm{mmol}, 5$ equiv) was stirred for 15 minutes in DMF ( $1 \mathrm{ml})$. The resin was washed with $\mathrm{DMF}(3 \mathrm{x})$ and $\mathrm{CH}_{2} \mathrm{Cl}_{2}(3 \mathrm{x})$ before the pre-stirred mixture was added. After 40 minutes of shaking, the excess of reagents was removed by filtration and the resin was washed with DMF (3x) and $\mathrm{CH}_{2} \mathrm{Cl}_{2}(3 \mathrm{x})$. The Kaiser test was applied to determine if the peptide coupling was finished. The same deprotection and coupling strategy was applied for the coupling of the following amino acids. Coupling of the Fmoc-L-Gln(tBu-nic)-OH L-4p was achieved overnight.

L-phenylalanyl- $N$-[(1S)-1-carboxyethyl]-L-glutaminyl-L-phenylalanyl-L-lysyl-L-phenylalanine 16.TFA from 15

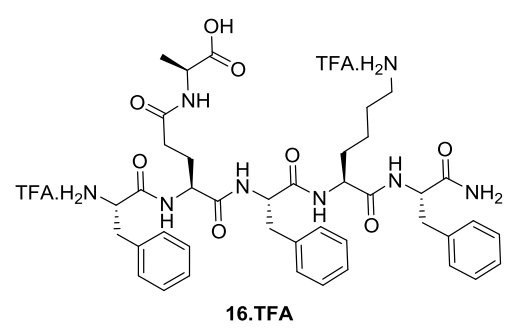

In the SPPS reactor, the resin with the assembled peptide $\mathbf{1 5}$ was rinsed with DMF (3x), $\mathrm{CH}_{2} \mathrm{Cl}_{2}(3 \mathrm{x})$ and THF $(3 \mathrm{x})$. The resin is swollen in THF for $5 \mathrm{~min}$, then carefully transferred in round-bottom shape microwave vial equipped with a magnet. Remaining beads in the SPPS reactor were recovered using additional THF. Subsequently, the beads were allowed to deposit at the bottom of the vial and the excess of THF supernatant was carefully removed by pipette to reduce the solvent to a minimal volume. $\mathrm{Zn}(\mathrm{OAc})_{2}(3.6 \mathrm{mg}, 0.02 \mathrm{mmol}, 20$ 
mol\%, $0.02 \mathrm{mmol}$ ), NaOAc (41 mg, $0.5 \mathrm{mmol}, 5$ equiv) and H-L-Ala-OtBu.HCl L-5i (91 mg, $0.5 \mathrm{mmol}$, 5 equiv) were added and the vial was sealed with crimp cap. The reaction was stirred over $48 \mathrm{~h}$ at $90^{\circ} \mathrm{C}$. After it was allowed to cool down to room temperature, the reacting mixture was diluted with THF and $\mathrm{CH}_{2} \mathrm{Cl}_{2}$ and then transferred to the SPPS syringe reactor. The resin is thoroughly washed with THF and $\mathrm{CH}_{2} \mathrm{Cl}_{2}$. The resin is then cleaved using TFA/TIS/ $\mathrm{H}_{2} \mathrm{O}(95: 2.5: 2.5)$ cocktail for 2 hours at room temperature. After filtration, the solvent is removed in vacuo and the crude cleaved product is lyophilized and purified using semi-preparative HPLC $\left(\mathrm{H}_{2} \mathrm{O}: \mathrm{CH}_{3} \mathrm{CHN}\right.$ 80:20 to 45:55 over $\left.20 \mathrm{~min}\right)$. This yielded, after lyophilization, the title compound 16.TFA with $50 \%$ (50 mg, 0.049 mmol) yield. HPLC $\mathrm{rt}=2.90 \mathrm{~min}$. HRMS (ESI+) $\mathrm{m} / \mathrm{z}$ calc for $\mathrm{C}_{41} \mathrm{H}_{54} \mathrm{~N}_{8} \mathrm{O}_{8} \mathrm{Na}[\mathrm{M}+\mathrm{Na}]^{+}: 809.3962$ found 809.4008. $[\alpha]_{\mathbf{D}}=-23\left(\mathrm{c} 5 \mathrm{mg} / \mathrm{ml}, \mathrm{CH}_{3} \mathrm{OH}, 21^{\circ} \mathrm{C}\right)$.

\subsection{Cyclization using Transamidation of $t$ Bu-nicotinate Linear Heptapeptide: Synthesis and Cyclization}

Synthesis of the tBu nicotinate bearing linear heptapeptide $\mathbf{1 7 . H C l}$

For the synthesis of the linear heptapeptide $17 . \mathbf{H C l}$, a covergent strategy assembeling fragment $\mathbf{L}, \mathbf{L}, \mathbf{L}, \mathbf{L}-$ S13 and L,L,L-S15 was used.

Synthesis of fragment $\mathbf{L}, \boldsymbol{L}, L, L-S 13$
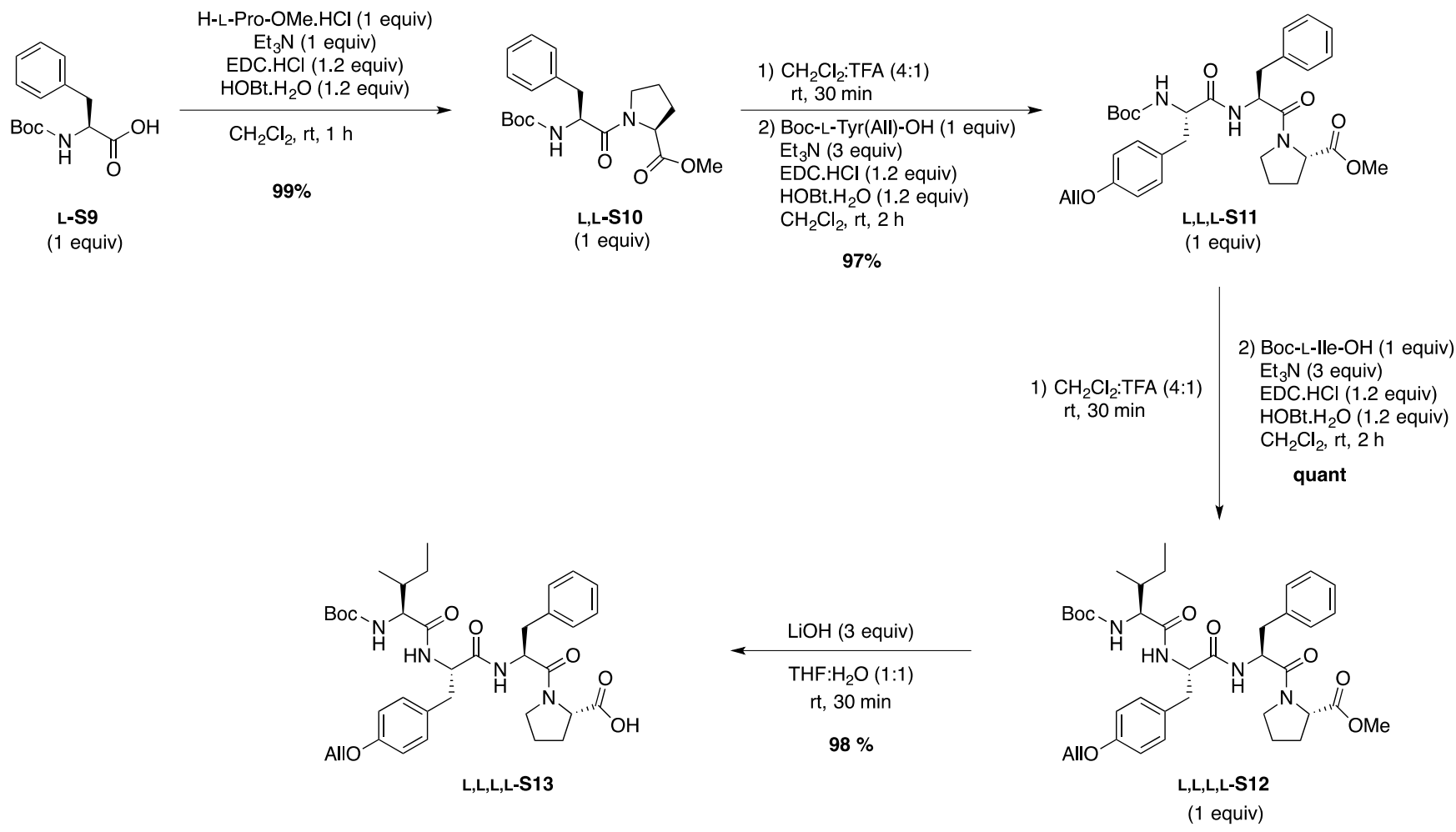
(S)-Methyl 1-((S)-2-((tert-butoxycarbonyl)amino)-3-phenylpropanoyl)pyrrolidine-2-carboxylate (BocL-Phe-L-Pro-OMe, KH_983) L,L-S10

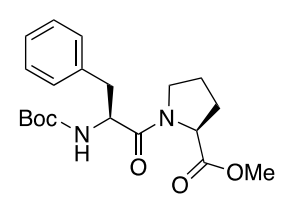

L,L-S10

The title compound was prepared using general procedure $\mathbf{D}$ from Boc-L-Phe-OH LS9 (796 mg, 3 mmol, 1 equiv), Pro-OMe. $\mathrm{HCl}$ (387 mg, 3 mmol, 1 equiv), EDC.HCl (690 mg, 3.6 mmol, 1.2 equiv), HOBt. $\mathrm{H}_{2} \mathrm{O}$ (551 mg, $3.6 \mathrm{mmol}, 1.2$ equiv) and $\mathrm{Et}_{3} \mathrm{~N}$ $(0.418 \mathrm{ml}, 3 \mathrm{mmol}, 1$ equiv). The reaction mixture was stirred for $1 \mathrm{~h}$. This yielded after extraction the title compound as a white powder with $99 \%$ yield (1.118 g, $2.97 \mathrm{mmol}) .{ }^{1} \mathbf{H}$ NMR (500 MHz, DMSO- $\boldsymbol{d}_{\boldsymbol{6}}$, mixture of rotamers) $\boldsymbol{\delta} 7.35-7.22(\mathrm{~m}, 4 \mathrm{H}), 7.22-7.17(\mathrm{~m}, 1 \mathrm{H}), 7.08(\mathrm{~d}, J=$ $8.4 \mathrm{~Hz}, 0.8 \mathrm{H}), 6.93(\mathrm{~d}, J=8.8 \mathrm{~Hz}, 0.1 \mathrm{H}), 6.71(\mathrm{~d}, J=7.7 \mathrm{~Hz}, 0.1 \mathrm{H}), 4.39-4.31(\mathrm{~m}, 1.6 \mathrm{H}), 4.29-4.22(\mathrm{~m}$, $0.3 \mathrm{H}), 4.14(\mathrm{dd}, J=2.5,7.9 \mathrm{~Hz}, 0.1 \mathrm{H}), 3.73-3.64(\mathrm{~m}, 1 \mathrm{H}), 3.63(\mathrm{~s}, 0.4 \mathrm{H}), 3.61(\mathrm{~s}, 2.6 \mathrm{H}), 3.54-3.47(\mathrm{~m}$, $0.7 \mathrm{H}), 3.45-3.37(\mathrm{~m}, 0.2 \mathrm{H}), 3.32-3.25(\mathrm{~m}, 0.1 \mathrm{H}), 2.89(\mathrm{dd}, J=4.5,14.2 \mathrm{~Hz}, 0.9 \mathrm{H}), 2.83(\mathrm{dd}, J=4.1$, $13.6 \mathrm{~Hz}, 0.1 \mathrm{H}), 2.74(\mathrm{dd}, J=9.8,14.1 \mathrm{~Hz}, 1 \mathrm{H}), 2.23-2.10(\mathrm{~m}, 1 \mathrm{H}), 1.95-1.89$ (m, 1.8H), 1.85-1.78 (m, $1 \mathrm{H}), 1.74-1.66(\mathrm{~m}, 0.2 \mathrm{H}), 1.29(\mathrm{~s}, 6.3 \mathrm{H}), 1.28(\mathrm{~s}, 1.4 \mathrm{H}), 1.22(\mathrm{~s}, 1.3 \mathrm{H}) \mathrm{ppm} .{ }^{\mathbf{1 3}} \mathbf{C}$ NMR (126 MHz, DMSO- $d_{\boldsymbol{\sigma}}$, mixture of rotamers) $\boldsymbol{\delta}[172.4,172.3,172.2](\mathrm{C}),[170.5,170.4,170.2](\mathrm{C}),[155.3,154.7$, 154.2] (C), [137.9, 137.6] (C), [129.9, 129.6, 129.4] (CH), [128.6, 128.1] (CH), [127.4, 126.4] (CH), $[78.3,78.1,78.0](\mathrm{C}),[59.0,58.7,58.5](\mathrm{CH}),[55.2,53.8,53.4](\mathrm{CH}),[52.2,52.0,51.8]\left(\mathrm{CH}_{3}\right),[46.6$, 46.5, 46.3, 46.1] $\left(\mathrm{CH}_{2}\right)$, [38.2, 36.9, 36.4, 36.2] $\left(\mathrm{CH}_{2}\right), 30.8\left(\mathrm{CH}_{2}\right)$, [28.6, 28.2, 27.9, 27.7] $\left(\mathrm{CH}_{3}\right), 24.8$ $\left(\mathrm{CH}_{2}\right)$ ppm. NMR-data was acquired at elevated temperature so that peaks arising from hindered rotation coalesced. ${ }^{1} \mathrm{H}$ NMR $\left(\mathbf{5 0 0} \mathbf{M H z}\right.$, DMSO-d $\boldsymbol{d}_{\boldsymbol{6}}, \mathbf{6 0}^{\circ} \mathrm{C}$, mixture of rotamers) $\boldsymbol{\delta}$ 7.32-7.24 (m, $3.7 \mathrm{H}), 7.23-7.18(\mathrm{~m}, 1.3 \mathrm{H}), 6.80($ br s, $1 \mathrm{H}) 4.48-4.38(\mathrm{~m}, 0.7 \mathrm{H}), 4.35$ (dd, $J=4.8,8.7 \mathrm{~Hz}, 1 \mathrm{H}), 4.33-$ $4.25(\mathrm{~m}, 0.3 \mathrm{H}), 3.93$ (br s, $1 \mathrm{H}), 3.63(\mathrm{~s}, 3 \mathrm{H}), 3.49-3.42(\mathrm{~m}, 0.8 \mathrm{H}), 3.42-3.37(\mathrm{~m}, 0.2 \mathrm{H}), 2.96-2.87(\mathrm{~m}$, $1 \mathrm{H}), 2.78(\mathrm{dd}, J=9.3,13.5 \mathrm{~Hz}, 1 \mathrm{H}), 2.27(\mathrm{td}, J=7.7,20.1 \mathrm{~Hz}, 1 \mathrm{H}), 1.92(\mathrm{td}, J=6.8,13.7 \mathrm{~Hz}, 2 \mathrm{H})$, 1.87-1.79 (m, 1H), 1.31 (s, 9H) ppm. HRMS (ESI+) m/z: calcd. $\mathrm{C}_{20} \mathrm{H}_{28} \mathrm{~N}_{2} \mathrm{O}_{5} \mathrm{Na}$ for $[\mathrm{M}+\mathrm{Na}]^{+}: 399.1891$ found 399.1867. No spectroscopic data are reported in literature. 
Methyl ((S)-2-((tert-butoxycarbonyl)amino)-3-(4-(((E)-prop-1-en-1-yl)oxy)phenyl)propanoyl)-Lphenylalanyl-L-prolinate (Boc-L-Tyr(All)-L-Phe-L-Pro-OMe, KH_985) L,L,L-S11

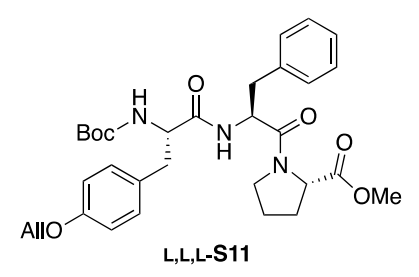

The title compound was obtained by means of a two step synthesis. To a solution of Boc-L-Phe-L-Pro-OMe L,L-S10 (1.118 g, 2.97 mmol, 1 equiv) in $\mathrm{CH}_{2} \mathrm{Cl}_{2}(20 \mathrm{ml})$ was trifluoroacetic acid $(5 \mathrm{ml})$ added and the mixture was stirred at room temperature for 30 minutes. After evaporation of the solvent in vacuo, the resulting crude solid material was used in the subsequent step without further purification. The peptide synthesis was performed following general procedure $\mathbf{D}$ forming the pre-activated mixture using Boc-L-Tyr(All)-OH (964 mg, 3 mmol, 1 equiv), EDC.HCl (690 mg, 3 mmol, 1.2 equiv) and HOBt. $\mathrm{H}_{2} \mathrm{O}$ (551 mg, $3.6 \mathrm{mmol}, 1.2$ equiv) in $\mathrm{CH}_{2} \mathrm{Cl}_{2}(20 \mathrm{ml})$. In parallel, to a flask containing the crude $\mathrm{H}$-L-Phe-L-Pro-OMe.TFA from the previous step, dissolved in $\mathrm{CH}_{2} \mathrm{Cl}_{2}(20 \mathrm{ml})$, was $\mathrm{Et}_{3} \mathrm{~N}$ added until the mixture reached $\mathrm{pH}$ 9. The two mixture were combined and allowed to stirred for $2 \mathrm{~h}$ at roomtemperature. This yielded, after extraction, the title compound as a white powder with $97 \%$ (1.686 g, $2.91 \mathrm{mmol}$ ) yield over the two steps. ${ }^{1} \mathbf{H}$ NMR (500 MHz, DMSO-d $\boldsymbol{d}_{\boldsymbol{\sigma}}$, mixture of rotamers) $\boldsymbol{\delta} 8.26$ $(\mathrm{d}, J=7.9 \mathrm{~Hz}, 0.1 \mathrm{H}), 8.14(\mathrm{~d}, J=8.0 \mathrm{~Hz}, 0.8 \mathrm{H}), 8.03(\mathrm{~d}, J=8.4 \mathrm{~Hz}, 0.1 \mathrm{H}), 7.31-7.25(\mathrm{~m}, 4 \mathrm{H}), 7.23-$ $7.18(\mathrm{~m}, 1 \mathrm{H}), 7.12(\mathrm{~d}, J=8.3 \mathrm{~Hz}, 0.5 \mathrm{H}), 7.07(\mathrm{~d}, J=8.5 \mathrm{~Hz}, 1.5 \mathrm{H}), 6.86-6.77(\mathrm{~m}, 3 \mathrm{H}), 6.02(\mathrm{ddt}, J=$ $5.0 \mathrm{~Hz}, 10.5 \mathrm{~Hz}, 17.2 \mathrm{~Hz}, 1 \mathrm{H}), 5.37(\mathrm{dd}, J=1.5,17.2 \mathrm{~Hz}, 1 \mathrm{H}), 5.23(\mathrm{dd}, J=1.3 \mathrm{~Hz}, 10.6 \mathrm{~Hz}, 1 \mathrm{H}), 4.70$ $(\mathrm{ddd}, \mathrm{J}=7.9,7.9,6.1 \mathrm{~Hz}, 0.9 \mathrm{H}), 4.57(\mathrm{ddd}, J=7.8,7.8,7.8 \mathrm{~Hz}, 0.1 \mathrm{H}), 4.51(\mathrm{~d}, J=5.0 \mathrm{~Hz}, 2 \mathrm{H}), 4.29$ $(\mathrm{dd}, J=4.8,8.6 \mathrm{~Hz}, 1 \mathrm{H}), 4.07$ (ddd, $J=4.9,4.9,13.9 \mathrm{~Hz}, 1 \mathrm{H}), 3.61$ (s, 2.5H), 3.59 (s, 0.5H), 3.60-2.56 (m, 1H), 3.41-3.35 (m, 1H), $3.01(\mathrm{dd}, J=5.9,14.1 \mathrm{~Hz}, 0.9 \mathrm{H}), 2.89$ (dd, $J=7.8,12.8 \mathrm{~Hz}, 0.1 \mathrm{H}), 2.84-$ $2.72(\mathrm{~m}, 2 \mathrm{H}), 2.56(\mathrm{dd}, J=10.3,13.5 \mathrm{~Hz}, 1 \mathrm{H}), 2.17-2.09(\mathrm{~m}, 1 \mathrm{H}), 1.92-1.84(\mathrm{~m}, 2 \mathrm{H}), 1.84-1.76(\mathrm{~m}$, 1H), 1.29 (s, 8H), $1.16(\mathrm{~s}, 1 \mathrm{H}) \mathrm{ppm} .{ }^{13} \mathrm{C}$ NMR (126 MHz, DMSO-d $\boldsymbol{d}_{6}$ mixture of rotamers) $\delta[172.1$, 171.8] (C), 171.3 (C), [169.6, 169.4] (C), 156.7 (C), 155.0 (C), 137.2 (C), 136.7 (C), 133.9 (CH), [130.1, 130.0] (CH), [129.4, 129.2] (CH), [128.3, 128.1] (CH), [126.7, 126.4] (CH), $117.2\left(\mathrm{CH}_{2}\right), 114.2$ $(\mathrm{CH}), 78.0(\mathrm{C}), 68.1\left(\mathrm{CH}_{2}\right)$, [58.6, 58.4] $(\mathrm{CH}), 55.9(\mathrm{CH}), 51.8(\mathrm{CH})$, [51.7, 51.5] $\left(\mathrm{CH}_{3}\right)$, [46.5, 45.9] $\left(\mathrm{CH}_{2}\right), 37.0\left(\mathrm{CH}_{2}\right), 36.7\left(\mathrm{CH}_{2}\right), 28.6\left(\mathrm{CH}_{2}\right)$, [28.1, 27.8] $\left(\mathrm{CH}_{3}\right), 24.6\left(\mathrm{CH}_{2}\right)$ ppm. NMR-data was acquired at elevated temperature so that peaks arising from hindered rotation coalesced. ${ }^{\mathbf{1}} \mathbf{H}$ NMR $(\mathbf{5 0 0}$ MHz, DMSO- $\boldsymbol{d}_{\boldsymbol{6}} \mathbf{6 0}^{\circ} \mathbf{C}$, mixture of rotamers) $\boldsymbol{\delta} 7.97$ (br s, 0.9H), 7.84 (br s, 0.1H), 7.31-7.24 (m, 4H), 7.23-7.18 (m, 1H), $7.10(\mathrm{~d}, J=8.0 \mathrm{~Hz}, 0.3 \mathrm{H}), 7.01(\mathrm{~d}, J=8.2 \mathrm{~Hz}, 1.7 \mathrm{H}), 6.84-6.78(\mathrm{~m}, 2 \mathrm{H}), 6.60(\mathrm{br} \mathrm{s}$, 1H), 6.02 (ddt, $J=5.0,10.5 \mathrm{~Hz}, 17.2 \mathrm{~Hz}, 1 \mathrm{H}), 5.37$ (dd, J = 1.5, $17.2 \mathrm{~Hz}, 1 \mathrm{H}), 5.23$ (dd, $J=1.3,10.6$ $\mathrm{Hz}, 1 \mathrm{H}), 4.74(\mathrm{dd}, J=7.7,14.4 \mathrm{~Hz}, 0.9 \mathrm{H}), 4.61(\mathrm{dd}, J=7.5,15.2 \mathrm{~Hz}, 0.1 \mathrm{H}), 4.51(\mathrm{~d}, J=5.2 \mathrm{~Hz}, 2 \mathrm{H})$, $4.31(\mathrm{dd}, J=4.7,8.6 \mathrm{~Hz}, 1 \mathrm{H}), 4.13-4.07(\mathrm{~m}, 1 \mathrm{H}), 3.62(\mathrm{~s}, 3 \mathrm{H}), 3.61-3.58(\mathrm{~m}, 1 \mathrm{H}), 3.40-3.33(\mathrm{~m}, 1 \mathrm{H})$, $3.02(\mathrm{dd}, J=6.0,13.8 \mathrm{~Hz}, 1 \mathrm{H}), 2.81(\mathrm{ddd}, J=14.2,14.2,6.3 \mathrm{~Hz}, 2 \mathrm{H}), 2.60(\mathrm{dd}, J=10.3,13.2 \mathrm{~Hz}, 1 \mathrm{H})$, 
2.19-2.09 (m, 1H), 1.92-1.76 (m, 3H),1.30 (s, 9H) ppm. HRMS (ESI+) m/z: calcd. $\mathrm{C}_{32} \mathrm{H}_{42} \mathrm{~N}_{3} \mathrm{O}_{7}$ for $[\mathrm{M}+\mathrm{H}]^{+}: 580.3017$ found 580.3020 . No spectroscopic data are reported in literature.

Methyl ((S)-2-((2S,3R)-2-((tert-butoxycarbonyl)amino)-3-methylpentanamido)-3-(4-(((E)-prop-1-en-1yl)oxy)phenyl)propanoyl)-L-phenylalanyl-L-prolinate (Boc-L-Ile-L-Tyr(All)-L-Phe-L-Pro-OMe, KH_987) L,L,L,L-S12

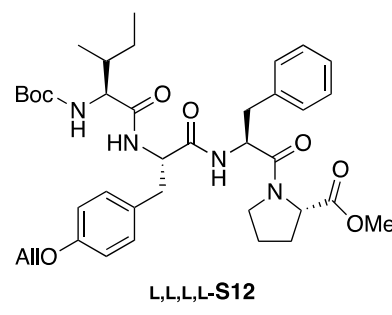

The title compound was obtained by means of a two step synthesis. To a solution of Boc-L-Tyr(All)-L-Phe-L-Pro-OMe L,L,L-S11 (1.686 g, 2.91 mmol, 1 equiv) in $\mathrm{CH}_{2} \mathrm{Cl}_{2}(20 \mathrm{ml})$, trifluoroacetic acid $(5 \mathrm{ml})$ was added and the mixture was stirred at room temperature for 30 minutes. After evaporation of the solvent in vacuo, the resulting crude solid material was used in the subsequent step without further purification. The peptide synthesis was performed following general procedure $\mathbf{D}$ forming the pre-activated mixture using Boc-L-Ile-OH.0.5 $\mathrm{H}_{2} \mathrm{O}(671 \mathrm{mg}, 2.9 \mathrm{mmol}$, 1 equiv), $\mathrm{EDC} . \mathrm{HCl}$ (667 mg, $3.5 \mathrm{mmol}, 1.2$ equiv) and $\mathrm{HOBt} . \mathrm{H}_{2} \mathrm{O}$ (533 mg, $3.5 \mathrm{mmol}, 1.2$ equiv) in $\mathrm{CH}_{2} \mathrm{Cl}_{2}(20 \mathrm{ml})$. In parallel, to a flask containing the crude H-L-Tyr(All)-L-Phe-L-Pro-OMe.TFA from the previous step, dissolved in $\mathrm{CH}_{2} \mathrm{Cl}_{2}(20 \mathrm{ml})$, was $\mathrm{Et}_{3} \mathrm{~N}$ added until the mixture reached $\mathrm{pH}$ 9. The two mixture were combined and allowed to stirred for $2 \mathrm{~h}$ at roomtemperature.This yielded, after extraction, the title compound as a white powder with $99 \%(1.987 \mathrm{~g}, 2.87 \mathrm{mmol})$ yield over the two steps. ${ }^{1} \mathbf{H}$ NMR (500 MHz, DMSO- $\boldsymbol{d}_{\boldsymbol{6}}$, mixture of rotamers) $\boldsymbol{\delta} 8.44$ (br s, $\left.0.1 \mathrm{H}\right), 8.33$ (d, $\left.J=7.8 \mathrm{~Hz}, 0.8 \mathrm{H}\right), 8.21$ $(\mathrm{d}, J=8 \mathrm{~Hz}, 0.1 \mathrm{H}), 7.78-7.70(\mathrm{~m}, 1 \mathrm{H}), 7.29-7.22(\mathrm{~m}, 4 \mathrm{H}), 7.21-7.17(\mathrm{~m}, 1 \mathrm{H}), 7.12(\mathrm{~d}, J=8.2 \mathrm{~Hz}$, $0.2 \mathrm{H}), 7.09(\mathrm{~d}, J=8.4 \mathrm{~Hz}, 1.8 \mathrm{H}), 6.77(\mathrm{~d}, J=8.4 \mathrm{~Hz}, 2 \mathrm{H}), 6.69(\mathrm{~d}, J=9.2 \mathrm{~Hz}, 1 \mathrm{H}), 6.02$ (ddt, $J=5.0$, 10.5, 17.2 Hz, 1H), 5.36 (dd, $J=1.5,17.2 \mathrm{~Hz}, 1 \mathrm{H}), 5.23(\mathrm{dd}, J=1.5,10.5 \mathrm{~Hz}, 1 \mathrm{H}), 4.65$ (q, $J=7.2 \mathrm{~Hz}$, $1 \mathrm{H}), 4.56-4.51(\mathrm{~m}, 1 \mathrm{H}), 4.49(\mathrm{~d}, J=5.0 \mathrm{~Hz}, 2 \mathrm{H}), 4.30-4.25(\mathrm{~m}, 1 \mathrm{H}), 3.84-3.82(\mathrm{~m}, 0.1 \mathrm{H}), 3.74(\mathrm{t}, J=$ $8.5 \mathrm{~Hz}, 0.9 \mathrm{H}), 3.60(\mathrm{~s}, 2.6 \mathrm{H}), 3.58(\mathrm{~s}, 0.4 \mathrm{H}), 3.53-3.49(\mathrm{~m}, 1 \mathrm{H}), 3.30-3.24(\mathrm{~m}, 1 \mathrm{H}), 2.99$ (dd, $J=6.3$, $13.9 \mathrm{~Hz}, 1 \mathrm{H}), 2.82(\mathrm{dd}, J=4.8,13.7 \mathrm{~Hz}, 1 \mathrm{H}), 2.78(\mathrm{dd}, J=7.4,13.8 \mathrm{~Hz}, 1 \mathrm{H}), 2.66(\mathrm{dd}, J=9.4,13.7$ $\mathrm{Hz}, 1 \mathrm{H}), 2.15-2.08(\mathrm{~m}, 1 \mathrm{H}), 1.84-1.77(\mathrm{~m}, 2.7 \mathrm{H}), 1.67-1.64(\mathrm{~m}, 0.3 \mathrm{H}), 1.57-1.52(\mathrm{~m}, 1 \mathrm{H}), 1.37(\mathrm{~s}, 9 \mathrm{H})$, 1.27-1.22 (m, 1H), 0.99-0.92 (m, 1H), $0.73(\mathrm{t}, J=7.4 \mathrm{~Hz}, 3 \mathrm{H}), 0.61(\mathrm{~d}, J=6.7 \mathrm{~Hz}, 3 \mathrm{H}) \mathrm{ppm} .{ }^{13} \mathbf{C} \mathbf{~ N M R}$ (126 MHz, DMSO-d $d_{6}$, mixture of rotamers) $\delta[172.2,171.9](\mathrm{C}), 170.9$ (C), [170.7, 170.4] (C), [169.5, 169.3] (C), 156.8 (C), 155.3 (C), 137.3 (C), 136.7 (C), 134.0 (CH), 130.3 (CH), [129.6, 129.3, 129.2] $(\mathrm{CH}),[128.4,128.2](\mathrm{CH}),[126.8,126.4](\mathrm{CH}), 117.2\left(\mathrm{CH}_{2}\right), 114.2(\mathrm{CH}), 78.1(\mathrm{C}), 68.1\left(\mathrm{CH}_{2}\right)$, $59.0(\mathrm{CH}),[58.7,58.4](\mathrm{CH}), 53.5(\mathrm{CH}),[52.4,51.9](\mathrm{CH}), 51.9\left(\mathrm{CH}_{3}\right),[46.5,46.0]\left(\mathrm{CH}_{2}\right), 37.0\left(\mathrm{CH}_{2}\right)$, $37.0\left(\mathrm{CH}_{2}\right), 36.6(\mathrm{CH})$, [30.5, 28.7] $\left(\mathrm{CH}_{2}\right)$, [28.2, 28.0] $\left(\mathrm{CH}_{3}\right)$, [24.6] $\left(\mathrm{CH}_{2}\right), 24.3\left(\mathrm{CH}_{2}\right)$, [21.9] $\left(\mathrm{CH}_{2}\right)$, $15.3\left(\mathrm{CH}_{3}\right), 10.9\left(\mathrm{CH}_{3}\right)$ ppm. NMR-data was acquired at elevated temperature so that peaks arising from hindered rotation coalesced. ${ }^{1} \mathrm{H}$ NMR $\left(500 \mathrm{MHz}\right.$, DMSO- $\boldsymbol{d}_{\boldsymbol{\sigma}}, \mathbf{6 0}^{\circ} \mathrm{C}$, mixture of rotamers) $\delta 8.11(\mathrm{~d}, J$ 
$=5.9 \mathrm{~Hz}, 0.9 \mathrm{H}), 7.93(\mathrm{~d}, J=8.1 \mathrm{~Hz}, 0.1 \mathrm{H}), 7.69(\mathrm{~d}, J=8.4 \mathrm{~Hz}, 0.1 \mathrm{H}), 7.64(\mathrm{~d}, J=8.4 \mathrm{~Hz}, 0.9 \mathrm{H}), 7.30$ $7.22(\mathrm{~m}, 4 \mathrm{H}), 7.21-7.15(\mathrm{~m}, 1 \mathrm{H}), 7.12(\mathrm{~d}, J=8.4 \mathrm{~Hz}, 0.3 \mathrm{H}), 7.08(\mathrm{~d}, J=8.5 \mathrm{~Hz}, 1.7 \mathrm{H}), 6.78(\mathrm{~d}, J=8.4$ $\mathrm{Hz}, 2 \mathrm{H}), 6.47$ (br s, 1H), 6.02 (ddt, $J=5.1,10.5,17.3 \mathrm{~Hz}, 1 \mathrm{H}), 5.36$ (dd, $J=1.5,17.3 \mathrm{~Hz}, 1 \mathrm{H}), 5.23$ $(\mathrm{dd}, J=1.5,10.5 \mathrm{~Hz}, 1 \mathrm{H}), 4.69(\mathrm{dd}, J=7.2,14.6 \mathrm{~Hz}, 1 \mathrm{H}) 4.61-4.51(\mathrm{~m}, 1 \mathrm{H}), 4.50(\mathrm{~d}, J=5.1 \mathrm{~Hz}, 2 \mathrm{H})$, $4.29(\mathrm{dd}, J=4.0,8.4 \mathrm{~Hz}, 1 \mathrm{H}), 3.86-3.83(\mathrm{~m}, 0.1 \mathrm{H}), 3.80-3.74(\mathrm{~m}, 0.9 \mathrm{H}), 3.61(\mathrm{~s}, 2.7 \mathrm{H}), 3.59(\mathrm{~s}, 0.3 \mathrm{H})$, 3.58-3.49 (m, 1H), 3.30-3.23 (m, 1H), $3.01(\mathrm{dd}, J=6.6,13.9 \mathrm{~Hz}, 1 \mathrm{H}), 2.86(\mathrm{dd}, J=5.1,14.1 \mathrm{~Hz}, 1 \mathrm{H})$, $2.80(\mathrm{dd}, J=6.9,13.9 \mathrm{~Hz}, 1 \mathrm{H}), 2.70(\mathrm{dd}, J=8.7,13.9 \mathrm{~Hz}, 1 \mathrm{H}), 2.17-2.07(\mathrm{~m}, 1 \mathrm{H}), 1.89-1.77(\mathrm{~m}, 3 \mathrm{H})$, $1.63-1.56(\mathrm{~m}, 1 \mathrm{H}), 1.37(\mathrm{~s}, 9 \mathrm{H}), 1.34-1.23(\mathrm{~m}, 1 \mathrm{H}), 1.06-0.97(\mathrm{~m}, 1 \mathrm{H}), 0.76(\mathrm{t}, J=7.4 \mathrm{~Hz}, 3 \mathrm{H}), 0.66(\mathrm{~d}$, $J=6.7 \mathrm{~Hz}, 3 \mathrm{H})$ ppm. HRMS $(\mathrm{ESI}+) \mathrm{m} / \mathrm{z}$ : calcd. $\mathrm{C}_{38} \mathrm{H}_{52} \mathrm{~N}_{4} \mathrm{O}_{8} \mathrm{Na}$ for $[\mathrm{M}+\mathrm{Na}]^{+}: 715.3677$ found 715.3718. No spectroscopic data are reported in literature.

((S)-2-((2S,3R)-2-((tert-Butoxycarbonyl)amino)-3-methylpentanamido)-3-(4-(((E)-prop-1-en-1yl)oxy)phenyl)propanoyl)-L-phenyl-alanyl-L-proline (Boc-L-Ile-L-Tyr(All)-L-Phe-L-Pro-OH，KH_988) L,L,L,L-S12

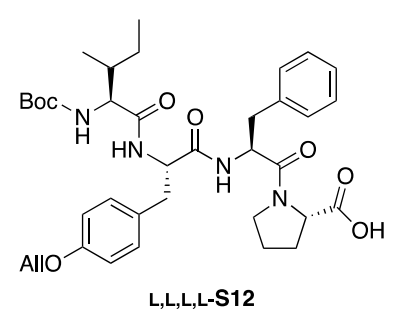

The title compound was obtained starting from Boc-L-Ile-L-Tyr(All)-L-Phe-LPro-OMe L,L,L,L-S11 (2.0 g, $2.99 \mathrm{mmol})$ solubilized in THF (22 ml). A solution of $\mathrm{LiOH}(0.119 \mathrm{~g}, 8.7 \mathrm{mmol}, 3$ equiv) in water $(22 \mathrm{ml})$ was added and the mixture was stirred at $\mathrm{rt}$ for 30 minutes and quenched with $\mathrm{HCl} 1 \mathrm{~N}$ until pH 4 was reached. The aqueous layer was then extracted with $\mathrm{CH}_{2} \mathrm{Cl}_{2}(3 \mathrm{x})$. The resulting organic layer was finally dried over $\mathrm{MgSO}_{4}$ and concentrated in vacuo giving a colorless oil in 98\% (1.93 g, $2.84 \mathrm{mmol}$ ) yield. ${ }^{1} \mathbf{H}$ NMR (500 MHz, DMSO- $\boldsymbol{d}_{\boldsymbol{6}}$, mixture of rotamers) $\boldsymbol{\delta} 12.52$ (br s, $1 \mathrm{H}), 8.43(\mathrm{~d}, J=7.1 \mathrm{~Hz}, 0.1 \mathrm{H}), 8.33(\mathrm{~d}, J=8.0 \mathrm{~Hz}, 0.8 \mathrm{H}), 8.05(\mathrm{~d}, J=8.0 \mathrm{~Hz}, 0.1 \mathrm{H}), 7.82(\mathrm{~d}, J=8.9$ $\mathrm{Hz}, 0.1 \mathrm{H}), 7.76(\mathrm{~d}, J=8.3 \mathrm{~Hz}, 0.8 \mathrm{H}), 7.70(\mathrm{~d}, J=8.0 \mathrm{~Hz}, 0.1 \mathrm{H}), 7.30-7.21(\mathrm{~m}, 4 \mathrm{H}), 9.19-7.14(\mathrm{~m}, 1 \mathrm{H})$, $7.09(\mathrm{~d}, J=8.6 \mathrm{~Hz}, 2 \mathrm{H}), 6.80-6.75(\mathrm{~m}, 2 \mathrm{H}), 6.73-6.65(\mathrm{~m}, 1 \mathrm{H}), 6.02(\mathrm{ddt}, J=5.1,10.5,17.2 \mathrm{~Hz}, 1 \mathrm{H})$, $5.36(\mathrm{dd}, J=1.5,17.2 \mathrm{~Hz}, 1 \mathrm{H}), 5.23(\mathrm{dd}, J=1.5,10.5 \mathrm{~Hz}, 1 \mathrm{H}), 4.64(\mathrm{dd}, J=7.3,14.0 \mathrm{~Hz}, 1 \mathrm{H}), 4.58$ $4.51(\mathrm{~m}, 1 \mathrm{H}), 4.48(\mathrm{~d}, J=5.1 \mathrm{~Hz}, 2 \mathrm{H}), 4.22(\mathrm{dd}, J=3.4,8.3 \mathrm{~Hz}, 1 \mathrm{H}), 3.77-3.69(\mathrm{~m}, 1 \mathrm{H}), 3.54-3.45(\mathrm{~m}$, $1 \mathrm{H}), 3.42-3.30(\mathrm{~m}, 1 \mathrm{H}$, overlap with $\mathrm{HDO}), 3.00(\mathrm{dd}, J=5.7,14.0 \mathrm{~Hz}, 1 \mathrm{H}), 2.83(\mathrm{dd}, J=4.5,13.7 \mathrm{~Hz}$, $1 \mathrm{H}), 2.77(\mathrm{dd}, J=7.7,13.9 \mathrm{~Hz}, 1 \mathrm{H}), 2.65(\mathrm{dd}, J=9.3,13.9 \mathrm{~Hz}, 1 \mathrm{H}), 2.13-2.05(\mathrm{~m}, 1 \mathrm{H}), 1.87-1.78(\mathrm{~m}$, 2.7H), 1.68-1.61 (m, 0.3H), 1.57-1.47 (m, 1H), $1.36(\mathrm{~s}, 8 \mathrm{H}), 1.26(\mathrm{~s}, 1 \mathrm{H}), 1.25-1.19(\mathrm{~m}, 1 \mathrm{H}), 1.00-0.91$ $(\mathrm{m}, 1 \mathrm{H}), 0.75-0.70(\mathrm{~m}, 3 \mathrm{H}), 0.63-0.58(\mathrm{~m}, 3 \mathrm{H}) \mathrm{ppm} .{ }^{13} \mathrm{C}$ NMR (126 MHz, DMSO-d $\boldsymbol{d}_{\boldsymbol{\sigma}}$, mixture of rotamers) $\delta[173.1,172.8](\mathrm{C}),[171.0,170.8](\mathrm{C}),[170.7,170.3](\mathrm{C}), 169.0(\mathrm{C}), 156.7(\mathrm{C}), 155.2(\mathrm{C})$, $137.3(\mathrm{C}), 136.7(\mathrm{C}), 133.9(\mathrm{CH}), 130.2(\mathrm{CH}),[129.5,129.3](\mathrm{CH}),[128.3,128.1](\mathrm{CH}),[126.7,126.3]$ $(\mathrm{CH}), 117.2\left(\mathrm{CH}_{2}\right), 114.1(\mathrm{CH}), 78.0(\mathrm{C}), 68.0\left(\mathrm{CH}_{2}\right), 58.9(\mathrm{CH}),[58.6,58.4](\mathrm{CH})$, [53.5, 53.4] $(\mathrm{CH})$, [52.1, 51.8] $(\mathrm{CH}),[46.4,45.8]\left(\mathrm{CH}_{2}\right), 37.0\left(\mathrm{CH}_{2}\right), 36.8\left(\mathrm{CH}_{2}\right), 36.6(\mathrm{CH})$, [30.5, 28.6] $\left(\mathrm{CH}_{2}\right)$, [28.2, 
27.9] $\left(\mathrm{CH}_{3}\right)$, [24.5] $\left(\mathrm{CH}_{2}\right), 24.2\left(\mathrm{CH}_{2}\right)$, [21.7] $\left(\mathrm{CH}_{2}\right), 15.2\left(\mathrm{CH}_{3}\right), 10.9\left(\mathrm{CH}_{3}\right)$ ppm. NMR-data was acquired at elevated temperature so that peaks arising from hindered rotation coalesced. ${ }^{\mathbf{1}} \mathbf{H}$ NMR $(\mathbf{5 0 0}$ MHz, DMSO- $d_{6}, \mathbf{6 0}^{\circ} \mathbf{C}$, mixture of rotamers) $\delta 12.32(\mathrm{br} \mathrm{s}, 1 \mathrm{H}), 8.08(\mathrm{~d}, J=6.5 \mathrm{~Hz}, 0.9 \mathrm{H}), 7.77(\mathrm{~d}, J$ $=7.6 \mathrm{~Hz}, 0.1 \mathrm{H}), 7.73(\mathrm{~d}, J=8.3 \mathrm{~Hz}, 0.1 \mathrm{H}), 7.63(\mathrm{~d}, J=8.3 \mathrm{~Hz}, 0.9 \mathrm{H}), 7.30-7.20(\mathrm{~m}, 4 \mathrm{H}), 7.19-7.14(\mathrm{~m}$, 1H), $7.08(\mathrm{~d}, J=8.1 \mathrm{~Hz}, 2 \mathrm{H}), 6.77$ (d, $J=7.8 \mathrm{~Hz}, 2 \mathrm{H}), 6.47$ (br s, 1H), 6.02 (ddt, $J=5.1,10.5,17.2 \mathrm{~Hz}$, $1 \mathrm{H}), 5.36(\mathrm{dd}, J=1.5,17.2 \mathrm{~Hz}, 1 \mathrm{H}), 5.23(\mathrm{dd}, J=1.5,10.5 \mathrm{~Hz}, 1 \mathrm{H}), 4.68(\mathrm{dd}, J=6.8,14.6 \mathrm{~Hz}, 1 \mathrm{H})$, $4.57-4.51(\mathrm{~m}, 1 \mathrm{H}), 4.50(\mathrm{~d}, J=5.5 \mathrm{~Hz}, 2 \mathrm{H}), 4.25(\mathrm{dd}, J=3.2,8.4 \mathrm{~Hz}, 1 \mathrm{H}), 3.79-3.71(\mathrm{~m}, 1 \mathrm{H}), 3.55-3.49$ (m, 1H), 3.35-3.26 (m, 1H), $3.03(\mathrm{dd}, J=6.3,13.9 \mathrm{~Hz}, 1 \mathrm{H}), 2.86(\mathrm{dd}, J=5.2,13.9 \mathrm{~Hz}, 1 \mathrm{H}), 2.79(\mathrm{dd}, J$ = 7.2, $13.9 \mathrm{~Hz}, 1 \mathrm{H}), 2.70(\mathrm{dd}, J=8.8,13.9 \mathrm{~Hz}, 1 \mathrm{H}), 2.15-2.05(\mathrm{~m}, 1 \mathrm{H}), 1.91-1.67(\mathrm{~m}, 3 \mathrm{H}), 1.63-1.55$ $(\mathrm{m}, 1 \mathrm{H}), 1.37(\mathrm{~s}, 9 \mathrm{H}), 1.33-1.26(\mathrm{~m}, 1 \mathrm{H}), 1.05-0.96(\mathrm{~m}, 1 \mathrm{H}), 0.76(\mathrm{t}, J=7.2 \mathrm{~Hz}, 3 \mathrm{H}), 0.70-0.64(\mathrm{~m}, 3 \mathrm{H})$ ppm. HRMS (ESI+) m/z: calcd. $\mathrm{C}_{37} \mathrm{H}_{50} \mathrm{~N}_{4} \mathrm{O}_{8} \mathrm{Na}$ for $[\mathrm{M}+\mathrm{Na}]^{+}: 701.3521$ found 701.3553. No spectroscopic data are reported in literature.

Synthesis of Fragment L,L,L-S15
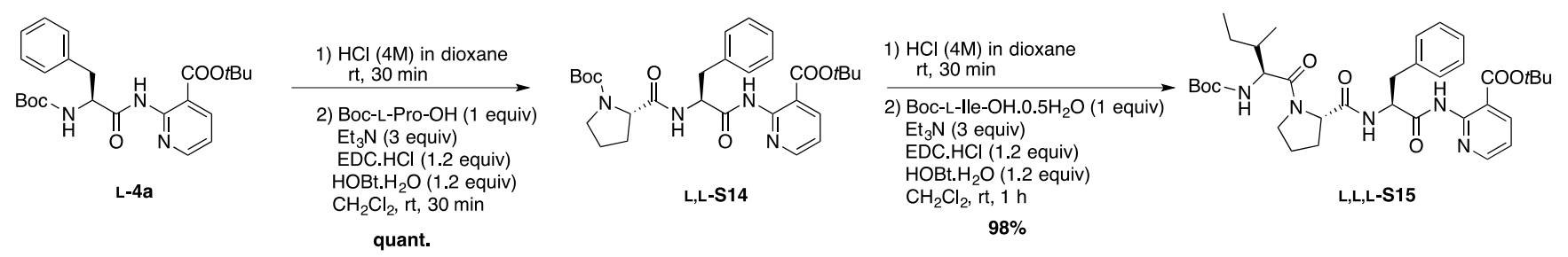

tert-Butyl 2-((2S)-2-(1-(tert-butoxycarbonyl)pyrrolidine-2-carboxamido)-3-phenylpropanamido) nicotinate (Boc-L-Pro-L-Phe-NH-tBu-nic, KH_1214) L,L-S14

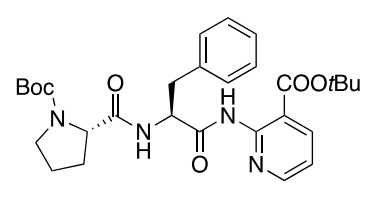

L,L-S14

For the synthesis of Boc-L-Pro-L-Phe-NH- $t$ Bu-nic L,L-S14 was started from Boc-L-Phe-NH- $t$ Bu-nic L-4a (442 mg, 1 mmol, 1 equiv) that was deprotected following general procedure $\mathbf{C}$ with $\mathrm{HCl} /$ dioxane $(40 \mathrm{ml}, 4 \mathrm{M}$ ). After removal of the solvent under vacuum, the resulting crude solid material $(\mathrm{H}-\mathrm{L}-\mathrm{Phe}-\mathrm{NH}-\mathrm{Bu}-$ nic. $\mathrm{HCl}$ ) was used in the subsequent step without further purification.

The next step was performed following general procedure D. The crude was dissolved in $\mathrm{CH}_{2} \mathrm{Cl}_{2}(20$ $\mathrm{ml}$ ) and $\mathrm{Et}_{3} \mathrm{~N}$ was added until the mixture reached $\mathrm{pH}$ 9. This was added to the pre-activated mixture of Boc-L-Pro-OH (215 mg, $1 \mathrm{mmol}, 1$ equiv), EDC.HCl (230 mg, 0.65 mmol, 1.2 equiv) and $\mathrm{HOBt}_{2} \mathrm{H}_{2} \mathrm{O}$ (184 mg, $1.2 \mathrm{mmol}, 1.2$ equiv). The reaction mixture was stirred for 30 minutes. After extraction, the title compound could be obtained as a white powder in quantitive yield (545 mg, $1 \mathrm{mmol}$ ) over the two steps. ${ }^{1} \mathbf{H}$ NMR (500 $\mathbf{M H z}, \mathbf{C D C l}_{3}$, mixture of rotamers) $\boldsymbol{\delta} 11.42$ (br s, 0.5H), 11.11 (br s, 0.5H), 8.64$8.50(\mathrm{~m}, 1 \mathrm{H}), 8.23(\mathrm{dd}, J=1.5,7.8 \mathrm{~Hz}, 1 \mathrm{H}), 7.60$ (br s, 0.5H), 7.25-7.19 (m, 4H), 7.18-7.14 (m, 1H), $7.06(\mathrm{dd}, J=4.8,7.5 \mathrm{~Hz}, 1 \mathrm{H}), 6.78$ (br s, 0.5H), 5.54-4.99 (m, 1H), 4.51-4.11 (m, 1H), 3.45- 3.16 (m, 
$3 \mathrm{H}), 3.02(\mathrm{dd}, J=8.6,13.9 \mathrm{~Hz}, 1 \mathrm{H}), 2.58-2.25(\mathrm{~m}, 1 \mathrm{H}), 2.07-1.88(\mathrm{~m}, 1 \mathrm{H}), 1.85-1.67(\mathrm{~m}, 2 \mathrm{H}), 1.57$ (s, 9H), 1.45 (s, 9H) ppm. ${ }^{13} \mathbf{C}$ NMR (126 $\mathbf{~ M H z , ~} \mathbf{C D C l}_{3}$, mixture of rotamers) $\delta 172.3(\mathrm{C}), 170.0(\mathrm{C})$, $165.7(\mathrm{C}), 152.2(\mathrm{CH}), 151.9(\mathrm{C}), 140.6(\mathrm{CH}), 137.0(\mathrm{C}), 129.5(\mathrm{CH}), 128.5(\mathrm{CH}), 126.8(\mathrm{CH}), 118.5$ $(\mathrm{CH}), 113.3(\mathrm{C}), 83.7(\mathrm{C}), 80.6(\mathrm{C})$, [61.0, 59.9] $(\mathrm{CH})$, [55.5, 55.2] $(\mathrm{CH}), 47.0\left(\mathrm{CH}_{2}\right)$, [38.8, 38.1] $\left(\mathrm{CH}_{2}\right), 30.5(\mathrm{C})$, [28.5] $\left(\mathrm{CH}_{3}\right), 28.3\left(\mathrm{CH}_{3}\right)$, [27.7] $\left(\mathrm{CH}_{3}\right), 24.6\left(\mathrm{CH}_{2}\right), 23.5\left(\mathrm{CH}_{2}\right)$ ppm. NMR-data was acquired at elevated temperature so that peaks arising from hindered rotation coalesced. ${ }^{\mathbf{1}} \mathbf{H}$ NMR (500 MHz, DMSO- $\left.\boldsymbol{d}_{\boldsymbol{6}}, \mathbf{8 0}^{\circ} \mathrm{C}\right) \boldsymbol{\delta} 10.38$ (br s, $\left.1 \mathrm{H}\right), 8.61$ (br s, $\left.1 \mathrm{H}\right), 8.10$ (d, $\left.J=7.6 \mathrm{~Hz}, 1 \mathrm{H}\right), 7.89$ (d, $J=7.2$ $\mathrm{Hz}, 1 \mathrm{H}), 7.34-7.21(\mathrm{~m}, 5 \mathrm{H}), 7.20-7.15(\mathrm{~m}, 1 \mathrm{H}), 5.00-4.89(\mathrm{~m}, 1 \mathrm{H}), 4.19-4.05(\mathrm{~m}, 1 \mathrm{H}), 3.33-3.24(\mathrm{~m}$, 2H), $3.20(\mathrm{dd}, J=14.0,3.8 \mathrm{~Hz}, 1 \mathrm{H}), 2.95(\mathrm{dd}, J=14.1,9.6 \mathrm{~Hz}, 1 \mathrm{H}), 2.05-1.92(\mathrm{~m}, 1 \mathrm{H}), 1.80-1.72(\mathrm{~m}$, $1 \mathrm{H}), 1.71-1.61(\mathrm{~m}, 2 \mathrm{H}), 1.54(\mathrm{~s}, 9 \mathrm{H}), 1.29$ (s, 9H) ppm. HRMS (ESI+) m/z: calcd. $\mathrm{C}_{29} \mathrm{H}_{39} \mathrm{~N}_{4} \mathrm{O}_{6}$ for $[\mathrm{M}+\mathrm{H}]^{+}: 539.2864$ found 539.2861. The spectroscopic data were in accordance with those previously reported. ${ }^{[1]}$

tert-Butyl 2-((S)-2-((S)-1-((2S,3S)-2-((tert-butoxycarbonyl)amino)-3-methylpentanoyl)pyrrolidine-2carboxamido)-3-phenylpropan-amido)nicotinate (Boc-L-Ile-L-Pro-L-Phe-NH- $t$ Bu-nic, KH_1039) L,L,LS15

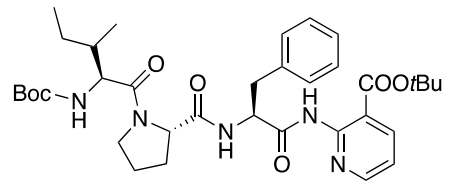

L,L,L-S15

For the synthesis of Boc-L-Ile-L-Pro-L-Phe-NH-tBu-nic L,L,L-S15 was started from Boc-L-Pro-L-Phe-NH-tBu-nic L,L-S14 (442 mg, 1 mmol, 1 equiv) that was deprotected following general procedure $\mathbf{C}$ with $\mathrm{HCl} /$ dioxane (40 ml, $4 \mathrm{M}$ ). After removal of the solvent under vacuum, the resulting crude solid material (H-L-Pro-L-Phe- $t \mathrm{Bu}-\mathrm{nic} . \mathrm{HCl})$ was used in the subsequent step without further purification.

The next step was performed following general procedure D. The crude was dissolved in $\mathrm{CH}_{2} \mathrm{Cl}_{2}(20$ $\mathrm{ml}$ ) and $\mathrm{Et}_{3} \mathrm{~N}$ was added until the mixture reached $\mathrm{pH}$ 9. This was added to the pre-activatede mixture of Boc-L-Ile-OH.0.5 $\mathrm{H}_{2} \mathrm{O}$ (231 mg, $1 \mathrm{mmol}, 1$ equiv), EDC. $\mathrm{HCl}$ (230 mg, $1.2 \mathrm{mmol}, 1.2$ equiv) and HOBt. $\mathrm{H}_{2} \mathrm{O}$ (184 mg, $1.2 \mathrm{mmol}, 1.2$ equiv). The reaction mixture was stirred for $1 \mathrm{~h}$. After extraction, the title compound could be obtained as a white powder in $98 \%$ yield ( $639 \mathrm{mg}, 0.98 \mathrm{mmol})$ over the two steps. ${ }^{1}$ H NMR (500 MHz, DMSO-d $\left.\boldsymbol{d}_{\boldsymbol{6}}\right) \boldsymbol{\delta} 10.73$ (s, 1H), 8.52 (dd, $\left.J=1.8,4.8 \mathrm{~Hz}, 1 \mathrm{H}\right), 8.11(\mathrm{~d}, J=7.8$ $\mathrm{Hz}, 1 \mathrm{H}), 8.06(\mathrm{dd}, J=1.8,7.7 \mathrm{~Hz}, 1 \mathrm{H}), 7.33(\mathrm{~d}, J=7.5 \mathrm{~Hz}, 2 \mathrm{H}) 7.31-7.23(\mathrm{~m}, 3 \mathrm{H}), 7.18(\mathrm{dd}, J=7.3$, $7.3 \mathrm{~Hz}, 1 \mathrm{H}), 6.81(\mathrm{~d}, J=8.7 \mathrm{~Hz}, 1 \mathrm{H}), 4.82-4.74(\mathrm{~m}, 1 \mathrm{H}), 4.42-4.37(\mathrm{~m}, 1 \mathrm{H}), 4.00(\mathrm{dd}, J=8.7,8.7 \mathrm{~Hz}$, $1 \mathrm{H}), 3.72-3.66(\mathrm{~m}, 1 \mathrm{H}), 3.53-3.46(\mathrm{~m}, 1 \mathrm{H}), 3.11(\mathrm{dd}, J=3.8,13.9 \mathrm{~Hz}, 1 \mathrm{H}), 2.90(\mathrm{dd}, J=9.2,13.8 \mathrm{~Hz}$, $1 \mathrm{H}), 2.01-1.94(\mathrm{~m}, 1 \mathrm{H}), 1.84-1.76(\mathrm{~m}, 3 \mathrm{H}), 1.71-1.61(\mathrm{~m}, 1 \mathrm{H}), 1.49(\mathrm{~s}, 9 \mathrm{H}), 1.36(\mathrm{~s}, 9 \mathrm{H}), 1.12-1.02(\mathrm{~m}$, $1 \mathrm{H}), 0.84-0.80(\mathrm{~m}, 4 \mathrm{H}), 0.78(\mathrm{t}, J=7.3 \mathrm{~Hz}, 3 \mathrm{H}) \mathrm{ppm}{ }^{13} \mathbf{C}$ NMR (126 MHz, DMSO-d $) \boldsymbol{\delta} 171.6(\mathrm{C})$, $170.5(\mathrm{C}), 170.1(\mathrm{C}), 164.9(\mathrm{C}), 155.4(\mathrm{C}), 150.7(\mathrm{CH}), 148.6(\mathrm{C}), 138.8(\mathrm{CH}), 137.5(\mathrm{C}), 129.2(\mathrm{CH})$, 
$128.0(\mathrm{CH}), 126.2(\mathrm{CH}), 120.3(\mathrm{C}), 120.0(\mathrm{CH}), 81.4(\mathrm{C}), 77.8(\mathrm{C}), 59.1(\mathrm{CH}), 55.9(\mathrm{CH}), 54.5(\mathrm{CH})$, $47.0\left(\mathrm{CH}_{2}\right), 36.9\left(\mathrm{CH}_{2}\right), 35.9(\mathrm{CH}), 28.9\left(\mathrm{CH}_{2}\right), 28.1\left(\mathrm{CH}_{3}\right), 27.7\left(\mathrm{CH}_{3}\right), 24.3\left(\mathrm{CH}_{2}\right), 24.2\left(\mathrm{CH}_{2}\right), 14.9$ $\left(\mathrm{CH}_{3}\right), 10.7\left(\mathrm{CH}_{3}\right)$ ppm. HRMS (ESI+) m/z: calcd. $\mathrm{C}_{35} \mathrm{H}_{49} \mathrm{~N}_{5} \mathrm{O}_{7} \mathrm{Na}$ for $[\mathrm{M}+\mathrm{Na}]^{+}: 674.3524$ found 674.3534. No spectroscopic data are reported in literature.

Assembling of the Fragments $\mathbf{L}, \mathbf{L}, \mathbf{L}, \mathbf{L}-\mathrm{S} 13$ and $\mathbf{L}, \mathbf{L}, \mathbf{L}-\mathrm{S} 15$ and Deprotection to Access Linear Heptamer $L, L, L, L, L, L, L-15$
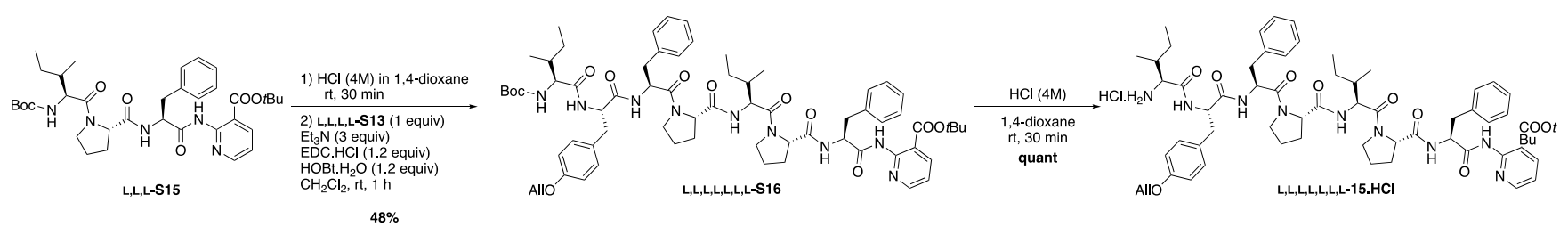

tert-Butyl 2-((S)-2-((S)-1-(((S)-2-((2S,3R)-2-((tert-butoxycarbonyl)amino)-3-methylpentanamido)-3-(4(((E)-prop-1-en-1-yl)oxy)phenyl) propanoyl)-L-phenylalanyl-L-prolyl-L-isoleucyl)pyrrolidine-2carboxamido)-3-phenylpropanamido)nicotinate (Boc-L-Ile-L-Tyr(All)-L-Phe-L-Pro-L-Ile-L-Pro-L-PheNH- $t$ Bu-nic, KH_1042) L,L,L,L,L,L,L,L-S16

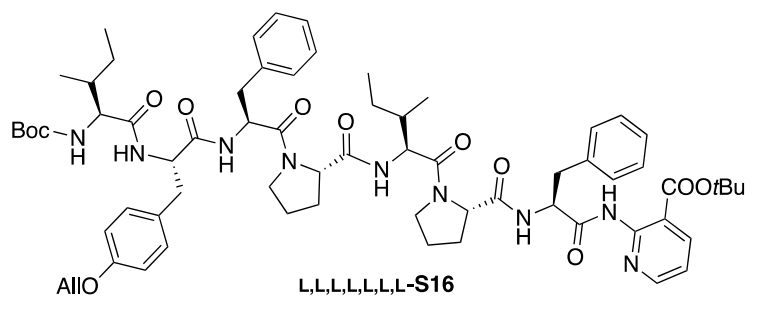

The title compound was obtained by means of a two step synthesis from Boc-L-Ile-L-Pro-L-Phe-NH- $t$ Bu-nic L,L,LS15 (433 mg, 0.664 mmol, 1 equiv) was deprotected following general procedure $\mathbf{C}$ with $\mathrm{HCl} /$ dioxane $(40 \mathrm{ml}$, $4 \mathrm{M})$. After removal of the solvent under vacuum, the resulting crude solid material (H-L-Ile-L-Pro-L-Phe-NH- $t \mathrm{Bu}-$ nic. $\mathrm{HCl}$ ) was used in the subsequent step without further purification. The peptide synthesis was performed following general procedure D forming the pre-activated mixture using Boc-L-Ile-L-Tyr(All)-L-Phe-L-Pro-OH L,L,L,L-S13 (461 mg, $0.66 \mathrm{mmol}, 1$ equiv), EDC. $\mathrm{HCl}$ (153 mg, $0.797 \mathrm{mmol}, 1.2$ equiv) and $\mathrm{HOBt} . \mathrm{H}_{2} \mathrm{O}$ (122 mg, $0.797 \mathrm{mmol}$, 1.2 equiv) in $\mathrm{CH}_{2} \mathrm{Cl}_{2}(20 \mathrm{ml})$. In parallel, to a flask containing the crude H-L-Ile-L-Pro-L-Phe-NH- $t \mathrm{Bu}-$ nic. $\mathrm{HCl}$ from the previous step, dissolved in $\mathrm{CH}_{2} \mathrm{Cl}_{2}(20 \mathrm{ml})$, was $\mathrm{Et}_{3} \mathrm{~N}$ added until the mixture reached $\mathrm{pH}$ 9. The two mixture were combined and allowed to stirred for $1 \mathrm{~h}$ at roomtemperature. This yielded, after extraction and Grace Reveleris ${ }^{\circledR}$ X2 Reverse Phase silicagel flash chromatography $\left(\mathrm{H}_{2} \mathrm{O}: \mathrm{CH}_{3} \mathrm{CN}\right.$, 97:3 to 0:100), the title compound as a white powder with $48 \%$ (385 $\mathrm{mg}, 0.318 \mathrm{mmol}$ ) yield over the two steps. ${ }^{1} \mathbf{H}$ NMR (500 MHz, DMSO-d $_{\text {) }} \boldsymbol{\delta} 10.74$ (br s, $\left.1 \mathrm{H}\right), 8.52$ (dd, $\left.J=1.6,4.8 \mathrm{~Hz}, 1 \mathrm{H}\right), 8.34-8.29$ $(\mathrm{m}, 1 \mathrm{H}), 8.11(\mathrm{~d}, J=8.6 \mathrm{~Hz}, 1 \mathrm{H}), 8.06(\mathrm{dd}, J=1.5,7.7 \mathrm{~Hz}, 1 \mathrm{H}), 7.93(\mathrm{~d}, J=8.3 \mathrm{~Hz}, 1 \mathrm{H}), 7.70(\mathrm{~d}, J=$ 8.6 Hz, 1H), $7.34(\mathrm{~d}, J=7.4 \mathrm{~Hz}, 2 \mathrm{H}), 7.31-7.20(\mathrm{~m}, 7 \mathrm{H}), 7.19-7.13(\mathrm{~m}, 2 \mathrm{H}), 7.10-7.06(\mathrm{~m}, 2 \mathrm{H}), 6.77$ (d, $J=8.3 \mathrm{~Hz}, 2 \mathrm{H}), 6.68(\mathrm{~d}, J=9.5 \mathrm{~Hz}, 1 \mathrm{H}), 6.02(\mathrm{ddt}, J=5.1,10.5,17.2 \mathrm{~Hz}, 1 \mathrm{H}), 5.36(\mathrm{dd}, J=1.5,17.2$ $\mathrm{Hz}, 1 \mathrm{H}), 5.23$ (dd, $J=1.5,10.5 \mathrm{~Hz}, 1 \mathrm{H}), 4.82-4.75(\mathrm{~m}, 1 \mathrm{H}), 4.67-4.61(\mathrm{~m}, 1 \mathrm{H}), 4.56-4.50(\mathrm{~m}, 1 \mathrm{H}), 4.48$ 
$(\mathrm{d}, J=5.1 \mathrm{~Hz}, 2 \mathrm{H}), 4.42-4.35(\mathrm{~m}, 3 \mathrm{H}), 3.76-3.63(\mathrm{~m}, 3 \mathrm{H}), 3.55-3.47(\mathrm{~m}, 1 \mathrm{H}), 3.44-3.38(\mathrm{~m}, 1 \mathrm{H}), 3.20$ $3.08(\mathrm{~m}, 1 \mathrm{H}), 3.03-2.96(\mathrm{~m}, 1 \mathrm{H}), 2.89(\mathrm{dd}, J=9.6,14.4 \mathrm{~Hz}, 1 \mathrm{H}), 2.83(\mathrm{dd}, J=3.5,13.5, \mathrm{~Hz}, 1 \mathrm{H}), 2.76$ $(\mathrm{dd}, J=7.9,14.2 \mathrm{~Hz}, 1 \mathrm{H}), 2.64(\mathrm{dd}, J=9.6,13.6 \mathrm{~Hz}, 1 \mathrm{H}), 2.04-1.91(\mathrm{~m}, 2 \mathrm{H}), 1.86-1.70(\mathrm{~m}, 6 \mathrm{H}), 1.57-$ $1.51(\mathrm{~m}, 1 \mathrm{H}), 1.50(\mathrm{~s}, 9 \mathrm{H}), 1.36(\mathrm{~s}, 9 \mathrm{H}), 1.27-1.20(\mathrm{~m}, 3 \mathrm{H}), 1.12-1.05(\mathrm{~m}, 1 \mathrm{H}), 0.90-0.88(\mathrm{~m}, 1 \mathrm{H}), 0.88-$ $0.82(\mathrm{~m}, 3 \mathrm{H}), 0.80(\mathrm{t}, J=7.3 \mathrm{~Hz}, 3 \mathrm{H}), 0.72(\mathrm{t}, J=7.3 \mathrm{~Hz}, 3 \mathrm{H}), 0.59(\mathrm{~d}, J=6.6 \mathrm{~Hz}, 3 \mathrm{H}) \mathrm{ppm} .{ }^{13} \mathbf{C} \mathbf{N M R}$ (126 MHz, DMSO-d $\left.\boldsymbol{d}_{\boldsymbol{6}}\right) \boldsymbol{\delta} 171.6$ (C), 171.1 (C), 170.8 (C), 170.7 (C), 170.2 (C), 169.9 (C), 169.3 (C), $164.9(\mathrm{C}), 156.7(\mathrm{C}), 155.2(\mathrm{C}), 150.7(\mathrm{CH}), 148.6(\mathrm{C}), 138.8(\mathrm{CH}), 137.5(\mathrm{C}), 137.5(\mathrm{C}), 133.9(\mathrm{CH})$, $130.2(\mathrm{CH}), 129.5(\mathrm{C}), 129.3(\mathrm{CH}), 129.3(\mathrm{CH}), 128.1(\mathrm{CH}), 128.0(\mathrm{CH}), 126.3(\mathrm{CH}), 126.3(\mathrm{CH})$, $120.6(\mathrm{C}), 120.1(\mathrm{CH}), 117.2\left(\mathrm{CH}_{2}\right), 114.0(\mathrm{CH}), 81.4(\mathrm{C}), 78.0(\mathrm{C}), 68.0\left(\mathrm{CH}_{2}\right), 59.2(\mathrm{CH}), 59.1(\mathrm{CH})$, $59.0(\mathrm{CH}), 54.5(\mathrm{CH}), 54.4(\mathrm{CH}), 53.3(\mathrm{CH}), 51.9(\mathrm{CH}), 47.1\left(\mathrm{CH}_{2}\right), 46.8\left(\mathrm{CH}_{2}\right), 37.0\left(\mathrm{CH}_{2}\right), 37.0$ $\left(\mathrm{CH}_{2}\right), 36.8\left(\mathrm{CH}_{2}\right), 36.5(\mathrm{CH}), 36.3(\mathrm{CH}), 29.0\left(\mathrm{CH}_{2}\right), 28.2\left(\mathrm{CH}_{3}\right), 27.7\left(\mathrm{CH}_{3}\right), 24.5\left(\mathrm{CH}_{2}\right), 24.4\left(\mathrm{CH}_{2}\right)$, $24.3\left(\mathrm{CH}_{2}\right), 24.2\left(\mathrm{CH}_{2}\right), 24.0\left(\mathrm{CH}_{2}\right), 15.2\left(\mathrm{CH}_{3}\right), 15.1\left(\mathrm{CH}_{3}\right), 10.9\left(\mathrm{CH}_{3}\right), 10.8\left(\mathrm{CH}_{3}\right)$ ppm. HRMS (ESI+) $\mathrm{m} / \mathrm{z}$ : calcd. $\mathrm{C}_{67} \mathrm{H}_{89} \mathrm{~N}_{9} \mathrm{O}_{12} \mathrm{Na}$ for $[\mathrm{M}+\mathrm{Na}]^{+}: 1234.6523$ found 1234.6475 . No spectroscopic data are reported in literature.

tert-Butyl 2-((S)-2-((S)-1-(((S)-2-((2S,3R)-2-amino-3-methylpentanamido)-3-(4-(((E)-prop-1-en-1yl)oxy)phenyl)propanoyl)-L-phenyl-alanyl-L-prolyl-L-isoleucyl)pyrrolidine-2-carboxamido)-3phenylpropanamido)nicotinate hydrochloride acid salt (H-L-Ile-L-Tyr(All)-L-Phe-L-Pro-L-Ile-L-Pro-LPhe-NH- $t$ Bu-nic.HCl, KH_1046) L,L,L,L,L,L,L-17.HCl

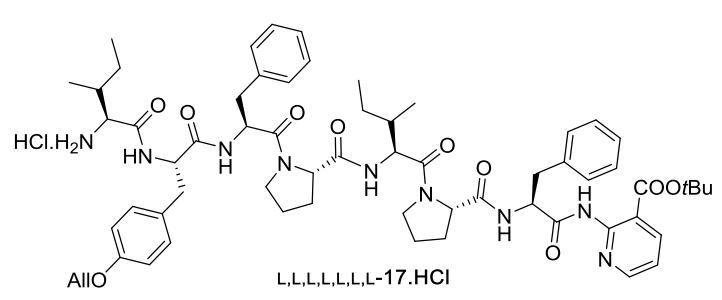

The title compound was prepared following general procedure D from Boc-L-Ile-L-Tyr(All)-L-Phe-L-Pro-L-IleL-Pro-L-Phe-NH- $t$ Bu-nic L,L,L,L,L,L,L-S16 (340 mg, 0.28 mmol, 1 equiv) in $\mathrm{HCl} /$ dioxane (10 $\mathrm{ml}, 4 \mathrm{M}$ ) by stirring 30 minutes at room temperature. After removal of solvent and

lyophilisation, the desired compound $\mathbf{L}, \mathbf{L}, \mathbf{L}, \mathbf{L}, \mathbf{L}, \mathbf{L}, \mathbf{L}-\mathbf{1 7 . H C l}$ was obtained in nearly quantitive yield (327 $\mathrm{mg}, 0.28 \mathrm{mmol}$ ) as a white powder. ${ }^{1} \mathbf{H}$ NMR (500 MHz, DMSO-d $\left.\boldsymbol{d}_{\boldsymbol{6}}\right) \boldsymbol{\delta} 10.8$ (br s, 1H), 8.55- 8.45 (m, 2H), 8.16-8.10 (m, 2H), 8.08-8.04 (m, 1H), $7.95(\mathrm{~d}, J=8.8 \mathrm{~Hz}, 1 \mathrm{H}), 7.34$ (d, $J=7.5 \mathrm{~Hz}, 1 \mathrm{H}), 7.30-7.11$ $(\mathrm{m}, 12 \mathrm{H}), 6.81(\mathrm{~d}, J=8.5 \mathrm{~Hz}, 2 \mathrm{H}), 6.02(\mathrm{ddt}, J=5.1,10.5,17.2 \mathrm{~Hz}, 1 \mathrm{H}), 5.36(\mathrm{~d}, J=17.2 \mathrm{~Hz}, 1 \mathrm{H})$, $5.23(\mathrm{~d}, J=10.5 \mathrm{~Hz}, 1 \mathrm{H}), 4.84-4.75(\mathrm{~m}, 1 \mathrm{H}), 4.69-4.61(\mathrm{~m}, 1 \mathrm{H}), 4.59-4.53(\mathrm{~m}, 1 \mathrm{H}), 4.52-4.47(\mathrm{~m}, 3 \mathrm{H})$, 4.43-4.30 (m, 3H), 3.89 (br s, 3H), 3.73-3.57 (m, 3H), 3.53-3.40 (m, 3H), $3.11(\mathrm{dd}, J=3.5,14.0 \mathrm{~Hz}$, $1 \mathrm{H}), 2.98(\mathrm{dd}, J=4.8,13.7 \mathrm{~Hz}, 1 \mathrm{H}), 2.93-2.84(\mathrm{~m}, 2 \mathrm{H}), 2.81-2.70(\mathrm{~m}, 2 \mathrm{H}), 2.03-1.89(\mathrm{~m}, 2 \mathrm{H}), 1.85-$ $1.67(\mathrm{~m}, 6 \mathrm{H}), 1.49$ (s, 9H), 1.44-1.34 (m, 2H),1.14-0.96 (m, 2H), 0.91-0.71 (m, 12H) ppm. ${ }^{13} \mathbf{C}$ NMR (126 MHz, DMSO-d d $_{\text {) }} \boldsymbol{\delta} 171.6(\mathrm{C}), 171.1$ (C), 170.2 (C), 169.9 (C), 169.2 (C), 167.5 (C), 164.9 (C), $156.8(\mathrm{C}), 150.6(\mathrm{CH}), 148.5(\mathrm{C}), 138.9(\mathrm{CH}), 137.6(\mathrm{C}), 137.5(\mathrm{C}), 133.9(\mathrm{CH}), 130.3(\mathrm{CH}), 129.3$ 
$(\mathrm{CH}), 129.3(\mathrm{C}), 129.2(\mathrm{CH}) 128.1(\mathrm{CH}), 128.0(\mathrm{CH}), 126.3(\mathrm{CH}), 126.2(\mathrm{CH}), 120.6(\mathrm{C}), 120.1(\mathrm{CH})$, $117.3\left(\mathrm{CH}_{2}\right), 114.2(\mathrm{CH}), 81.4(\mathrm{C}), 68.1\left(\mathrm{CH}_{2}\right), 59.1(\mathrm{CH}), 59.1(\mathrm{CH}), 56.3(\mathrm{CH}), 54.5(\mathrm{CH}), 54.4(\mathrm{CH})$, $53.9(\mathrm{CH}), 51.9(\mathrm{CH}), 47.1\left(\mathrm{CH}_{2}\right), 46.8\left(\mathrm{CH}_{2}\right), 37.0\left(\mathrm{CH}_{2}\right), 36.8\left(\mathrm{CH}_{2}\right), 36.7\left(\mathrm{CH}_{2}\right), 36.3(\mathrm{CH}), 36.3$ $(\mathrm{CH}), 29.0\left(\mathrm{CH}_{2}\right), 27.7\left(\mathrm{CH}_{3}\right), 24.5\left(\mathrm{CH}_{2}\right), 24.4\left(\mathrm{CH}_{2}\right) 24.3\left(\mathrm{CH}_{2}\right), 24.0\left(\mathrm{CH}_{2}\right), 23.6\left(\mathrm{CH}_{2}\right), 15.1\left(\mathrm{CH}_{3}\right)$, $14.6\left(\mathrm{CH}_{3}\right), 11.2\left(\mathrm{CH}_{3}\right), 10.9\left(\mathrm{CH}_{3}\right)$ ppm. HRMS (ESI+) m/z: calcd. $\mathrm{C}_{62} \mathrm{H}_{82} \mathrm{~N}_{9} \mathrm{O}_{10}$ for $[\mathrm{M}+\mathrm{H}]^{+}$: 1112.6179 found 1112.6151. No spectroscopic data are reported in literature.

\section{Cyclization of $\mathbf{L}, L, L, L, L, L, L-17 . H C l$ by means of Transamidation}

$(6 S, 9 S, 12 S, 15 S, 17 \mathrm{a} S, 23 S, 25 \mathrm{a} S)-6,15-\mathrm{Dibenzyl}-12,23-\mathrm{di}((S)$-sec-butyl)-9-(4-(((E)-prop-1-en-1yl)oxy)benzyl)octadecahydro-5H-dipyrrolo[1,2-a:1',2'-g][1,4,7,10,13,16,19]heptaazacyclohenicosine-

5,8,11,14,17,22,25-heptaone (c[-L-Ile-L-Tyr(All)-L-Phe-L-Pro-L-Ile-L-Pro-L-Phe-],

KH_1236)

L,L,L,L,L,L,L-18

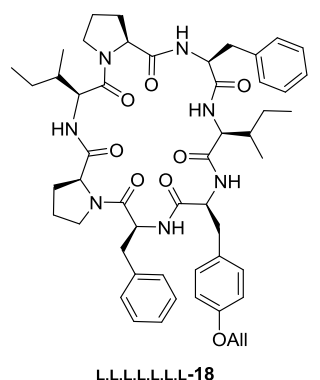

L,L,L,L,L,L,L-18

The title compound was prepared using general procedure B from H-L-Ile-LTyr(All)-L-Phe-L-Pro-L-Ile-L-Pro-L-Phe-NH-tBu-nic $\quad$ L,L,L,L,L,L,L-17.HCl $\quad(120$ mg, 0.105 mmol, 1 equiv), NaOAc (9 mg, 0.15 mmol, 1 equiv) and $\mathrm{Zn}(\mathrm{OAc})_{2}$ (4 $\mathrm{mg}, 0.02 \mathrm{mmol}, 20 \mathrm{~mol} \%)$ in THF $(0.3 \mathrm{ml})$. This yielded, after semi-preparative HPLC $\left(\mathrm{H}_{2} \mathrm{O}: \mathrm{CH}_{3} \mathrm{CH}\right.$ 70:30 to 40:60 over 10 min followed by 0:100 over $\left.20 \mathrm{~min}\right)$, the desired compound as a white powder in $26 \%(25 \mathrm{mg}, 0.027 \mathrm{mmol})$ yield.

${ }^{1}$ H NMR (500 MHz, DMSO-d 6 ) $\boldsymbol{\delta} 9.39$ (d, $\left.J=8.6 \mathrm{~Hz}, 1 \mathrm{H}\right), 8.93(\mathrm{~d}, J=8.6 \mathrm{~Hz}, 1 \mathrm{H}), 8.76$ (br s, 1H), $7.39(\mathrm{~d}, J=10.3 \mathrm{~Hz}, 1 \mathrm{H}), 7.36$ (d, $J=8.6 \mathrm{~Hz}, 2 \mathrm{H}), 7.33$ (d, $J=7.5 \mathrm{~Hz}, 2 \mathrm{H}), 7.30-7.28$ (m, 1H), 7.27$7.21(\mathrm{~m}, 7 \mathrm{H}), 7.20-7.16(\mathrm{~m}, 1 \mathrm{H}), 6.89(\mathrm{~d}, J=8.6 \mathrm{~Hz}, 2 \mathrm{H}), 6.04(\mathrm{ddt}, J=5.0,10.5,17.2 \mathrm{~Hz}, 1 \mathrm{H}), 5.40$ $(\mathrm{dd}, J=1.5,17.2 \mathrm{~Hz}, 1 \mathrm{H}), 5.24(\mathrm{dd}, J=1.5,10.5 \mathrm{~Hz}, 1 \mathrm{H}), 4.56(\mathrm{~d}, J=5.0 \mathrm{~Hz}, 2 \mathrm{H}), 4.27-4.24(\mathrm{~m}, 2 \mathrm{H})$, 4.21-4.17 (m, 2H), 4.12-4.10 (m, 1H), 4.10-4.06 (m, 1H), $4.04(\mathrm{dd}, J=4.6,11.4 \mathrm{~Hz}, 1 \mathrm{H}), 3.52(\mathrm{td}, J=$ 7.7, $9.5 \mathrm{~Hz}, 1 \mathrm{H}), 3.44(\mathrm{~d}, J=7.7 \mathrm{~Hz}, 1 \mathrm{H}), 3.39(\mathrm{dd}, J=2.3,14.0 \mathrm{~Hz}, 1 \mathrm{H}), 3.29(\mathrm{td}, J=9.3,11.5 \mathrm{~Hz}$, $1 \mathrm{H}), 3.23-3.17(\mathrm{~m}, 1 \mathrm{H}), 3.15(\mathrm{dd}, J=2.1,13.2 \mathrm{~Hz}, 1 \mathrm{H}), 2.89(\mathrm{dd}, J=4.5,12.2 \mathrm{~Hz}, 1 \mathrm{H}), 2.73(\mathrm{dd}, J=$ 9.5, 13.2 Hz, 1H), 2.70-2.62 (m, 2H), 2.04-1.94 (m, 2H), 1.93-1.86 (m, 1H), 1.83-1.76 (m, 1H), 1.75$1.67(\mathrm{~m}, 1 \mathrm{H}), 1.65-1.57(\mathrm{~m}, 1 \mathrm{H}), 1.48-1.37(\mathrm{~m}, 2 \mathrm{H}), 1.36-1.29(\mathrm{~m}, 1 \mathrm{H}), 1.28-1.20(\mathrm{~m}, 1 \mathrm{H}), 1.09-1.00$ $(\mathrm{m}, 1 \mathrm{H}), 0.98(\mathrm{~m}, 6 \mathrm{H}), 0.83(\mathrm{~d}, J=6.7 \mathrm{~Hz}, 2 \mathrm{H}), 0.75-0.73(\mathrm{~m}, 6 \mathrm{H}), 0.62-0.57(\mathrm{~m}, 1 \mathrm{H}) \mathrm{ppm} .{ }^{13} \mathbf{C}$ NMR (126 MHz, DMSO-d d $_{\text {) }} \boldsymbol{\delta} 171.9$ (C), 170.6 (C), 170.1 (C), 169.9 (C), 169.0 (C), 168.3 (C), 157.0 (C), $138.6(\mathrm{C}), 135.2(\mathrm{C}), 133.9(\mathrm{CH}), 131.3(\mathrm{CH}), 129.6(\mathrm{CH}), 128.8(\mathrm{CH}), 128.8(\mathrm{C}), 128.5(\mathrm{CH}), 128.1$ $(\mathrm{CH}), 127.2(\mathrm{CH}), 126.2(\mathrm{CH}), 117.2\left(\mathrm{CH}_{2}\right), 114.1(\mathrm{CH}), 68.1\left(\mathrm{CH}_{2}\right), 60.5(\mathrm{CH}), 60.0(\mathrm{CH}), 59.4(\mathrm{CH})$, $55.2(\mathrm{CH}), 54.5(\mathrm{CH}), 54.1(\mathrm{CH}), 53.8(\mathrm{CH}), 48.1\left(\mathrm{CH}_{2}\right), 45.6\left(\mathrm{CH}_{2}\right), 37.1\left(\mathrm{CH}_{2}\right), 36.6\left(\mathrm{CH}_{2}\right), 35.3$ $\left(\mathrm{CH}_{2}\right), 34.8(\mathrm{CH}), 29.4\left(\mathrm{CH}_{2}\right), 28.9\left(\mathrm{CH}_{2}\right), 24.5\left(\mathrm{CH}_{2}\right), 24.0(\mathrm{CH}), 21.6\left(\mathrm{CH}_{2}\right), 17.8\left(\mathrm{CH}_{3}\right), 14.6\left(\mathrm{CH}_{2}\right)$, $14.5\left(\mathrm{CH}_{2}\right), 12.1\left(\mathrm{CH}_{3}\right), 9.8\left(\mathrm{CH}_{3}\right), 9.7\left(\mathrm{CH}_{3}\right)$ ppm. HRMS (ESI+) m/z: calcd. $\mathrm{C}_{52} \mathrm{H}_{66} \mathrm{~N}_{7} \mathrm{O}_{8} \mathrm{Na}_{\text {for }}$ 
$[\mathrm{M}+\mathrm{Na}]^{+}: 940.4943$ found $940.4918 .[\boldsymbol{\alpha}]_{\mathbf{D}}=-185\left(c 1 \mathrm{mg} / \mathrm{ml}, \mathrm{CHCl}_{3}, 27^{\circ} \mathrm{C}\right)$. No spectroscopic data are reported in literature. 


\subsection{Synthesis of the Chemoselectivity Building Block L,L-15 and Transamidation}

Starting from the Boc-L-Phe-NH- $t$ Bu-nic amide L-4a, oligoamide tert-butyl 2-\{[(3S)-3-\{[(3S)-6-amino3-4-[methyl(phenyl)carbamoyl]-benzamido \}-6-oxohex-1-en-2-yl]amino-4-phenylbut-1-en-

2yl]amino \}pyridine-3-carboxylate L,L-15 was built up using a convergent strategy assembling 4[methyl(phenyl)carbamoyl]benzoic acid S17 and Boc-L-Gln-L-Phe-NH- $t$ Bu-nic amide L,L-S18.

Synthesis of 4-[methyl(phenyl)carbamoyl]benzoic acid (KH_808) S17

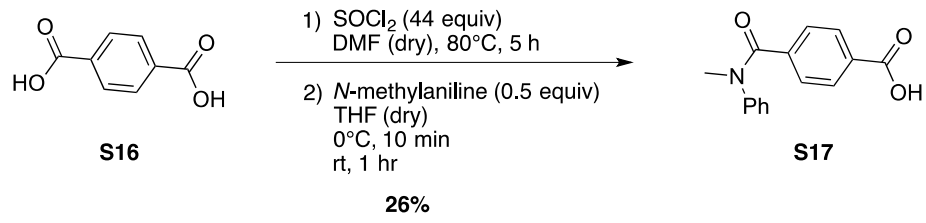

The synthesis of 4-[methyl(phenyl)carbamoyl]benzoic acid S17 was done following a literature procedure. ${ }^{[8]}$ A flame-dried microwave vial, charged with terephthalic acid S16 (0.498 g, 3.0 mmol, 2.0 equiv), thionylchloride (9.60 ml, $132.0 \mathrm{mmol}, 44.0$ equiv) and anhydrous DMF $(0.50 \mathrm{ml})$ was stirred at $80^{\circ} \mathrm{C}$ for $5 \mathrm{~h}$ under argon atmosphere. After removal of the solvent under vacuum, the resulting crude solid material was used in the subsequent step without further purification. To a flask containing the crude material from the previous step was added anhydrous THF $(30.0 \mathrm{ml})$. The mixture was then cooled to $0^{\circ} \mathrm{C}$ and $N$-methylaniline $(0.164 \mathrm{ml}, 1.50 \mathrm{mmol}, 1.0$ equiv) was added dropwise and the mixture was stirred for another $15 \mathrm{~min}$ at $0^{\circ} \mathrm{C}$, before being allowed to warm up to room temperature, followed by stirring for $2 \mathrm{~h}$ at room temperature. The reaction was quenched with saturated aqueous $\mathrm{NaHCO}_{3}(50 \mathrm{~mL})$ and extracted with EtOAc $(3 \times 50 \mathrm{ml})$. Subsequently, the resulting aqueous layer was acidified with $37 \% \mathrm{HCl}$ (aq.) until $\mathrm{pH}=3$ and extracted with DCM $(3 \times 50 \mathrm{ml})$. The organic layer was dried over $\mathrm{MgSO}_{4}$ and the solvent was removed under vacuum. Purification via Grace® Reveleris X2 Normal Phase Silicagel Flash Chromatography (Petroleum ether (1\% AcOH):EtOAc (1\% AcOH), 100:0 to 70:30, $40.0 \mathrm{~mL} / \mathrm{min})$, yielded the title compound $\mathbf{S 1 7}$ in 26\% (0.196 g, $0.769 \mathrm{mmol})$ yield as white crystals. ${ }^{1} \mathbf{H}$ NMR (400 MHz, $\left.\mathbf{C D C l}_{3}\right) \boldsymbol{\delta} 11.47$ (br s, 1H), $7.86(\mathrm{~d}, J=8.2 \mathrm{H}, 2 \mathrm{H}), 7.36$ (d, $J=8.2$ $\mathrm{Hz}, 2 \mathrm{H}), 7.20$ (t, $J=7.6 \mathrm{~Hz}, 2 \mathrm{H}), 7.12$ (t, $J=7.4 \mathrm{~Hz}, 1 \mathrm{H}), 7.02$ (d, $J=7.1 \mathrm{~Hz}, 2 \mathrm{H}), 3.50$ (s, 3H) ppm. ${ }^{13}$ C NMR (100 MHz, CDCl $)$ ) 170.6 (C), 170.0 (C), 144.1 (C), 140.7 (C), 130.4 (C), $129.6(\mathrm{CH})$, $129.4(\mathrm{CH}), 128.7(\mathrm{CH}), 127.1(\mathrm{CH}), 127.0(\mathrm{CH}), 38.5\left(\mathrm{CH}_{3}\right) \mathrm{ppm}$. HRMS (ESI+) m/z: calcd for $\mathrm{C}_{15} \mathrm{H}_{13} \mathrm{NO}_{3}[\mathrm{M}+\mathrm{H}]^{+}:$256.0968, found 256.0962 The spectroscopic data were in accordance with those previously reported. ${ }^{[1]}$ 
tert-Butyl 2-\{[(3S)-3-\{[(3S)-6-amino-3-\{4-[methyl(phenyl)carbamoyl]-benzamido $\}-6-o x o-h e x-1-e n-2-$ yl] amino \}-4-phenylbut-1-en-2yl]amino \} pyridine-3-carboxylate L,L-19
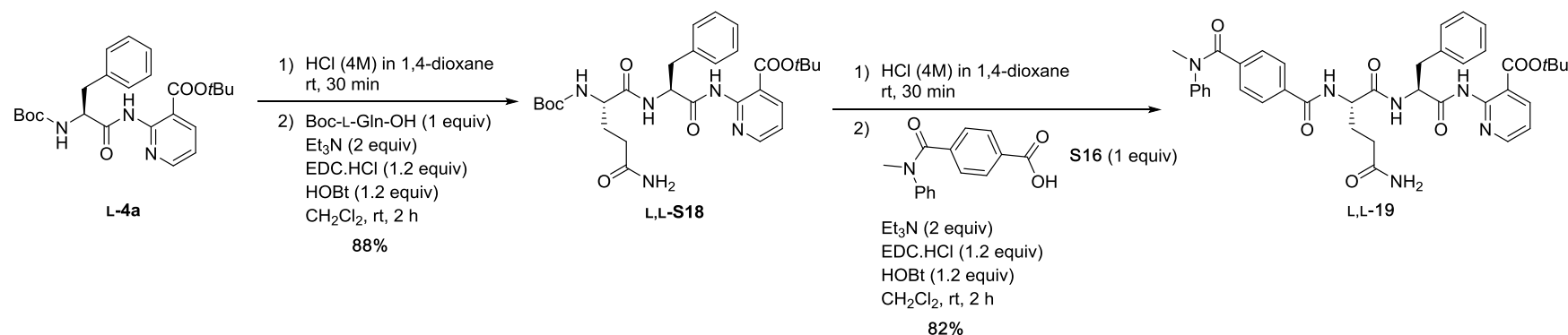

$\mathrm{EDC} . \mathrm{HCl}(1.2$ equiv) $\mathrm{H}_{2} \mathrm{Cl}_{2}, \mathrm{rt}, 2 \mathrm{~h}$

$82 \%$

tert-Butyl 2-\{[(3S)-3-(\{[(3S)-6-amino-3-[\{tert-butoxycarbonyl)amino)-6-oxohex-1-en-2-

yl]amino \}pyridine-3-carboxylate (Boc-L-Gln-L-Phe-NH- $t$ Bu-nic, KH_905) L,L-S18

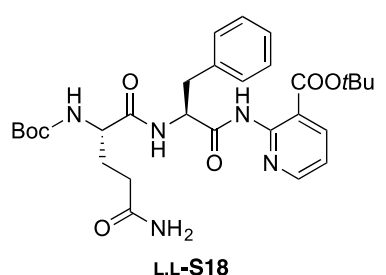

L,L-S18

For the synthesis of Boc-L-Gln-L-Phe-NH- $t$ Bu-nic L,L-S18 was started from Boc-L-Phe-NH-tBu-nic L-4a (1.14 g, 2.58 mmol, 1 equiv) that was deprotected following general procedure $\mathbf{C}$ with $\mathrm{HCl} /$ dioxane $(50 \mathrm{ml}, 4 \mathrm{M})$. After removal of the solvent under vacuum, the resulting crude solid material (H-L-Phe-NH$t \mathrm{Bu}-\mathrm{nic} . \mathrm{HCl})$ was used in the subsequent step without further purification. The next step was performed following general procedure D. The crude was dissolved in $\mathrm{CH}_{2} \mathrm{Cl}_{2}(20 \mathrm{ml})$ and $\mathrm{Et}_{3} \mathrm{~N}$ was added until the mixture reached $\mathrm{pH}$ 9. This was added to the pre-activatede mixture of Boc-L-Gln-OH (635 mg, 2.58 mmol, 1 equiv), EDC. $\mathrm{HCl}$ (584 mg, $3.1 \mathrm{mmol}, 1.2$ equiv) and HOBt. $\mathrm{H}_{2} \mathrm{O}$ (423 mg, $3.1 \mathrm{mmol}, 1.2$ equiv). The reaction mixture was stirred for $2 \mathrm{~h}$. After extraction with citric acid in water $(3 \times 50 \mathrm{ml}, 10 \%)$ and $\mathrm{NaHCO}_{3}(3 \times 50 \mathrm{ml}$, satuerated), the title compound could be obtained as a white powder in $84 \%$ yield $(1.24 \mathrm{~g}, 2.18 \mathrm{mmol})$ over the two steps. ${ }^{1} \mathbf{H}$ NMR (500 MHz, DMSO-d $\boldsymbol{d}_{\boldsymbol{6}}$, mixture of rotamers) $\delta 10.83(\mathrm{~s}, 1 \mathrm{H}), 8.53(\mathrm{dd}, J=4.9,1.8 \mathrm{~Hz}, 1 \mathrm{H}), 8.10-8.04(\mathrm{~m}, 2 \mathrm{H}), 7.33-7.28(\mathrm{~m}$, $3 \mathrm{H}), 7.24$ (t, $J=7.4 \mathrm{~Hz}, 2 \mathrm{H}), 7.20-7.15(\mathrm{~m}, 2 \mathrm{H}), 6.83(\mathrm{~d}, J=8.4 \mathrm{~Hz}, 1 \mathrm{H}), 6.73$ (br s, 1H), 4.91-4.85 (m, $1 \mathrm{H}), 3.94-3.87$ (m, 1H), $3.14(\mathrm{dd}, J=13.9,3.7 \mathrm{~Hz}, 1 \mathrm{H}), 2.84(\mathrm{dd}, J=13.9,10.1 \mathrm{~Hz}, 1 \mathrm{H}), 2.08-1.92$ (m, $2 \mathrm{H}), 1.78-1.70(\mathrm{~m}, 1 \mathrm{H}), 1.62-1.55(\mathrm{~m}, 1 \mathrm{H}), 1.48(\mathrm{~s}, 8 \mathrm{H}), 1.36(\mathrm{~s}, 8 \mathrm{H}), 1.23(\mathrm{~s}, 1 \mathrm{H}), 1.18(\mathrm{~s}, 1 \mathrm{H}) \mathrm{ppm}$. ${ }^{13}$ C NMR (126 MHz, DMSO-d $)$ ) 173.8 (C), 171.6 (C), 170.2 (C), $164.9(\mathrm{C}), 155.1(\mathrm{C}), 150.6(\mathrm{CH})$, $148.4(\mathrm{C}), 138.8(\mathrm{CH}), 137.4(\mathrm{C}), 129.3(\mathrm{CH}), 128.0(\mathrm{CH}), 126.3(\mathrm{CH}), 121.0(\mathrm{C}), 120.2(\mathrm{CH}), 81.4$ (C), $78.1(\mathrm{C}), 54.1(\mathrm{CH}), 54.0(\mathrm{CH}), 37.4\left(\mathrm{CH}_{2}\right), 31.5\left(\mathrm{CH}_{2}\right), 28.2\left(\mathrm{CH}_{2}\right), 28.1\left(\mathrm{CH}_{3}\right), 27.7\left(\mathrm{CH}_{3}\right)$ ppm. NMR-data were also acquired at elevated temperature so that peaks arising from hindered rotation around the amide bond coalesced. ${ }^{1} \mathbf{H}$ NMR $\left(500 \mathrm{MHz}\right.$, DMSO-d $\left.\boldsymbol{d}_{\boldsymbol{6}}, \mathbf{6 0}^{\circ} \mathbf{C}\right) \boldsymbol{\delta} 10.60(\mathrm{~s}, 1 \mathrm{H}), 8.51(\mathrm{dd}, J=$ 4.8, $1.8 \mathrm{~Hz}, 1 \mathrm{H}), 8.08(\mathrm{dd}, J=7.7,1.7 \mathrm{~Hz}, 1 \mathrm{H}), 7.92(\mathrm{~d}, J=8.3 \mathrm{~Hz}, 1 \mathrm{H}), 7.30-7.26(\mathrm{~m}, 3 \mathrm{H}), 7.23(\mathrm{t}, J=$ $7.4 \mathrm{~Hz}, 2 \mathrm{H}), 7.18-7.15$ (m, 1H), 7.02 (br s, 1H), 6.64 (br s, 1H), 6.55 (br s, 1H), 4.93 (td, $J=8.9 \mathrm{~Hz}, 4.1$ $\mathrm{Hz}, 1 \mathrm{H}), 3.92$ (dd, $J=8.5,7.9 \mathrm{~Hz}, 1 \mathrm{H}), 3.20-3.17$ (m, 1H, overlap with HDO), 2.89 (dd, $J=14.0,9.5$ $\mathrm{Hz}, 1 \mathrm{H}), 2.10-1.97(\mathrm{~m}, 2 \mathrm{H}), 1.82-1.75(\mathrm{~m}, 1 \mathrm{H}), 1.68-1.60(\mathrm{~m}, 1 \mathrm{H}), 1.51(\mathrm{~s}, 9 \mathrm{H}), 1.35(\mathrm{~s}, 9 \mathrm{H}) \mathrm{ppm}$. 
HRMS (ES+) m/z: calc for $\mathrm{C}_{29} \mathrm{H}_{40} \mathrm{~N}_{5} \mathrm{O}_{7}[\mathrm{M}+\mathrm{H}]^{+}$570.2922, found 570.2927. The spectroscopic data were in accordance with those previously reported. ${ }^{[1]}$

tert-Butyl 2-\{[(3S)-3-\{[(3S)-6-amino-3-\{4-[methyl(phenyl)carbamoyl]-benzamido $\}-6-o x o-h e x-1-e n-2-$ yl]amino \}-4-phenylbut-1-en-2yl] amino \} pyridine-3-carboxylate (KH_907) L,L-19

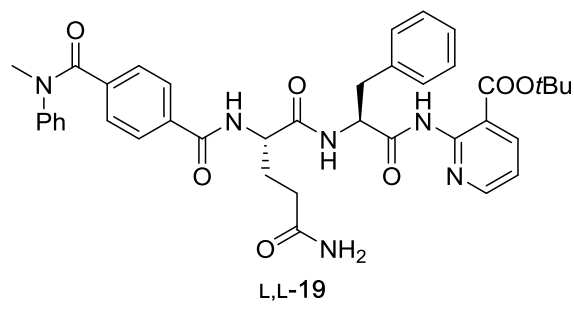

For the synthesis of L,L-19 was started from Boc-L-Gln-L-Phe$t$ Bu-nic L-S18 (1.14 g, 2 mmol, 1 equiv) that was deprotected following general procedure $\mathbf{C}$ with $\mathrm{HCl} /$ dioxane $(40 \mathrm{ml}, 4 \mathrm{M})$. After removal of the solvent under vacuum, the resulting crude solid material (H-L-Gln-L-Phe-NH- $t \mathrm{Bu}-$ nic.HCl) was used in the subsequent step without further purification. The next step was performed following general procedure D. The crude was dissolved in $\mathrm{CH}_{2} \mathrm{Cl}_{2}(20 \mathrm{ml})$ and $\mathrm{Et}_{3} \mathrm{~N}$ was added until the mixture reached $\mathrm{pH}$ 9. This was added to the pre-activated mixture of $\mathbf{S 1 7}$ (511 mg, $2 \mathrm{mmol}, 1$ equiv), EDC.HCl (460 mg, 2.4 mmol, 1.2 equiv) and $\mathrm{HOBt} . \mathrm{H}_{2} \mathrm{O}$ (368 mg, $2.4 \mathrm{mmol}, 1.2$ equiv). The reaction mixture was stirred for 2 h. After extraction with citric acid in water $(3 \times 70 \mathrm{ml}, 10 \%)$ and $\mathrm{NaHCO}_{3}(3 \times 70 \mathrm{ml}$, saturated), the title compound was obtained as a white powder in $95 \%$ yield $(1.34 \mathrm{~g}, 1.89 \mathrm{mmol})$ over the two steps. ${ }^{1}$ H-NMR (500 MHz, DMSO-d $\left.)_{6}\right) \boldsymbol{\delta} 10.78(\mathrm{~s}, 1 \mathrm{H}), 8.54(\mathrm{~d}, J=7.8 \mathrm{~Hz}, 1 \mathrm{H}), 8.52$ (dd, $J=4.8,1.8 \mathrm{~Hz}$, $1 \mathrm{H}), 8.14(\mathrm{~d}, J=8.3 \mathrm{~Hz}, 1 \mathrm{H}), 8.06(\mathrm{dd}, J=7.7,1.9 \mathrm{~Hz}, 1 \mathrm{H}), 7.69(\mathrm{~d}, J=8.0 \mathrm{~Hz}, 2 \mathrm{H}), 7.34(\mathrm{~d}, J=7.9$ $\mathrm{Hz}, 2 \mathrm{H}), 7.31-7.15$ (m, 9H), 7.14-7.10 (m, 3H), 6.77 (br s, 1H), 4.83 (td, $J=10.4,3.5 \mathrm{~Hz}, 1 \mathrm{H}), 4.38-$ $4.34(\mathrm{~m}, 1 \mathrm{H}), 3.39$ (s, 3H), $3.12(\mathrm{dd}, J=13.9,3.7 \mathrm{~Hz}, 1 \mathrm{H}), 2.84(\mathrm{dd}, J=13.9 \mathrm{~Hz}, 10.0 \mathrm{~Hz}, 1 \mathrm{H}), 2.13-$ $2.00(\mathrm{~m}, 2 \mathrm{H}), 1.92-1.86(\mathrm{~m}, 1 \mathrm{H}), 1.81-1.73(\mathrm{~m}, 1 \mathrm{H}), 1.45$ (s, 9H) ppm. ${ }^{\mathbf{1 3}}$ C-NMR (126 MHz, DMSOd $\boldsymbol{d}_{\boldsymbol{6}} \boldsymbol{\delta} 174.1(\mathrm{C}), 171.4(\mathrm{C}), 170.2(\mathrm{C}), 169.0(\mathrm{C}), 165.7$ (C), $165.0(\mathrm{C}), 150.7(\mathrm{CH}), 148.4(\mathrm{C}), 144.3(\mathrm{C})$, $139.1(\mathrm{C}), 138.9(\mathrm{CH}), 137.4(\mathrm{C}), 134.4(\mathrm{C}), 129.3(\mathrm{CH}), 129.3(\mathrm{CH}), 128.1(\mathrm{CH}), 128.0(\mathrm{CH}), 127.2$ $(\mathrm{CH}), 127.1(\mathrm{CH}), 126.8(\mathrm{CH}), 126.4(\mathrm{CH}), 120.9(\mathrm{C}), 120.3(\mathrm{CH}), 81.5(\mathrm{C}), 54.3(\mathrm{CH}), 53.2(\mathrm{CH}), 37.9$ $\left(\mathrm{CH}_{3}\right), 37.2\left(\mathrm{CH}_{2}\right), 31.6\left(\mathrm{CH}_{2}\right), 27.8\left(\mathrm{CH}_{3}\right), 26.9\left(\mathrm{CH}_{2}\right)$ ppm. HRMS (ESI+) m/z: calcd. for $\mathrm{C}_{39} \mathrm{H}_{42} \mathrm{~N}_{6} \mathrm{O}_{7} \mathrm{Na}[\mathrm{M}+\mathrm{Na}]^{+}:$729.3007, found 729.3035. $[\boldsymbol{\alpha}]_{\mathbf{D}}=16$ (c $\left.5 \mathrm{mg} / \mathrm{ml}, \mathrm{CDCl}_{3}, 20^{\circ} \mathrm{C}\right)$. The spectroscopic data were in accordance with those previously reported. ${ }^{[1]}$ 
Methyl (4-(methyl(phenyl)carbamoyl)benzoyl)-L-glutaminyl-L-phenylalanyl-L-alaninate (KH_1234) L,L,L-20

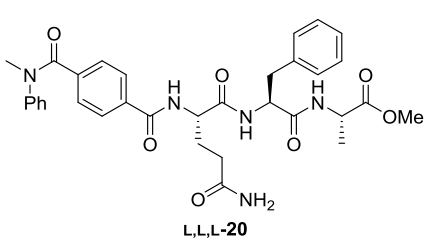

The title compound was prepared using general procedure $\mathbf{B}$, from compound L,L-19 (353 mg, 0.5 mmol, 1 equiv), H-L-Ala-OMe hydrochloric acid salt L-5j.HCl (80 mg, 0.55 mmol, 1.1 equiv), NaOAc (46 mg, 0.55 mmol, 1.1 equiv) and $\mathrm{Zn}(\mathrm{OAc})_{2}(18 \mathrm{mg}, 0.1 \mathrm{mmol}, 20 \mathrm{~mol} \%)$ in THF (1 ml). This yielded, after Grace Reveleris ${ }^{\circledR}$ X2 Normal Phase silicagel flash chromatography $\left(\mathrm{CH}_{2} \mathrm{Cl}_{2}: \mathrm{MeOH}, 100: 0\right.$ to $90: 10$ over $\left.17 \mathrm{CV}\right)$, the desired compound $\mathbf{L}, \mathbf{L}, \mathbf{L}-\mathbf{2 0}$ as a white powder in $93 \%$ (288 mg, $0.467 \mathrm{mmol})$ yield. ${ }^{1} \mathrm{H}$ NMR (500 MHz, DMSO- $\boldsymbol{d}_{6}$, mixture of diastereomers 94:6 (L,L,L: L,D,L), mixture of rotamers) $\delta 8.70(\mathrm{~d}, J=6.6 \mathrm{~Hz}, 0.1 \mathrm{H}), 8.65(\mathrm{~d}, J=7.1 \mathrm{~Hz}, 0.1 \mathrm{H}), 8.61(\mathrm{~d}, J=7.2$ $\mathrm{Hz}, 0.8 \mathrm{H}), 8.31(\mathrm{~d}, J=7.0 \mathrm{~Hz}, 0.8 \mathrm{H}), 8.29-8.22(\mathrm{~m}, 0.2 \mathrm{H}), 7.98-7.91(\mathrm{~m}, 1 \mathrm{H}), 7.72-7.66(\mathrm{~m}, 2 \mathrm{H}), 7.33$ $(\mathrm{d}, J=7.9 \mathrm{~Hz}, 2 \mathrm{H}), 7.30-7.24(\mathrm{~m}, 3 \mathrm{H}), 7.21-7.08(\mathrm{~m}, 8 \mathrm{H}), 6.80(\mathrm{br} \mathrm{s}, 1 \mathrm{H}), 4.51$ (ddd, $J=8.7,8.74 .3$ $\mathrm{Hz}, 1 \mathrm{H}), 4.30-4.21(\mathrm{~m}, 2 \mathrm{H}), 3.63(\mathrm{~s}, 0.4 \mathrm{H}), 3.61(\mathrm{~s}, 2.3 \mathrm{H}), 3.58(\mathrm{~s}, 0.3 \mathrm{H}), 3.39(\mathrm{~s}, 3 \mathrm{H}), 3.03(\mathrm{dd}, J=$ $4.2,14.0 \mathrm{~Hz}, 0.9 \mathrm{H}) 2.96(\mathrm{dd}, J=5.0,13.8 \mathrm{~Hz}, 0.1 \mathrm{H}), 2.82-1.72(\mathrm{~m}, 1 \mathrm{H}), 2.16-2.02(\mathrm{~m}, 2 \mathrm{H}), 1.92-1.82$ $(\mathrm{m}, 1 \mathrm{H}), 1.81-1.71(\mathrm{~m}, 1 \mathrm{H}), 1.27(\mathrm{~d}, J=7.3 \mathrm{~Hz}, 2.2 \mathrm{H}), 1.23(\mathrm{~d}, J=7.4 \mathrm{~Hz}, 0.4 \mathrm{H}), 1.21(\mathrm{~d}, J=7.4 \mathrm{~Hz}$, 0.4H) ppm. ${ }^{13} \mathrm{C}$ NMR (126 MHz, DMSO-d $\boldsymbol{d}_{6}$, mixture of diastereomer 94:6 (L,L,L: L,D,L), mixture of rotamers) $\delta[174.1,173.9](\mathrm{C}),[172.8,172.7](\mathrm{C}), 171.0(\mathrm{C}), 170.8(\mathrm{C}), 168.8(\mathrm{C}),[165.8,165.7](\mathrm{C})$, $144.2(\mathrm{C}), 139.1(\mathrm{C}), 137.6(\mathrm{C}), 134.3(\mathrm{C}), 129.2(\mathrm{CH}), 127.9(\mathrm{CH}), 127.9(\mathrm{CH}), 127.1(\mathrm{CH}), 126.9$ $(\mathrm{CH}), 126.8(\mathrm{CH}), 126.7(\mathrm{CH}),[126.2,126.1](\mathrm{CH}), 53.5(\mathrm{CH}), 53.3(\mathrm{CH}), 51.8\left(\mathrm{CH}_{3}\right)$, [47.6, 47.5] $(\mathrm{CH})$, [37.8, 37.6] $\left(\mathrm{CH}_{3}\right), 37.2\left(\mathrm{CH}_{2}\right), 31.5\left(\mathrm{CH}_{2}\right), 26.8\left(\mathrm{CH}_{2}\right)$, [17.0, 16.8] $\left(\mathrm{CH}_{3}\right)$ ppm. NMR-data was acquired at elevated temperature so that peaks arising from hindered rotation coalesced. ${ }^{\mathbf{1}} \mathbf{H}$ NMR $(\mathbf{5 0 0}$ MHz, DMSO- $\boldsymbol{d}_{\boldsymbol{\sigma}}, \mathbf{8 0}^{\circ} \mathrm{C}$, mixture of diastereomer 94:6 (L,L,L: L,D,L)) $\boldsymbol{\delta} 8.47$ (d, $\left.J=7.2 \mathrm{~Hz}, 0.06 \mathrm{H}\right)$, 8.45-8.8.37 (m, 0.94H), 8.06-7.94 (m, 1H), 7.74-7.66 (m, 3H), 7.35 (d, J = 8.0 Hz, 2H), 7.31-7.24 (m, 2H), 7.23-7.07 (m, 8H), $7.00($ br s, 1H), 6.56 (br s, 1H), 4.58-4.52 (m, 1H), 4.35-4.26 (m, 2H), $3.63(\mathrm{~s}$, $2.8 \mathrm{H}), 3.60(\mathrm{~s}, 0.2 \mathrm{H}), 3.39(\mathrm{~s}, 3 \mathrm{H}), 3.09-3.04(\mathrm{~m}, 1 \mathrm{H}), 2.84(\mathrm{dd}, J=8.8,13.8 \mathrm{~Hz}, 1 \mathrm{H}), 2.17-2.04(\mathrm{~m}$, 2H), 1.97-1.96 (m, 1H), 1.89-1.80 (m, 1H), 1.28-1.22 (m, 3H) ppm. ${ }^{13}$ C NMR (126 MHz, DMSO- $\boldsymbol{d}_{\boldsymbol{\sigma}}$, $\mathbf{8 0}^{\circ} \mathrm{C}$, mixture of diastereomer 94:6 (L,L,L: L,D,L)) $\delta 173.7$ (C), 172.2 (C), 170.5 (C), 170.2 (C), 168.5 (C), 165.5 (C), 143.9 (C), $138.8(\mathrm{C}), 137.1(\mathrm{C}), 134.2$ (C), $128.7(\mathrm{CH}), 127.5(\mathrm{CH}), 127.5(\mathrm{CH}), 126.7$ $(\mathrm{CH}), 126.5(\mathrm{CH}), 126.4(\mathrm{CH}), 126.2(\mathrm{CH}), 125.7(\mathrm{CH}), 53.3(\mathrm{CH}), 53.1(\mathrm{CH}), 51.3\left(\mathrm{CH}_{3}\right), 47.3(\mathrm{CH})$, $37.4\left(\mathrm{CH}_{3}\right), 37.0\left(\mathrm{CH}_{2}\right), 31.2\left(\mathrm{CH}_{2}\right), 26.5\left(\mathrm{CH}_{2}\right)$, [16.6, 16.5] $\left(\mathrm{CH}_{3}\right)$ ppm. HRMS (ESI+) m/z: calcd. for $\mathrm{C}_{33} \mathrm{H}_{37} \mathrm{~N}_{5} \mathrm{O}_{7} \mathrm{Na}[\mathrm{M}+\mathrm{Na}]^{+}: 638.2585$ found $638.2584 .[\alpha]_{\mathbf{D}}=-44\left(\right.$ c $\left.5 \mathrm{mg} / \mathrm{ml}, \mathrm{DMSO}, 21^{\circ} \mathrm{C}\right)$. No spectroscopic data are reported in literature 
LC-CD analysis on a Chiralpak IA column of the batch Boc-L-Phe-NH- $t$ Bu-nic L-4a (retention time = $3.94 \mathrm{~min}, 250 \mathrm{~nm}$, $n$-hexane:EtOH $65: 35,1.0 \mathrm{~mL} / \mathrm{min}$ ) used in this synthesis, showed to contain $6 \%$ Boc-D-Phe-NH- $t$ Bu-nic D-4a. 


\section{Amide resonance energy calculations}

Amide resonance energies $\left(E_{R}\right)$ were computed using the density functional theory (DFT) methodology reported by Greenberg and Venanzi by computing the isodesmic equation in Scheme S3. ${ }^{9}$ To this end, the electronic energies of the most stable amide conformation and corresponding amine, ketone and hydrocarbon low-energy gas phase conformation were computed. In the initial phase of the calculations, conformational analyses were performed using the MMFF force field. Subsequently, for all conformations obtained in a $7.5 \mathrm{kcal} \mathrm{mol}^{-1}$ cut-off window, an intermediate geometry optimization at the QM level was performed in the gas phase at the B3LYP/6-31G* level. Finally, for all conformations obtained in a $1.0 \mathrm{kcal} \mathrm{mol}^{-1}$ cut-off window, a full geometry optimization at the QM level was performed in the gas phase at the B3LYP/6-311++G(d,p) level of theory as recommended by Szostak et al. ${ }^{10}$<smiles>CCCCc1cccc(C(=O)Nc2ncccc2C(=O)OC(C)(C)C)c1</smiles>

Scheme S3. Computation of the amide resonance energy $\left(E_{R}\right)$ of e.g. tert-butyl 2-benzamidonicotinate using an isodesmic reaction.

Conformational analyses were performed using PCmodel version $10 .{ }^{11}$ Density functional calculations were performed using Gaussian $16 .{ }^{12}$ For all DFT calculations, the default ultrafine grid having 99 radial shells and 590 angular points per shell was used. For the most stable conformations of the compounds studied, the Cartesian coordinates of the equilibrium geometry, the B3LYP/ 6-311++G(d,p) energy, the lowest frequencies obtained by diagonalizing the Hessian, and the thermodynamic properties derived from them are given below. For the most stable amide conformation, the resonance energies estimated from the isodesmic equation are compared with the NBO based second-order perturbation energies ${ }^{13,14}$ involving the nitrogen lone pair and the CO $\pi^{*}$ orbital. While the isodesmic equation resonance energies were in accordance with the observed experimental results, NBO based second-order perturbation energies were not. 


\section{Cartesian coordinates for the optimized structures}

\section{Oligoamide L,L-19}

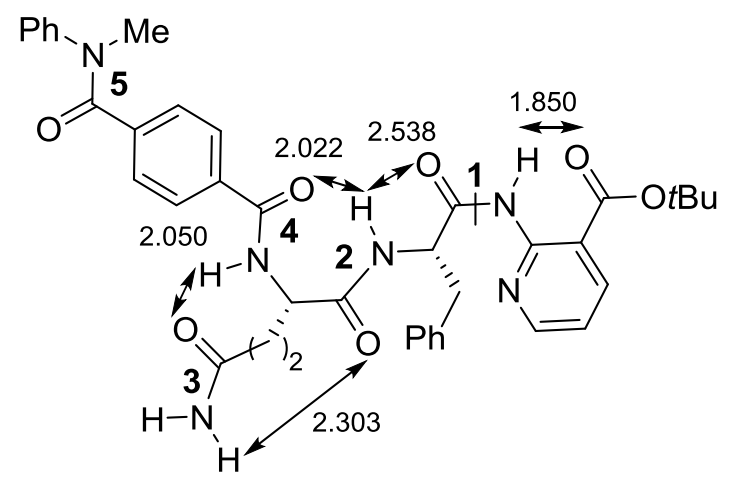

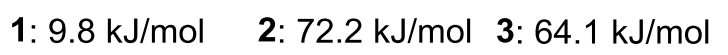

4: $67.9 \mathrm{~kJ} / \mathrm{mol} \quad 5: 62.4 \mathrm{~kJ} / \mathrm{mol}$

$\begin{array}{ll}\text { Stoichiometry } & \text { C39H42N6O7 } \\ \text { Framework group } & \text { C1 }[\mathrm{X}(\mathrm{C} 39 \mathrm{H} 42 \mathrm{~N} 607)]\end{array}$

Standard orientation:

\begin{tabular}{|c|c|c|c|c|c|}
\hline \multirow{2}{*}{$\begin{array}{l}\text { Center } \\
\text { Number }\end{array}$} & \multirow[t]{2}{*}{ Atom } & \multirow{2}{*}{$\begin{array}{l}\text { Atomic } \\
\text { Type }\end{array}$} & \multicolumn{3}{|c|}{ Coordinates (Angstroms) } \\
\hline & & & $\mathrm{x}$ & $\mathrm{Y}$ & $\mathrm{z}$ \\
\hline 1 & C & 0 & -7.253891 & -0.246185 & 1.687467 \\
\hline 2 & C & 0 & -5.970709 & -0.274757 & 0.901850 \\
\hline 3 & C & 0 & -3.496354 & -0.160041 & -0.424336 \\
\hline 4 & C & 0 & -5.482865 & 0.940790 & 0.411544 \\
\hline 5 & C & 0 & -5.199215 & -1.430421 & 0.741626 \\
\hline 6 & C & 0 & -3.970534 & -1.368955 & 0.096064 \\
\hline 7 & C & 0 & -4.267366 & 0.999342 & -0.260363 \\
\hline 8 & 0 & 0 & -7.485280 & 0.684811 & 2.448035 \\
\hline 9 & $\mathrm{~N}$ & 0 & -8.126490 & -1.312880 & 1.578841 \\
\hline 10 & C & 0 & -9.291548 & -1.295841 & 2.474604 \\
\hline 11 & C & 0 & -8.198217 & -2.170755 & 0.433249 \\
\hline 12 & C & 0 & -8.392281 & -3.868868 & -1.782732 \\
\hline 13 & C & 0 & -8.285784 & -1.639180 & -0.855801 \\
\hline 14 & $\mathrm{C}$ & 0 & -8.224659 & -3.558140 & 0.607662 \\
\hline 15 & $\mathrm{C}$ & 0 & -8.325226 & -4.401197 & -0.495148 \\
\hline 16 & $\mathrm{C}$ & 0 & -8.370051 & -2.487147 & -1.958294 \\
\hline 17 & $\mathrm{C}$ & 0 & -2.160578 & -0.191183 & -1.116476 \\
\hline 18 & 0 & 0 & -1.462183 & -1.208238 & -1.106259 \\
\hline 19 & $\mathrm{~N}$ & 0 & -1.763804 & 0.943909 & -1.742846 \\
\hline 20 & $\mathrm{C}$ & 0 & -0.549039 & 1.013152 & -2.564247 \\
\hline 21 & $\mathrm{C}$ & 0 & -0.738748 & 2.020193 & -3.714406 \\
\hline 22 & $\mathrm{C}$ & 0 & -0.705441 & 3.537495 & -3.396598 \\
\hline 23 & $\mathrm{C}$ & 0 & -1.450162 & 3.920010 & -2.125609 \\
\hline 24 & 0 & 0 & -2.667331 & 3.763994 & -2.013844 \\
\hline 25 & $\mathrm{~N}$ & 0 & -0.684454 & 4.428541 & -1.129340 \\
\hline 26 & C & 0 & 0.723125 & 1.268114 & -1.707491 \\
\hline 27 & $\mathrm{~N}$ & 0 & 1.033645 & 0.250983 & -0.874483 \\
\hline 28 & 0 & 0 & 1.382786 & 2.302017 & -1.800340 \\
\hline 29 & $\mathrm{C}$ & 0 & 2.174940 & 0.296134 & 0.020923 \\
\hline 30 & $\mathrm{C}$ & 0 & 3.049500 & -0.934878 & -0.236621 \\
\hline 31 & O & 0 & 2.565137 & -1.966530 & -0.658498 \\
\hline
\end{tabular}




\begin{tabular}{|c|c|c|c|c|c|}
\hline 32 & $\mathrm{C}$ & 0 & 1.742762 & 0.287853 & 1.515530 \\
\hline 33 & $\mathrm{C}$ & 0 & 0.912916 & 1.487793 & 1.915400 \\
\hline 34 & $\mathrm{C}$ & 0 & -0.620617 & 3.718334 & 2.681225 \\
\hline 35 & $\mathrm{C}$ & 0 & 1.512839 & 2.739011 & 2.105462 \\
\hline 36 & $\mathrm{C}$ & 0 & -0.466716 & 1.375215 & 2.111884 \\
\hline 37 & $\mathrm{C}$ & 0 & -1.230165 & 2.479545 & 2.491761 \\
\hline 38 & $\mathrm{C}$ & 0 & 0.756039 & 3.844580 & 2.485639 \\
\hline 39 & $\mathrm{~N}$ & 0 & 4.416578 & -0.927993 & 0.052048 \\
\hline 40 & $\mathrm{C}$ & 0 & 5.308833 & 0.054169 & 0.448542 \\
\hline 41 & $\mathrm{C}$ & 0 & 7.057204 & 2.041252 & 1.229087 \\
\hline 42 & C & 0 & 6.707929 & -0.237168 & 0.496837 \\
\hline 43 & $\mathrm{~N}$ & 0 & 4.831388 & 1.256549 & 0.785316 \\
\hline 44 & $\mathrm{C}$ & 0 & 5.680109 & 2.214310 & 1.154506 \\
\hline 45 & $\mathrm{C}$ & 0 & 7.562686 & 0.792178 & 0.896761 \\
\hline 46 & $\mathrm{C}$ & 0 & 7.258218 & -1.576502 & 0.141634 \\
\hline 47 & 0 & 0 & 6.571693 & -2.528417 & -0.190597 \\
\hline 48 & 0 & 0 & 8.592161 & -1.611750 & 0.232614 \\
\hline 49 & $\mathrm{C}$ & 0 & 9.381418 & -2.838728 & -0.067778 \\
\hline 50 & $\mathrm{C}$ & 0 & 10.814660 & -2.369771 & 0.179901 \\
\hline 51 & $\mathrm{C}$ & 0 & 9.182537 & -3.237337 & -1.531125 \\
\hline 52 & $\mathrm{C}$ & 0 & 8.996148 & -3.954285 & 0.905381 \\
\hline 53 & $\mathrm{H}$ & 0 & -6.062323 & 1.841955 & 0.570335 \\
\hline 54 & $\mathrm{H}$ & 0 & -5.548884 & -2.377280 & 1.132784 \\
\hline 55 & $\mathrm{H}$ & 0 & -3.357068 & -2.253728 & -0.018093 \\
\hline 56 & $\mathrm{H}$ & 0 & -3.934674 & 1.962186 & -0.632318 \\
\hline 57 & $\mathrm{H}$ & 0 & -8.983450 & -0.952064 & 3.459999 \\
\hline 58 & $\mathrm{H}$ & 0 & -10.067323 & -0.618988 & 2.100900 \\
\hline 59 & $\mathrm{H}$ & 0 & -9.701855 & -2.302228 & 2.542176 \\
\hline 60 & $\mathrm{H}$ & 0 & -8.462889 & -4.526689 & -2.641182 \\
\hline 61 & $\mathrm{H}$ & 0 & -8.284415 & -0.564422 & -0.992806 \\
\hline 62 & $\mathrm{H}$ & 0 & -8.152226 & -3.971818 & 1.607323 \\
\hline 63 & $\mathrm{H}$ & 0 & -8.339424 & -5.475387 & -0.349164 \\
\hline 64 & $\mathrm{H}$ & 0 & -8.428582 & -2.063960 & -2.954620 \\
\hline 65 & $\mathrm{H}$ & 0 & -2.375707 & 1.750503 & -1.762809 \\
\hline 66 & $\mathrm{H}$ & 0 & -0.424497 & 0.020242 & -3.008350 \\
\hline 67 & $\mathrm{H}$ & 0 & 0.039030 & 1.828924 & -4.457690 \\
\hline 68 & $\mathrm{H}$ & 0 & -1.691538 & 1.769459 & -4.188413 \\
\hline 69 & $\mathrm{H}$ & 0 & -1.198933 & 4.064978 & -4.217917 \\
\hline 70 & $\mathrm{H}$ & 0 & 0.326342 & 3.875999 & -3.343493 \\
\hline 71 & $\mathrm{H}$ & 0 & 0.307432 & 4.230529 & -1.143659 \\
\hline 72 & $\mathrm{H}$ & 0 & -1.116904 & 4.539945 & -0.222327 \\
\hline 73 & $\mathrm{H}$ & 0 & 0.450129 & -0.583625 & -0.895751 \\
\hline 74 & $\mathrm{H}$ & 0 & 2.726826 & 1.207284 & -0.188154 \\
\hline 75 & $\mathrm{H}$ & 0 & 2.649358 & 0.250664 & 2.125001 \\
\hline 76 & $\mathrm{H}$ & 0 & 1.182527 & -0.632195 & 1.705717 \\
\hline 77 & $\mathrm{H}$ & 0 & -1.209516 & 4.574962 & 2.990943 \\
\hline 78 & $\mathrm{H}$ & 0 & 2.582368 & 2.840613 & 1.956232 \\
\hline 79 & $\mathrm{H}$ & 0 & -0.949152 & 0.413491 & 1.972959 \\
\hline 80 & $\mathrm{H}$ & 0 & -2.297846 & 2.368013 & 2.644341 \\
\hline 81 & $\mathrm{H}$ & 0 & 1.240229 & 4.803207 & 2.639226 \\
\hline 82 & $\mathrm{H}$ & 0 & 4.857711 & -1.824948 & -0.156064 \\
\hline 83 & $\mathrm{H}$ & 0 & 7.704339 & 2.851511 & 1.538675 \\
\hline 84 & $\mathrm{H}$ & 0 & 5.229848 & 3.170428 & 1.407714 \\
\hline 85 & $\mathrm{H}$ & 0 & 8.625050 & 0.595139 & 0.939988 \\
\hline 86 & $\mathrm{H}$ & 0 & 11.070712 & -1.540494 & -0.483243 \\
\hline 87 & $\mathrm{H}$ & 0 & 11.510778 & -3.190174 & -0.009149 \\
\hline 88 & $\mathrm{H}$ & 0 & 10.941515 & -2.041371 & 1.213900 \\
\hline 89 & $\mathrm{H}$ & 0 & 9.404294 & -2.394527 & -2.190680 \\
\hline 90 & $\mathrm{H}$ & 0 & 9.873110 & -4.048485 & -1.776848 \\
\hline 91 & $\mathrm{H}$ & 0 & 8.166221 & -3.578644 & -1.720035 \\
\hline 92 & $\mathrm{H}$ & 0 & 7.978603 & -4.301927 & 0.735585 \\
\hline
\end{tabular}

S88 


$\begin{array}{llllll}93 & \mathrm{H} & \mathrm{O} & 9.087863 & -3.609347 & 1.938436 \\ 94 & \mathrm{H} & \mathrm{O} & 9.678815 & -4.797385 & 0.769814\end{array}$

Standard basis: $6-311++G(d, p) \quad(5 D, 7 F)$

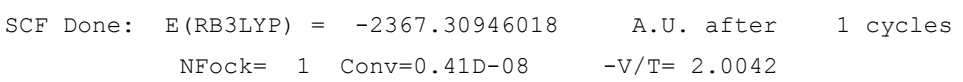

Full mass-weighted force constant matrix:

\begin{tabular}{|c|c|c|c|c|c|c|c|}
\hline Low & frequencies --- & -0.0013 & -0.0003 & 0.0003 & 0.7993 & 1.7110 & 3.3320 \\
\hline Low & frequencies --- & 6.7484 & 8.5881 & 8.6743 & & & \\
\hline
\end{tabular}

\section{Zero-point correction=}

Thermal correction to Energy=

Thermal correction to Enthalpy=

Thermal correction to Gibbs Free Energy=

Sum of electronic and zero-point Energies=

Sum of electronic and thermal Energies=

Sum of electronic and thermal Enthalpies=

Sum of electronic and thermal Free Energies=
0.762219 (Hartree/Particle)

0.811884

0.812828

0.668953

$-2366.547241$

$-2366.497576$

$-2366.496632$

$-2366.640507$ 
Amide 1 A

Stoichiometry C40H43N5O7

Framework group C1[X(C40H43N507)]

Standard orientation:

\begin{tabular}{|c|c|c|c|c|c|}
\hline \multirow{2}{*}{$\begin{array}{l}\text { Center } \\
\text { Number }\end{array}$} & \multirow[t]{2}{*}{ Atom } & \multirow{2}{*}{$\begin{array}{l}\text { Atomic } \\
\text { Type }\end{array}$} & \multicolumn{3}{|c|}{ Coordinates (Angstroms) } \\
\hline & & & $\mathrm{X}$ & $\mathrm{Y}$ & Z \\
\hline 1 & $\mathrm{C}$ & 0 & -7.564158 & 1.009090 & 1.228072 \\
\hline 2 & $\mathrm{C}$ & 0 & -6.060571 & 0.973356 & 1.157032 \\
\hline 3 & $\mathrm{C}$ & 0 & -3.253882 & 0.930092 & 1.246723 \\
\hline 4 & $\mathrm{C}$ & 0 & -5.311463 & 1.810662 & 0.323364 \\
\hline 5 & $\mathrm{C}$ & 0 & -5.392705 & 0.139049 & 2.058972 \\
\hline 6 & C & 0 & -4.003962 & 0.103738 & 2.092735 \\
\hline 7 & $\mathrm{C}$ & 0 & -3.923305 & 1.799611 & 0.380268 \\
\hline 8 & 0 & 0 & -8.125366 & 0.925055 & 2.312823 \\
\hline 9 & $\mathrm{~N}$ & 0 & -8.284490 & 1.196137 & 0.064005 \\
\hline 10 & $\mathrm{C}$ & 0 & -9.737054 & 1.362279 & 0.217191 \\
\hline 11 & C & 0 & -7.802689 & 0.844152 & -1.239156 \\
\hline 12 & $\mathrm{C}$ & 0 & -6.915885 & 0.165485 & -3.804939 \\
\hline 13 & C & 0 & -7.253886 & -0.417074 & -1.483788 \\
\hline 14 & C & 0 & -7.920836 & 1.758309 & -2.290966 \\
\hline 15 & C & 0 & -7.482975 & 1.417603 & -3.567402 \\
\hline 16 & C & 0 & -6.801601 & -0.748057 & -2.759585 \\
\hline 17 & $\mathrm{C}$ & 0 & -1.751063 & 0.958760 & 1.237662 \\
\hline 18 & $\mathrm{O}$ & 0 & -1.144067 & 1.938966 & 0.805180 \\
\hline 19 & $\mathrm{~N}$ & 0 & -1.128327 & -0.141316 & 1.735437 \\
\hline 20 & C & 0 & 0.307342 & -0.381409 & 1.659825 \\
\hline 21 & $\mathrm{C}$ & 0 & 1.058757 & 0.082189 & 2.937159 \\
\hline 22 & C & 0 & 1.241755 & 1.608590 & 3.044369 \\
\hline 23 & $\mathrm{C}$ & 0 & 2.224710 & 2.118491 & 1.990790 \\
\hline 24 & O & 0 & 3.434947 & 1.952121 & 2.119784 \\
\hline 25 & $\mathrm{~N}$ & 0 & 1.668676 & 2.731008 & 0.917245 \\
\hline 26 & C & 0 & 0.512266 & -1.890003 & 1.433991 \\
\hline 27 & C & 0 & 1.891049 & -2.402716 & 1.055631 \\
\hline 28 & O & 0 & -0.424457 & -2.652788 & 1.576825 \\
\hline 29 & C & 0 & 2.167666 & -2.478884 & -0.480160 \\
\hline 30 & C & 0 & 2.164714 & -1.079701 & -1.084947 \\
\hline 31 & $\mathrm{O}$ & 0 & 1.193903 & -0.644817 & -1.681240 \\
\hline 32 & C & 0 & 1.199767 & -3.409941 & -1.245952 \\
\hline 33 & C & 0 & 1.424993 & -4.875118 & -0.932450 \\
\hline 34 & C & 0 & 1.874324 & -7.594889 & -0.363669 \\
\hline 35 & C & 0 & 2.469263 & -5.576681 & -1.549013 \\
\hline 36 & C & 0 & 0.608442 & -5.561891 & -0.026763 \\
\hline 37 & C & 0 & 0.830546 & -6.910086 & 0.254929 \\
\hline 38 & $\mathrm{C}$ & 0 & 2.694720 & -6.922517 & -1.269209 \\
\hline 39 & $\mathrm{~N}$ & 0 & 3.260803 & -0.227773 & -0.931607 \\
\hline 40 & C & 0 & 4.531117 & -0.367660 & -0.385369 \\
\hline 41 & C & 0 & 7.053625 & -0.695456 & 0.677558 \\
\hline 42 & C & 0 & 5.420416 & 0.748695 & -0.370229 \\
\hline 43 & $\mathrm{~N}$ & 0 & 4.887979 & -1.555580 & 0.108789 \\
\hline 44 & C & 0 & 6.108255 & -1.710818 & 0.622226 \\
\hline 45 & $\mathrm{C}$ & 0 & 6.688809 & 0.546049 & 0.174725 \\
\hline 46 & C & 0 & 5.033878 & 2.094785 & -0.879015 \\
\hline 47 & $\mathrm{O}$ & 0 & 3.935994 & 2.360958 & -1.348741 \\
\hline 48 & $\mathrm{O}$ & 0 & 6.031726 & 2.972431 & -0.774143 \\
\hline 49 & $\mathrm{C}$ & 0 & 5.914711 & 4.392679 & -1.204686 \\
\hline 50 & C & 0 & 5.680742 & 4.452702 & -2.714913 \\
\hline 51 & C & 0 & 7.290226 & 4.951279 & -0.840754 \\
\hline 52 & $\mathrm{C}$ & 0 & 4.815602 & 5.096496 & -0.407439 \\
\hline
\end{tabular}




\begin{tabular}{|c|c|c|c|c|c|}
\hline 53 & $\mathrm{H}$ & 0 & -5.810230 & 2.483319 & -0.362316 \\
\hline 54 & $\mathrm{H}$ & 0 & -5.975101 & -0.467293 & 2.741634 \\
\hline 55 & $\mathrm{H}$ & 0 & -3.514936 & -0.545676 & 2.809730 \\
\hline 56 & $\mathrm{H}$ & 0 & -3.337113 & 2.460007 & -0.246023 \\
\hline 57 & $\mathrm{H}$ & 0 & -9.938832 & 1.997341 & 1.077499 \\
\hline 58 & $\mathrm{H}$ & 0 & -10.138452 & 1.819072 & -0.686015 \\
\hline 59 & $\mathrm{H}$ & 0 & -10.229870 & 0.397572 & 0.378147 \\
\hline 60 & $\mathrm{H}$ & 0 & -6.567591 & -0.095460 & -4.797483 \\
\hline 61 & $\mathrm{H}$ & 0 & -7.179388 & -1.134474 & -0.675237 \\
\hline 62 & $\mathrm{H}$ & 0 & -8.343305 & 2.738742 & -2.101258 \\
\hline 63 & $\mathrm{H}$ & 0 & -7.574223 & 2.135381 & -4.374767 \\
\hline 64 & $\mathrm{H}$ & 0 & -6.368160 & -1.725818 & -2.936172 \\
\hline 65 & $\mathrm{H}$ & 0 & -1.668424 & -0.988017 & 1.869421 \\
\hline 66 & $\mathrm{H}$ & 0 & 0.703630 & 0.148259 & 0.789002 \\
\hline 67 & $\mathrm{H}$ & 0 & 2.049500 & -0.376801 & 2.973863 \\
\hline 68 & $\mathrm{H}$ & 0 & 0.501576 & -0.282537 & 3.805026 \\
\hline 69 & $\mathrm{H}$ & 0 & 0.280514 & 2.119151 & 2.970324 \\
\hline 70 & $\mathrm{H}$ & 0 & 1.677717 & 1.835799 & 4.019717 \\
\hline 71 & $\mathrm{H}$ & 0 & 2.273920 & 2.928215 & 0.131319 \\
\hline 72 & $\mathrm{H}$ & 0 & 0.667449 & 2.654485 & 0.758528 \\
\hline 73 & $\mathrm{H}$ & 0 & 2.673617 & -1.793290 & 1.508671 \\
\hline 74 & $\mathrm{H}$ & 0 & 1.974698 & -3.415402 & 1.454772 \\
\hline 75 & $\mathrm{H}$ & 0 & 3.181012 & -2.872041 & -0.551515 \\
\hline 76 & $\mathrm{H}$ & 0 & 1.353307 & -3.242680 & -2.315342 \\
\hline 77 & $\mathrm{H}$ & 0 & 0.164577 & -3.132923 & -1.042628 \\
\hline 78 & $\mathrm{H}$ & 0 & 2.044803 & -8.643612 & -0.147140 \\
\hline 79 & $\mathrm{H}$ & 0 & 3.106606 & -5.065485 & -2.264636 \\
\hline 80 & $\mathrm{H}$ & 0 & -0.200585 & -5.032487 & 0.464100 \\
\hline 81 & $\mathrm{H}$ & 0 & 0.184383 & -7.425147 & 0.957558 \\
\hline 82 & $\mathrm{H}$ & 0 & 3.504823 & -7.448168 & -1.763081 \\
\hline 83 & $\mathrm{H}$ & 0 & 3.082589 & 0.703195 & -1.308765 \\
\hline 84 & $\mathrm{H}$ & 0 & 8.031965 & -0.867782 & 1.106971 \\
\hline 85 & $\mathrm{H}$ & 0 & 6.332898 & -2.701004 & 1.009275 \\
\hline 86 & $\mathrm{H}$ & 0 & 7.377837 & 1.378407 & 0.205183 \\
\hline 87 & $\mathrm{H}$ & 0 & 6.461942 & 3.903347 & -3.246448 \\
\hline 88 & $\mathrm{H}$ & 0 & 4.709658 & 4.041007 & -2.985597 \\
\hline 89 & $\mathrm{H}$ & 0 & 5.718847 & 5.495376 & -3.041630 \\
\hline 90 & $\mathrm{H}$ & 0 & 7.465861 & 4.874244 & 0.234438 \\
\hline 91 & $\mathrm{H}$ & 0 & 7.350047 & 6.004016 & -1.126258 \\
\hline 92 & $\mathrm{H}$ & 0 & 8.079587 & 4.406444 & -1.363444 \\
\hline 93 & $\mathrm{H}$ & 0 & 4.933268 & 4.905063 & 0.661325 \\
\hline 94 & $\mathrm{H}$ & 0 & 4.892282 & 6.174518 & -0.572875 \\
\hline 95 & $\mathrm{H}$ & 0 & 3.823414 & 4.772940 & -0.715776 \\
\hline
\end{tabular}

Standard basis: $6-311++G(d, p) \quad(5 D, 7 F)$

$\begin{array}{llr}\mathrm{SCF} \text { Done: } & \mathrm{E}(\mathrm{RB} 3 \mathrm{LYP})=-2351.24318025 & \mathrm{~A} . \mathrm{U} \text {. after } 2 \text { cycles } \\ & \text { NFock }=2 \text { Conv=0.11D-08 } & -\mathrm{V} / \mathrm{T}=2.0042\end{array}$

Full mass-weighted force constant matrix:

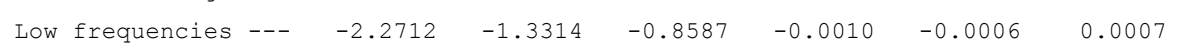

Low frequencies --- $\quad 7.3346 \quad 9.3816 \quad 14.3196$

Zero-point correction=

Thermal correction to Energy=

Thermal correction to Enthalpy=

Thermal correction to Gibbs Free Energy=
0.773134 (Hartree/Particle)

0.822802

0.823747

0.681208 
Sum of electronic and zero-point Energies=

Sum of electronic and thermal Energies=

Sum of electronic and thermal Enthalpies=

Sum of electronic and thermal Free Energies=
$-2350.470046$

$-2350.420378$

$-2350.419434$

$-2350.561972$ 
Amide 1 B

Stoichiometry C39H44N606

Framework group C1[X(C39H44N6O6)]

Standard orientation:

\begin{tabular}{|c|c|c|c|c|c|}
\hline \multirow{2}{*}{$\begin{array}{l}\text { Center } \\
\text { Number }\end{array}$} & \multirow[t]{2}{*}{ Atom } & \multirow{2}{*}{$\begin{array}{l}\text { Atomic } \\
\text { Type }\end{array}$} & \multicolumn{3}{|c|}{ Coordinates (Angstroms) } \\
\hline & & & $\mathrm{X}$ & $\mathrm{Y}$ & Z \\
\hline 1 & $\mathrm{C}$ & 0 & -5.493988 & -3.115616 & -0.464337 \\
\hline 2 & C & 0 & -4.699324 & -1.879268 & -0.783359 \\
\hline 3 & C & 0 & -3.241150 & 0.384177 & -1.582040 \\
\hline 4 & $\mathrm{C}$ & 0 & -5.236191 & -0.981475 & -1.713319 \\
\hline 5 & $\mathrm{C}$ & 0 & -3.413874 & -1.651257 & -0.284033 \\
\hline 6 & C & 0 & -2.685786 & -0.534232 & -0.683599 \\
\hline 7 & C & 0 & -4.518834 & 0.141682 & -2.100842 \\
\hline 8 & 0 & 0 & -6.129066 & -3.683395 & -1.343287 \\
\hline 9 & $\mathrm{~N}$ & 0 & -5.442776 & -3.627803 & 0.820568 \\
\hline 10 & C & 0 & -6.121278 & -4.913468 & 1.035634 \\
\hline 11 & C & 0 & -5.167517 & -2.836873 & 1.982868 \\
\hline 12 & C & 0 & -4.654703 & -1.342066 & 4.293332 \\
\hline 13 & C & 0 & -4.243783 & -3.293337 & 2.928590 \\
\hline 14 & C & 0 & -5.843291 & -1.635001 & 2.209148 \\
\hline 15 & C & 0 & -5.577419 & -0.886841 & 3.354139 \\
\hline 16 & C & 0 & -3.992847 & -2.551016 & 4.078801 \\
\hline 17 & $\mathrm{C}$ & 0 & -2.524417 & 1.619260 & -2.057726 \\
\hline 18 & 0 & 0 & -2.884406 & 2.184581 & -3.096528 \\
\hline 19 & $\mathrm{~N}$ & 0 & -1.508546 & 2.075484 & -1.289373 \\
\hline 20 & $\mathrm{C}$ & 0 & -0.615393 & 3.176560 & -1.663336 \\
\hline 21 & C & 0 & -1.030475 & 4.515556 & -1.020457 \\
\hline 22 & C & 0 & -2.336989 & 5.125079 & -1.563075 \\
\hline 23 & $\mathrm{C}$ & 0 & -2.155728 & 5.726436 & -2.956947 \\
\hline 24 & o & 0 & -1.663242 & 6.837118 & -3.111238 \\
\hline 25 & $\mathrm{~N}$ & 0 & -2.569222 & 4.945943 & -3.989259 \\
\hline 26 & C & 0 & 0.814827 & 2.773028 & -1.269162 \\
\hline 27 & $\mathrm{~N}$ & 0 & 0.916084 & 2.533793 & 0.173483 \\
\hline 28 & C & 0 & 2.032641 & 1.696465 & 0.589544 \\
\hline 29 & C & 0 & 1.702670 & 0.226361 & 0.291646 \\
\hline 30 & 0 & 0 & 0.554109 & -0.170928 & 0.213485 \\
\hline 31 & C & 0 & 2.259416 & 1.831332 & 2.118799 \\
\hline 32 & C & 0 & 2.604756 & 3.241699 & 2.547581 \\
\hline 33 & C & 0 & 3.244612 & 5.866827 & 3.330608 \\
\hline 34 & C & 0 & 1.702082 & 4.008062 & 3.292502 \\
\hline 35 & C & 0 & 3.837104 & 3.813093 & 2.203121 \\
\hline 36 & $\mathrm{C}$ & 0 & 4.154986 & 5.112161 & 2.589674 \\
\hline 37 & C & 0 & 2.017679 & 5.310508 & 3.682162 \\
\hline 38 & $\mathrm{~N}$ & 0 & 2.713259 & -0.723155 & 0.121778 \\
\hline 39 & $\mathrm{C}$ & 0 & 4.098396 & -0.676314 & 0.083637 \\
\hline 40 & C & 0 & 6.854288 & -0.555119 & 0.011323 \\
\hline 41 & C & 0 & 4.831455 & -1.846284 & -0.288555 \\
\hline 42 & $\mathrm{~N}$ & 0 & 4.715732 & 0.464801 & 0.404945 \\
\hline 43 & C & 0 & 6.046872 & 0.519149 & 0.363864 \\
\hline 44 & $\mathrm{C}$ & 0 & 6.223905 & -1.748158 & -0.314649 \\
\hline 45 & C & 0 & 4.162496 & -3.134198 & -0.636452 \\
\hline 46 & 0 & 0 & 2.956248 & -3.309302 & -0.583527 \\
\hline 47 & 0 & 0 & 5.039699 & -4.070262 & -1.008479 \\
\hline 48 & C & 0 & 4.623443 & -5.445887 & -1.406929 \\
\hline 49 & $\mathrm{C}$ & 0 & 5.962323 & -6.106142 & -1.732804 \\
\hline 50 & $\mathrm{C}$ & 0 & 3.731396 & -5.376610 & -2.647251 \\
\hline 51 & C & 0 & 3.942815 & -6.142391 & -0.227583 \\
\hline 52 & $\mathrm{H}$ & 0 & -6.214035 & -1.183047 & -2.133521 \\
\hline
\end{tabular}




\begin{tabular}{|c|c|c|c|c|c|}
\hline 53 & $\mathrm{H}$ & 0 & -2.963807 & -2.354642 & 0.404760 \\
\hline 54 & $\mathrm{H}$ & 0 & -1.676910 & -0.413507 & -0.308123 \\
\hline 55 & $\mathrm{H}$ & 0 & -4.927353 & 0.841480 & -2.818797 \\
\hline 56 & $\mathrm{H}$ & 0 & -5.926816 & -5.569399 & 0.189402 \\
\hline 57 & $\mathrm{H}$ & 0 & -5.743918 & -5.365166 & 1.951859 \\
\hline 58 & $\mathrm{H}$ & 0 & -7.204744 & -4.779168 & 1.124574 \\
\hline 59 & $\mathrm{H}$ & 0 & -4.453846 & -0.761758 & 5.186474 \\
\hline 60 & $\mathrm{H}$ & 0 & -3.714344 & -4.222813 & 2.751401 \\
\hline 61 & $\mathrm{H}$ & 0 & -6.572596 & -1.287328 & 1.487305 \\
\hline 62 & $\mathrm{H}$ & 0 & -6.102147 & 0.048063 & 3.515145 \\
\hline 63 & $\mathrm{H}$ & 0 & -3.270865 & -2.911932 & 4.802679 \\
\hline 64 & $\mathrm{H}$ & 0 & -1.232231 & 1.571388 & -0.455043 \\
\hline 65 & $\mathrm{H}$ & 0 & -0.653544 & 3.269181 & -2.751213 \\
\hline 66 & $\mathrm{H}$ & 0 & -1.137235 & 4.363093 & 0.058097 \\
\hline 67 & $\mathrm{H}$ & 0 & -0.224008 & 5.242820 & -1.166403 \\
\hline 68 & $\mathrm{H}$ & 0 & -2.634972 & 5.950869 & -0.913869 \\
\hline 69 & $\mathrm{H}$ & 0 & -3.136035 & 4.380786 & -1.554033 \\
\hline 70 & $\mathrm{H}$ & 0 & -2.393700 & 5.279784 & -4.924918 \\
\hline 71 & $\mathrm{H}$ & 0 & -2.804112 & 3.967707 & -3.838601 \\
\hline 72 & $\mathrm{H}$ & 0 & 1.518388 & 3.541240 & -1.624353 \\
\hline 73 & $\mathrm{H}$ & 0 & 1.060159 & 1.844050 & -1.790411 \\
\hline 74 & $\mathrm{H}$ & 0 & 0.963151 & 3.414221 & 0.673346 \\
\hline 75 & $\mathrm{H}$ & 0 & 2.969972 & 1.952100 & 0.084482 \\
\hline 76 & $\mathrm{H}$ & 0 & 3.067277 & 1.153810 & 2.403169 \\
\hline 77 & $\mathrm{H}$ & 0 & 1.349505 & 1.501567 & 2.627306 \\
\hline 78 & $\mathrm{H}$ & 0 & 3.491779 & 6.878603 & 3.631231 \\
\hline 79 & $\mathrm{H}$ & 0 & 0.745570 & 3.579697 & 3.574756 \\
\hline 80 & $\mathrm{H}$ & 0 & 4.549400 & 3.229488 & 1.629601 \\
\hline 81 & $\mathrm{H}$ & 0 & 5.114264 & 5.537341 & 2.315363 \\
\hline 82 & $\mathrm{H}$ & 0 & 1.304127 & 5.887570 & 4.259798 \\
\hline 83 & $\mathrm{H}$ & 0 & 2.333930 & -1.649280 & -0.083040 \\
\hline 84 & $\mathrm{H}$ & 0 & 7.932228 & -0.460457 & -0.002976 \\
\hline 85 & $\mathrm{H}$ & 0 & 6.487828 & 1.474915 & 0.634058 \\
\hline 86 & $\mathrm{H}$ & 0 & 6.799040 & -2.620042 & -0.594489 \\
\hline 87 & $\mathrm{H}$ & 0 & 5.798492 & -7.139371 & -2.047214 \\
\hline 88 & $\mathrm{H}$ & 0 & 6.615217 & -6.111905 & -0.857129 \\
\hline 89 & $\mathrm{H}$ & 0 & 6.468778 & -5.575860 & -2.542256 \\
\hline 90 & $\mathrm{H}$ & 0 & 3.550471 & -6.390766 & -3.013039 \\
\hline 91 & $\mathrm{H}$ & 0 & 4.225898 & -4.814844 & -3.443671 \\
\hline 92 & $\mathrm{H}$ & 0 & 2.771641 & -4.912202 & -2.427505 \\
\hline 93 & $\mathrm{H}$ & 0 & 2.984105 & -5.684853 & 0.010409 \\
\hline 94 & $\mathrm{H}$ & 0 & 3.772997 & -7.191548 & -0.483539 \\
\hline 95 & $\mathrm{H}$ & 0 & 4.583464 & -6.111122 & 0.657406 \\
\hline
\end{tabular}

Standard basis: $6-311++G(d, p) \quad(5 D, 7 F)$

$\begin{array}{llr}\text { SCF Done: } & \text { E (RB3LYP }=-2293.24522782 & \text { A.U. after } 2 \text { cycles } \\ & \text { NFock }=2 \text { Conv=0.77D-09 } \quad-\mathrm{V} / \mathrm{T}=2.0042\end{array}$

Full mass-weighted force constant matrix:

$\begin{array}{lrrrrrr}\text { Low frequencies --- } & -2.3342 & -1.0289 & -0.0009 & -0.0008 & -0.0005 & 0.8558 \\ \text { Low frequencies - -- } & 4.5434 & 6.2745 & 9.2519 & & \end{array}$ 
Thermal correction to Energy=

Thermal correction to Enthalpy=

Thermal correction to Gibbs Free Energy=

Sum of electronic and zero-point Energies=

Sum of electronic and thermal Energies=

Sum of electronic and thermal Enthalpies=

Sum of electronic and thermal Free Energies=
0.829683

0.830627

0.686781

$-2292.464721$

$-2292.415545$

$-2292.414600$

$-2292.558446$ 
Amide $1 \mathrm{C}$

Stoichiometry C40H45N506

Framework group C1[X(C40H45N506)]

Standard orientation:

\begin{tabular}{|c|c|c|c|c|c|}
\hline \multirow{2}{*}{$\begin{array}{l}\text { Center } \\
\text { Number }\end{array}$} & \multirow[t]{2}{*}{ Atom } & \multirow{2}{*}{$\begin{array}{l}\text { Atomic } \\
\text { Type }\end{array}$} & \multicolumn{3}{|c|}{ Coordinates (Angstroms) } \\
\hline & & & $\mathrm{X}$ & Y & z \\
\hline 1 & C & 0 & -4.350882 & 0.960094 & 0.666157 \\
\hline 2 & $\mathrm{C}$ & 0 & -3.263421 & -0.079035 & 0.666439 \\
\hline 3 & $\mathrm{C}$ & 0 & -1.137791 & -1.908804 & 0.808088 \\
\hline 4 & C & 0 & -2.865643 & -0.610617 & 1.898370 \\
\hline 5 & C & 0 & -2.576999 & -0.456329 & -0.490776 \\
\hline 6 & $\mathrm{C}$ & 0 & -1.514735 & -1.352119 & -0.418811 \\
\hline 7 & $\mathrm{C}$ & 0 & -1.832777 & -1.535532 & 1.964421 \\
\hline 8 & 0 & 0 & -4.363931 & 1.834676 & 1.525150 \\
\hline 9 & $\mathrm{~N}$ & 0 & -5.291529 & 0.935583 & -0.343243 \\
\hline 10 & C & 0 & -6.266699 & 2.034608 & -0.363334 \\
\hline 11 & C & 0 & -5.618401 & -0.236902 & -1.102459 \\
\hline 12 & C & 0 & -6.309926 & -2.491706 & -2.606338 \\
\hline 13 & C & 0 & -5.655614 & -0.168311 & -2.498386 \\
\hline 14 & C & 0 & -5.944655 & -1.435303 & -0.463485 \\
\hline 15 & C & 0 & -6.277259 & -2.560409 & -1.215337 \\
\hline 16 & C & 0 & -6.003471 & -1.290216 & -3.245218 \\
\hline 17 & C & 0 & -0.034610 & -2.922010 & 0.959091 \\
\hline 18 & o & 0 & -0.011732 & -3.670297 & 1.940843 \\
\hline 19 & $\mathrm{~N}$ & 0 & 0.904070 & -2.955971 & -0.016846 \\
\hline 20 & C & 0 & 2.077503 & -3.844065 & -0.009442 \\
\hline 21 & C & 0 & 1.854067 & -5.074737 & -0.910022 \\
\hline 22 & C & 0 & 0.771063 & -6.057452 & -0.424420 \\
\hline 23 & C & 0 & 1.248607 & -6.909698 & 0.751680 \\
\hline 24 & 0 & 0 & 1.930334 & -7.910966 & 0.575153 \\
\hline 25 & $\mathrm{~N}$ & 0 & 0.870546 & -6.479367 & 1.985059 \\
\hline 26 & C & 0 & 3.318073 & -3.051399 & -0.455958 \\
\hline 27 & C & 0 & 3.704303 & -1.879642 & 0.480328 \\
\hline 28 & C & 0 & 3.805147 & -0.472607 & -0.169944 \\
\hline 29 & C & 0 & 2.434680 & -0.014968 & -0.648936 \\
\hline 30 & 0 & 0 & 1.932638 & -0.471644 & -1.671763 \\
\hline 31 & C & 0 & 4.822874 & -0.428925 & -1.337476 \\
\hline 32 & C & 0 & 5.134362 & 0.973292 & -1.817105 \\
\hline 33 & $\mathrm{C}$ & 0 & 5.695006 & 3.594563 & -2.673189 \\
\hline 34 & $\mathrm{C}$ & 0 & 4.483937 & 1.518251 & -2.930045 \\
\hline 35 & $\mathrm{C}$ & 0 & 6.072405 & 1.764904 & -1.142644 \\
\hline 36 & C & 0 & 6.352128 & 3.063007 & -1.564046 \\
\hline 37 & C & 0 & 4.760698 & 2.817328 & -3.354783 \\
\hline 38 & $\mathrm{~N}$ & 0 & 1.714504 & 0.954680 & 0.034206 \\
\hline 39 & $\mathrm{C}$ & 0 & 1.847779 & 1.522569 & 1.295124 \\
\hline 40 & C & 0 & 2.090731 & 2.571996 & 3.829277 \\
\hline 41 & $\mathrm{C}$ & 0 & 1.094339 & 2.686018 & 1.629814 \\
\hline 42 & $\mathrm{~N}$ & 0 & 2.656932 & 0.926777 & 2.175359 \\
\hline 43 & $\mathrm{C}$ & 0 & 2.777771 & 1.443173 & 3.397616 \\
\hline 44 & $\mathrm{C}$ & 0 & 1.239135 & 3.190064 & 2.924042 \\
\hline 45 & C & 0 & 0.175408 & 3.349675 & 0.658459 \\
\hline 46 & 0 & 0 & -0.081550 & 2.903389 & -0.447511 \\
\hline 47 & 0 & 0 & -0.326359 & 4.486176 & 1.146840 \\
\hline 48 & C & 0 & -1.267814 & 5.346806 & 0.376475 \\
\hline 49 & $\mathrm{C}$ & 0 & -2.533219 & 4.564662 & 0.022992 \\
\hline 50 & C & 0 & -0.550440 & 5.899730 & -0.856008 \\
\hline 51 & C & 0 & -1.577556 & 6.459846 & 1.376836 \\
\hline 52 & $\mathrm{H}$ & 0 & -3.376365 & -0.291508 & 2.798947 \\
\hline
\end{tabular}




\begin{tabular}{|c|c|c|c|c|c|}
\hline 53 & $\mathrm{H}$ & 0 & -2.860545 & -0.046175 & -1.451396 \\
\hline 54 & $\mathrm{H}$ & 0 & -1.002695 & -1.621813 & -1.334769 \\
\hline 55 & $\mathrm{H}$ & 0 & -1.543330 & -1.980572 & 2.907998 \\
\hline 56 & $\mathrm{H}$ & 0 & -7.068525 & 1.863918 & 0.362989 \\
\hline 57 & $\mathrm{H}$ & 0 & -6.701111 & 2.105544 & -1.359360 \\
\hline 58 & $\mathrm{H}$ & 0 & -5.765800 & 2.966055 & -0.108204 \\
\hline 59 & $\mathrm{H}$ & 0 & -6.573968 & -3.366486 & -3.189138 \\
\hline 60 & $\mathrm{H}$ & 0 & -5.398652 & 0.761012 & -2.994494 \\
\hline 61 & $\mathrm{H}$ & 0 & -5.933559 & -1.485650 & 0.618728 \\
\hline 62 & $\mathrm{H}$ & 0 & -6.520106 & -3.488260 & -0.710201 \\
\hline 63 & $\mathrm{H}$ & 0 & -6.024747 & -1.228578 & -4.327377 \\
\hline 64 & $\mathrm{H}$ & 0 & 0.882421 & -2.245722 & -0.738020 \\
\hline 65 & $\mathrm{H}$ & 0 & 2.209587 & -4.168552 & 1.025363 \\
\hline 66 & $\mathrm{H}$ & 0 & 2.798989 & -5.618697 & -1.005351 \\
\hline 67 & $\mathrm{H}$ & 0 & 1.592120 & -4.721812 & -1.913802 \\
\hline 68 & $\mathrm{H}$ & 0 & -0.148847 & -5.522205 & -0.176914 \\
\hline 69 & $\mathrm{H}$ & 0 & 0.544831 & -6.757920 & -1.230602 \\
\hline 70 & $\mathrm{H}$ & 0 & 1.240488 & -6.976218 & 2.781382 \\
\hline 71 & $\mathrm{H}$ & 0 & 0.462946 & -5.557951 & 2.119123 \\
\hline 72 & $\mathrm{H}$ & 0 & 4.149210 & -3.757731 & -0.524185 \\
\hline 73 & $\mathrm{H}$ & 0 & 3.141938 & -2.691562 & -1.473351 \\
\hline 74 & $\mathrm{H}$ & 0 & 4.679031 & -2.083339 & 0.933909 \\
\hline 75 & $\mathrm{H}$ & 0 & 2.998682 & -1.811640 & 1.312684 \\
\hline 76 & $\mathrm{H}$ & 0 & 4.144668 & 0.203641 & 0.611299 \\
\hline 77 & $\mathrm{H}$ & 0 & 4.454405 & -1.028524 & -2.171249 \\
\hline 78 & $\mathrm{H}$ & 0 & 5.743946 & -0.901644 & -0.980408 \\
\hline 79 & $\mathrm{H}$ & 0 & 5.913984 & 4.603098 & -3.005806 \\
\hline 80 & $\mathrm{H}$ & 0 & 3.753427 & 0.919772 & -3.463508 \\
\hline 81 & $\mathrm{H}$ & 0 & 6.596221 & 1.356877 & -0.283197 \\
\hline 82 & $\mathrm{H}$ & 0 & 7.087225 & 3.656674 & -1.031480 \\
\hline 83 & $\mathrm{H}$ & 0 & 4.247747 & 3.220092 & -4.221378 \\
\hline 84 & $\mathrm{H}$ & 0 & 0.935099 & 1.331995 & -0.505883 \\
\hline 85 & $\mathrm{H}$ & 0 & 2.212875 & 2.945455 & 4.837756 \\
\hline 86 & $\mathrm{H}$ & 0 & 3.451160 & 0.914822 & 4.066981 \\
\hline 87 & $\mathrm{H}$ & 0 & 0.674692 & 4.068763 & 3.204315 \\
\hline 88 & $\mathrm{H}$ & 0 & -2.336364 & 3.808456 & -0.734931 \\
\hline 89 & $\mathrm{H}$ & 0 & -3.278809 & 5.263520 & -0.367185 \\
\hline 90 & $\mathrm{H}$ & 0 & -2.953484 & 4.076056 & 0.904693 \\
\hline 91 & $\mathrm{H}$ & 0 & 0.370199 & 6.413131 & -0.567261 \\
\hline 92 & $\mathrm{H}$ & 0 & -1.200320 & 6.624521 & -1.353739 \\
\hline 93 & $\mathrm{H}$ & 0 & -0.308951 & 5.108746 & -1.564747 \\
\hline 94 & $\mathrm{H}$ & 0 & -2.253405 & 7.188077 & 0.922504 \\
\hline 95 & $\mathrm{H}$ & 0 & -2.058486 & 6.053616 & 2.269274 \\
\hline 96 & $\mathrm{H}$ & 0 & -0.663305 & 6.977311 & 1.676258 \\
\hline
\end{tabular}

Standard basis: $6-311++G(d, p) \quad(5 D, 7 F)$

SCF Done: E (RB3LYP) $=-2277.20641002$ A.U. after 2 cycles $\mathrm{NFOCk}=2$ Conv $=0.36 \mathrm{D}-08 \quad-\mathrm{V} / \mathrm{T}=2.0043$

Full mass-weighted force constant matrix:

\begin{tabular}{|c|c|c|c|c|c|c|}
\hline Low & frequencies --- & -0.8607 & -0.0008 & -0.0001 & 0.0003 & 1.4635 \\
\hline Low & frequencies --- & 7.7474 & 10.4313 & 13.6588 & & \\
\hline
\end{tabular}




\section{Zero-point correction=}

Thermal correction to Energy=

Thermal correction to Enthalpy=

Thermal correction to Gibbs Free Energy=

Sum of electronic and zero-point Energies=

Sum of electronic and thermal Energies=

Sum of electronic and thermal Enthalpies=

Sum of electronic and thermal Free Energies=
0.791789 (Hartree/Particle)

0.841091

0.842035

0.699780

$-2276.414621$

$-2276.365319$

$-2276.364375$

$-2276.506630$ 
$\begin{array}{ll}\text { Stoichiometry } & \mathrm{C} 40 \mathrm{H} 43 \mathrm{~N} 5 \mathrm{O} 7 \\ \text { Framework group } & \mathrm{C} 1[\mathrm{X}(\mathrm{C} 40 \mathrm{H} 43 \mathrm{~N} 507)]\end{array}$

Standard orientation:

\begin{tabular}{|c|c|c|c|c|c|}
\hline \multirow{2}{*}{$\begin{array}{l}\text { Center } \\
\text { Number }\end{array}$} & \multirow[t]{2}{*}{ Atom } & \multirow{2}{*}{$\begin{array}{l}\text { Atomic } \\
\text { Type }\end{array}$} & \multicolumn{3}{|c|}{ Coordinates (Angstroms) } \\
\hline & & & $\mathrm{X}$ & Y & z \\
\hline 1 & $\mathrm{C}$ & 0 & -7.965294 & 2.411839 & 0.773085 \\
\hline 2 & $\mathrm{C}$ & 0 & -6.686735 & 1.646819 & 0.554564 \\
\hline 3 & $\mathrm{C}$ & 0 & -4.194315 & 0.384221 & 0.249987 \\
\hline 4 & $\mathrm{C}$ & 0 & -5.850416 & 1.450149 & 1.660835 \\
\hline 5 & $\mathrm{C}$ & 0 & -6.255411 & 1.230374 & -0.707842 \\
\hline 6 & $\mathrm{C}$ & 0 & -5.017782 & 0.613211 & -0.859271 \\
\hline 7 & $\mathrm{C}$ & 0 & -4.627832 & 0.813164 & 1.512557 \\
\hline 8 & 0 & 0 & -7.982615 & 3.360796 & 1.545571 \\
\hline 9 & $\mathrm{~N}$ & 0 & -9.086223 & 2.060763 & 0.045207 \\
\hline 10 & C & 0 & -10.260881 & 2.931419 & 0.197373 \\
\hline 11 & $\mathrm{C}$ & 0 & -9.310013 & 0.751550 & -0.492887 \\
\hline 12 & $\mathrm{C}$ & 0 & -9.803969 & -1.790082 & -1.554977 \\
\hline 13 & C & 0 & -9.726921 & 0.610559 & -1.820456 \\
\hline 14 & $\mathrm{C}$ & 0 & -9.158531 & -0.386208 & 0.303670 \\
\hline 15 & C & 0 & -9.393173 & -1.651428 & -0.231128 \\
\hline 16 & C & 0 & -9.976017 & -0.653958 & -2.345922 \\
\hline 17 & C & 0 & -2.860991 & -0.298396 & 0.145252 \\
\hline 18 & 0 & 0 & -2.170924 & -0.453851 & 1.137883 \\
\hline 19 & $\mathrm{C}$ & 0 & -2.404207 & -0.799700 & -1.217034 \\
\hline 20 & C & 0 & -1.023645 & -1.463127 & -1.203897 \\
\hline 21 & C & 0 & -0.752153 & -2.170366 & -2.552084 \\
\hline 22 & $\mathrm{C}$ & 0 & 0.577708 & -2.939353 & -2.612568 \\
\hline 23 & C & 0 & 0.600105 & -4.120609 & -1.643667 \\
\hline 24 & 0 & 0 & -0.256353 & -4.993994 & -1.682827 \\
\hline 25 & $\mathrm{~N}$ & 0 & 1.619751 & -4.129698 & -0.744042 \\
\hline 26 & C & 0 & 0.057387 & -0.406724 & -0.946971 \\
\hline 27 & $\mathrm{~N}$ & 0 & 1.009070 & -0.762756 & -0.041477 \\
\hline 28 & C & 0 & 2.120728 & 0.089097 & 0.314723 \\
\hline 29 & C & 0 & 3.435436 & -0.594320 & -0.070528 \\
\hline 30 & 0 & 0 & 3.497010 & -1.796208 & -0.283626 \\
\hline 31 & C & 0 & 2.086090 & 0.471713 & 1.827610 \\
\hline 32 & C & 0 & 2.056455 & -0.700619 & 2.783729 \\
\hline 33 & C & 0 & 1.995234 & -2.891615 & 4.546415 \\
\hline 34 & C & 0 & 0.837669 & -1.274385 & 3.170924 \\
\hline 35 & $\mathrm{C}$ & 0 & 3.240991 & -1.240009 & 3.300243 \\
\hline 36 & C & 0 & 3.213301 & -2.325331 & 4.173417 \\
\hline 37 & C & 0 & 0.808289 & -2.362159 & 4.043224 \\
\hline 38 & $\mathrm{~N}$ & 0 & 4.623904 & 0.124510 & -0.144128 \\
\hline 39 & $\mathrm{C}$ & 0 & 4.914826 & 1.482573 & -0.182662 \\
\hline 40 & C & 0 & 5.443005 & 4.185758 & -0.271496 \\
\hline 41 & C & 0 & 6.259153 & 1.912179 & -0.400315 \\
\hline 42 & $\mathrm{~N}$ & 0 & 3.916573 & 2.352303 & -0.017624 \\
\hline 43 & $\mathrm{C}$ & 0 & 4.173012 & 3.658355 & -0.065511 \\
\hline 44 & $\mathrm{C}$ & 0 & 6.489532 & 3.289803 & -0.438074 \\
\hline 45 & C & 0 & 7.384848 & 0.953191 & -0.591076 \\
\hline 46 & 0 & 0 & 7.252277 & -0.259921 & -0.588582 \\
\hline 47 & 0 & 0 & 8.552950 & 1.578299 & -0.765537 \\
\hline 48 & $\mathrm{C}$ & 0 & 9.830524 & 0.843102 & -0.985396 \\
\hline 49 & C & 0 & 9.742919 & 0.027873 & -2.276566 \\
\hline 50 & $\mathrm{C}$ & 0 & 10.148628 & -0.019277 & 0.237148 \\
\hline 51 & $\mathrm{C}$ & 0 & 10.838586 & 1.982894 & -1.126567 \\
\hline
\end{tabular}




\begin{tabular}{|c|c|c|c|c|c|}
\hline 52 & 0 & 0 & 0.074606 & 0.656297 & -1.556002 \\
\hline 53 & $\mathrm{H}$ & 0 & -6.171224 & 1.811597 & 2.630232 \\
\hline 54 & $\mathrm{H}$ & 0 & -6.877338 & 1.396733 & -1.577847 \\
\hline 55 & $\mathrm{H}$ & 0 & -4.701359 & 0.314099 & -1.850436 \\
\hline 56 & $\mathrm{H}$ & 0 & -3.982319 & 0.643782 & 2.365356 \\
\hline 57 & $\mathrm{H}$ & 0 & -10.793664 & 2.716031 & 1.129655 \\
\hline 58 & $\mathrm{H}$ & 0 & -10.934789 & 2.767515 & -0.642019 \\
\hline 59 & $\mathrm{H}$ & 0 & -9.938792 & 3.970573 & 0.219310 \\
\hline 60 & $\mathrm{H}$ & 0 & -9.991800 & -2.774580 & -1.967381 \\
\hline 61 & $\mathrm{H}$ & 0 & -9.842033 & 1.492634 & -2.440464 \\
\hline 62 & $\mathrm{H}$ & 0 & -8.855732 & -0.278899 & 1.338433 \\
\hline 63 & $\mathrm{H}$ & 0 & -9.264516 & -2.527995 & 0.393532 \\
\hline 64 & $\mathrm{H}$ & 0 & -10.294785 & -0.752519 & -3.377454 \\
\hline 65 & $\mathrm{H}$ & 0 & -3.152879 & -1.507530 & -1.591280 \\
\hline 66 & $\mathrm{H}$ & 0 & -2.391624 & 0.042805 & -1.916626 \\
\hline 67 & $\mathrm{H}$ & 0 & -1.002375 & -2.207335 & -0.402926 \\
\hline 68 & $\mathrm{H}$ & 0 & -1.564242 & -2.877999 & -2.740467 \\
\hline 69 & $\mathrm{H}$ & 0 & -0.769553 & -1.423741 & -3.351848 \\
\hline 70 & $\mathrm{H}$ & 0 & 0.691398 & -3.361501 & -3.615673 \\
\hline 71 & $\mathrm{H}$ & 0 & 1.427054 & -2.273590 & -2.443507 \\
\hline 72 & $\mathrm{H}$ & 0 & 1.673753 & -4.906357 & -0.102308 \\
\hline 73 & $\mathrm{H}$ & 0 & 2.307769 & -3.387569 & -0.677169 \\
\hline 74 & $\mathrm{H}$ & 0 & 0.903562 & -1.638490 & 0.448267 \\
\hline 75 & $\mathrm{H}$ & 0 & 2.021597 & 1.008729 & -0.257790 \\
\hline 76 & $\mathrm{H}$ & 0 & 1.188696 & 1.079924 & 1.962548 \\
\hline 77 & $\mathrm{H}$ & 0 & 2.942338 & 1.114168 & 2.037696 \\
\hline 78 & $\mathrm{H}$ & 0 & 1.971603 & -3.734697 & 5.227892 \\
\hline 79 & $\mathrm{H}$ & 0 & -0.092937 & -0.866677 & 2.789347 \\
\hline 80 & $\mathrm{H}$ & 0 & 4.194988 & -0.803185 & 3.021814 \\
\hline 81 & $\mathrm{H}$ & 0 & 4.141992 & -2.726188 & 4.564597 \\
\hline 82 & $\mathrm{H}$ & 0 & -0.144663 & -2.791493 & 4.332386 \\
\hline 83 & $\mathrm{H}$ & 0 & 5.432043 & -0.472146 & -0.328571 \\
\hline 84 & $\mathrm{H}$ & 0 & 5.602928 & 5.255703 & -0.300543 \\
\hline 85 & $\mathrm{H}$ & 0 & 3.314827 & 4.310630 & 0.069246 \\
\hline 86 & $\mathrm{H}$ & 0 & 7.498932 & 3.641326 & -0.602013 \\
\hline 87 & $\mathrm{H}$ & 0 & 10.727824 & -0.389634 & -2.501966 \\
\hline 88 & $\mathrm{H}$ & 0 & 9.031877 & -0.791628 & -2.187275 \\
\hline 89 & $\mathrm{H}$ & 0 & 9.448344 & 0.666438 & -3.113176 \\
\hline 90 & $\mathrm{H}$ & 0 & 9.440846 & -0.839303 & 0.345428 \\
\hline 91 & $\mathrm{H}$ & 0 & 11.152099 & -0.438650 & 0.126159 \\
\hline 92 & $\mathrm{H}$ & 0 & 10.135528 & 0.586549 & 1.146732 \\
\hline 93 & $\mathrm{H}$ & 0 & 10.863291 & 2.592617 & -0.220671 \\
\hline 94 & $\mathrm{H}$ & 0 & 10.581564 & 2.624798 & -1.972073 \\
\hline 95 & $\mathrm{H}$ & 0 & 11.837807 & 1.574782 & -1.294894 \\
\hline
\end{tabular}

Standard basis: $6-311++G(d, p) \quad(5 D, 7 F)$

$\begin{array}{llr}\mathrm{SCF} \text { Done: } & \mathrm{E}(\mathrm{RB} 3 \mathrm{LYP})=-2351.24730824 & \mathrm{~A} . \mathrm{U} \text {. after } 2 \text { cycles } \\ & \text { NFock }=2 \text { Conv=0.16D-08 } & -\mathrm{V} / \mathrm{T}=2.0042\end{array}$

Full mass-weighted force constant matrix:

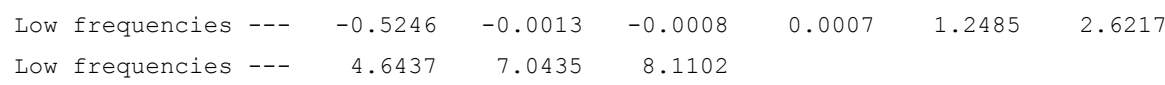


Thermal correction to Enthalpy=

Thermal correction to Gibbs Free Energy= Sum of electronic and zero-point Energies= Sum of electronic and thermal Energies= Sum of electronic and thermal Enthalpies= Sum of electronic and thermal Free Energies=
0.823277

0.677444

$-2350.475114$

$-2350.424976$

$-2350.424031$

$-2350.569864$ 
Amide 2 B

Stoichiometry C39H44N606

Framework group C1[X(C39H44N6O6)]

Standard orientation:

\begin{tabular}{|c|c|c|c|c|c|}
\hline \multirow{2}{*}{$\begin{array}{l}\text { Center } \\
\text { Number }\end{array}$} & \multirow[t]{2}{*}{ Atom } & \multirow{2}{*}{$\begin{array}{l}\text { Atomic } \\
\text { Type }\end{array}$} & \multicolumn{3}{|c|}{ Coordinates (Angstroms) } \\
\hline & & & $\mathrm{X}$ & $\mathrm{Y}$ & Z \\
\hline 1 & $\mathrm{C}$ & 0 & -5.937452 & 3.589687 & 0.412677 \\
\hline 2 & $\mathrm{C}$ & 0 & -4.832937 & 2.842697 & -0.283400 \\
\hline 3 & $\mathrm{C}$ & 0 & -2.660549 & 1.539901 & -1.521338 \\
\hline 4 & $\mathrm{C}$ & 0 & -5.016774 & 2.131184 & -1.474321 \\
\hline 5 & $\mathrm{C}$ & 0 & -3.546911 & 2.938997 & 0.253481 \\
\hline 6 & C & 0 & -2.478435 & 2.282117 & -0.350770 \\
\hline 7 & $\mathrm{C}$ & 0 & -3.940727 & 1.499667 & -2.086805 \\
\hline 8 & 0 & 0 & -5.723488 & 4.695159 & 0.895921 \\
\hline 9 & $\mathrm{~N}$ & 0 & -7.196990 & 3.023681 & 0.459208 \\
\hline 10 & $\mathrm{C}$ & 0 & -8.257629 & 3.849527 & 1.052434 \\
\hline 11 & C & 0 & -7.450102 & 1.616592 & 0.338367 \\
\hline 12 & $\mathrm{C}$ & 0 & -7.977321 & -1.119745 & 0.097099 \\
\hline 13 & C & 0 & -8.466778 & 1.168152 & -0.511096 \\
\hline 14 & $\mathrm{C}$ & 0 & -6.715125 & 0.687251 & 1.078680 \\
\hline 15 & C & 0 & -6.964159 & -0.678103 & 0.946575 \\
\hline 16 & C & 0 & -8.732270 & -0.193966 & -0.623758 \\
\hline 17 & $\mathrm{C}$ & 0 & -1.519608 & 0.756945 & -2.145672 \\
\hline 18 & $\mathrm{~N}$ & 0 & -1.591831 & -0.707479 & -1.959784 \\
\hline 19 & C & 0 & -1.562378 & -1.181849 & -0.571986 \\
\hline 20 & C & 0 & -2.295557 & -2.540278 & -0.486260 \\
\hline 21 & $\mathrm{C}$ & 0 & -2.323392 & -3.218614 & 0.896077 \\
\hline 22 & C & 0 & -3.086102 & -2.412464 & 1.946384 \\
\hline 23 & $\mathrm{O}$ & 0 & -4.304175 & -2.492750 & 2.062476 \\
\hline 24 & $\mathrm{~N}$ & 0 & -2.315521 & -1.617955 & 2.734466 \\
\hline 25 & C & 0 & -0.114782 & -1.301482 & -0.058373 \\
\hline 26 & $\mathrm{~N}$ & 0 & 0.817777 & -1.462250 & -1.019023 \\
\hline 27 & C & 0 & 2.238925 & -1.534702 & -0.744964 \\
\hline 28 & C & 0 & 2.914279 & -0.278246 & -1.309935 \\
\hline 29 & O & 0 & 2.438615 & 0.298985 & -2.271116 \\
\hline 30 & C & 0 & 2.885749 & -2.784117 & -1.399417 \\
\hline 31 & $\mathrm{C}$ & 0 & 2.289199 & -4.092544 & -0.929093 \\
\hline 32 & C & 0 & 1.193219 & -6.532426 & -0.059786 \\
\hline 33 & C & 0 & 1.590253 & -4.919414 & -1.813195 \\
\hline 34 & C & 0 & 2.430629 & -4.508268 & 0.400394 \\
\hline 35 & C & 0 & 1.887687 & -5.715367 & 0.832678 \\
\hline 36 & C & 0 & 1.046901 & -6.131011 & -1.385356 \\
\hline 37 & $\mathrm{~N}$ & 0 & 4.099192 & 0.216388 & -0.771605 \\
\hline 38 & C & 0 & 4.866908 & -0.132405 & 0.329789 \\
\hline 39 & C & 0 & 6.367042 & -0.858071 & 2.526375 \\
\hline 40 & C & 0 & 5.947261 & 0.710309 & 0.734149 \\
\hline 41 & $\mathrm{~N}$ & 0 & 4.576251 & -1.263121 & 0.979266 \\
\hline 42 & C & 0 & 5.299992 & -1.606615 & 2.043845 \\
\hline 43 & C & 0 & 6.685298 & 0.311733 & 1.850795 \\
\hline 44 & C & 0 & 6.294955 & 1.967161 & 0.010233 \\
\hline 45 & O & 0 & 5.723733 & 2.351701 & -0.996898 \\
\hline 46 & O & 0 & 7.306152 & 2.618157 & 0.592746 \\
\hline 47 & $\mathrm{C}$ & 0 & 7.844361 & 3.902319 & 0.060772 \\
\hline 48 & C & 0 & 8.412200 & 3.684084 & -1.342591 \\
\hline 49 & $\mathrm{C}$ & 0 & 8.958081 & 4.222782 & 1.056740 \\
\hline 50 & $\mathrm{C}$ & 0 & 6.753856 & 4.974167 & 0.096705 \\
\hline 51 & O & 0 & 0.135988 & -1.280816 & 1.149354 \\
\hline 52 & $\mathrm{H}$ & 0 & -5.998699 & 2.073494 & -1.926638 \\
\hline
\end{tabular}




\begin{tabular}{|c|c|c|c|c|c|}
\hline 53 & $\mathrm{H}$ & 0 & -3.396641 & 3.529577 & 1.149240 \\
\hline 54 & $\mathrm{H}$ & 0 & -1.489553 & 2.349520 & 0.091774 \\
\hline 55 & $\mathrm{H}$ & 0 & -4.101813 & 0.963005 & -3.017468 \\
\hline 56 & $\mathrm{H}$ & 0 & -9.225911 & 3.451540 & 0.753823 \\
\hline 57 & $\mathrm{H}$ & 0 & -8.153509 & 4.876364 & 0.706948 \\
\hline 58 & $\mathrm{H}$ & 0 & -8.193641 & 3.847106 & 2.145842 \\
\hline 59 & $\mathrm{H}$ & 0 & -8.177551 & -2.180581 & -0.001430 \\
\hline 60 & $\mathrm{H}$ & 0 & -9.036247 & 1.886232 & -1.090809 \\
\hline 61 & $\mathrm{H}$ & 0 & -5.938052 & 1.031856 & 1.750321 \\
\hline 62 & $\mathrm{H}$ & 0 & -6.359545 & -1.386389 & 1.502088 \\
\hline 63 & $\mathrm{H}$ & 0 & -9.521541 & -0.532052 & -1.286280 \\
\hline 64 & $\mathrm{H}$ & 0 & -1.487051 & 0.936563 & -3.223915 \\
\hline 65 & $\mathrm{H}$ & 0 & -0.563355 & 1.103872 & -1.745686 \\
\hline 66 & $\mathrm{H}$ & 0 & -2.412942 & -1.072426 & -2.429487 \\
\hline 67 & $\mathrm{H}$ & 0 & -2.056692 & -0.480233 & 0.110622 \\
\hline 68 & $\mathrm{H}$ & 0 & -1.834040 & -3.228392 & -1.202007 \\
\hline 69 & $\mathrm{H}$ & 0 & -3.327713 & -2.374152 & -0.813706 \\
\hline 70 & $\mathrm{H}$ & 0 & -2.849436 & -4.169810 & 0.796015 \\
\hline 71 & $\mathrm{H}$ & 0 & -1.307946 & -3.427803 & 1.236450 \\
\hline 72 & $\mathrm{H}$ & 0 & -1.355209 & -1.420718 & 2.461447 \\
\hline 73 & $\mathrm{H}$ & 0 & -2.787455 & -0.999202 & 3.376709 \\
\hline 74 & $\mathrm{H}$ & 0 & 0.489880 & -1.327869 & -1.969906 \\
\hline 75 & $\mathrm{H}$ & 0 & 2.364858 & -1.581566 & 0.332643 \\
\hline 76 & $\mathrm{H}$ & 0 & 2.783526 & -2.701026 & -2.485818 \\
\hline 77 & $\mathrm{H}$ & 0 & 3.954788 & -2.761116 & -1.171685 \\
\hline 78 & $\mathrm{H}$ & 0 & 0.771345 & -7.472951 & 0.276267 \\
\hline 79 & $\mathrm{H}$ & 0 & 1.472916 & -4.615034 & -2.848451 \\
\hline 80 & $\mathrm{H}$ & 0 & 2.965974 & -3.876851 & 1.101188 \\
\hline 81 & $\mathrm{H}$ & 0 & 2.006238 & -6.019932 & 1.866799 \\
\hline 82 & $\mathrm{H}$ & 0 & 0.510613 & -6.759225 & -2.088198 \\
\hline 83 & $\mathrm{H}$ & 0 & 4.419431 & 1.062967 & -1.244831 \\
\hline 84 & $\mathrm{H}$ & 0 & 6.926664 & -1.182202 & 3.394065 \\
\hline 85 & $\mathrm{H}$ & 0 & 5.010874 & -2.533908 & 2.530748 \\
\hline 86 & $\mathrm{H}$ & 0 & 7.507237 & 0.934005 & 2.177145 \\
\hline 87 & $\mathrm{H}$ & 0 & 8.930685 & 4.591892 & -1.662287 \\
\hline 88 & $\mathrm{H}$ & 0 & 7.627472 & 3.461602 & -2.063570 \\
\hline 89 & $\mathrm{H}$ & 0 & 9.136806 & 2.865837 & -1.339384 \\
\hline 90 & $\mathrm{H}$ & 0 & 9.711785 & 3.432225 & 1.062932 \\
\hline 91 & $\mathrm{H}$ & 0 & 8.555695 & 4.329135 & 2.066542 \\
\hline 92 & $\mathrm{H}$ & 0 & 9.444594 & 5.160561 & 0.778926 \\
\hline 93 & $\mathrm{H}$ & 0 & 6.328006 & 5.053508 & 1.100085 \\
\hline 94 & $\mathrm{H}$ & 0 & 5.955966 & 4.759909 & -0.612227 \\
\hline 95 & $\mathrm{H}$ & 0 & 7.195600 & 5.940873 & -0.159174 \\
\hline
\end{tabular}

Standard basis: $6-311++G(d, p) \quad(5 D, 7 F)$

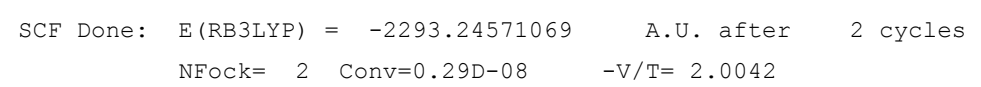

Full mass-weighted force constant matrix:

$\begin{array}{lrrrrrr}\text { Low frequencies --- } & -1.0194 & -0.6559 & -0.0013 & -0.0010 & 0.0002 & 1.8308 \\ \text { Low frequencies --- } & 4.5480 & 6.7881 & 11.3031 & & \end{array}$


Thermal correction to Enthalpy=

Thermal correction to Gibbs Free Energy= Sum of electronic and zero-point Energies=

Sum of electronic and thermal Energies=

Sum of electronic and thermal Enthalpies=

Sum of electronic and thermal Free Energies=
0.830634

0.687150

$-2292.465090$

$-2292.416021$

$-2292.415077$

$-2292.558561$ 


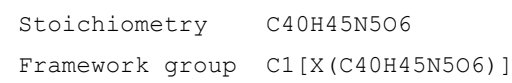

Standard orientation:

\begin{tabular}{|c|c|c|c|c|c|}
\hline \multirow{2}{*}{$\begin{array}{l}\text { Center } \\
\text { Number }\end{array}$} & \multirow[t]{2}{*}{ Atom } & \multirow{2}{*}{$\begin{array}{l}\text { Atomic } \\
\text { Type }\end{array}$} & \multicolumn{3}{|c|}{ Coordinates (Angstroms) } \\
\hline & & & $\mathrm{X}$ & $\mathrm{Y}$ & $\mathrm{z}$ \\
\hline 1 & $\mathrm{C}$ & 0 & -6.730310 & -1.106784 & -0.901221 \\
\hline 2 & $\mathrm{C}$ & 0 & -5.621466 & -1.064242 & 0.111220 \\
\hline 3 & $\mathrm{C}$ & 0 & -3.455636 & -1.077300 & 1.924087 \\
\hline 4 & $\mathrm{C}$ & 0 & -4.424073 & -1.707343 & -0.212426 \\
\hline 5 & $\mathrm{C}$ & 0 & -5.747556 & -0.476858 & 1.374970 \\
\hline 6 & C & 0 & -4.682324 & -0.494316 & 2.267569 \\
\hline 7 & $\mathrm{C}$ & 0 & -3.352824 & -1.701111 & 0.676317 \\
\hline 8 & o & 0 & -6.910955 & -2.110980 & -1.581102 \\
\hline 9 & $\mathrm{~N}$ & 0 & -7.561128 & -0.007983 & -1.028584 \\
\hline 10 & $\mathrm{C}$ & 0 & -8.690974 & -0.155935 & -1.955700 \\
\hline 11 & $\mathrm{C}$ & 0 & -7.199496 & 1.324225 & -0.639547 \\
\hline 12 & C & 0 & -6.521720 & 3.927278 & 0.132103 \\
\hline 13 & C & 0 & -5.964035 & 1.869606 & -0.998507 \\
\hline 14 & $\mathrm{C}$ & 0 & -8.104799 & 2.100652 & 0.091387 \\
\hline 15 & C & 0 & -7.768583 & 3.398614 & 0.466739 \\
\hline 16 & C & 0 & -5.616527 & 3.158671 & -0.597870 \\
\hline 17 & C & 0 & -2.273352 & -0.993823 & 2.864946 \\
\hline 18 & C & 0 & -1.682934 & 0.428935 & 2.979165 \\
\hline 19 & $\mathrm{C}$ & 0 & -1.052346 & 0.979882 & 1.685517 \\
\hline 20 & C & 0 & -0.793677 & 2.497720 & 1.820954 \\
\hline 21 & C & 0 & -0.166983 & 3.167880 & 0.586718 \\
\hline 22 & $\mathrm{C}$ & 0 & -1.078445 & 3.127100 & -0.636619 \\
\hline 23 & O & 0 & -2.197613 & 3.624755 & -0.627689 \\
\hline 24 & $\mathrm{~N}$ & 0 & -0.568162 & 2.492543 & -1.727832 \\
\hline 25 & C & 0 & 0.262736 & 0.252676 & 1.389382 \\
\hline 26 & $\mathrm{~N}$ & 0 & 0.369771 & -0.306555 & 0.144217 \\
\hline 27 & C & 0 & 1.609880 & -0.906510 & -0.310149 \\
\hline 28 & C & 0 & 2.551196 & 0.177315 & -0.862797 \\
\hline 29 & O & 0 & 2.104780 & 1.165809 & -1.430758 \\
\hline 30 & C & 0 & 1.361681 & -1.954063 & -1.417880 \\
\hline 31 & C & 0 & 0.489120 & -3.120102 & -1.000668 \\
\hline 32 & $\mathrm{C}$ & 0 & -1.104315 & -5.325858 & -0.286328 \\
\hline 33 & $\mathrm{C}$ & 0 & -0.632630 & -3.470482 & -1.758043 \\
\hline 34 & C & 0 & 0.801662 & -3.895331 & 0.122709 \\
\hline 35 & C & 0 & 0.011947 & -4.985975 & 0.478284 \\
\hline 36 & $\mathrm{C}$ & 0 & -1.423719 & -4.565120 & -1.407918 \\
\hline 37 & $\mathrm{~N}$ & 0 & 3.926102 & 0.069289 & -0.754314 \\
\hline 38 & C & 0 & 4.762804 & -0.876923 & -0.173862 \\
\hline 39 & C & 0 & 6.389923 & -2.782102 & 0.969407 \\
\hline 40 & C & 0 & 6.144112 & -0.574490 & 0.015037 \\
\hline 41 & $\mathrm{~N}$ & 0 & 4.245319 & -2.057719 & 0.171240 \\
\hline 42 & C & 0 & 5.034510 & -2.974736 & 0.730310 \\
\hline 43 & C & 0 & 6.939568 & -1.562684 & 0.599117 \\
\hline 44 & C & 0 & 6.739712 & 0.731568 & -0.392978 \\
\hline 45 & o & 0 & 6.125539 & 1.600342 & -0.990195 \\
\hline 46 & 0 & 0 & 8.021651 & 0.831110 & -0.033453 \\
\hline 47 & C & 0 & 8.846405 & 2.038095 & -0.328708 \\
\hline 48 & C & 0 & 8.954807 & 2.233496 & -1.841728 \\
\hline 49 & $\mathrm{C}$ & 0 & 10.199307 & 1.658891 & 0.271793 \\
\hline 50 & $\mathrm{C}$ & 0 & 8.253223 & 3.254480 & 0.383623 \\
\hline 51 & o & 0 & 1.167583 & 0.179858 & 2.210922 \\
\hline
\end{tabular}




\begin{tabular}{|c|c|c|c|c|c|}
\hline 52 & $\mathrm{H}$ & 0 & -4.342059 & -2.207483 & -1.170044 \\
\hline 53 & $\mathrm{H}$ & 0 & -6.677328 & -0.003572 & 1.664434 \\
\hline 54 & $\mathrm{H}$ & 0 & -4.803154 & -0.035366 & 3.243985 \\
\hline 55 & $\mathrm{H}$ & 0 & -2.426536 & -2.192061 & 0.397480 \\
\hline 56 & $\mathrm{H}$ & 0 & -9.140666 & -1.138673 & -1.826368 \\
\hline 57 & $\mathrm{H}$ & 0 & -9.425200 & 0.620402 & -1.747890 \\
\hline 58 & $\mathrm{H}$ & 0 & -8.361494 & -0.064590 & -2.996381 \\
\hline 59 & $\mathrm{H}$ & 0 & -6.255030 & 4.931747 & 0.440300 \\
\hline 60 & $\mathrm{H}$ & 0 & -5.265379 & 1.276477 & -1.576027 \\
\hline 61 & $\mathrm{H}$ & 0 & -9.062094 & 1.679758 & 0.378323 \\
\hline 62 & $\mathrm{H}$ & 0 & -8.476609 & 3.990887 & 1.036062 \\
\hline 63 & $\mathrm{H}$ & 0 & -4.636260 & 3.549256 & -0.846980 \\
\hline 64 & $\mathrm{H}$ & 0 & -2.582731 & -1.312226 & 3.866420 \\
\hline 65 & $\mathrm{H}$ & 0 & -1.496322 & -1.694070 & 2.544064 \\
\hline 66 & $\mathrm{H}$ & 0 & -2.474870 & 1.116741 & 3.292443 \\
\hline 67 & $\mathrm{H}$ & 0 & -0.919094 & 0.439201 & 3.762007 \\
\hline 68 & $\mathrm{H}$ & 0 & -1.750037 & 0.820691 & 0.856262 \\
\hline 69 & $\mathrm{H}$ & 0 & -0.136033 & 2.662937 & 2.679488 \\
\hline 70 & $\mathrm{H}$ & 0 & -1.747671 & 2.986097 & 2.036145 \\
\hline 71 & $\mathrm{H}$ & 0 & 0.809303 & 2.733949 & 0.359014 \\
\hline 72 & $\mathrm{H}$ & 0 & -0.005490 & 4.226743 & 0.810998 \\
\hline 73 & $\mathrm{H}$ & 0 & -1.103592 & 2.523073 & -2.582495 \\
\hline 74 & $\mathrm{H}$ & 0 & 0.374912 & 2.117858 & -1.740063 \\
\hline 75 & $\mathrm{H}$ & 0 & -0.339393 & -0.084262 & -0.538749 \\
\hline 76 & $\mathrm{H}$ & 0 & 2.080512 & -1.379206 & 0.546067 \\
\hline 77 & $\mathrm{H}$ & 0 & 2.339294 & -2.327581 & -1.736431 \\
\hline 78 & $\mathrm{H}$ & 0 & 0.916080 & -1.455045 & -2.284809 \\
\hline 79 & $\mathrm{H}$ & 0 & -1.720647 & -6.172776 & -0.007287 \\
\hline 80 & $\mathrm{H}$ & 0 & -0.890366 & -2.883693 & -2.634353 \\
\hline 81 & $\mathrm{H}$ & 0 & 1.667841 & -3.642469 & 0.724252 \\
\hline 82 & $\mathrm{H}$ & 0 & 0.267242 & -5.571835 & 1.354564 \\
\hline 83 & $\mathrm{H}$ & 0 & -2.290925 & -4.817362 & -2.007920 \\
\hline 84 & $\mathrm{H}$ & 0 & 4.417453 & 0.898385 & -1.093690 \\
\hline 85 & $\mathrm{H}$ & 0 & 6.990392 & -3.560065 & 1.422598 \\
\hline 86 & $\mathrm{H}$ & 0 & 4.554484 & -3.913946 & 0.990399 \\
\hline 87 & $\mathrm{H}$ & 0 & 7.989471 & -1.357137 & 0.756457 \\
\hline 88 & $\mathrm{H}$ & 0 & 9.675216 & 3.029681 & -2.047175 \\
\hline 89 & $\mathrm{H}$ & 0 & 9.316672 & 1.320309 & -2.321026 \\
\hline 90 & $\mathrm{H}$ & 0 & 7.998093 & 2.510889 & -2.280896 \\
\hline 91 & $\mathrm{H}$ & 0 & 10.911008 & 2.474425 & 0.124613 \\
\hline 92 & $\mathrm{H}$ & 0 & 10.107916 & 1.470170 & 1.343643 \\
\hline 93 & $\mathrm{H}$ & 0 & 10.598843 & 0.762438 & -0.207630 \\
\hline 94 & $\mathrm{H}$ & 0 & 8.939895 & 4.098615 & 0.278661 \\
\hline 95 & $\mathrm{H}$ & 0 & 8.129701 & 3.051074 & 1.450222 \\
\hline 96 & $\mathrm{H}$ & 0 & 7.290270 & 3.537468 & -0.038030 \\
\hline
\end{tabular}

Standard basis: $6-311++G(d, p) \quad(5 D, 7 F)$

SCF Done: $\mathrm{E}(\mathrm{RB} 3 \mathrm{LYP})=-2277.20942629 \quad \mathrm{~A} . \mathrm{U} \cdot$ after 2 cycles $\mathrm{NFOCk}=2$ Conv $=0.35 \mathrm{D}-08 \quad-\mathrm{V} / \mathrm{T}=2.0043$

Full mass-weighted force constant matrix:

$\begin{array}{lrrrrrr}\text { Low frequencies --- } & -2.5557 & -0.9252 & -0.4490 & -0.0011 & -0.0007 & 0.0010 \\ \text { Low frequencies - -- } & 5.2065 & 8.9061 & 13.4394 & & \end{array}$

Zero-point correction=

Thermal correction to Energy=
0.791602 (Hartree/Particle)

0.840901 
Thermal correction to Enthalpy=

Thermal correction to Gibbs Free Energy= Sum of electronic and zero-point Energies= Sum of electronic and thermal Energies= Sum of electronic and thermal Enthalpies= Sum of electronic and thermal Free Energies=
0.841845

0.699719

$-2276.417825$

$-2276.368525$

$-2276.367581$

$-2276.509707$ 
Stoichiometry C40H43N5O7

Framework group C1[X(C40H43N507)]

Standard orientation:

\begin{tabular}{|c|c|c|c|c|c|}
\hline \multirow{2}{*}{$\begin{array}{l}\text { Center } \\
\text { Number }\end{array}$} & \multirow[t]{2}{*}{ Atom } & \multirow{2}{*}{$\begin{array}{l}\text { Atomic } \\
\text { Type }\end{array}$} & \multicolumn{3}{|c|}{ Coordinates (Angstroms) } \\
\hline & & & $\mathrm{X}$ & Y & z \\
\hline 1 & C & 0 & 8.281751 & 1.588753 & -1.285774 \\
\hline 2 & $\mathrm{C}$ & 0 & 6.895030 & 1.413809 & -0.725379 \\
\hline 3 & $\mathrm{C}$ & 0 & 4.271169 & 1.307577 & 0.269938 \\
\hline 4 & C & 0 & 6.644787 & 0.929013 & 0.562792 \\
\hline 5 & $\mathrm{C}$ & 0 & 5.824273 & 1.874234 & -1.498092 \\
\hline 6 & $\mathrm{C}$ & 0 & 4.523566 & 1.806790 & -1.014060 \\
\hline 7 & $\mathrm{C}$ & 0 & 5.347845 & 0.892086 & 1.059639 \\
\hline 8 & 0 & 0 & 8.554769 & 2.590575 & -1.933749 \\
\hline 9 & $\mathrm{~N}$ & 0 & 9.241412 & 0.635618 & -1.003749 \\
\hline 10 & $\mathrm{C}$ & 0 & 10.607768 & 0.932710 & -1.457738 \\
\hline 11 & C & 0 & 8.941408 & -0.730878 & -0.692841 \\
\hline 12 & C & 0 & 8.416693 & -3.413739 & -0.094874 \\
\hline 13 & $\mathrm{C}$ & 0 & 9.556122 & -1.342175 & 0.404633 \\
\hline 14 & C & 0 & 8.075468 & -1.475333 & -1.497688 \\
\hline 15 & C & 0 & 7.806456 & -2.807719 & -1.190719 \\
\hline 16 & C & 0 & 9.297168 & -2.677775 & 0.698247 \\
\hline 17 & C & 0 & 2.894557 & 1.221318 & 0.865700 \\
\hline 18 & $\mathrm{~N}$ & 0 & 1.859123 & 1.241313 & -0.011964 \\
\hline 19 & C & 0 & 0.469205 & 0.987506 & 0.353165 \\
\hline 20 & C & 0 & -0.341126 & 2.285908 & 0.572659 \\
\hline 21 & C & 0 & 0.030926 & 3.056982 & 1.853419 \\
\hline 22 & C & 0 & -0.459500 & 2.342965 & 3.113214 \\
\hline 23 & 0 & 0 & -1.644727 & 2.355615 & 3.431789 \\
\hline 24 & $\mathrm{~N}$ & 0 & 0.493106 & 1.706027 & 3.837787 \\
\hline 25 & $\mathrm{C}$ & 0 & -0.122020 & 0.119393 & -0.782764 \\
\hline 26 & $\mathrm{~N}$ & 0 & -1.372167 & -0.348583 & -0.578528 \\
\hline 27 & C & 0 & -2.004894 & -1.264734 & -1.518907 \\
\hline 28 & C & 0 & -3.512089 & -0.992030 & -1.662743 \\
\hline 29 & 0 & 0 & -4.277968 & -1.885081 & -1.943139 \\
\hline 30 & C & 0 & -1.674097 & -2.753213 & -1.237578 \\
\hline 31 & C & 0 & -1.958152 & -3.222406 & 0.173504 \\
\hline 32 & C & 0 & -2.459978 & -4.083883 & 2.806172 \\
\hline 33 & $\mathrm{C}$ & 0 & -3.220533 & -3.705785 & 0.541592 \\
\hline 34 & C & 0 & -0.951889 & -3.188088 & 1.146647 \\
\hline 35 & $\mathrm{C}$ & 0 & -1.197520 & -3.612178 & 2.451614 \\
\hline 36 & $\mathrm{C}$ & 0 & -3.468864 & -4.131644 & 1.845353 \\
\hline 37 & C & 0 & -4.017630 & 0.448557 & -1.522359 \\
\hline 38 & C & 0 & -4.639400 & 0.662372 & -0.154798 \\
\hline 39 & $\mathrm{C}$ & 0 & -5.532568 & 0.988687 & 2.430255 \\
\hline 40 & C & 0 & -6.021687 & 0.814708 & 0.071944 \\
\hline 41 & $\mathrm{~N}$ & 0 & -3.759895 & 0.655220 & 0.861383 \\
\hline 42 & $\mathrm{C}$ & 0 & -4.186302 & 0.836429 & 2.114834 \\
\hline 43 & C & 0 & -6.454701 & 0.960658 & 1.396340 \\
\hline 44 & C & 0 & -7.031037 & 0.873052 & -1.039993 \\
\hline 45 & 0 & 0 & -6.795343 & 1.261223 & -2.163118 \\
\hline 46 & 0 & 0 & -8.233974 & 0.464315 & -0.607862 \\
\hline 47 & C & 0 & -9.430497 & 0.446543 & -1.490263 \\
\hline 48 & C & 0 & -9.755703 & 1.867241 & -1.956167 \\
\hline 49 & $\mathrm{C}$ & 0 & -10.517499 & -0.083017 & -0.555296 \\
\hline 50 & C & 0 & -9.198274 & -0.516563 & -2.656084 \\
\hline
\end{tabular}




\begin{tabular}{|c|c|c|c|c|c|}
\hline 51 & 0 & 0 & 0.536587 & -0.130973 & -1.789677 \\
\hline 52 & 0 & 0 & 2.741676 & 1.145342 & 2.087297 \\
\hline 53 & $\mathrm{H}$ & 0 & 7.463430 & 0.593744 & 1.186364 \\
\hline 54 & $\mathrm{H}$ & 0 & 6.026437 & 2.299518 & -2.473331 \\
\hline 55 & $\mathrm{H}$ & 0 & 3.715176 & 2.180117 & -1.631769 \\
\hline 56 & $\mathrm{H}$ & 0 & 5.149029 & 0.540955 & 2.064226 \\
\hline 57 & $\mathrm{H}$ & 0 & 10.719882 & 0.733413 & -2.528777 \\
\hline 58 & $\mathrm{H}$ & 0 & 11.308275 & 0.308042 & -0.905729 \\
\hline 59 & $\mathrm{H}$ & 0 & 10.825802 & 1.983885 & -1.280288 \\
\hline 60 & $\mathrm{H}$ & 0 & 8.211057 & -4.451913 & 0.138641 \\
\hline 61 & $\mathrm{H}$ & 0 & 10.225733 & -0.764279 & 1.031812 \\
\hline 62 & $\mathrm{H}$ & 0 & 7.613674 & -1.010167 & -2.360448 \\
\hline 63 & $\mathrm{H}$ & 0 & 7.126832 & -3.373809 & -1.817452 \\
\hline 64 & $\mathrm{H}$ & 0 & 9.775677 & -3.141049 & 1.553685 \\
\hline 65 & $\mathrm{H}$ & 0 & 2.047628 & 1.022128 & -0.985121 \\
\hline 66 & $\mathrm{H}$ & 0 & 0.456498 & 0.390255 & 1.270407 \\
\hline 67 & $\mathrm{H}$ & 0 & -0.185048 & 2.932233 & -0.296568 \\
\hline 68 & $\mathrm{H}$ & 0 & -1.407380 & 2.050183 & 0.613760 \\
\hline 69 & $\mathrm{H}$ & 0 & 1.105713 & 3.241133 & 1.896320 \\
\hline 70 & $\mathrm{H}$ & 0 & -0.476763 & 4.023919 & 1.833574 \\
\hline 71 & $\mathrm{H}$ & 0 & 0.187559 & 1.162819 & 4.631502 \\
\hline 72 & $\mathrm{H}$ & 0 & 1.407818 & 1.525980 & 3.432549 \\
\hline 73 & $\mathrm{H}$ & 0 & -1.925397 & -0.038973 & 0.222381 \\
\hline 74 & $\mathrm{H}$ & 0 & -1.588175 & -1.027605 & -2.507540 \\
\hline 75 & $\mathrm{H}$ & 0 & -0.612855 & -2.887750 & -1.459775 \\
\hline 76 & $\mathrm{H}$ & 0 & -2.237788 & -3.352111 & -1.955405 \\
\hline 77 & $\mathrm{H}$ & 0 & -2.653344 & -4.418981 & 3.819158 \\
\hline 78 & $\mathrm{H}$ & 0 & -4.009045 & -3.747905 & -0.200503 \\
\hline 79 & $\mathrm{H}$ & 0 & 0.039487 & -2.839022 & 0.875700 \\
\hline 80 & $\mathrm{H}$ & 0 & -0.401181 & -3.581379 & 3.187368 \\
\hline 81 & $\mathrm{H}$ & 0 & -4.451831 & -4.506818 & 2.108920 \\
\hline 82 & $\mathrm{H}$ & 0 & -3.193605 & 1.154799 & -1.638278 \\
\hline 83 & $\mathrm{H}$ & 0 & -4.748765 & 0.617664 & -2.307514 \\
\hline 84 & $\mathrm{H}$ & 0 & -5.841821 & 1.129091 & 3.458341 \\
\hline 85 & $\mathrm{H}$ & 0 & -3.418268 & 0.886202 & 2.878893 \\
\hline 86 & $\mathrm{H}$ & 0 & -7.513012 & 1.066204 & 1.594704 \\
\hline 87 & $\mathrm{H}$ & 0 & -10.711554 & 1.859665 & -2.486975 \\
\hline 88 & $\mathrm{H}$ & 0 & -9.852029 & 2.538953 & -1.099135 \\
\hline 89 & $\mathrm{H}$ & 0 & -8.989820 & 2.254380 & -2.626145 \\
\hline 90 & $\mathrm{H}$ & 0 & -10.653985 & 0.584680 & 0.298521 \\
\hline 91 & $\mathrm{H}$ & 0 & -10.255026 & -1.075127 & -0.181878 \\
\hline 92 & $\mathrm{H}$ & 0 & -11.466525 & -0.154406 & -1.091891 \\
\hline 93 & $\mathrm{H}$ & 0 & -8.429603 & -0.148189 & -3.333267 \\
\hline 94 & $\mathrm{H}$ & 0 & -8.901165 & -1.500485 & -2.285226 \\
\hline 95 & $\mathrm{H}$ & 0 & -10.130333 & -0.632103 & -3.215915 \\
\hline
\end{tabular}

Standard basis: $6-311++G(d, p) \quad(5 D, 7 F)$

SCF Done: $\mathrm{E}(\mathrm{RB} 3 \mathrm{LYP})=-2351.24077207$ A.U. after 2 cycles NFock $=2$ Conv=0.19D-0XBig12 $=4.84 \mathrm{D}-14 \quad 4.83 \mathrm{D}-09$.

Full mass-weighted force constant matrix:

$\begin{array}{lrrrrrr}\text { Low frequencies --- } & -2.5988 & -0.9715 & -0.0016 & -0.0010 & 0.0010 & 1.0734 \\ \text { Low frequencies --- } & 5.3837 & 6.5684 & 7.8196 & & \end{array}$


Zero-point correction=

Thermal correction to Energy=

Thermal correction to Enthalpy=

Thermal correction to Gibbs Free Energy=

Sum of electronic and zero-point Energies=

Sum of electronic and thermal Energies=

Sum of electronic and thermal Enthalpies=

Sum of electronic and thermal Free Energies=
0.772715 (Hartree/Particle)

0.822526

0.823470

0.678227

$-2350.468057$

$-2350.418247$

$-2350.417302$

$-2350.562545$ 
Stoichiometry C39H44N6O6

Framework group C1[X(C39H44N6O6)]

Standard orientation:

\begin{tabular}{|c|c|c|c|c|c|}
\hline \multirow{2}{*}{$\begin{array}{l}\text { Center } \\
\text { Number }\end{array}$} & \multirow[t]{2}{*}{ Atom } & Atomic & \multicolumn{3}{|c|}{ Coordinates (Angstroms) } \\
\hline & & Type & $\mathrm{x}$ & Y & $\mathrm{z}$ \\
\hline 1 & $\mathrm{C}$ & 0 & -8.352464 & 0.145950 & -0.509286 \\
\hline 2 & $\mathrm{C}$ & 0 & -6.920887 & -0.313505 & -0.429485 \\
\hline 3 & $\mathrm{C}$ & 0 & -4.283253 & -1.278114 & -0.478285 \\
\hline 4 & $\mathrm{C}$ & 0 & -6.395958 & -0.997906 & 0.671844 \\
\hline 5 & $\mathrm{C}$ & 0 & -6.124644 & -0.144499 & -1.566650 \\
\hline 6 & $\mathrm{C}$ & 0 & -4.814171 & -0.605989 & -1.586436 \\
\hline 7 & $\mathrm{C}$ & 0 & -5.096250 & -1.487940 & 0.639919 \\
\hline 8 & 0 & 0 & -8.976621 & 0.017723 & -1.554329 \\
\hline 9 & $\mathrm{~N}$ & 0 & -8.956272 & 0.652295 & 0.625939 \\
\hline 10 & C & 0 & -10.392449 & 0.948604 & 0.521709 \\
\hline 11 & C & 0 & -8.240390 & 1.268921 & 1.703355 \\
\hline 12 & C & 0 & -6.896718 & 2.495572 & 3.829685 \\
\hline 13 & C & 0 & -8.525873 & 0.903689 & 3.022907 \\
\hline 14 & C & 0 & -7.290135 & 2.262860 & 1.456308 \\
\hline 15 & C & 0 & -6.613776 & 2.863532 & 2.516269 \\
\hline 16 & C & 0 & -7.859637 & 1.517668 & 4.079431 \\
\hline 17 & $\mathrm{C}$ & 0 & -2.881910 & -1.820559 & -0.432012 \\
\hline 18 & $\mathrm{~N}$ & 0 & -2.006323 & -1.299675 & -1.325055 \\
\hline 19 & C & 0 & -0.572223 & -1.566070 & -1.334835 \\
\hline 20 & C & 0 & -0.177463 & -2.662340 & -2.352852 \\
\hline 21 & C & 0 & -0.650238 & -4.079658 & -1.981635 \\
\hline 22 & C & 0 & 0.157694 & -4.664559 & -0.825046 \\
\hline 23 & 0 & 0 & 1.304215 & -5.070836 & -0.993572 \\
\hline 24 & $\mathrm{~N}$ & 0 & -0.468503 & -4.696837 & 0.376576 \\
\hline 25 & C & 0 & 0.107982 & -0.226712 & -1.699459 \\
\hline 26 & $\mathrm{~N}$ & 0 & 1.429996 & -0.152404 & -1.462546 \\
\hline 27 & C & 0 & 2.217097 & 1.024150 & -1.841725 \\
\hline 28 & C & 0 & 3.686321 & 0.621174 & -2.065655 \\
\hline 29 & C & 0 & 2.037876 & 2.170551 & -0.817648 \\
\hline 30 & C & 0 & 2.636036 & 3.482697 & -1.275763 \\
\hline 31 & C & 0 & 3.744506 & 5.911085 & -2.167687 \\
\hline 32 & C & 0 & 2.017794 & 4.224753 & -2.291478 \\
\hline 33 & C & 0 & 3.815044 & 3.983324 & -0.712494 \\
\hline 34 & C & 0 & 4.366078 & 5.186898 & -1.152931 \\
\hline 35 & C & 0 & 2.565847 & 5.426117 & -2.734740 \\
\hline 36 & $\mathrm{~N}$ & 0 & 4.470308 & 0.338768 & -0.866904 \\
\hline 37 & C & 0 & 4.599264 & -0.886939 & -0.293839 \\
\hline 38 & C & 0 & 4.798373 & -3.436397 & 0.763333 \\
\hline 39 & C & 0 & 5.633072 & -1.167103 & 0.659890 \\
\hline 40 & $\mathrm{~N}$ & 0 & 3.706580 & -1.833353 & -0.654585 \\
\hline 41 & C & 0 & 3.816216 & -3.066498 & -0.151556 \\
\hline 42 & C & 0 & 5.700772 & -2.464720 & 1.172149 \\
\hline 43 & C & 0 & 6.602728 & -0.129575 & 1.074674 \\
\hline 44 & o & 0 & 6.592878 & 1.022434 & 0.661055 \\
\hline 45 & 0 & 0 & 7.499623 & -0.592690 & 1.959413 \\
\hline 46 & C & 0 & 8.577418 & 0.255618 & 2.524704 \\
\hline 47 & C & 0 & 9.506492 & 0.734109 & 1.406719 \\
\hline 48 & C & 0 & 9.303827 & -0.717110 & 3.454032 \\
\hline 49 & $\mathrm{C}$ & 0 & 7.969706 & 1.413576 & 3.319635 \\
\hline 50 & o & 0 & -0.550155 & 0.690036 & -2.192318 \\
\hline
\end{tabular}




\begin{tabular}{|c|c|c|c|c|c|}
\hline 51 & 0 & 0 & -2.574453 & -2.696286 & 0.381788 \\
\hline 52 & $\mathrm{H}$ & 0 & -7.005577 & -1.163088 & 1.550792 \\
\hline 53 & $\mathrm{H}$ & 0 & -6.550508 & 0.337810 & -2.437772 \\
\hline 54 & $\mathrm{H}$ & 0 & -4.225722 & -0.466889 & -2.485862 \\
\hline 55 & $\mathrm{H}$ & 0 & -4.690657 & -2.039084 & 1.478785 \\
\hline 56 & $\mathrm{H}$ & 0 & -10.563224 & 1.894434 & -0.003278 \\
\hline 57 & $\mathrm{H}$ & 0 & -10.885020 & 0.154185 & -0.035406 \\
\hline 58 & $\mathrm{H}$ & 0 & -10.813482 & 1.017958 & 1.523442 \\
\hline 59 & $\mathrm{H}$ & 0 & -6.373937 & 2.968203 & 4.653066 \\
\hline 60 & $\mathrm{H}$ & 0 & -9.260557 & 0.129866 & 3.215567 \\
\hline 61 & $\mathrm{H}$ & 0 & -7.081359 & 2.562261 & 0.436153 \\
\hline 62 & $\mathrm{H}$ & 0 & -5.872601 & 3.627958 & 2.312643 \\
\hline 63 & $\mathrm{H}$ & 0 & -8.085372 & 1.224159 & 5.098391 \\
\hline 64 & $\mathrm{H}$ & 0 & -2.240009 & -0.438831 & -1.809789 \\
\hline 65 & $\mathrm{H}$ & 0 & -0.266417 & -1.864480 & -0.328205 \\
\hline 66 & $\mathrm{H}$ & 0 & 0.910038 & -2.676336 & -2.465087 \\
\hline 67 & $\mathrm{H}$ & 0 & -0.596599 & -2.384062 & -3.324541 \\
\hline 68 & $\mathrm{H}$ & 0 & -0.478077 & -4.737015 & -2.836826 \\
\hline 69 & $\mathrm{H}$ & 0 & -1.719307 & -4.084888 & -1.762210 \\
\hline 70 & $\mathrm{H}$ & 0 & 0.069365 & -5.005695 & 1.172699 \\
\hline 71 & $\mathrm{H}$ & 0 & -1.310333 & -4.146667 & 0.528624 \\
\hline 72 & $\mathrm{H}$ & 0 & 1.960726 & -0.959994 & -1.128602 \\
\hline 73 & $\mathrm{H}$ & 0 & 1.833011 & 1.369111 & -2.806907 \\
\hline 74 & $\mathrm{H}$ & 0 & 4.181461 & 1.451704 & -2.571276 \\
\hline 75 & $\mathrm{H}$ & 0 & 3.717526 & -0.243291 & -2.734164 \\
\hline 76 & $\mathrm{H}$ & 0 & 2.480267 & 1.863757 & 0.133697 \\
\hline 77 & $\mathrm{H}$ & 0 & 0.964266 & 2.294065 & -0.657885 \\
\hline 78 & $\mathrm{H}$ & 0 & 4.170236 & 6.847504 & -2.510401 \\
\hline 79 & $\mathrm{H}$ & 0 & 1.093429 & 3.860826 & -2.729298 \\
\hline 80 & $\mathrm{H}$ & 0 & 4.304096 & 3.431418 & 0.083989 \\
\hline 81 & $\mathrm{H}$ & 0 & 5.279110 & 5.557991 & -0.700313 \\
\hline 82 & $\mathrm{H}$ & 0 & 2.069585 & 5.987534 & -3.518861 \\
\hline 83 & $\mathrm{H}$ & 0 & 5.173805 & 1.009766 & -0.580678 \\
\hline 84 & $\mathrm{H}$ & 0 & 4.840638 & -4.448521 & 1.143270 \\
\hline 85 & $\mathrm{H}$ & 0 & 3.075319 & -3.786793 & -0.488896 \\
\hline 86 & $\mathrm{H}$ & 0 & 6.476459 & -2.696546 & 1.889789 \\
\hline 87 & $\mathrm{H}$ & 0 & 9.869512 & -0.115584 & 0.822912 \\
\hline 88 & $\mathrm{H}$ & 0 & 10.372323 & 1.234415 & 1.848937 \\
\hline 89 & $\mathrm{H}$ & 0 & 9.003700 & 1.433022 & 0.740481 \\
\hline 90 & $\mathrm{H}$ & 0 & 9.697145 & -1.567136 & 2.892110 \\
\hline 91 & $\mathrm{H}$ & 0 & 10.138613 & -0.210653 & 3.944236 \\
\hline 92 & $\mathrm{H}$ & 0 & 8.627191 & -1.093283 & 4.224558 \\
\hline 93 & $\mathrm{H}$ & 0 & 7.263395 & 1.036206 & 4.063517 \\
\hline 94 & $\mathrm{H}$ & 0 & 7.456201 & 2.119344 & 2.669031 \\
\hline 95 & $\mathrm{H}$ & 0 & 8.766743 & 1.942800 & 3.848938 \\
\hline
\end{tabular}

Standard basis: $6-311++G(d, p) \quad(5 D, 7 F)$

SCF Done: E(RB3LYP) $=-2293.27267065$ A.U. after 2 cycles $\mathrm{NFOCk}=2$ Conv $=0.15 \mathrm{D}-08 \quad-\mathrm{V} / \mathrm{T}=2.0042$

Full mass-weighted force constant matrix:

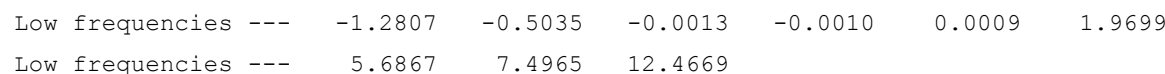

Diagonal vibrational polarizability:
230.2711108
284.5712477
229.2931176 
Zero-point correction=

Thermal correction to Energy=

Thermal correction to Enthalpy=

Thermal correction to Gibbs Free Energy=

Sum of electronic and zero-point Energies=

Sum of electronic and thermal Energies=

Sum of electronic and thermal Enthalpies=

Sum of electronic and thermal Free Energies=
0.781331 (Hartree/Particle)

0.830161

0.831105

0.689730

$-2292.491340$

$-2292.442510$

$-2292.441566$

$-2292.582941$ 
Stoichiometry C40H45N5O6

Framework group C1[X(C40H45N5O6)]

Standard orientation:

\begin{tabular}{|c|c|c|c|c|c|}
\hline \multirow{2}{*}{$\begin{array}{l}\text { Center } \\
\text { Number }\end{array}$} & \multirow[t]{2}{*}{ Atom } & Atomic & \multicolumn{3}{|c|}{ Coordinates (Angstroms) } \\
\hline & & Type & $\mathrm{x}$ & Y & Z \\
\hline 1 & C & 0 & -7.219930 & 0.162420 & 1.741341 \\
\hline 2 & C & 0 & -5.794279 & 0.379382 & 1.308830 \\
\hline 3 & $\mathrm{C}$ & 0 & -3.073653 & 0.779923 & 0.735171 \\
\hline 4 & C & 0 & -4.826003 & -0.492400 & 1.816727 \\
\hline 5 & C & 0 & -5.388070 & 1.460679 & 0.519529 \\
\hline 6 & C & 0 & -4.042001 & 1.657589 & 0.237581 \\
\hline 7 & C & 0 & -3.480684 & -0.303954 & 1.523172 \\
\hline 8 & 0 & 0 & -7.460918 & -0.207264 & 2.882979 \\
\hline 9 & $\mathrm{~N}$ & 0 & -8.240207 & 0.446491 & 0.852931 \\
\hline 10 & C & 0 & -9.608271 & 0.354130 & 1.383056 \\
\hline 11 & C & 0 & -8.090856 & 0.413280 & -0.571735 \\
\hline 12 & C & 0 & -7.861823 & 0.350096 & -3.360250 \\
\hline 13 & C & 0 & -7.496853 & -0.681279 & -1.205001 \\
\hline 14 & C & 0 & -8.584613 & 1.469406 & -1.344427 \\
\hline 15 & C & 0 & -8.473575 & 1.434537 & -2.731319 \\
\hline 16 & C & 0 & -7.372986 & -0.704573 & -2.592715 \\
\hline 17 & C & 0 & -1.639042 & 1.045840 & 0.371769 \\
\hline 18 & $\mathrm{~N}$ & 0 & -0.703536 & 0.524046 & 1.196283 \\
\hline 19 & C & 0 & 0.725771 & 0.455132 & 0.915355 \\
\hline 20 & C & 0 & 1.524775 & 1.577744 & 1.616870 \\
\hline 21 & C & 0 & 1.265526 & 2.995199 & 1.072427 \\
\hline 22 & C & 0 & 1.900341 & 3.221580 & -0.298584 \\
\hline 23 & 0 & 0 & 3.098093 & 3.471292 & -0.417290 \\
\hline 24 & $\mathrm{~N}$ & 0 & 1.057657 & 3.125295 & -1.354885 \\
\hline 25 & C & 0 & 1.194254 & -0.924286 & 1.435590 \\
\hline 26 & $\mathrm{~N}$ & 0 & 2.337846 & -1.391889 & 0.897028 \\
\hline 27 & C & 0 & 2.904889 & -2.698975 & 1.237187 \\
\hline 28 & C & 0 & 4.195187 & -2.588571 & 2.074236 \\
\hline 29 & C & 0 & 3.097549 & -3.548771 & -0.042864 \\
\hline 30 & C & 0 & 1.813878 & -3.840928 & -0.788183 \\
\hline 31 & C & 0 & -0.590385 & -4.368435 & -2.151099 \\
\hline 32 & C & 0 & 1.477646 & -3.136298 & -1.948702 \\
\hline 33 & C & 0 & 0.924588 & -4.817572 & -0.324066 \\
\hline 34 & C & 0 & -0.266284 & -5.080504 & -0.996570 \\
\hline 35 & C & 0 & 0.286215 & -3.395416 & -2.625912 \\
\hline 36 & C & 0 & 5.373776 & -1.800576 & 1.451265 \\
\hline 37 & C & 0 & 5.405740 & -0.326140 & 1.806990 \\
\hline 38 & C & 0 & 5.683836 & 2.275851 & 2.683482 \\
\hline 39 & C & 0 & 5.390954 & 0.726294 & 0.868444 \\
\hline 40 & $\mathrm{~N}$ & 0 & 5.542501 & -0.079881 & 3.118640 \\
\hline 41 & C & 0 & 5.663443 & 1.178336 & 3.540243 \\
\hline 42 & C & 0 & 5.550235 & 2.040495 & 1.323656 \\
\hline 43 & C & 0 & 5.154378 & 0.499938 & -0.591557 \\
\hline 44 & 0 & 0 & 4.309961 & -0.264931 & -1.030228 \\
\hline 45 & 0 & 0 & 5.964832 & 1.248955 & -1.333849 \\
\hline 46 & C & 0 & 5.880665 & 1.313990 & -2.820744 \\
\hline 47 & C & 0 & 6.266345 & -0.044661 & -3.406763 \\
\hline 48 & C & 0 & 6.928307 & 2.375006 & -3.154896 \\
\hline 49 & C & 0 & 4.485733 & 1.772598 & -3.248133 \\
\hline 50 & $\mathrm{O}$ & 0 & 0.543295 & -1.504997 & 2.301348 \\
\hline
\end{tabular}




\begin{tabular}{|c|c|c|c|c|c|}
\hline 51 & 0 & 0 & -1.360964 & 1.729171 & -0.619618 \\
\hline 52 & $\mathrm{H}$ & 0 & -5.139327 & -1.315224 & 2.447323 \\
\hline 53 & $\mathrm{H}$ & 0 & -6.120391 & 2.157009 & 0.131879 \\
\hline 54 & $\mathrm{H}$ & 0 & -3.721395 & 2.490891 & -0.374725 \\
\hline 55 & $\mathrm{H}$ & 0 & -2.761018 & -1.022732 & 1.898959 \\
\hline 56 & $\mathrm{H}$ & 0 & -9.636528 & 0.779893 & 2.384050 \\
\hline 57 & $\mathrm{H}$ & 0 & -9.939028 & -0.688204 & 1.442835 \\
\hline 58 & $\mathrm{H}$ & 0 & -10.280992 & 0.902444 & 0.725496 \\
\hline 59 & $\mathrm{H}$ & 0 & -7.769677 & 0.327489 & -4.439893 \\
\hline 60 & $\mathrm{H}$ & 0 & -7.131321 & -1.510076 & -0.610547 \\
\hline 61 & $\mathrm{H}$ & 0 & -9.042566 & 2.321063 & -0.853877 \\
\hline 62 & $\mathrm{H}$ & 0 & -8.855640 & 2.260512 & -3.320538 \\
\hline 63 & $\mathrm{H}$ & 0 & -6.902536 & -1.554970 & -3.072964 \\
\hline 64 & $\mathrm{H}$ & 0 & -0.980588 & -0.079929 & 1.961700 \\
\hline 65 & $\mathrm{H}$ & 0 & 0.867189 & 0.509845 & -0.166574 \\
\hline 66 & $\mathrm{H}$ & 0 & 1.272178 & 1.551323 & 2.681115 \\
\hline 67 & $\mathrm{H}$ & 0 & 2.592263 & 1.358418 & 1.539218 \\
\hline 68 & $\mathrm{H}$ & 0 & 0.193786 & 3.201845 & 1.046128 \\
\hline 69 & $\mathrm{H}$ & 0 & 1.731430 & 3.715640 & 1.748044 \\
\hline 70 & $\mathrm{H}$ & 0 & 0.125006 & 2.732377 & -1.237120 \\
\hline 71 & $\mathrm{H}$ & 0 & 1.453576 & 3.196682 & -2.279933 \\
\hline 72 & $\mathrm{H}$ & 0 & 2.812954 & -0.860648 & 0.171793 \\
\hline 73 & $\mathrm{H}$ & 0 & 2.154578 & -3.184549 & 1.863937 \\
\hline 74 & $\mathrm{H}$ & 0 & 4.530295 & -3.610954 & 2.277302 \\
\hline 75 & $\mathrm{H}$ & 0 & 3.956944 & -2.145729 & 3.044188 \\
\hline 76 & $\mathrm{H}$ & 0 & 3.568312 & -4.490747 & 0.257532 \\
\hline 77 & $\mathrm{H}$ & 0 & 3.798321 & -3.044313 & -0.713483 \\
\hline 78 & $\mathrm{H}$ & 0 & -1.516191 & -4.573361 & -2.676966 \\
\hline 79 & $\mathrm{H}$ & 0 & 2.158127 & -2.380470 & -2.327620 \\
\hline 80 & $\mathrm{H}$ & 0 & 1.166944 & -5.379911 & 0.572610 \\
\hline 81 & $\mathrm{H}$ & 0 & -0.939967 & -5.843076 & -0.621282 \\
\hline 82 & $\mathrm{H}$ & 0 & 0.044678 & -2.838142 & -3.524509 \\
\hline 83 & $\mathrm{H}$ & 0 & 5.414821 & -1.943371 & 0.372362 \\
\hline 84 & $\mathrm{H}$ & 0 & 6.299551 & -2.215672 & 1.861606 \\
\hline 85 & $\mathrm{H}$ & 0 & 5.791282 & 3.281026 & 3.072259 \\
\hline 86 & $\mathrm{H}$ & 0 & 5.754201 & 1.313550 & 4.614843 \\
\hline 87 & $\mathrm{H}$ & 0 & 5.521391 & 2.856852 & 0.614662 \\
\hline 88 & $\mathrm{H}$ & 0 & 7.249664 & -0.354579 & -3.044024 \\
\hline 89 & $\mathrm{H}$ & 0 & 5.534716 & -0.810125 & -3.151057 \\
\hline 90 & $\mathrm{H}$ & 0 & 6.317254 & 0.034943 & -4.496019 \\
\hline 91 & $\mathrm{H}$ & 0 & 6.659504 & 3.334616 & -2.708627 \\
\hline 92 & $\mathrm{H}$ & 0 & 7.910485 & 2.079404 & -2.779772 \\
\hline 93 & $\mathrm{H}$ & 0 & 6.994186 & 2.502419 & -4.238031 \\
\hline 94 & $\mathrm{H}$ & 0 & 3.743613 & 0.989946 & -3.096787 \\
\hline 95 & $\mathrm{H}$ & 0 & 4.187875 & 2.655407 & -2.677890 \\
\hline 96 & $\mathrm{H}$ & 0 & 4.509483 & 2.030315 & -4.310718 \\
\hline
\end{tabular}

Standard basis: $6-311++G(d, p) \quad(5 D, 7 F)$

SCF Done: $\mathrm{E}(\mathrm{RB} 3 \mathrm{LYP})=-2277.20772117 \quad$ A.U. after 2 cycles

Full mass-weighted force constant matrix:

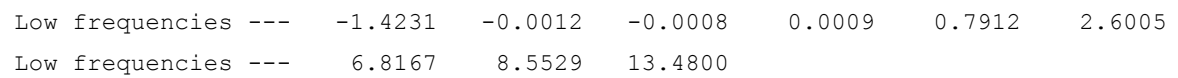


Thermal correction to Enthalpy=

Thermal correction to Gibbs Free Energy= Sum of electronic and zero-point Energies= Sum of electronic and thermal Energies= Sum of electronic and thermal Enthalpies= Sum of electronic and thermal Free Energies=
0.842195

0.700825

$-2276.415618$

$-2276.366471$

$-2276.365527$

$-2276.506896$ 
$\begin{array}{ll}\text { Stoichiometry } & \mathrm{C} 40 \mathrm{H} 43 \mathrm{~N} 5 \mathrm{O} 7 \\ \text { Framework group } & \mathrm{C} 1[\mathrm{X}(\mathrm{C} 40 \mathrm{H} 43 \mathrm{~N} 507)]\end{array}$

Standard orientation:

\begin{tabular}{|c|c|c|c|c|c|}
\hline \multirow{2}{*}{$\begin{array}{l}\text { Center } \\
\text { Number }\end{array}$} & \multirow[t]{2}{*}{ Atom } & \multirow{2}{*}{$\begin{array}{l}\text { Atomic } \\
\text { Type }\end{array}$} & \multicolumn{3}{|c|}{ Coordinates (Angstroms) } \\
\hline & & & $\mathrm{x}$ & Y & $\mathrm{Z}$ \\
\hline 1 & C & 0 & -7.538425 & 0.362590 & 0.420196 \\
\hline 2 & C & 0 & -6.112574 & 0.067407 & 0.793316 \\
\hline 3 & C & 0 & -3.460523 & -0.612064 & 1.444389 \\
\hline 4 & C & 0 & -5.598218 & -1.197326 & 0.475052 \\
\hline 5 & C & 0 & -5.278658 & 0.988823 & 1.439750 \\
\hline 6 & C & 0 & -3.967430 & 0.653569 & 1.754817 \\
\hline 7 & C & 0 & -4.292007 & -1.539178 & 0.798073 \\
\hline 8 & 0 & 0 & -8.176374 & -0.439739 & -0.235709 \\
\hline 9 & C & 0 & -9.711490 & 1.552535 & 0.943451 \\
\hline 10 & C & 0 & -7.765245 & 2.822420 & -0.067000 \\
\hline 11 & C & 0 & -7.083061 & 4.955277 & -1.766000 \\
\hline 12 & C & 0 & -7.838879 & 2.679228 & -1.457638 \\
\hline 13 & C & 0 & -7.347222 & 4.050290 & 0.456929 \\
\hline 14 & C & 0 & -7.009821 & 5.110056 & -0.383170 \\
\hline 15 & C & 0 & -7.499331 & 3.736462 & -2.299363 \\
\hline 16 & C & 0 & -2.029493 & -0.885356 & 1.828362 \\
\hline 17 & 0 & 0 & -1.316922 & 0.007516 & 2.293407 \\
\hline 18 & $\mathrm{~N}$ & 0 & -1.569364 & -2.145038 & 1.637001 \\
\hline 19 & C & 0 & -0.243657 & -2.581496 & 2.092989 \\
\hline 20 & C & 0 & -0.279282 & -4.068875 & 2.492542 \\
\hline 21 & C & 0 & -0.330435 & -5.144717 & 1.377291 \\
\hline 22 & C & 0 & -1.264138 & -4.799749 & 0.226218 \\
\hline 23 & 0 & 0 & -2.478418 & -4.669094 & 0.391121 \\
\hline 24 & $\mathrm{~N}$ & 0 & -0.670000 & -4.636617 & -0.980986 \\
\hline 25 & C & 0 & 0.873299 & -2.230687 & 1.069720 \\
\hline 26 & $\mathrm{~N}$ & 0 & 1.072244 & -0.903792 & 0.909992 \\
\hline 27 & 0 & 0 & 1.516356 & -3.094891 & 0.476798 \\
\hline 28 & C & 0 & 2.065517 & -0.368419 & -0.003368 \\
\hline 29 & C & 0 & 2.983527 & 0.579714 & 0.774282 \\
\hline 30 & 0 & 0 & 2.575602 & 1.180196 & 1.749089 \\
\hline 31 & C & 0 & 1.414547 & 0.427007 & -1.171504 \\
\hline 32 & C & 0 & 0.519808 & -0.413700 & -2.054941 \\
\hline 33 & C & 0 & -1.137928 & -1.965300 & -3.715962 \\
\hline 34 & C & 0 & -0.872334 & -0.326534 & -1.960091 \\
\hline 35 & C & 0 & 1.069350 & -1.294812 & -2.994751 \\
\hline 36 & C & 0 & 0.250998 & -2.063066 & -3.819108 \\
\hline 37 & C & 0 & -1.697109 & -1.094948 & -2.782197 \\
\hline 38 & $\mathrm{~N}$ & 0 & 4.296258 & 0.820394 & 0.362704 \\
\hline 39 & C & 0 & 5.109104 & 0.276405 & -0.618169 \\
\hline 40 & C & 0 & 6.699498 & -0.838482 & -2.577958 \\
\hline 41 & C & 0 & 6.494882 & 0.625294 & -0.663657 \\
\hline 42 & $\mathrm{~N}$ & 0 & 4.567570 & -0.561466 & -1.507889 \\
\hline 43 & C & 0 & 5.341228 & -1.102649 & -2.447446 \\
\hline 44 & C & 0 & 7.269015 & 0.042352 & -1.669088 \\
\hline 45 & C & 0 & 7.113488 & 1.570839 & 0.309317 \\
\hline 46 & 0 & 0 & 6.493868 & 2.142343 & 1.190947 \\
\hline 47 & 0 & 0 & 8.425764 & 1.719754 & 0.099565 \\
\hline 48 & C & 0 & 9.272906 & 2.614279 & 0.93711 \\
\hline 49 & C & 0 & 10.653492 & 2.435126 & 0.306791 \\
\hline 50 & C & 0 & 9.266751 & 2.125444 & 2.386491 \\
\hline 51 & C & 0 & 8.793346 & 4.060307 & 0.799801 \\
\hline
\end{tabular}




\begin{tabular}{|c|c|c|c|c|c|}
\hline 52 & $\mathrm{H}$ & 0 & -6.244093 & -1.906477 & -0.027368 \\
\hline 53 & $\mathrm{H}$ & 0 & -5.637374 & 1.979410 & 1.685503 \\
\hline 54 & $\mathrm{H}$ & 0 & -3.313022 & 1.363150 & 2.244978 \\
\hline 55 & $\mathrm{H}$ & 0 & -3.940739 & -2.530096 & 0.531941 \\
\hline 56 & $\mathrm{H}$ & 0 & -10.128661 & 1.291621 & -0.029481 \\
\hline 57 & $\mathrm{H}$ & 0 & -9.997841 & 0.770628 & 1.651292 \\
\hline 58 & $\mathrm{H}$ & 0 & -10.150278 & 2.497937 & 1.270436 \\
\hline 59 & $\mathrm{H}$ & 0 & -6.817919 & 5.776603 & -2.422084 \\
\hline 60 & $\mathrm{H}$ & 0 & -8.161981 & 1.736022 & -1.884423 \\
\hline 61 & $\mathrm{H}$ & 0 & -7.290495 & 4.182024 & 1.533297 \\
\hline 62 & $\mathrm{H}$ & 0 & -6.689402 & 6.054288 & 0.043167 \\
\hline 63 & $\mathrm{H}$ & 0 & -7.559195 & 3.606458 & -3.374302 \\
\hline 64 & $\mathrm{H}$ & 0 & -2.186519 & -2.862200 & 1.274968 \\
\hline 65 & $\mathrm{H}$ & 0 & -0.026004 & -1.999566 & 2.994346 \\
\hline 66 & $\mathrm{H}$ & 0 & -1.142023 & -4.188959 & 3.153198 \\
\hline 67 & $\mathrm{H}$ & 0 & 0.607282 & -4.273269 & 3.097643 \\
\hline 68 & $\mathrm{H}$ & 0 & 0.673410 & -5.330596 & 1.003499 \\
\hline 69 & $\mathrm{H}$ & 0 & -0.709454 & -6.070795 & 1.819140 \\
\hline 70 & $\mathrm{H}$ & 0 & -1.233492 & -4.252411 & -1.727262 \\
\hline 71 & $\mathrm{H}$ & 0 & 0.319466 & -4.428020 & -0.999965 \\
\hline 72 & $\mathrm{H}$ & 0 & 0.524964 & -0.261629 & 1.480423 \\
\hline 73 & $\mathrm{H}$ & 0 & 2.630281 & -1.202425 & -0.408189 \\
\hline 74 & $\mathrm{H}$ & 0 & 2.220193 & 0.858000 & -1.771382 \\
\hline 75 & $\mathrm{H}$ & 0 & 0.843665 & 1.256869 & -0.744992 \\
\hline 76 & $\mathrm{H}$ & 0 & -1.776894 & -2.552556 & -4.366674 \\
\hline 77 & $\mathrm{H}$ & 0 & -1.316833 & 0.354270 & -1.241876 \\
\hline 78 & $\mathrm{H}$ & 0 & 2.147948 & -1.372139 & -3.079031 \\
\hline 79 & $\mathrm{H}$ & 0 & 0.695107 & -2.731499 & -4.549091 \\
\hline 80 & $\mathrm{H}$ & 0 & -2.774390 & -1.007293 & -2.695468 \\
\hline 81 & $\mathrm{H}$ & 0 & 4.776792 & 1.480499 & 0.975842 \\
\hline 82 & $\mathrm{H}$ & 0 & 7.284049 & -1.301695 & -3.362101 \\
\hline 83 & $\mathrm{H}$ & 0 & 4.842841 & -1.781300 & -3.134492 \\
\hline 84 & $\mathrm{H}$ & 0 & 8.320125 & 0.290614 & -1.722440 \\
\hline 85 & $\mathrm{H}$ & 0 & 11.385334 & 3.048481 & 0.837414 \\
\hline 86 & $\mathrm{H}$ & 0 & 10.972349 & 1.391965 & 0.362933 \\
\hline 87 & $\mathrm{H}$ & 0 & 10.643301 & 2.740657 & -0.741850 \\
\hline 88 & $\mathrm{H}$ & 0 & 10.000044 & 2.699189 & 2.959472 \\
\hline 89 & $\mathrm{H}$ & 0 & 9.550703 & 1.071193 & 2.435858 \\
\hline 90 & $\mathrm{H}$ & 0 & 8.289618 & 2.252406 & 2.849283 \\
\hline 91 & $\mathrm{H}$ & 0 & 9.505530 & 4.721266 & 1.300931 \\
\hline 92 & $\mathrm{H}$ & 0 & 8.748201 & 4.350129 & -0.253078 \\
\hline 93 & $\mathrm{H}$ & 0 & 7.812629 & 4.202944 & 1.250304 \\
\hline 94 & C & 0 & -8.181516 & 1.686569 & 0.865783 \\
\hline 95 & $\mathrm{H}$ & 0 & -7.805071 & 1.925447 & 1.864608 \\
\hline
\end{tabular}

Standard basis: $6-311++G(d, p) \quad(5 D, 7 F)$

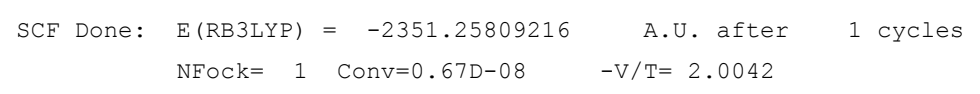

Full mass-weighted force constant matrix:

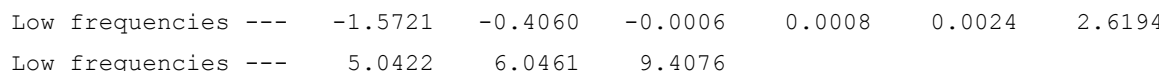




\section{Zero-point correction=}

Thermal correction to Energy=

Thermal correction to Enthalpy=

Thermal correction to Gibbs Free Energy=

Sum of electronic and zero-point Energies=

Sum of electronic and thermal Energies=

Sum of electronic and thermal Enthalpies=

Sum of electronic and thermal Free Energies=
0.773770 (Hartree/Particle)

0.823485

0.824429

0.679311

$-2350.484322$

$-2350.434607$

$-2350.433663$

$-2350.578781$ 
Amide 4 B

Stoichiometry C40H45N5O6

Framework group C1[X(C40H45N506)]

Standard orientation:

\begin{tabular}{|c|c|c|c|c|c|}
\hline \multirow{2}{*}{$\begin{array}{l}\text { Center } \\
\text { Number }\end{array}$} & \multirow[t]{2}{*}{ Atom } & \multirow{2}{*}{$\begin{array}{l}\text { Atomic } \\
\text { Type }\end{array}$} & \multicolumn{3}{|c|}{ Coordinates (Angstroms) } \\
\hline & & & $\mathrm{X}$ & $\mathrm{Y}$ & Z \\
\hline 1 & $\mathrm{C}$ & 0 & -6.522497 & -1.206867 & -0.578745 \\
\hline 2 & C & 0 & -3.842533 & -1.384603 & 0.302744 \\
\hline 3 & C & 0 & -6.217521 & -1.385446 & 0.777495 \\
\hline 4 & $\mathrm{C}$ & 0 & -5.459386 & -1.134730 & -1.484493 \\
\hline 5 & $\mathrm{C}$ & 0 & -4.137819 & -1.220549 & -1.054866 \\
\hline 6 & C & 0 & -4.902138 & -1.478316 & 1.212582 \\
\hline 7 & C & 0 & -7.933500 & 1.440510 & -1.446501 \\
\hline 8 & C & 0 & -10.117151 & 0.228859 & -0.990136 \\
\hline 9 & $\mathrm{C}$ & 0 & -12.891662 & 0.124644 & -1.471547 \\
\hline 10 & C & 0 & -10.617574 & 0.071547 & -2.289315 \\
\hline 11 & C & 0 & -11.035634 & 0.332784 & 0.059688 \\
\hline 12 & C & 0 & -12.409677 & 0.282432 & -0.174520 \\
\hline 13 & C & 0 & -11.988740 & 0.019121 & -2.529112 \\
\hline 14 & $\mathrm{C}$ & 0 & -2.446332 & -1.490542 & 0.843244 \\
\hline 15 & 0 & 0 & -2.241497 & -1.943395 & 1.973570 \\
\hline 16 & $\mathrm{~N}$ & 0 & -1.448062 & -1.076279 & 0.021827 \\
\hline 17 & $\mathrm{C}$ & 0 & -0.055479 & -0.930244 & 0.428187 \\
\hline 18 & C & 0 & 0.800437 & -2.180970 & 0.119894 \\
\hline 19 & C & 0 & 0.557522 & -3.365050 & 1.072567 \\
\hline 20 & $\mathrm{C}$ & 0 & 1.058277 & -3.059425 & 2.483410 \\
\hline 21 & o & 0 & 2.258890 & -2.981609 & 2.732211 \\
\hline 22 & $\mathrm{~N}$ & 0 & 0.095177 & -2.883336 & 3.421216 \\
\hline 23 & $\mathrm{C}$ & 0 & 0.477480 & 0.325056 & -0.305193 \\
\hline 24 & $\mathrm{~N}$ & 0 & 1.723116 & 0.707616 & 0.056806 \\
\hline 25 & o & 0 & -0.215840 & 0.911045 & -1.128809 \\
\hline 26 & C & 0 & 2.365945 & 1.903230 & -0.457130 \\
\hline 27 & C & 0 & 3.519996 & 1.652118 & -1.442394 \\
\hline 28 & 0 & 0 & 3.513390 & 2.230288 & -2.512105 \\
\hline 29 & C & 0 & 2.832599 & 2.857885 & 0.674973 \\
\hline 30 & C & 0 & 1.690898 & 3.374702 & 1.522009 \\
\hline 31 & C & 0 & -0.446416 & 4.323827 & 3.081888 \\
\hline 32 & C & 0 & 0.828233 & 4.361358 & 1.030170 \\
\hline 33 & C & 0 & 1.465775 & 2.874281 & 2.807933 \\
\hline 34 & C & 0 & 0.406419 & 3.343824 & 3.584235 \\
\hline 35 & C & 0 & -0.231135 & 4.832493 & 1.801173 \\
\hline 36 & $\mathrm{~N}$ & 0 & 4.640198 & 0.859236 & -1.158823 \\
\hline 37 & C & 0 & 5.075021 & 0.070306 & -0.105629 \\
\hline 38 & C & 0 & 5.918474 & -1.534395 & 1.980468 \\
\hline 39 & $\mathrm{C}$ & 0 & 6.405864 & -0.451620 & -0.127529 \\
\hline 40 & $\mathrm{~N}$ & 0 & 4.238542 & -0.202729 & 0.903401 \\
\hline 41 & $\mathrm{C}$ & 0 & 4.643979 & -0.987842 & 1.907263 \\
\hline 42 & $\mathrm{C}$ & 0 & 6.798817 & -1.254367 & 0.946457 \\
\hline 43 & C & 0 & 7.355210 & -0.179185 & -1.244179 \\
\hline 44 & 0 & 0 & 7.067231 & 0.455595 & -2.245757 \\
\hline 45 & o & 0 & 8.559573 & -0.715887 & -1.021424 \\
\hline 46 & C & 0 & 9.680435 & -0.597410 & -1.995063 \\
\hline 47 & $\mathrm{C}$ & 0 & 10.055575 & 0.874361 & -2.176894 \\
\hline 48 & C & 0 & 9.297802 & -1.275934 & -3.311561 \\
\hline 49 & $\mathrm{C}$ & 0 & 10.800904 & -1.363005 & -1.292281 \\
\hline 50 & $\mathrm{H}$ & 0 & -7.023413 & -1.462681 & 1.500720 \\
\hline 51 & $\mathrm{H}$ & 0 & -5.666368 & -1.018053 & -2.543090 \\
\hline 52 & $\mathrm{H}$ & 0 & -3.346754 & -1.183137 & -1.794946 \\
\hline
\end{tabular}




\begin{tabular}{|c|c|c|c|c|c|}
\hline 53 & $\mathrm{H}$ & 0 & -4.671819 & -1.629188 & 2.259790 \\
\hline 54 & $\mathrm{H}$ & 0 & -8.404795 & 2.391447 & -1.186558 \\
\hline 55 & $\mathrm{H}$ & 0 & -6.875669 & 1.500575 & -1.180004 \\
\hline 56 & $\mathrm{H}$ & 0 & -7.998703 & 1.323147 & -2.532370 \\
\hline 57 & $\mathrm{H}$ & 0 & -13.958919 & 0.085150 & -1.658066 \\
\hline 58 & $\mathrm{H}$ & 0 & -9.932808 & -0.008823 & -3.127005 \\
\hline 59 & $\mathrm{H}$ & 0 & -10.670709 & 0.457317 & 1.074631 \\
\hline 60 & $\mathrm{H}$ & 0 & -13.101246 & 0.367481 & 0.656602 \\
\hline 61 & $\mathrm{H}$ & 0 & -12.353111 & -0.102781 & -3.543374 \\
\hline 62 & $\mathrm{H}$ & 0 & -1.679228 & -0.473969 & -0.762535 \\
\hline 63 & $\mathrm{H}$ & 0 & -0.026964 & -0.722529 & 1.502654 \\
\hline 64 & $\mathrm{H}$ & 0 & 0.592871 & -2.489326 & -0.908883 \\
\hline 65 & $\mathrm{H}$ & 0 & 1.860694 & -1.921750 & 0.167449 \\
\hline 66 & $\mathrm{H}$ & 0 & -0.495935 & -3.646934 & 1.081389 \\
\hline 67 & $\mathrm{H}$ & 0 & 1.135661 & -4.221826 & 0.717384 \\
\hline 68 & $\mathrm{H}$ & 0 & -0.855858 & -2.679525 & 3.124260 \\
\hline 69 & $\mathrm{H}$ & 0 & 0.393299 & -2.593939 & 4.341196 \\
\hline 70 & $\mathrm{H}$ & 0 & 2.306914 & 0.133783 & 0.659560 \\
\hline 71 & $\mathrm{H}$ & 0 & 1.623404 & 2.412152 & -1.069204 \\
\hline 72 & $\mathrm{H}$ & 0 & 3.559685 & 2.342126 & 1.305733 \\
\hline 73 & $\mathrm{H}$ & 0 & 3.350285 & 3.697042 & 0.199198 \\
\hline 74 & $\mathrm{H}$ & 0 & -1.270365 & 4.691179 & 3.683251 \\
\hline 75 & $\mathrm{H}$ & 0 & 0.986792 & 4.764796 & 0.034865 \\
\hline 76 & $\mathrm{H}$ & 0 & 2.129811 & 2.115154 & 3.208838 \\
\hline 77 & $\mathrm{H}$ & 0 & 0.249694 & 2.944981 & 4.580507 \\
\hline 78 & $\mathrm{H}$ & 0 & -0.888124 & 5.597723 & 1.402910 \\
\hline 79 & $\mathrm{H}$ & 0 & 5.331424 & 0.932748 & -1.908447 \\
\hline 80 & $\mathrm{H}$ & 0 & 6.197068 & -2.171248 & 2.809335 \\
\hline 81 & $\mathrm{H}$ & 0 & 3.900438 & -1.215627 & 2.663119 \\
\hline 82 & $\mathrm{H}$ & 0 & 7.801542 & -1.659114 & 0.948179 \\
\hline 83 & $\mathrm{H}$ & 0 & 10.966459 & 0.938570 & -2.778070 \\
\hline 84 & $\mathrm{H}$ & 0 & 10.257244 & 1.339975 & -1.208865 \\
\hline 85 & $\mathrm{H}$ & 0 & 9.267524 & 1.430423 & -2.681722 \\
\hline 86 & $\mathrm{H}$ & 0 & 8.504650 & -0.736337 & -3.826183 \\
\hline 87 & $\mathrm{H}$ & 0 & 8.971936 & -2.303557 & -3.131648 \\
\hline 88 & $\mathrm{H}$ & 0 & 10.174882 & -1.309846 & -3.963348 \\
\hline 89 & $\mathrm{H}$ & 0 & 11.037773 & -0.905059 & -0.329386 \\
\hline 90 & $\mathrm{H}$ & 0 & 11.701489 & -1.351977 & -1.910513 \\
\hline 91 & $\mathrm{H}$ & 0 & 10.512241 & -2.402613 & -1.122172 \\
\hline 92 & $\mathrm{C}$ & 0 & -8.621692 & 0.276820 & -0.713626 \\
\hline 93 & $\mathrm{H}$ & 0 & -8.500620 & 0.446016 & 0.362124 \\
\hline 94 & $\mathrm{C}$ & 0 & -7.958989 & -1.094285 & -1.035706 \\
\hline 95 & $\mathrm{H}$ & 0 & -8.551721 & -1.879528 & -0.555891 \\
\hline 96 & $\mathrm{H}$ & 0 & -8.016166 & -1.274470 & -2.113658 \\
\hline
\end{tabular}

Standard basis: $6-311++G(d, p) \quad(5 D, 7 F)$

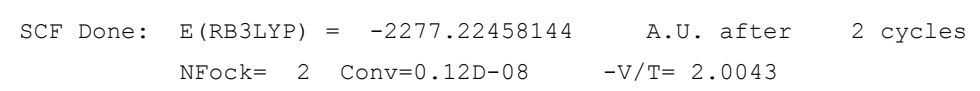

Full mass-weighted force constant matrix:

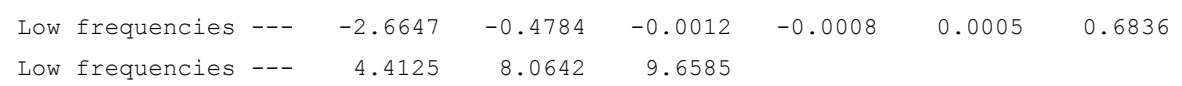

Zero-point correction=

Thermal correction to Energy=

Thermal correction to Enthalpy=
0.792199 (Hartree/Particle)

0.841338

0.842282 
Thermal correction to Gibbs Free Energy=

Sum of electronic and zero-point Energies=

Sum of electronic and thermal Energies=

Sum of electronic and thermal Enthalpies=

Sum of electronic and thermal Free Energies=
0.697789

$-2276.432383$

$-2276.383244$

$-2276.382300$

$-2276.526792$ 
$\begin{array}{ll}\text { Stoichiometry } & \text { C39H44N606 } \\ \text { Framework group } & \text { C1 [X(C39H44N6O6)] }\end{array}$

Standard orientation:

\begin{tabular}{|c|c|c|c|c|c|}
\hline \multirow{2}{*}{$\begin{array}{l}\text { Center } \\
\text { Number }\end{array}$} & \multirow[t]{2}{*}{ Atom } & \multirow{2}{*}{$\begin{array}{l}\text { Atomic } \\
\text { Type }\end{array}$} & \multicolumn{3}{|c|}{ Coordinates (Angstroms) } \\
\hline & & & $x$ & $Y$ & Z \\
\hline 1 & C & 0 & 5.441121 & -1.437933 & -0.467416 \\
\hline 2 & $\mathrm{C}$ & 0 & 3.094247 & -0.210841 & -1.434150 \\
\hline 3 & $\mathrm{C}$ & 0 & 4.250049 & -2.161512 & -0.584344 \\
\hline 4 & $\mathrm{C}$ & 0 & 5.445580 & -0.093975 & -0.847153 \\
\hline 5 & $\mathrm{C}$ & 0 & 4.287183 & 0.510918 & -1.322565 \\
\hline 6 & $\mathrm{C}$ & 0 & 3.088032 & -1.561225 & -1.057993 \\
\hline 7 & $\mathrm{~N}$ & 0 & 7.945306 & -1.487429 & -0.262040 \\
\hline 8 & C & 0 & 8.512237 & -1.925256 & -1.526221 \\
\hline 9 & $\mathrm{C}$ & 0 & 8.769620 & -0.926381 & 0.716015 \\
\hline 10 & $\mathrm{C}$ & 0 & 10.426264 & 0.266748 & 2.682066 \\
\hline 11 & $\mathrm{C}$ & 0 & 8.222595 & -0.349395 & 1.881928 \\
\hline 12 & $\mathrm{C}$ & 0 & 10.169185 & -0.879158 & 0.560196 \\
\hline 13 & C & 0 & 10.975816 & -0.286967 & 1.529206 \\
\hline 14 & $\mathrm{C}$ & 0 & 9.041458 & 0.227260 & 2.845227 \\
\hline 15 & C & 0 & 1.893894 & 0.522726 & -1.964100 \\
\hline 16 & 0 & 0 & 1.943392 & 1.710378 & -2.261572 \\
\hline 17 & $\mathrm{~N}$ & 0 & 0.728817 & -0.195971 & -2.079181 \\
\hline 18 & C & 0 & -0.416879 & 0.366400 & -2.784894 \\
\hline 19 & $\mathrm{C}$ & 0 & -1.201575 & -0.723405 & -3.555152 \\
\hline 20 & C & 0 & -2.111788 & -1.641383 & -2.724547 \\
\hline 21 & C & 0 & -1.400655 & -2.568085 & -1.743431 \\
\hline 22 & 0 & 0 & -0.221525 & -2.900048 & -1.882786 \\
\hline 23 & $\mathrm{~N}$ & 0 & -2.164883 & -3.036001 & -0.732740 \\
\hline 24 & C & 0 & -1.391515 & 1.152465 & -1.891942 \\
\hline 25 & $\mathrm{~N}$ & 0 & -1.269385 & 0.966589 & -0.558823 \\
\hline 26 & o & 0 & -2.269226 & 1.841073 & -2.413371 \\
\hline 27 & C & 0 & -2.076138 & 1.591380 & 0.497133 \\
\hline 28 & C & 0 & -3.526650 & 1.067216 & 0.584306 \\
\hline 29 & o & 0 & -3.985971 & 0.733452 & 1.663438 \\
\hline 30 & C & 0 & -2.044187 & 3.146230 & 0.479872 \\
\hline 31 & C & 0 & -0.706907 & 3.706408 & 0.912071 \\
\hline 32 & C & 0 & 1.785393 & 4.693054 & 1.756028 \\
\hline 33 & C & 0 & -0.478408 & 4.020243 & 2.257925 \\
\hline 34 & C & 0 & 0.332754 & 3.896639 & -0.004510 \\
\hline 35 & $\mathrm{C}$ & 0 & 1.570487 & 4.382579 & 0.414835 \\
\hline 36 & C & 0 & 0.755410 & 4.511840 & 2.678510 \\
\hline 37 & $\mathrm{~N}$ & 0 & -4.237246 & 1.057234 & -0.589253 \\
\hline 38 & C & 0 & -5.613950 & 0.811904 & -0.714111 \\
\hline 39 & $\mathrm{C}$ & 0 & -8.332823 & 0.642609 & -1.016623 \\
\hline 40 & C & 0 & -6.308097 & -0.177602 & 0.012289 \\
\hline 41 & $\mathrm{~N}$ & 0 & -6.220212 & 1.615072 & -1.591550 \\
\hline 42 & $\mathrm{C}$ & 0 & -7.538569 & 1.515497 & -1.753859 \\
\hline 43 & $\mathrm{C}$ & 0 & -7.697374 & -0.213623 & -0.126672 \\
\hline 44 & $\mathrm{C}$ & 0 & -5.628243 & -1.258962 & 0.792322 \\
\hline 45 & 0 & 0 & -4.650485 & -1.859691 & 0.388415 \\
\hline 46 & 0 & 0 & -6.293890 & -1.532613 & 1.912399 \\
\hline 47 & $\mathrm{C}$ & 0 & -5.875713 & -2.594751 & 2.866521 \\
\hline 48 & C & 0 & -4.469284 & -2.301227 & 3.390308 \\
\hline 49 & C & 0 & -5.984702 & -3.963288 & 2.191678 \\
\hline 50 & C & 0 & -6.910429 & -2.452091 & 3.982313 \\
\hline 51 & $\mathrm{H}$ & 0 & 4.228340 & -3.211045 & -0.306367 \\
\hline
\end{tabular}




\begin{tabular}{|c|c|c|c|c|c|}
\hline 52 & $\mathrm{H}$ & 0 & 6.365913 & 0.473637 & -0.773775 \\
\hline 53 & $\mathrm{H}$ & 0 & 4.285140 & 1.552734 & -1.617738 \\
\hline 54 & $\mathrm{H}$ & 0 & 2.192203 & -2.167335 & -1.132351 \\
\hline 55 & $\mathrm{H}$ & 0 & 7.699584 & -2.107465 & -2.231191 \\
\hline 56 & $\mathrm{H}$ & 0 & 9.103709 & -2.849868 & -1.434937 \\
\hline 57 & $\mathrm{H}$ & 0 & 9.149050 & -1.147707 & -1.952522 \\
\hline 58 & $\mathrm{H}$ & 0 & 11.059099 & 0.722938 & 3.433733 \\
\hline 59 & $\mathrm{H}$ & 0 & 7.149145 & -0.322765 & 2.022785 \\
\hline 60 & $\mathrm{H}$ & 0 & 10.636630 & -1.316126 & -0.311789 \\
\hline 61 & $\mathrm{H}$ & 0 & 12.050036 & -0.271163 & 1.378585 \\
\hline 62 & $\mathrm{H}$ & 0 & 8.586478 & 0.665030 & 3.727322 \\
\hline 63 & $\mathrm{H}$ & 0 & 0.748715 & -1.209268 & -2.015163 \\
\hline 64 & $\mathrm{H}$ & 0 & -0.044476 & 1.099303 & -3.504437 \\
\hline 65 & $\mathrm{H}$ & 0 & -0.481961 & -1.322447 & -4.118180 \\
\hline 66 & $\mathrm{H}$ & 0 & -1.832615 & -0.206777 & -4.280535 \\
\hline 67 & $\mathrm{H}$ & 0 & -2.875496 & -1.064095 & -2.196563 \\
\hline 68 & $\mathrm{H}$ & 0 & -2.659143 & -2.294828 & -3.414269 \\
\hline 69 & $\mathrm{H}$ & 0 & -3.075213 & -2.648822 & -0.508199 \\
\hline 70 & $\mathrm{H}$ & 0 & -1.727197 & -3.653928 & -0.065914 \\
\hline 71 & $\mathrm{H}$ & 0 & -0.471299 & 0.418151 & -0.269580 \\
\hline 72 & $\mathrm{H}$ & 0 & -1.634356 & 1.260381 & 1.436617 \\
\hline 73 & $\mathrm{H}$ & 0 & -2.310804 & 3.498508 & -0.517516 \\
\hline 74 & $\mathrm{H}$ & 0 & -2.821149 & 3.490136 & 1.168477 \\
\hline 75 & $\mathrm{H}$ & 0 & 2.746734 & 5.075550 & 2.080628 \\
\hline 76 & $\mathrm{H}$ & 0 & -1.277166 & 3.885601 & 2.981316 \\
\hline 77 & $\mathrm{H}$ & 0 & 0.186314 & 3.660017 & -1.051936 \\
\hline 78 & $\mathrm{H}$ & 0 & 2.363904 & 4.513010 & -0.311918 \\
\hline 79 & $\mathrm{H}$ & 0 & 0.910817 & 4.756287 & 3.723703 \\
\hline 80 & $\mathrm{H}$ & 0 & -3.811845 & 1.542751 & -1.384637 \\
\hline 81 & $\mathrm{H}$ & 0 & -9.406962 & 0.621428 & -1.150722 \\
\hline 82 & $\mathrm{H}$ & 0 & -7.975926 & 2.174627 & -2.498038 \\
\hline 83 & $\mathrm{H}$ & 0 & -8.263226 & -0.936517 & 0.447283 \\
\hline 84 & $\mathrm{H}$ & 0 & -3.715264 & -2.439004 & 2.617533 \\
\hline 85 & $\mathrm{H}$ & 0 & -4.250684 & -2.979750 & 4.219502 \\
\hline 86 & $\mathrm{H}$ & 0 & -4.404254 & -1.274196 & 3.753594 \\
\hline 87 & $\mathrm{H}$ & 0 & -6.990983 & -4.117081 & 1.793450 \\
\hline 88 & $\mathrm{H}$ & 0 & -5.792231 & -4.745300 & 2.931141 \\
\hline 89 & $\mathrm{H}$ & 0 & -5.264749 & -4.069162 & 1.381398 \\
\hline 90 & $\mathrm{H}$ & 0 & -7.920517 & -2.609030 & 3.597062 \\
\hline 91 & $\mathrm{H}$ & 0 & -6.719599 & -3.192264 & 4.762974 \\
\hline 92 & $\mathrm{H}$ & 0 & -6.859699 & -1.456289 & 4.426989 \\
\hline 93 & $\mathrm{C}$ & 0 & 6.681721 & -2.114693 & 0.097856 \\
\hline 94 & $\mathrm{H}$ & 0 & 6.692365 & -3.162311 & -0.233341 \\
\hline 95 & $\mathrm{H}$ & 0 & 6.602547 & -2.154586 & 1.186909 \\
\hline
\end{tabular}

Standard basis: $6-311++G(d, p) \quad(5 D, 7 F)$

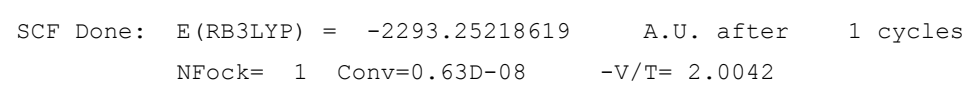

Full mass-weighted force constant matrix:

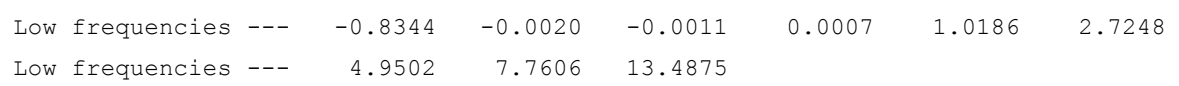


Thermal correction to Gibbs Free Energy=

Sum of electronic and zero-point Energies=

Sum of electronic and thermal Energies=

Sum of electronic and thermal Enthalpies=

Sum of electronic and thermal Free Energies=
0.688727

$-2292.471491$

$-2292.422695$

$-2292.421751$

$-2292.563459$ 
Stoichiometry C39H44N606

Framework group C1[X(C39H44N606)]

Standard orientation

\begin{tabular}{|c|c|c|c|c|c|}
\hline \multirow{2}{*}{$\begin{array}{l}\text { Center } \\
\text { Number }\end{array}$} & \multirow[t]{2}{*}{ Atom } & \multirow{2}{*}{$\begin{array}{l}\text { Atomic } \\
\text { Type }\end{array}$} & \multicolumn{3}{|c|}{ Coordinates (Angstroms) } \\
\hline & & & $\mathrm{X}$ & Y & Z \\
\hline 1 & C & 0 & 7.602460 & -1.384721 & -1.917943 \\
\hline 2 & C & 0 & 6.474954 & -0.703494 & -1.192028 \\
\hline 3 & $\mathrm{C}$ & 0 & 4.231580 & 0.393813 & 0.099495 \\
\hline 4 & C & 0 & 6.547275 & -0.317010 & 0.150914 \\
\hline 5 & $\mathrm{C}$ & 0 & 5.266026 & -0.548143 & -1.877695 \\
\hline 6 & C & 0 & 4.160215 & 0.006992 & -1.244179 \\
\hline 7 & C & 0 & 5.436832 & 0.223659 & 0.787355 \\
\hline 8 & 0 & 0 & 7.367285 & -2.301811 & -2.694392 \\
\hline 9 & $\mathrm{~N}$ & 0 & 8.903966 & -0.999975 & -1.647491 \\
\hline 10 & C & 0 & 9.961759 & -1.803743 & -2.276120 \\
\hline 11 & C & 0 & 9.270916 & 0.302960 & -1.178451 \\
\hline 12 & C & 0 & 10.048561 & 2.835825 & -0.275535 \\
\hline 13 & C & 0 & 8.781454 & 1.453973 & -1.801115 \\
\hline 14 & C & 0 & 10.165364 & 0.427928 & -0.110401 \\
\hline 15 & C & 0 & 10.554289 & 1.687974 & 0.334626 \\
\hline 16 & C & 0 & 9.160605 & 2.713640 & -1.341876 \\
\hline 17 & C & 0 & 3.080892 & 1.005764 & 0.857624 \\
\hline 18 & 0 & 0 & 3.268982 & 1.636758 & 1.895022 \\
\hline 19 & $\mathrm{~N}$ & 0 & 1.843425 & 0.818308 & 0.333088 \\
\hline 20 & C & 0 & 0.637549 & 1.320064 & 0.976998 \\
\hline 21 & C & 0 & 0.378618 & 2.789737 & 0.559608 \\
\hline 22 & C & 0 & -0.779249 & 3.535914 & 1.244032 \\
\hline 23 & $\mathrm{~N}$ & 0 & -1.290618 & 2.470495 & 3.445679 \\
\hline 24 & C & 0 & -0.505820 & 0.384505 & 0.552131 \\
\hline 25 & $\mathrm{~N}$ & 0 & -1.491013 & 0.255658 & 1.468140 \\
\hline 26 & o & 0 & -0.485942 & -0.175472 & -0.541270 \\
\hline 27 & C & 0 & -2.689718 & -0.520954 & 1.221105 \\
\hline 28 & C & 0 & -3.908977 & 0.407237 & 1.257087 \\
\hline 29 & 0 & 0 & -3.923137 & 1.394227 & 1.976263 \\
\hline 30 & C & 0 & -2.891297 & -1.629366 & 2.294305 \\
\hline 31 & C & 0 & -1.758322 & -2.628905 & 2.344084 \\
\hline 32 & C & 0 & 0.357709 & -4.478076 & 2.417802 \\
\hline 33 & C & 0 & -0.785037 & -2.563336 & 3.345421 \\
\hline 34 & C & 0 & -1.655025 & -3.637895 & 1.379659 \\
\hline 35 & C & 0 & -0.607824 & -4.554799 & 1.414299 \\
\hline 36 & C & 0 & 0.265752 & -3.479303 & 3.384246 \\
\hline 37 & $\mathrm{~N}$ & 0 & -5.049588 & 0.143575 & 0.506322 \\
\hline 38 & C & 0 & -5.372907 & -0.825736 & -0.433338 \\
\hline 39 & C & 0 & -5.972136 & -2.765281 & -2.297956 \\
\hline 40 & C & 0 & -6.569094 & -0.693570 & -1.202603 \\
\hline 41 & $\mathrm{~N}$ & 0 & -4.553211 & -1.869048 & -0.581715 \\
\hline 42 & C & 0 & -4.841259 & -2.799554 & -1.490134 \\
\hline 43 & C & 0 & -6.840805 & -1.695159 & -2.137780 \\
\hline 44 & C & 0 & -7.507513 & 0.452528 & -1.032071 \\
\hline 45 & 0 & 0 & -7.362201 & 1.333523 & -0.200484 \\
\hline 46 & 0 & 0 & -8.527807 & 0.397953 & -1.894041 \\
\hline 47 & C & 0 & -9.606676 & 1.424830 & -1.924410 \\
\hline 48 & C & 0 & -10.503823 & 0.917572 & -3.052722 \\
\hline 49 & C & 0 & -9.011465 & 2.790362 & -2.271838 \\
\hline 50 & $\mathrm{C}$ & 0 & -10.355972 & 1.429339 & -0.590872 \\
\hline
\end{tabular}




\begin{tabular}{|c|c|c|c|c|c|}
\hline 51 & $\mathrm{H}$ & 0 & 7.467581 & -0.446954 & 0.705712 \\
\hline 52 & $\mathrm{H}$ & 0 & 5.202975 & -0.872210 & -2.909259 \\
\hline 53 & $\mathrm{H}$ & 0 & 3.249007 & 0.145803 & -1.814687 \\
\hline 54 & $\mathrm{H}$ & 0 & 5.483809 & 0.527976 & 1.825437 \\
\hline 55 & $\mathrm{H}$ & 0 & 9.696364 & -2.857548 & -2.218843 \\
\hline 56 & $\mathrm{H}$ & 0 & 10.086460 & -1.537603 & -3.331290 \\
\hline 57 & $\mathrm{H}$ & 0 & 10.900076 & -1.626723 & -1.752750 \\
\hline 58 & $\mathrm{H}$ & 0 & 10.345651 & 3.816571 & 0.077343 \\
\hline 59 & $\mathrm{H}$ & 0 & 8.102240 & 1.360630 & -2.640008 \\
\hline 60 & $\mathrm{H}$ & 0 & 10.541971 & -0.464271 & 0.377264 \\
\hline 61 & $\mathrm{H}$ & 0 & 11.243749 & 1.773160 & 1.167008 \\
\hline 62 & $\mathrm{H}$ & 0 & 8.766612 & 3.599804 & -1.826316 \\
\hline 63 & $\mathrm{H}$ & 0 & 1.682475 & 0.200879 & -0.451364 \\
\hline 64 & $\mathrm{H}$ & 0 & 0.780935 & 1.271673 & 2.057143 \\
\hline 65 & $\mathrm{H}$ & 0 & 1.310151 & 3.327856 & 0.759790 \\
\hline 66 & $\mathrm{H}$ & 0 & 0.227987 & 2.807475 & -0.525177 \\
\hline 67 & $\mathrm{H}$ & 0 & -1.744757 & 3.095648 & 0.973870 \\
\hline 68 & $\mathrm{H}$ & 0 & -0.787973 & 4.550057 & 0.830287 \\
\hline 69 & $\mathrm{H}$ & 0 & -2.304188 & 2.492662 & 3.350381 \\
\hline 70 & $\mathrm{H}$ & 0 & -1.078949 & 2.481532 & 4.438518 \\
\hline 71 & $\mathrm{H}$ & 0 & -1.427459 & 0.842822 & 2.305703 \\
\hline 72 & $\mathrm{H}$ & 0 & -2.589696 & -0.983816 & 0.243922 \\
\hline 73 & $\mathrm{H}$ & 0 & -3.006422 & -1.147355 & 3.269988 \\
\hline 74 & $\mathrm{H}$ & 0 & -3.829934 & -2.143224 & 2.071323 \\
\hline 75 & $\mathrm{H}$ & 0 & 1.174799 & -5.190207 & 2.444422 \\
\hline 76 & $\mathrm{H}$ & 0 & -0.849165 & -1.788552 & 4.102578 \\
\hline 77 & $\mathrm{H}$ & 0 & -2.400839 & -3.698322 & 0.594294 \\
\hline 78 & $\mathrm{H}$ & 0 & -0.543016 & -5.328906 & 0.657479 \\
\hline 79 & $\mathrm{H}$ & 0 & 1.012095 & -3.410185 & 4.167957 \\
\hline 80 & H & 0 & -5.762768 & 0.866604 & 0.612735 \\
\hline 81 & $\mathrm{H}$ & 0 & -6.164294 & -3.548677 & -3.019440 \\
\hline 82 & $\mathrm{H}$ & 0 & -4.129651 & -3.616755 & -1.569225 \\
\hline 83 & $\mathrm{H}$ & 0 & -7.739612 & -1.617087 & -2.733867 \\
\hline 84 & $\mathrm{H}$ & 0 & -9.951798 & 0.868540 & -3.993884 \\
\hline 85 & $\mathrm{H}$ & 0 & -11.351337 & 1.594078 & -3.184702 \\
\hline 86 & $\mathrm{H}$ & 0 & -10.890054 & -0.077929 & -2.822774 \\
\hline 87 & $\mathrm{H}$ & 0 & -8.429234 & 2.730250 & -3.194776 \\
\hline 88 & $\mathrm{H}$ & 0 & -8.373871 & 3.165683 & -1.473237 \\
\hline 89 & $\mathrm{H}$ & 0 & -9.824481 & 3.503506 & -2.431946 \\
\hline 90 & $\mathrm{H}$ & 0 & -9.729373 & 1.794827 & 0.220860 \\
\hline 91 & $\mathrm{H}$ & 0 & -11.230288 & 2.080203 & -0.675401 \\
\hline 92 & $\mathrm{H}$ & 0 & -10.707144 & 0.423763 & -0.345802 \\
\hline 93 & C & 0 & -0.693779 & 3.645747 & 2.776037 \\
\hline 94 & $\mathrm{H}$ & 0 & 0.354573 & 3.712599 & 3.083133 \\
\hline 95 & $\mathrm{H}$ & 0 & -1.178020 & 4.579305 & 3.094089 \\
\hline
\end{tabular}

Standard basis: $6-311++G(d, p) \quad(5 D, 7 F)$

SCF Done: E (RB3LYP $)=-2293.24453695$ A.U. after 1 cycles $\mathrm{NFOCk}=1$ Conv $=0.37 \mathrm{D}-08 \quad-\mathrm{V} / \mathrm{T}=2.0042$

Full mass-weighted force constant matrix:

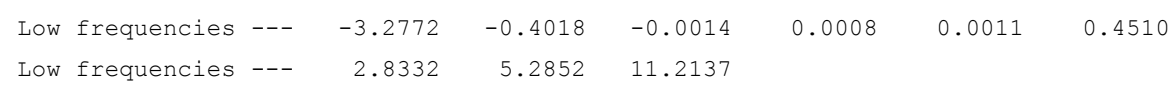


Zero-point correction=

Thermal correction to Energy=

Thermal correction to Enthalpy=

Thermal correction to Gibbs Free Energy=

Sum of electronic and zero-point Energies=

Sum of electronic and thermal Energies=

Sum of electronic and thermal Enthalpies=

Sum of electronic and thermal Free Energies=
0.780404 (Hartree/Particle)

0.829810

0.830754

0.685173

$-2292.464133$

$-2292.414727$

$-2292.413783$

$-2292.559364$ 
Amide 5 B

Stoichiometry C40H43N507

Framework group C1[X(C40H43N5O7)]

Standard orientation:

\begin{tabular}{|c|c|c|c|c|c|}
\hline \multirow{2}{*}{$\begin{array}{l}\text { Center } \\
\text { Number }\end{array}$} & \multirow[t]{2}{*}{ Atom } & \multirow{2}{*}{$\begin{array}{l}\text { Atomic } \\
\text { Type }\end{array}$} & \multicolumn{3}{|c|}{ Coordinates (Angstroms) } \\
\hline & & & $\mathrm{X}$ & $\mathrm{Y}$ & z \\
\hline 1 & C & 0 & -7.412465 & -1.210956 & 0.822659 \\
\hline 2 & $\mathrm{C}$ & 0 & -6.082047 & -0.666218 & 0.379253 \\
\hline 3 & $\mathrm{C}$ & 0 & -3.508179 & 0.272055 & -0.247541 \\
\hline 4 & C & 0 & -5.384432 & 0.149467 & 1.275904 \\
\hline 5 & C & 0 & -5.470499 & -1.027375 & -0.826013 \\
\hline 6 & $\mathrm{C}$ & 0 & -4.192615 & -0.573127 & -1.127455 \\
\hline 7 & $\mathrm{C}$ & 0 & -4.118727 & 0.629862 & 0.961289 \\
\hline 8 & 0 & 0 & -7.581636 & -1.540648 & 1.989609 \\
\hline 9 & $\mathrm{~N}$ & 0 & -8.410098 & -1.387646 & -0.118928 \\
\hline 10 & C & 0 & -9.633160 & -2.056515 & 0.346731 \\
\hline 11 & C & 0 & -8.504270 & -0.632507 & -1.332920 \\
\hline 12 & C & 0 & -8.748268 & 0.821641 & -3.711866 \\
\hline 13 & C & 0 & -8.406849 & 0.761171 & -1.319738 \\
\hline 14 & C & 0 & -8.740483 & -1.293111 & -2.542857 \\
\hline 15 & C & 0 & -8.865686 & -0.568243 & -3.724369 \\
\hline 16 & C & 0 & -8.516680 & 1.481814 & -2.507253 \\
\hline 17 & C & 0 & -2.133277 & 0.722374 & -0.659601 \\
\hline 18 & o & 0 & -1.553902 & 0.186790 & -1.610626 \\
\hline 19 & $\mathrm{~N}$ & 0 & -1.570137 & 1.728629 & 0.050719 \\
\hline 20 & C & 0 & -0.314974 & 2.367936 & -0.350511 \\
\hline 21 & C & 0 & -0.286478 & 3.854398 & 0.047871 \\
\hline 22 & C & 0 & -0.142504 & 4.234903 & 1.533850 \\
\hline 23 & C & 0 & -1.357380 & 4.134530 & 2.426533 \\
\hline 24 & 0 & 0 & -2.353831 & 3.492694 & 2.135228 \\
\hline 25 & C & 0 & 0.948960 & 1.597234 & 0.124035 \\
\hline 26 & $\mathrm{~N}$ & 0 & 1.019123 & 0.323561 & -0.321968 \\
\hline 27 & 0 & 0 & 1.815712 & 2.122659 & 0.817064 \\
\hline 28 & C & 0 & 2.171731 & -0.514703 & -0.055982 \\
\hline 29 & C & 0 & 3.237008 & -0.287260 & -1.142578 \\
\hline 30 & 0 & 0 & 2.924463 & 0.043998 & -2.268425 \\
\hline 31 & C & 0 & 1.771714 & -2.010984 & -0.049157 \\
\hline 32 & C & 0 & 0.786936 & -2.369133 & 1.043007 \\
\hline 33 & $\mathrm{C}$ & 0 & -1.033302 & -3.027466 & 3.082184 \\
\hline 34 & $\mathrm{C}$ & 0 & 1.209008 & -2.473078 & 2.373848 \\
\hline 35 & $\mathrm{C}$ & 0 & -0.560238 & -2.602078 & 0.751272 \\
\hline 36 & C & 0 & -1.465149 & -2.927645 & 1.761630 \\
\hline 37 & C & 0 & 0.309011 & -2.799164 & 3.385445 \\
\hline 38 & $\mathrm{~N}$ & 0 & 4.597324 & -0.483057 & -0.879171 \\
\hline 39 & $\mathrm{C}$ & 0 & 5.318972 & -0.782440 & 0.262051 \\
\hline 40 & C & 0 & 6.723468 & -1.381194 & 2.562048 \\
\hline 41 & $\mathrm{C}$ & 0 & 6.746860 & -0.698026 & 0.242258 \\
\hline 42 & $\mathrm{~N}$ & 0 & 4.655725 & -1.159637 & 1.360495 \\
\hline 43 & C & 0 & 5.337647 & -1.439862 & 2.469093 \\
\hline 44 & $\mathrm{C}$ & 0 & 7.423827 & -1.007793 & 1.423766 \\
\hline 45 & C & 0 & 7.506610 & -0.301915 & -0.976814 \\
\hline 46 & 0 & 0 & 6.990570 & -0.068993 & -2.057029 \\
\hline 47 & 0 & 0 & 8.824356 & -0.236219 & -0.748696 \\
\hline 48 & C & 0 & 9.800202 & 0.131789 & -1.810030 \\
\hline 49 & $\mathrm{C}$ & 0 & 11.131961 & 0.063419 & -1.063158 \\
\hline 50 & C & 0 & 9.522446 & 1.554252 & -2.299893 \\
\hline 51 & C & 0 & 9.754698 & -0.899816 & -2.938842 \\
\hline 52 & $\mathrm{H}$ & 0 & -5.840729 & 0.392828 & 2.227531 \\
\hline
\end{tabular}




\begin{tabular}{|c|c|c|c|c|c|}
\hline 53 & $\mathrm{H}$ & 0 & -5.983634 & -1.675994 & -1.524419 \\
\hline 54 & $\mathrm{H}$ & 0 & -3.702269 & -0.863977 & -2.047834 \\
\hline 55 & $\mathrm{H}$ & 0 & -3.614785 & 1.265542 & 1.678857 \\
\hline 56 & $\mathrm{H}$ & 0 & -10.273129 & -1.365544 & 0.905926 \\
\hline 57 & $\mathrm{H}$ & 0 & -10.183447 & -2.430095 & -0.515459 \\
\hline 58 & $\mathrm{H}$ & 0 & -9.365069 & -2.881600 & 1.003593 \\
\hline 59 & $\mathrm{H}$ & 0 & -8.838393 & 1.384317 & -4.633790 \\
\hline 60 & $\mathrm{H}$ & 0 & -8.242104 & 1.277135 & -0.381386 \\
\hline 61 & $\mathrm{H}$ & 0 & -8.811454 & -2.375005 & -2.555120 \\
\hline 62 & $\mathrm{H}$ & 0 & -9.043364 & -1.090797 & -4.657529 \\
\hline 63 & $\mathrm{H}$ & 0 & -8.430681 & 2.562286 & -2.486475 \\
\hline 64 & $\mathrm{H}$ & 0 & -2.095000 & 2.211432 & 0.775031 \\
\hline 65 & $\mathrm{H}$ & 0 & -0.283260 & 2.326295 & -1.445307 \\
\hline 66 & $\mathrm{H}$ & 0 & -1.180324 & 4.326689 & -0.372058 \\
\hline 67 & $\mathrm{H}$ & 0 & 0.570588 & 4.292398 & -0.468498 \\
\hline 68 & $\mathrm{H}$ & 0 & 0.224418 & 5.266086 & 1.601858 \\
\hline 69 & $\mathrm{H}$ & 0 & 0.649818 & 3.628701 & 1.988945 \\
\hline 70 & $\mathrm{H}$ & 0 & 0.323704 & 0.028690 & -1.004040 \\
\hline 71 & $\mathrm{H}$ & 0 & 2.578315 & -0.242200 & 0.913487 \\
\hline 72 & $\mathrm{H}$ & 0 & 2.685292 & -2.597789 & 0.072784 \\
\hline 73 & $\mathrm{H}$ & 0 & 1.353244 & -2.263209 & -1.028465 \\
\hline 74 & $\mathrm{H}$ & 0 & -1.735750 & -3.281409 & 3.868169 \\
\hline 75 & $\mathrm{H}$ & 0 & 2.252388 & -2.296593 & 2.612326 \\
\hline 76 & $\mathrm{H}$ & 0 & -0.907583 & -2.521170 & -0.273387 \\
\hline 77 & $\mathrm{H}$ & 0 & -2.507040 & -3.099783 & 1.515771 \\
\hline 78 & $\mathrm{H}$ & 0 & 0.654661 & -2.878431 & 4.410673 \\
\hline 79 & $\mathrm{H}$ & 0 & 5.178553 & -0.265188 & -1.689149 \\
\hline 80 & $\mathrm{H}$ & 0 & 7.230512 & -1.623572 & 3.486919 \\
\hline 81 & $\mathrm{H}$ & 0 & 4.739943 & -1.733554 & 3.328064 \\
\hline 82 & $\mathrm{H}$ & 0 & 8.503546 & -0.949020 & 1.432173 \\
\hline 83 & $\mathrm{H}$ & 0 & 11.143359 & 0.766757 & -0.227550 \\
\hline 84 & $\mathrm{H}$ & 0 & 11.950449 & 0.318488 & -1.740285 \\
\hline 85 & $\mathrm{H}$ & 0 & 11.306710 & -0.942339 & -0.674450 \\
\hline 86 & $\mathrm{H}$ & 0 & 10.327732 & 1.864376 & -2.971239 \\
\hline 87 & $\mathrm{H}$ & 0 & 8.578600 & 1.616212 & -2.838761 \\
\hline 88 & $\mathrm{H}$ & 0 & 9.498328 & 2.250530 & -1.457779 \\
\hline 89 & $\mathrm{H}$ & 0 & 10.570937 & -0.700026 & -3.638259 \\
\hline 90 & $\mathrm{H}$ & 0 & 9.891297 & -1.908524 & -2.540705 \\
\hline 91 & $\mathrm{H}$ & 0 & 8.813209 & -0.857369 & -3.483669 \\
\hline 92 & C & 0 & -1.283764 & 4.882557 & 3.740163 \\
\hline 93 & $\mathrm{H}$ & 0 & -2.068825 & 4.538964 & 4.412458 \\
\hline 94 & $\mathrm{H}$ & 0 & -0.302803 & 4.765209 & 4.207736 \\
\hline 95 & $\mathrm{H}$ & 0 & -1.423167 & 5.952273 & 3.548398 \\
\hline
\end{tabular}

Standard basis: $6-311++G(d, p) \quad$ (5D, 7F)

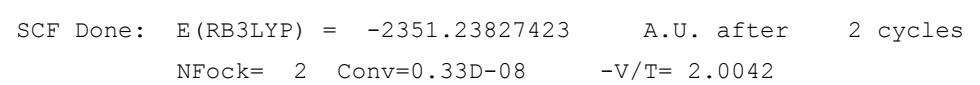

Full mass-weighted force constant matrix:

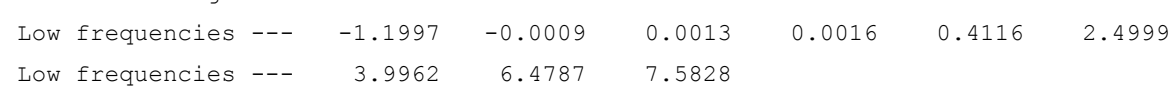

Zero-point correction=

Thermal correction to Energy=

Thermal correction to Enthalpy=

Thermal correction to Gibbs Free Energy=

Sum of electronic and zero-point Energies=

Sum of electronic and thermal Energies=
0.770904 (Hartree/Particle)

0.821347

0.822292

0.674258

$-2350.467370$

$-2350.416927$ 
Sum of electronic and thermal Enthalpies=

Sum of electronic and thermal Free Energies=
$-2350.415983$

$-2350.564017$ 
Stoichiometry C40H45N5O6

Framework group C1[X(C40H45N5O6)]

Standard orientation:

\begin{tabular}{|c|c|c|c|c|c|}
\hline \multirow{2}{*}{$\begin{array}{l}\text { Center } \\
\text { Number }\end{array}$} & \multirow[t]{2}{*}{ Atom } & \multirow{2}{*}{$\begin{array}{l}\text { Atomic } \\
\text { Type }\end{array}$} & \multicolumn{3}{|c|}{ Coordinates (Angstroms) } \\
\hline & & & $\mathrm{X}$ & $\mathrm{Y}$ & $\mathrm{Z}$ \\
\hline 1 & $\mathrm{C}$ & 0 & 7.134471 & -2.119132 & -0.029975 \\
\hline 2 & $\mathrm{C}$ & 0 & 5.887594 & -1.283103 & 0.080478 \\
\hline 3 & $\mathrm{C}$ & 0 & 3.442001 & 0.095259 & 0.186302 \\
\hline 4 & $\mathrm{C}$ & 0 & 5.425587 & -0.749693 & 1.288717 \\
\hline 5 & $\mathrm{C}$ & 0 & 5.099099 & -1.147613 & -1.066576 \\
\hline 6 & $\mathrm{C}$ & 0 & 3.896816 & -0.452173 & -1.019292 \\
\hline 7 & C & 0 & 4.208083 & -0.082160 & 1.342416 \\
\hline 8 & $\mathrm{O}$ & 0 & 7.141882 & -3.111980 & -0.746418 \\
\hline 9 & $\mathrm{~N}$ & 0 & 8.236423 & -1.782142 & 0.733099 \\
\hline 10 & $\mathrm{C}$ & 0 & 9.367974 & -2.719680 & 0.692341 \\
\hline 11 & C & 0 & 8.508164 & -0.455063 & 1.200589 \\
\hline 12 & $\mathrm{C}$ & 0 & 9.098376 & 2.118804 & 2.127040 \\
\hline 13 & C & 0 & 8.866439 & -0.251247 & 2.537019 \\
\hline 14 & $\mathrm{C}$ & 0 & 8.463251 & 0.634998 & 0.327925 \\
\hline 15 & C & 0 & 8.745922 & 1.917251 & 0.794502 \\
\hline 16 & C & 0 & 9.163435 & 1.028948 & 2.995241 \\
\hline 17 & C & 0 & 2.143104 & 0.839630 & 0.322205 \\
\hline 18 & $\mathrm{O}$ & 0 & 1.560298 & 0.917484 & 1.402107 \\
\hline 19 & $\mathrm{~N}$ & 0 & 1.663076 & 1.438777 & -0.800536 \\
\hline 20 & C & 0 & 0.435773 & 2.239256 & -0.813958 \\
\hline 21 & C & 0 & 0.530297 & 3.363859 & -1.846124 \\
\hline 22 & $\mathrm{C}$ & 0 & 1.585934 & 4.424243 & -1.513243 \\
\hline 23 & C & 0 & -0.796143 & 1.332848 & -1.070457 \\
\hline 24 & $\mathrm{~N}$ & 0 & -1.120527 & 0.546090 & -0.012124 \\
\hline 25 & O & 0 & -1.394877 & 1.337717 & -2.136711 \\
\hline 26 & C & 0 & -2.257855 & -0.353205 & -0.028344 \\
\hline 27 & C & 0 & -3.305700 & 0.147325 & 0.974762 \\
\hline 28 & $\mathrm{O}$ & 0 & -2.973888 & 0.770106 & 1.964816 \\
\hline 29 & C & 0 & -1.858223 & -1.800634 & 0.358095 \\
\hline 30 & $\mathrm{C}$ & 0 & -0.854642 & -2.435132 & -0.580933 \\
\hline 31 & C & 0 & 0.993375 & -3.665682 & -2.307495 \\
\hline 32 & $\mathrm{C}$ & 0 & -1.164242 & -2.643605 & -1.930599 \\
\hline 33 & C & 0 & 0.396517 & -2.848541 & -0.114318 \\
\hline 34 & C & 0 & 1.314686 & -3.461050 & -0.967769 \\
\hline 35 & $\mathrm{C}$ & 0 & -0.250000 & -3.251765 & -2.786727 \\
\hline 36 & $\mathrm{~N}$ & 0 & -4.665101 & -0.126532 & 0.804413 \\
\hline 37 & C & 0 & -5.398850 & -0.741380 & -0.196167 \\
\hline 38 & C & 0 & -6.823122 & -1.976241 & -2.211045 \\
\hline 39 & C & 0 & -6.827462 & -0.700699 & -0.156214 \\
\hline 40 & $\mathrm{~N}$ & 0 & -4.742835 & -1.372028 & -1.175385 \\
\hline 41 & C & 0 & -5.434330 & -1.961412 & -2.148829 \\
\hline 42 & C & 0 & -7.515033 & -1.336221 & -1.192666 \\
\hline 43 & $\mathrm{C}$ & 0 & -7.576916 & -0.018146 & 0.936146 \\
\hline 44 & $\mathrm{O}$ & 0 & -7.047040 & 0.517535 & 1.895387 \\
\hline 45 & $\mathrm{O}$ & 0 & -8.900368 & -0.064769 & 0.742818 \\
\hline 46 & $\mathrm{C}$ & 0 & -9.868445 & 0.542630 & 1.696515 \\
\hline 47 & C & 0 & -11.211693 & 0.224835 & 1.040396 \\
\hline 48 & C & 0 & -9.642636 & 2.053734 & 1.772820 \\
\hline 49 & C & 0 & -9.752257 & -0.140629 & 3.060316 \\
\hline 50 & $\mathrm{H}$ & 0 & 6.006606 & -0.869447 & 2.194006 \\
\hline
\end{tabular}




\begin{tabular}{|c|c|c|c|c|c|}
\hline 51 & $\mathrm{H}$ & 0 & 5.430134 & -1.611821 & -1.987400 \\
\hline 52 & $\mathrm{H}$ & 0 & 3.296948 & -0.388801 & -1.920261 \\
\hline 53 & $\mathrm{H}$ & 0 & 3.829967 & 0.309105 & 2.278606 \\
\hline 54 & $\mathrm{H}$ & 0 & 8.991512 & -3.740331 & 0.716804 \\
\hline 55 & $\mathrm{H}$ & 0 & 10.009603 & -2.538191 & 1.553162 \\
\hline 56 & $\mathrm{H}$ & 0 & 9.954847 & -2.590873 & -0.223332 \\
\hline 57 & $\mathrm{H}$ & 0 & 9.323233 & 3.116069 & 2.486908 \\
\hline 58 & $\mathrm{H}$ & 0 & 8.897910 & -1.096039 & 3.215987 \\
\hline 59 & $\mathrm{H}$ & 0 & 8.205795 & 0.476782 & -0.712596 \\
\hline 60 & $\mathrm{H}$ & 0 & 8.700790 & 2.757161 & 0.110581 \\
\hline 61 & $\mathrm{H}$ & 0 & 9.435100 & 1.176712 & 4.034321 \\
\hline 62 & $\mathrm{H}$ & 0 & 2.171429 & 1.350060 & -1.666331 \\
\hline 63 & $\mathrm{H}$ & 0 & 0.337670 & 2.662114 & 0.190085 \\
\hline 64 & $\mathrm{H}$ & 0 & -0.455722 & 3.830476 & -1.918541 \\
\hline 65 & $\mathrm{H}$ & 0 & 0.715833 & 2.929007 & -2.835196 \\
\hline 66 & $\mathrm{H}$ & 0 & 2.573606 & 3.957506 & -1.416638 \\
\hline 67 & $\mathrm{H}$ & 0 & 1.362362 & 4.861978 & -0.531992 \\
\hline 68 & $\mathrm{H}$ & 0 & -0.591910 & 0.655204 & 0.846465 \\
\hline 69 & $\mathrm{H}$ & 0 & -2.666971 & -0.342714 & -1.033870 \\
\hline 70 & $\mathrm{H}$ & 0 & -1.455701 & -1.790657 & 1.375545 \\
\hline 71 & $\mathrm{H}$ & 0 & -2.771190 & -2.402800 & 0.378235 \\
\hline 72 & $\mathrm{H}$ & 0 & 1.704380 & -4.143131 & -2.972701 \\
\hline 73 & $\mathrm{H}$ & 0 & -2.126755 & -2.321070 & -2.312048 \\
\hline 74 & $\mathrm{H}$ & 0 & 0.656315 & -2.689642 & 0.927239 \\
\hline 75 & $\mathrm{H}$ & 0 & 2.279908 & -3.774045 & -0.585554 \\
\hline 76 & $\mathrm{H}$ & 0 & -0.507772 & -3.403861 & -3.829347 \\
\hline 77 & $\mathrm{H}$ & 0 & -5.237210 & 0.283771 & 1.543402 \\
\hline 78 & $\mathrm{H}$ & 0 & -7.338682 & -2.472918 & -3.022644 \\
\hline 79 & $\mathrm{H}$ & 0 & -4.841960 & -2.452459 & -2.916126 \\
\hline 80 & $\mathrm{H}$ & 0 & -8.596190 & -1.317468 & -1.184300 \\
\hline 81 & $\mathrm{H}$ & 0 & -12.025657 & 0.624497 & 1.649641 \\
\hline 82 & $\mathrm{H}$ & 0 & -11.272446 & 0.673276 & 0.046298 \\
\hline 83 & $\mathrm{H}$ & 0 & -11.350271 & -0.854430 & 0.944917 \\
\hline 84 & $\mathrm{H}$ & 0 & -10.445883 & 2.505418 & 2.361111 \\
\hline 85 & $\mathrm{H}$ & 0 & -9.667646 & 2.494971 & 0.773219 \\
\hline 86 & $\mathrm{H}$ & 0 & -8.690975 & 2.294322 & 2.243492 \\
\hline 87 & $\mathrm{H}$ & 0 & -9.852915 & -1.223887 & 2.955152 \\
\hline 88 & $\mathrm{H}$ & 0 & -10.560838 & 0.211924 & 3.706236 \\
\hline 89 & $\mathrm{H}$ & 0 & -8.801769 & 0.082882 & 3.541669 \\
\hline 90 & $\mathrm{C}$ & 0 & 2.705374 & 6.610619 & -2.228311 \\
\hline 91 & $\mathrm{H}$ & 0 & 2.732849 & 7.392677 & -2.992103 \\
\hline 92 & $\mathrm{H}$ & 0 & 2.489901 & 7.091070 & -1.268744 \\
\hline 93 & $\mathrm{H}$ & 0 & 3.707974 & 6.176103 & -2.161647 \\
\hline 94 & C & 0 & 1.659281 & 5.542495 & -2.559614 \\
\hline 95 & $\mathrm{H}$ & 0 & 0.673437 & 6.013202 & -2.654201 \\
\hline 96 & $\mathrm{H}$ & 0 & 1.881008 & 5.105234 & -3.540798 \\
\hline
\end{tabular}

Standard basis: $6-311++G(d, p) \quad(5 D, 7 F)$

$\begin{array}{llr}\text { SCF Done: } & \text { E (RB3LYP }=-2277.19574124 & \text { A.U. after } 2 \text { cycles } \\ & \text { NFock }=2 \text { Conv=0.37D-09 } \quad-\mathrm{V} / \mathrm{T}=2.0043\end{array}$

Full mass-weighted force constant matrix:

$\begin{array}{ccccccc}\text { Low frequencies --- } & -1.7036 & -0.0009 & 0.0008 & 0.0019 & 1.2297 & 1.7771 \\ \text { Low frequencies --- } & 3.5469 & 6.8960 & 8.1273 & & \\ \text { Diagonal vibrational polarizability: } & & & \\ 407.0984171 & 816.7210318 & 545.4044106 & \end{array}$


Zero-point correction=

Thermal correction to Energy=

Thermal correction to Enthalpy=

Thermal correction to Gibbs Free Energy=

Sum of electronic and zero-point Energies=

Sum of electronic and thermal Energies=

Sum of electronic and thermal Enthalpies=

Sum of electronic and thermal Free Energies=
0.789362 (Hartree/Particle)

0.839707

0.840651

0.692363

$-2276.406380$

$-2276.356035$

$-2276.355091$

$-2276.503378$ 
Model Fragment A

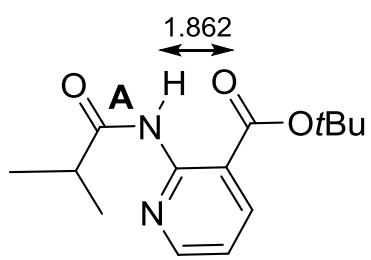

\section{A: $27.4 \mathrm{~kJ} / \mathrm{mol}$}

$\begin{array}{ll}\text { Stoichiometry } & \mathrm{C} 14 \mathrm{H} 20 \mathrm{~N} 2 \mathrm{O} 3 \\ \text { Framework group } & \mathrm{C} 1[\mathrm{X}(\mathrm{C} 14 \mathrm{H} 20 \mathrm{~N} 2 \mathrm{O} 3)]\end{array}$

Standard orientation:

\begin{tabular}{|c|c|c|c|c|c|}
\hline \multirow{2}{*}{$\begin{array}{l}\text { Center } \\
\text { Number }\end{array}$} & \multirow[t]{2}{*}{ Atom } & \multirow{2}{*}{$\begin{array}{l}\text { Atomic } \\
\text { Type }\end{array}$} & \multicolumn{3}{|c|}{ Coordinates (Angstroms) } \\
\hline & & & $\mathrm{X}$ & Y & z \\
\hline 1 & $\mathrm{~N}$ & 0 & 1.407509 & -0.649817 & -0.005082 \\
\hline 2 & $\mathrm{C}$ & 0 & 0.949502 & 0.653171 & -0.052673 \\
\hline 3 & $\mathrm{C}$ & 0 & 0.069424 & 3.271535 & -0.147949 \\
\hline 4 & $\mathrm{C}$ & 0 & -0.459177 & 0.912471 & -0.050899 \\
\hline 5 & $\mathrm{~N}$ & 0 & 1.840756 & 1.650642 & -0.093726 \\
\hline 6 & $\mathrm{C}$ & 0 & 1.411966 & 2.909562 & -0.142917 \\
\hline 7 & C & 0 & -0.866078 & 2.247698 & -0.099209 \\
\hline 8 & $\mathrm{C}$ & 0 & -1.473248 & -0.177596 & 0.002628 \\
\hline 9 & 0 & 0 & -2.725434 & 0.298250 & -0.022140 \\
\hline 10 & $\mathrm{C}$ & 0 & -3.917092 & -0.590454 & 0.025575 \\
\hline 11 & C & 0 & -3.931444 & -1.371415 & 1.341114 \\
\hline 12 & C & 0 & -5.070495 & 0.411253 & -0.025260 \\
\hline 13 & C & 0 & -3.934360 & -1.503744 & -1.201751 \\
\hline 14 & O & 0 & -1.198859 & -1.364316 & 0.063403 \\
\hline 15 & C & 0 & 2.678334 & -1.250759 & -0.066847 \\
\hline 16 & C & 0 & 3.948344 & -0.414458 & -0.141408 \\
\hline 17 & C & 0 & 5.068398 & -1.209589 & -0.821650 \\
\hline 18 & C & 0 & 4.348314 & 0.014949 & 1.285545 \\
\hline 19 & 0 & 0 & 2.706155 & -2.466537 & -0.030366 \\
\hline 20 & $\mathrm{H}$ & 0 & 0.663194 & -1.345168 & 0.047287 \\
\hline 21 & $\mathrm{H}$ & 0 & -0.227959 & 4.311468 & -0.185611 \\
\hline 22 & $\mathrm{H}$ & 0 & 2.189146 & 3.668797 & -0.176271 \\
\hline 23 & $\mathrm{H}$ & 0 & -1.925237 & 2.465593 & -0.098161 \\
\hline 24 & $\mathrm{H}$ & 0 & -3.851701 & -0.689230 & 2.191372 \\
\hline 25 & $\mathrm{H}$ & 0 & -4.879838 & -1.908491 & 1.427438 \\
\hline 26 & $\mathrm{H}$ & 0 & -3.118414 & -2.093826 & 1.387912 \\
\hline 27 & $\mathrm{H}$ & 0 & -6.024785 & -0.119824 & 0.002608 \\
\hline 28 & $\mathrm{H}$ & 0 & -5.029604 & 1.092178 & 0.827872 \\
\hline 29 & $\mathrm{H}$ & 0 & -5.030498 & 1.000998 & -0.943812 \\
\hline 30 & $\mathrm{H}$ & 0 & -3.856906 & -0.913487 & -2.118394 \\
\hline 31 & $\mathrm{H}$ & 0 & -4.882734 & -2.047048 & -1.229559 \\
\hline 32 & $\mathrm{H}$ & 0 & -3.121164 & -2.227002 & -1.175350 \\
\hline 33 & $\mathrm{H}$ & 0 & 3.733103 & 0.489638 & -0.709824 \\
\hline 34 & $\mathrm{H}$ & 0 & 5.301466 & -2.119894 & -0.266785 \\
\hline 35 & $\mathrm{H}$ & 0 & 5.971070 & -0.594923 & -0.879668 \\
\hline 36 & $\mathrm{H}$ & 0 & 4.790224 & -1.502490 & -1.837067 \\
\hline 37 & $\mathrm{H}$ & 0 & 4.534865 & -0.861091 & 1.913202 \\
\hline 38 & $\mathrm{H}$ & 0 & 5.265667 & 0.608554 & 1.248609 \\
\hline 39 & $\mathrm{H}$ & 0 & 3.573017 & 0.624553 & 1.752895 \\
\hline
\end{tabular}

Standard basis: $6-311++G(d, p)$ (5D, 7F) 
SCF Done: E (RB3LYP $)=-881.011683052$

$\mathrm{NFOCk}=1$ Conv $=0.69 \mathrm{D}-08$
A.U. after 1 cycles

$-\mathrm{V} / \mathrm{T}=2.0042$

Full mass-weighted force constant matrix:

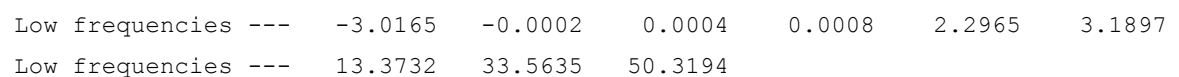

\section{Zero-point correction=}

Thermal correction to Energy=

Thermal correction to Enthalpy=

Thermal correction to Gibbs Free Energy=

Sum of electronic and zero-point Energies=

Sum of electronic and thermal Energies=

Sum of electronic and thermal Enthalpies=

Sum of electronic and thermal Free Energies=

\author{
0.325403 (Hartree/Particle) \\ 0.345377 \\ 0.346321 \\ 0.275889 \\ $-880.686280$ \\ $-880.666307$ \\ $-880.665362$ \\ $-880.735794$
}


Stoichiometry

C15H23NO2

Framework group

C1 [X (C15H23NO2) ]

Standard orientation:

\begin{tabular}{|c|c|c|c|c|c|}
\hline \multirow{2}{*}{$\begin{array}{l}\text { Center } \\
\text { Number }\end{array}$} & \multirow[t]{2}{*}{ Atom } & \multirow{2}{*}{$\begin{array}{l}\text { Atomic } \\
\text { Type }\end{array}$} & \multicolumn{3}{|c|}{ Coordinates (Angstroms) } \\
\hline & & & $\mathrm{X}$ & $\mathrm{Y}$ & z \\
\hline 1 & C & 0 & -0.989921 & 1.338233 & 0.336195 \\
\hline 2 & C & 0 & 0.432221 & 3.572044 & -0.458878 \\
\hline 3 & $\mathrm{C}$ & 0 & 0.378506 & 1.203714 & -0.002335 \\
\hline 4 & $\mathrm{~N}$ & 0 & -1.602693 & 2.532511 & 0.271323 \\
\hline 5 & C & 0 & -0.917854 & 3.605165 & -0.122265 \\
\hline 6 & C & 0 & 1.083766 & 2.350775 & -0.387653 \\
\hline 7 & $\mathrm{C}$ & 0 & 1.081596 & -0.119743 & 0.023394 \\
\hline 8 & 0 & 0 & 2.418715 & 0.043173 & 0.069381 \\
\hline 9 & C & 0 & 3.356137 & -1.105204 & 0.059199 \\
\hline 10 & C & 0 & 4.720362 & -0.416159 & 0.102478 \\
\hline 11 & C & 0 & 3.142535 & -1.962842 & 1.308825 \\
\hline 12 & C & 0 & 3.192983 & -1.905595 & -1.235250 \\
\hline 13 & 0 & 0 & 0.525681 & -1.195804 & -0.005641 \\
\hline 14 & C & 0 & -3.544375 & -1.617861 & 0.009517 \\
\hline 15 & C & 0 & -4.101911 & -2.302969 & -1.246417 \\
\hline 16 & C & 0 & -4.686384 & -1.204688 & 0.949110 \\
\hline 17 & $\mathrm{H}$ & 0 & 0.951499 & 4.472687 & -0.763189 \\
\hline 18 & $\mathrm{H}$ & 0 & -1.475970 & 4.537051 & -0.163813 \\
\hline 19 & $\mathrm{H}$ & 0 & 2.134171 & 2.267403 & -0.631853 \\
\hline 20 & $\mathrm{H}$ & 0 & 4.814223 & 0.196848 & 1.001555 \\
\hline 21 & $\mathrm{H}$ & 0 & 5.516150 & -1.164841 & 0.108796 \\
\hline 22 & $\mathrm{H}$ & 0 & 4.857749 & 0.225563 & -0.770881 \\
\hline 23 & $\mathrm{H}$ & 0 & 3.927154 & -2.722524 & 1.362446 \\
\hline 24 & $\mathrm{H}$ & 0 & 3.206868 & -1.346552 & 2.209224 \\
\hline 25 & $\mathrm{H}$ & 0 & 2.175451 & -2.462447 & 1.289441 \\
\hline 26 & $\mathrm{H}$ & 0 & 2.228178 & -2.408277 & -1.276117 \\
\hline 27 & $\mathrm{H}$ & 0 & 3.982476 & -2.659919 & -1.292814 \\
\hline 28 & $\mathrm{H}$ & 0 & 3.289474 & -1.249701 & -2.104414 \\
\hline 29 & $\mathrm{H}$ & 0 & -2.915992 & -2.347839 & 0.537380 \\
\hline 30 & $\mathrm{H}$ & 0 & -4.732679 & -1.614195 & -1.819556 \\
\hline 31 & $\mathrm{H}$ & 0 & -4.713033 & -3.172361 & -0.985482 \\
\hline 32 & $\mathrm{H}$ & 0 & -3.296841 & -2.643595 & -1.904080 \\
\hline 33 & $\mathrm{H}$ & 0 & -5.325822 & -0.451233 & 0.475529 \\
\hline 34 & $\mathrm{H}$ & 0 & -5.315435 & -2.064414 & 1.199175 \\
\hline 35 & $\mathrm{H}$ & 0 & -4.317702 & -0.784955 & 1.888271 \\
\hline 36 & C & 0 & -1.882127 & 0.207898 & 0.790659 \\
\hline 37 & $\mathrm{H}$ & 0 & -2.592706 & 0.644646 & 1.495096 \\
\hline 38 & $\mathrm{H}$ & 0 & -1.305771 & -0.562538 & 1.302552 \\
\hline 39 & C & 0 & -2.643296 & -0.433943 & -0.387596 \\
\hline 40 & $\mathrm{H}$ & 0 & -1.910834 & -0.784479 & -1.120815 \\
\hline 41 & $\mathrm{H}$ & 0 & -3.250345 & 0.336214 & -0.879543 \\
\hline
\end{tabular}

Standard basis: $6-311++G(d, p) \quad(5 D, 7 F)$

SCF Done: $\mathrm{E}(\mathrm{RB} 3 \mathrm{LYP})=-790.901155082$

A.U. after 1 cycles

NFock $=1$ Conv $=0.15 \mathrm{D}-08$

$-\mathrm{V} / \mathrm{T}=2.0045$ 
Full mass-weighted force constant matrix:

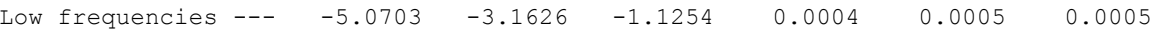

Low frequencies --- $\quad \begin{array}{llll}17.9779 & 28.3952 \quad 35.1810\end{array}$

Zero-point correction=

Thermal correction to Energy=

Thermal correction to Enthalpy=

Thermal correction to Gibbs Free Energy=

Sum of electronic and zero-point Energies=

Sum of electronic and thermal Energies=

Sum of electronic and thermal Enthalpies=

Sum of electronic and thermal Free Energies=

0.354769 (Hartree/Particle)
0.374476
0.375420
0.305267
$\quad-790.546386$
-790.526679
-790.525735
-790.595889


Stoichiometry C14H22N2O2

Framework group C1[X(C14H22N2O2)]

Standard orientation:

\begin{tabular}{|c|c|c|c|c|c|}
\hline \multirow{2}{*}{$\begin{array}{l}\text { Center } \\
\text { Number }\end{array}$} & \multirow[t]{2}{*}{ Atom } & Atomic & \multicolumn{3}{|c|}{ Coordinates (Angstroms) } \\
\hline & & Type & $\mathrm{x}$ & Y & z \\
\hline 1 & $\mathrm{~N}$ & 0 & 1.606962 & -0.416747 & -0.642196 \\
\hline 2 & $\mathrm{C}$ & 0 & 1.100936 & 0.790524 & -0.298755 \\
\hline 3 & C & 0 & 0.180552 & 3.313374 & 0.376724 \\
\hline 4 & $\mathrm{C}$ & 0 & -0.303316 & 0.982691 & -0.056900 \\
\hline 5 & $\mathrm{~N}$ & 0 & 1.975681 & 1.815075 & -0.198702 \\
\hline 6 & C & 0 & 1.520093 & 3.020163 & 0.121978 \\
\hline 7 & C & 0 & -0.728422 & 2.269219 & 0.282110 \\
\hline 8 & C & 0 & -1.262279 & -0.135593 & -0.162300 \\
\hline 9 & 0 & 0 & -2.526269 & 0.244382 & 0.100133 \\
\hline 10 & C & 0 & -3.666017 & -0.700207 & 0.059408 \\
\hline 11 & C & 0 & -4.848712 & 0.198359 & 0.423120 \\
\hline 12 & C & 0 & -3.830164 & -1.264759 & -1.353942 \\
\hline 13 & C & 0 & -3.477066 & -1.797179 & 1.110283 \\
\hline 14 & 0 & 0 & -0.950923 & -1.282559 & -0.454242 \\
\hline 15 & C & 0 & 3.861666 & -0.955675 & 0.355223 \\
\hline 16 & C & 0 & 5.340495 & -1.074334 & -0.033228 \\
\hline 17 & C & 0 & 3.375867 & -2.206725 & 1.096179 \\
\hline 18 & $\mathrm{H}$ & 0 & 0.932841 & -1.169442 & -0.703631 \\
\hline 19 & $\mathrm{H}$ & 0 & -0.129717 & 4.316503 & 0.638642 \\
\hline 20 & $\mathrm{H}$ & 0 & 2.271940 & 3.803894 & 0.183088 \\
\hline 21 & $\mathrm{H}$ & 0 & -1.781392 & 2.433287 & 0.468578 \\
\hline 22 & $\mathrm{H}$ & 0 & -4.955541 & 1.007881 & -0.302433 \\
\hline 23 & $\mathrm{H}$ & 0 & -5.772511 & -0.385091 & 0.429163 \\
\hline 24 & $\mathrm{H}$ & 0 & -4.709894 & 0.636595 & 1.414005 \\
\hline 25 & $\mathrm{H}$ & 0 & -4.756111 & -1.844249 & -1.404632 \\
\hline 26 & $\mathrm{H}$ & 0 & -3.899340 & -0.452862 & -2.082493 \\
\hline 27 & $\mathrm{H}$ & 0 & -2.998216 & -1.912825 & -1.624057 \\
\hline 28 & $\mathrm{H}$ & 0 & -2.642558 & -2.450043 & 0.860364 \\
\hline 29 & $\mathrm{H}$ & 0 & -4.387543 & -2.399963 & 1.168972 \\
\hline 30 & $\mathrm{H}$ & 0 & -3.300425 & -1.355295 & 2.094316 \\
\hline 31 & $\mathrm{H}$ & 0 & 3.748419 & -0.090533 & 1.017802 \\
\hline 32 & $\mathrm{H}$ & 0 & 5.501862 & -1.911670 & -0.721976 \\
\hline 33 & $\mathrm{H}$ & 0 & 5.965068 & -1.248042 & 0.847503 \\
\hline 34 & $\mathrm{H}$ & 0 & 5.698989 & -0.164023 & -0.522813 \\
\hline 35 & $\mathrm{H}$ & 0 & 3.482668 & -3.100126 & 0.469567 \\
\hline 36 & $\mathrm{H}$ & 0 & 3.956740 & -2.371794 & 2.008269 \\
\hline 37 & $\mathrm{H}$ & 0 & 2.324693 & -2.120742 & 1.383355 \\
\hline 38 & C & 0 & 3.017497 & -0.668438 & -0.902755 \\
\hline 39 & $\mathrm{H}$ & 0 & 3.432435 & 0.195185 & -1.425286 \\
\hline 40 & $\mathrm{H}$ & 0 & 3.069853 & -1.528054 & -1.580015 \\
\hline
\end{tabular}

Standard basis: $6-311++G(d, p) \quad(5 D, 7 F)$

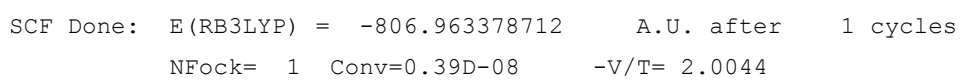

Full mass-weighted force constant matrix:

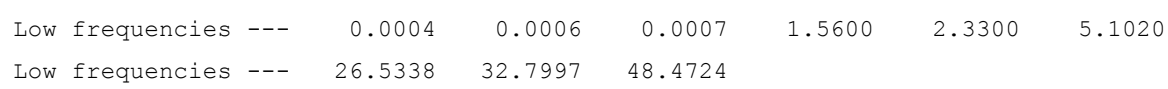




\section{Zero-point correction=}

Thermal correction to Energy=

Thermal correction to Enthalpy=

Thermal correction to Gibbs Free Energy=

Sum of electronic and zero-point Energies=

Sum of electronic and thermal Energies=

Sum of electronic and thermal Enthalpies=

Sum of electronic and thermal Free Energies=
0.343917 (Hartree/Particle)

0.363341

0.364285

0.295474

$-806.619462$

$-806.600038$

$-806.599094$

$-806.667905$ 
Framework group C1[X(C15H21NO3)]

Standard orientation:

\begin{tabular}{|c|c|c|c|c|c|}
\hline \multirow{2}{*}{$\begin{array}{l}\text { Center } \\
\text { Number }\end{array}$} & \multirow[t]{2}{*}{ Atom } & \multirow{2}{*}{$\begin{array}{l}\text { Atomic } \\
\text { Type }\end{array}$} & \multicolumn{3}{|c|}{ Coordinates (Angstroms) } \\
\hline & & & $\mathrm{x}$ & Y & $\mathrm{Z}$ \\
\hline 1 & C & 0 & -1.011142 & 1.531428 & 0.462634 \\
\hline 2 & $\mathrm{C}$ & 0 & 0.384959 & 3.691864 & -0.518068 \\
\hline 3 & C & 0 & 0.370507 & 1.394204 & 0.207508 \\
\hline 4 & $\mathrm{~N}$ & 0 & -1.655602 & 2.685331 & 0.240614 \\
\hline 5 & $\mathrm{C}$ & 0 & -0.974182 & 3.732869 & -0.227277 \\
\hline 6 & C & 0 & 1.059762 & 2.498878 & -0.300676 \\
\hline 7 & C & 0 & 1.105483 & 0.118815 & 0.492680 \\
\hline 8 & 0 & 0 & 2.245723 & 0.052660 & -0.213332 \\
\hline 9 & C & 0 & 3.169212 & -1.104852 & -0.121804 \\
\hline 10 & C & 0 & 2.453671 & -2.376966 & -0.581339 \\
\hline 11 & C & 0 & 4.275362 & -0.719778 & -1.104367 \\
\hline 12 & C & 0 & 3.721058 & -1.221676 & 1.300904 \\
\hline 13 & 0 & 0 & 0.730409 & -0.725843 & 1.277286 \\
\hline 14 & C & 0 & -2.156027 & -0.681085 & -0.097793 \\
\hline 15 & C & 0 & -3.304082 & -1.638241 & 0.231505 \\
\hline 16 & C & 0 & -3.003548 & -3.060574 & -0.251740 \\
\hline 17 & C & 0 & -4.597899 & -1.084377 & -0.401896 \\
\hline 18 & 0 & 0 & -1.516797 & -0.776651 & -1.121232 \\
\hline 19 & $\mathrm{H}$ & 0 & 0.895627 & 4.565912 & -0.903726 \\
\hline 20 & $\mathrm{H}$ & 0 & -1.546707 & 4.644161 & -0.377005 \\
\hline 21 & $\mathrm{H}$ & 0 & 2.116016 & 2.410428 & -0.516716 \\
\hline 22 & $\mathrm{H}$ & 0 & 1.672902 & -2.670690 & 0.117984 \\
\hline 23 & $\mathrm{H}$ & 0 & 2.005336 & -2.227352 & -1.566242 \\
\hline 24 & $\mathrm{H}$ & 0 & 3.181027 & -3.190065 & -0.656288 \\
\hline 25 & $\mathrm{H}$ & 0 & 5.033886 & -1.505528 & -1.137719 \\
\hline 26 & $\mathrm{H}$ & 0 & 3.868398 & -0.587498 & -2.109113 \\
\hline 27 & $\mathrm{H}$ & 0 & 4.757420 & 0.211984 & -0.799271 \\
\hline 28 & $\mathrm{H}$ & 0 & 4.499144 & -1.989688 & 1.322367 \\
\hline 29 & $\mathrm{H}$ & 0 & 2.941816 & -1.496174 & 2.009994 \\
\hline 30 & $\mathrm{H}$ & 0 & 4.171443 & -0.276503 & 1.615383 \\
\hline 31 & $\mathrm{H}$ & 0 & -3.433302 & -1.642533 & 1.320125 \\
\hline 32 & $\mathrm{H}$ & 0 & -2.837335 & -3.071868 & -1.330710 \\
\hline 33 & $\mathrm{H}$ & 0 & -3.841678 & -3.724055 & -0.021402 \\
\hline 34 & $\mathrm{H}$ & 0 & -2.108468 & -3.463030 & 0.229262 \\
\hline 35 & $\mathrm{H}$ & 0 & -4.509865 & -1.065752 & -1.491309 \\
\hline 36 & $\mathrm{H}$ & 0 & -4.820144 & -0.069375 & -0.061778 \\
\hline 37 & $\mathrm{H}$ & 0 & -5.446720 & -1.720602 & -0.137762 \\
\hline 38 & C & 0 & -1.890881 & 0.408542 & 0.943977 \\
\hline 39 & $\mathrm{H}$ & 0 & -2.846138 & 0.836964 & 1.256754 \\
\hline 40 & $\mathrm{H}$ & 0 & -1.451647 & -0.101696 & 1.805621 \\
\hline
\end{tabular}

Standard basis: $6-311++G(d, p) \quad(5 D, 7 F)$

$\begin{array}{llr}\text { SCF Done: } & \text { E (RB3LYP }=-864.939006781 & \text { A.U. after } 1 \text { cycles } \\ \text { NFock }=1 \text { Conv }=0.48 \mathrm{D}-08 & -\mathrm{V} / \mathrm{T}=2.0043\end{array}$

Full mass-weighted force constant matrix:

$\begin{array}{lllllll}\text { Low frequencies --- } & -4.4024 & -3.2738 & -1.2288 & 0.0005 & 0.0006 & 0.0008 \\ \text { Low frequencies --- } & 15.3575 & 17.9514 & 37.4215 & & \end{array}$


Zero-point correction=

Thermal correction to Energy=

Thermal correction to Enthalpy=

Thermal correction to Gibbs Free Energy=

Sum of electronic and zero-point Energies=

Sum of electronic and thermal Energies=

Sum of electronic and thermal Enthalpies=

Sum of electronic and thermal Free Energies=
0.335510 (Hartree/Particle)

0.355965

0.356910

0.284411

$-864.603497$

$-864.583041$

$-864.582097$

$-864.654595$ 


\section{Model Fragment B}

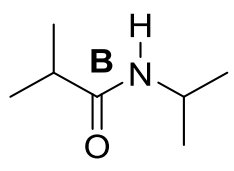

\section{B: $84.1 \mathrm{~kJ} / \mathrm{mol}$}

$\begin{array}{ll}\text { Stoichiometry } & \text { C7H15NO } \\ \text { Framework group } & \text { C1[X(C7H15NO)] }\end{array}$

Standard orientation:

\begin{tabular}{|c|c|c|c|c|c|}
\hline \multirow{2}{*}{$\begin{array}{l}\text { Center } \\
\text { Number }\end{array}$} & \multirow[t]{2}{*}{ Atom } & \multirow{2}{*}{$\begin{array}{l}\text { Atomic } \\
\text { Type }\end{array}$} & \multicolumn{3}{|c|}{ Coordinates (Angstroms) } \\
\hline & & & $\mathrm{x}$ & $\mathrm{Y}$ & Z \\
\hline 1 & $\mathrm{C}$ & 0 & -2.568140 & -1.144584 & 0.650670 \\
\hline 2 & $\mathrm{C}$ & 0 & -1.822229 & 0.189408 & 0.489904 \\
\hline 3 & $\mathrm{C}$ & 0 & -2.684338 & 1.230053 & -0.241590 \\
\hline 4 & C & 0 & -0.523174 & -0.040001 & -0.290069 \\
\hline 5 & $\mathrm{~N}$ & 0 & 0.628513 & 0.269180 & 0.374323 \\
\hline 6 & C & 0 & 1.964151 & 0.086137 & -0.202416 \\
\hline 7 & $\mathrm{C}$ & 0 & 2.898140 & 1.183738 & 0.310821 \\
\hline 8 & $\mathrm{C}$ & 0 & 2.506778 & -1.320713 & 0.079713 \\
\hline 9 & 0 & 0 & -0.527376 & -0.464142 & -1.437830 \\
\hline 10 & $\mathrm{H}$ & 0 & -1.970859 & -1.875448 & 1.203208 \\
\hline 11 & $\mathrm{H}$ & 0 & -2.797497 & -1.566907 & -0.330029 \\
\hline 12 & $\mathrm{H}$ & 0 & -3.505908 & -0.994055 & 1.192591 \\
\hline 13 & $\mathrm{H}$ & 0 & -1.577203 & 0.576458 & 1.486769 \\
\hline 14 & $\mathrm{H}$ & 0 & -2.914782 & 0.883490 & -1.251261 \\
\hline 15 & $\mathrm{H}$ & 0 & -3.623950 & 1.391061 & 0.294121 \\
\hline 16 & $\mathrm{H}$ & 0 & -2.170236 & 2.191860 & -0.322319 \\
\hline 17 & $\mathrm{H}$ & 0 & 0.561926 & 0.566542 & 1.335947 \\
\hline 18 & $\mathrm{H}$ & 0 & 1.832683 & 0.194967 & -1.281196 \\
\hline 19 & $\mathrm{H}$ & 0 & 3.018965 & 1.123592 & 1.398655 \\
\hline 20 & $\mathrm{H}$ & 0 & 3.890244 & 1.079179 & -0.134793 \\
\hline 21 & $\mathrm{H}$ & 0 & 2.513959 & 2.175908 & 0.062040 \\
\hline 22 & $\mathrm{H}$ & 0 & 2.636531 & -1.481841 & 1.155073 \\
\hline 23 & $\mathrm{H}$ & 0 & 1.820730 & -2.077729 & -0.304776 \\
\hline 24 & $\mathrm{H}$ & 0 & 3.477687 & -1.462417 & -0.403852 \\
\hline
\end{tabular}

Standard basis: $6-311++G(d, p) \quad(5 D, 7 F)$

SCF Done: E (RB3LYP $)=-405.908895155$ A.U. after 1 cycles NFock $=1$ Conv $=0.33 \mathrm{D}-08 \quad-\mathrm{V} / \mathrm{T}=2.0046$

Full mass-weighted force constant matrix:

Low frequencies --- $\quad-2.3103 \quad-0.0006 \quad 0.0006 \quad 0.0009 \quad 2.5254 \quad 6.0099$

Low frequencies --- $22.2098 \quad 36.0468 \quad 83.6840$

\section{Zero-point correction=}

Thermal correction to Energy=

Thermal correction to Enthalpy=

Thermal correction to Gibbs Free Energy=

Sum of electronic and zero-point Energies=

Sum of electronic and thermal Energies=

Sum of electronic and thermal Enthalpies=

Sum of electronic and thermal Free Energies=

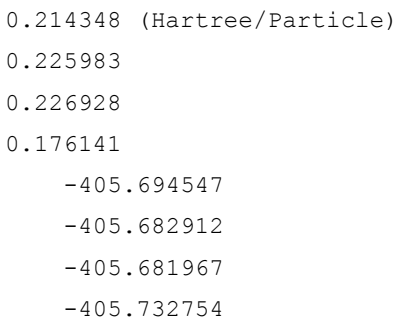


$\begin{array}{ll}\text { Stoichiometry } & \mathrm{C} 8 \mathrm{H} 18 \\ \text { Framework group } & \mathrm{C} 1[\mathrm{X}(\mathrm{C} 8 \mathrm{H} 18)]\end{array}$

Standard orientation:

\begin{tabular}{|c|c|c|c|c|c|}
\hline \multirow{2}{*}{$\begin{array}{l}\text { Center } \\
\text { Number }\end{array}$} & \multirow[t]{2}{*}{ Atom } & \multirow{2}{*}{$\begin{array}{l}\text { Atomic } \\
\text { Type }\end{array}$} & \multicolumn{3}{|c|}{ Coordinates (Angstroms) } \\
\hline & & & $\mathrm{X}$ & $\mathrm{Y}$ & z \\
\hline 1 & C & 0 & 2.280338 & -1.110860 & 0.904142 \\
\hline 2 & $\mathrm{C}$ & 0 & 1.976208 & -0.212345 & -0.303491 \\
\hline 3 & $\mathrm{C}$ & 0 & 3.087545 & 0.829977 & -0.491578 \\
\hline 4 & $\mathrm{C}$ & 0 & -1.976208 & 0.212345 & -0.303491 \\
\hline 5 & C & 0 & -3.087546 & -0.829976 & -0.491577 \\
\hline 6 & C & 0 & -2.280336 & 1.110860 & 0.904142 \\
\hline 7 & $\mathrm{H}$ & 0 & 1.547168 & -1.913338 & 1.015303 \\
\hline 8 & $\mathrm{H}$ & 0 & 2.280510 & -0.526789 & 1.831634 \\
\hline 9 & $\mathrm{H}$ & 0 & 3.265589 & -1.576514 & 0.806413 \\
\hline 10 & $\mathrm{H}$ & 0 & 1.958504 & -0.847816 & -1.199954 \\
\hline 11 & $\mathrm{H}$ & 0 & 3.148931 & 1.496224 & 0.376226 \\
\hline 12 & $\mathrm{H}$ & 0 & 4.064522 & 0.352136 & -0.611339 \\
\hline 13 & $\mathrm{H}$ & 0 & 2.907572 & 1.450475 & -1.374651 \\
\hline 14 & $\mathrm{H}$ & 0 & -1.958504 & 0.847816 & -1.199954 \\
\hline 15 & $\mathrm{H}$ & 0 & -3.148930 & -1.496225 & 0.376226 \\
\hline 16 & $\mathrm{H}$ & 0 & -4.064523 & -0.352135 & -0.611336 \\
\hline 17 & $\mathrm{H}$ & 0 & -2.907575 & -1.450473 & -1.374651 \\
\hline 18 & $\mathrm{H}$ & 0 & -2.280509 & 0.526789 & 1.831634 \\
\hline 19 & $\mathrm{H}$ & 0 & -1.547165 & 1.913337 & 1.015303 \\
\hline 20 & $\mathrm{H}$ & 0 & -3.265586 & 1.576516 & 0.806414 \\
\hline 21 & C & 0 & -0.601667 & -0.476239 & -0.195039 \\
\hline 22 & $\mathrm{H}$ & 0 & -0.594397 & -1.095038 & 0.710935 \\
\hline 23 & $\mathrm{H}$ & 0 & -0.496408 & -1.170725 & -1.038781 \\
\hline 24 & C & 0 & 0.601666 & 0.476239 & -0.195037 \\
\hline 25 & $\mathrm{H}$ & 0 & 0.594396 & 1.095036 & 0.710937 \\
\hline 26 & $\mathrm{H}$ & 0 & 0.496408 & 1.170725 & -1.038779 \\
\hline
\end{tabular}

Standard basis: $6-311++G(d, p) \quad(5 D, 7 F)$

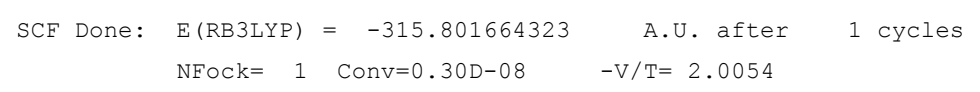

Full mass-weighted force constant matrix:

$\begin{array}{lrrrrrr}\text { Low frequencies --- } & -7.0637 & -0.0004 & -0.0000 & 0.0006 & 0.5386 & 2.2222 \\ \text { Low frequencies --- } & 37.9900 & 77.4015 & 121.3289 & & \end{array}$

Zero-point correction=

Thermal correction to Energy=

Thermal correction to Enthalpy=

Thermal correction to Gibbs Free Energy=

Sum of electronic and zero-point Energies=

Sum of electronic and thermal Energies=

Sum of electronic and thermal Enthalpies=

Sum of electronic and thermal Free Energies=

\author{
0.243840 (Hartree/Particle) \\ 0.254861 \\ 0.255805 \\ 0.207568 \\ $-315.557824$ \\ $-315.546804$ \\ $-315.545859$ \\ $-315.594097$
}


Stoichiometry C7H17N

Framework group $\mathrm{C} 1[\mathrm{X}(\mathrm{C} 7 \mathrm{H} 17 \mathrm{~N})]$

Standard orientation:

\begin{tabular}{|c|c|c|c|c|c|}
\hline \multirow{2}{*}{$\begin{array}{l}\text { Center } \\
\text { Number }\end{array}$} & \multirow[t]{2}{*}{ Atom } & \multirow{2}{*}{$\begin{array}{l}\text { Atomic } \\
\text { Type }\end{array}$} & \multicolumn{3}{|c|}{ Coordinates (Angstroms) } \\
\hline & & & $\mathrm{x}$ & $\mathrm{Y}$ & $\mathrm{Z}$ \\
\hline 1 & C & 0 & -3.071192 & 0.643628 & -0.612130 \\
\hline 2 & $\mathrm{C}$ & 0 & -1.904862 & -0.281292 & -0.240282 \\
\hline 3 & $\mathrm{C}$ & 0 & -2.112343 & -0.908480 & 1.144171 \\
\hline 4 & $\mathrm{~N}$ & 0 & 0.579339 & -0.413024 & -0.169800 \\
\hline 5 & $\mathrm{C}$ & 0 & 1.896714 & 0.227025 & -0.294799 \\
\hline 6 & $\mathrm{C}$ & 0 & 2.223642 & 1.051809 & 0.954713 \\
\hline 7 & $\mathrm{C}$ & 0 & 2.953965 & -0.854931 & -0.524239 \\
\hline 8 & $\mathrm{H}$ & 0 & -2.955687 & 1.053848 & -1.619862 \\
\hline 9 & $\mathrm{H}$ & 0 & -3.139392 & 1.485555 & 0.085693 \\
\hline 10 & $\mathrm{H}$ & 0 & -4.023977 & 0.107991 & -0.575580 \\
\hline 11 & $\mathrm{H}$ & 0 & -1.872869 & -1.093470 & -0.981588 \\
\hline 12 & $\mathrm{H}$ & 0 & -2.197955 & -0.129053 & 1.909861 \\
\hline 13 & $\mathrm{H}$ & 0 & -3.030356 & -1.503223 & 1.171638 \\
\hline 14 & $\mathrm{H}$ & 0 & -1.273295 & -1.551190 & 1.415419 \\
\hline 15 & $\mathrm{H}$ & 0 & 0.511894 & -1.157102 & -0.858462 \\
\hline 16 & $\mathrm{H}$ & 0 & 1.912456 & 0.909890 & -1.165403 \\
\hline 17 & $\mathrm{H}$ & 0 & 2.233686 & 0.408164 & 1.839011 \\
\hline 18 & $\mathrm{H}$ & 0 & 3.205365 & 1.523564 & 0.854383 \\
\hline 19 & $\mathrm{H}$ & 0 & 1.493454 & 1.846897 & 1.121888 \\
\hline 20 & $\mathrm{H}$ & 0 & 2.973990 & -1.553237 & 0.317661 \\
\hline 21 & $\mathrm{H}$ & 0 & 2.747221 & -1.425263 & -1.435926 \\
\hline 22 & $\mathrm{H}$ & 0 & 3.947522 & -0.411904 & -0.628362 \\
\hline 23 & C & 0 & -0.571023 & 0.475980 & -0.332233 \\
\hline 24 & $\mathrm{H}$ & 0 & -0.532689 & 1.024041 & -1.291981 \\
\hline 25 & $\mathrm{H}$ & 0 & -0.544155 & 1.233220 & 0.458999 \\
\hline
\end{tabular}

Standard basis: $6-311++G(d, p) \quad(5 D, 7 F)$

SCF Done: E (RB3LYP $)=-331.838594977$ A.U. after 1 cycles $\mathrm{NFOCk}=1$ Conv $=0.32 \mathrm{D}-08 \quad-\mathrm{V} / \mathrm{T}=2.0051$

\begin{tabular}{|c|c|c|c|c|c|c|c|c|c|c|c|c|}
\hline Alpha & virt. & - eigenvalues & s -- & 3.7 & 79743 & 3.8 & 1178 & 3.826 & 697 & 3.8497 & 3.85776 & \\
\hline Alpha & virt. & - eigenvalues & s -- & 3.8 & 87416 & 4.0 & 0651 & 4.038 & 838 & $4.2208^{\circ}$ & 4.28359 & \\
\hline Alpha & virt. & - eigenvalues & s -- & 4.3 & 31035 & 4.34 & 4057 & 4.809 & 972 & 4.93258 & 23.81265 & \\
\hline Alpha & virt. & - eigenvalues & $s--$ & 23.8 & 83903 & 23.8 & 9418 & 23.968 & 881 & 23.9777 & 23.98430 & \\
\hline Alpha & virt. & - eigenvalues & s -- & 23.9 & 98961 & 35.5 & 9934 & & & & & \\
\hline & & ondensed to at & atoms & (all & elect & rons) & & & & & & \\
\hline & & 1 & 2 & & 3 & & 4 & & & 5 & 6 & \\
\hline 1 & C & 5.597953 & -0.0043 & 4353 & -0.16 & 6679 & -0.00 & 09634 & -0 & 049176 & -0.001062 & \\
\hline 2 & C & -0.004353 & 5.8142 & 4246 & 0.12 & 9014 & -0.08 & 87312 & -0 & 143585 & 0.062246 & \\
\hline 3 & $\mathrm{C}$ & $-0.17 \mathrm{H}$ & -0.033 & 33336 & 0.01 & 14528 & -0.0 & 002887 & & .000185 & -0.007348 & 0.006558 \\
\hline
\end{tabular}

Full mass-weighted force constant matrix:

Low frequencies --- $\quad-2.7421 \quad-0.0002 \quad 0.0005 \quad 0.0006 \quad 2.5698 \quad 7.7496$

Low frequencies --- $\quad 38.4055 \quad 71.1661 \quad 126.3518$

Diagonal vibrational polarizability:

$$
8.4307964 \quad 6.8694582 \quad 3.4472416
$$


Zero-point correction=

Thermal correction to Energy=

Thermal correction to Enthalpy=

Thermal correction to Gibbs Free Energy=

Sum of electronic and zero-point Energies=

Sum of electronic and thermal Energies=

Sum of electronic and thermal Enthalpies=

Sum of electronic and thermal Free Energies=
0.232615 (Hartree/Particle)

0.243512

0.244456

0.196447

$-331.605980$

$-331.595083$

$-331.594139$

$-331.642148$ 
Stoichiometry C8H160

Framework group C1[X(C8H160)]

Standard orientation:

\begin{tabular}{|c|c|c|c|c|c|}
\hline \multirow{2}{*}{$\begin{array}{l}\text { Center } \\
\text { Number }\end{array}$} & \multirow[t]{2}{*}{ Atom } & Atomic & \multicolumn{3}{|c|}{ Coordinates (Angstroms) } \\
\hline & & Type & $\mathrm{X}$ & Y & z \\
\hline 1 & $\mathrm{C}$ & 0 & 3.049727 & -0.848497 & 0.151795 \\
\hline 2 & $\mathrm{C}$ & 0 & 1.889836 & -0.106791 & -0.519496 \\
\hline 3 & $\mathrm{C}$ & 0 & 2.173146 & 1.406675 & -0.626497 \\
\hline 4 & $\mathrm{C}$ & 0 & 0.575994 & -0.300659 & 0.244945 \\
\hline 5 & $\mathrm{C}$ & 0 & -2.011585 & -0.214767 & 0.169126 \\
\hline 6 & $\mathrm{C}$ & 0 & -2.199286 & 1.225046 & 0.667135 \\
\hline 7 & $\mathrm{C}$ & 0 & -3.196792 & -0.656567 & -0.700130 \\
\hline 8 & 0 & 0 & 0.554927 & -0.392043 & 1.454189 \\
\hline 9 & $\mathrm{H}$ & 0 & 2.867092 & -1.925743 & 0.187228 \\
\hline 10 & $\mathrm{H}$ & 0 & 3.184364 & -0.500967 & 1.177870 \\
\hline 11 & $\mathrm{H}$ & 0 & 3.979326 & -0.679530 & -0.398603 \\
\hline 12 & $\mathrm{H}$ & 0 & 1.746169 & -0.495454 & -1.534971 \\
\hline 13 & $\mathrm{H}$ & 0 & 2.325020 & 1.836500 & 0.367164 \\
\hline 14 & $\mathrm{H}$ & 0 & 3.078074 & 1.579531 & -1.214889 \\
\hline 15 & $\mathrm{H}$ & 0 & 1.353115 & 1.945840 & -1.108875 \\
\hline 16 & $\mathrm{H}$ & 0 & -1.959866 & -0.869251 & 1.045499 \\
\hline 17 & $\mathrm{H}$ & 0 & -1.379444 & 1.530728 & 1.320309 \\
\hline 18 & $\mathrm{H}$ & 0 & -2.250755 & 1.925840 & -0.174411 \\
\hline 19 & $\mathrm{H}$ & 0 & -3.129690 & 1.321917 & 1.233926 \\
\hline 20 & $\mathrm{H}$ & 0 & -3.278809 & -0.039364 & -1.601969 \\
\hline 21 & $\mathrm{H}$ & 0 & -3.095558 & -1.698983 & -1.017641 \\
\hline 22 & $\mathrm{H}$ & 0 & -4.138916 & -0.565380 & -0.152033 \\
\hline 23 & $\mathrm{C}$ & 0 & -0.691560 & -0.375311 & -0.596532 \\
\hline 24 & $\mathrm{H}$ & 0 & -0.655147 & -1.358096 & -1.089775 \\
\hline 25 & $\mathrm{H}$ & 0 & -0.621269 & 0.353981 & -1.414413 \\
\hline
\end{tabular}

Standard basis: $6-311++G(d, p) \quad(5 D, 7 F)$

SCF Done: E (RB3LYP $)=-389.839727583 \quad$ A.U. after 1 cycles $\mathrm{NFOCk}=1$ Conv $=0.35 \mathrm{D}-08 \quad-\mathrm{V} / \mathrm{T}=2.0048$

Full mass-weighted force constant matrix:

$\begin{array}{lllllll}\text { Low frequencies --- } & -4.9329 & -1.5565 & -0.0007 & -0.0007 & -0.0006 & 3.1098 \\ \text { Low frequencies --- } & 30.6891 & 48.5470 & 94.5766 & & \end{array}$

Zero-point correction=

Thermal correction to Energy=

Thermal correction to Enthalpy=

Thermal correction to Gibbs Free Energy=

Sum of electronic and zero-point Energies=

Sum of electronic and thermal Energies=

Sum of electronic and thermal Enthalpies=

Sum of electronic and thermal Free Energies=

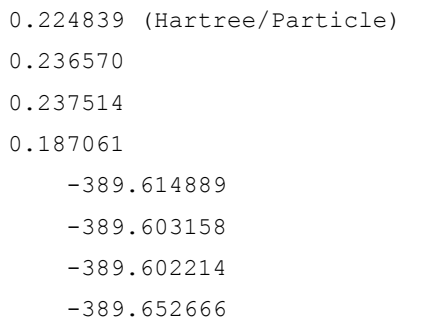


Model Fragment C

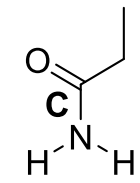

\section{C: $82.6 \mathrm{~kJ} / \mathrm{mol}$}

Stoichiometry C3H7NO

Framework group $\mathrm{C} 1[\mathrm{X}(\mathrm{C} 3 \mathrm{H} 7 \mathrm{NO})]$

Standard orientation:

\begin{tabular}{|c|c|c|c|c|c|}
\hline \multirow{2}{*}{$\begin{array}{l}\text { Center } \\
\text { Number }\end{array}$} & \multirow[t]{2}{*}{ Atom } & \multirow{2}{*}{$\begin{array}{l}\text { Atomic } \\
\text { Type }\end{array}$} & \multicolumn{3}{|c|}{ Coordinates (Angstroms) } \\
\hline & & & $\mathrm{X}$ & $\mathrm{Y}$ & z \\
\hline 1 & C & 0 & 1.966392 & 0.034042 & 0.192188 \\
\hline 2 & C & 0 & 0.698705 & -0.697356 & -0.248738 \\
\hline 3 & C & 0 & -0.564208 & 0.136880 & -0.048204 \\
\hline 4 & $\mathrm{~N}$ & 0 & -1.711242 & -0.582581 & 0.151768 \\
\hline 5 & 0 & 0 & -0.566076 & 1.354323 & -0.084786 \\
\hline 6 & $\mathrm{H}$ & 0 & 2.040676 & 1.001942 & -0.304724 \\
\hline 7 & $\mathrm{H}$ & 0 & 2.853450 & -0.555425 & -0.051692 \\
\hline 8 & $\mathrm{H}$ & 0 & 1.962133 & 0.214144 & 1.269980 \\
\hline 9 & $\mathrm{H}$ & 0 & 0.752380 & -0.928375 & -1.319792 \\
\hline 10 & $\mathrm{H}$ & 0 & 0.600815 & -1.657217 & 0.269357 \\
\hline 11 & $\mathrm{H}$ & 0 & -1.722038 & -1.588282 & 0.162822 \\
\hline 12 & $\mathrm{H}$ & 0 & -2.585446 & -0.084702 & 0.218485 \\
\hline
\end{tabular}

Standard basis: $6-311++G(d, p) \quad(5 D, 7 F)$

$\begin{array}{llrr}\text { SCF Done: } & \text { E (RB3LYP }= & -248.613046929 & \text { A.U. after } 1 \text { cycles } \\ \text { NFock }=1 \text { Conv=0.17D-08 } & -\mathrm{V} / \mathrm{T}=2.0042 & \end{array}$

Full mass-weighted force constant matrix:

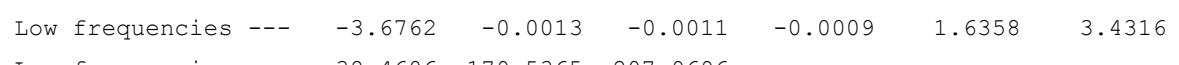

Low frequencies --- $\quad 38.4606 \quad 179.5365 \quad 207.9696$ 
Stoichiometry

$\mathrm{C} 4 \mathrm{H} 10$

Framework group

$\mathrm{C} 1[\mathrm{X}(\mathrm{C} 4 \mathrm{H} 10)]$

Standard orientation:

\begin{tabular}{|c|c|c|c|c|c|}
\hline \multirow{2}{*}{$\begin{array}{l}\text { Center } \\
\text { Number }\end{array}$} & \multirow[t]{2}{*}{ Atom } & \multirow{2}{*}{$\begin{array}{l}\text { Atomic } \\
\text { Type }\end{array}$} & \multicolumn{3}{|c|}{ Coordinates (Angstroms) } \\
\hline & & & $\mathrm{x}$ & $\mathrm{Y}$ & z \\
\hline 1 & C & 0 & -1.962432 & -0.121045 & -0.000004 \\
\hline 2 & $\mathrm{C}$ & 0 & -0.568390 & 0.514612 & 0.000001 \\
\hline 3 & $\mathrm{H}$ & 0 & -2.109650 & -0.750712 & -0.883198 \\
\hline 4 & $\mathrm{H}$ & 0 & -2.748776 & 0.639042 & 0.000012 \\
\hline 5 & $\mathrm{H}$ & 0 & -2.109650 & -0.750715 & 0.883195 \\
\hline 6 & $\mathrm{H}$ & 0 & -0.463838 & 1.165399 & -0.876739 \\
\hline 7 & $\mathrm{H}$ & 0 & -0.463848 & 1.165397 & 0.876740 \\
\hline 8 & C & 0 & 0.568392 & -0.514603 & 0.000002 \\
\hline 9 & $\mathrm{H}$ & 0 & 0.463828 & -1.165389 & -0.876735 \\
\hline 10 & $\mathrm{H}$ & 0 & 0.463829 & -1.165390 & 0.876741 \\
\hline 11 & C & 0 & 1.962428 & 0.121035 & 0.000000 \\
\hline 12 & $\mathrm{H}$ & 0 & 2.748781 & -0.639045 & 0.000005 \\
\hline 13 & $\mathrm{H}$ & 0 & 2.109670 & 0.750699 & -0.883200 \\
\hline 14 & $\mathrm{H}$ & 0 & 2.109663 & 0.750718 & 0.883186 \\
\hline
\end{tabular}

Standard basis: $6-311++G(d, p) \quad(5 D, 7 F)$

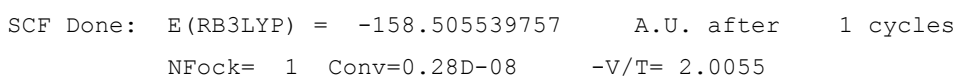

Full mass-weighted force constant matrix:

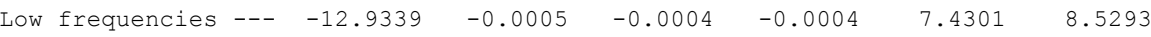

Low frequencies --- $\quad \begin{array}{lll}118.8223 & 221.1609 & 257.8792\end{array}$

Zero-point correction=

Thermal correction to Energy=

Thermal correction to Enthalpy=

Thermal correction to Gibbs Free Energy=

Sum of electronic and zero-point Energies=

Sum of electronic and thermal Energies=

Sum of electronic and thermal Enthalpies=

Sum of electronic and thermal Free Energies=
0.131280 (Hartree/Particle)

0.137084

0.138028

0.103234

$-158.374260$

$-158.368456$

$-158.367512$

$-158.402306$ 
Stoichiometry $\mathrm{C} 4 \mathrm{H} 8 \mathrm{O}$

Framework group $\mathrm{C} 1[\mathrm{X}(\mathrm{C} 4 \mathrm{H} 8 \mathrm{O})]$

Standard orientation:

\begin{tabular}{|c|c|c|c|c|c|}
\hline \multirow{2}{*}{$\begin{array}{l}\text { Center } \\
\text { Number }\end{array}$} & \multirow[t]{2}{*}{ Atom } & Atomic & \multicolumn{3}{|c|}{ Coordinates (Angstroms) } \\
\hline & & Type & $\mathrm{X}$ & Y & z \\
\hline 1 & $\mathrm{C}$ & 0 & 2.023757 & -0.020786 & -0.043883 \\
\hline 2 & $\mathrm{C}$ & 0 & 0.685651 & -0.747563 & 0.053931 \\
\hline 3 & $\mathrm{C}$ & 0 & -0.529426 & 0.170040 & 0.017902 \\
\hline 4 & 0 & 0 & -0.424293 & 1.377319 & 0.020266 \\
\hline 5 & $\mathrm{H}$ & 0 & 2.102239 & 0.532420 & -0.982119 \\
\hline 6 & $\mathrm{H}$ & 0 & 2.851667 & -0.732189 & 0.006020 \\
\hline 7 & $\mathrm{H}$ & 0 & 2.137618 & 0.699806 & 0.767857 \\
\hline 8 & $\mathrm{H}$ & 0 & 0.573342 & -1.487588 & -0.748703 \\
\hline 9 & $\mathrm{H}$ & 0 & 0.621506 & -1.327823 & 0.984246 \\
\hline 10 & C & 0 & -1.885851 & -0.509439 & -0.030809 \\
\hline 11 & $\mathrm{H}$ & 0 & -2.030834 & -0.957972 & -1.019663 \\
\hline 12 & $\mathrm{H}$ & 0 & -1.951129 & -1.319280 & 0.701316 \\
\hline 13 & $\mathrm{H}$ & 0 & -2.674853 & 0.220561 & 0.146074 \\
\hline
\end{tabular}

Standard basis: $6-311++G(d, p) \quad(5 D, 7 F)$

SCF Done: E (RB3LYP $)=-232.543050999 \quad$ A.U. after 1 cycles $\mathrm{NFOCk}=1$ Conv=0.90D-09 $-\mathrm{V} / \mathrm{T}=2.0044$

Full mass-weighted force constant matrix:

\begin{tabular}{|c|c|c|c|c|c|c|c|}
\hline Low & frequencies --- & -0.4118 & -0.0007 & 0.0005 & 0.0006 & 2.2267 & 3.6217 \\
\hline Low & frequencies --- & 50.9428 & 92.5757 & 199.5930 & & & \\
\hline
\end{tabular}

\section{Zero-point correction=}

Thermal correction to Energy=

Thermal correction to Enthalpy=

Thermal correction to Gibbs Free Energy=

Sum of electronic and zero-point Energies=

Sum of electronic and thermal Energies=

Sum of electronic and thermal Enthalpies=

Sum of electronic and thermal Free Energies=

0.111753 (Hartree/Particle)
0.118377
0.119322
0.081332
-232.431298
-232.424674
-232.423729
-232.461719


Stoichiometry C3H9N

Framework group C1[X(C3H9N)]

Standard orientation:

\begin{tabular}{|c|c|c|c|c|c|}
\hline \multirow{2}{*}{$\begin{array}{l}\text { Center } \\
\text { Number }\end{array}$} & \multirow[t]{2}{*}{ Atom } & Atomic & \multicolumn{3}{|c|}{ Coordinates (Angstroms) } \\
\hline & & Type & $\mathrm{X}$ & Y & z \\
\hline 1 & C & 0 & 1.930685 & 0.117058 & -0.000000 \\
\hline 2 & $\mathrm{C}$ & 0 & 0.540389 & -0.525866 & 0.000001 \\
\hline 3 & $\mathrm{~N}$ & 0 & -1.961829 & -0.027528 & -0.000000 \\
\hline 4 & $\mathrm{H}$ & 0 & 2.075263 & 0.747726 & -0.882927 \\
\hline 5 & $\mathrm{H}$ & 0 & 2.720762 & -0.638397 & -0.000002 \\
\hline 6 & $\mathrm{H}$ & 0 & 2.075264 & 0.747725 & 0.882928 \\
\hline 7 & $\mathrm{H}$ & 0 & 0.432065 & -1.175192 & -0.877807 \\
\hline 8 & $\mathrm{H}$ & 0 & 0.432066 & -1.175192 & 0.877808 \\
\hline 9 & $\mathrm{H}$ & 0 & -2.126567 & -0.607422 & 0.816899 \\
\hline 10 & $\mathrm{H}$ & 0 & -2.126565 & -0.607427 & -0.816897 \\
\hline 11 & C & 0 & -0.597265 & 0.506322 & -0.000000 \\
\hline 12 & $\mathrm{H}$ & 0 & -0.496169 & 1.157894 & -0.875359 \\
\hline 13 & $\mathrm{H}$ & 0 & -0.496170 & 1.157896 & 0.875358 \\
\hline
\end{tabular}

Standard basis: $6-311++G(d, p) \quad(5 D, 7 F)$

$\begin{array}{llrr}\mathrm{SCF} \text { Done: } & \mathrm{E}(\mathrm{RB} 3 \mathrm{LYP})=-174.545971426 & \mathrm{~A} . \mathrm{U} \text {. after } & 1 \text { cycles } \\ & \text { NFock }=1 \text { Conv=0.19D-08 } & -\mathrm{V} / \mathrm{T}=2.0050 & \end{array}$

Full mass-weighted force constant matrix:

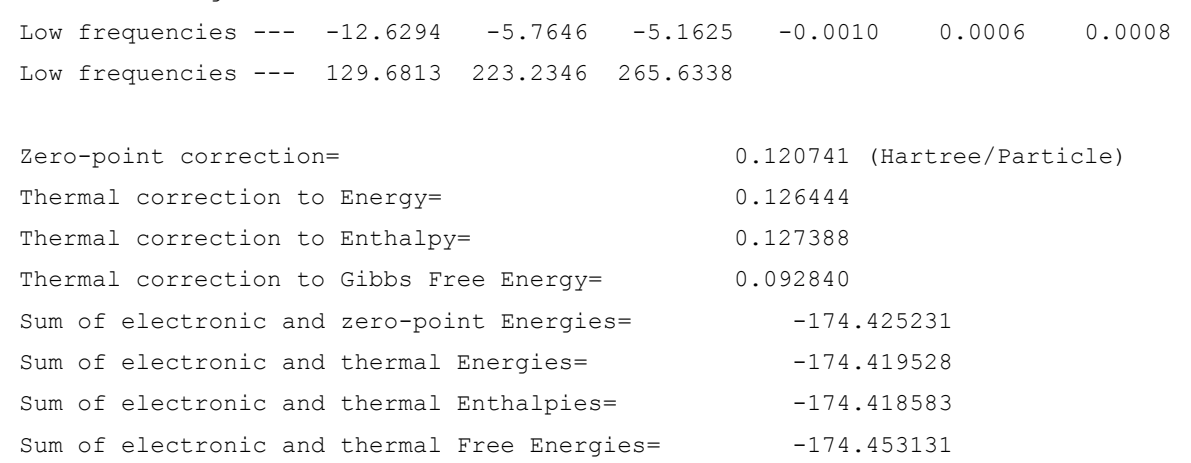




\section{Model Fragments D and E}

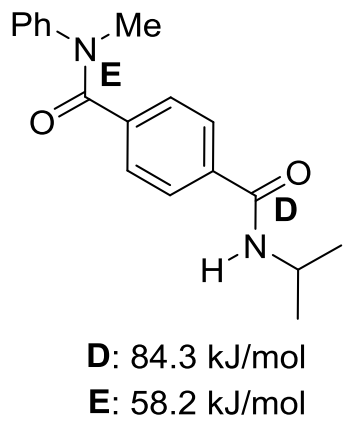

Stoichiometry C18H20N2O2

Framework group C1[X(C18H20N2O2)]

Standard orientation:

\begin{tabular}{|c|c|c|c|c|c|}
\hline \multirow{2}{*}{$\begin{array}{l}\text { Center } \\
\text { Number }\end{array}$} & \multirow[t]{2}{*}{ Atom } & \multirow{2}{*}{$\begin{array}{l}\text { Atomic } \\
\text { Type }\end{array}$} & \multicolumn{3}{|c|}{ Coordinates (Angstroms) } \\
\hline & & & $x$ & $\mathrm{Y}$ & $\mathrm{Z}$ \\
\hline 1 & C & 0 & 2.161484 & -1.893613 & -0.097961 \\
\hline 2 & C & 0 & 0.845801 & -1.162240 & -0.098534 \\
\hline 3 & $\mathrm{C}$ & 0 & -1.718899 & -0.012690 & -0.133649 \\
\hline 4 & C & 0 & -0.164161 & -1.655420 & 0.733423 \\
\hline 5 & $\mathrm{C}$ & 0 & 0.558099 & -0.093846 & -0.955218 \\
\hline 6 & C & 0 & -0.710282 & 0.473562 & -0.970784 \\
\hline 7 & C & 0 & -1.429189 & -1.079121 & 0.726036 \\
\hline 8 & O & 0 & 2.181333 & -3.111845 & 0.024339 \\
\hline 9 & $\mathrm{~N}$ & 0 & 3.327330 & -1.178712 & -0.297253 \\
\hline 10 & C & 0 & 4.559661 & -1.968977 & -0.430901 \\
\hline 11 & C & 0 & 3.477412 & 0.210817 & 0.019865 \\
\hline 12 & C & 0 & 3.831379 & 2.918323 & 0.632345 \\
\hline 13 & C & 0 & 3.086089 & 0.708040 & 1.265406 \\
\hline 14 & C & 0 & 4.062830 & 1.074152 & -0.911774 \\
\hline 15 & C & 0 & 4.241768 & 2.419637 & -0.604019 \\
\hline 16 & C & 0 & 3.252281 & 2.059065 & 1.563294 \\
\hline 17 & C & 0 & -3.063919 & 0.663195 & -0.196128 \\
\hline 18 & $\mathrm{~N}$ & 0 & -4.133246 & -0.087800 & 0.200542 \\
\hline 19 & o & 0 & -3.176835 & 1.821250 & -0.578621 \\
\hline 20 & C & 0 & -5.511374 & 0.412675 & 0.151681 \\
\hline 21 & $\mathrm{C}$ & 0 & -6.131418 & 0.216498 & -1.237901 \\
\hline 22 & C & 0 & -6.332933 & -0.255943 & 1.255440 \\
\hline 23 & $\mathrm{H}$ & 0 & 0.054248 & -2.495870 & 1.380760 \\
\hline 24 & $\mathrm{H}$ & 0 & 1.320888 & 0.291521 & -1.619324 \\
\hline 25 & $\mathrm{H}$ & 0 & -0.938554 & 1.303914 & -1.627338 \\
\hline 26 & $\mathrm{H}$ & 0 & -2.179428 & -1.453195 & 1.413846 \\
\hline 27 & $\mathrm{H}$ & 0 & 4.920856 & -2.305664 & 0.546688 \\
\hline 28 & $\mathrm{H}$ & 0 & 5.326061 & -1.353415 & -0.899169 \\
\hline 29 & $\mathrm{H}$ & 0 & 4.360984 & -2.846508 & -1.042943 \\
\hline 30 & $\mathrm{H}$ & 0 & 3.963885 & 3.967916 & 0.867610 \\
\hline 31 & $\mathrm{H}$ & 0 & 2.649985 & 0.037572 & 1.996317 \\
\hline 32 & $\mathrm{H}$ & 0 & 4.363271 & 0.690114 & -1.880250 \\
\hline 33 & $\mathrm{H}$ & 0 & 4.691659 & 3.081556 & -1.335331 \\
\hline 34 & $\mathrm{H}$ & 0 & 2.936221 & 2.436288 & 2.529171 \\
\hline 35 & $\mathrm{H}$ & 0 & -4.001056 & -1.079888 & 0.327256 \\
\hline 36 & $\mathrm{H}$ & 0 & -5.439805 & 1.484096 & 0.349358 \\
\hline 37 & $\mathrm{H}$ & 0 & -6.204982 & -0.847329 & -1.486827 \\
\hline 38 & $\mathrm{H}$ & 0 & -5.525856 & 0.711478 & -1.999112 \\
\hline 39 & $\mathrm{H}$ & 0 & -7.138310 & 0.642421 & -1.271652 \\
\hline
\end{tabular}




$\begin{array}{lllrrr}40 & \mathrm{H} & 0 & -6.391845 & -1.339805 & 1.103474 \\ 41 & \mathrm{H} & 0 & -5.895647 & -0.069982 & 2.239248 \\ 42 & \mathrm{H} & 0 & -7.354916 & 0.130433 & 1.255731\end{array}$

Standard basis: $6-311++G(d, p) \quad(5 D, 7 F)$

$\begin{array}{rlrr}\text { SCF Done: } & \text { E (RB3LYP })=-958.210260014 & \text { A.U. after } 1 \text { cycles } \\ \text { NFock }=1 \text { Conv }=0.59 \mathrm{D}-08 & -\mathrm{V} / \mathrm{T}=2.0043\end{array}$

Full mass-weighted force constant matrix:

$\begin{array}{lllllll}\text { Low frequencies --- } & -1.9476 & -1.7214 & -0.0003 & 0.0008 & 0.0008 & 1.6146 \\ \text { Low frequencies --- } & 17.9789 & 27.3663 & 41.6783 & & \end{array}$

Zero-point correction=

Thermal correction to Energy=

Thermal correction to Enthalpy=

Thermal correction to Gibbs Free Energy=

Sum of electronic and zero-point Energies=

Sum of electronic and thermal Energies=

Sum of electronic and thermal Enthalpies=

Sum of electronic and thermal Free Energies=
0.345865 (Hartree/Particle)

0.367118

0.368062

0.292976

$-957.864395$

$-957.843142$

$-957.842198$

$-957.917284$ 
Stoichiometry

C18H22N2O

Framework group

$\mathrm{C} 1[\mathrm{X}(\mathrm{C} 18 \mathrm{H} 22 \mathrm{~N} 2 \mathrm{O})]$

Standard orientation:

\begin{tabular}{|c|c|c|c|c|c|}
\hline \multirow{2}{*}{$\begin{array}{l}\text { Center } \\
\text { Number }\end{array}$} & \multirow[t]{2}{*}{ Atom } & \multirow{2}{*}{$\begin{array}{l}\text { Atomic } \\
\text { Type }\end{array}$} & \multicolumn{3}{|c|}{ Coordinates (Angstroms) } \\
\hline & & & $\mathrm{X}$ & $\mathrm{Y}$ & z \\
\hline 1 & $\mathrm{C}$ & 0 & 2.189752 & -1.822404 & -0.158904 \\
\hline 2 & $\mathrm{C}$ & 0 & 0.803922 & -1.309312 & 0.113000 \\
\hline 3 & $\mathrm{C}$ & 0 & -1.871038 & -0.564544 & 0.601138 \\
\hline 4 & C & 0 & 0.203756 & -0.281820 & -0.624625 \\
\hline 5 & $\mathrm{C}$ & 0 & 0.045454 & -1.974736 & 1.080113 \\
\hline 6 & C & 0 & -1.270734 & -1.596860 & 1.327826 \\
\hline 7 & C & 0 & -1.118219 & 0.078650 & -0.388416 \\
\hline 8 & O & 0 & 2.428604 & -3.021478 & -0.090233 \\
\hline 9 & $\mathrm{~N}$ & 0 & 3.172808 & -0.926463 & -0.547160 \\
\hline 10 & $\mathrm{C}$ & 0 & 4.461041 & -1.510652 & -0.946429 \\
\hline 11 & C & 0 & 3.176135 & 0.459543 & -0.186720 \\
\hline 12 & C & 0 & 3.251682 & 3.172833 & 0.500776 \\
\hline 13 & C & 0 & 3.448810 & 1.427620 & -1.158939 \\
\hline 14 & C & 0 & 2.956593 & 0.858667 & 1.134343 \\
\hline 15 & C & 0 & 2.983219 & 2.210331 & 1.471548 \\
\hline 16 & C & 0 & 3.490117 & 2.775636 & -0.814912 \\
\hline 17 & $\mathrm{~N}$ & 0 & -4.082205 & -0.021292 & -0.346053 \\
\hline 18 & C & 0 & -5.503648 & 0.304001 & -0.153667 \\
\hline 19 & C & 0 & -5.677424 & 1.770391 & 0.255622 \\
\hline 20 & C & 0 & -6.256463 & 0.006341 & -1.451751 \\
\hline 21 & $\mathrm{H}$ & 0 & 0.763650 & 0.230804 & -1.396534 \\
\hline 22 & $\mathrm{H}$ & 0 & 0.493926 & -2.798978 & 1.621450 \\
\hline 23 & $\mathrm{H}$ & 0 & -1.843000 & -2.119746 & 2.087591 \\
\hline 24 & $\mathrm{H}$ & 0 & -1.582367 & 0.859559 & -0.979167 \\
\hline 25 & $\mathrm{H}$ & 0 & 5.019777 & -0.776476 & -1.524923 \\
\hline 26 & $\mathrm{H}$ & 0 & 4.282607 & -2.400181 & -1.547260 \\
\hline 27 & $\mathrm{H}$ & 0 & 5.052954 & -1.800161 & -0.071388 \\
\hline 28 & $\mathrm{H}$ & 0 & 3.278239 & 4.223315 & 0.766375 \\
\hline 29 & $\mathrm{H}$ & 0 & 3.614793 & 1.120266 & -2.185411 \\
\hline 30 & $\mathrm{H}$ & 0 & 2.762865 & 0.110372 & 1.893473 \\
\hline 31 & $\mathrm{H}$ & 0 & 2.804420 & 2.508342 & 2.498494 \\
\hline 32 & $\mathrm{H}$ & 0 & 3.698393 & 3.517340 & -1.577828 \\
\hline 33 & $\mathrm{H}$ & 0 & -4.005229 & -0.888821 & -0.868828 \\
\hline 34 & $\mathrm{H}$ & 0 & -5.937908 & -0.325579 & 0.645427 \\
\hline 35 & $\mathrm{H}$ & 0 & -5.171463 & 1.996809 & 1.197156 \\
\hline 36 & $\mathrm{H}$ & 0 & -6.737200 & 2.004646 & 0.389785 \\
\hline 37 & $\mathrm{H}$ & 0 & -5.269692 & 2.428657 & -0.516876 \\
\hline 38 & $\mathrm{H}$ & 0 & -5.859458 & 0.612641 & -2.271116 \\
\hline 39 & $\mathrm{H}$ & 0 & -6.164066 & -1.048085 & -1.731488 \\
\hline 40 & $\mathrm{H}$ & 0 & -7.320701 & 0.228813 & -1.342312 \\
\hline 41 & C & 0 & -3.297924 & -0.145562 & 0.883959 \\
\hline 42 & $\mathrm{H}$ & 0 & -3.287653 & 0.829442 & 1.383415 \\
\hline 43 & $\mathrm{H}$ & 0 & -3.746126 & -0.855617 & 1.600537 \\
\hline
\end{tabular}

Standard basis: $6-311++G(d, p) \quad(5 D, 7 F)$

SCF Done: E (RB3LYP) $=-884.140209582$

A.U. after 2 cycles

$\mathrm{NFock}=2$ Conv $=0.62 \mathrm{D}-08$

$-\mathrm{V} / \mathrm{T}=2.0045$ 
Full mass-weighted force constant matrix:

$\begin{array}{lllllll}\text { Low frequencies --- } & -4.5743 & -2.1054 & -1.6565 & -0.0005 & 0.0003 & 0.0006 \\ \text { Low frequencies --- } & 13.4334 & 26.3183 & 39.7215 & & \end{array}$

\section{Zero-point correction=}

Thermal correction to Energy=

Thermal correction to Enthalpy=

Thermal correction to Gibbs Free Energy=

Sum of electronic and zero-point Energies=

Sum of electronic and thermal Energies=

Sum of electronic and thermal Enthalpies=

Sum of electronic and thermal Free Energies=
0.364165 (Hartree/Particle)

0.384807

0.385751

0.311743

$-883.776045$

$-883.755403$

$-883.754459$

$-883.828467$ 
Stoichiometry C19H23NO

Framework group C1[X(C19H23NO)]

Standard orientation:

\begin{tabular}{|c|c|c|c|c|c|}
\hline \multirow{2}{*}{$\begin{array}{l}\text { Center } \\
\text { Number }\end{array}$} & \multirow[t]{2}{*}{ Atom } & \multirow{2}{*}{$\begin{array}{l}\text { Atomic } \\
\text { Type }\end{array}$} & \multicolumn{3}{|c|}{ Coordinates (Angstroms) } \\
\hline & & & $\mathrm{x}$ & Y & $\mathrm{Z}$ \\
\hline 1 & C & 0 & 2.438369 & -1.730221 & -0.134816 \\
\hline 2 & $\mathrm{C}$ & 0 & 0.972823 & -1.457702 & 0.048141 \\
\hline 3 & $\mathrm{C}$ & 0 & -1.816612 & -1.181228 & 0.401762 \\
\hline 4 & C & 0 & 0.460992 & -0.634856 & 1.056460 \\
\hline 5 & C & 0 & 0.076628 & -2.158553 & -0.766326 \\
\hline 6 & $\mathrm{C}$ & 0 & -1.295234 & -2.009005 & -0.600100 \\
\hline 7 & $\mathrm{C}$ & 0 & -0.913651 & -0.508426 & 1.232315 \\
\hline 8 & 0 & 0 & 2.824292 & -2.848954 & -0.449433 \\
\hline 9 & $\mathrm{~N}$ & 0 & 3.347655 & -0.714848 & 0.118251 \\
\hline 10 & C & 0 & 4.767510 & -1.092569 & 0.074105 \\
\hline 11 & $\mathrm{C}$ & 0 & 3.045874 & 0.678379 & -0.016114 \\
\hline 12 & C & 0 & 2.525537 & 3.417464 & -0.279565 \\
\hline 13 & C & 0 & 2.401721 & 1.167676 & -1.155688 \\
\hline 14 & C & 0 & 3.441210 & 1.572613 & 0.984435 \\
\hline 15 & C & 0 & 3.185696 & 2.934238 & 0.850174 \\
\hline 16 & C & 0 & 2.133644 & 2.529399 & -1.278962 \\
\hline 17 & $\mathrm{C}$ & 0 & -5.389623 & 0.343759 & -0.220704 \\
\hline 18 & C & 0 & -5.877887 & 0.731876 & 1.182510 \\
\hline 19 & C & 0 & -5.848154 & 1.380902 & -1.255852 \\
\hline 20 & $\mathrm{H}$ & 0 & 1.132480 & -0.105021 & 1.720024 \\
\hline 21 & $\mathrm{H}$ & 0 & 0.471233 & -2.830696 & -1.518774 \\
\hline 22 & $\mathrm{H}$ & 0 & -1.973595 & -2.556581 & -1.247118 \\
\hline 23 & $\mathrm{H}$ & 0 & -1.291083 & 0.119679 & 2.033247 \\
\hline 24 & $\mathrm{H}$ & 0 & 5.353402 & -0.332103 & 0.588122 \\
\hline 25 & $\mathrm{H}$ & 0 & 5.122734 & -1.178733 & -0.958477 \\
\hline 26 & $\mathrm{H}$ & 0 & 4.899501 & -2.056409 & 0.561694 \\
\hline 27 & $\mathrm{H}$ & 0 & 2.322156 & 4.477280 & -0.380586 \\
\hline 28 & $\mathrm{H}$ & 0 & 2.109816 & 0.480670 & -1.940881 \\
\hline 29 & $\mathrm{H}$ & 0 & 3.937129 & 1.194683 & 1.871504 \\
\hline 30 & $\mathrm{H}$ & 0 & 3.493802 & 3.617150 & 1.633945 \\
\hline 31 & $\mathrm{H}$ & 0 & 1.627234 & 2.896019 & -2.164713 \\
\hline 32 & $\mathrm{H}$ & 0 & -5.850132 & -0.618542 & -0.484494 \\
\hline 33 & $\mathrm{H}$ & 0 & -5.423262 & 1.675864 & 1.503964 \\
\hline 34 & $\mathrm{H}$ & 0 & -5.636011 & -0.027488 & 1.930177 \\
\hline 35 & $\mathrm{H}$ & 0 & -6.963355 & 0.867186 & 1.193716 \\
\hline 36 & $\mathrm{H}$ & 0 & -5.412533 & 2.363490 & -1.043262 \\
\hline 37 & $\mathrm{H}$ & 0 & -5.546539 & 1.095342 & -2.267944 \\
\hline 38 & $\mathrm{H}$ & 0 & -6.936388 & 1.492905 & -1.249359 \\
\hline 39 & C & 0 & -3.308240 & -0.999068 & 0.564575 \\
\hline 40 & $\mathrm{H}$ & 0 & -3.534545 & -0.826333 & 1.620573 \\
\hline 41 & $\mathrm{H}$ & 0 & -3.821350 & -1.924447 & 0.279661 \\
\hline 42 & C & 0 & -3.861484 & 0.162126 & -0.286597 \\
\hline 43 & $\mathrm{H}$ & 0 & -3.570378 & -0.005883 & -1.329919 \\
\hline 44 & $\mathrm{H}$ & 0 & -3.372940 & 1.096714 & 0.018084 \\
\hline
\end{tabular}

Standard basis: $6-311++G(d, p) \quad(5 D, 7 F)$

SCF Done: E (RB3LYP $)=-868.104837741$ 
Full mass-weighted force constant matrix:
Low frequencies --- $\quad-0.0005$
0.0001
0.0003
0.5895
2.2386
3.9580
Low frequencies --- $\quad 15.7600$
$24.7513 \quad 34.8214$

\section{Zero-point correction=}

Thermal correction to Energy=

Thermal correction to Enthalpy=

Thermal correction to Gibbs Free Energy=

Sum of electronic and zero-point Energies=

Sum of electronic and thermal Energies=

Sum of electronic and thermal Enthalpies=

Sum of electronic and thermal Free Energies=
0.375393 (Hartree/Particle)

0.396164

0.397108

0.322994

$-867.729444$

$-867.708674$

$-867.707730$

$-867.781844$ 
Stoichiometry

C19H21NO2

Framework group

$\mathrm{C} 1[\mathrm{X}(\mathrm{C} 19 \mathrm{H} 21 \mathrm{NO})]$

Standard orientation:

\begin{tabular}{|c|c|c|c|c|c|}
\hline \multirow{2}{*}{$\begin{array}{l}\text { Center } \\
\text { Number }\end{array}$} & \multirow[t]{2}{*}{ Atom } & \multirow{2}{*}{$\begin{array}{l}\text { Atomic } \\
\text { Type }\end{array}$} & \multicolumn{3}{|c|}{ Coordinates (Angstroms) } \\
\hline & & & $\mathrm{X}$ & $\mathrm{Y}$ & Z \\
\hline 1 & $\mathrm{C}$ & 0 & 2.334940 & -1.863668 & -0.041501 \\
\hline 2 & $\mathrm{C}$ & 0 & 0.961317 & -1.268933 & 0.125215 \\
\hline 3 & $\mathrm{C}$ & 0 & -1.684754 & -0.377079 & 0.459759 \\
\hline 4 & C & 0 & -0.068665 & -1.795412 & -0.659932 \\
\hline 5 & $\mathrm{C}$ & 0 & 0.654201 & -0.308254 & 1.096663 \\
\hline 6 & C & 0 & -0.653924 & 0.123221 & 1.266336 \\
\hline 7 & C & 0 & -1.374245 & -1.343330 & -0.506664 \\
\hline 8 & O & 0 & 2.456990 & -3.063395 & -0.251504 \\
\hline 9 & $\mathrm{~N}$ & 0 & 3.439787 & -1.047911 & 0.107983 \\
\hline 10 & $\mathrm{C}$ & 0 & 4.750120 & -1.713794 & 0.077252 \\
\hline 11 & C & 0 & 3.423093 & 0.367322 & -0.117235 \\
\hline 12 & C & 0 & 3.452870 & 3.130520 & -0.557412 \\
\hline 13 & C & 0 & 4.002151 & 1.225105 & 0.823416 \\
\hline 14 & C & 0 & 2.874263 & 0.899957 & -1.286329 \\
\hline 15 & C & 0 & 2.879695 & 2.277343 & -1.497586 \\
\hline 16 & C & 0 & 4.019492 & 2.598867 & 0.601123 \\
\hline 17 & C & 0 & -3.080178 & 0.138274 & 0.687335 \\
\hline 18 & O & 0 & -3.295683 & 0.942150 & 1.575173 \\
\hline 19 & C & 0 & -5.557632 & 0.317294 & -0.014585 \\
\hline 20 & C & 0 & -5.532319 & 1.737679 & -0.596444 \\
\hline 21 & C & 0 & -6.682098 & -0.514861 & -0.646495 \\
\hline 22 & $\mathrm{H}$ & 0 & 0.165515 & -2.568775 & -1.381119 \\
\hline 23 & $\mathrm{H}$ & 0 & 1.434902 & 0.092978 & 1.729962 \\
\hline 24 & $\mathrm{H}$ & 0 & -0.902456 & 0.855049 & 2.024913 \\
\hline 25 & $\mathrm{H}$ & 0 & -2.149166 & -1.760477 & -1.137349 \\
\hline 26 & $\mathrm{H}$ & 0 & 4.693452 & -2.646779 & 0.634362 \\
\hline 27 & $\mathrm{H}$ & 0 & 5.492406 & -1.055725 & 0.526189 \\
\hline 28 & $\mathrm{H}$ & 0 & 5.052069 & -1.943592 & -0.950079 \\
\hline 29 & $\mathrm{H}$ & 0 & 3.461087 & 4.201084 & -0.725943 \\
\hline 30 & $\mathrm{H}$ & 0 & 4.425167 & 0.812819 & 1.732670 \\
\hline 31 & $\mathrm{H}$ & 0 & 2.443826 & 0.235711 & -2.026279 \\
\hline 32 & $\mathrm{H}$ & 0 & 2.444164 & 2.680863 & -2.404593 \\
\hline 33 & $\mathrm{H}$ & 0 & 4.466375 & 3.255638 & 1.338857 \\
\hline 34 & $\mathrm{H}$ & 0 & -5.746804 & 0.397346 & 1.060645 \\
\hline 35 & $\mathrm{H}$ & 0 & -5.342447 & 1.713111 & -1.675966 \\
\hline 36 & $\mathrm{H}$ & 0 & -4.760610 & 2.348089 & -0.124216 \\
\hline 37 & $\mathrm{H}$ & 0 & -6.493452 & 2.236500 & -0.442158 \\
\hline 38 & $\mathrm{H}$ & 0 & -6.526256 & -0.639394 & -1.724094 \\
\hline 39 & $\mathrm{H}$ & 0 & -6.743627 & -1.512442 & -0.200717 \\
\hline 40 & $\mathrm{H}$ & 0 & -7.651777 & -0.028130 & -0.508654 \\
\hline 41 & C & 0 & -4.206353 & -0.390629 & -0.193493 \\
\hline 42 & $\mathrm{H}$ & 0 & -3.894737 & -0.346149 & -1.244294 \\
\hline 43 & $\mathrm{H}$ & 0 & -4.308425 & -1.460123 & 0.036109 \\
\hline
\end{tabular}

Standard basis: $6-311++G(d, p) \quad(5 D, 7 F)$

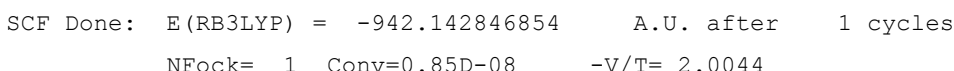

Full mass-weighted force constant matrix:

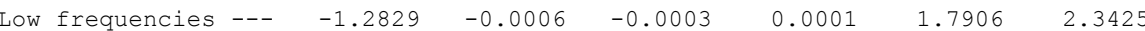


Low frequencies --- $\quad \begin{array}{lll}15.8940 \quad 29.0760 \quad 39.8904\end{array}$

Zero-point correction=

Thermal correction to Energy=

Thermal correction to Enthalpy=

Thermal correction to Gibbs Free Energy=

Sum of electronic and zero-point Energies=

Sum of electronic and thermal Energies=

Sum of electronic and thermal Enthalpies=

Sum of electronic and thermal Free Energies=
0.356581 (Hartree/Particle)

0.377902

0.378846

0.303740

$-941.786266$

$-941.764945$

$-941.764001$

$-941.839107$ 
Stoichiometry

C18H22N2O

Framework group

$\mathrm{C} 1[\mathrm{X}(\mathrm{C} 18 \mathrm{H} 22 \mathrm{~N} 2 \mathrm{O})]$

Standard orientation:

\begin{tabular}{|c|c|c|c|c|c|}
\hline \multirow{2}{*}{$\begin{array}{l}\text { Center } \\
\text { Number }\end{array}$} & \multirow[t]{2}{*}{ Atom } & \multirow{2}{*}{$\begin{array}{l}\text { Atomic } \\
\text { Type }\end{array}$} & \multicolumn{3}{|c|}{ Coordinates (Angstroms) } \\
\hline & & & $\mathrm{x}$ & Y & z \\
\hline 1 & C & 0 & -0.644217 & -1.315120 & -0.339994 \\
\hline 2 & C & 0 & 2.120343 & -0.808757 & -0.120642 \\
\hline 3 & C & 0 & -0.176656 & -0.247483 & 0.426076 \\
\hline 4 & C & 0 & 0.289361 & -2.137795 & -0.982085 \\
\hline 5 & C & 0 & 1.651685 & -1.893593 & -0.870803 \\
\hline 6 & C & 0 & 1.190144 & 0.004499 & 0.533699 \\
\hline 7 & $\mathrm{~N}$ & 0 & -2.999417 & -0.957078 & 0.473035 \\
\hline 8 & C & 0 & -3.271414 & -1.733100 & 1.671209 \\
\hline 9 & C & 0 & -3.846420 & 0.089890 & 0.108715 \\
\hline 10 & C & 0 & -5.536514 & 2.249549 & -0.609379 \\
\hline 11 & C & 0 & -5.034556 & 0.355027 & 0.819355 \\
\hline 12 & C & 0 & -3.525063 & 0.944349 & -0.967797 \\
\hline 13 & C & 0 & -4.362212 & 1.997057 & -1.318174 \\
\hline 14 & C & 0 & -5.857124 & 1.420485 & 0.462182 \\
\hline 15 & C & 0 & 3.609847 & -0.609524 & -0.037786 \\
\hline 16 & $\mathrm{~N}$ & 0 & 4.034191 & 0.651571 & 0.273355 \\
\hline 17 & 0 & 0 & 4.389253 & -1.533880 & -0.236292 \\
\hline 18 & C & 0 & 5.453980 & 1.011419 & 0.345960 \\
\hline 19 & C & 0 & 6.012472 & 1.378783 & -1.035111 \\
\hline 20 & C & 0 & 5.641509 & 2.135302 & 1.366891 \\
\hline 21 & $\mathrm{H}$ & 0 & -0.888352 & 0.379745 & 0.949879 \\
\hline 22 & $\mathrm{H}$ & 0 & -0.054782 & -2.983574 & -1.570301 \\
\hline 23 & $\mathrm{H}$ & 0 & 2.375872 & -2.539761 & -1.351496 \\
\hline 24 & $\mathrm{H}$ & 0 & 1.520351 & 0.823189 & 1.163969 \\
\hline 25 & $\mathrm{H}$ & 0 & -3.459437 & -1.075767 & 2.522782 \\
\hline 26 & $\mathrm{H}$ & 0 & -4.132305 & -2.410049 & 1.558144 \\
\hline 27 & $\mathrm{H}$ & 0 & -2.392374 & -2.333888 & 1.908734 \\
\hline 28 & $\mathrm{H}$ & 0 & -6.182540 & 3.074448 & -0.884544 \\
\hline 29 & $\mathrm{H}$ & 0 & -5.330005 & -0.277949 & 1.645054 \\
\hline 30 & $\mathrm{H}$ & 0 & -2.602274 & 0.804288 & -1.516676 \\
\hline 31 & $\mathrm{H}$ & 0 & -4.082787 & 2.635216 & -2.149720 \\
\hline 32 & $\mathrm{H}$ & 0 & -6.766340 & 1.592848 & 1.028295 \\
\hline 33 & $\mathrm{H}$ & 0 & 3.362849 & 1.403818 & 0.244204 \\
\hline 34 & $\mathrm{H}$ & 0 & 5.965089 & 0.112466 & 0.696297 \\
\hline 35 & $\mathrm{H}$ & 0 & 5.513222 & 2.267597 & -1.435186 \\
\hline 36 & $\mathrm{H}$ & 0 & 5.869791 & 0.554040 & -1.735572 \\
\hline 37 & $\mathrm{H}$ & 0 & 7.083414 & 1.592139 & -0.971472 \\
\hline 38 & $\mathrm{H}$ & 0 & 5.108352 & 3.042299 & 1.059410 \\
\hline 39 & $\mathrm{H}$ & 0 & 5.273655 & 1.837657 & 2.351654 \\
\hline 40 & $\mathrm{H}$ & 0 & 6.699292 & 2.393031 & 1.459200 \\
\hline 41 & C & 0 & -2.132390 & -1.593353 & -0.506961 \\
\hline 42 & $\mathrm{H}$ & 0 & -2.291404 & -2.678633 & -0.468985 \\
\hline 43 & $\mathrm{H}$ & 0 & -2.443385 & -1.289379 & -1.510192 \\
\hline
\end{tabular}

Standard basis: $6-311++G(d, p) \quad(5 D, 7 F)$

$\begin{array}{llrr}\text { SCF Done: } & \text { E }(\text { RB3LYP })=-884.151433911 & \text { A.U. after } 1 \text { cycles } \\ \text { NFock }=1 \text { Conv=0.58D-08 } & -\mathrm{V} / \mathrm{T}=2.0045 & \end{array}$ 
Full mass-weighted force constant matrix:

\begin{tabular}{|c|c|c|c|c|c|c|}
\hline Low & frequencies --- & -2.6723 & -0.0001 & 0.0007 & 0.0008 & 1.0589 \\
\hline Low & frequencies --- & 8.1586 & 17.8000 & 34.1174 & & \\
\hline
\end{tabular}

\section{Zero-point correction=}

Thermal correction to Energy=

Thermal correction to Enthalpy=

Thermal correction to Gibbs Free Energy=

Sum of electronic and zero-point Energies=

Sum of electronic and thermal Energies=

Sum of electronic and thermal Enthalpies=

Sum of electronic and thermal Free Energies=
0.364476 (Hartree/Particle)

0.385053

0.385997

0.311277

$-883.786958$

$-883.766381$

$-883.765436$

$-883.840157$ 
Stoichiometry C19H23NO

Framework group C1[X(C19H23NO)]

Standard orientation:

\begin{tabular}{|c|c|c|c|c|c|}
\hline \multirow{2}{*}{$\begin{array}{l}\text { Center } \\
\text { Number }\end{array}$} & \multirow[t]{2}{*}{ Atom } & \multirow{2}{*}{$\begin{array}{l}\text { Atomic } \\
\text { Type }\end{array}$} & \multicolumn{3}{|c|}{ Coordinates (Angstroms) } \\
\hline & & & $\mathrm{x}$ & Y & z \\
\hline 1 & C & 0 & -1.060067 & -1.574984 & -0.518339 \\
\hline 2 & $\mathrm{C}$ & 0 & 1.697696 & -1.069637 & -0.161548 \\
\hline 3 & C & 0 & -0.405850 & -0.595196 & -1.270954 \\
\hline 4 & C & 0 & -0.303531 & -2.308701 & 0.405070 \\
\hline 5 & $\mathrm{C}$ & 0 & 1.053572 & -2.067314 & 0.577879 \\
\hline 6 & C & 0 & 0.952643 & -0.343224 & -1.096265 \\
\hline 7 & $\mathrm{C}$ & 0 & -4.874842 & -1.672538 & 0.306322 \\
\hline 8 & $\mathrm{C}$ & 0 & -3.374442 & 0.373378 & 0.304607 \\
\hline 9 & C & 0 & -3.270725 & 3.186098 & 0.169384 \\
\hline 10 & C & 0 & -2.811639 & 1.124237 & 1.341133 \\
\hline 11 & C & 0 & -3.885287 & 1.061088 & -0.804098 \\
\hline 12 & $\mathrm{C}$ & 0 & -3.834866 & 2.451377 & -0.873365 \\
\hline 13 & C & 0 & -2.759034 & 2.516613 & 1.278131 \\
\hline 14 & $\mathrm{C}$ & 0 & 3.168942 & -0.863632 & 0.075381 \\
\hline 15 & $\mathrm{~N}$ & 0 & 3.670957 & 0.359227 & -0.273320 \\
\hline 16 & 0 & 0 & 3.870846 & -1.748113 & 0.551515 \\
\hline 17 & $\mathrm{C}$ & 0 & 5.074303 & 0.726020 & -0.060457 \\
\hline 18 & C & 0 & 5.505377 & 1.729558 & -1.131863 \\
\hline 19 & C & 0 & 5.309024 & 1.254233 & 1.360974 \\
\hline 20 & $\mathrm{H}$ & 0 & -0.962975 & -0.025814 & -2.006871 \\
\hline 21 & $\mathrm{H}$ & 0 & -0.783532 & -3.088176 & 0.989184 \\
\hline 22 & $\mathrm{H}$ & 0 & 1.639504 & -2.649909 & 1.278228 \\
\hline 23 & $\mathrm{H}$ & 0 & 1.429679 & 0.402087 & -1.723478 \\
\hline 24 & $\mathrm{H}$ & 0 & -5.321346 & -1.467101 & -0.670910 \\
\hline 25 & $\mathrm{H}$ & 0 & -5.506281 & -1.204468 & 1.065344 \\
\hline 26 & $\mathrm{H}$ & 0 & -4.899643 & -2.755157 & 0.461637 \\
\hline 27 & $\mathrm{H}$ & 0 & -3.233434 & 4.268457 & 0.117672 \\
\hline 28 & $\mathrm{H}$ & 0 & -2.406721 & 0.612423 & 2.208245 \\
\hline 29 & $\mathrm{H}$ & 0 & -4.332027 & 0.508964 & -1.624623 \\
\hline 30 & $\mathrm{H}$ & 0 & -4.239178 & 2.962186 & -1.740635 \\
\hline 31 & $\mathrm{H}$ & 0 & -2.318192 & 3.076036 & 2.096051 \\
\hline 32 & $\mathrm{H}$ & 0 & 3.020630 & 1.101497 & -0.481964 \\
\hline 33 & $\mathrm{H}$ & 0 & 5.639583 & -0.200285 & -0.181139 \\
\hline 34 & $\mathrm{H}$ & 0 & 4.928557 & 2.658749 & -1.058519 \\
\hline 35 & $\mathrm{H}$ & 0 & 5.367425 & 1.317849 & -2.134430 \\
\hline 36 & $\mathrm{H}$ & 0 & 6.559842 & 1.988364 & -1.009096 \\
\hline 37 & $\mathrm{H}$ & 0 & 4.744029 & 2.176357 & 1.533718 \\
\hline 38 & $\mathrm{H}$ & 0 & 4.999007 & 0.512227 & 2.098879 \\
\hline 39 & $\mathrm{H}$ & 0 & 6.369214 & 1.472212 & 1.519367 \\
\hline 40 & $\mathrm{C}$ & 0 & -2.539625 & -1.837559 & -0.678596 \\
\hline 41 & $\mathrm{H}$ & 0 & -2.719756 & -2.916144 & -0.616263 \\
\hline 42 & $\mathrm{H}$ & 0 & -2.866889 & -1.523522 & -1.674720 \\
\hline 43 & C & 0 & -3.431374 & -1.144892 & 0.390597 \\
\hline 44 & $\mathrm{H}$ & 0 & -3.032617 & -1.426058 & 1.371483 \\
\hline
\end{tabular}

Standard basis: $6-311++G(d, p) \quad(5 D, 7 F)$

SCF Done: E (RB3LYP $)=-868.122410774$ A.U. after 2 cycles 
Full mass-weighted force constant matrix:

Low frequencies --- $\quad-3.4039 \quad-2.0932 \quad-0.0004$

Low frequencies --- $\quad 14.6223 \quad 19.7786 \quad 31.0446$

$\begin{array}{lll}-0.0001 & 0.0003 & 1.2897\end{array}$

\section{Zero-point correction=}

Thermal correction to Energy=

Thermal correction to Enthalpy=

Thermal correction to Gibbs Free Energy=

Sum of electronic and zero-point Energies=

Sum of electronic and thermal Energies=

Sum of electronic and thermal Enthalpies=

Sum of electronic and thermal Free Energies=
0.376009 (Hartree/Particle)

0.396677

0.397621

0.323248

$-867.746402$

$-867.725734$

$-867.724790$

$-867.799163$ 
Stoichiometry

C19H21NO2

Framework group

$\mathrm{C} 1[\mathrm{X}(\mathrm{C} 19 \mathrm{H} 21 \mathrm{NO})]$

Standard orientation:

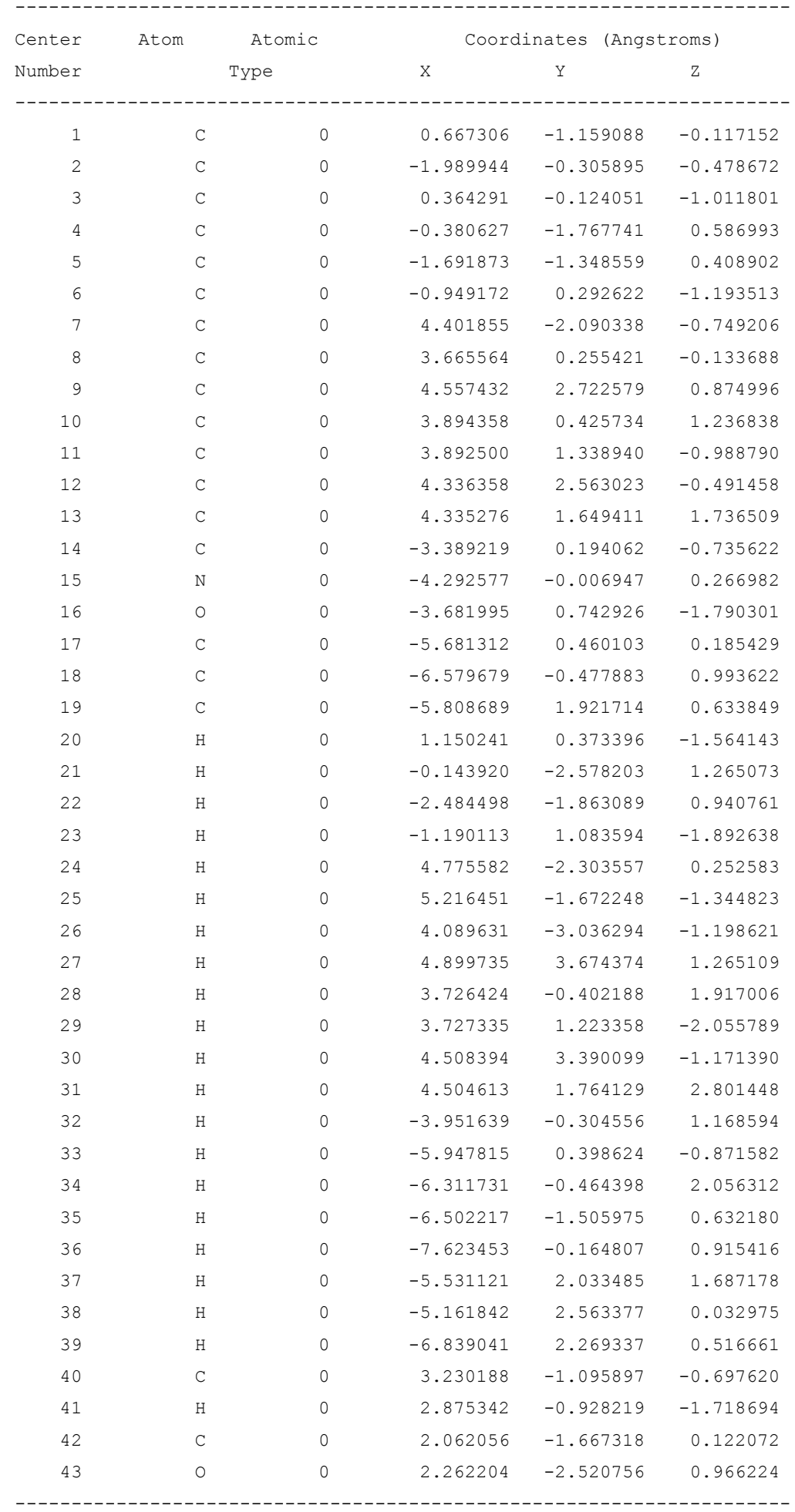

Standard basis: $6-311++G(d, p) \quad(5 D, 7 F)$

$\begin{array}{llr}\mathrm{SCF} \text { Done: } & \mathrm{E}(\mathrm{RB} 3 \mathrm{LYP})=-942.159052588 \quad \mathrm{~A} . \mathrm{U} \text {. after } 1 \text { cycles } \\ & \text { NFOck }=1 \text { Conv }=0.32 \mathrm{D}-08 \quad-\mathrm{V} / \mathrm{T}=2.0044 & \end{array}$

Full mass-weighted force constant matrix:

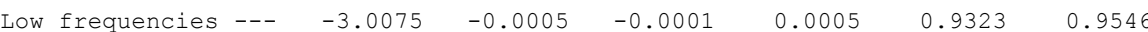


Low frequencies --- $\quad \begin{array}{llll}13.0875 & 19.5289 & 34.2263\end{array}$

\section{Zero-point correction=}

Thermal correction to Energy=

Thermal correction to Enthalpy=

Thermal correction to Gibbs Free Energy=

Sum of electronic and zero-point Energies=

Sum of electronic and thermal Energies=

Sum of electronic and thermal Enthalpies=

Sum of electronic and thermal Free Energies=
0.357464 (Hartree/Particle)

0.378746

0.379690

0.303920

$-941.801589$

$-941.780306$

$-941.779362$

$-941.855133$ 
N A T URALA T O M I C ORB I T A L A N D

N A T U R A L B O N D OR B I T A L A N A L Y S I S

Second Order Perturbation Theory Analysis of Fock Matrix in NBO Basis

Threshold for printing: $0.50 \mathrm{kcal} / \mathrm{mol}$

$E(2) \quad E(j)-E(i) \quad E(i, j)$

Donor NBO (i)

Acceptor NBO $(j)$

$\mathrm{kcal} / \mathrm{mol} \quad \mathrm{a} \cdot \mathrm{u}$. a.u.

\begin{tabular}{|c|c|c|c|c|c|c|c|c|c|c|c|c|}
\hline 170. LP ( & 1) $\mathrm{N}$ & 9 & $/ * * * \cdot \mathrm{BD}^{*}($ & 2) & C & 1 & -0 & 8 & 36.94 & 0.32 & 0.098 & $(\mathrm{E})$ \\
\hline \multicolumn{13}{|l|}{$\cdots$} \\
\hline 173. LP & 1) $\mathrm{N}$ & 19 & $/ * \star \star \cdot \cdot \mathrm{BD}^{\star}($ & 2) & C & 17 & -0 & 18 & 68.71 & 0.27 & 0.122 & (D) \\
\hline \multicolumn{13}{|l|}{$\cdots$} \\
\hline 176. LP & 1) & 25 & $/ * \star \star \cdot . \quad \mathrm{BD}^{\star}($ & 2) & $\mathrm{C}$ & 23 & -0 & 24 & 49.81 & 0.31 & 0.111 & (C) \\
\hline \multicolumn{13}{|l|}{$\cdots$} \\
\hline 177. LP & 1) $\mathrm{N}$ & 27 & $/ * \star \star \cdot \cdot \mathrm{BD}^{\star}($ & 2) & $\mathrm{C}$ & 26 & -0 & 28 & 78.71 & 0.25 & 0.126 & (B) \\
\hline \multicolumn{13}{|l|}{$\cdots$} \\
\hline 182. LP ( & 1) $\mathrm{N}$ & 39 & $/ * \star \star \cdot . \mathrm{BD}^{\star}($ & 2) & $\mathrm{C}$ & 30 & -0 & 31 & 61.85 & 0.23 & 0.111 & (A) \\
\hline
\end{tabular}

Comparison of the Resonance Energy (estimated from the isodesmic equation (REIE) and thee second-order pertubrbation energies (SOPE) involving the nitrogen lone pair and the CO $\Pi^{\star}$ orbital.

$\mathrm{REIE} / \mathrm{kJ} \mathrm{mol} \quad \mathrm{SOPE} / \mathrm{kJ} \mathrm{mol}^{-1}$

$\begin{array}{lrr}\text { (A) } & \mathbf{9 . 8} & 258.1 \\ \text { (B) } & 72.2 & 329.4 \\ \text { (C) } & 64.1 & 208.5 \\ \text { (D) } & 67.9 & 287.6 \\ \text { (E) } & 62.4 & \mathbf{1 5 4 . 6}\end{array}$


7 Copies of the NMR spectra 
tert-Butyl 2-\{[N-(tert-butoxycarbonyl)-L-phenylalanyl $]$ amino $\}$ nicotinate $\quad$ (Boc-L-Phe-NH- $t$ Bu-nic, ER489) L-4a - ${ }^{1}$ H NMR (400 MHz, $\mathbf{C D C l}_{3}$ )

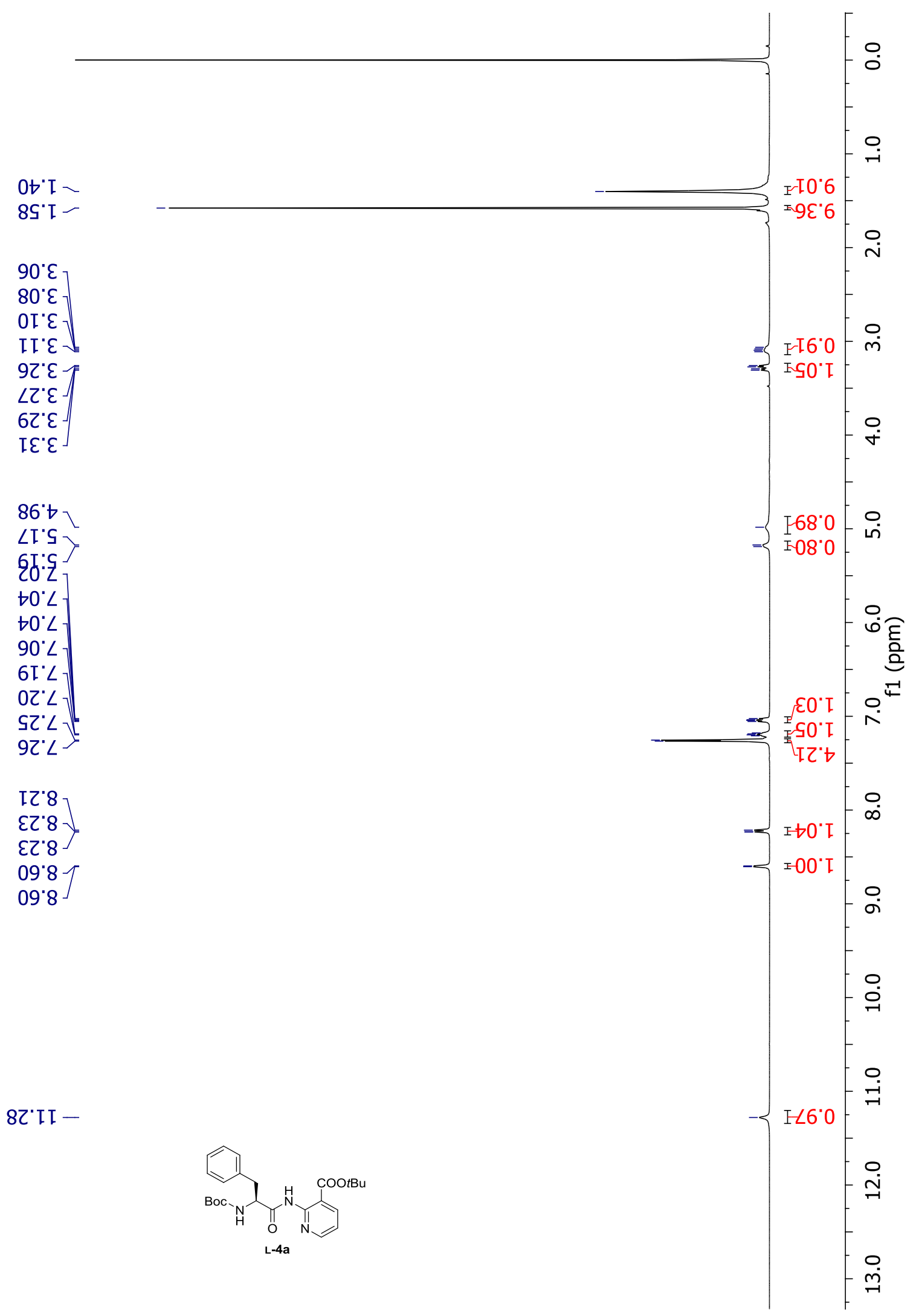


tert-Butyl 2-\{[N-(tert-butoxycarbonyl)-L-phenylalanyl $]$ amino $\}$ nicotinate $\quad$ (Boc-L-Phe-NH- $t$ Bu-nic, ER489) L-4a - ${ }^{13}$ C NMR (101 MHz, $\left.\mathbf{C D C l}_{3}\right)$

$\left.\begin{array}{l}\tau \cdot 8 Z \\ Z \cdot 8 Z\end{array}\right\}$

ع6 $8 \varepsilon-$

68.95-

$78^{\circ} 9 L$
$\left.9 I^{\circ} \angle L\right]$

$85^{\circ} \angle L$

$26^{\circ} 6 \mathrm{~L}$

เ७' $\varepsilon 8$

08'ZII -

$0 \mathrm{t}^{\circ} 8 \mathrm{II}$ -

$\angle 88^{\circ} 9 \mathrm{ZI}$

$09^{\circ} 8 Z \mathrm{I}$

$8 \mathrm{~S}^{\circ} 6 \mathrm{LI}-$

9L'9عI -

$\angle I^{\circ} 0 b I$ -

$\angle Z$ ZSI

$\angle 9^{\circ} \mathrm{ZSI}$

Ot'SSI $\digamma$

6L'ง9I -

$6 S^{\circ} 0 \angle I-$

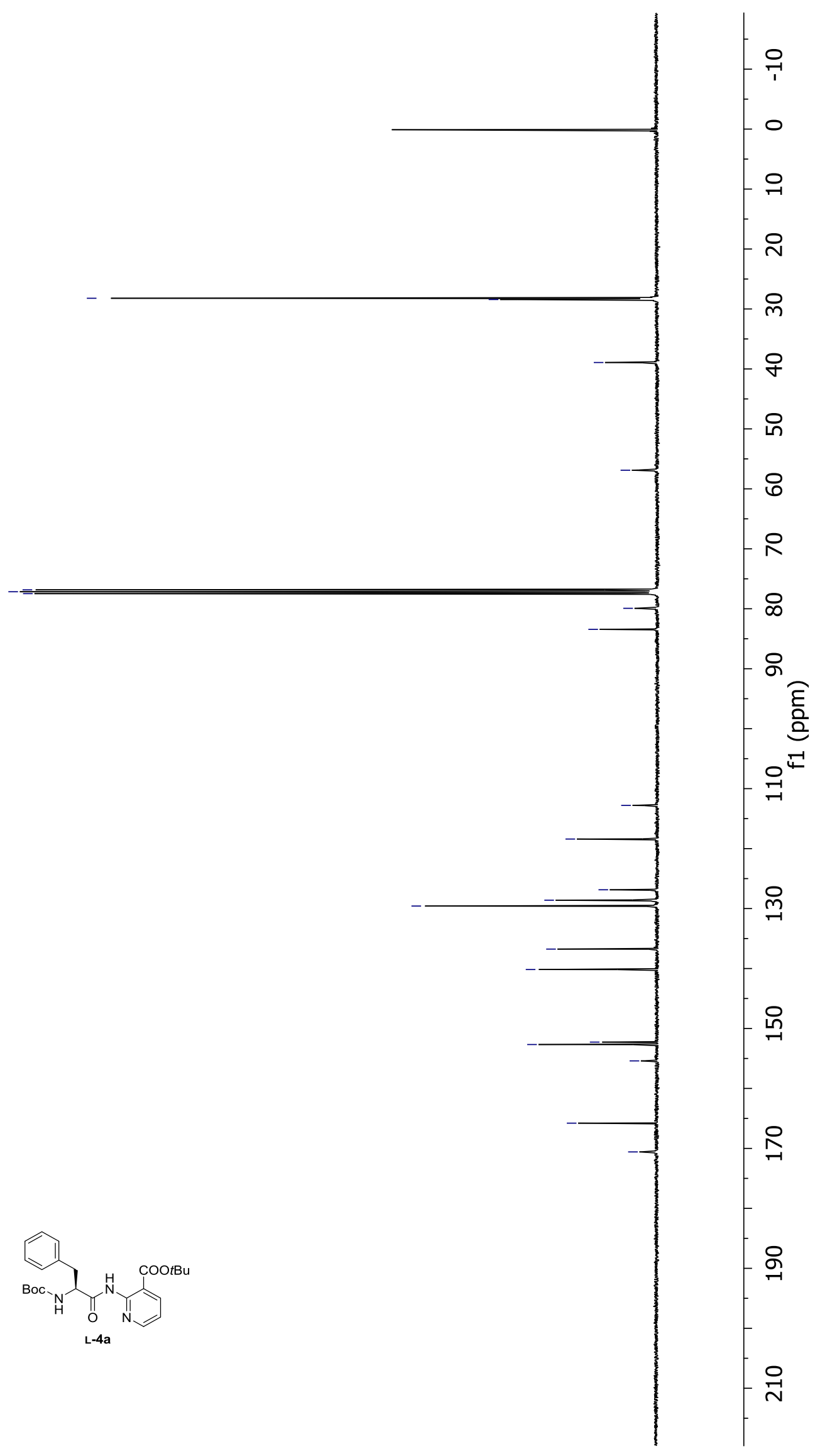


tert-Butyl ER804) L-4b - ${ }^{1} \mathbf{H}$ NMR (500 MHz, $\mathbf{C D C l}_{3}$ )
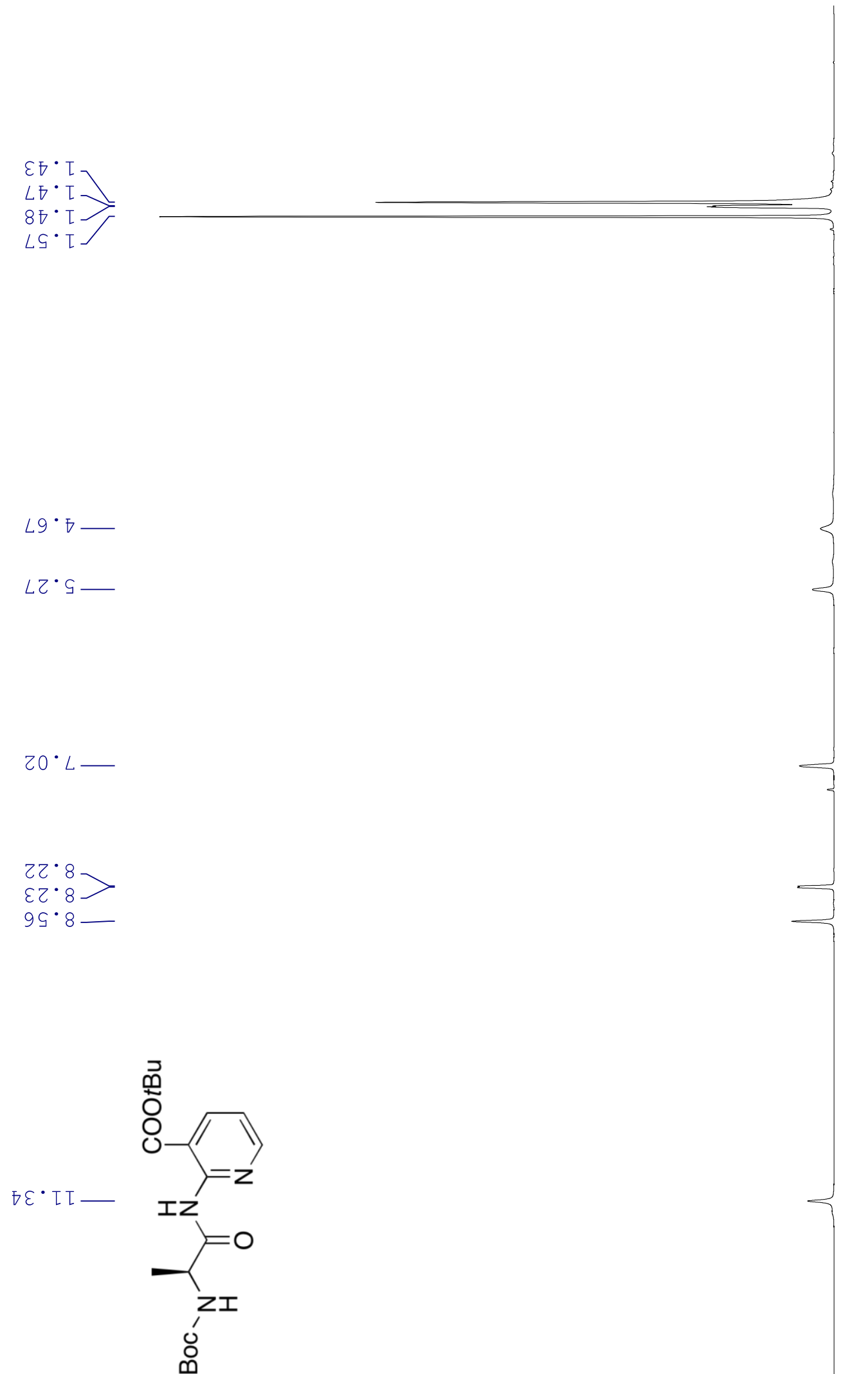
tert-Butyl ER804) L-4b - ${ }^{\mathbf{1 3}} \mathrm{C}$ NMR (126 $\mathbf{M H z} \mathrm{CDCl}_{3}$ )

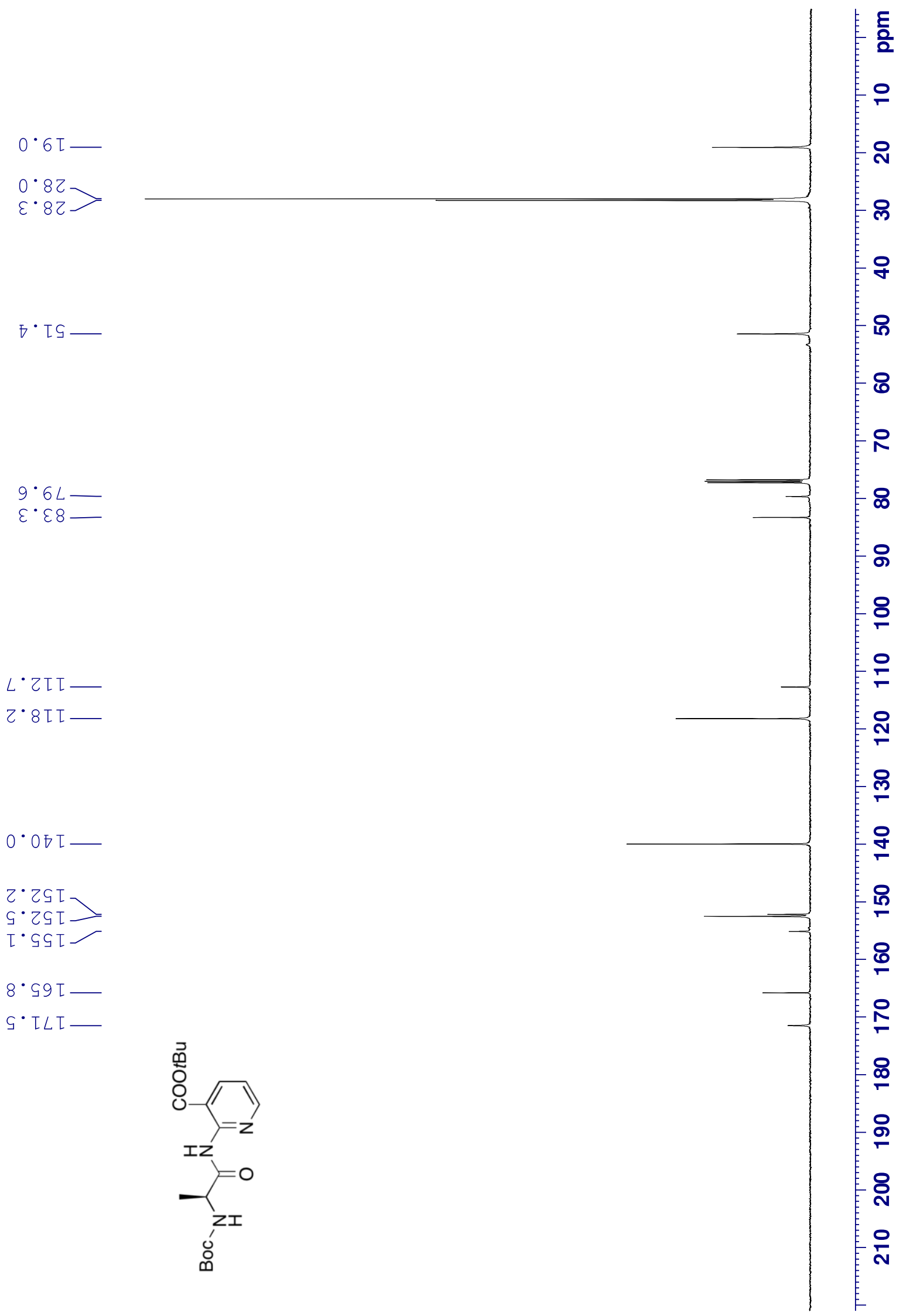


tert-Butyl

(S)-2-(3-(4-(tert-butoxy)phenyl)-2-((tert-butoxycarbonyl)amino)propanamido)nicotinate (Boc-L-Tyr( $t$ Bu)-NH- $t$ Bu-nic, ER803) L-4c - ${ }^{1}$ H NMR (500 MHz, CDCl $\left.\mathbf{~}_{3}\right)$

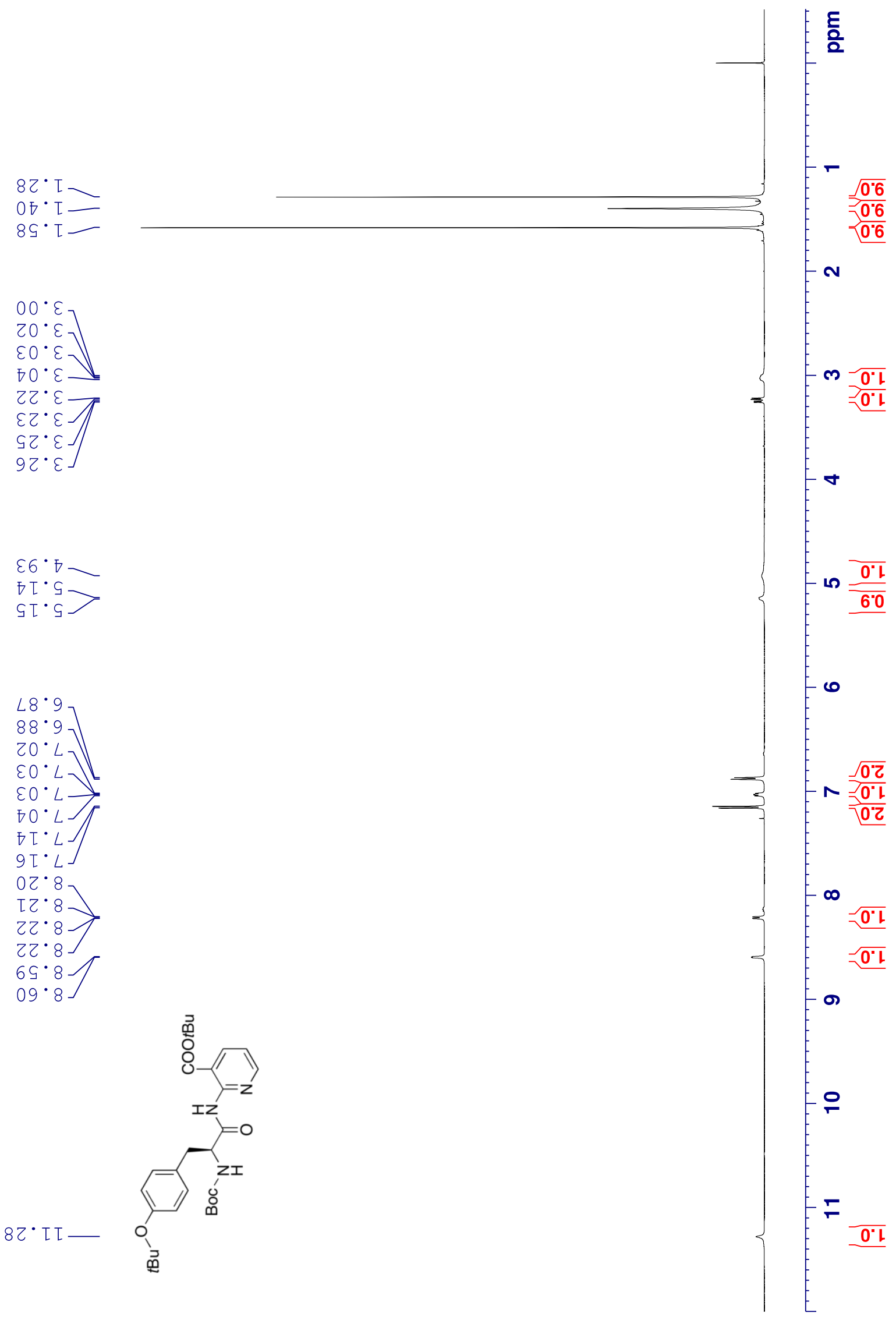


tert-Butyl

(S)-2-(3-(4-(tert-butoxy)phenyl)-2-((tert-butoxycarbonyl)amino)propanamido)nicotinate (Boc-L-Tyr $t$ Bu)-NH- $t$ Bu-nic, ER803) L-4c - ${ }^{13}$ C NMR (126 MHz, CDCl $)$

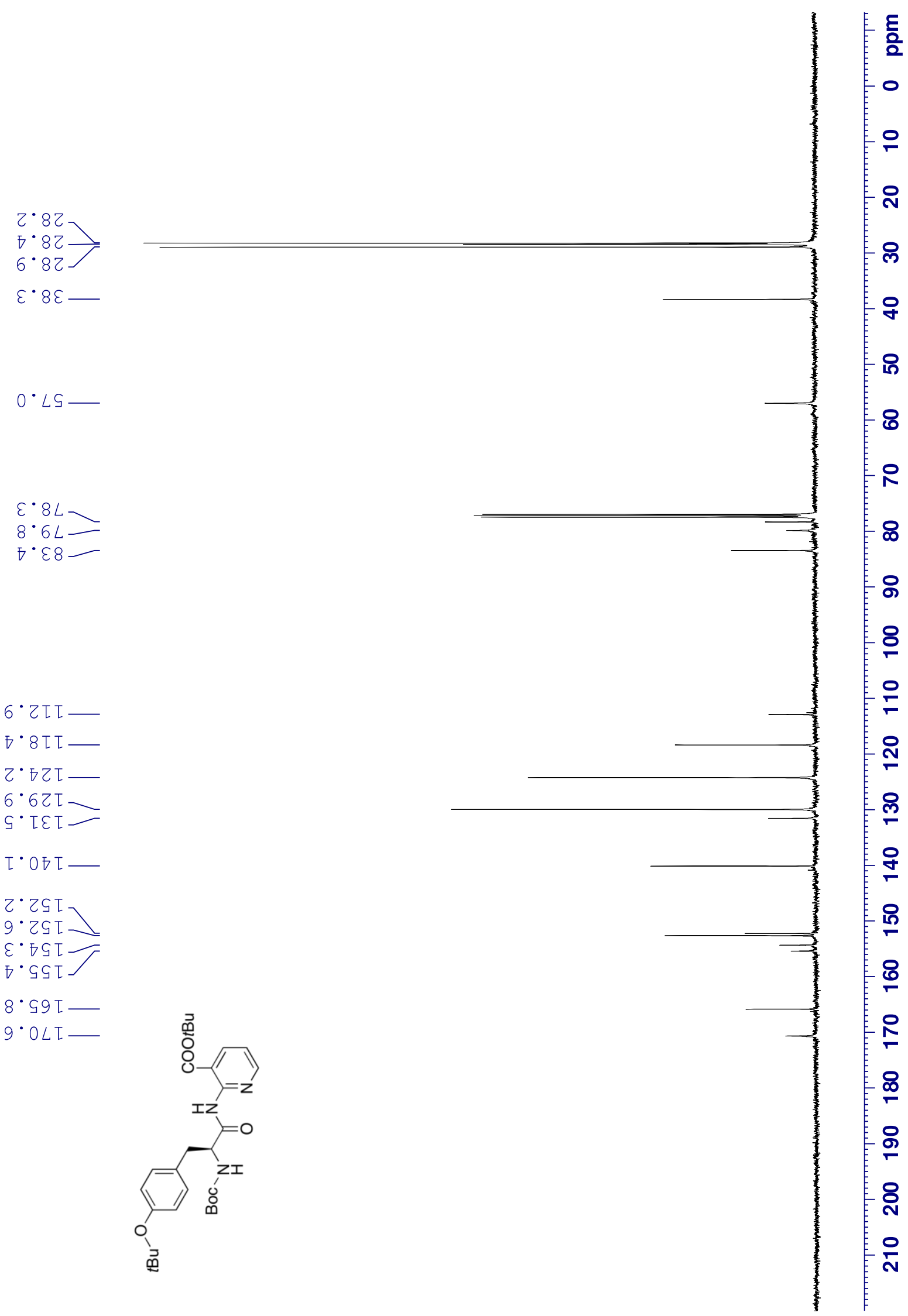


tert-Butyl 2-\{[1-(tert-butoxycarbonyl)-L-prolyl]amino\}nicotinate (Boc-L-Pro-NH-tBu-nic, CW-1061) L-4d L - ${ }^{1} \mathrm{H}$ NMR (400 MHz, $\mathrm{CDCl}_{3}$ )

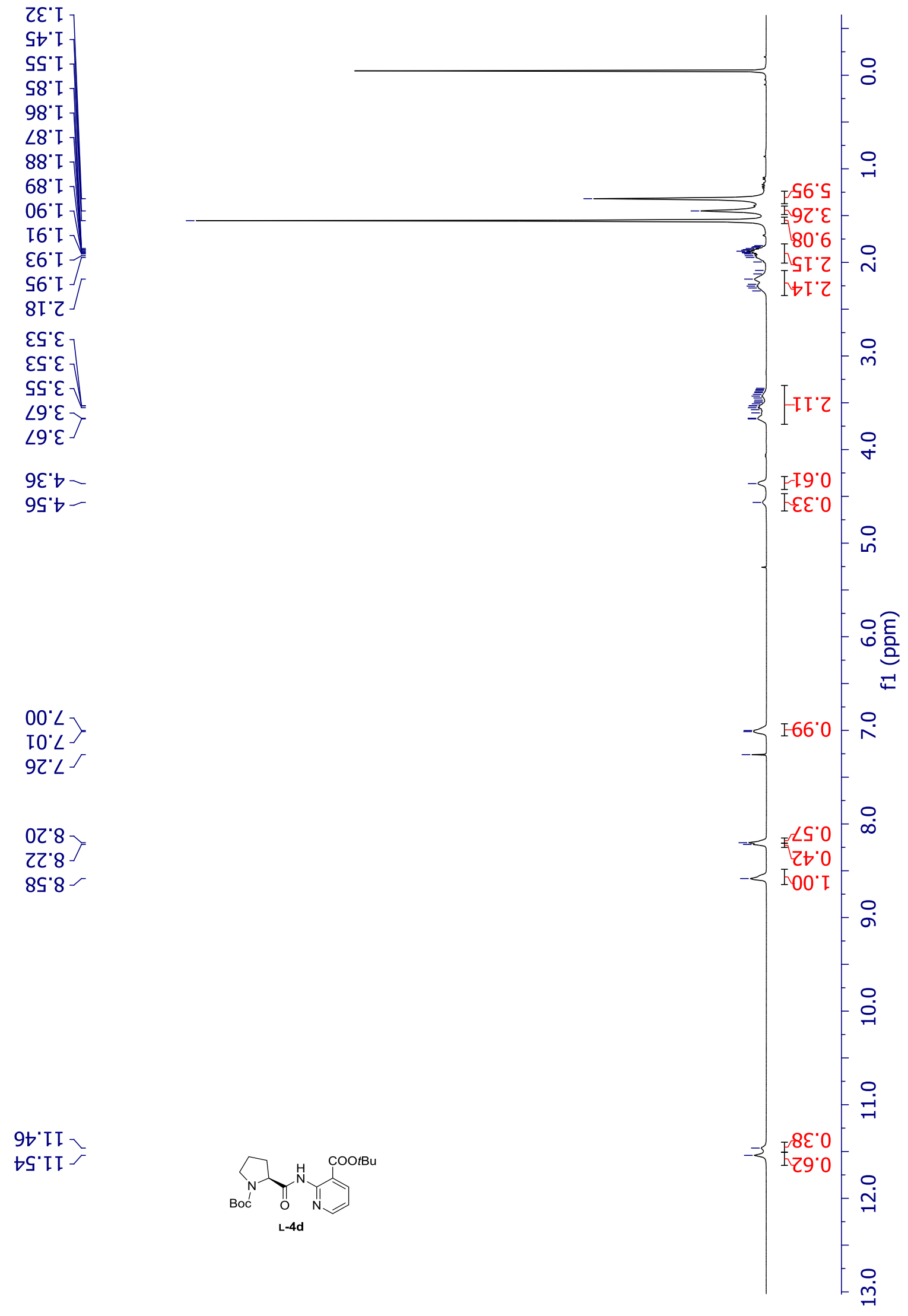


tert-Butyl 2-\{[1-(tert-butoxycarbonyl)-L-prolyl]amino\}nicotinate (Boc-L-Pro-NH-tBu-nic, CW-1061) L-4d - ${ }^{13}$ C NMR (101 MHz, $\mathrm{CDCl}_{3}$ )

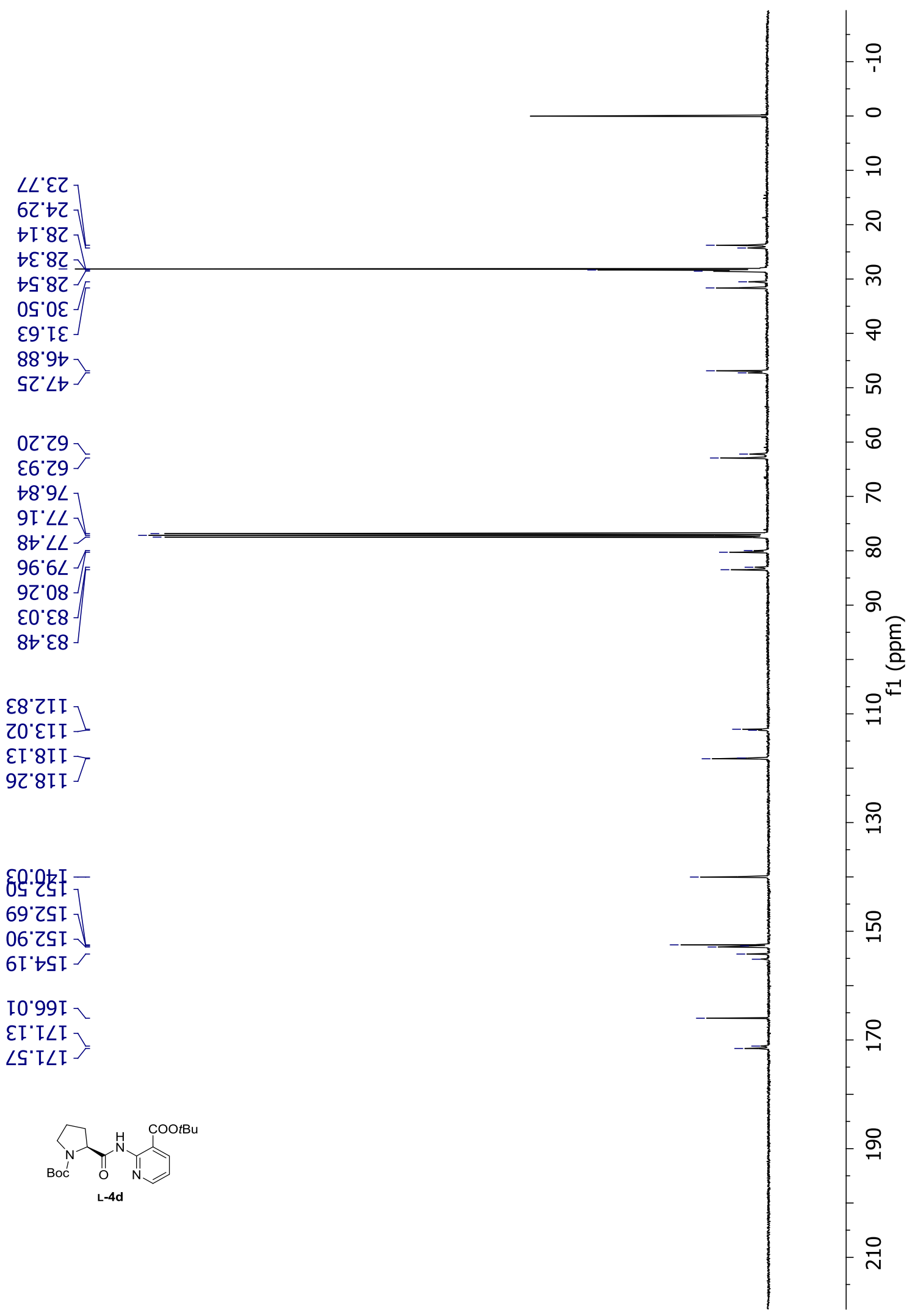


tert-Butyl 2-\{[N-(tert-butoxycarbonyl)-L-methionyl]amino $\}$ nicotinate (Boc-L-Met-NH- $t$ Bu-nic, ER1558) L-4e - ${ }^{1}$ H NMR (400 MHz, $\left.\mathrm{CDCl}_{3}\right)$

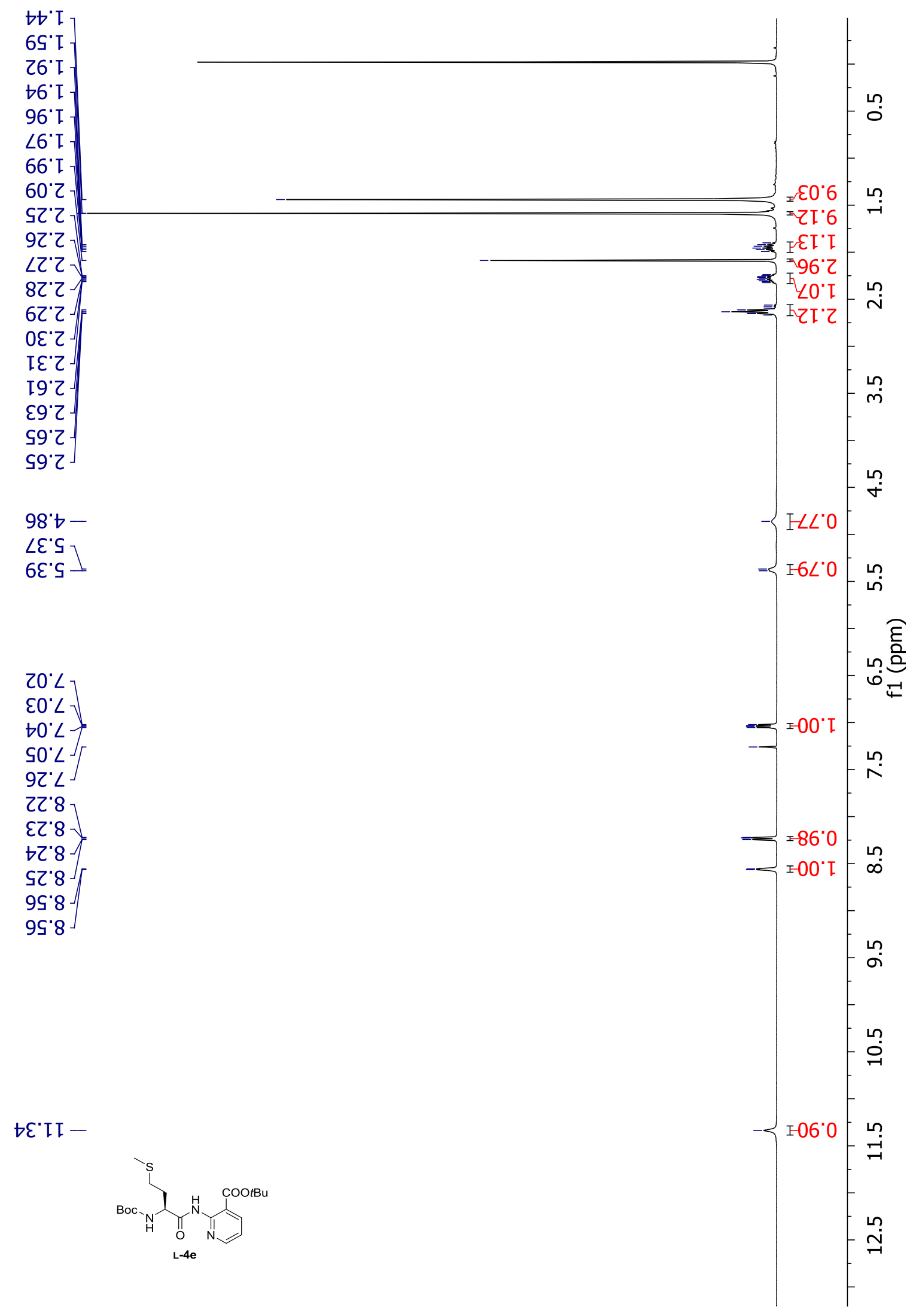


tert-Butyl ER1558) L-4e - ${ }^{13} \mathrm{C}$ NMR (101 MHz, $\left.\mathrm{CDCl}_{3}\right)$

OS'SI -

$02 \cdot 82$

St" 82

$\angle \varepsilon^{\circ} 0 \varepsilon^{\top}$

$\varepsilon 8^{\circ} Z \varepsilon$

EI'SS-

$t 8^{\circ} 9 L$

$\left.9 I^{\circ} \angle L\right]$

$86^{\circ} \angle L$

20.08

$09^{\circ} \varepsilon 8$

96'ZII-

OS' 8 I I -

IZ'0†I-

$\downarrow Z \cdot Z S I$

†9'ZSI

¿9. SSI $\leftrightharpoons$

ธ6.ร9I -

ع8. $0 \angle I-$

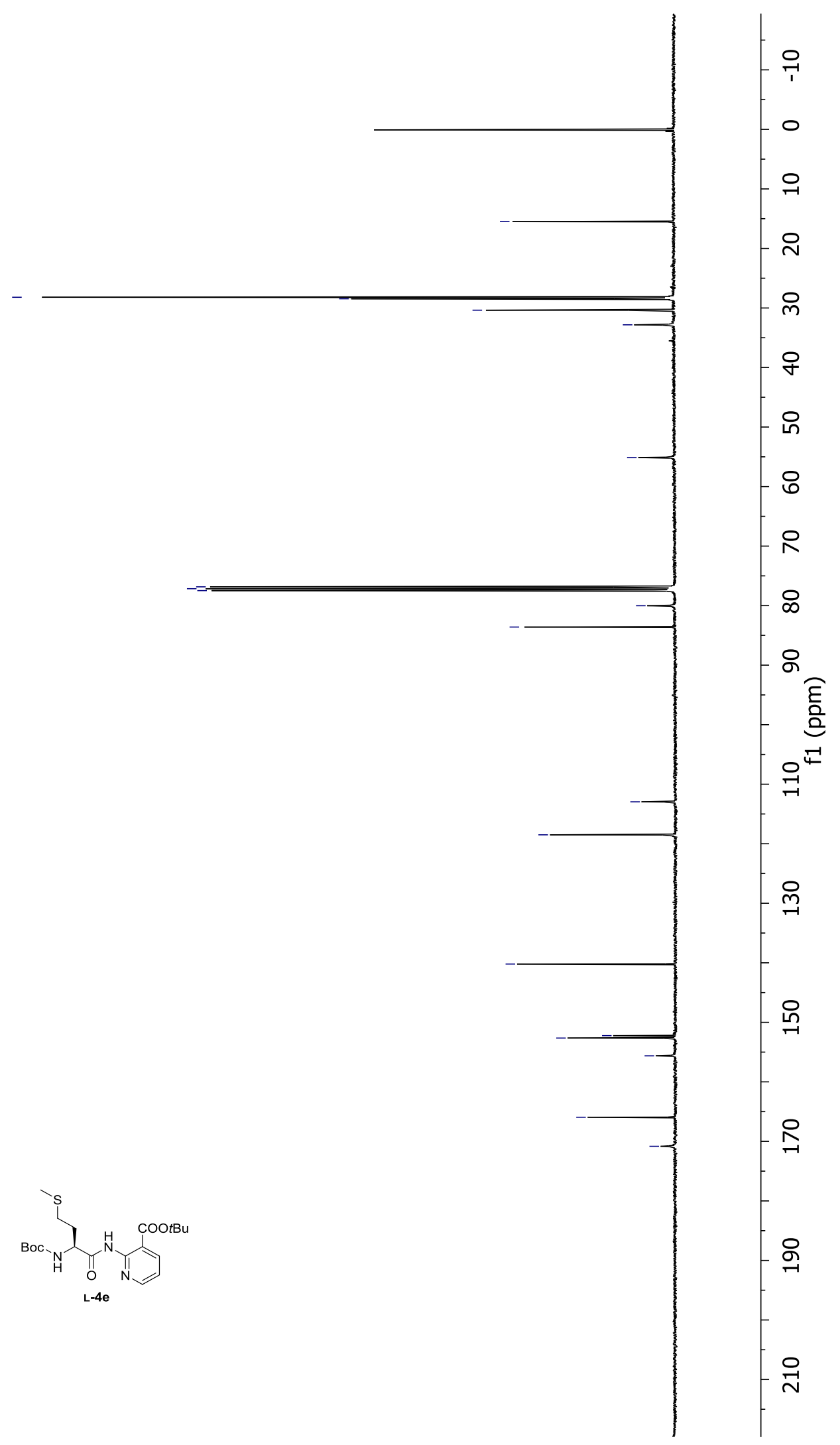


tert-Butyl 2-\{3-[(tert-butoxycarbonyl)amino]propanamido\}nicotinate (Boc- $\beta$ Ala-NH-tBu-nic, CW1618) 4f - ${ }^{1} \mathbf{H}$ NMR (400 MHz, $\mathrm{CDCl}_{3}$ )

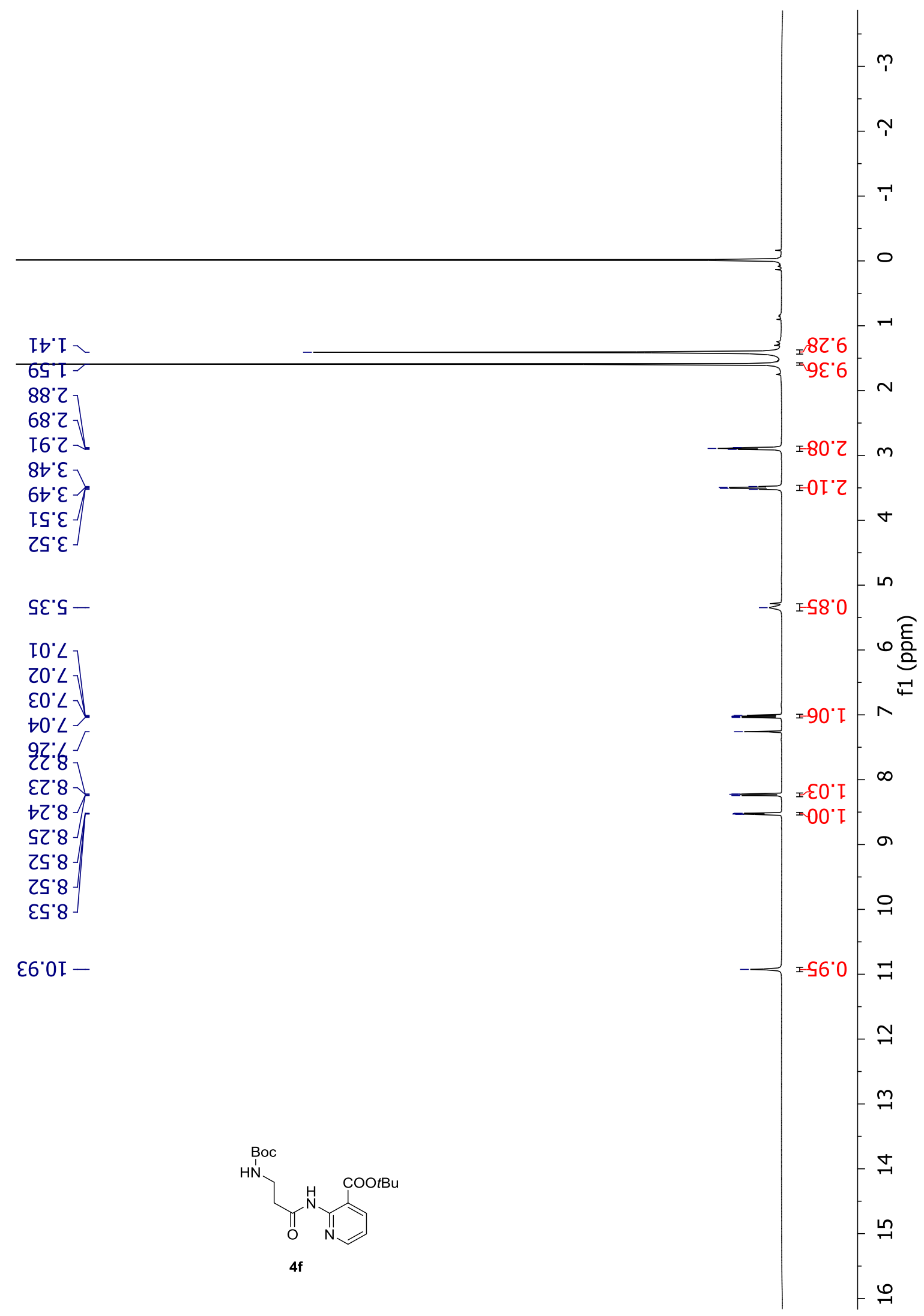


tert-Butyl 2-\{3-[(tert-butoxycarbonyl)amino]propanamido\}nicotinate (Boc- $\beta$ Ala-NH-tBu-nic, CW1618) $4 \mathrm{f}-{ }^{13} \mathrm{C}$ NMR (101 MHz, $\left.\mathrm{CDCl}_{3}\right)$

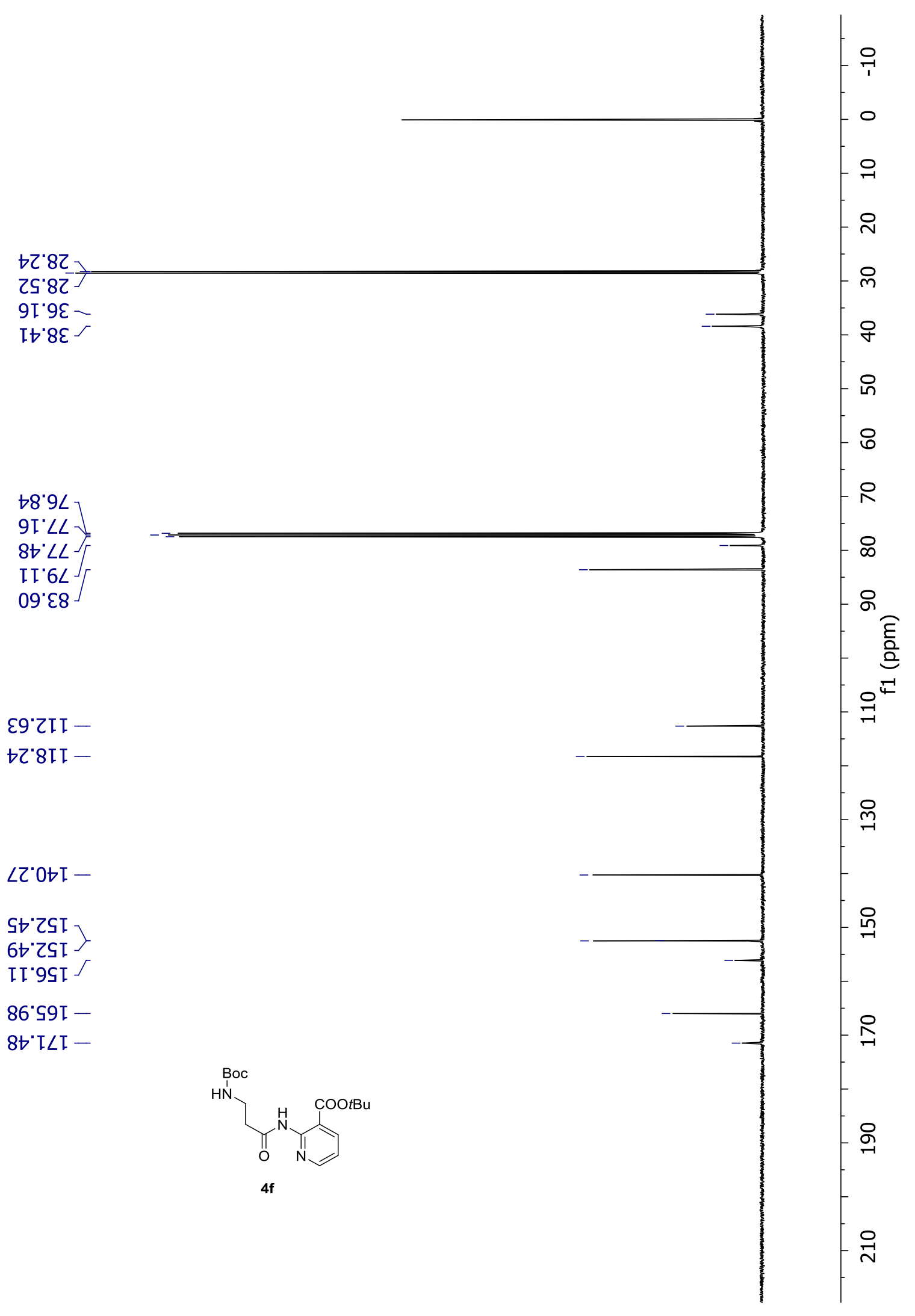


tert-Butyl 2-\{[N-(tert-butoxycarbonyl)glycyl]amino\}nicotinate (Boc-Gly-NH-tBu-nic, CW-1124) $4 \mathrm{~g}$ ${ }^{1} \mathrm{H}$ NMR (400 MHz, $\left.\mathrm{CDCl}_{3}\right)$

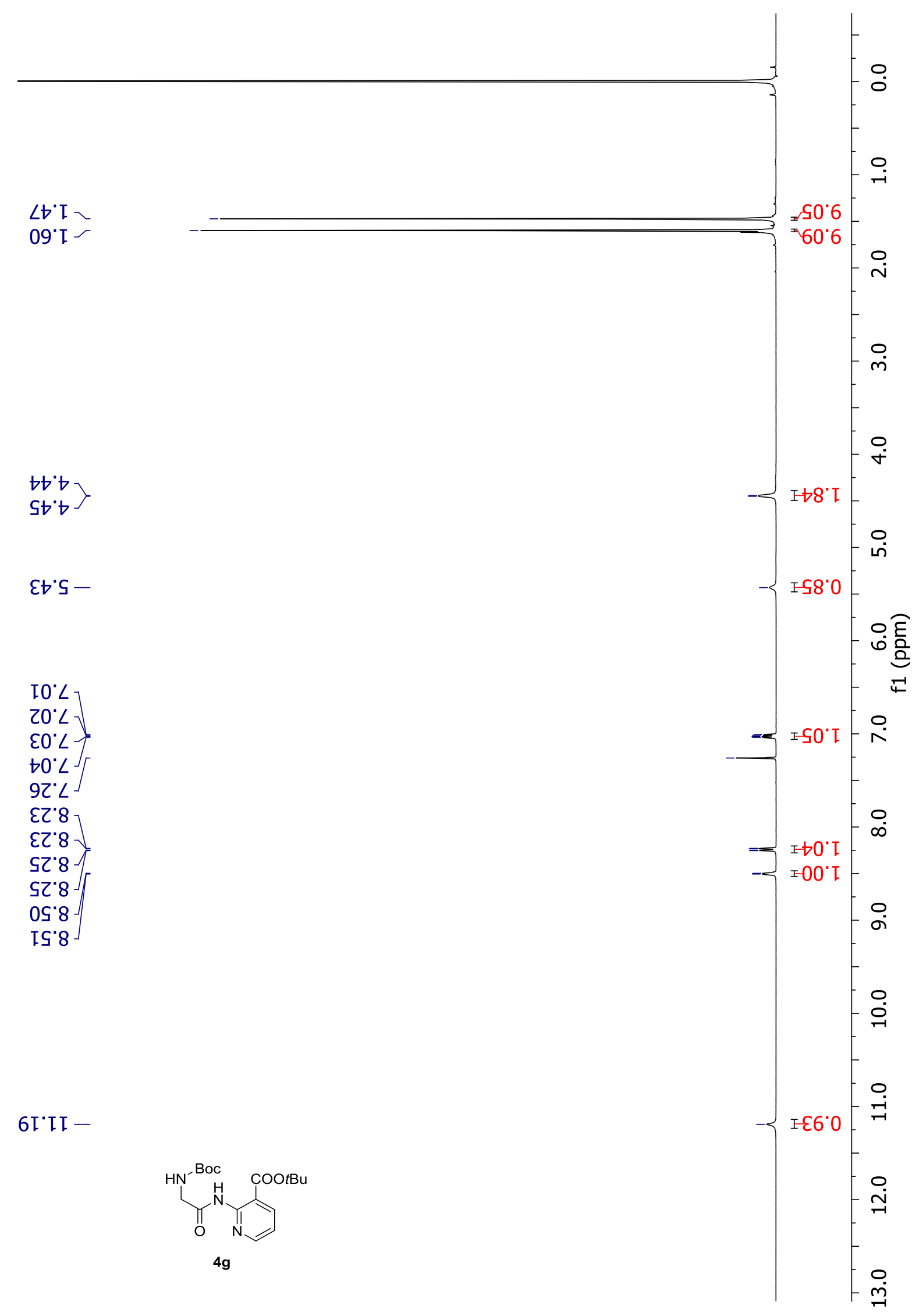


tert-Butyl 2-\{[N-(tert-butoxycarbonyl)glycyl]amino\}nicotinate (Boc-Gly-NH-tBu-nic, CW-1124) 4g ${ }^{13} \mathrm{C}$ NMR (101 MHz, $\left.\mathrm{CDCl}_{3}\right)$

$\left.\begin{array}{l}S{ }^{\circ} 8 Z \\ \varepsilon S^{\prime} 8 Z\end{array}\right\}$

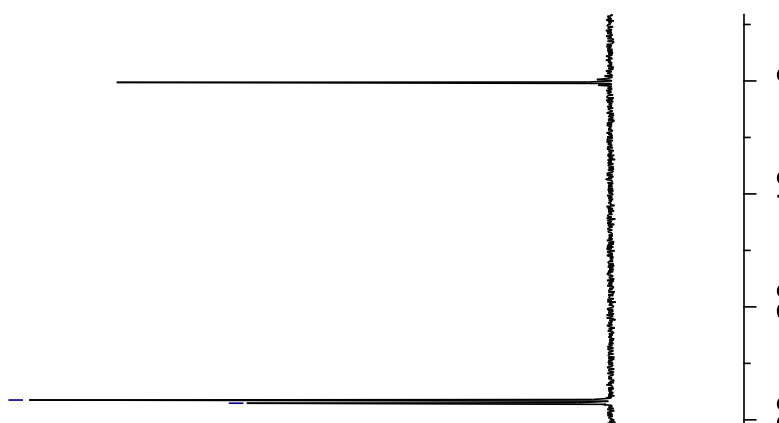

$\varepsilon 8^{\circ} 9 t-$

$78^{\circ} 9 L$

9I' $\angle L$

$8 b^{\circ} \angle L$

$\angle 8^{\circ} 6 \angle$

$29^{\circ} \varepsilon 8$

SZ'ZII -

$\varepsilon Z^{\prime} 8 \mathrm{II}-$

$8 \varepsilon^{\prime} 0 \nrightarrow \mathrm{I}-$

SZ'ZSI

$8 \varepsilon^{\prime} \mathrm{ZSI} \zeta$

20.9SI

โ8. $991-$

โ6.69I-

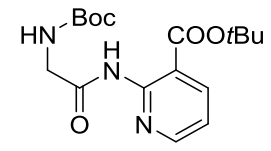

$4 g$

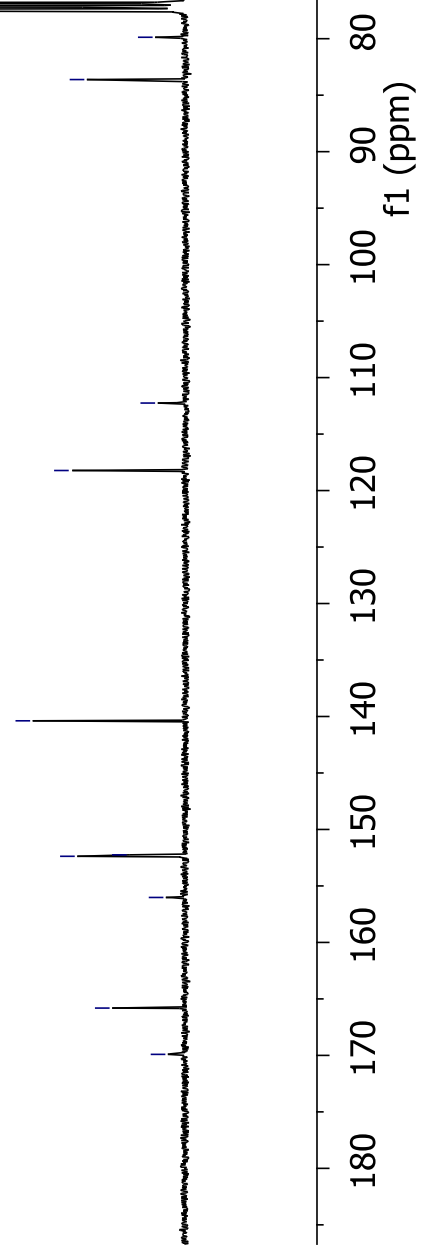


tert-Butyl 2-\{[N-(tert-butoxycarbonyl)-L-valyl]amino $\}$ nicotinate (Boc-L-Val-NH-tBu-nic, ER1538) L4h - ${ }^{1}$ H NMR (400 MHz, CDCl $)$

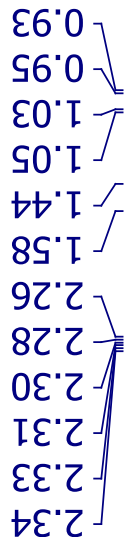

$\varepsilon S^{\prime} \vdash-$

$\nabla Z^{\prime} S$

$9 Z^{\prime} \mathcal{S}^{\prime}$

TO० $\angle$

$20^{\circ} \angle \sqrt{ }$

$\varepsilon 0^{\circ} \angle \mathcal{J}$

$\circ 0^{\circ} \angle$
$9 C^{\circ} \angle$

IZ'8

โZ'8

ع '8-

$\varepsilon \mathrm{C}^{\circ} 8$

95. 8

$\angle S^{\prime} 8$ -

$85^{\circ} 8$ -

85.8

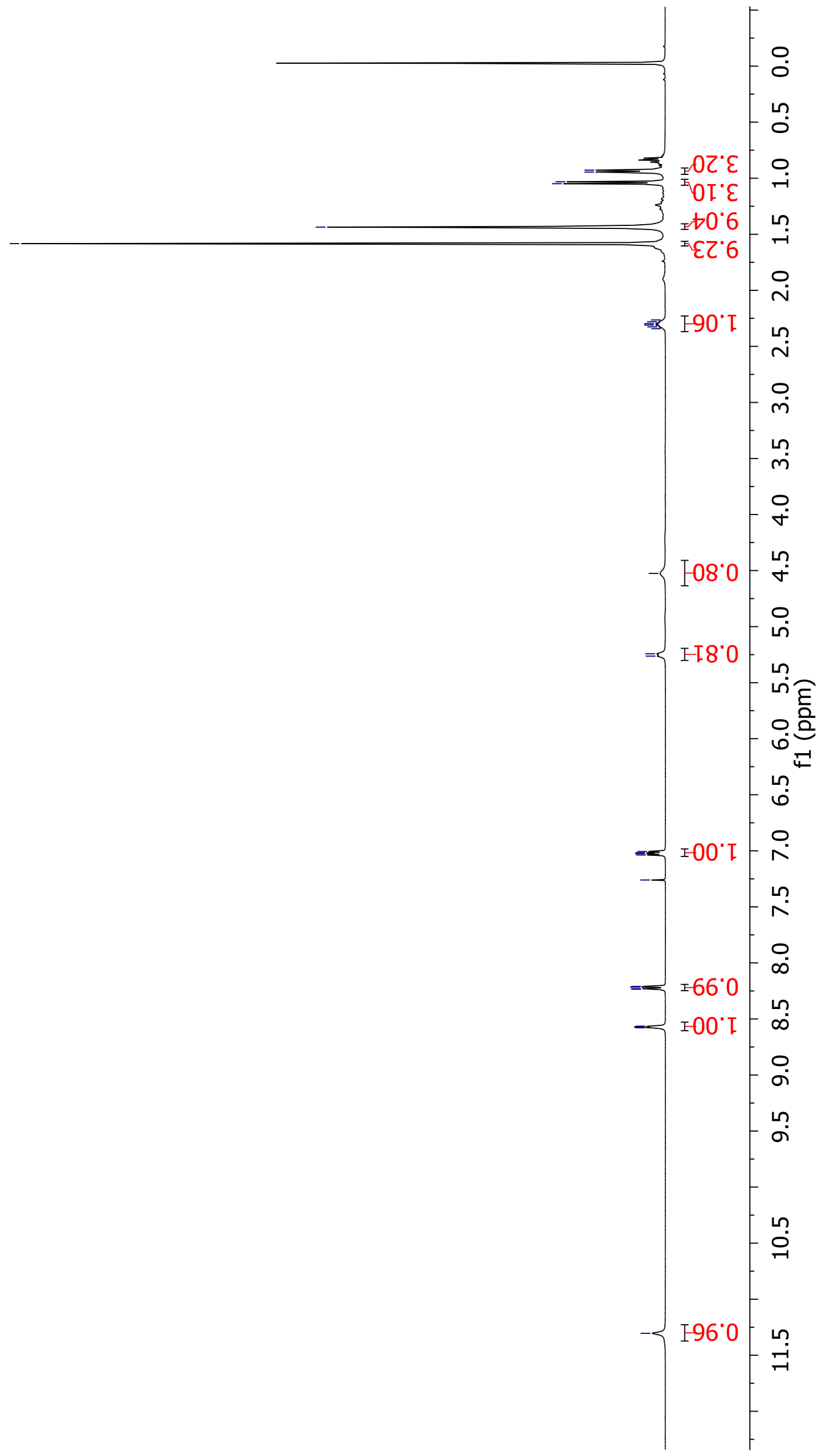

OE'II - 
tert-Butyl 2-\{[N-(tert-butoxycarbonyl)-L-valyl]amino\}nicotinate (Boc-L-Val-NH-tBu-nic, ER1538) L4h - ${ }^{13} \mathrm{C}$ NMR (101 MHz, $\left.\mathrm{CDCl}_{3}\right)$

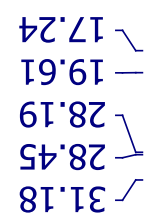

SL'09-

$78^{\circ} 9 L$

$91^{\circ} \angle L$

$86^{\circ} \angle L J$

$\varepsilon L^{\prime} 6 L$ ]

OS' $\varepsilon 8$

06'ZII -

$8 \varepsilon^{\circ} 8 I$ I -

OI'OtI-

IE'ZST

$\varepsilon L$ 'ZSI

00'9SI

I0.99I -

09.0 L I -

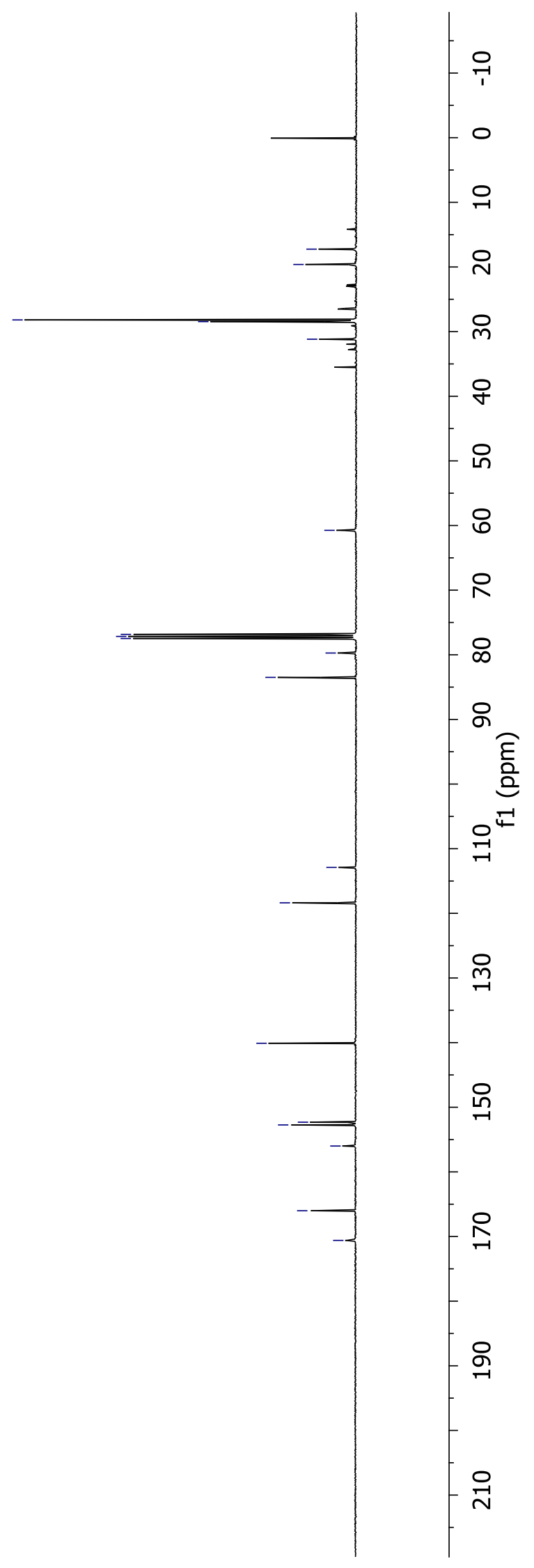


tert-Butyl 2-\{[N-(tert-butoxycarbonyl)-L-isoleucyl]amino $\}$ nicotinate (Boc-L-Ile-NH-tBu-nic, DM679) L-4i - ${ }^{1}$ H NMR (400 MHz, $\mathrm{CDCl}_{3}$ )

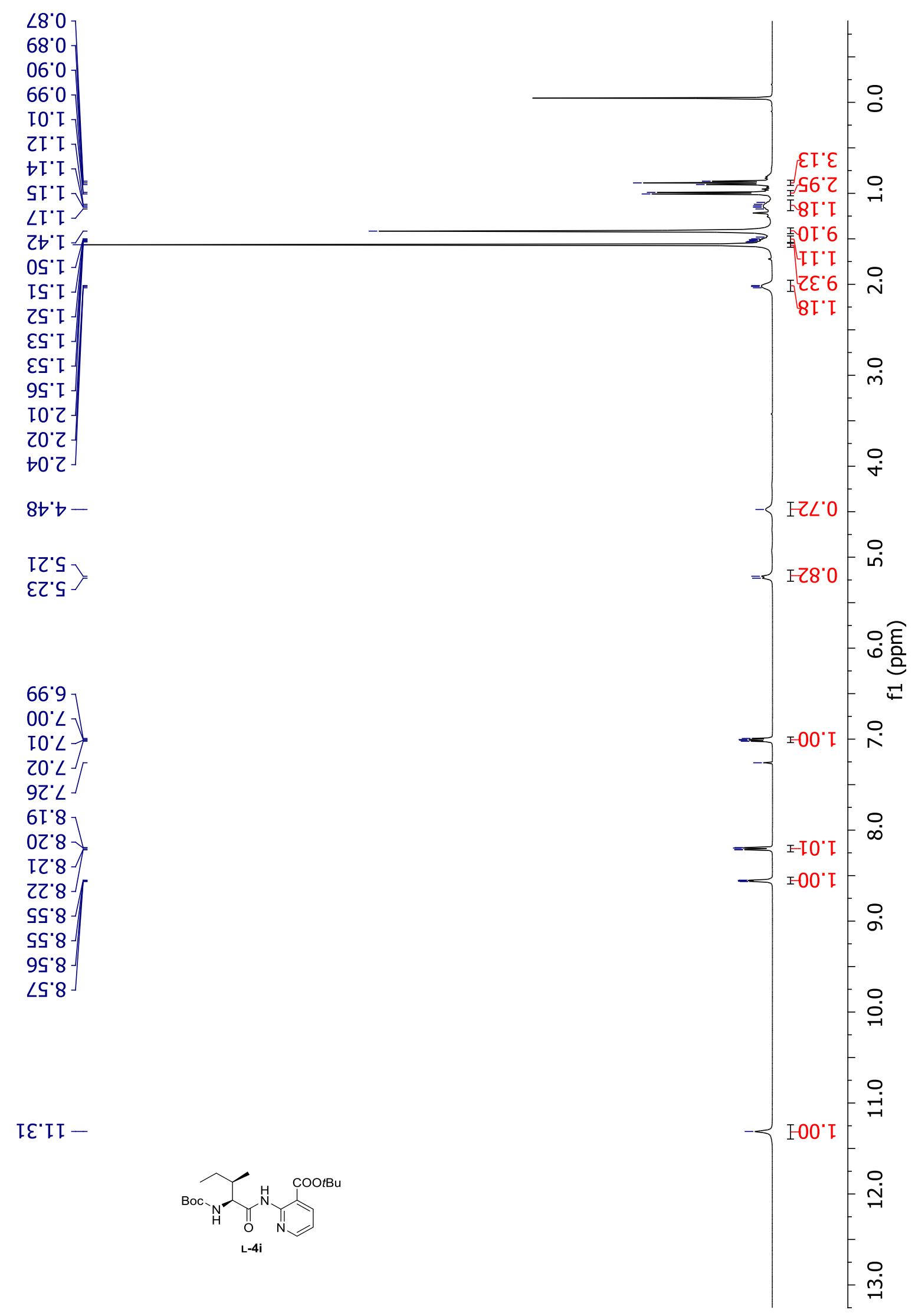


tert-Butyl 2-\{[N-(tert-butoxycarbonyl)-L-isoleucyl]amino\}nicotinate (Boc-L-Ile-NH- $t$ Bu-nic, DM679) L-4i - ${ }^{13} \mathrm{C}$ NMR (101 MHz, $\left.\mathrm{CDCl}_{3}\right)$

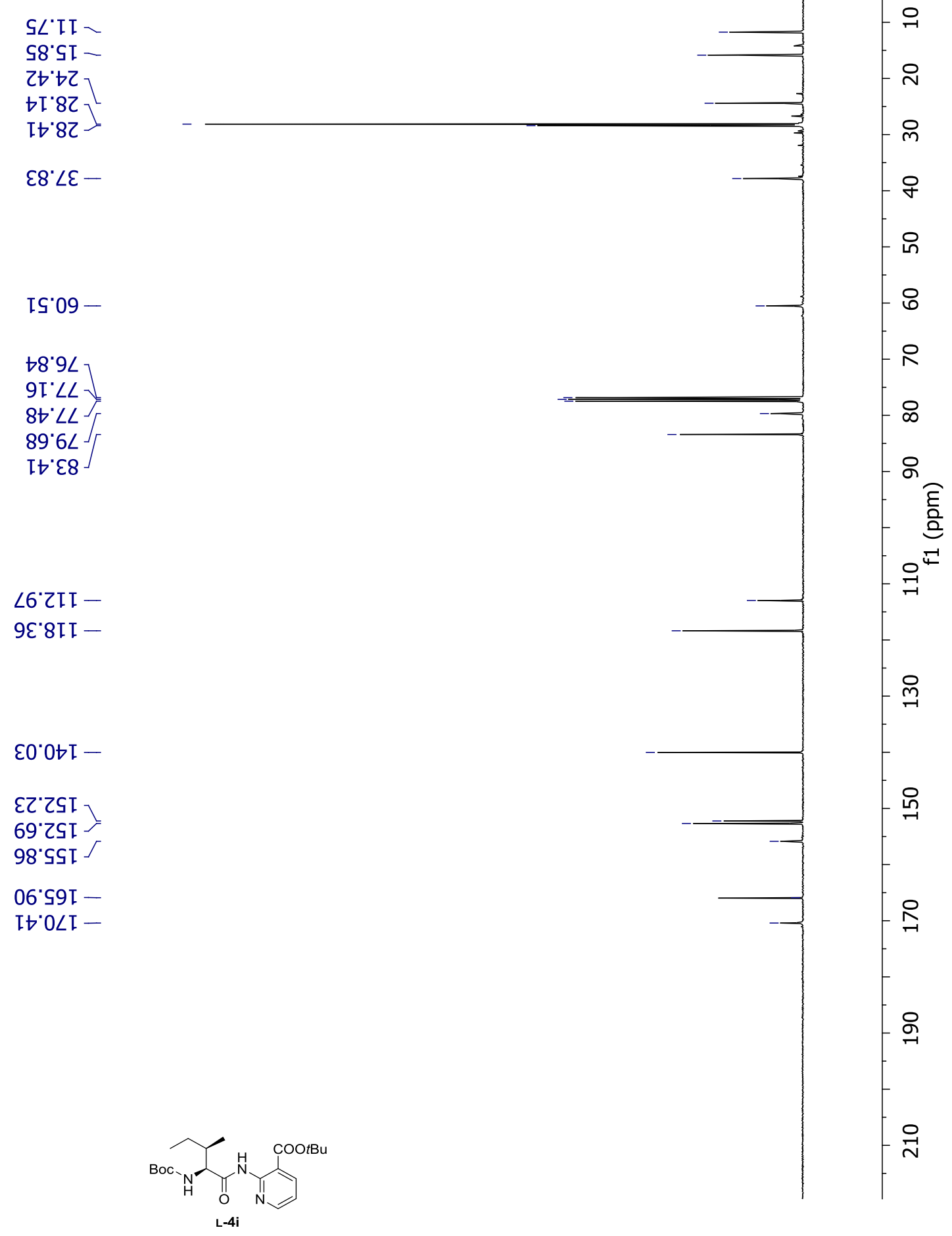


tert-Butyl 2-\{[N-(tert-butoxycarbonyl)-O-tert-butyl-L-seryl]amino $\}$ nicotinate (Boc-L-Ser $(t \mathrm{Bu})-\mathrm{NH}$ tBu-nic, LVR0603) L-4j - ${ }^{\mathbf{H}} \mathbf{H}$ NMR (400 MHz, $\left.\mathbf{C D C l}_{3}\right)$

OI'I -

Zt. I

$t S^{\prime} I-$

$\mathrm{Zs} \varepsilon-$

$\angle 8^{\circ} \varepsilon-$

$8 b^{\circ} \bullet-$

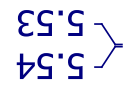

$\angle 6^{\circ} 9$

$86^{\circ} 9$

$66.9-$

$00^{\circ} \angle$

$9 C^{\circ} \angle$

$8 \mathrm{I}^{\circ} 8$

6I:8

$t S^{\prime} 8>$

${ }^{t} \mathrm{~S}^{\circ} 8$

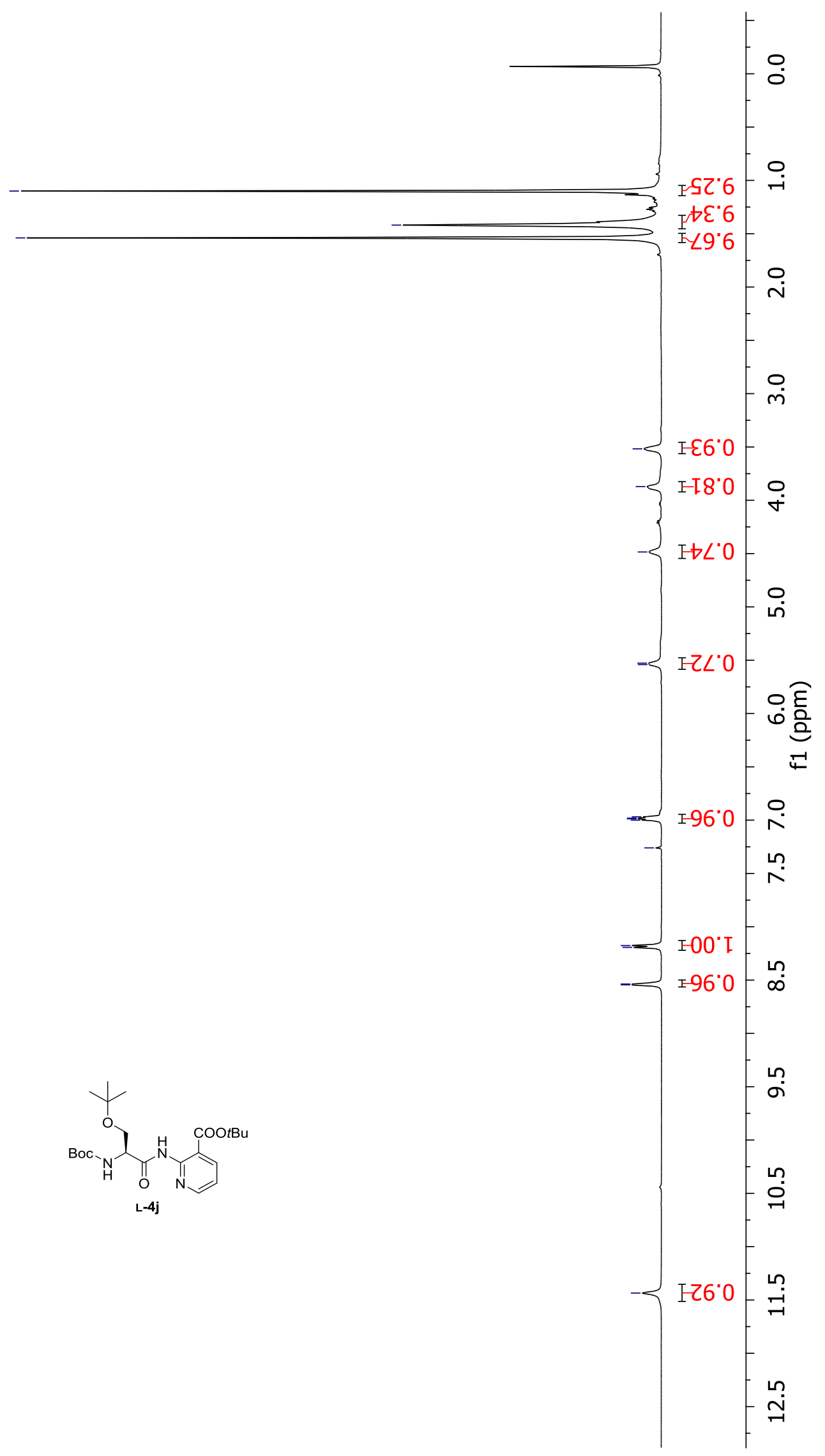

tt'II- 
tert-Butyl 2-\{[N-(tert-butoxycarbonyl)-O-tert-butyl-L-seryl]amino\} nicotinate (Boc-L-Ser $(t \mathrm{Bu})-\mathrm{NH}$ ${ }^{t} \mathrm{Bu}$-nic, LVR0603) L-4j - ${ }^{13} \mathrm{C}$ NMR (101 MHz, $\mathbf{C D C l}_{3}$ )

$\left.\begin{array}{l}t \varepsilon^{\prime} \angle Z \\ 60^{\circ} 8 乙 \\ \angle \varepsilon^{\prime} 8 乙\end{array}\right]$

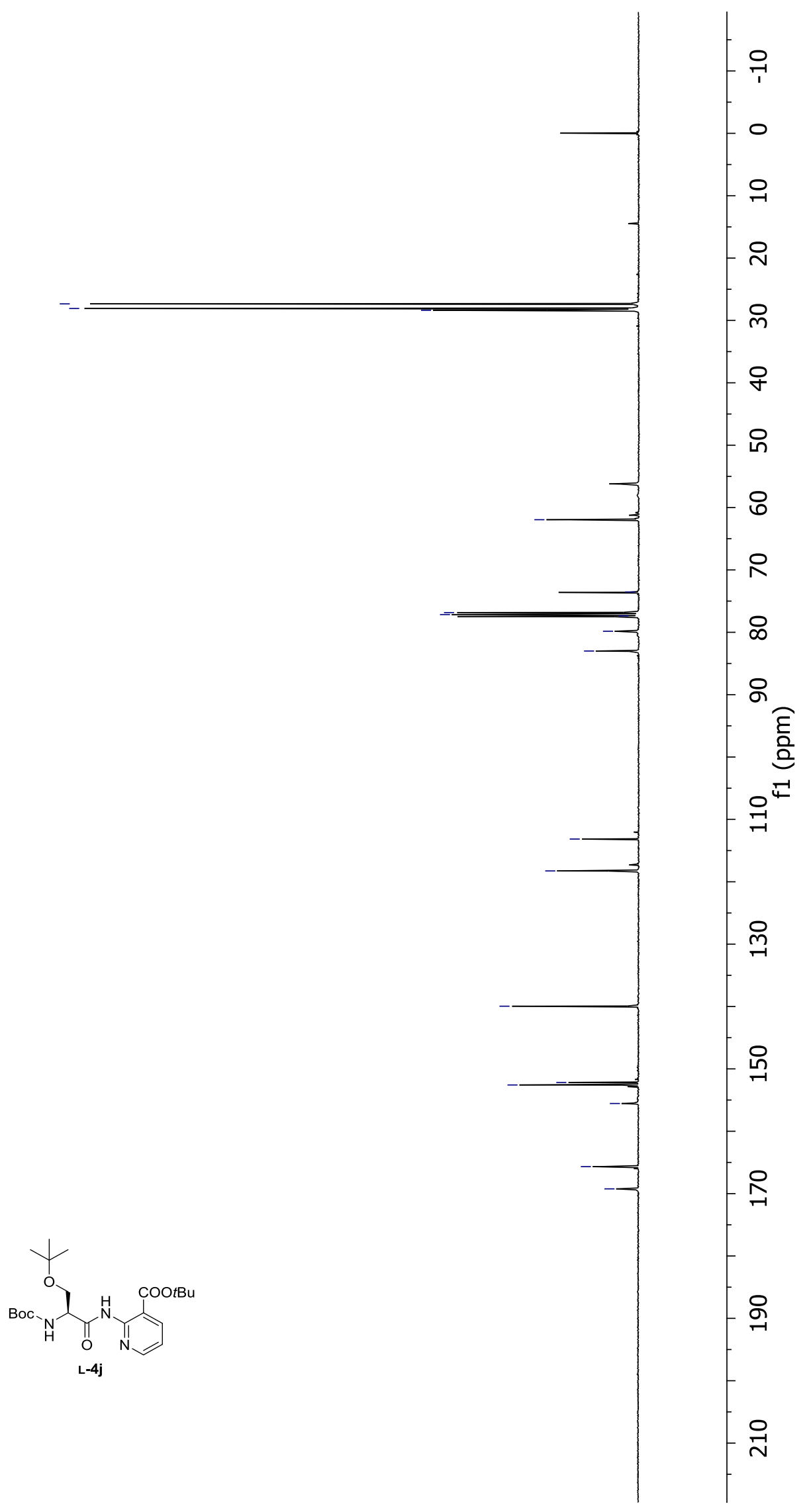


tert-Butyl 2-\{[ $N^{2}, N^{6}$-bis(tert-butoxycarbonyl)-L-lysyl]amino $\}$ nicotinate (Boc-L-Lys(Boc)-NH-tBu-nic, ER1718) L-4k - ${ }^{1} \mathrm{H}$ NMR (400 MHz, $\mathrm{CDCl}_{3}$ )

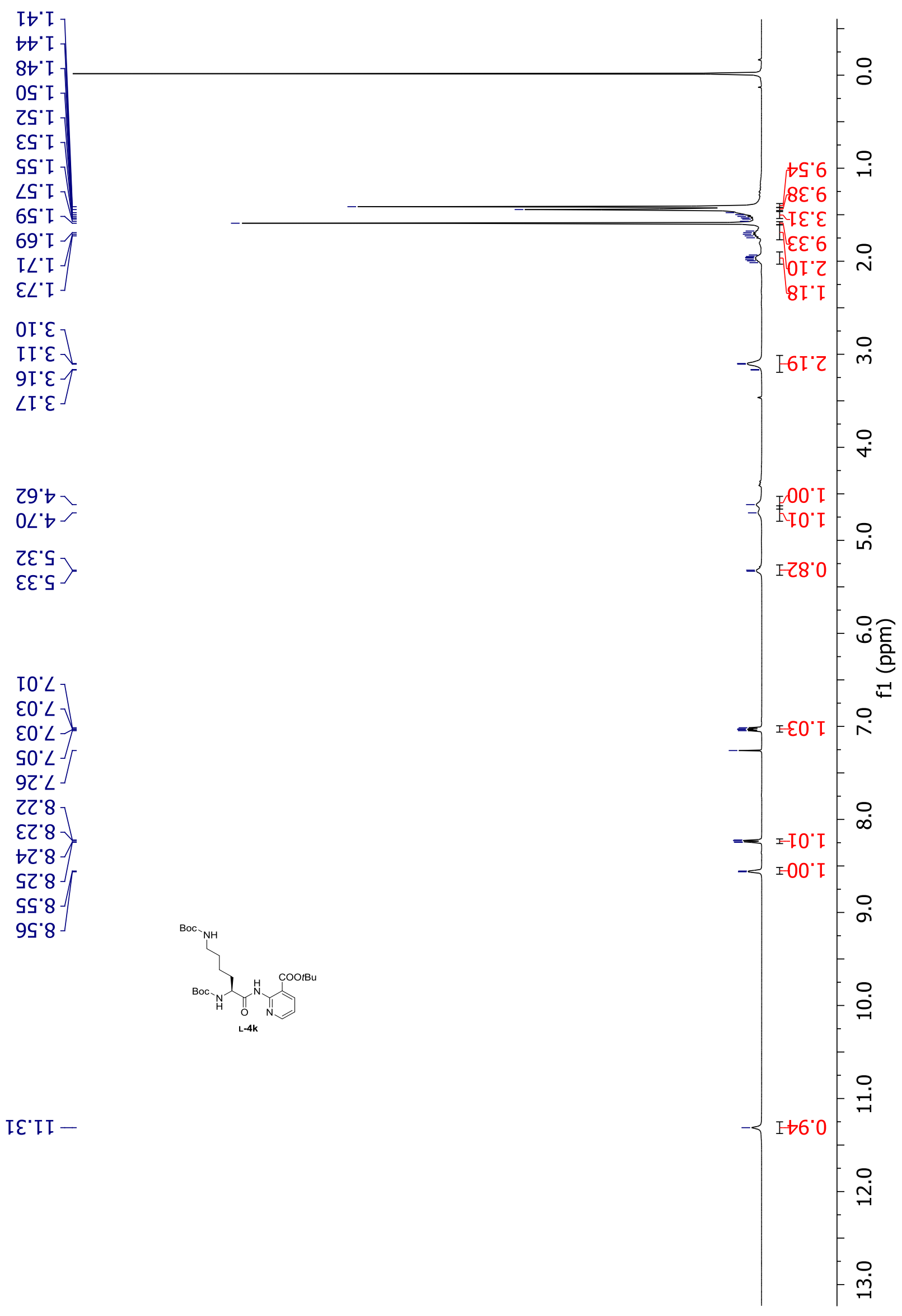


tert-Butyl 2-\{[ $N^{2}, N^{6}$-bis(tert-butoxycarbonyl) L-lysyl]amino $\}$ nicotinate (Boc-L-Lys(Boc)-NH-tBu-nic, ER1718) L-4k - ${ }^{13} \mathrm{C}$ NMR (101 MHz, $\left.\mathrm{CDCl}_{3}\right)$

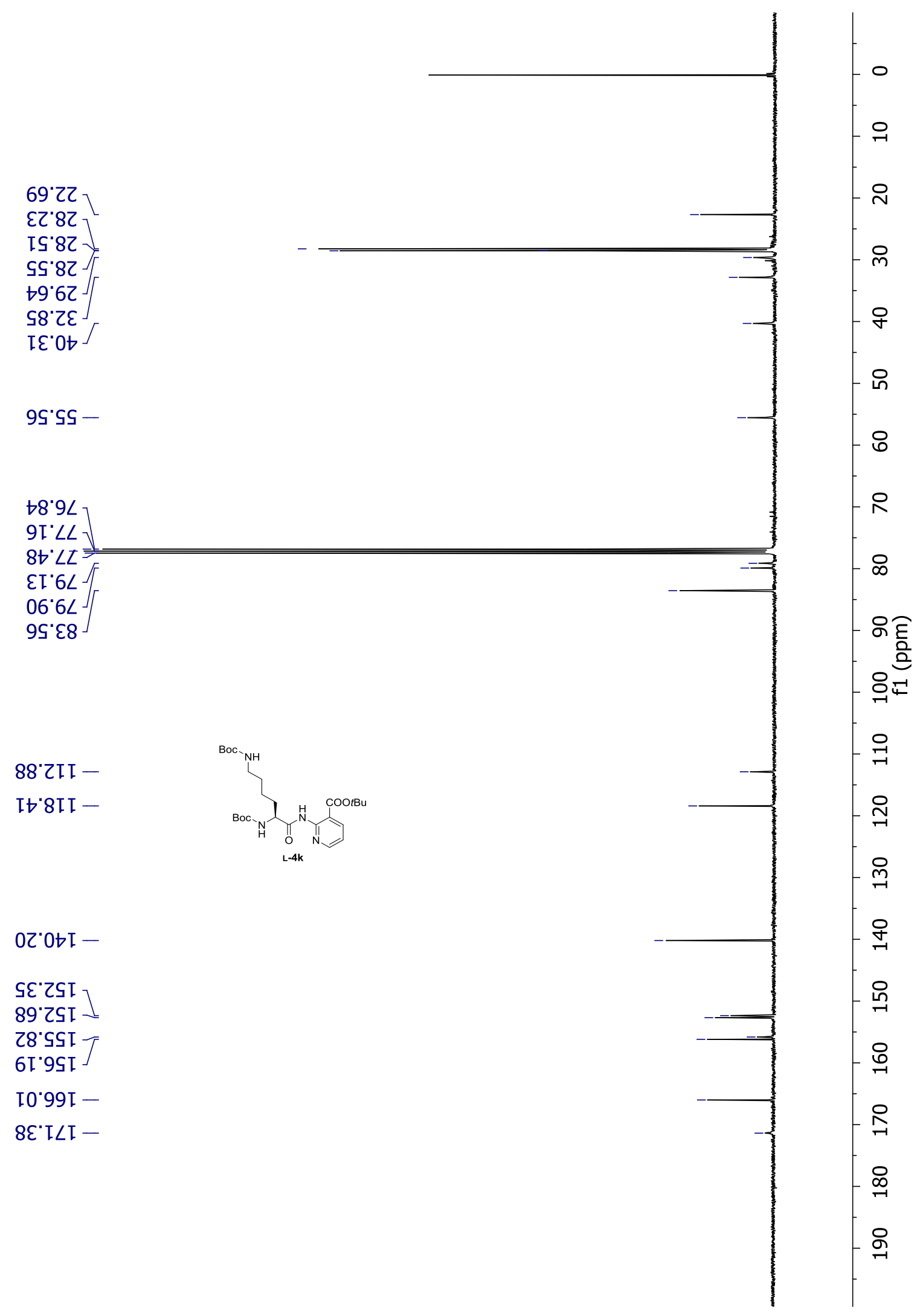


tert-Butyl (R)-2-(2-((tert-butoxycarbonyl)amino)-3-(tert-butylthio)propanamido)nicotinate (Boc-LCys $(t \mathrm{Bu})-\mathrm{NH}-t \mathrm{Bu}-\mathrm{nic}$, ER1625/ER1676) L-4l - ${ }^{\mathbf{1}} \mathbf{H}$ NMR (400 MHz, CDCl $\mathbf{3}$ )

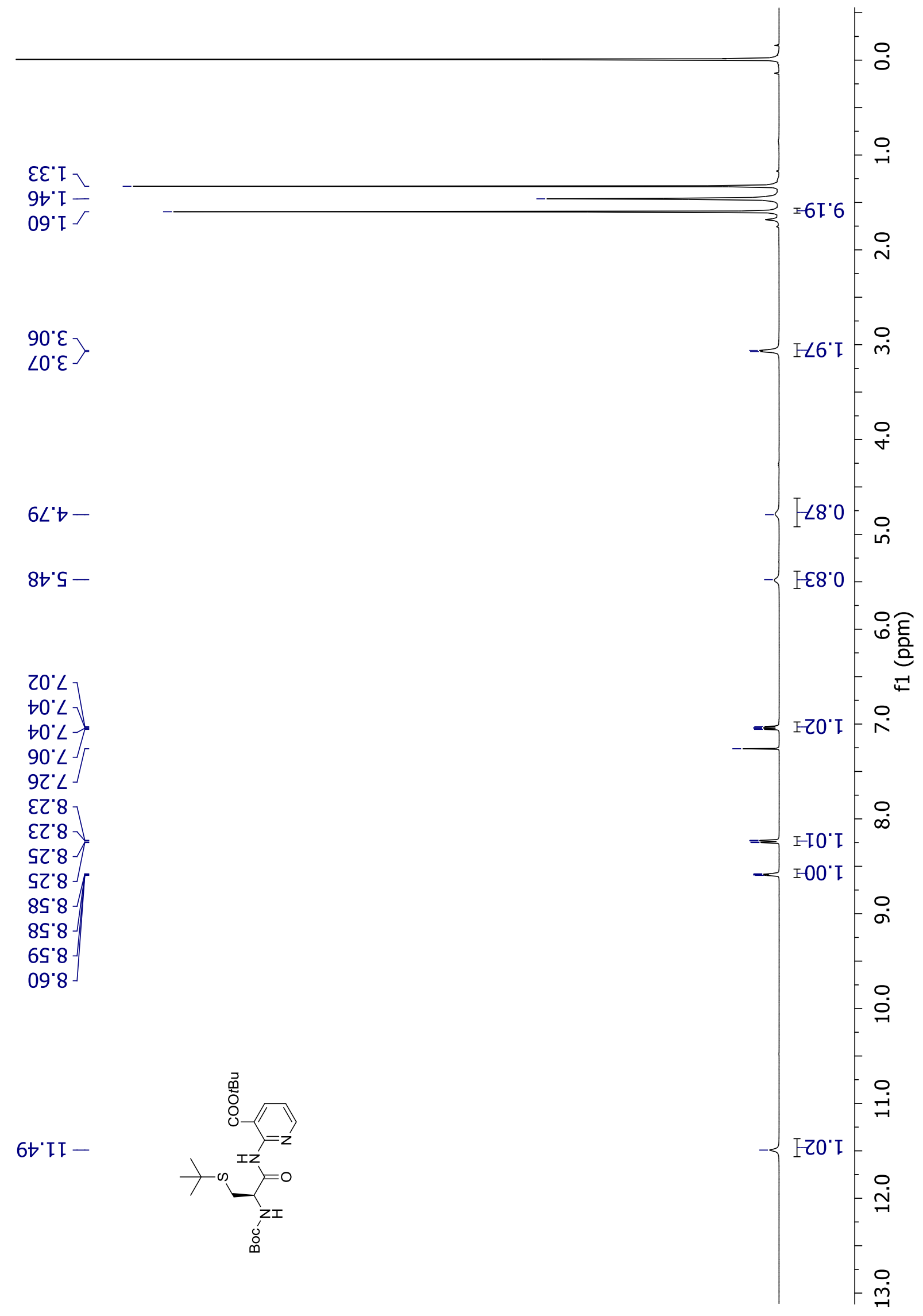


tert-Butyl (R)-2-(2-((tert-butoxycarbonyl)amino)-3-(tert-butylthio)propanamido)nicotinate (Boc-LCys( $t \mathrm{Bu})-\mathrm{NH}-t \mathrm{Bu}-n i c$, ER1625/ER1676) L-4l $-{ }^{13}$ C NMR (101 MHz, CDCl 3 )

52.82

05: 82$]$

SO'TE

$8 \tau^{\circ} \mathrm{T \varepsilon}$

$88^{\circ} 乙 \succ-$

$\downarrow L ' S S-$

$78^{\circ} 9 L$

$9 I^{\prime} \angle L$

$8 t^{\circ} \angle t$

$\varepsilon \tau^{\prime} 08$ -

98. โ8

Sเ" $\varepsilon 8$

9I'ยI -

โS'8I I -

$\angle Z$ ZSI

9L'ZSI $Y$

S8. S9I -

\&S.69I -

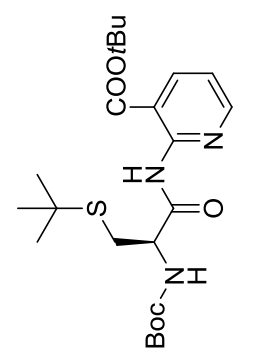

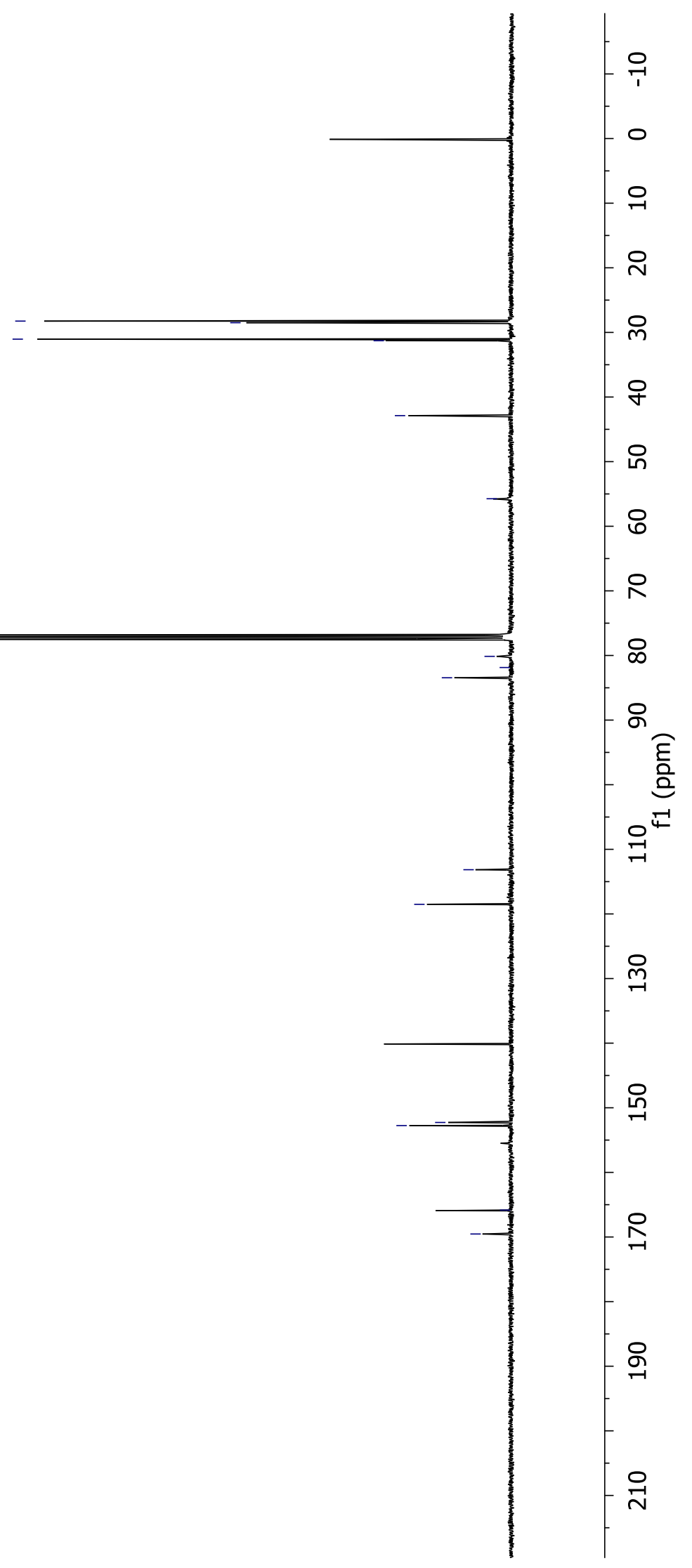


tert-Butyl 2-\{[N-(tert-butoxycarbonyl)-1-(triphenylmethyl)-L-histidyl $]$ amino $\}$ pyridine-3-carboxylate (Boc-L-His(Trt)-NH-tBu-nic, ER1738) L-4m - ${ }^{\mathbf{H}} \mathbf{H}$ NMR (400 MHz, CDCl $\left.\mathbf{3}_{3}\right)$

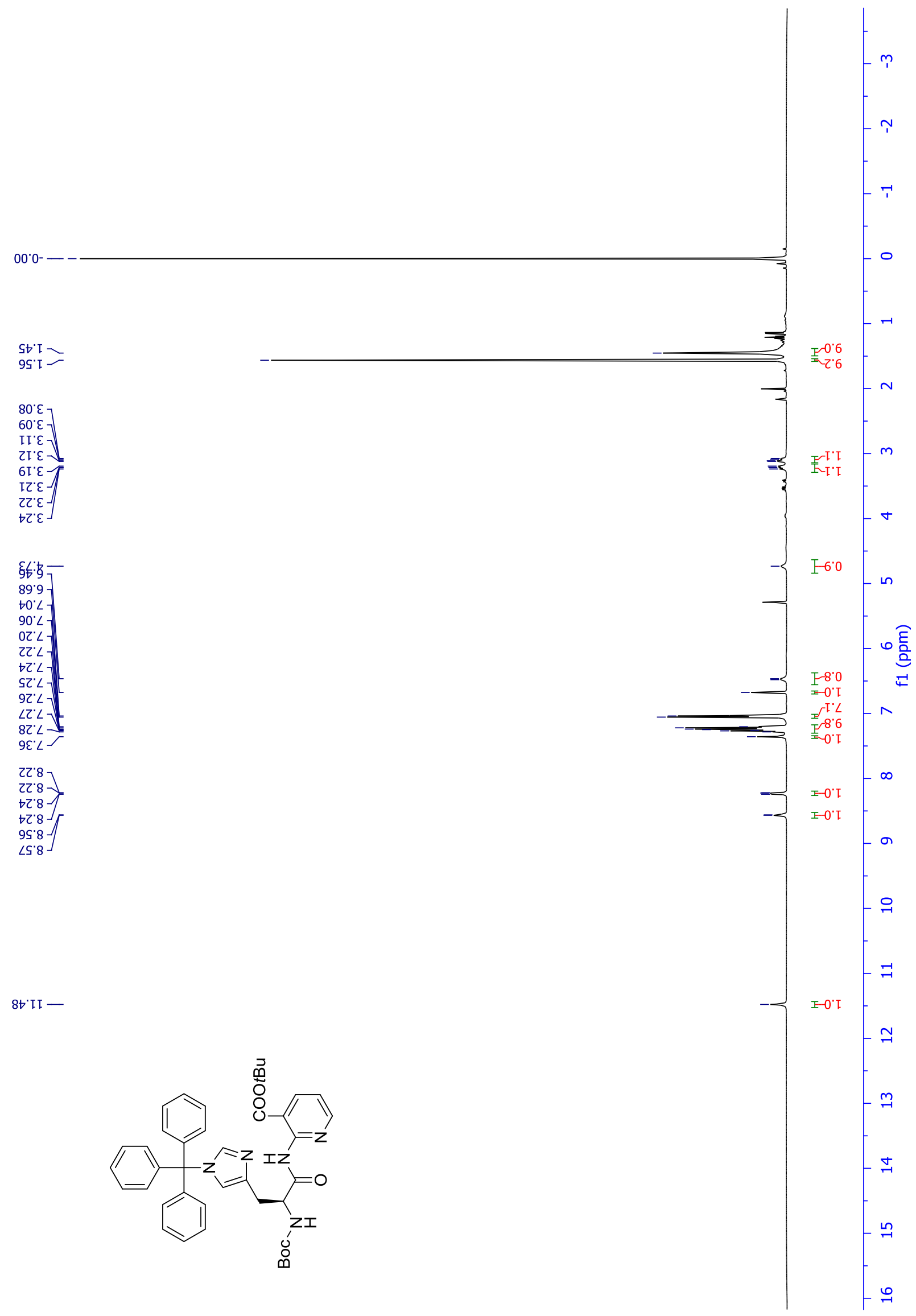


tert-Butyl 2-\{[N-(tert-butoxycarbonyl)-1-(triphenylmethyl)-L-histidyl $]$ amino $\}$ pyridine-3-carboxylate (Boc-L-His(Trt)-NH- $t$ Bu-nic, ER1738) L-4m - ${ }^{\mathbf{1}}$ H NMR (400 MHz, CDCl 3 )

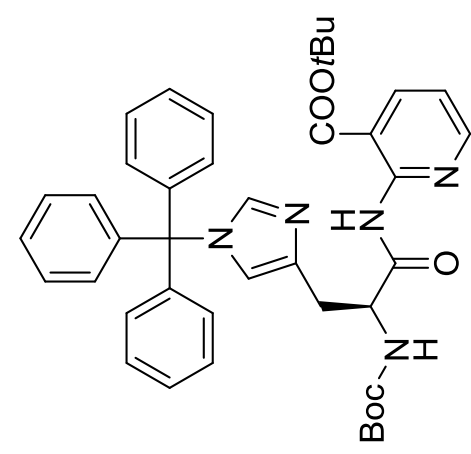


tert-Butoxycarbonyl)-L-prolyl-L-leucyl- $N$-[3-tert-butyoxycarbonyl)pyridin-2-yl)]glycinamide (Boc-LPro-L-Leu-Gly-NH-tBu-nic, CW-1843) L,L-4n- ${ }^{1}$ H NMR (400 MHz, CDCl $)$

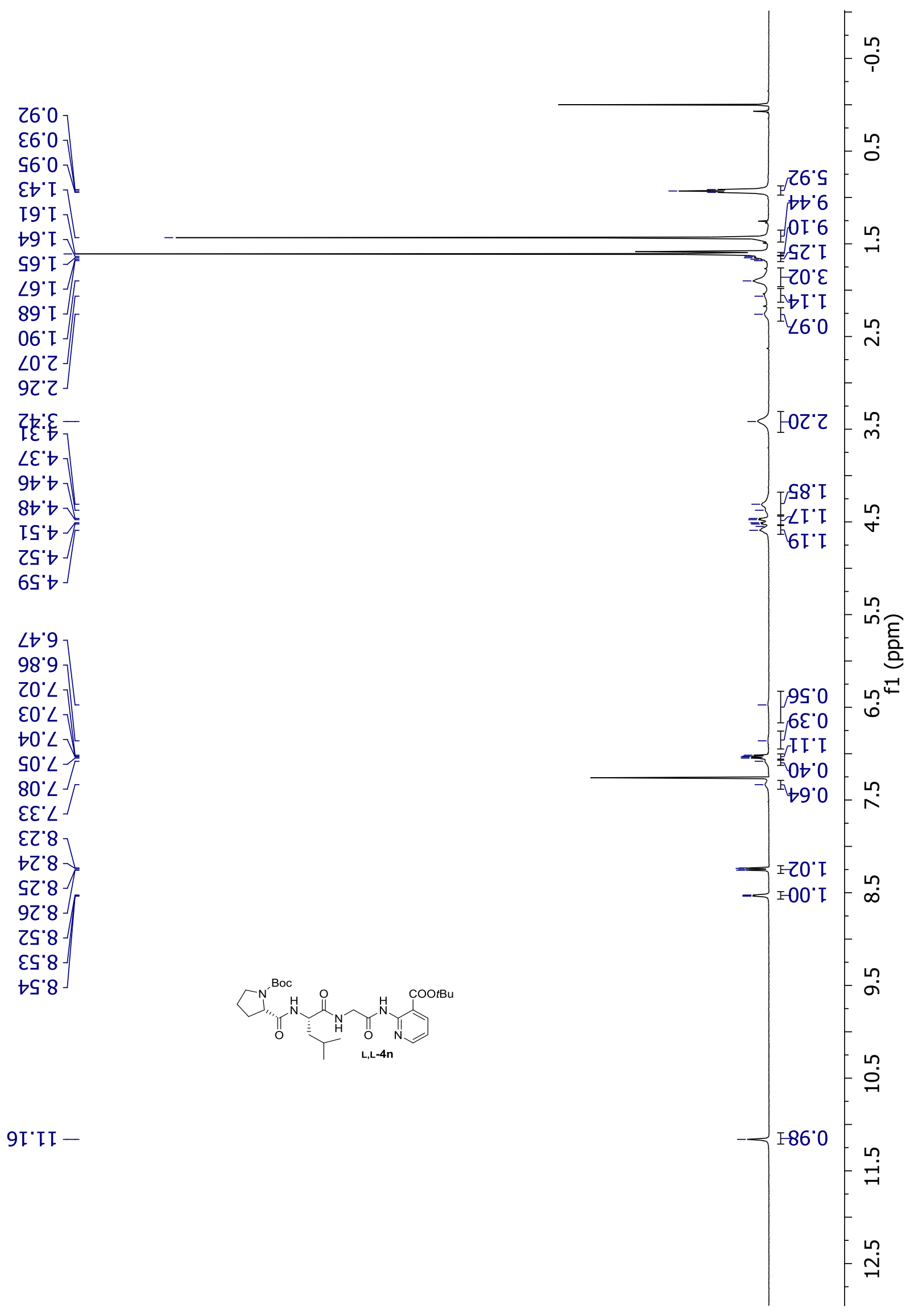


1-(tert-Butoxycarbonyl)-L-prolyl-L-leucyl- $N$-[3-tert-butyoxycarbonyl)pyridin-2-yl)]glycinamide (BocL-Pro-L-LeU-Gly-NH-tBu-nic, CW-1843) L,L-4n - ${ }^{13}$ C NMR (101 MHz, $\left.\mathbf{C D C l}_{3}\right)$
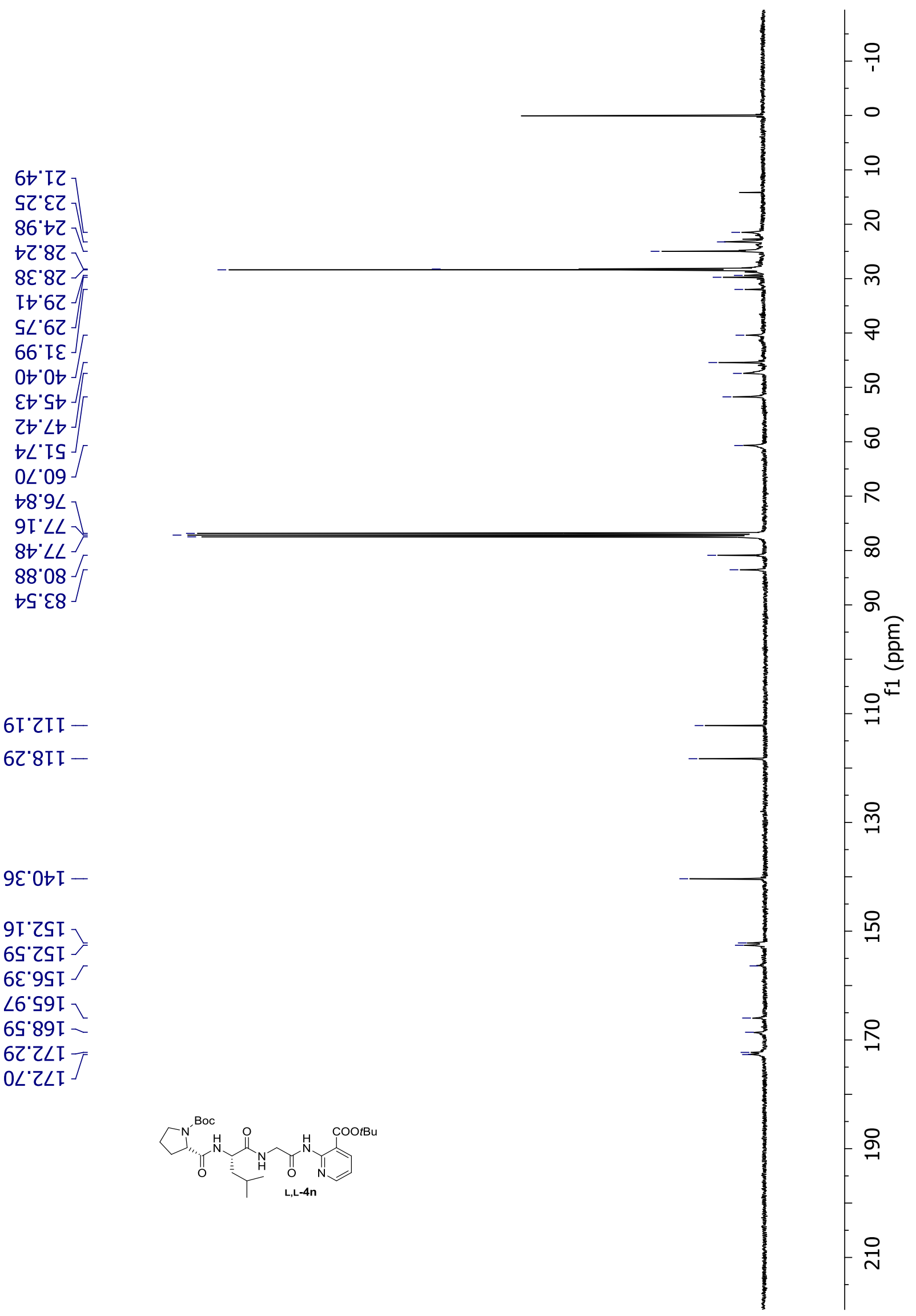
$N^{2}$-(tert-Butoxycarbonyl)- $N^{5}$-(3-(tert-butoxycarbonyl)pyridin-2-yl)-L-glutamine $\quad$ (Boc-L-Gln(NH-tBunic)-OH, DM613) L-4o - ${ }^{1}$ H NMR (400 MHz, DMSO-d d

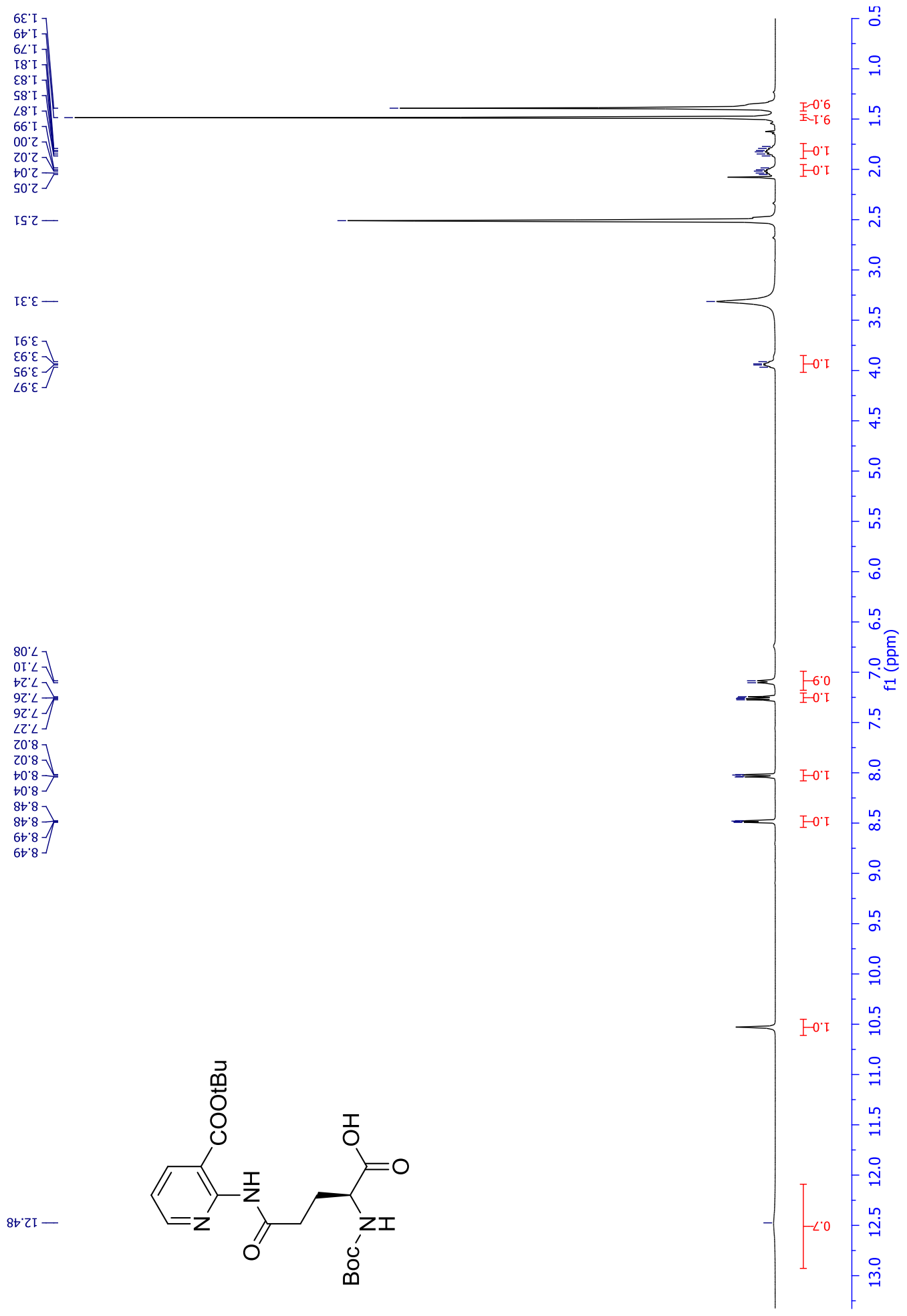


$N^{2}$-(tert-Butoxycarbonyl)- $N^{5}$-(3-(tert-butoxycarbonyl)pyridin-2-yl)-L-glutamine $\quad$ (Boc-L-Gln(NH-tBunic)-OH, DM613) L-4o - ${ }^{13}$ C NMR (100 MHz, DMSO-d 6 )

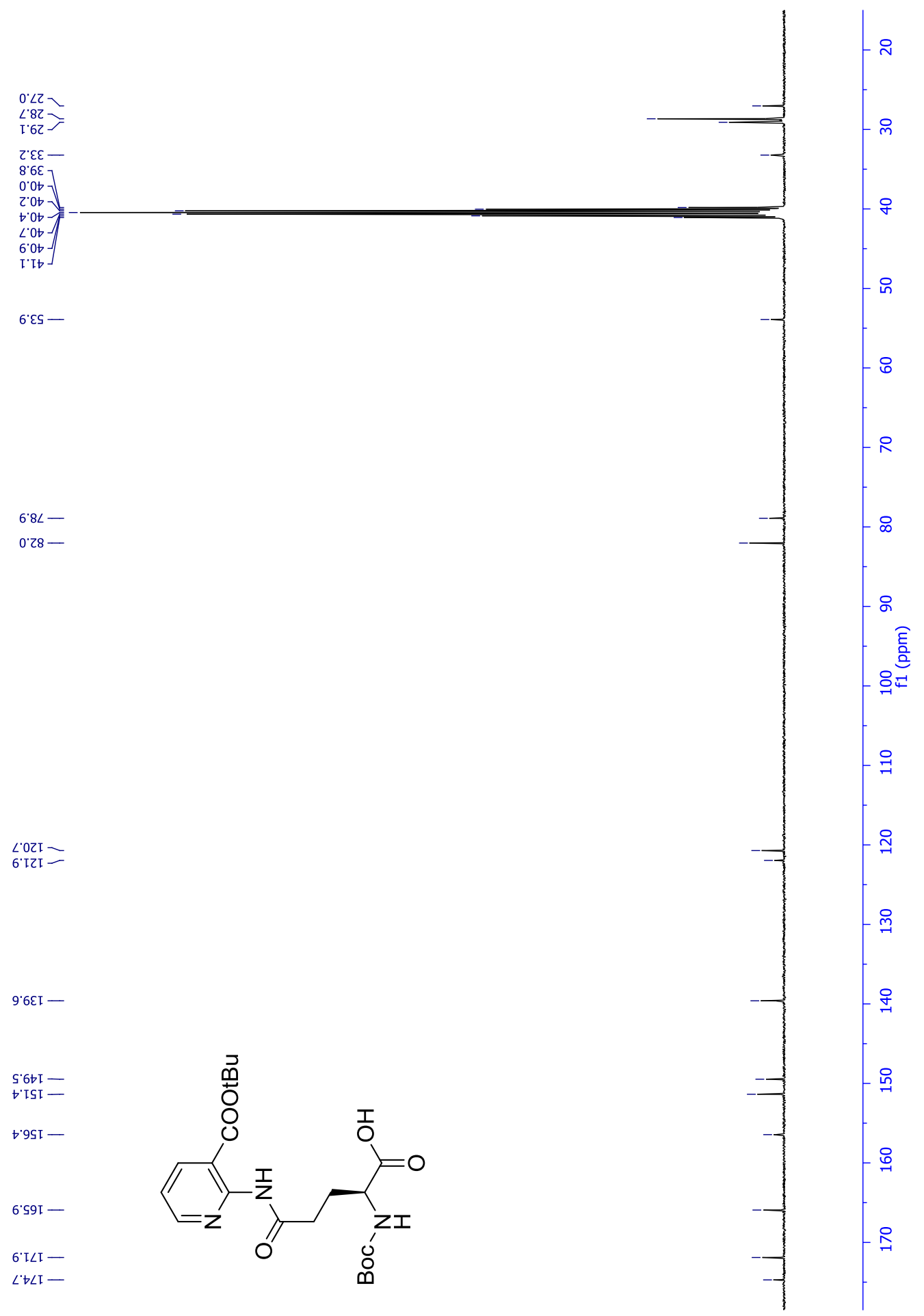


$N$-[3-(tert-butoxycarbonyl)pyridin-2-yl]- $N$-2-\{[(9H-fluoren-9-yl)methoxy]carbonyl $\}$-L-glutamine (Fmoc-L-Gln( $t$ Bu-nic)-OH, DM674) L-4p - ${ }^{1}$ H NMR (400 MHz, DMSO-d ${ }_{6}$ )

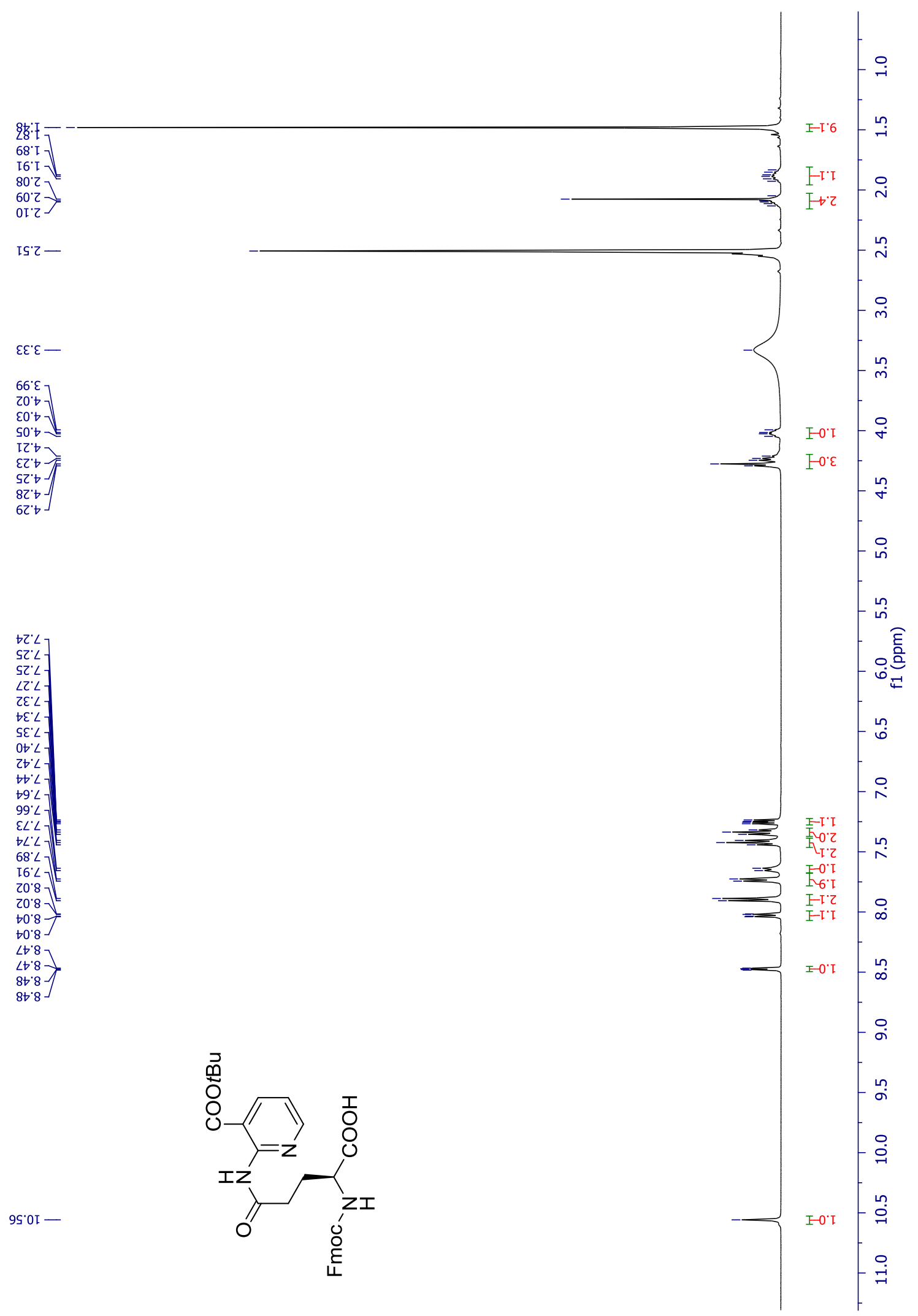


$N$-[3-(tert-butoxycarbonyl)pyridin-2-yl]- $N$-2-\{[(9H-fluoren-9-yl)methoxy]carbonyl $\}$-L-glutamine (Fmoc-L-Gln( $t$ Bu-nic)-OH, DM674) L-4p - ${ }^{13}$ C NMR (101 MHz, DMSO-d $)$

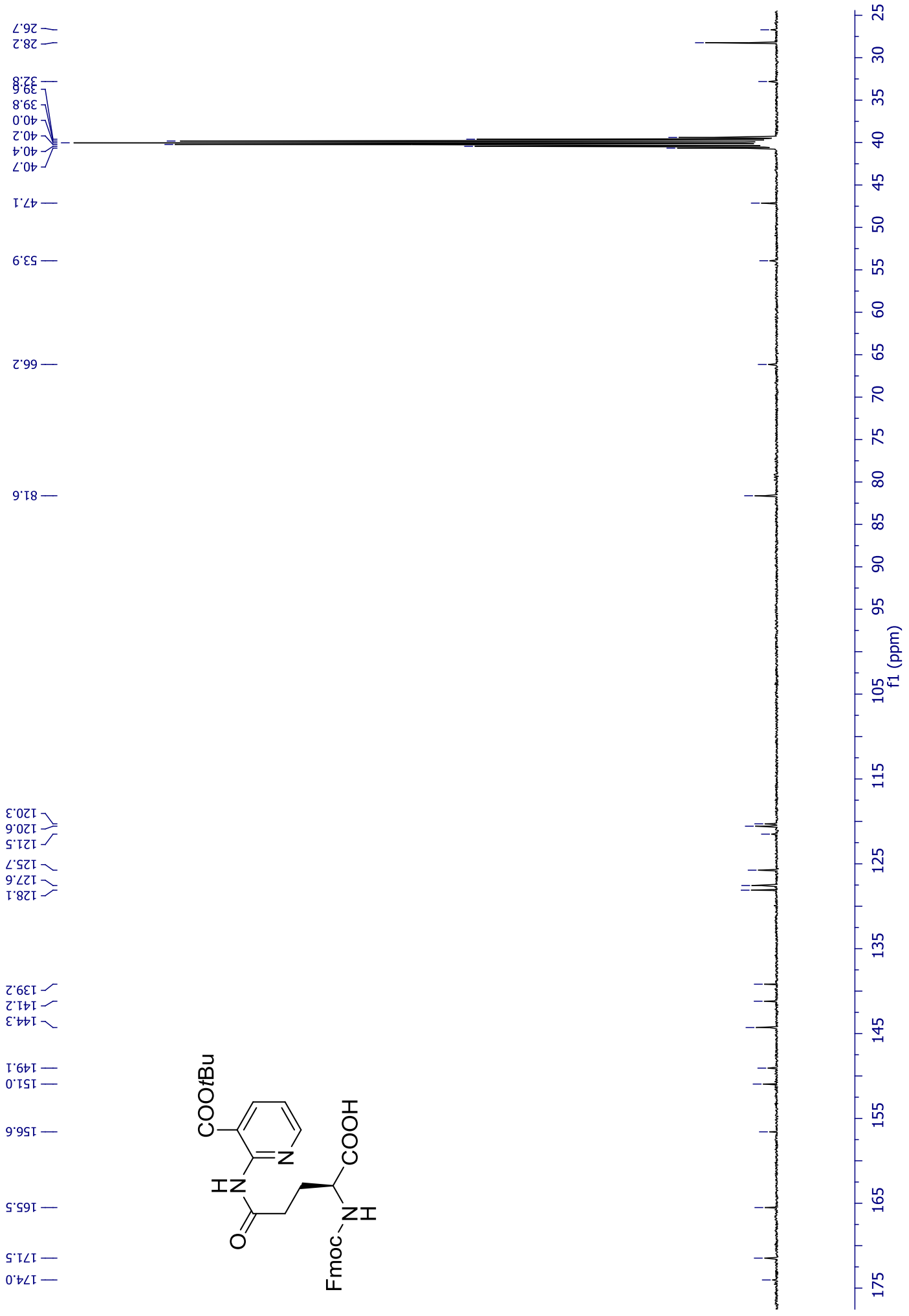


$N^{2}$-(tert-Butoxycarbonyl)- $N^{5}$-(3-(tert-butoxycarbonyl)pyridin-2-yl)-D-glutamine $\quad$ (Boc-D-Gln(NH- $t \mathrm{Bu}-$ nic)-OH, DM602) D-4o - ${ }^{1}$ H NMR (400 MHz, DMSO-d $)$

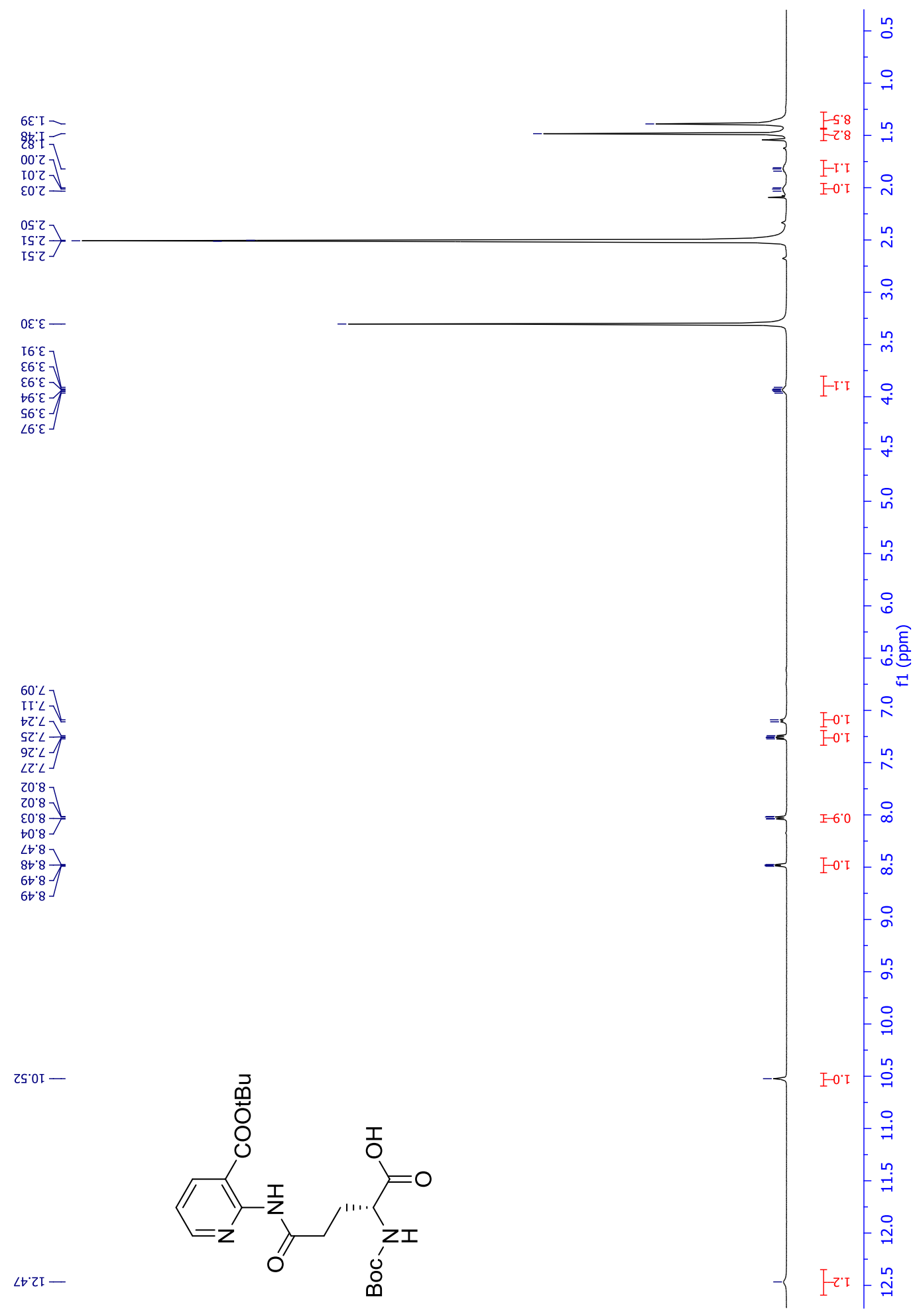


$N^{2}$-(tert-Butoxycarbonyl)- $N^{5}$-(3-(tert-butoxycarbonyl)pyridin-2-yl)-D-glutamine $\quad$ (Boc-D-Gln(NH- $t \mathrm{Bu}-$ nic)-OH, DM602) D-4o - ${ }^{13}$ C NMR (100 MHz, DMSO-d $)$

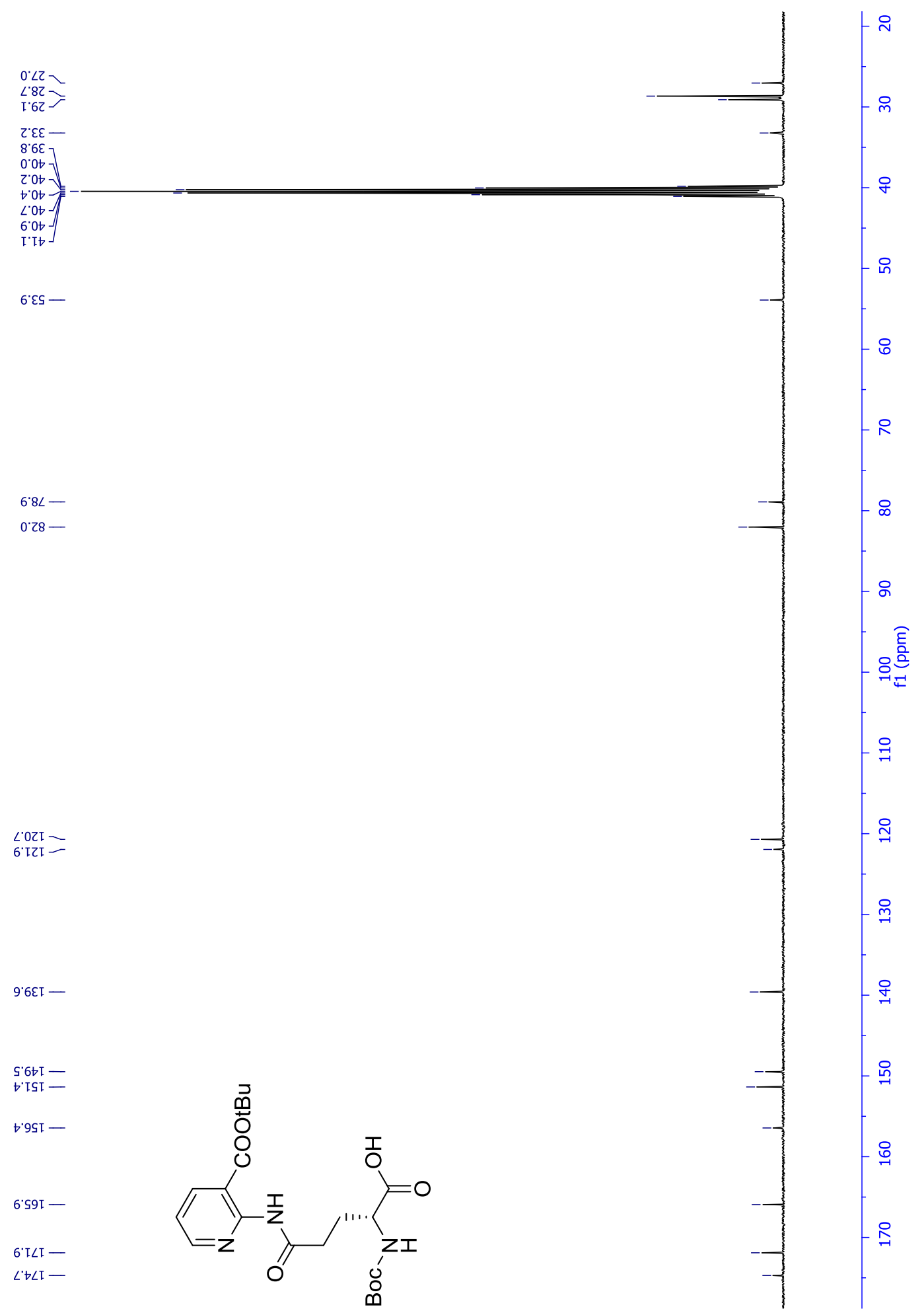


Methyl $N^{2}$-(tert-butoxycarbonyl)-N-[3-(tert-butoxycarbonyl)pyridin-2yl]-L-glutaminyl-L-valinate (BocL-Gln( $t$ Bu-nic)-L-Val-OMe, DM58) L,L-21 - ${ }^{1}$ H NMR (400 MHz, CDCl 3 )
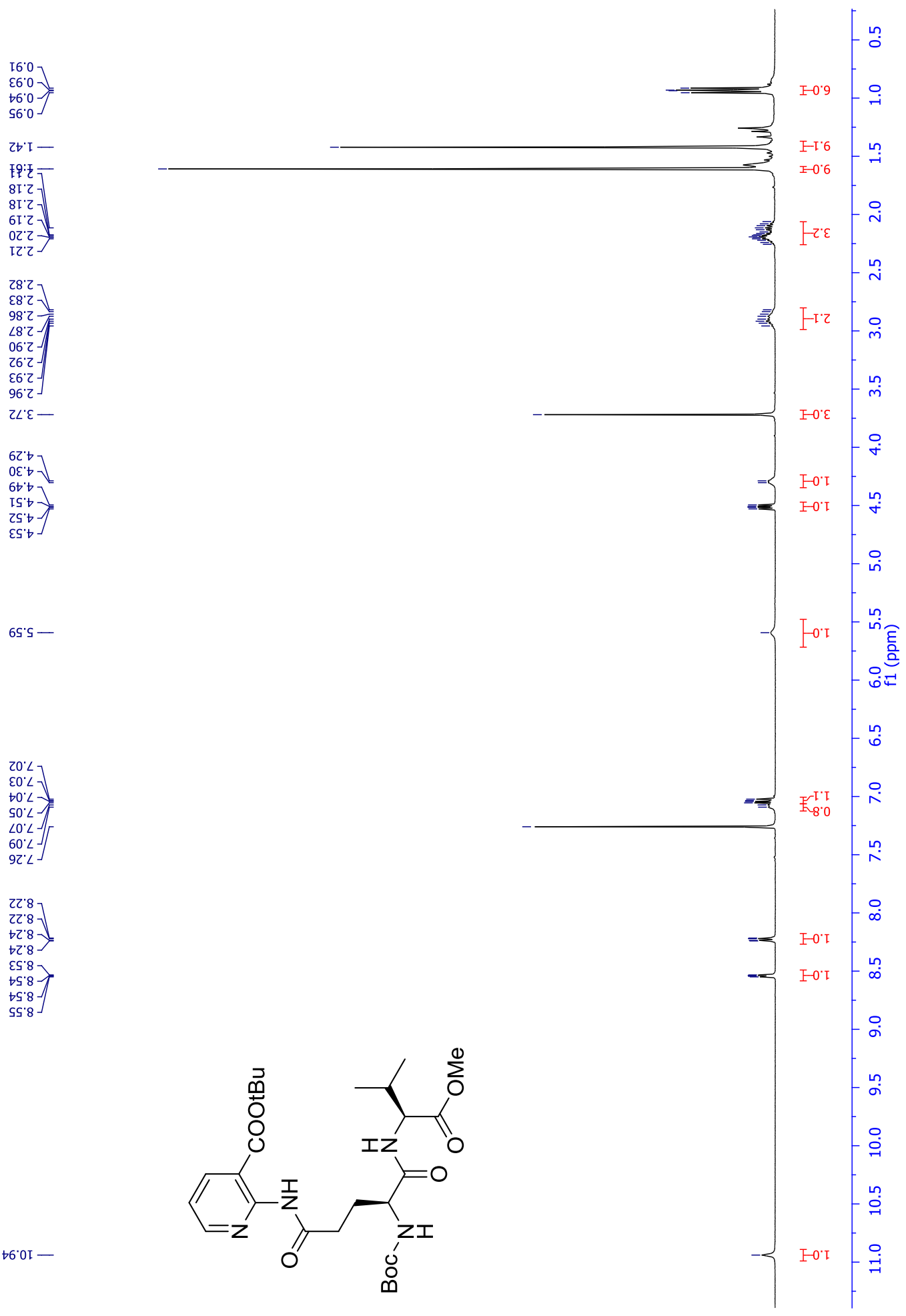
Methyl $N^{2}$-(tert-butoxycarbonyl)-N-[3-(tert-butoxycarbonyl)pyridin-2yl]-L-glutaminyl-L-valinate (BocL-Gln( $t$ Bu-nic)-L-Val-OMe, DM584) L,L-21 - ${ }^{\mathbf{1}} \mathbf{H}$ NMR (400 MHz, CDCl $\left.\mathbf{~}_{3}\right)$

$28^{\circ} \angle \mathrm{I}-$

$2821=$

$\varepsilon\ulcorner\cdot 8 Z$ 厂

$06^{\circ} 0 \varepsilon^{\circ} \digamma$

$8 \varepsilon^{\prime} \downarrow \varepsilon-$

\&o'Zs -

Z๐ $\angle s-$

$00^{\circ} \angle L$

乙E' $\angle L]$

乙๖・ย8-

0Z'8II -

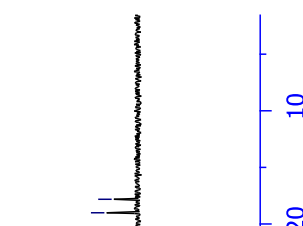

오

(

${ }_{50} 0 \mathrm{OI}$

$\left.\begin{array}{l}\text { SO'ZSI } \\ \text { IZ'ZSI }\end{array}\right\rangle$

๖8. $99 \mathrm{I}$

โ6 $[\angle[$

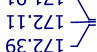

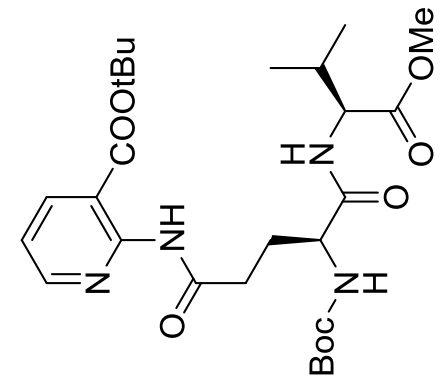


Methyl $N^{2}$-(tert-butoxycarbonyl)- $N$-[3-(tert-butoxycarbonyl)pyridin-2yl]-D-glutaminyl-L-valinate (BocD-Gln(tBu-nic)-L-Val-OMe, DM593) D,L-21 - ${ }^{\mathbf{H}}$ NMR (400 MHz, CDCl $)$

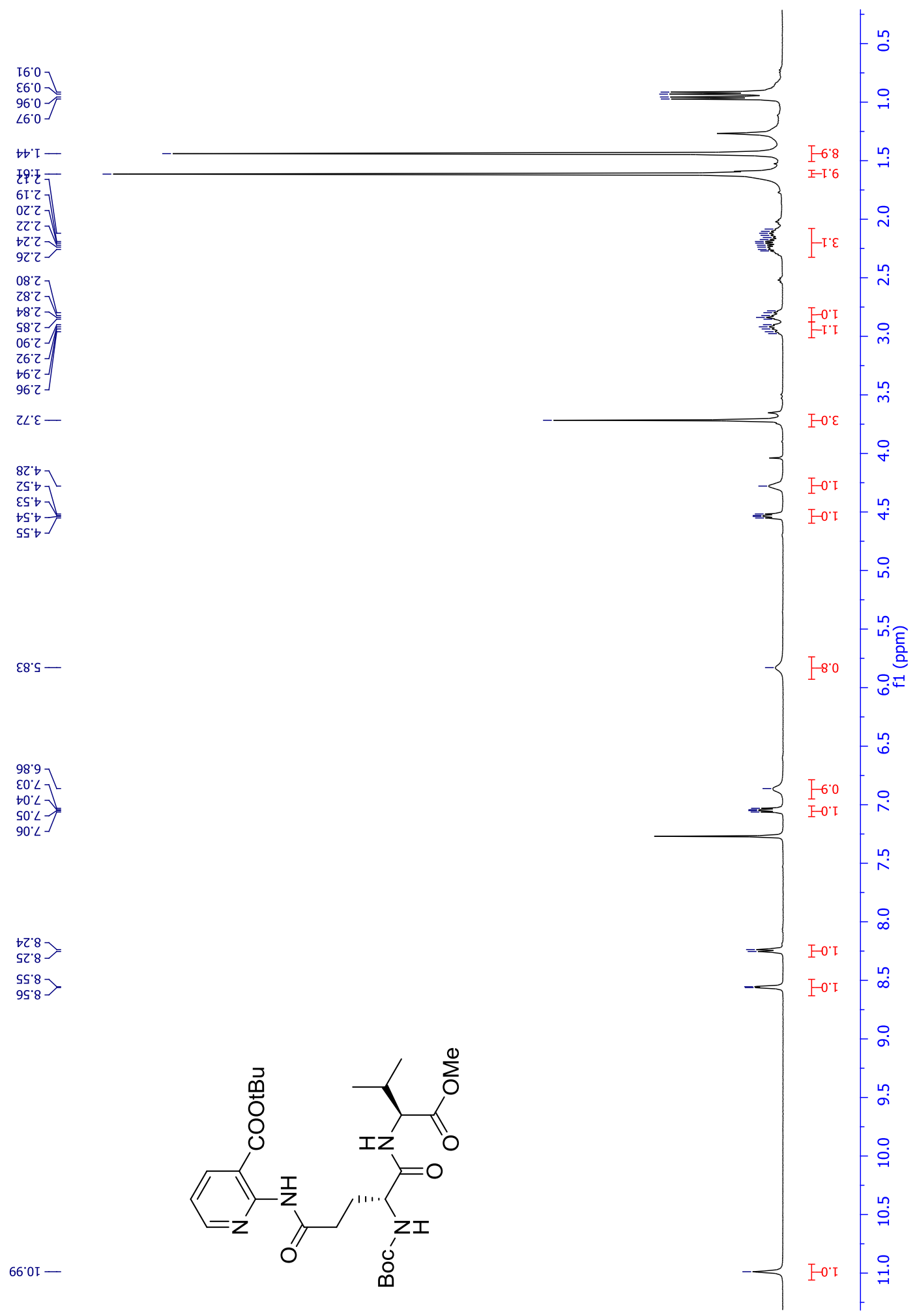


Methyl $N^{2}$-(tert-butoxycarbonyl)- $N$-[3-(tert-butoxycarbonyl)pyridin-2yl]-D-glutaminyl-L-valinate (BocD-Gln( $t$ Bu-nic)-L-Val-OMe, DM593) D,L-21 - ${ }^{1}$ H NMR (400 MHz, CDCl ) $^{2}$

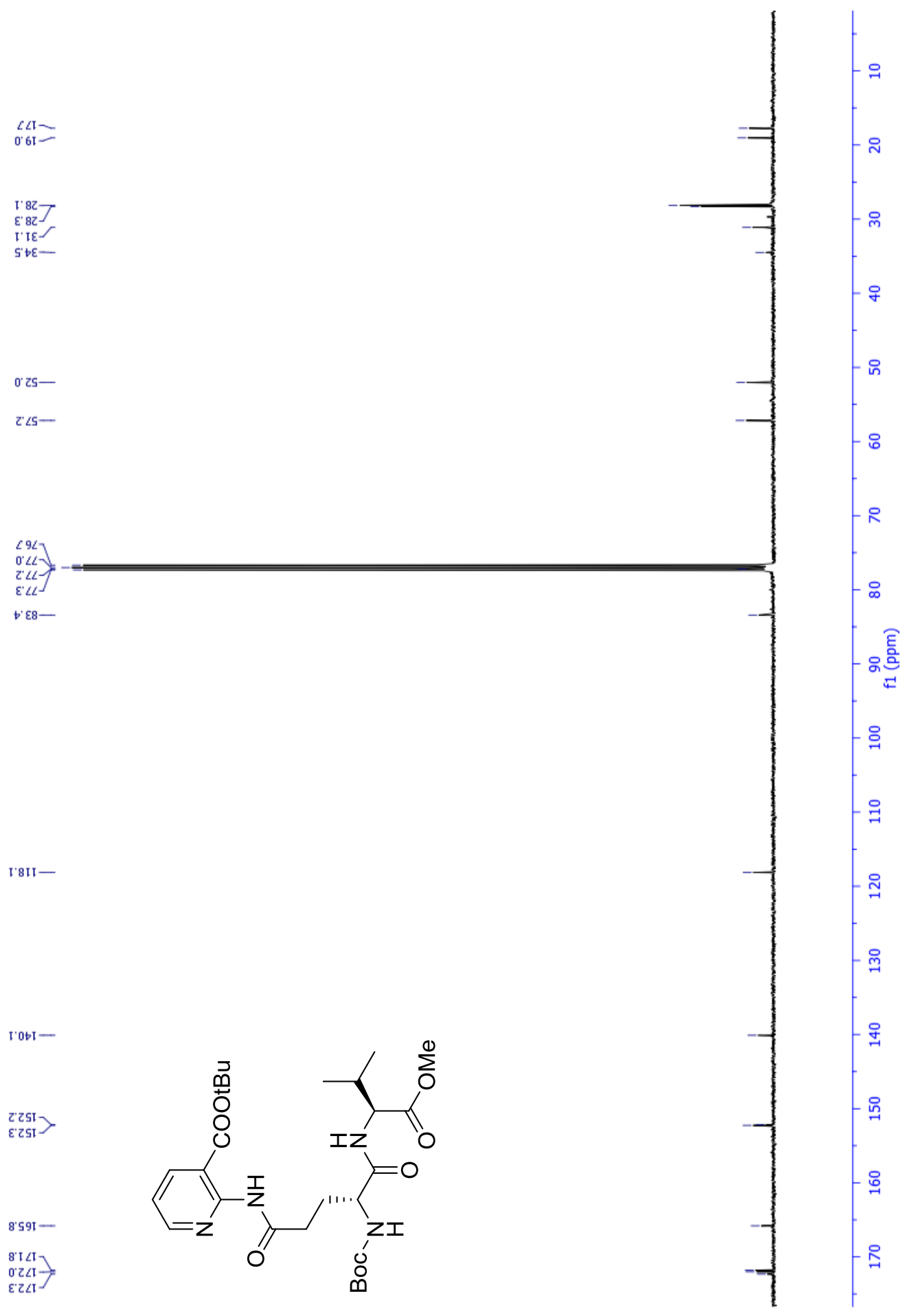


Methyl

$N$-[3-(tert-butoxycarbonyl)pyridin-2-yl]- $N^{2} \sim-\{[(9 H$-fluoren-9-yl)methoxy]carbonyl $\}$-Lglutaminyl-L-valinate (Fmoc-L-Gln(tBu-nic)-L-Val-OMe) L,L-23 - ${ }^{\mathbf{1}}$ H NMR (400 MHz, CDCl N $^{2}$
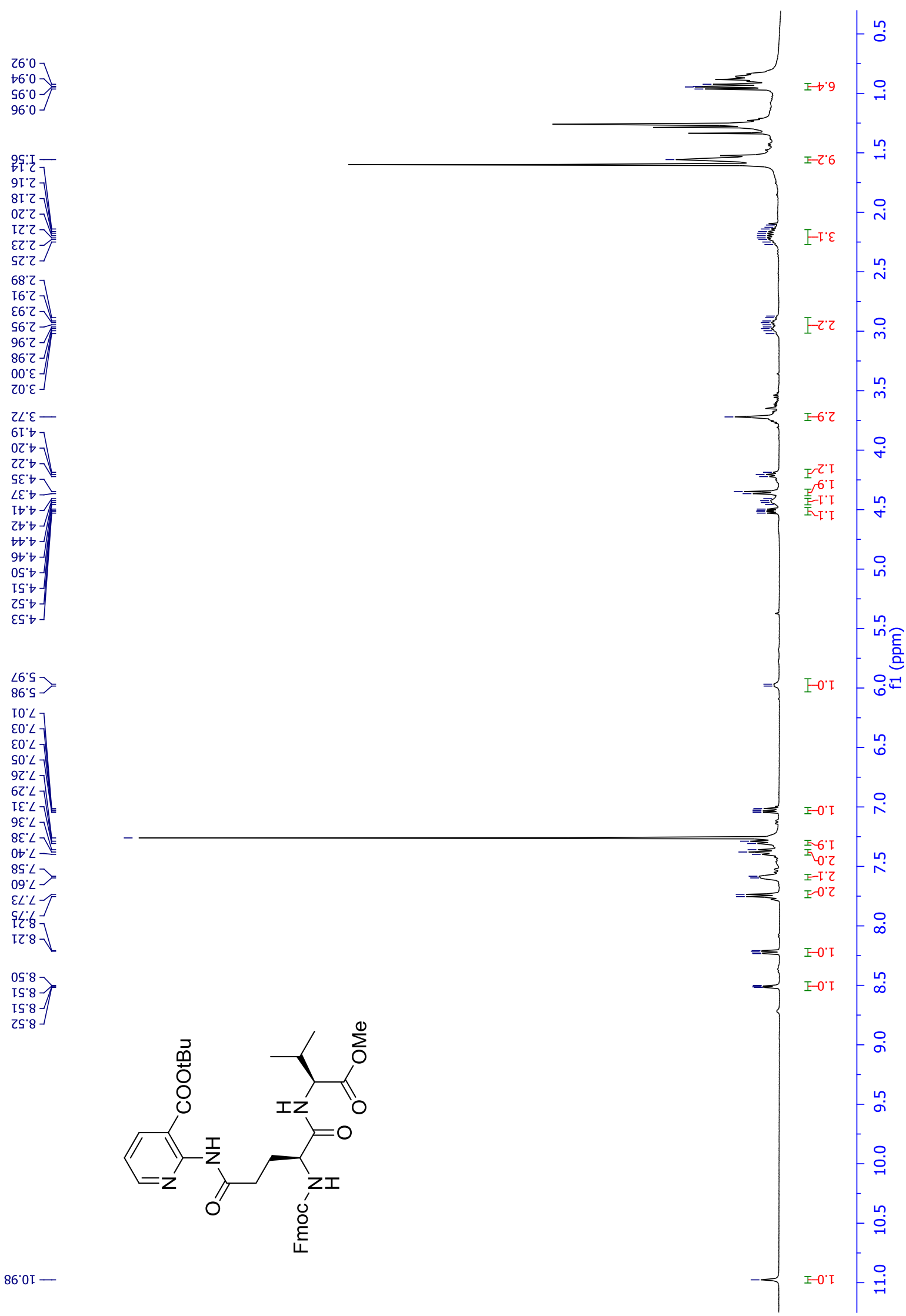

$86^{\circ} 01-$ 
Methyl

$N$-[3-(tert-butoxycarbonyl)pyridin-2-yl]- $N^{2} \sim-\{[(9 H$-fluoren-9-yl)methoxy]carbonyl $\}$-Lglutaminyl-L-valinate (Fmoc-L-Gln( $t$ Bu-nic)-L-Val-OMe) L,L-23 - ${ }^{\mathbf{1 3}} \mathbf{C}$ NMR (101 MHz, CDCl $)$

0.0-

${ }_{0.6 I}^{6} \angle \mathrm{I} \simeq$

$[\cdot 82]$

$\angle 262$

$8.0 \varepsilon \frac{1}{5}$

$\varepsilon+\llcorner\varepsilon$

T: $\angle b-$

I'ZSר

o.tS-

$L^{\circ} 94$

$0: \angle L$
$\varepsilon: \angle L$

ऽ' $\varepsilon 8-$

E.8II

$66 \mathrm{II}-$

Z'SZI

I. $\angle 21$

9:LZI

$0.0 \mathrm{tI}$

$\varepsilon$ 'ItI

$\varepsilon \cdot \mathrm{ItT} J$

8 \& $E$ I

6. TSI

Z'ZSI

8.59I -

$\angle ' T \angle T$

I' $2 \angle I]$

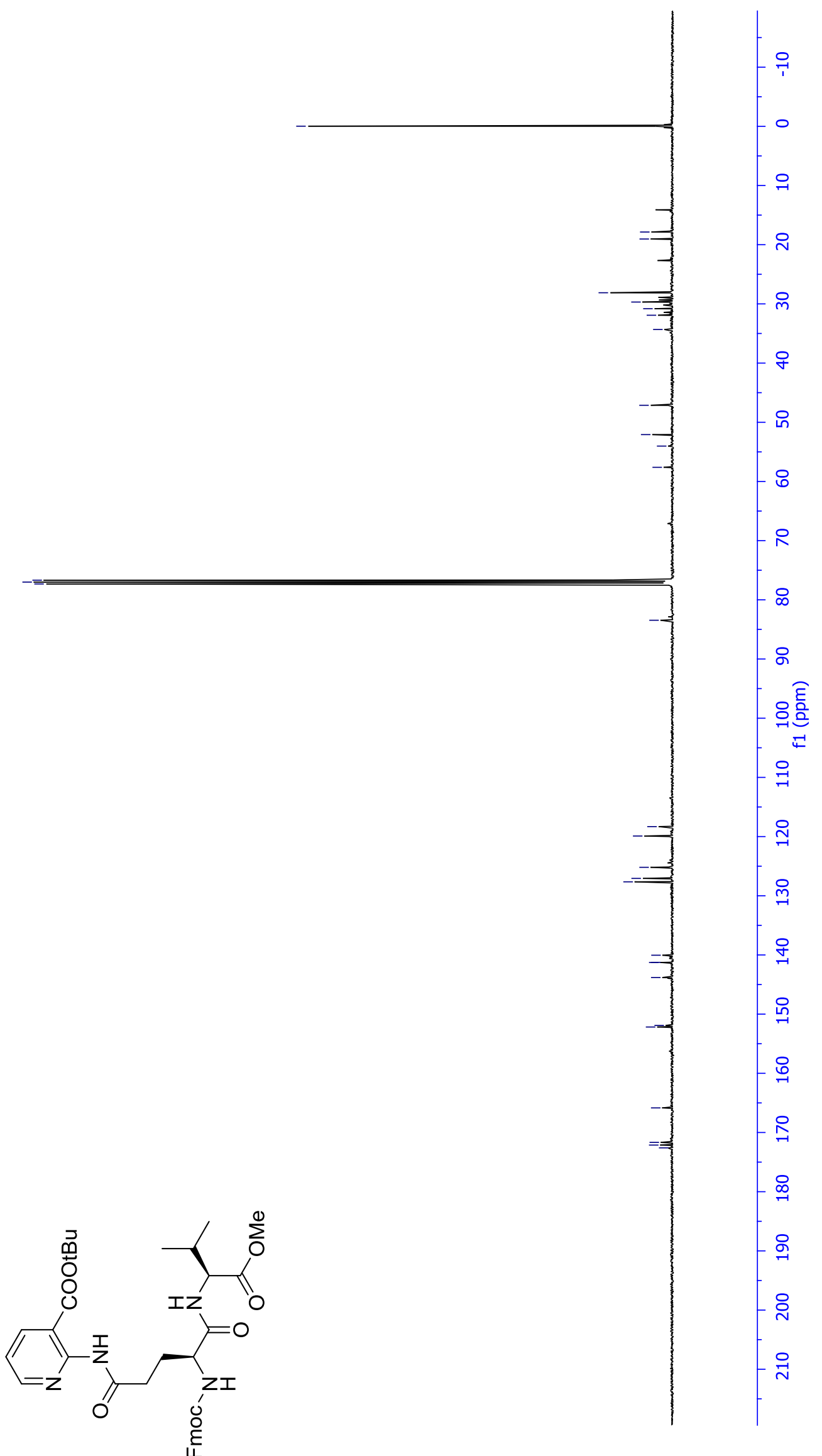


Methyl (tert-butoxycarbonyl)-L-phenylalanyl-L-phenylalaninate (Boc-L-Phe-L-Phe-OMe, KH_1160) L,L-6a - ${ }^{1}$ H NMR (500 MHz, $\mathrm{CDCl}_{3}$ )

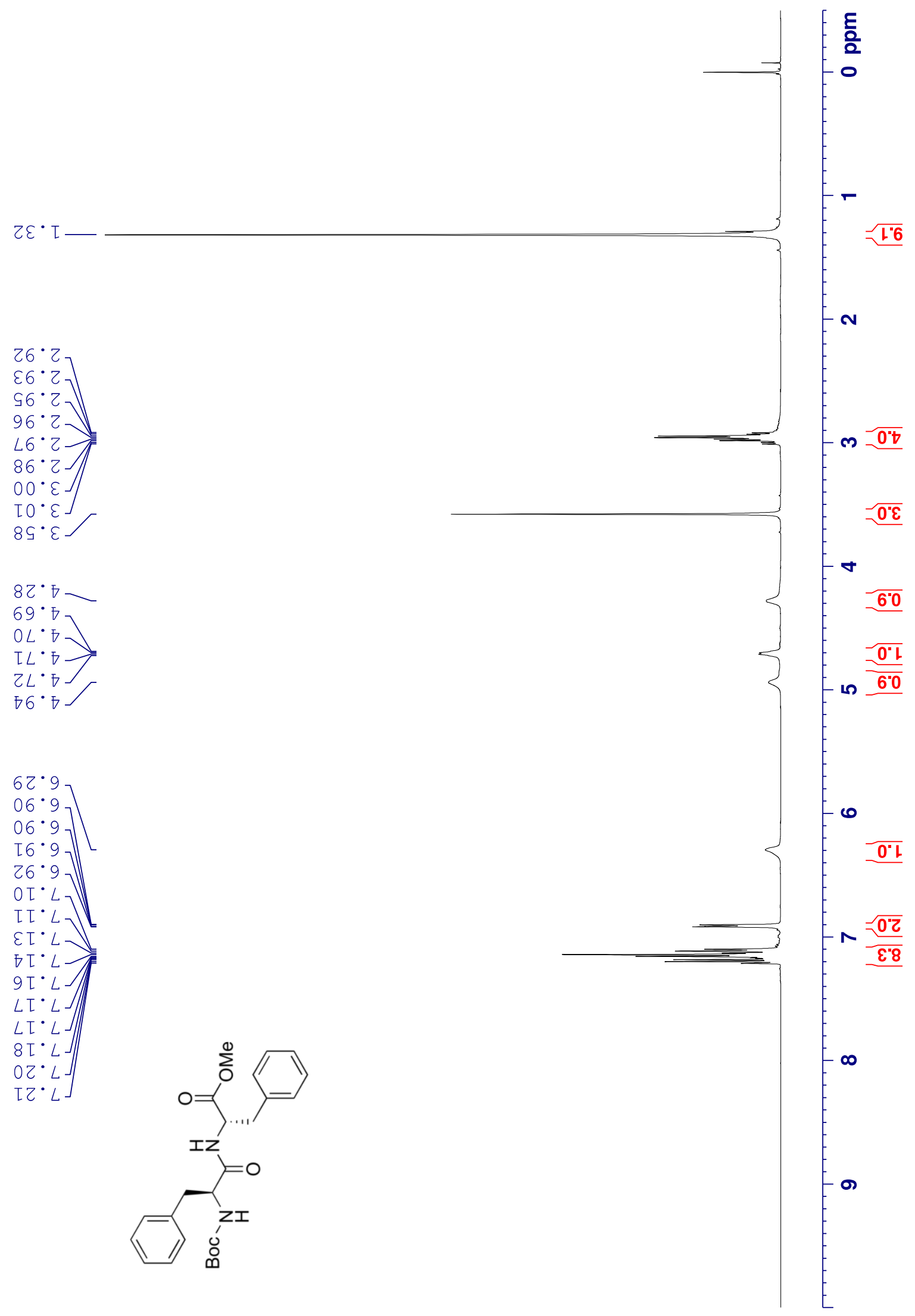


Methyl (tert-butoxycarbonyl)-L-phenylalanyl-L-phenylalaninate (Boc-L-Phe-L-Phe-OMe, KH_1160) L,L-6a - ${ }^{13} \mathrm{C}$ NMR (126 MHz, $\left.\mathrm{CDCl}_{3}\right)$

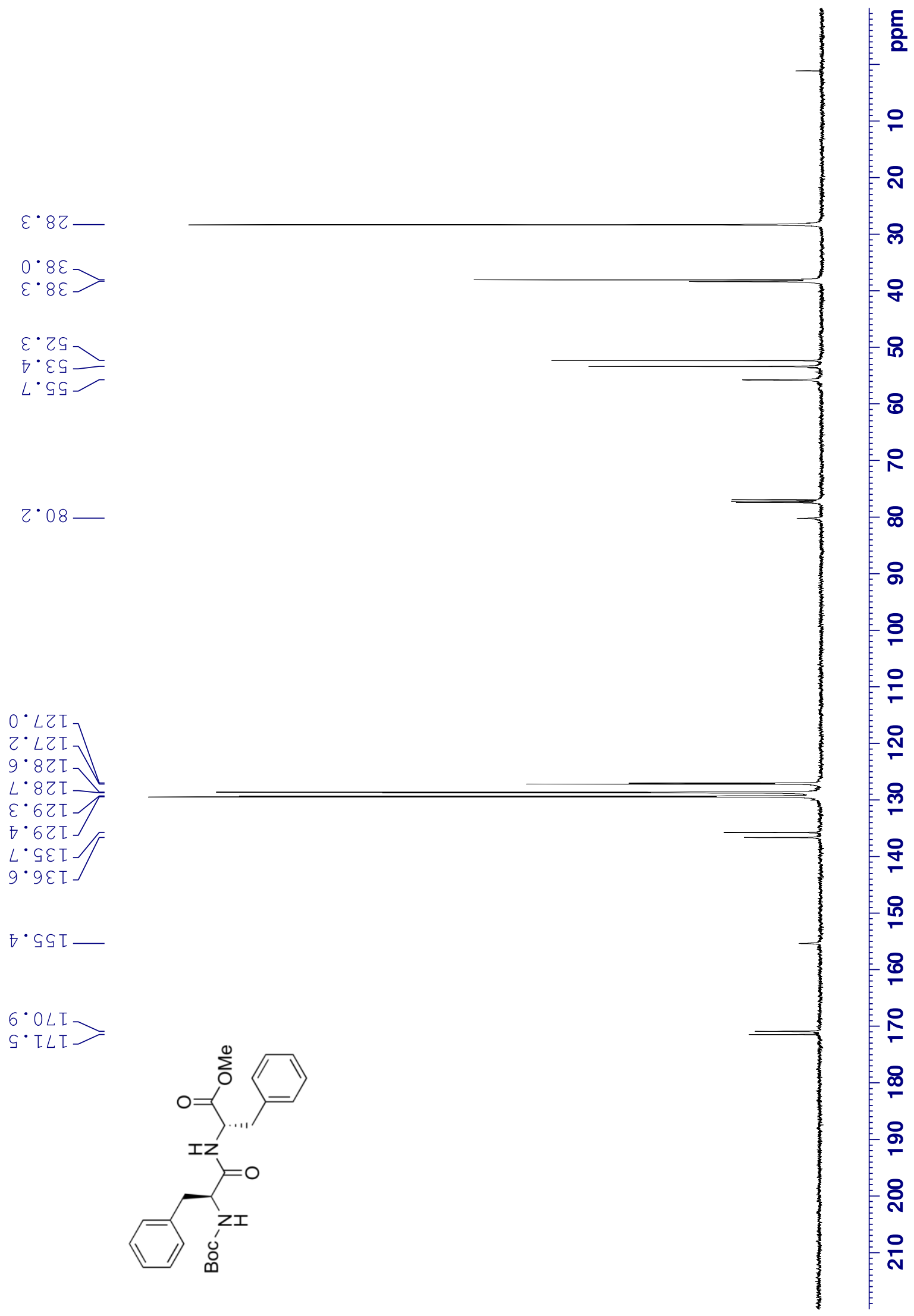


Prop-1-en-1-yl (tert-butoxycarbonyl)-L-alanyl-L-leucinate (Boc-L-Ala-L-Leu-OAll, KH_1197) L,L6b - ${ }^{1} \mathrm{H}$ NMR (500MHz, $\left.\mathrm{CDCl}_{3}\right)$

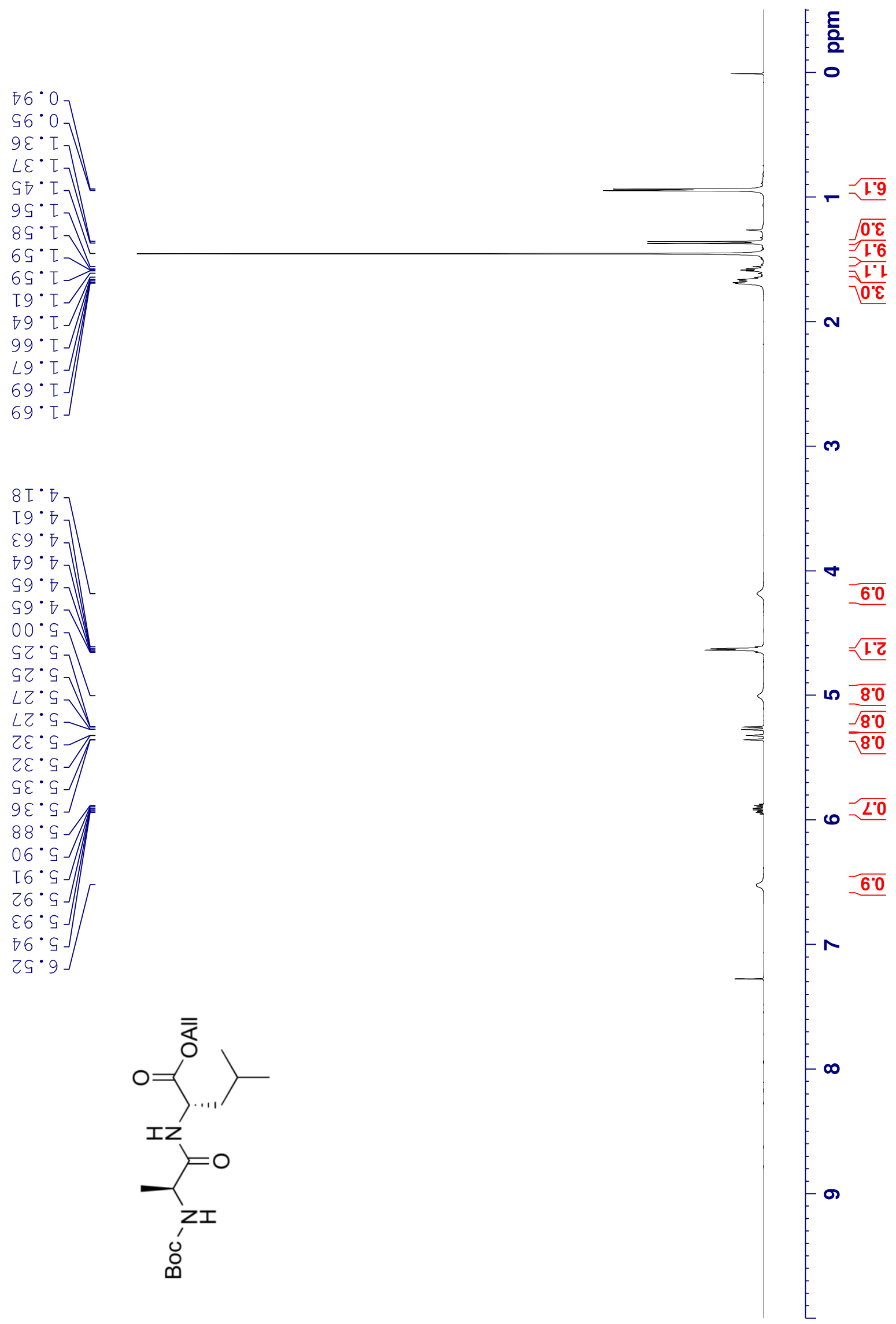


Prop-1-en-1-yl (tert-butoxycarbonyl)-L-alanyl-L-leucinate (Boc-L-Ala-L-Leu-OAll, KH_1197) L,L6b - ${ }^{13} \mathrm{C}$ NMR (126MHz, $\left.\mathrm{CDCl}_{3}\right)$

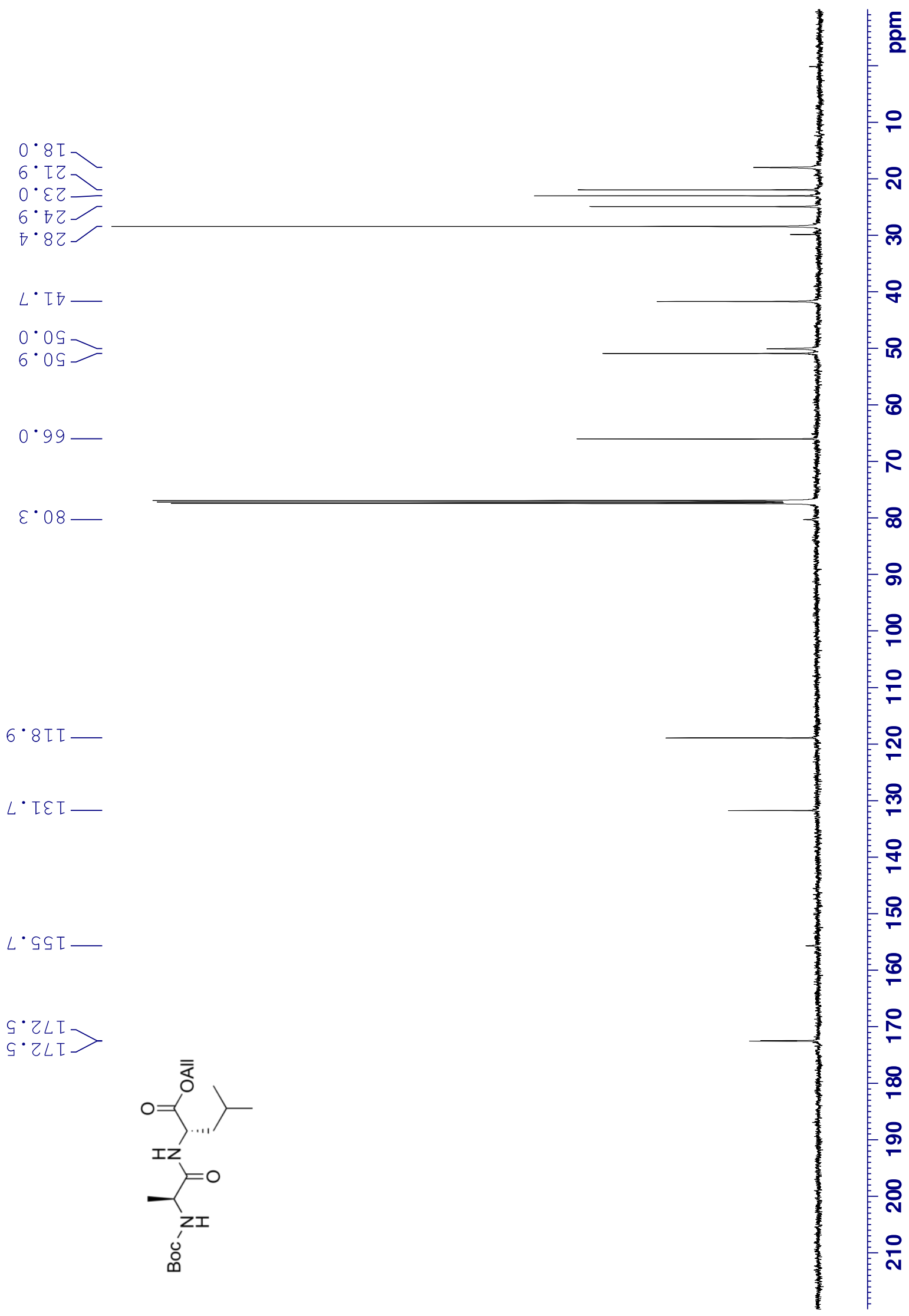


tert-Butyl ((S)-3-(4-(tert-butoxy)phenyl)-2-((tert-butoxycarbonyl)amino)propanoyl)-L-valinate (Boc-L$\left.\operatorname{Tyr}(t \mathrm{Bu})-\mathrm{L}-\mathrm{Val}-\mathrm{O} t \mathrm{Bu}, \mathrm{KH} \_1245\right)$ L,L-6c - ${ }^{\mathbf{1}} \mathbf{H}$ NMR (500 MHz, DMSO-d $\boldsymbol{\sigma}_{\boldsymbol{6}}$, mixture of rotamers)

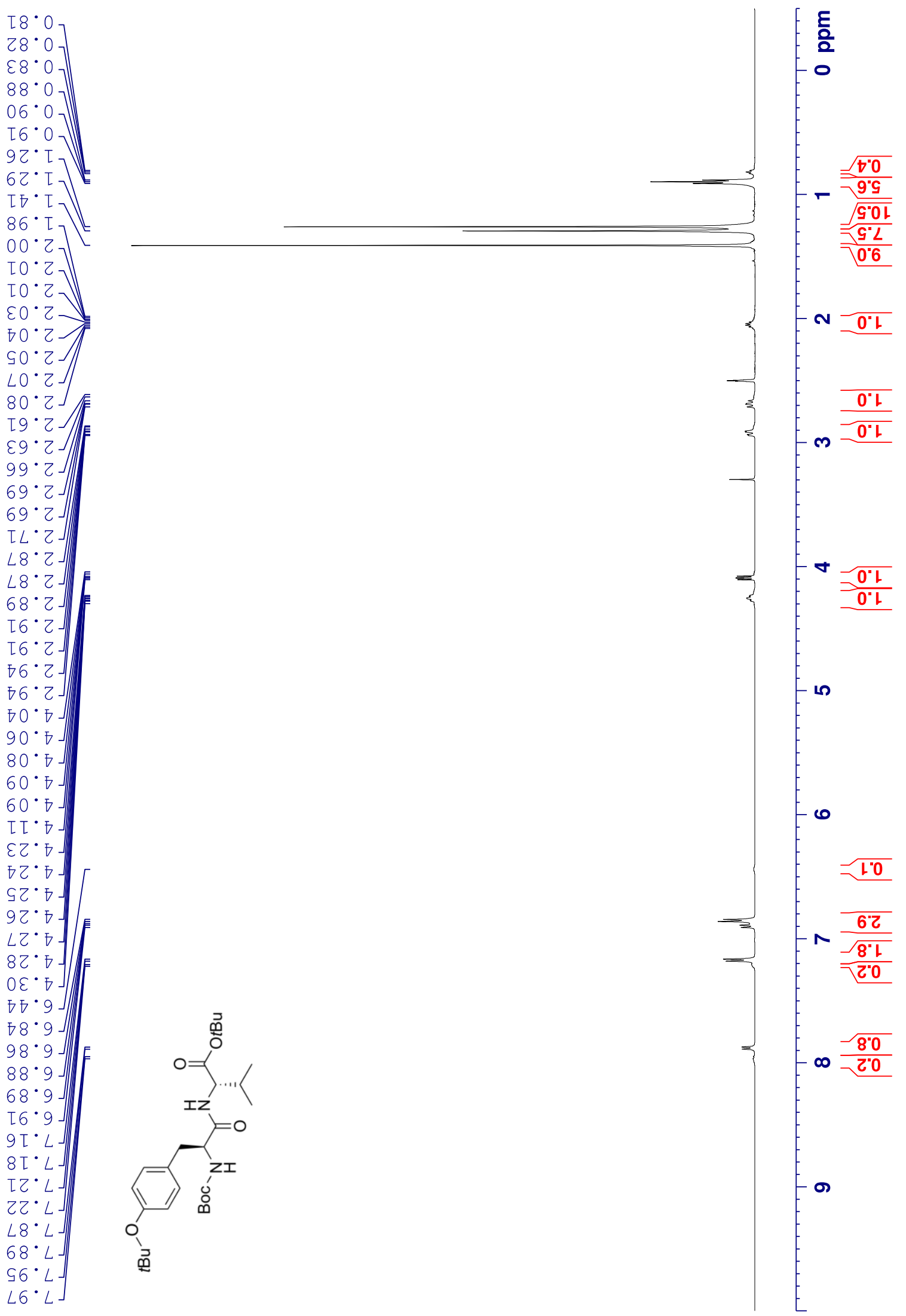


tert-Butyl ((S)-3-(4-(tert-butoxy)phenyl)-2-((tert-butoxycarbonyl)amino)propanoyl)-L-valinate (Boc-L$\left.\operatorname{Tyr}(t \mathrm{Bu})-\mathrm{L}-\mathrm{Val}-\mathrm{O} t \mathrm{Bu}, \mathrm{KH} \_1245\right)$ L,L-6c - ${ }^{13} \mathrm{C}$ NMR (126 MHz, DMSO-d $\boldsymbol{6}$, mixture of rotamers)
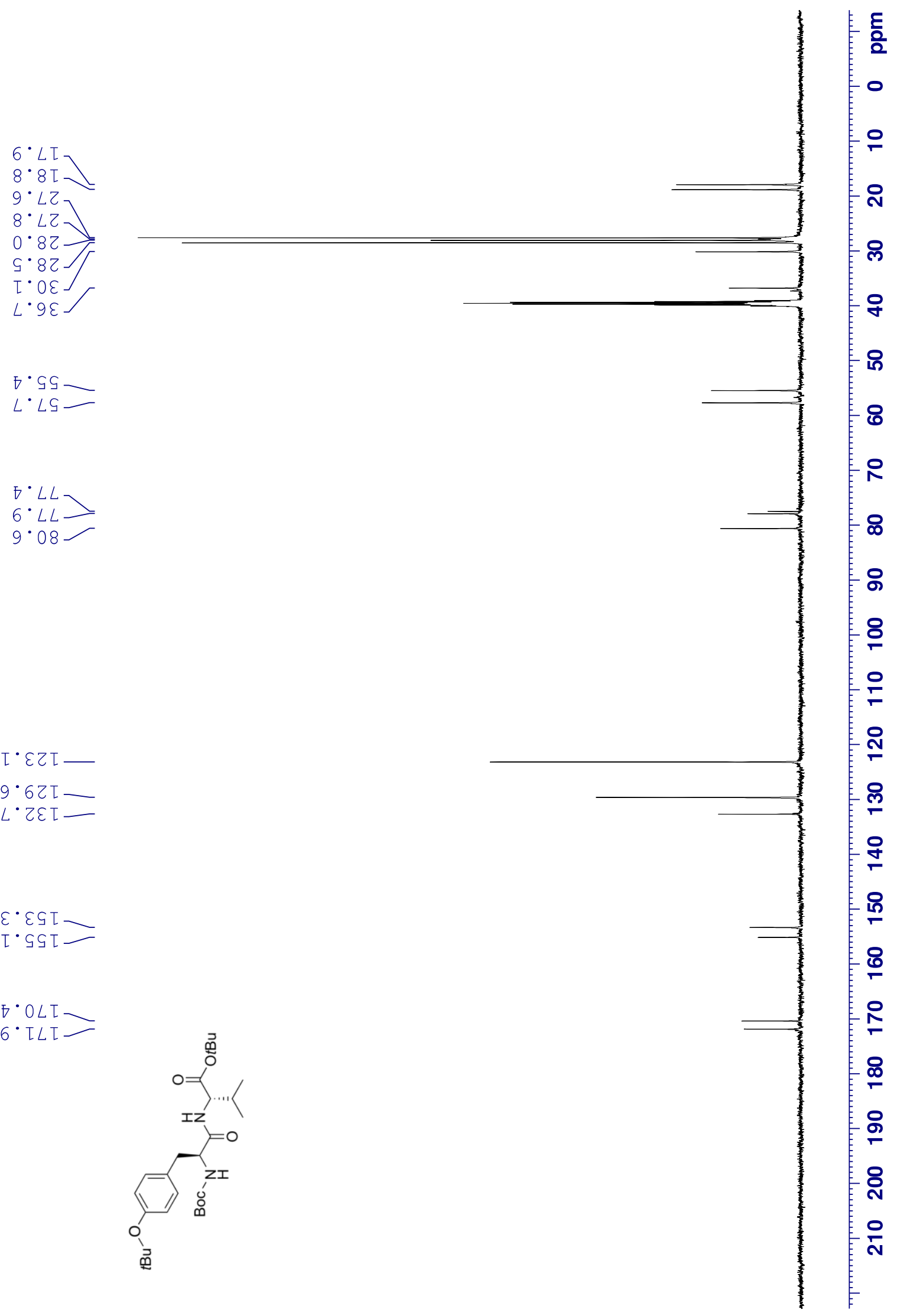
tert-Butyl ((S)-3-(4-(tert-butoxy)phenyl)-2-((tert-butoxycarbonyl)amino)propanoyl)-L-valinate (Boc-L$\left.\operatorname{Tyr}(t \mathrm{Bu})-\mathrm{L}-\mathrm{Val}-\mathrm{O} t \mathrm{Bu}, \mathrm{KH} \_1245\right)$ L,L-6c - ${ }^{1} \mathbf{H}$ NMR $\left(500\right.$ MHz, DMSO-d 6 , 60 $\left.{ }^{\circ} \mathbf{C}\right)$
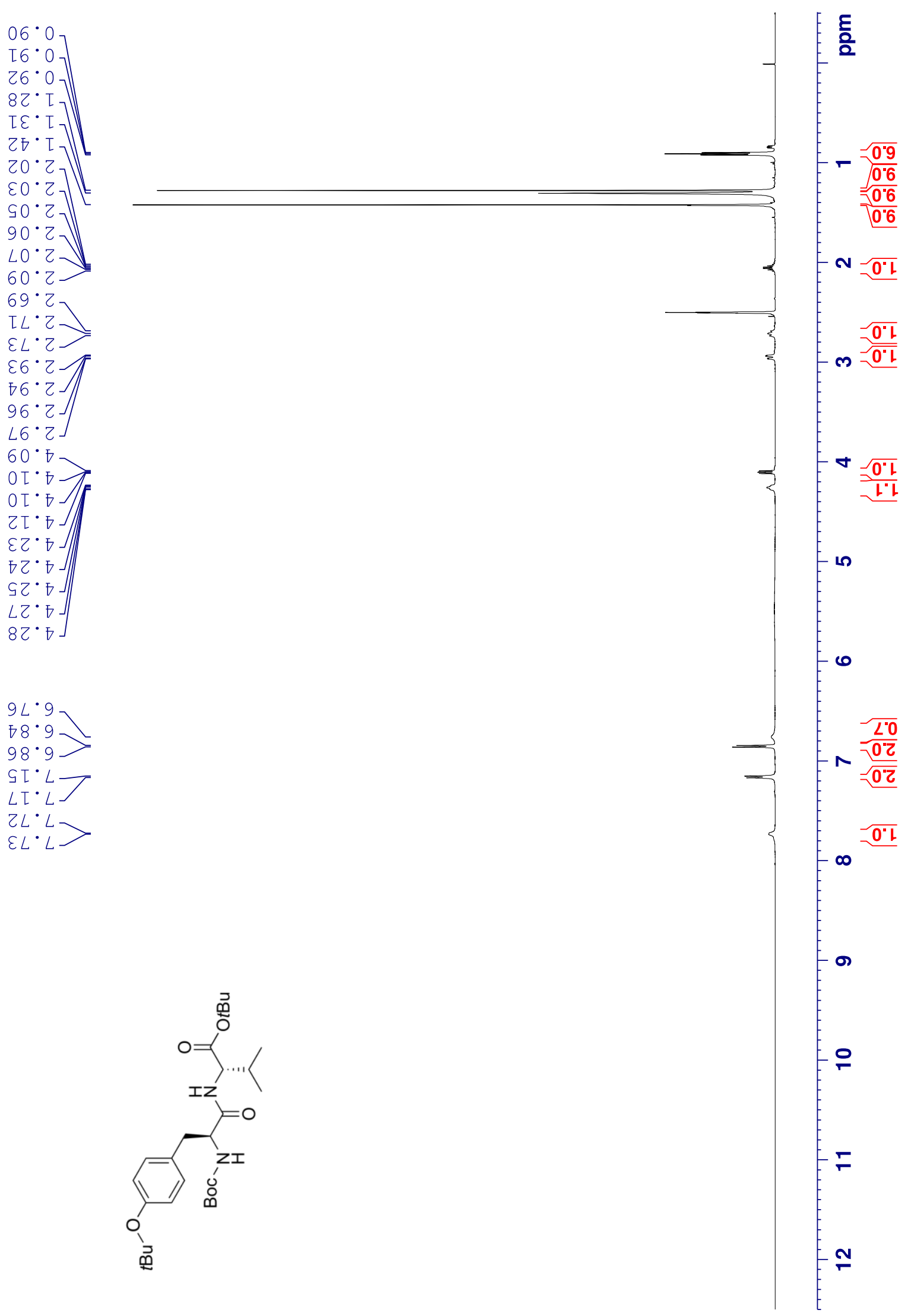
tert-Butyl ((S)-3-(4-(tert-butoxy)phenyl)-2-((tert-butoxycarbonyl)amino)propanoyl)-L-valinate (Boc-L$\left.\operatorname{Tyr}(t \mathrm{Bu})-\mathrm{L}-\mathrm{Val}-\mathrm{O} t \mathrm{Bu}, \mathrm{KH} \_1245\right)$ L,L-6c $-{ }^{13} \mathrm{C}$ NMR (126 MHz, DMSO-d $\left.6,60^{\circ} \mathrm{C}\right)$

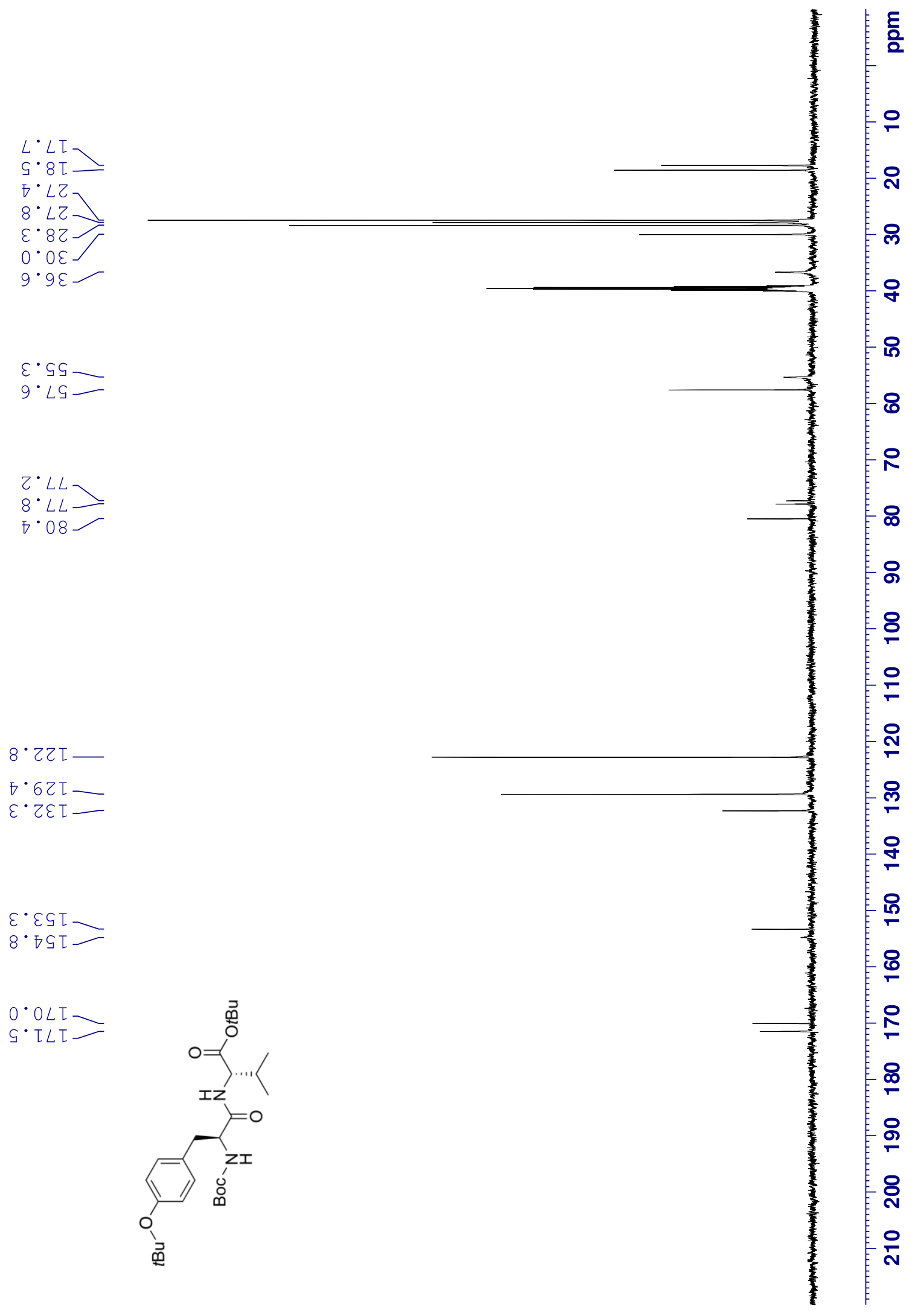


tert-Butyl

(S)-2-(((S)-1-methoxy-4-methyl-1-oxopentan-2-yl)carbamoyl)pyrrolidine-1-carboxylate (Boc-L-Pro-L-Leu-OMe, KH_1196) L,L-6d - ${ }^{\mathbf{H}} \mathbf{H}$ NMR (500 MHz, DMSO-d $\boldsymbol{6}$, mixture of rotamers)
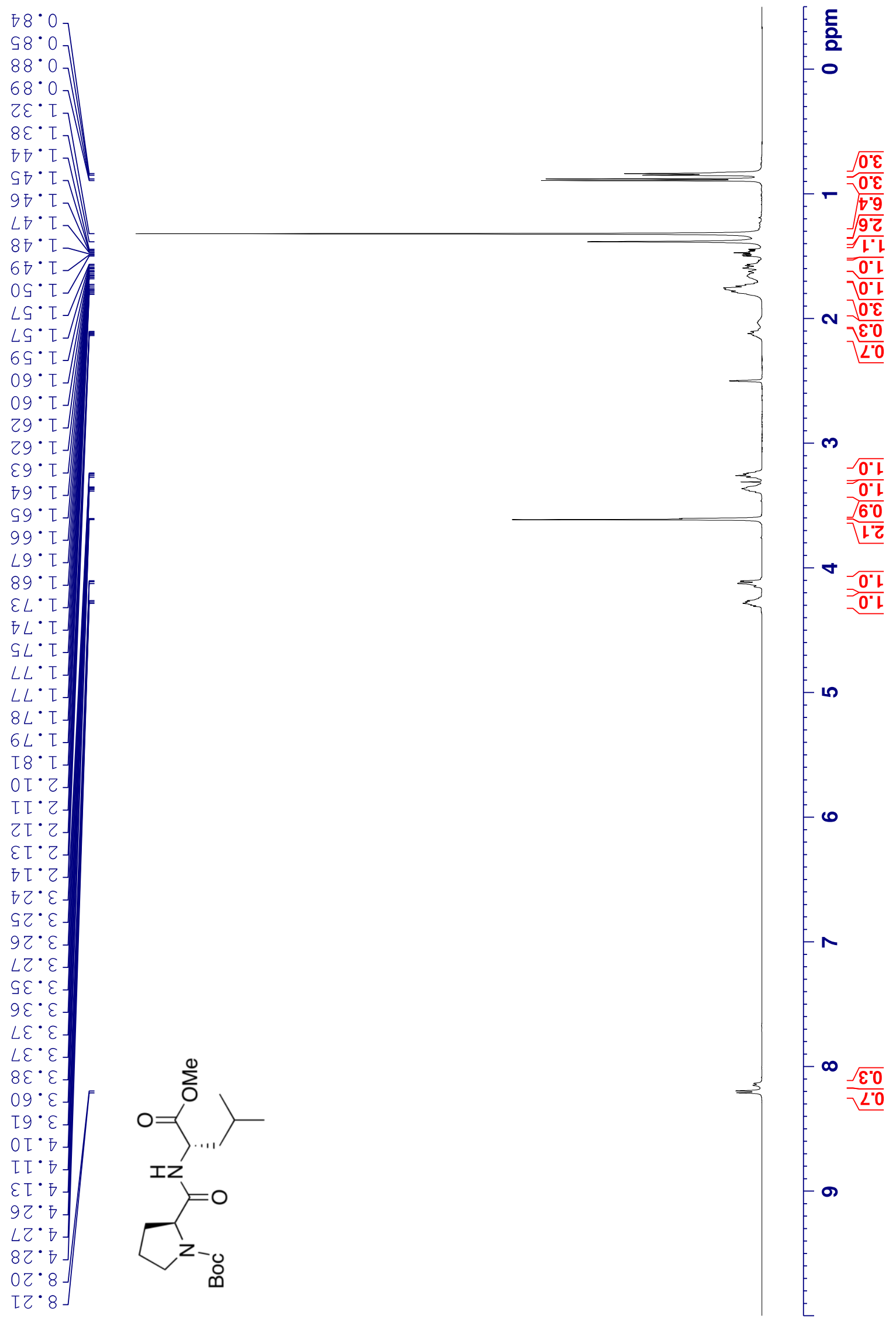
tert-Butyl

(S)-2-(((S)-1-methoxy-4-methyl-1-oxopentan-2-yl)carbamoyl)pyrrolidine-1-carboxylate (Boc-L-Pro-L-Leu-OMe, KH_1196) L,L-6d - ${ }^{13}$ C NMR (126 MHz, DMSO-d $\boldsymbol{d}_{\boldsymbol{6}}$, mixture of rotamers)
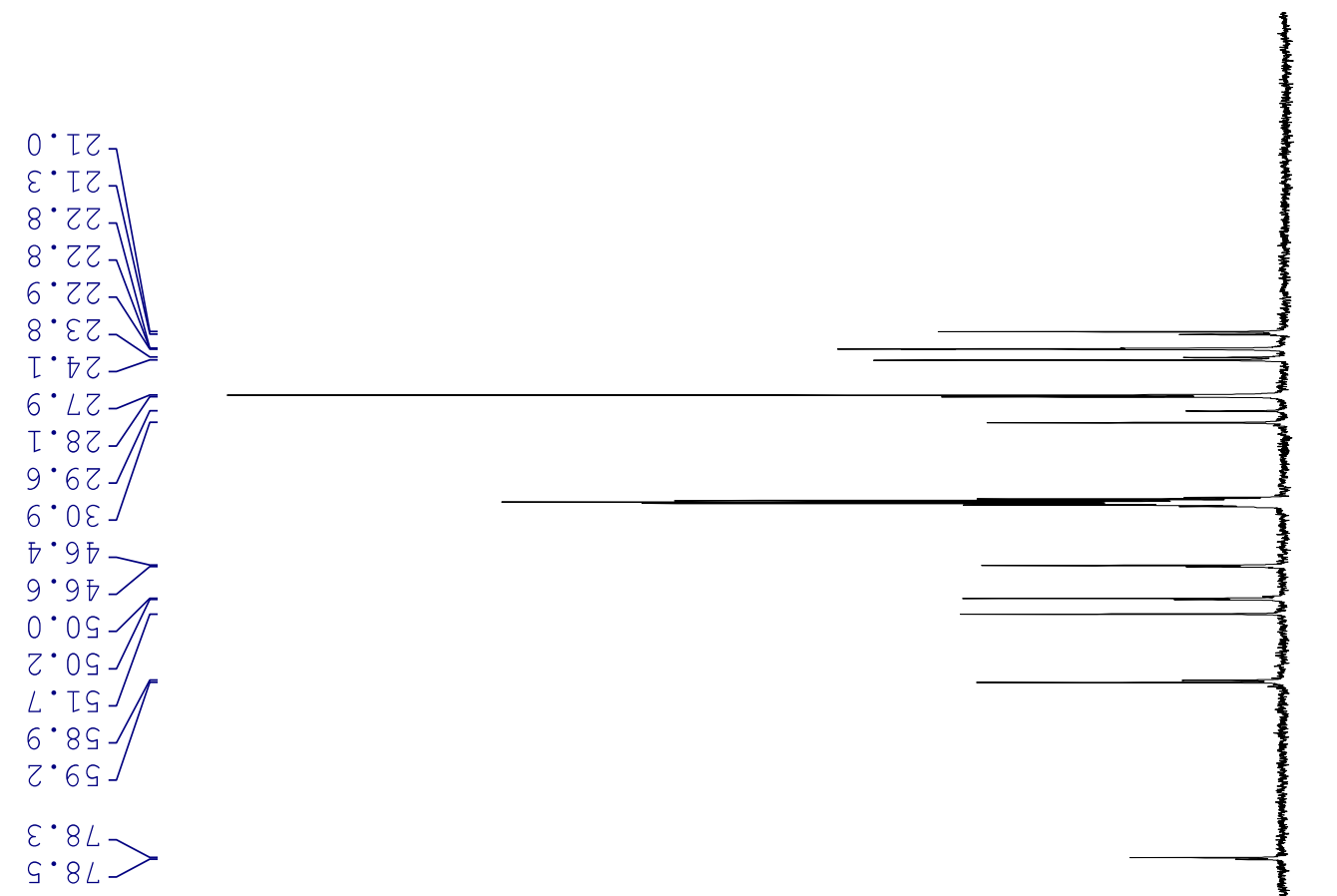

छ

응

o

으

ㅇำ

요

우

오

8

운

๑

8

8

오

오

으

움

$\varepsilon \cdot \varepsilon S[$

움

S. $\varepsilon S I>$

8

$\varepsilon \cdot Z L I$

$L \cdot 2 L I>$

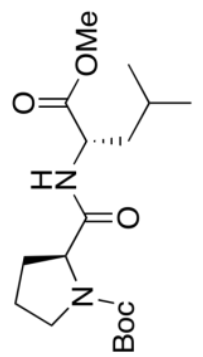

움

오

은 
tert-Butyl

(S)-2-(((S)-1-methoxy-4-methyl-1-oxopentan-2-yl)carbamoyl)pyrrolidine-1-carboxylate (Boc-L-Pro-L-Leu-OMe, KH_1196) L,L-6d - ${ }^{\mathbf{1}}$ H NMR (500 MHz, DMSO-d $\boldsymbol{6}, \mathbf{6 0}^{\circ} \mathrm{C}$ )
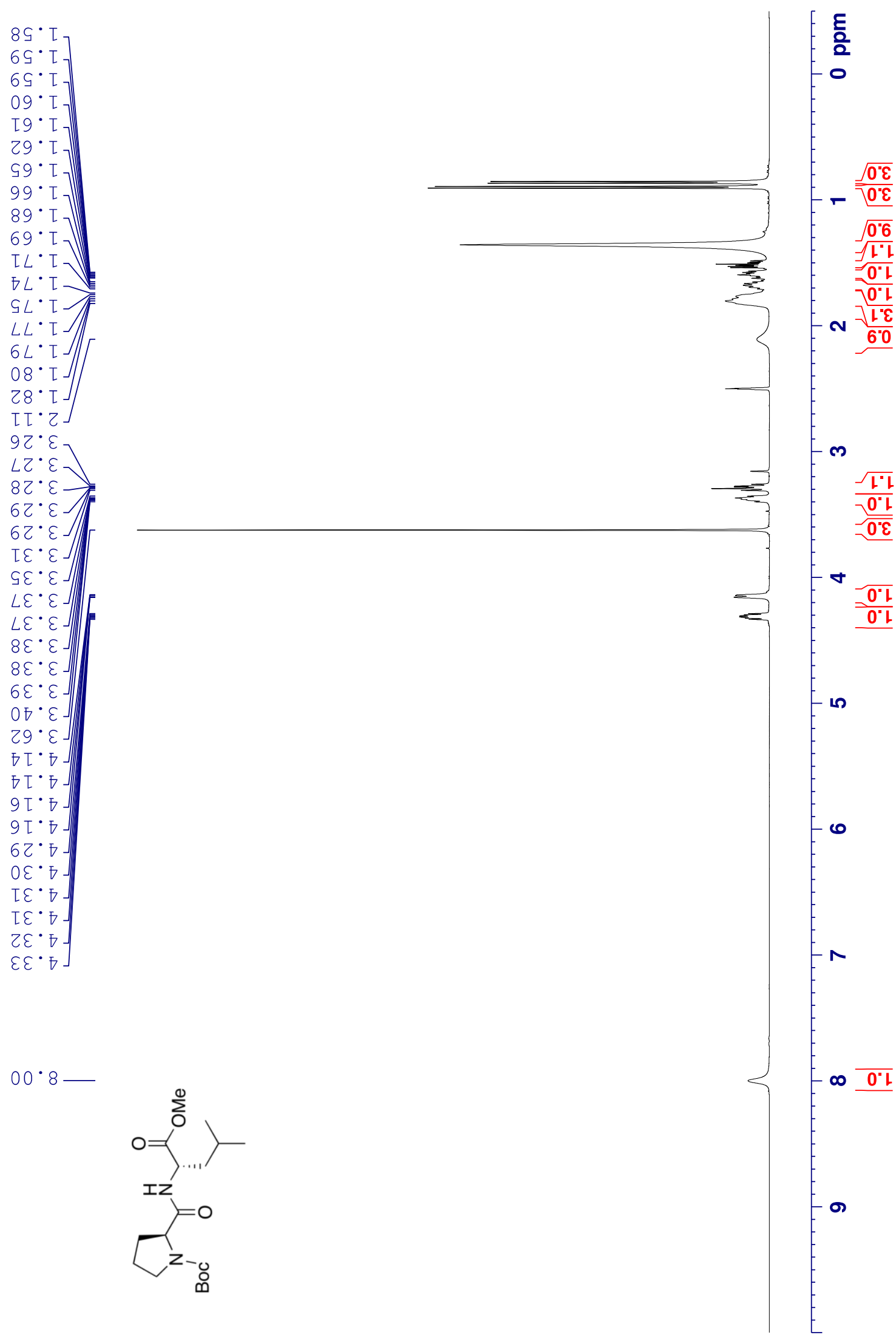
tert-Butyl

(S)-2-(((S)-1-methoxy-4-methyl-1-oxopentan-2-yl)carbamoyl)pyrrolidine-1-carboxylate (Boc-L-Pro-L-Leu-OMe, KH_1196) L,L-6d - ${ }^{13}$ C NMR (126 MHz, DMSO-d 6 , 60 ${ }^{\circ}$ C)
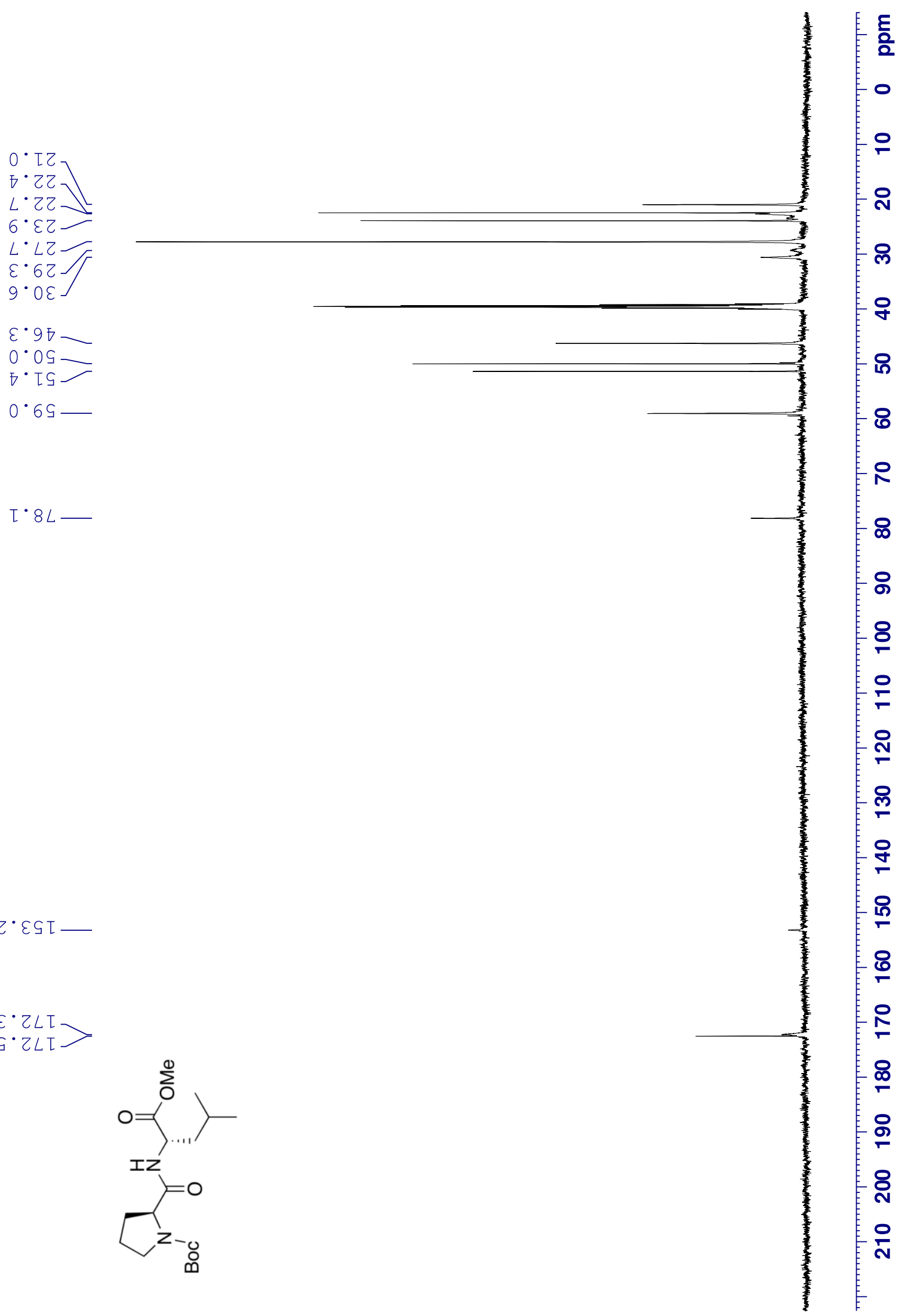
Benzyl (tert-butoxycarbonyl)-L-methionyl-L-tryptophanate (Boc-L-Met-L-Trp-OBn, DM769) L,L6e - ${ }^{1}$ H NMR (400 MHz, DMSO-d ${ }_{6}$ )

$$
\begin{aligned}
& \text { Ot' } I \\
& 202 \\
& \varepsilon t^{\prime} Z \\
& \text { St' } \\
& \downarrow I^{\prime} \varepsilon \\
& \left.\begin{array}{l}
\quad t \cdot \varepsilon \\
9 I^{\prime} \varepsilon \\
8 I^{\prime} \varepsilon \\
6 I^{\prime} \varepsilon
\end{array}\right] \\
& \begin{array}{l}
8 I^{\circ} \varepsilon \\
6 I^{\prime} \varepsilon \\
-\varepsilon \cdot \varepsilon
\end{array} \\
& \text { I } 2 \cdot \varepsilon- \\
& \text { 乙Z' } \varepsilon-1 \\
& \left.\begin{array}{l}
b Z^{\prime} \varepsilon \\
9 Z^{\prime} \varepsilon
\end{array}\right] \\
& \text { OI' } \\
& \text { 2I. } 5 \text {. } \\
& \text { की: } \\
& \begin{array}{l}
99^{\circ} \circ \\
\angle 9^{\circ} \circ
\end{array} \\
& 66^{\circ} \mathrm{\gamma} \\
& 20.5 \\
& \text { 60's } 90 \\
& \text { QI: } \\
& \varepsilon 0 . \angle \text { - } \\
& 60 \% \\
& 60 \circ \\
& \text { II } \angle \\
& 8 I^{\circ} \mathrm{L}- \\
& 6 \mathrm{I} \cdot 2 \\
& 612 \\
& \begin{array}{l}
0 Z^{\circ} \angle \\
I Z \cdot \angle]
\end{array} \\
& \text { โ } \left.\varepsilon^{\prime} L\right] \\
& \tau \varepsilon^{\prime} \angle \\
& \text { 乙E' } L \\
& \varepsilon \varepsilon: \angle \\
& 88^{\circ} \angle T \\
& \text { IS } \angle \text { - } \\
& \text { ¿S. } L \\
& \begin{array}{l}
82^{\prime} 8 \\
0 \varepsilon^{\prime} 8
\end{array}
\end{aligned}
$$

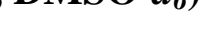

$$
\text { d) }
$$


Benzyl (tert-butoxycarbonyl)-L-methionyl-L-tryptophanate (Boc-L-Met-L-Trp-OBn, DM769) L,L6e - ${ }^{13}$ C NMR (400 MHz, DMSO-d 6 ,
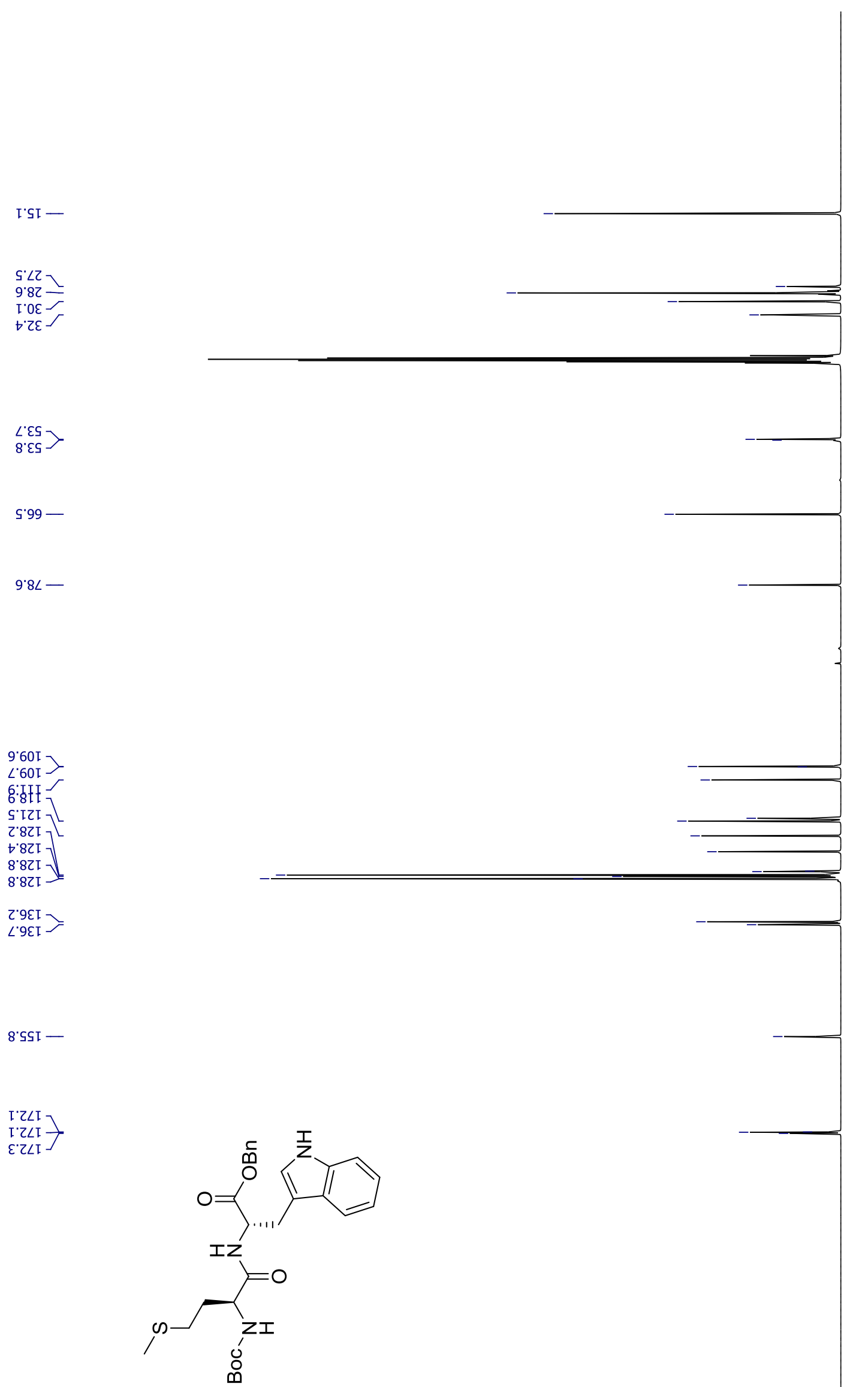
tert-Butyl (S)-(3-((1-amino-1-oxo-3-phenylpropan-2-yl)amino)-3-oxopropyl)carbamate (Boc- $\beta$ Ala-LPhe-NH 2 , DM332) L-6f - ${ }^{1}$ H NMR (400 MHz, DMSO-d )

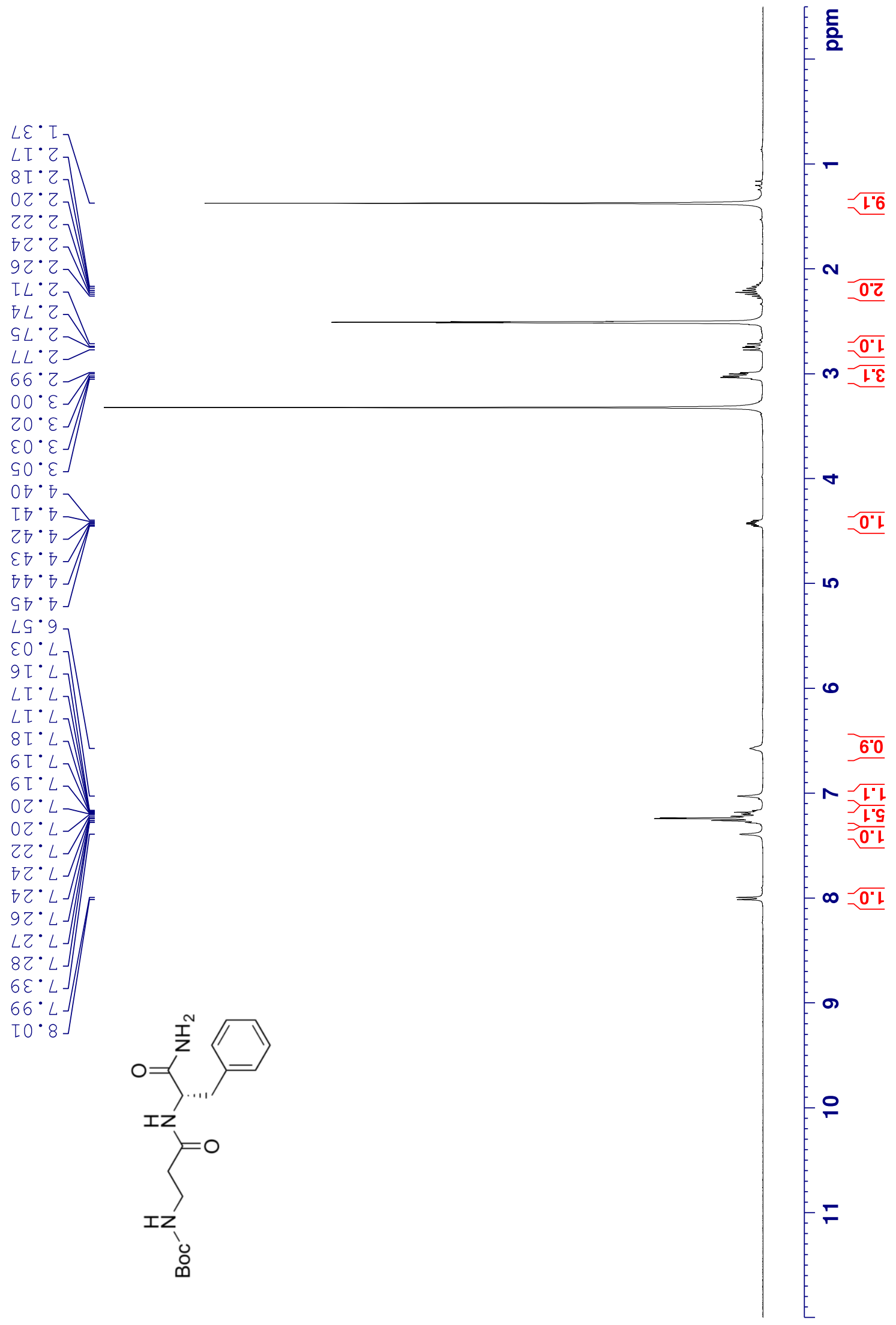


tert-Butyl (S)-(3-((1-amino-1-oxo-3-phenylpropan-2-yl)amino)-3-oxopropyl)carbamate (Boc- $\beta$ Ala-LPhe-NH ${ }_{2}$, DM332) L-6f - ${ }^{\mathbf{1 3}}$ C NMR (100 MHz, DMSO-d $\left._{\boldsymbol{6}}\right)$

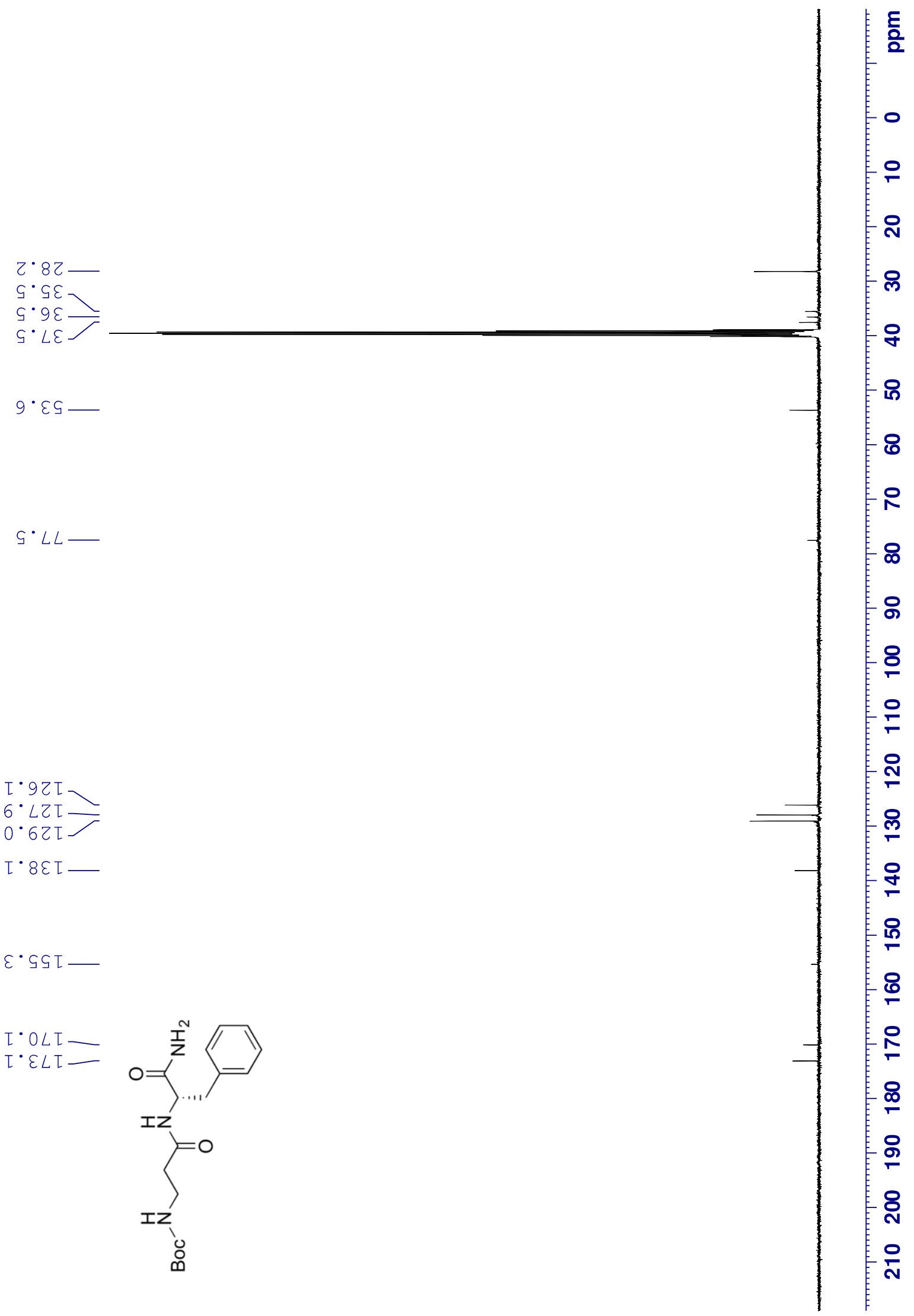


tert-Butyl

((S)-1-(((S)-1-amino-1-oxo-3-phenylpropan-2-yl)amino)-4-(methylthio)-1-oxobutan-2yl)carbamate (Boc-L-Met-L-Phe-NH 2 , DM770) L,L-6g - ${ }^{\mathbf{1}}$ H NMR (400 MHz, DMSO-d $)$

$6 Z \cdot T-$

$8 \varepsilon^{\prime} \mathrm{T}-$

69. $T$

$[\angle \cdot]$

\&L.T

66.5

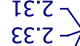

$\downarrow \varepsilon \cdot \tau]$

$28 \cdot Z]$

$58 \cdot Z 7$

$98^{\circ} \mathrm{Z}$

乙० $\mathrm{\circ}$ '

$\varepsilon 0^{\circ} \varepsilon-$

ธด $0^{\circ} \varepsilon$

$\left\lceil 6^{\circ} \varepsilon\right]$

น6. $\varepsilon$

$\downarrow \sigma^{\circ} \varepsilon-\bar{\tau}$

$96^{\circ} \varepsilon-$

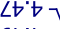

$6 t^{\circ} t$

$0 s^{\prime} t$ -

2st
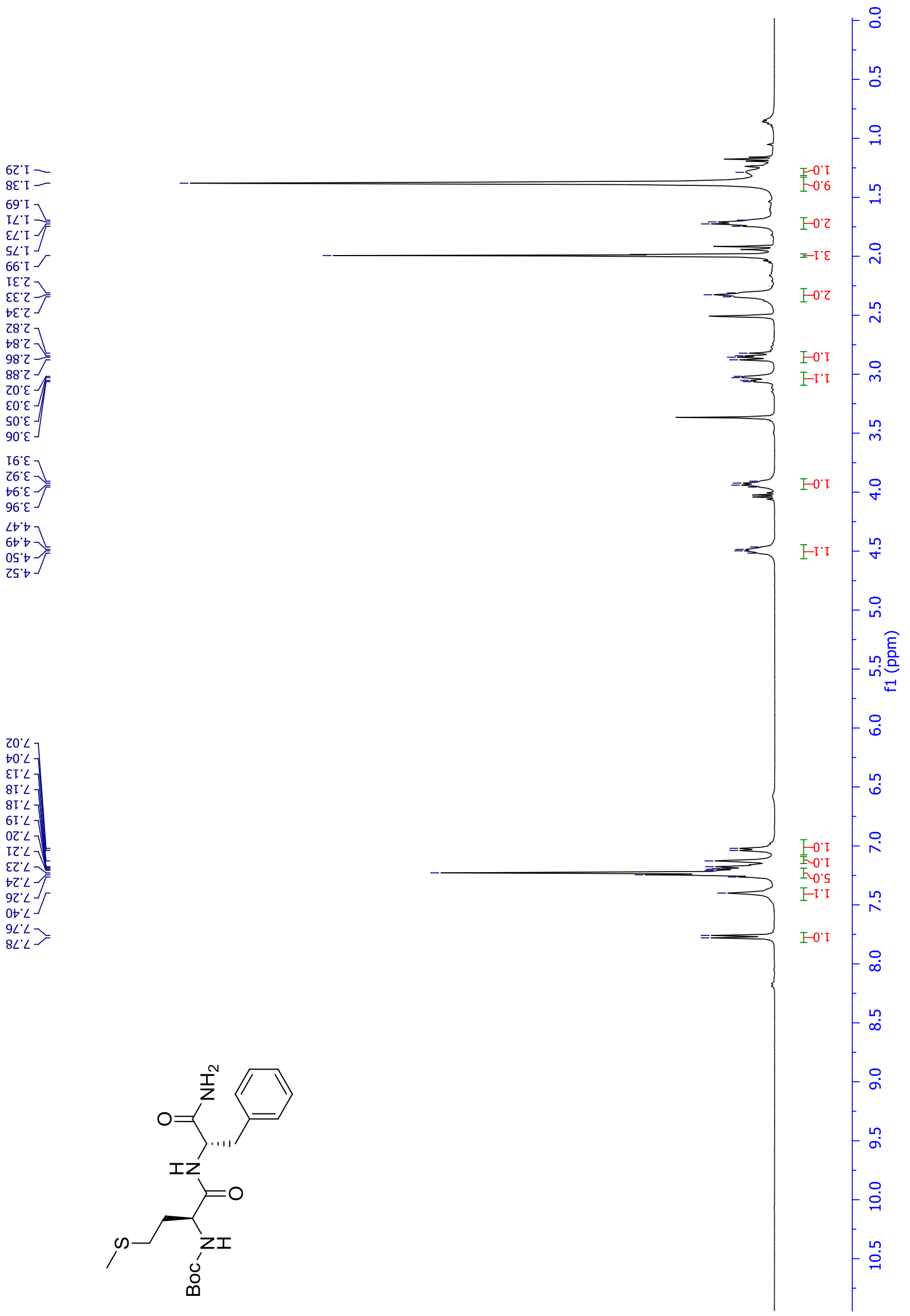
tert-Butyl

((S)-1-(((S)-1-amino-1-oxo-3-phenylpropan-2-yl)amino)-4-(methylthio)-1-oxobutan-2yl)carbamate (Boc-L-Met-L-Phe-NH 2, DM770) L,L-6g - ${ }^{13}$ C NMR (100 MHz, DMSO-d ) $^{2}$

0'SI -

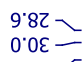

$0.0 \varepsilon-$

$0.8 \varepsilon-$

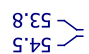

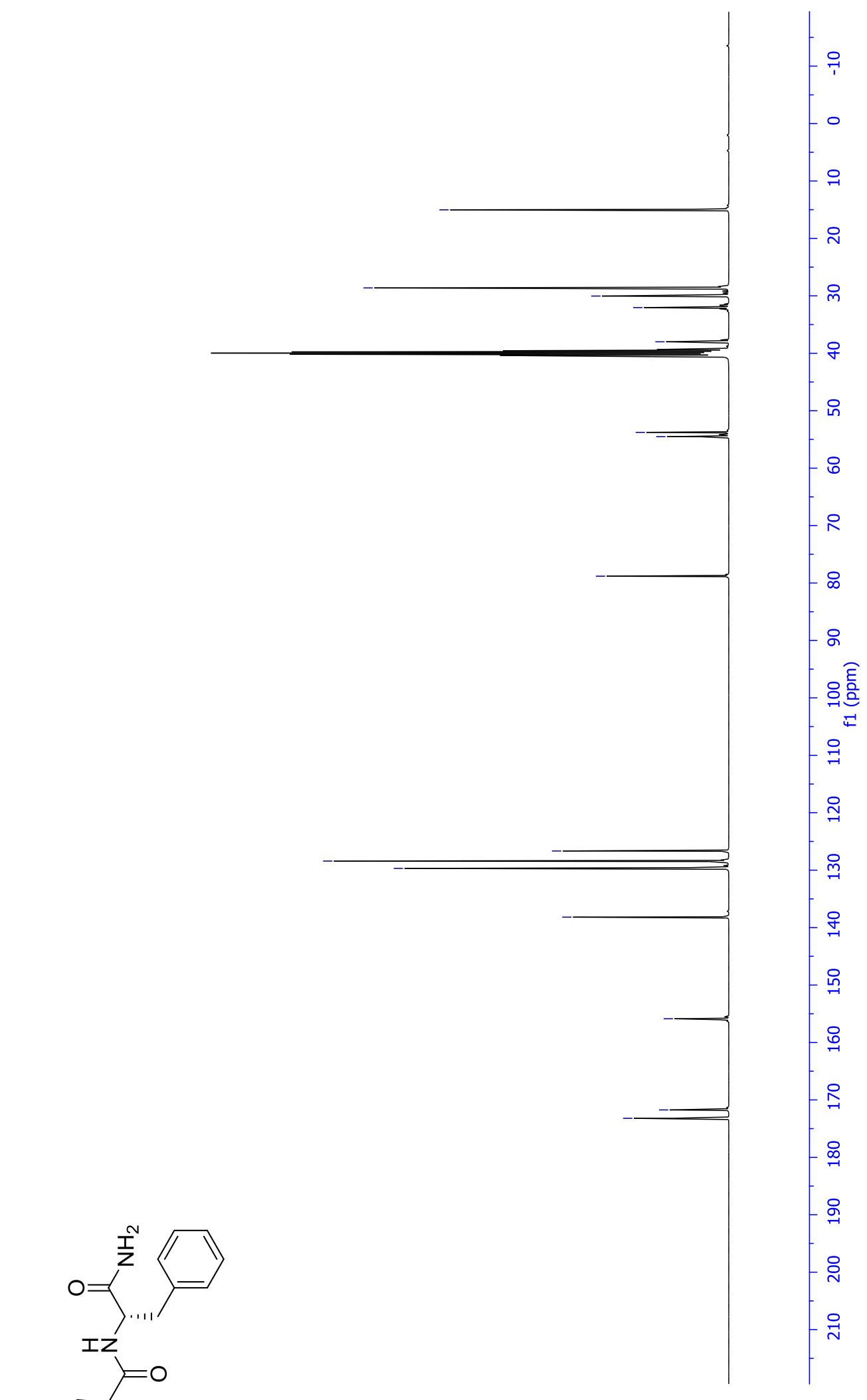

$\angle \cdot 92 \mathrm{~T}$

$\mathrm{t}^{\circ} 8 \mathrm{ZI}$

乙.8عI -

6.SSI -

$\angle \cdot T \angle I=$
Z'ELI $=$

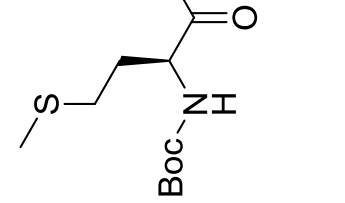


Methyl (tert-butoxycarbonyl)-L-alanyl-L-prolinate (Boc-L-Ala-L-Pro-OMe，KH_1204) L,L-6h - ${ }^{\mathbf{1}} \mathbf{H}$ NMR (500 MHz, $\mathbf{C D C l}_{3}$ )

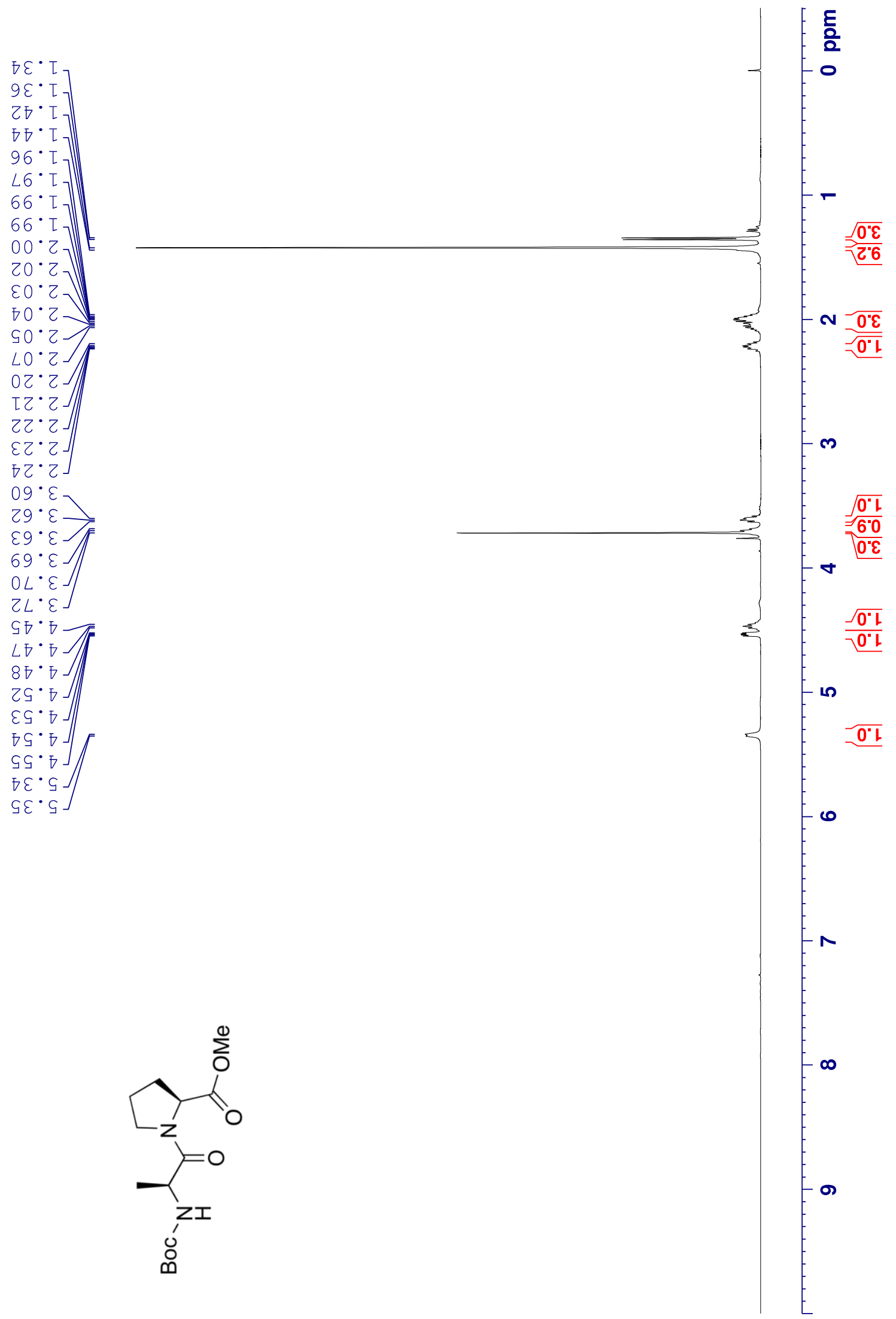


Methyl (tert-butoxycarbonyl)-L-alanyl-L-prolinate (Boc-L-Ala-L-Pro-OMe, KH_1204) L,L-6h - ${ }^{\mathbf{1 3}} \mathbf{C}$ NMR (126 MHz, CDCl $\left.{ }_{3}\right)$

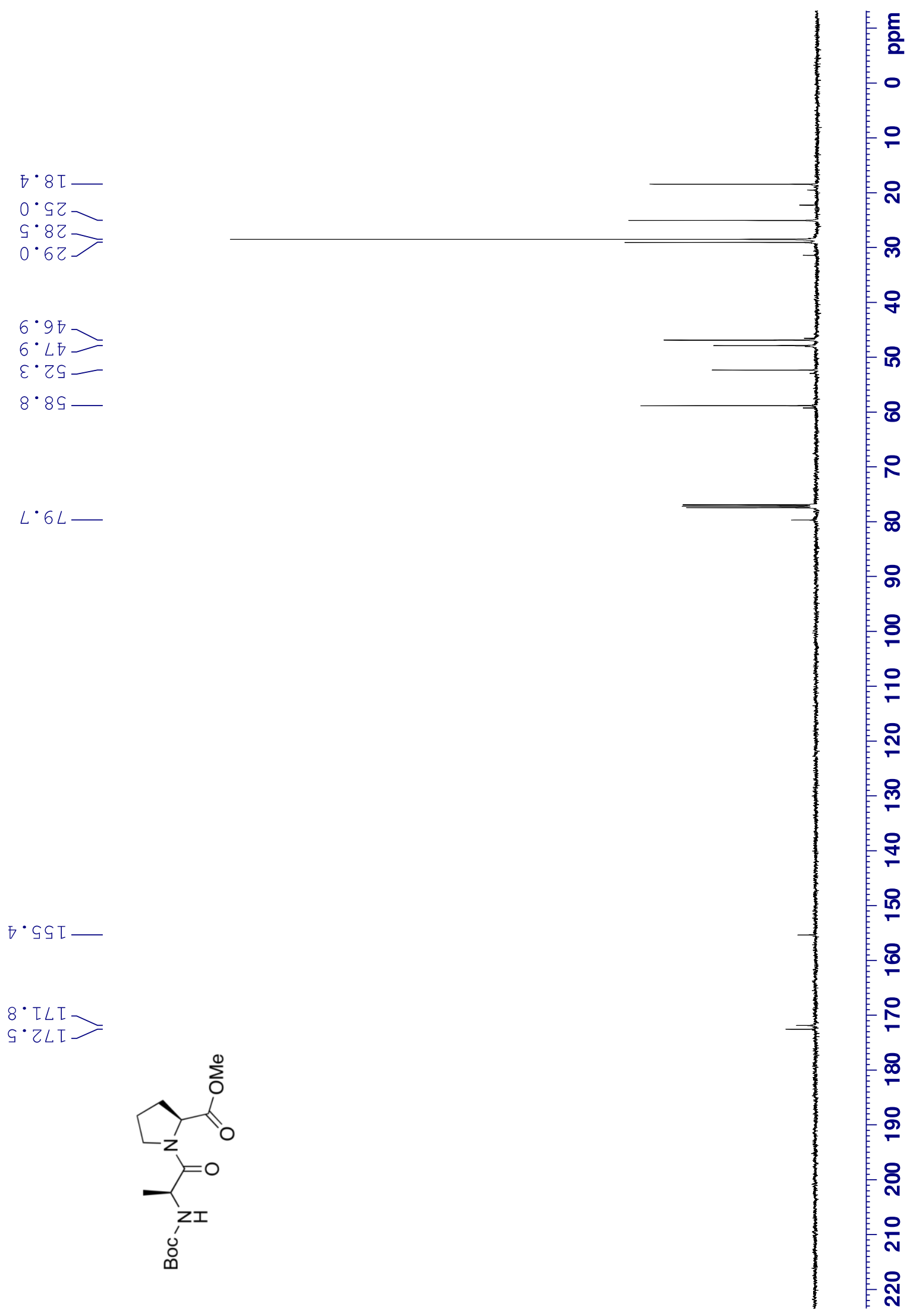


methyl $\quad N^{6}$-((benzyloxy)carbonyl)- $N^{2}$-(N-(tert-butoxycarbonyl)-S-(tert-butyl)-L-cysteinyl)-L-lysinate (Boc-L-Cys $\left.(t \mathrm{Bu})-\mathrm{L}-\mathrm{Lys}(\mathrm{Cbz})-\mathrm{OMe}, \mathrm{KH} \_1315\right)$ L,L-6i - ${ }^{\mathbf{1}} \mathbf{H}$ NMR (500 MHz, CDCl $\left.\mathbf{3}\right)$
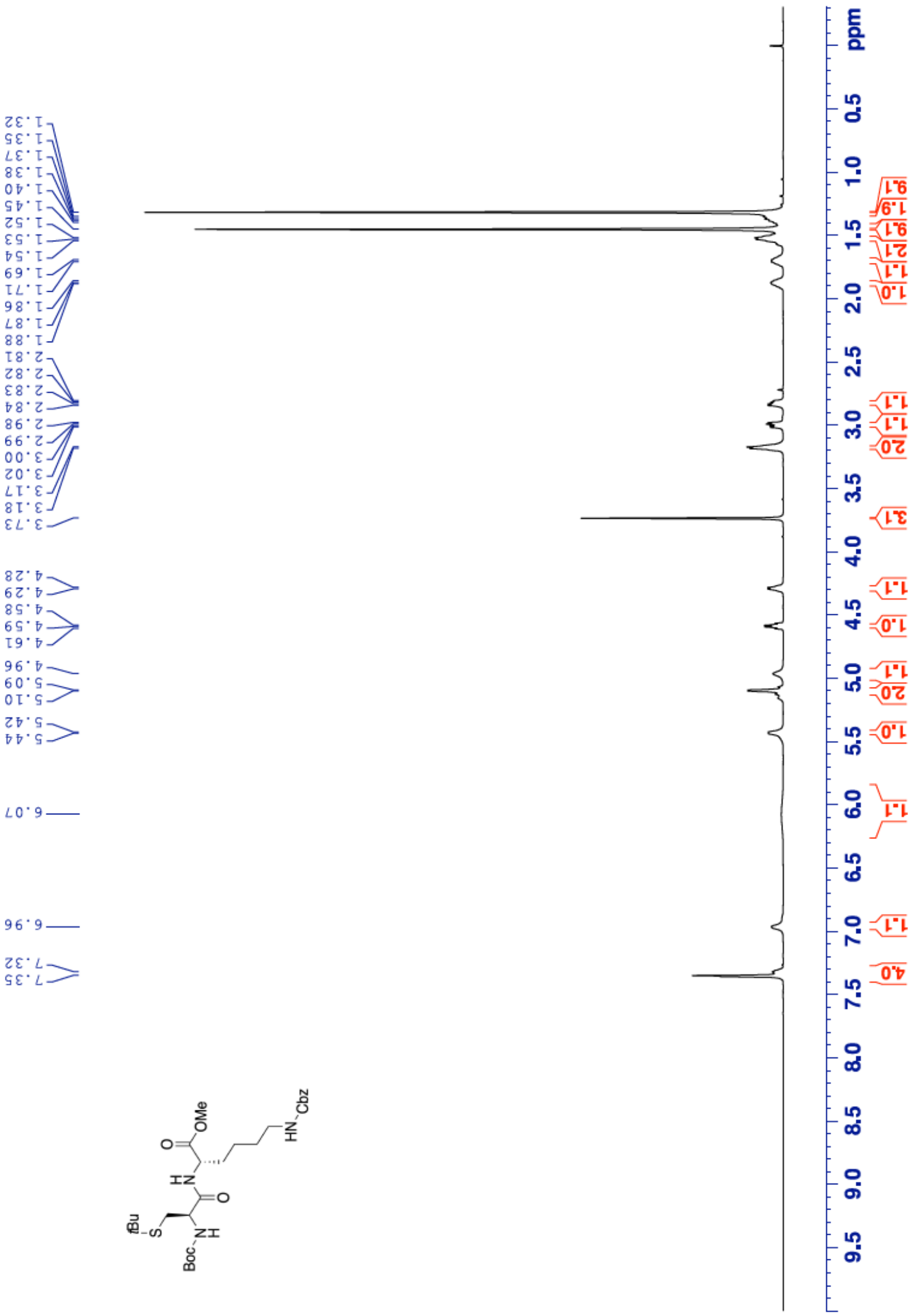
methyl $\quad N^{6}$-((benzyloxy)carbonyl)- $N^{2}$-(N-(tert-butoxycarbonyl)-S-(tert-butyl)-L-cysteinyl)-L-lysinate (Boc-L-Cys( $t$ Bu $)$-L-Lys(Cbz)-OMe, KH_1315) L,L-6i $-{ }^{13}$ C APT NMR (126 MHz, CDCl $)$

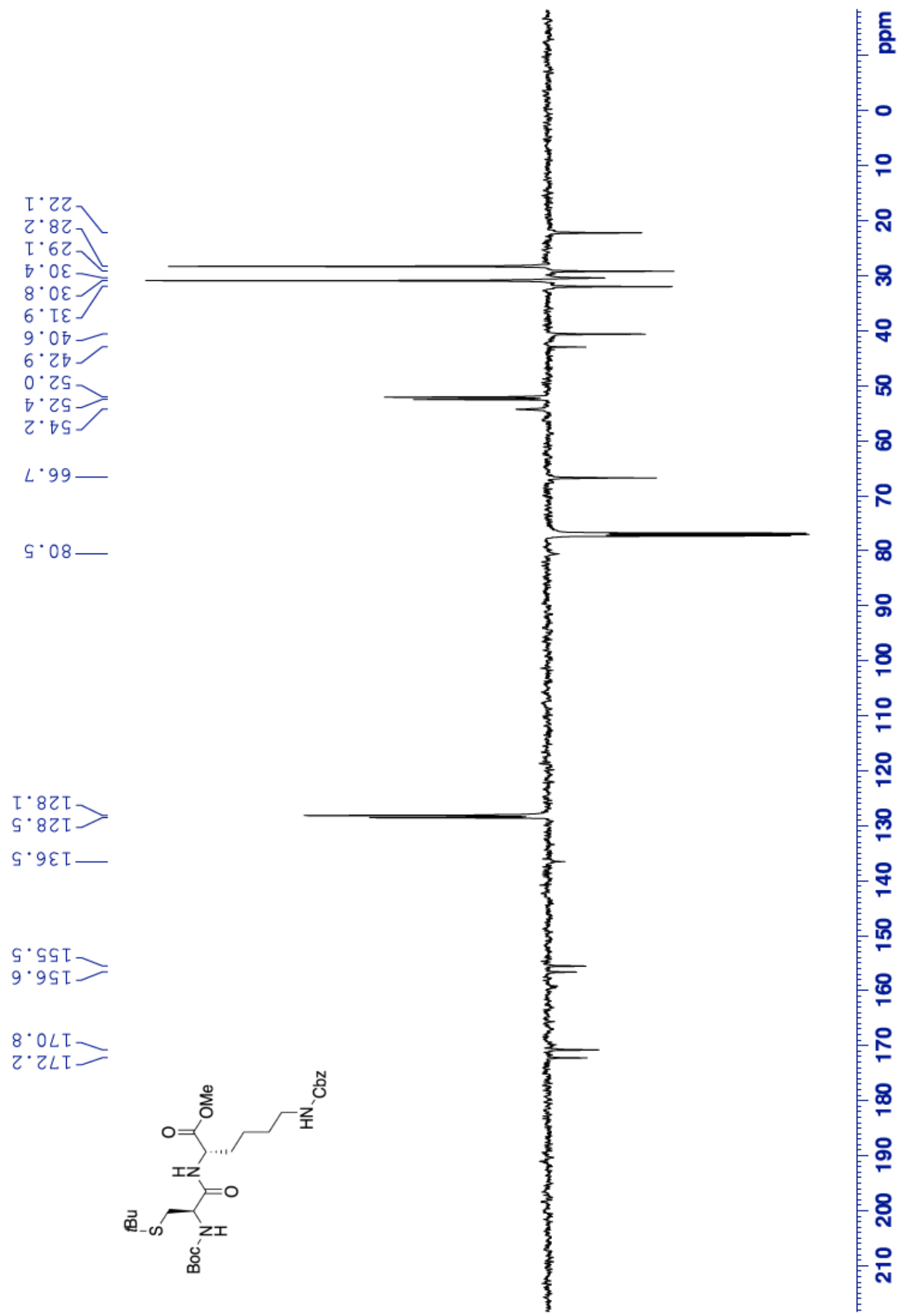


Methyl $N^{6}$-((benzyloxy)carbonyl)- $N^{2}$-(N'-(tert-butoxycarbonyl)- $N^{\tau}$-trityl-L-histidyl)-L-lysinate (Boc-LHis(Trt)-L-Lys(Cbz)-OMe, KH_1308) L,L-6j - ${ }^{\mathbf{1}} \mathbf{H}$ NMR (500 MHz, MeOD)
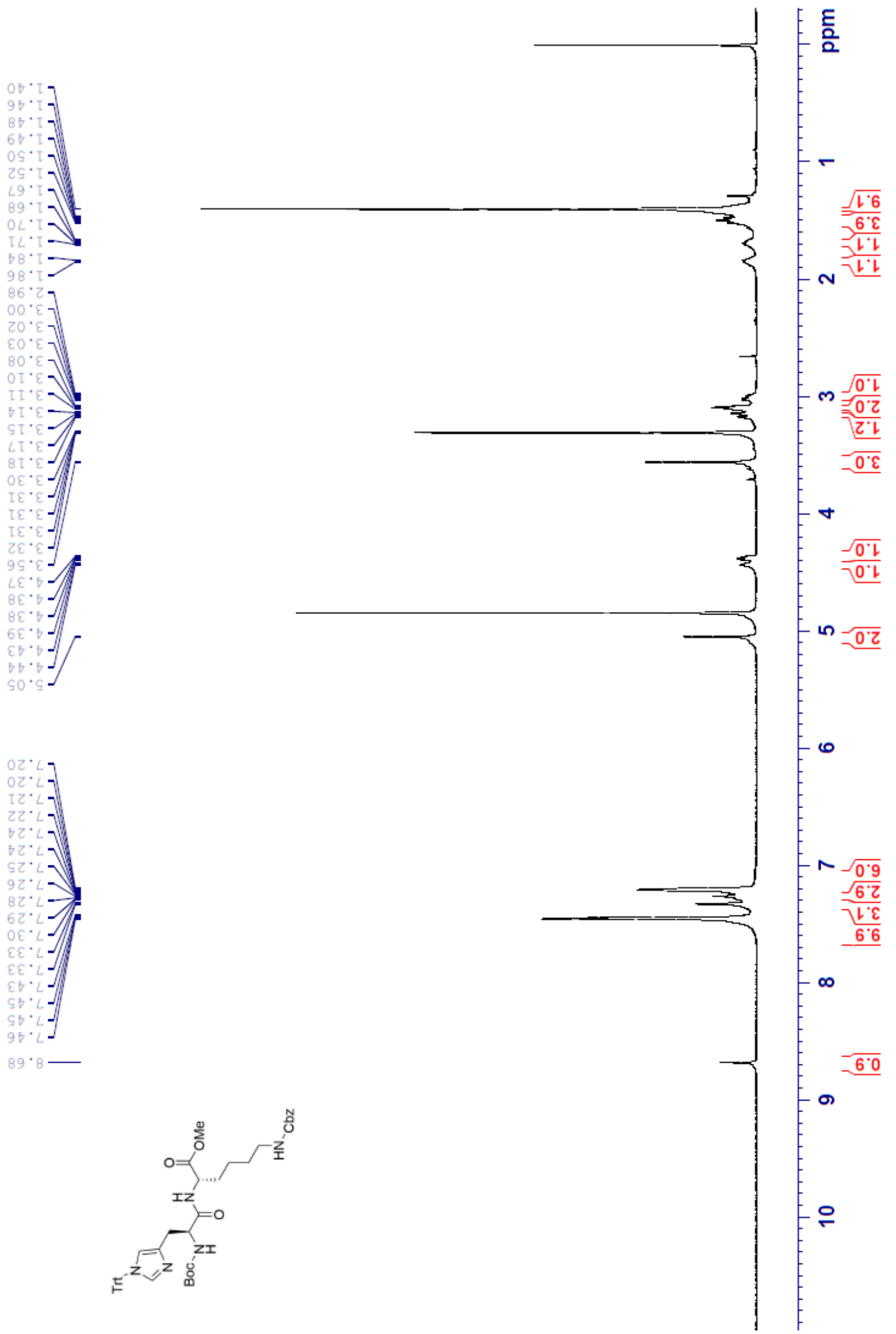
Methyl $N^{6}$-((benzyloxy)carbonyl)- $N^{2}-\left(N^{\prime}\right.$-(tert-butoxycarbonyl)- $N^{\tau}$-trityl-L-histidyl)-L-lysinate (Boc-LHis(Trt)-L-Lys(Cbz)-OMe, KH_1308) L,L-6j - ${ }^{\mathbf{1 3}}$ C APT NMR (126 MHz, MeOD)
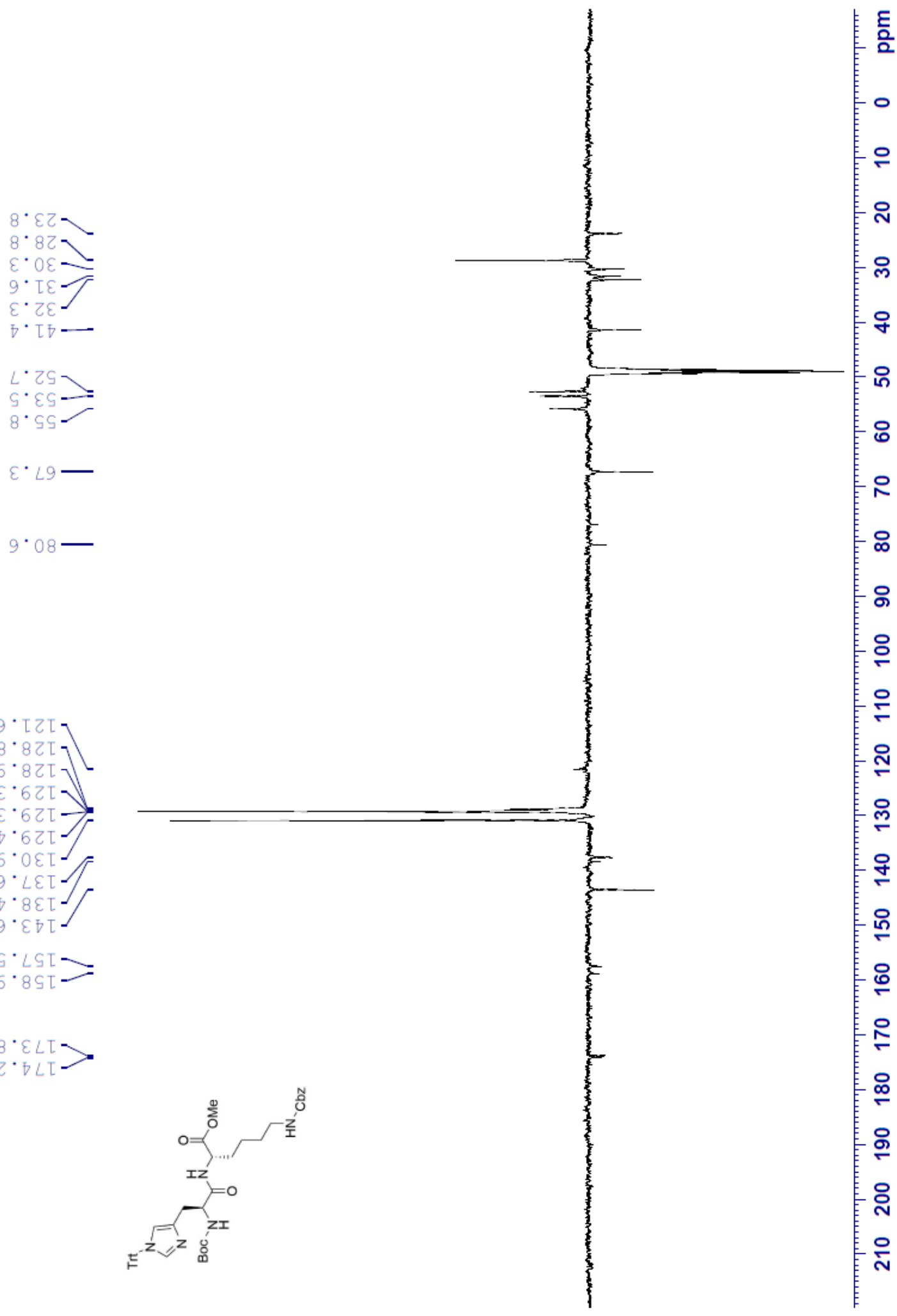
tert-Butyl (S)-2-(((S)-1-((2-(((S)-1-methoxy-1-oxo-3-phenylpropan-2-yl)amino)-2-oxoethyl)amino)-4methyl-1-oxopentan-2-yl)carbamoyl)pyrrolidine-1-carboxylate

(Boc-L-Pro-L-Leu-Gly-L-Phe-OMe, KH_1265) L,L,L-6k - ${ }^{1}$ H NMR (500 MHz, DMSO-d ${ }_{6}$, mixture of rotamers)

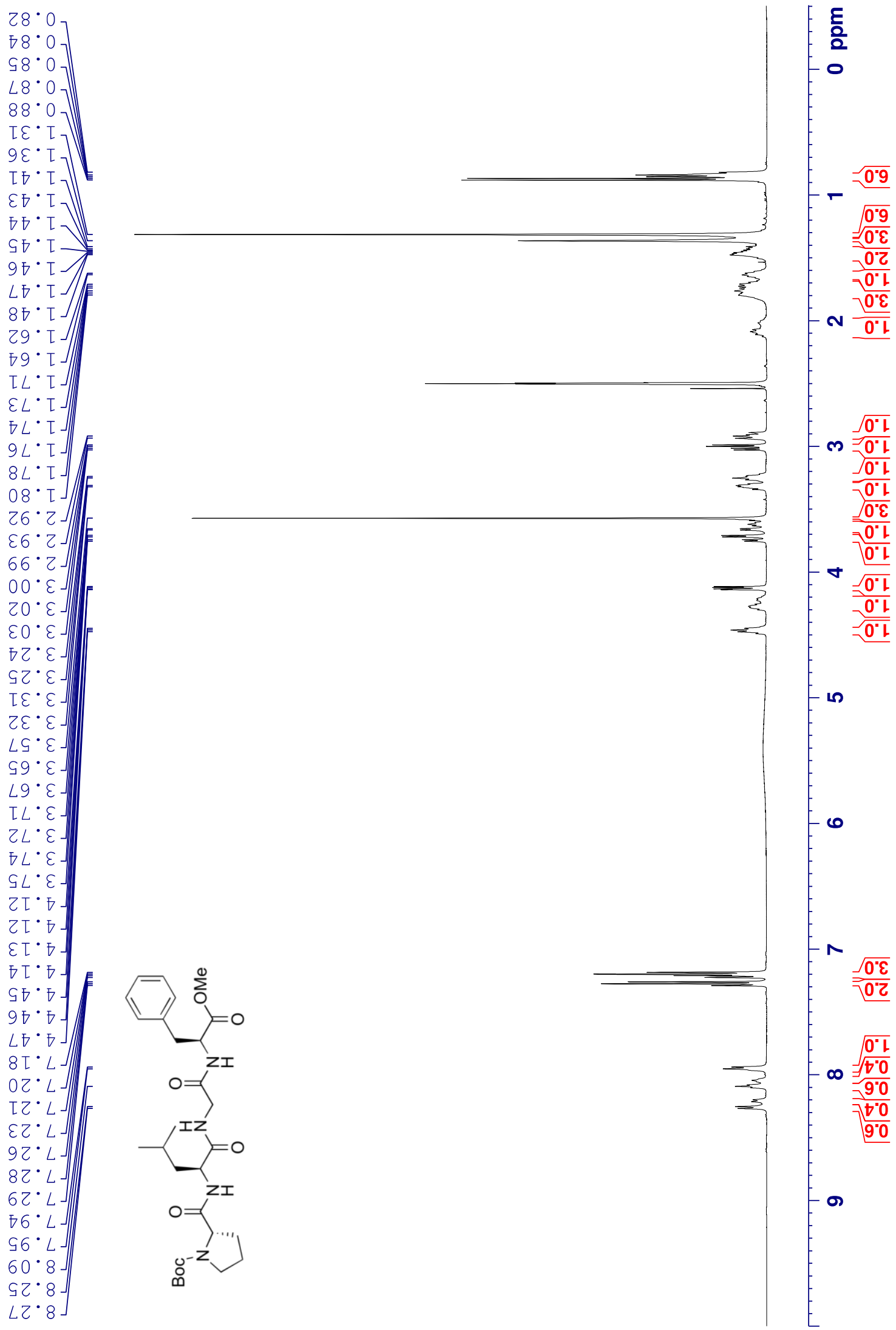


tert-Butyl $\quad(S)-2-(((S)-1-((2-(((S)-1-m e t h o x y-1-o x o-3-p h e n y l p r o p a n-2-y l) a m i n o)-2-o x o e t h y l) a m i n o)-4-$ methyl-1-oxopentan-2-yl)carbamoyl)pyrrolidine-1-carboxylate

(Boc-L-Pro-L-Leu-Gly-L-Phe-OMe, KH_1265) L,L,L-6k - ${ }^{13} \mathrm{C}$ NMR (126 MHz, DMSO-d ${ }_{6}$, mixture of rotamers)

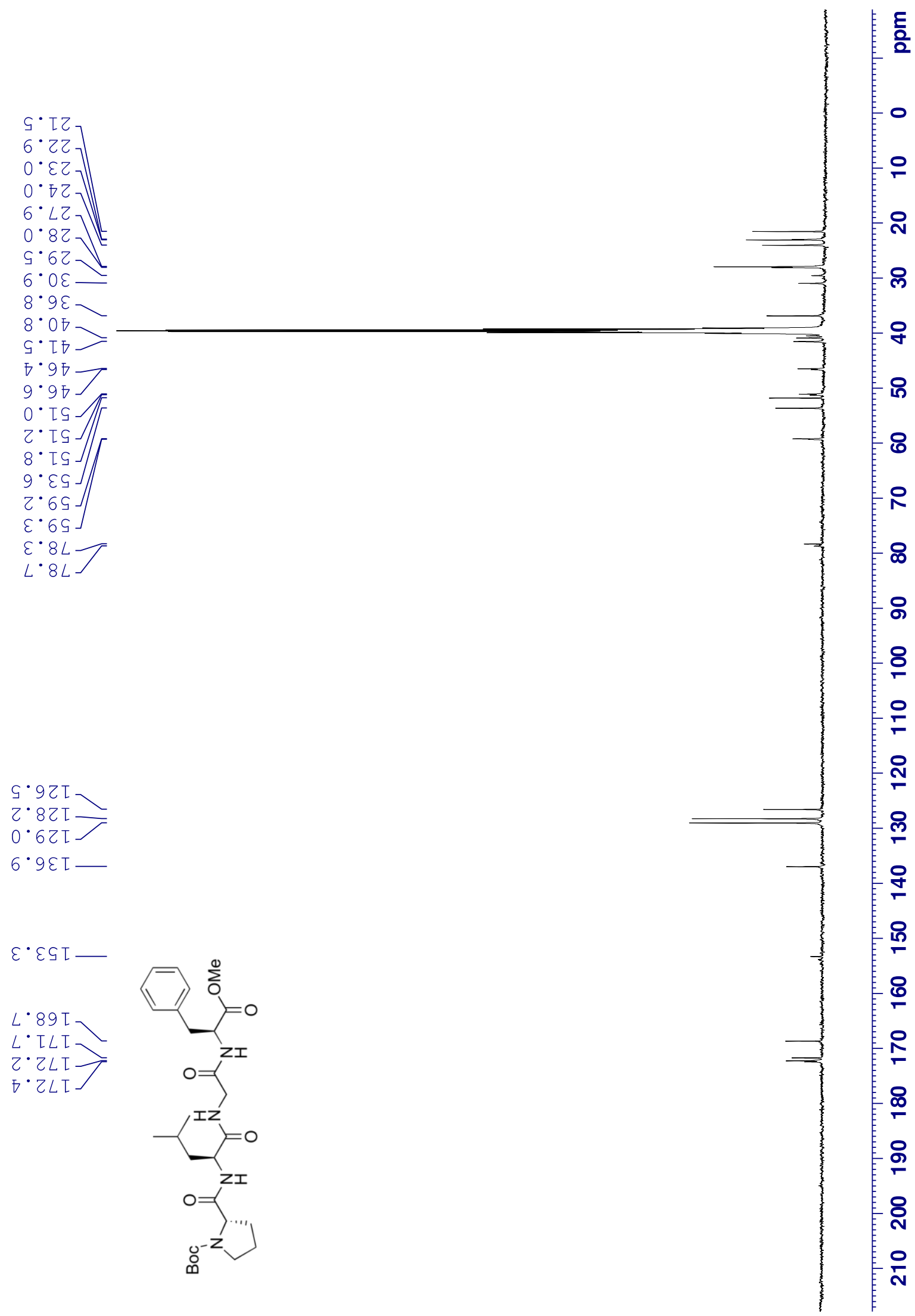


tert-Butyl (S)-2-(((S)-1-((2-(((S)-1-methoxy-1-oxo-3-phenylpropan-2-yl)amino)-2-oxoethyl)amino)-4methyl-1-oxopentan-2-yl)carbamoyl)pyrrolidine-1-carboxylate

(Boc-L-Pro-L-Leu-Gly-L-Phe-OMe, KH_1265) L,L,L-6k- ${ }^{1}$ H NMR $\left(500\right.$ MHz, DMSO-d 6 , 80 $\left.{ }^{\circ} \mathrm{C}\right)$

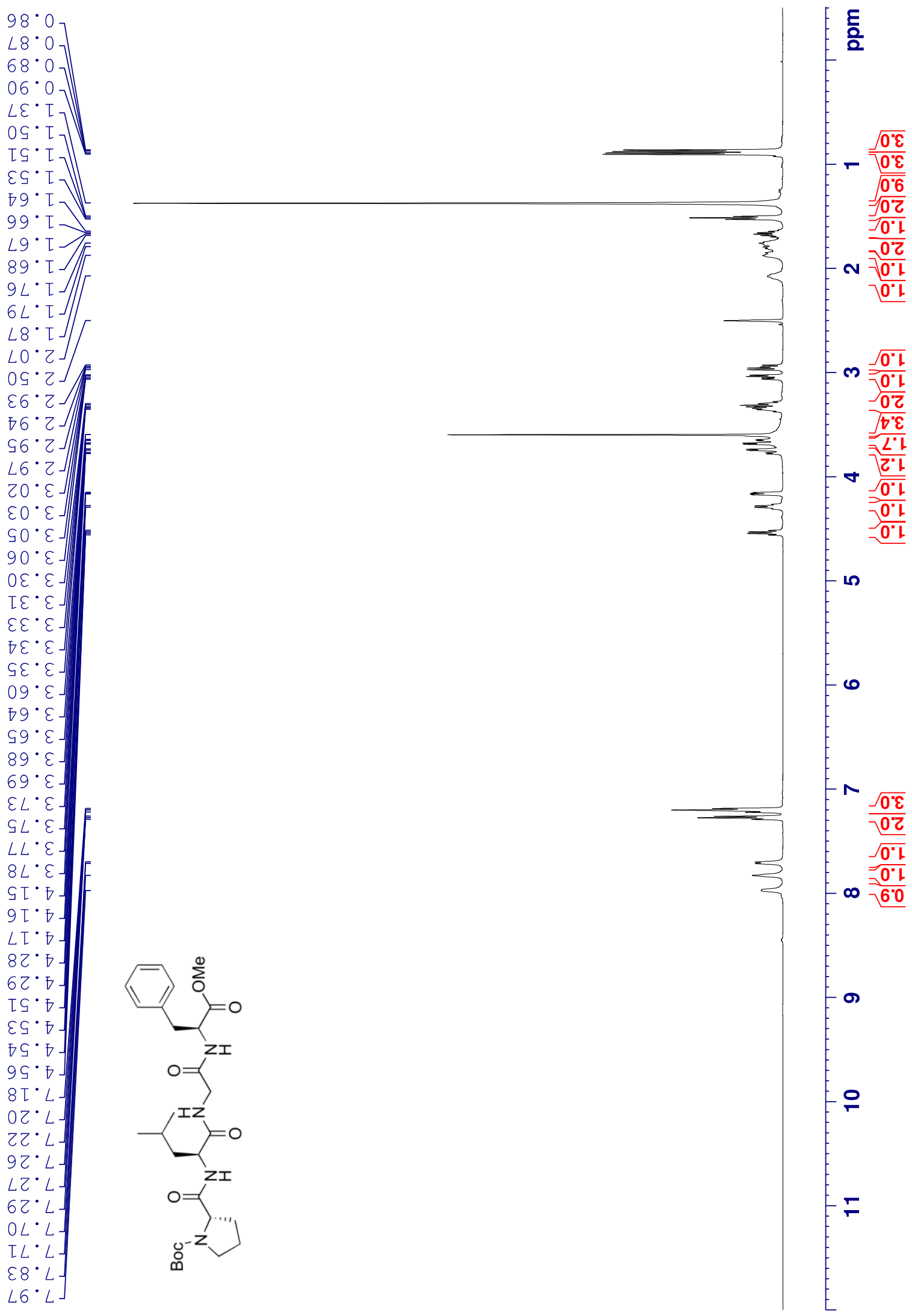


tert-Butyl (S)-2-(((S)-1-((2-(((S)-1-methoxy-1-oxo-3-phenylpropan-2-yl)amino)-2-oxoethyl)amino)-4methyl-1-oxopentan-2-yl)carbamoyl)pyrrolidine-1-carboxylate KH_1265) L,L,L-6k - ${ }^{13} \mathrm{C}$ NMR $\left(126\right.$ MHz, DMSO-d $\left.6,8^{\circ} \mathrm{C}\right)$
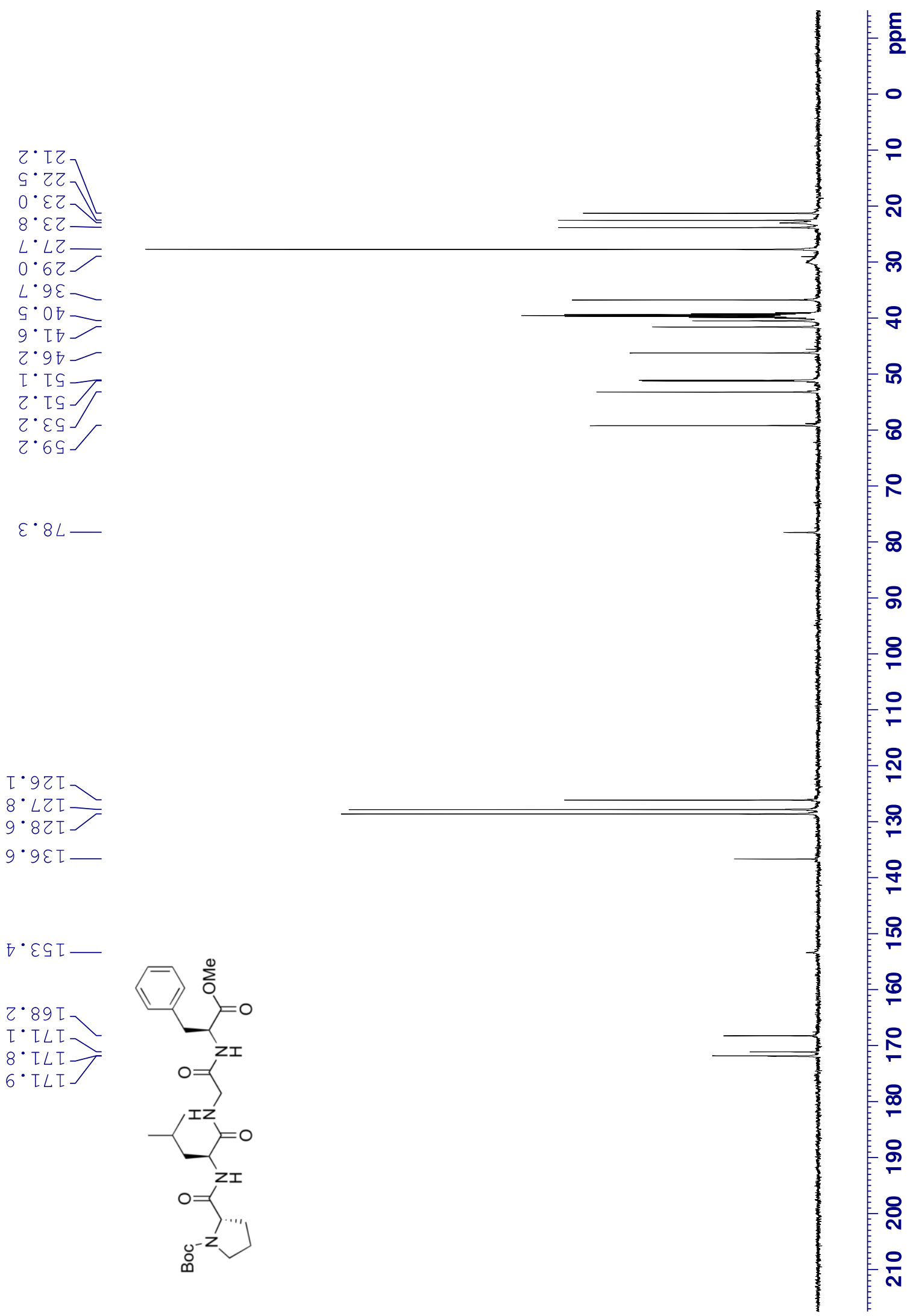

(Boc-L-Pro-L-Leu-Gly-L-Phe-OMe, 
tert-Butyl 2-((S)-2-((S)-2-((tert-butoxycarbonyl)amino)-4-methylpentanamido)-3phenylpropanamido)nicotinate (Boc-L-Leu-L-Phe-NH-tBu-nicn KH_549) L,L-7 - ${ }^{\mathbf{1}}$ H NMR (500 MHz, DMSO- $\left.d_{6}\right)$
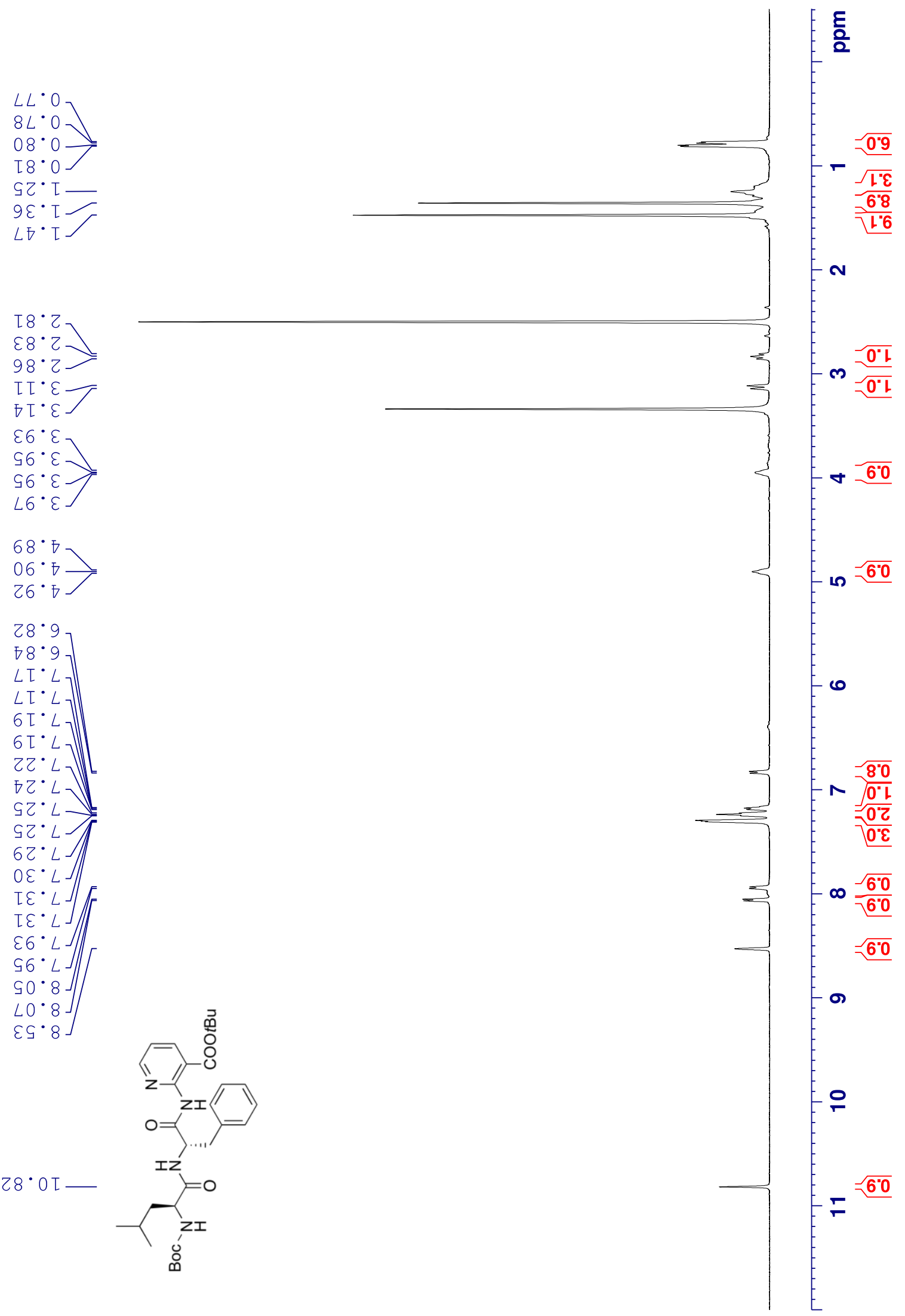
tert-Butyl 2-((S)-2-((S)-2-((tert-butoxycarbonyl)amino)-4-methylpentanamido)-3phenylpropanamido)nicotinate (Boc-L-Leu-L-Phe-NH-tBu-nic, KH_549) L,L-7 - 13 C NMR (126 MHz, DMSO- $\left.d_{6}\right)$

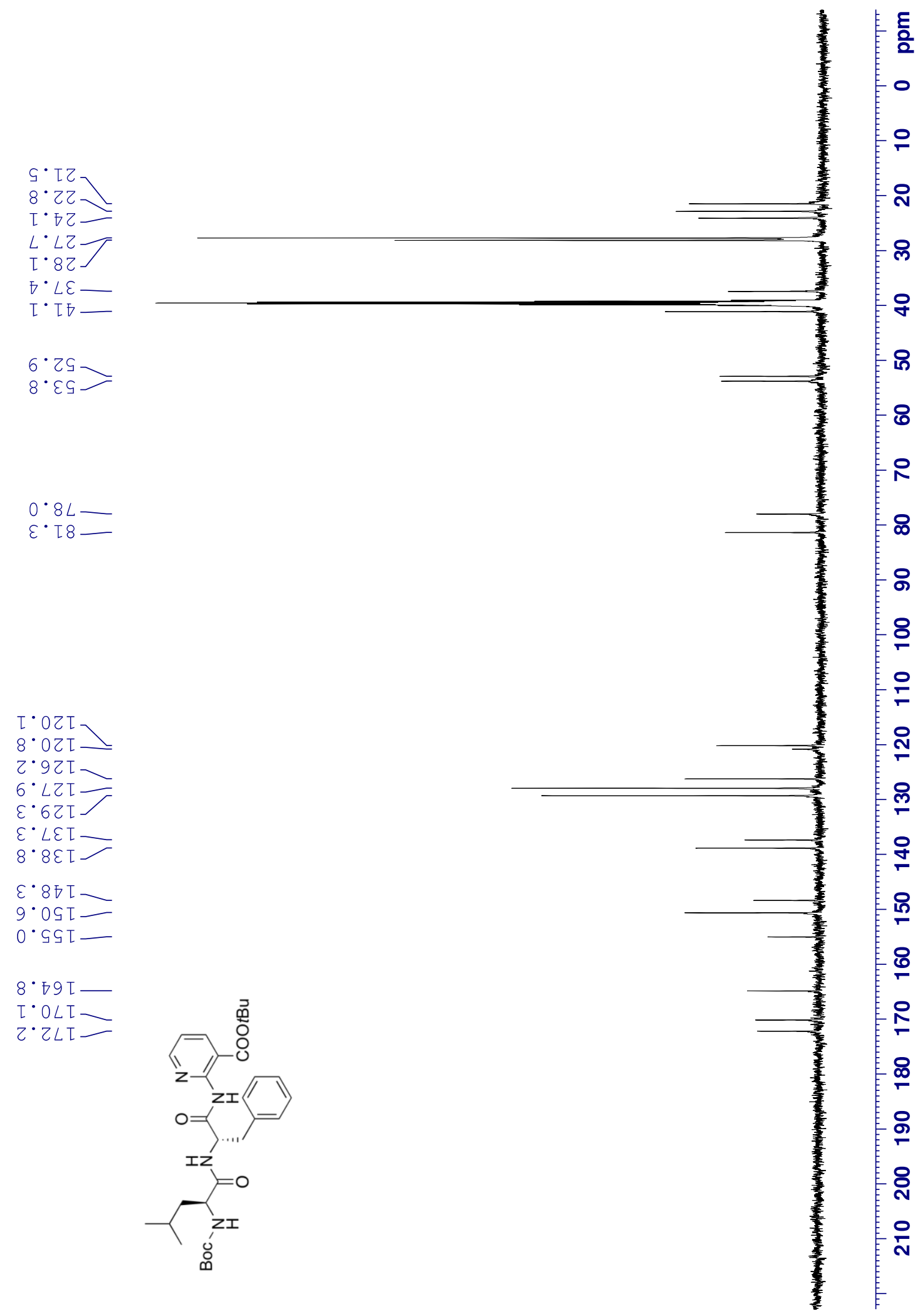


tert-Butyl 2-((S)-2-((S)-2-((tert-butoxycarbonyl)amino)-4-methylpentanamido)-3-

phenylpropanamido)nicotinate (Boc-L-Leu-L-Phe-NH $\left.\mathrm{NH}_{2}, \mathrm{KH}_{-} 1281\right)$ L,L-S19 - ${ }^{\mathbf{1}} \mathbf{H}$ NMR (500 MHz, DMSO- $d_{6}$ )
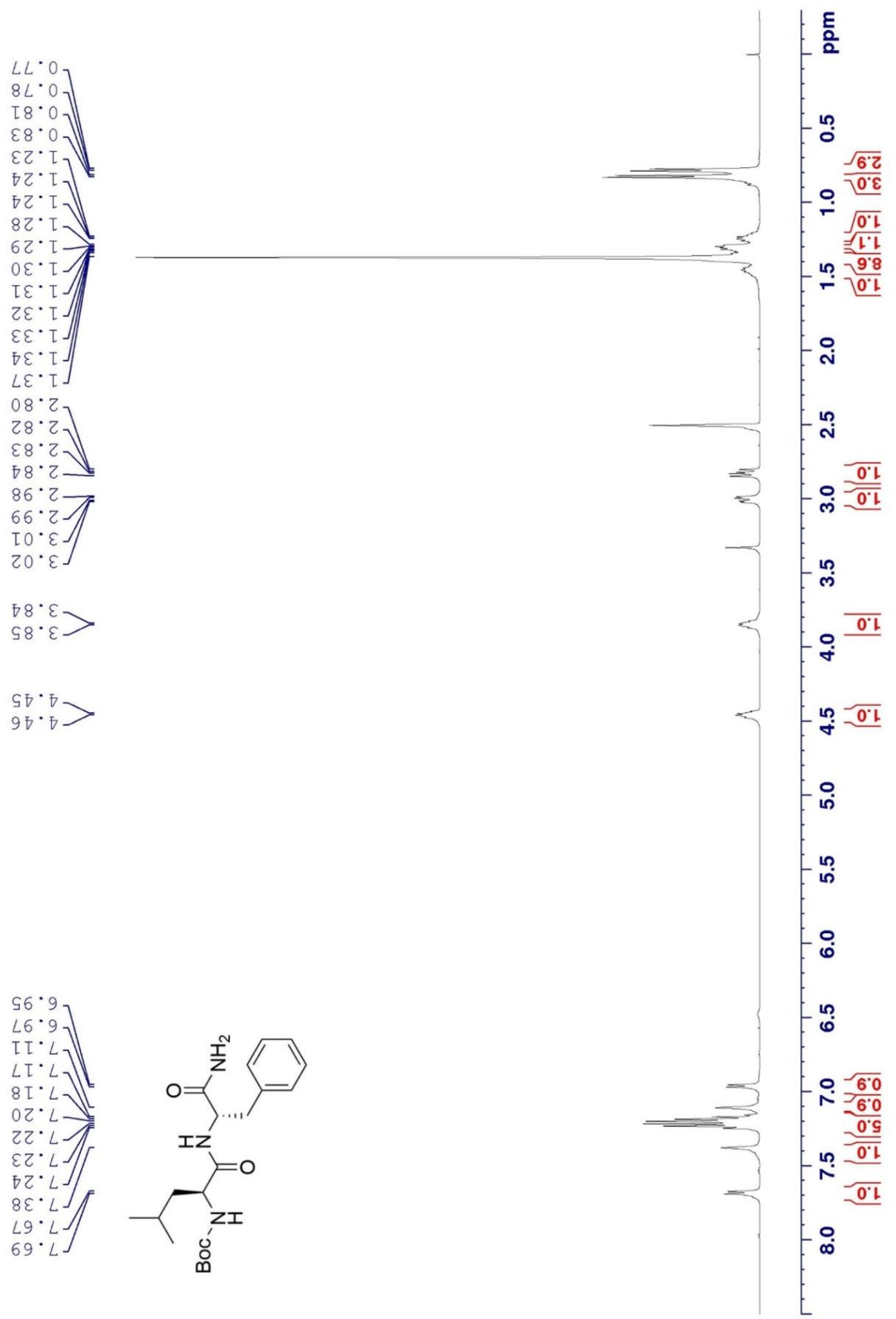
tert-Butyl 2-((S)-2-((S)-2-((tert-butoxycarbonyl)amino)-4-methylpentanamido)-3-

phenylpropanamido)nicotinate (Boc-L-Leu-L-Phe-NH 2, KH_1281) ) L,L-S19 ${ }^{13}$ C APT NMR (126 MHz, DMSO- $d_{6}$ )
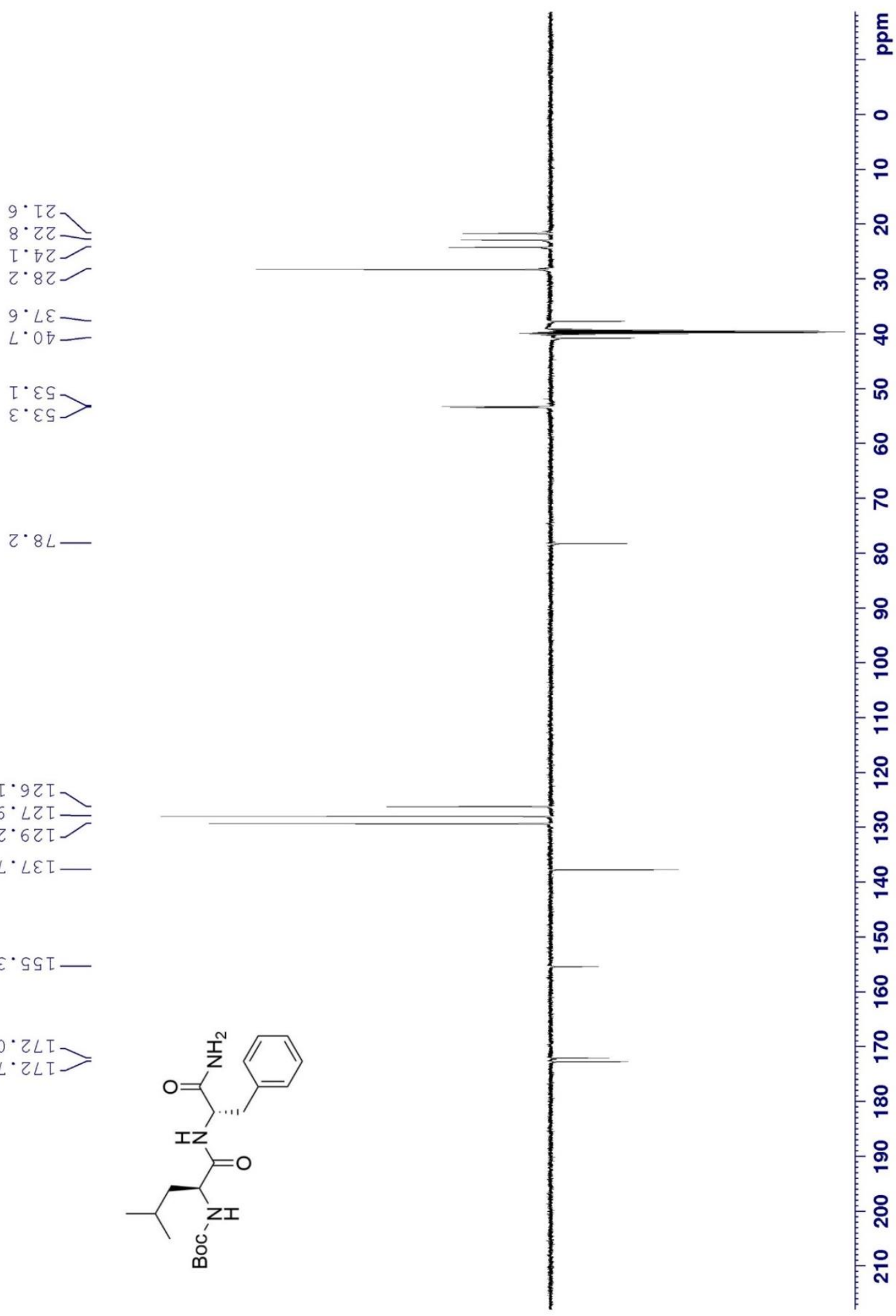
tert-Butyl 2-((S)-2-((S)-3-(tert-butoxy)-2-((tert-butoxycarbonyl)amino)propanamido)propanamido) nicotinate (Boc-L-Ser( $t$ Bu)-L-Ala-NH- $t$ Bu-nic, KH_635) L,L-8 - ${ }^{1} \mathbf{H}$ NMR (500 MHz, CDCl $)$
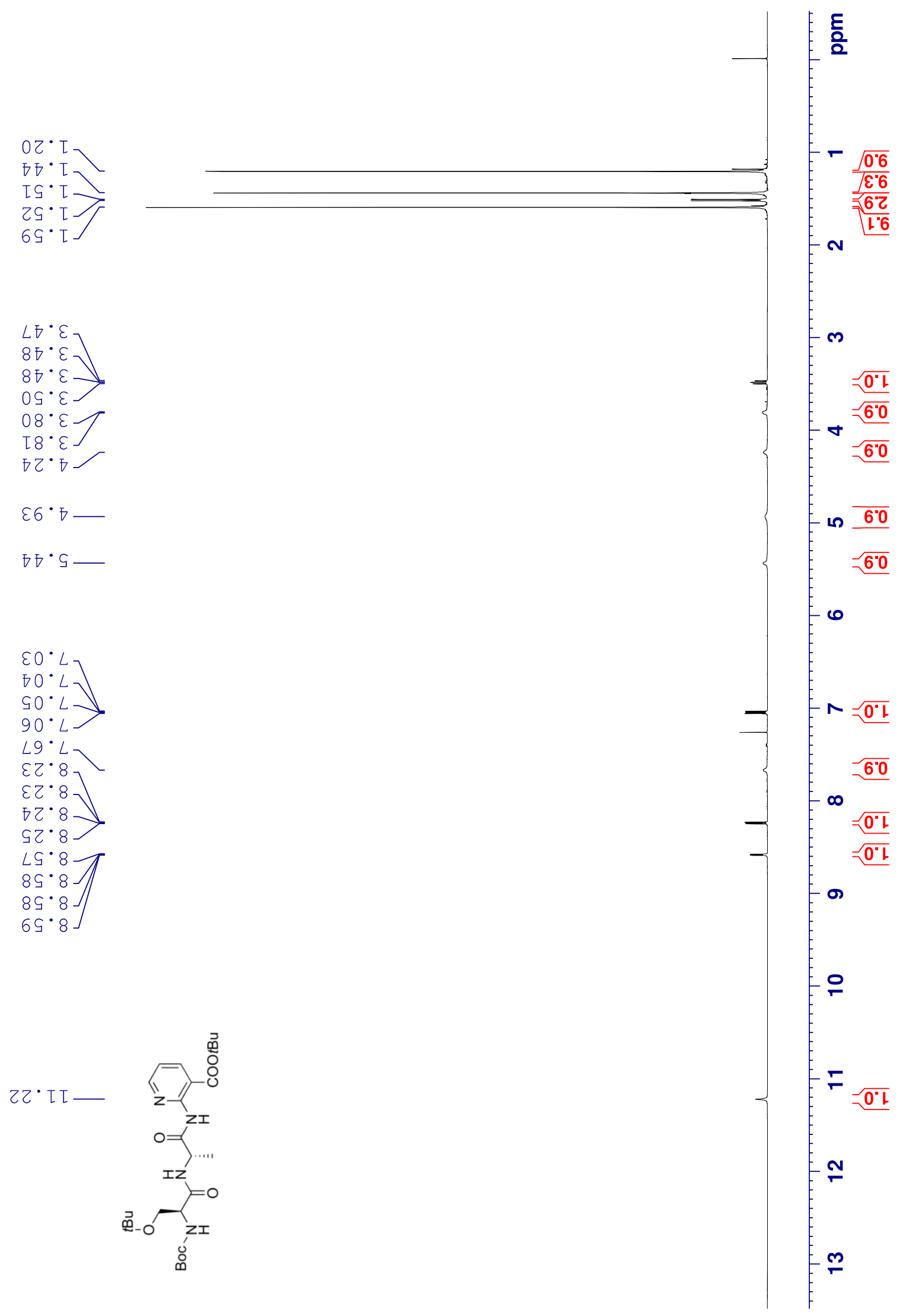
tert-Butyl 2-((S)-2-((S)-3-(tert-butoxy)-2-((tert-butoxycarbonyl)amino)propanamido)propanamido) nicotinate (Boc-L-Ser( $t$ Bu) $)$ L-Ala-NH- $t$ Bu-nic, KH_635) L,L-8 - ${ }^{13}$ C NMR (126 MHz, CDCl $)$

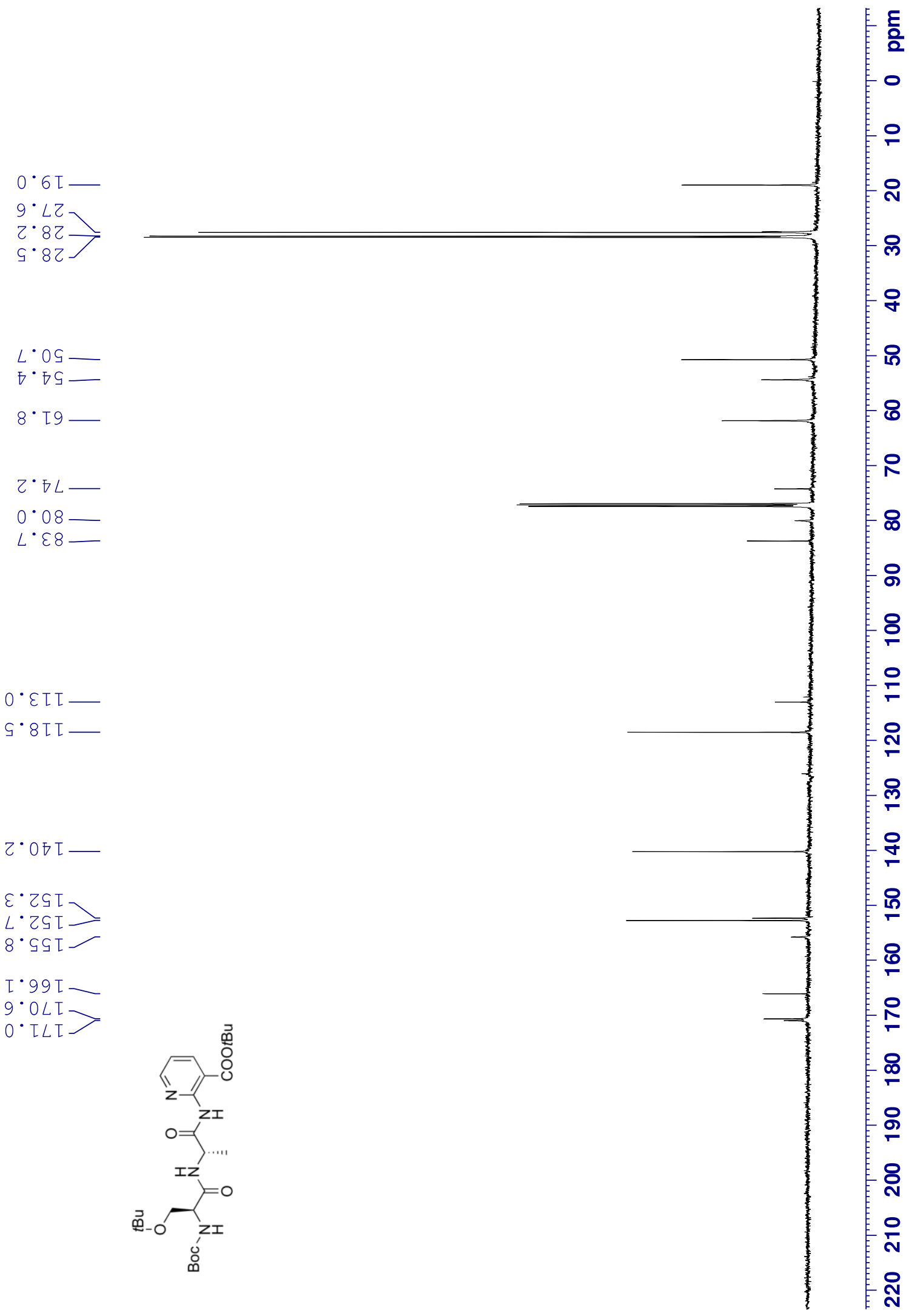


tert-Butyl L-leucyl-L-valinate hydrochloric acid salt (H-L-Leu-L-Val-OtBu.HCl, KH_562) L,L-9.HCl ${ }^{1}$ H NMR (500 MHz, DMSO-d ${ }_{6}$ )
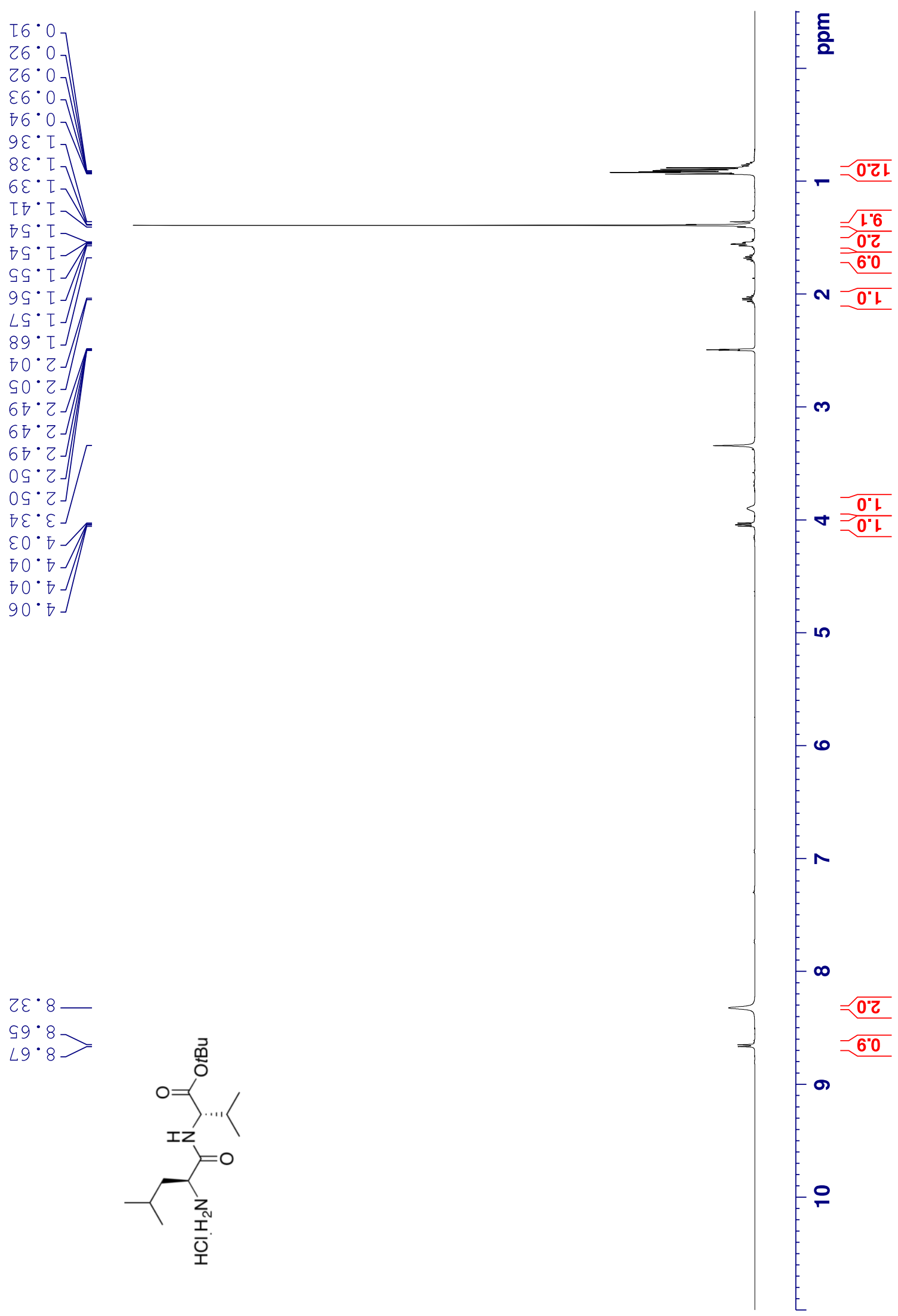
tert-Butyl L-leucyl-L-valinate hydrochloric acid salt (H-L-Leu-L-Val-OtBu.HCl, KH_562) L,L-9.HCl ${ }^{13}$ C NMR (126 MHz, DMSO- $d_{6}$ )

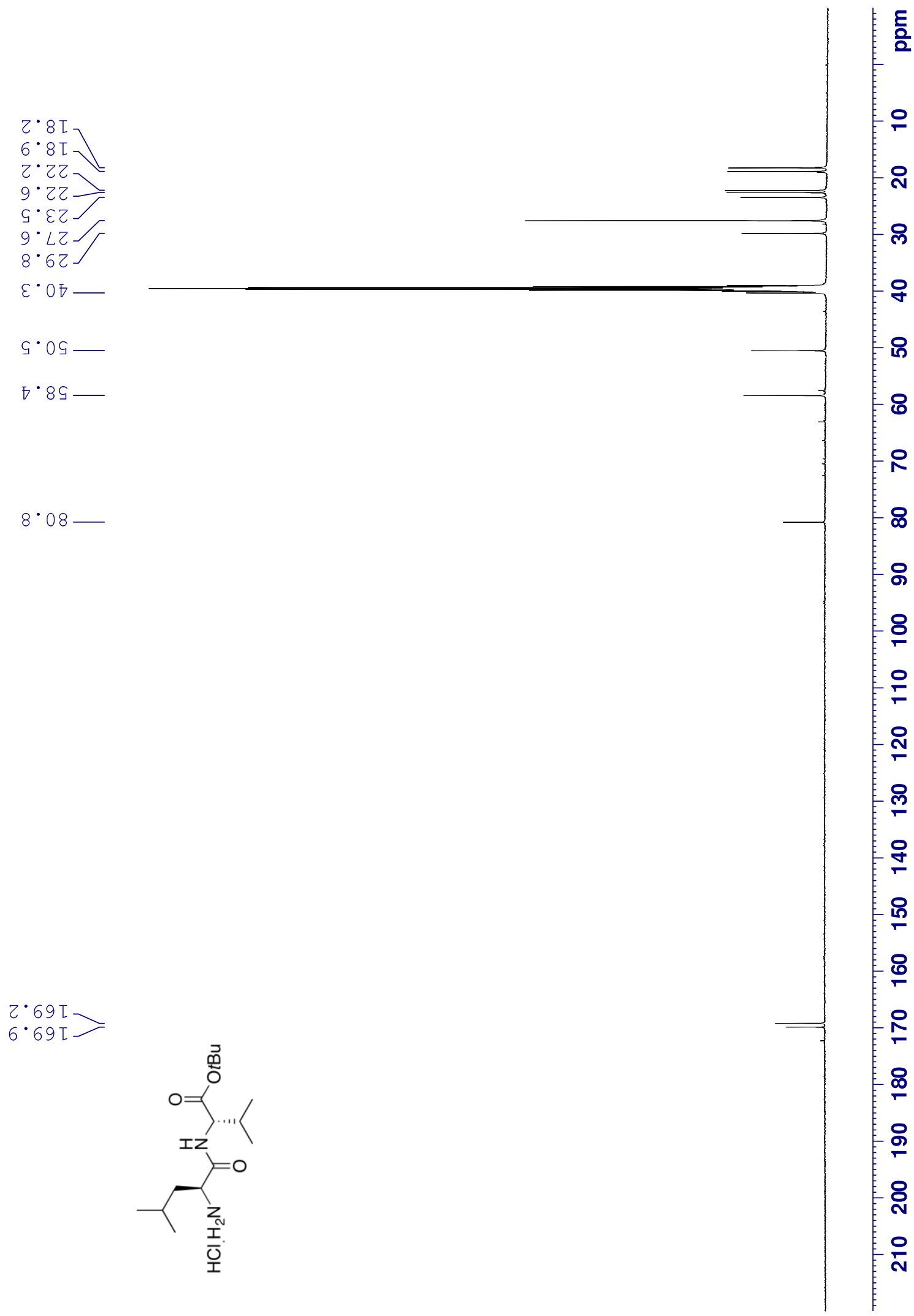


tert-Butyl L-alanyl-L-phenylalaninate hydrochloride (H-L-Ala-L-Phe-OtBu.HCl, KH_782) L,L-10.HCl ${ }^{1} \mathrm{H}$ NMR (500 MHz, $\left.\mathrm{CDCl}_{3}\right)$

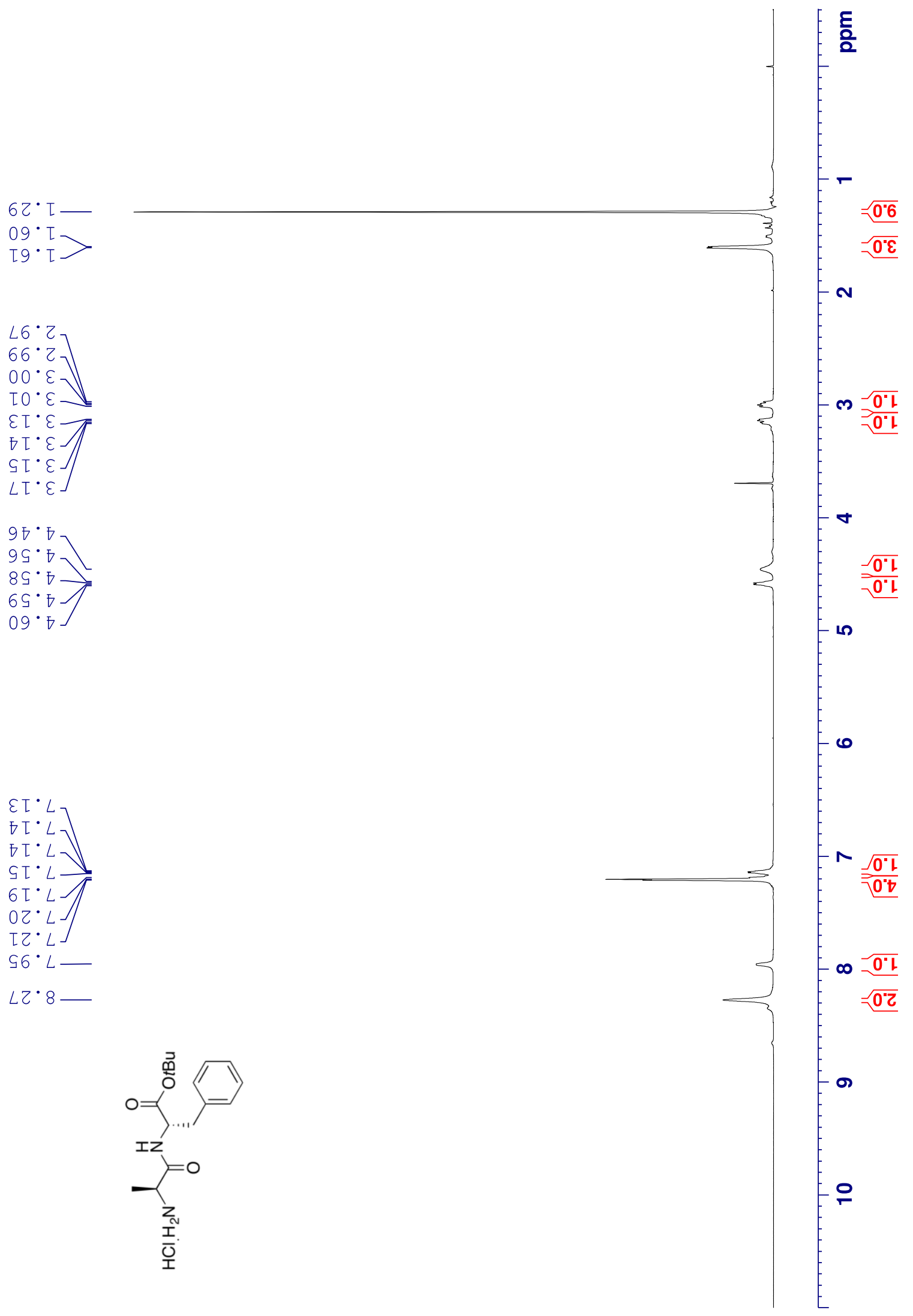


tert-Butyl L-alanyl-L-phenylalaninate hydrochloride (H-L-Ala-L-Phe-OtBu.HCl, KH_782) L,L-10.HCl ${ }^{13} \mathrm{C}$ NMR (500 MHz, $\mathrm{CDCl}_{3}$ )

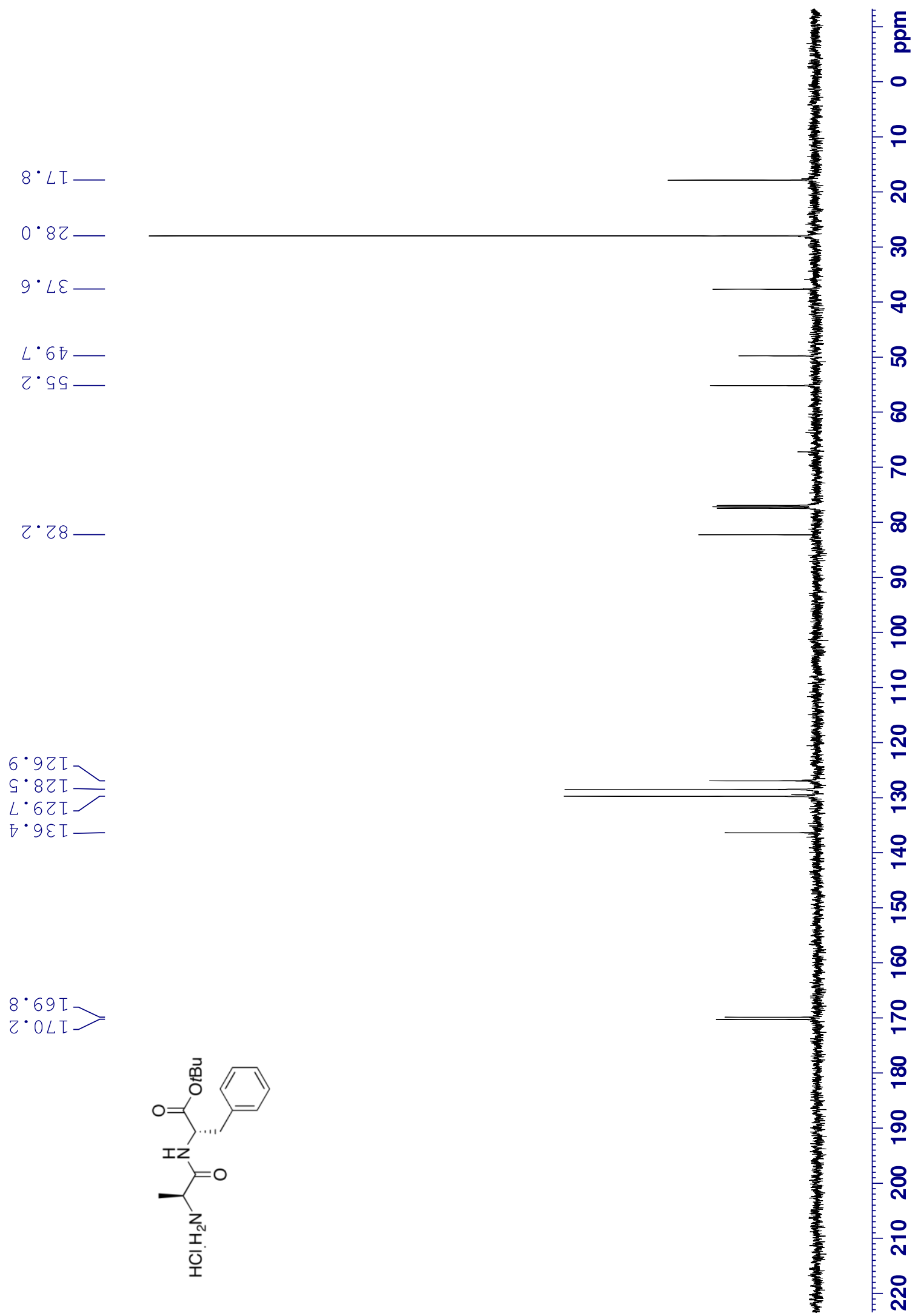


tert-Butyl (tert-butoxycarbonyl)-L-leucyl-L-phenylalanyl-L-leucyl-L-valinate (Boc-L-Leu-L-Phe-L-LeuL-Val-OtBu, KH_1224) L,L,L,L-11- ${ }^{1} \mathbf{H}$ NMR (500 MHz, $\left.\mathbf{C D C l}_{3}\right)$

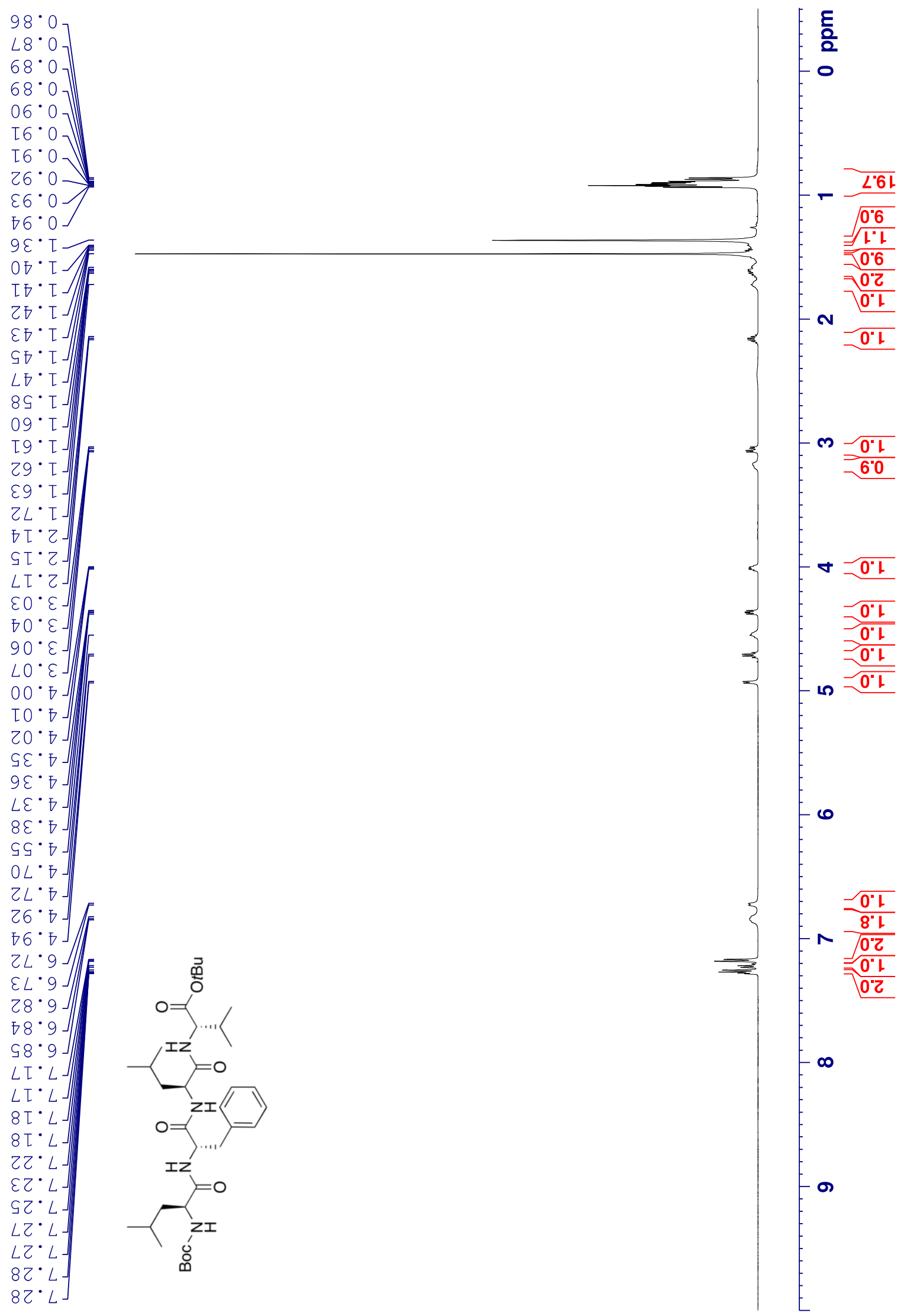


tert-Butyl (tert-butoxycarbonyl)-L-leucyl-L-phenylalanyl-L-leucyl-L-valinate (Boc-L-Leu-L-Phe-L-LeuL-Val-OtBu, KH_1224) L,L,L,L-11- ${ }^{13} \mathbf{C}$ NMR (126 MHz, CDCl $)$

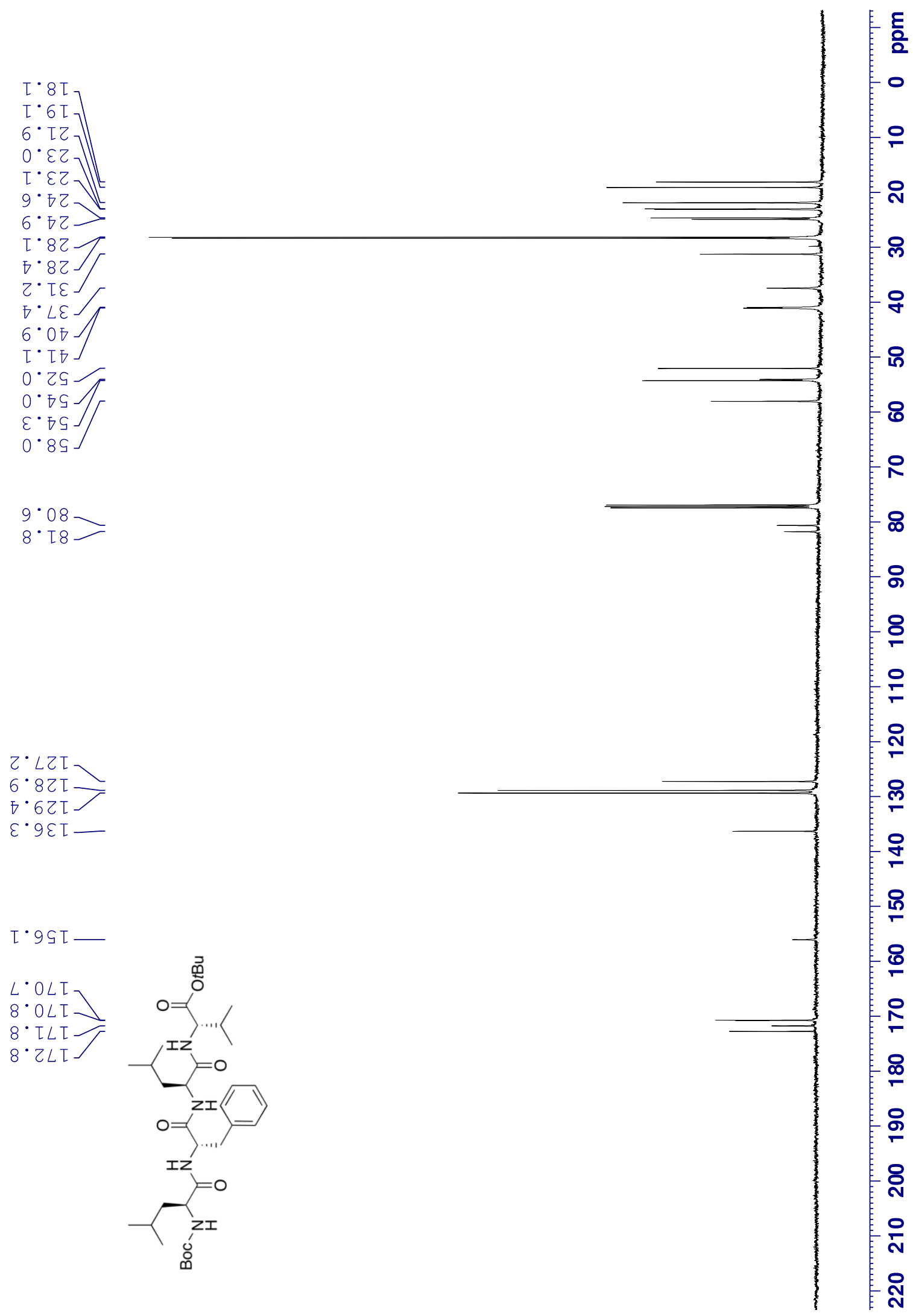


tert-Butyl $N$-(tert-butoxycarbonyl)-O-(tert-butyl)-L-seryl-L-alanyl-L-alanyl-L-phenylalaninate (Boc-L$\operatorname{Ser}(t \mathrm{Bu})$-L-Ala-L-Ala-L-Phe-OtBu, KH_1229), L,L,L,L-12 - ${ }^{1}$ H NMR (500 MHz, DMSO-d )

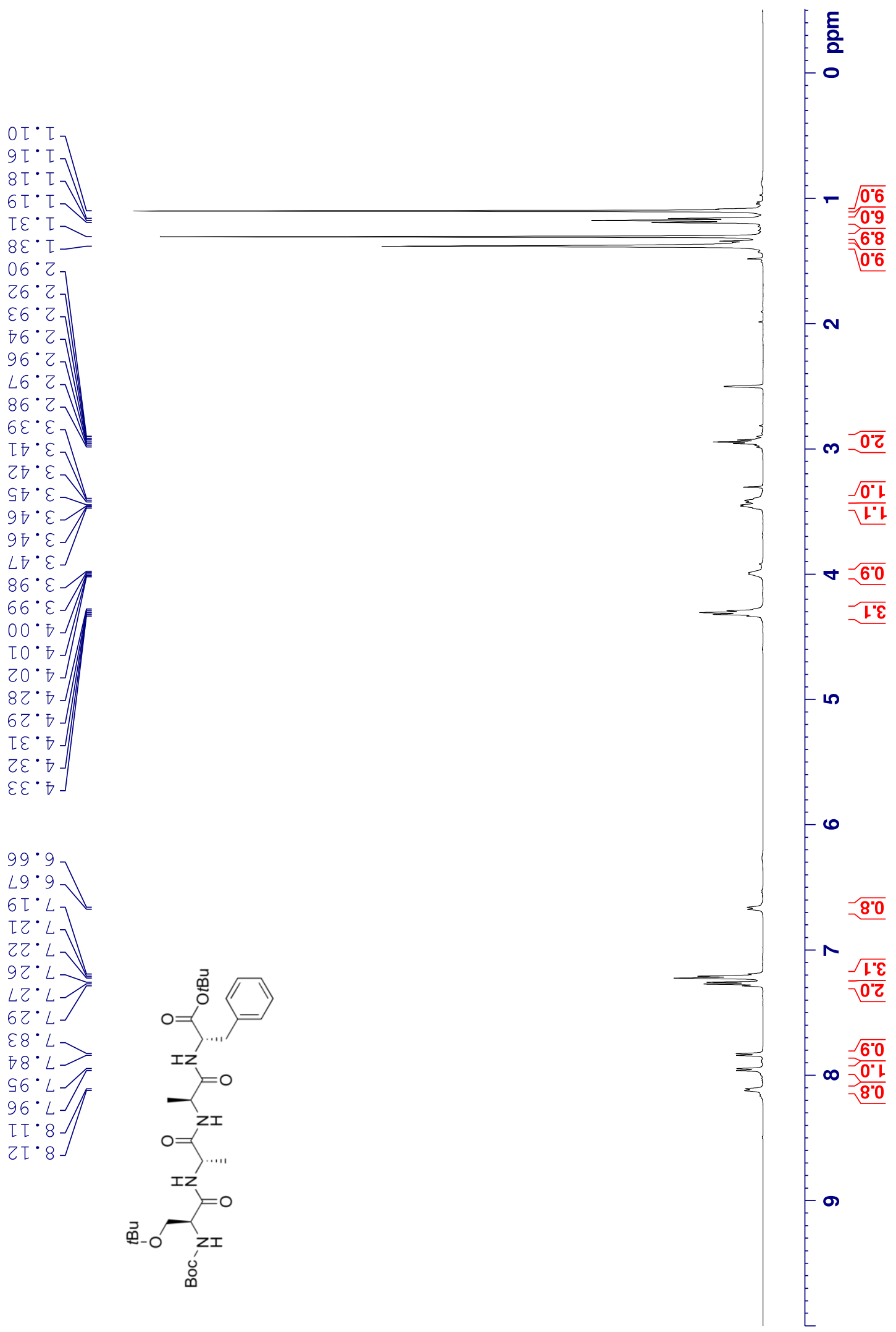


tert-Butyl $N$-(tert-butoxycarbonyl)-O-(tert-butyl)-L-seryl-L-alanyl-L-alanyl-L-phenylalaninate (Boc-L$\operatorname{Ser}(t \mathrm{Bu})$-L-Ala-L-Ala-L-Phe-O $t$ Bu, KH_1229) L,L,L,L-12 - ${ }^{\mathbf{1 3}}$ C NMR (126 MHz, DMSO-d )
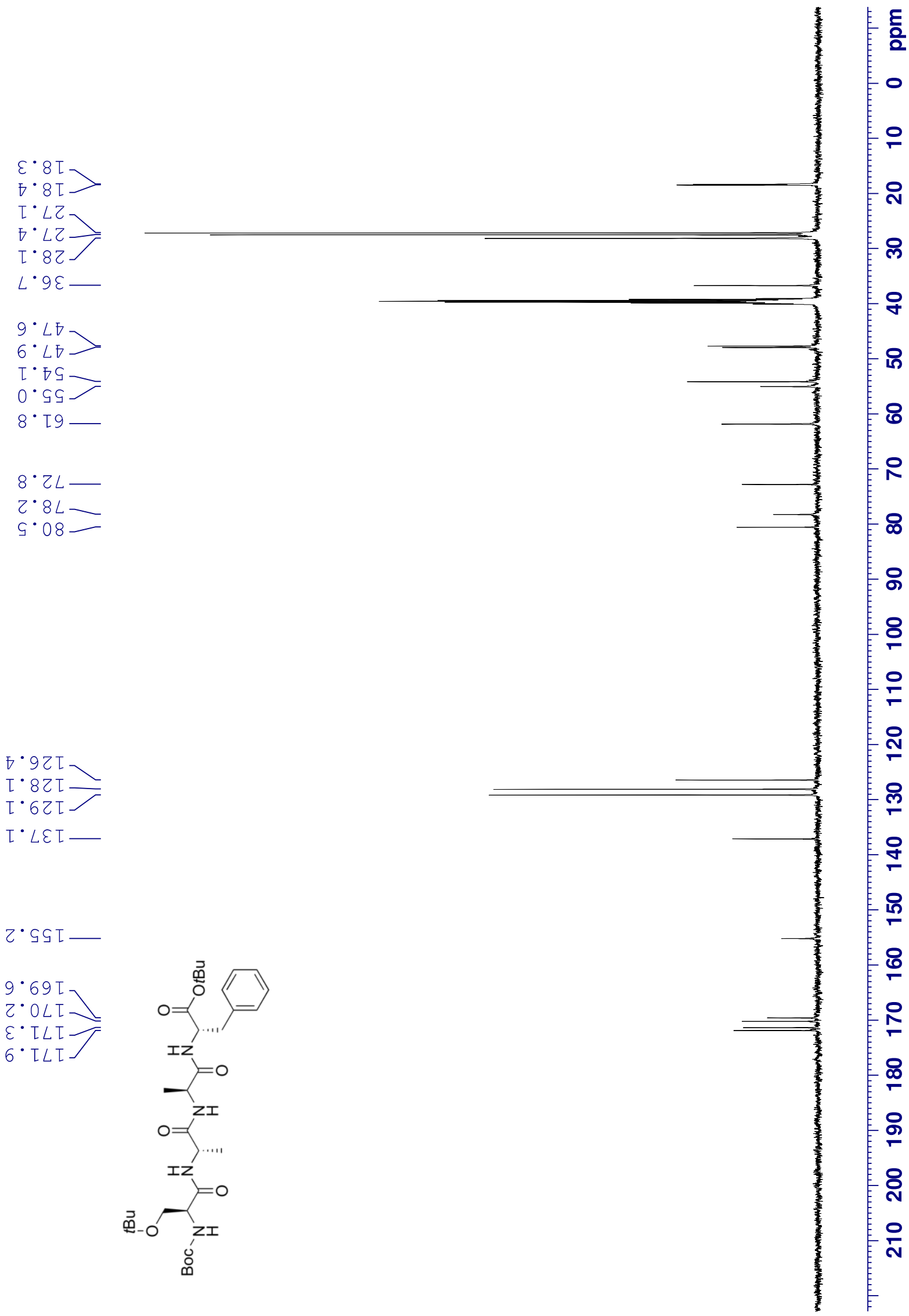

8

里

$\stackrel{\infty}{\infty}$

옹

:

은 
(S)-Methyl 1-((S)-2-((tert-butoxycarbonyl)amino)-3-phenylpropanoyl)pyrrolidine-2-carboxylate (BocL-Phe-L-Pro-OMe, KH_983) L,L-S10 - ${ }^{1}$ H NMR (500 MHz, DMSO-d 6 , mixture of rotamers)

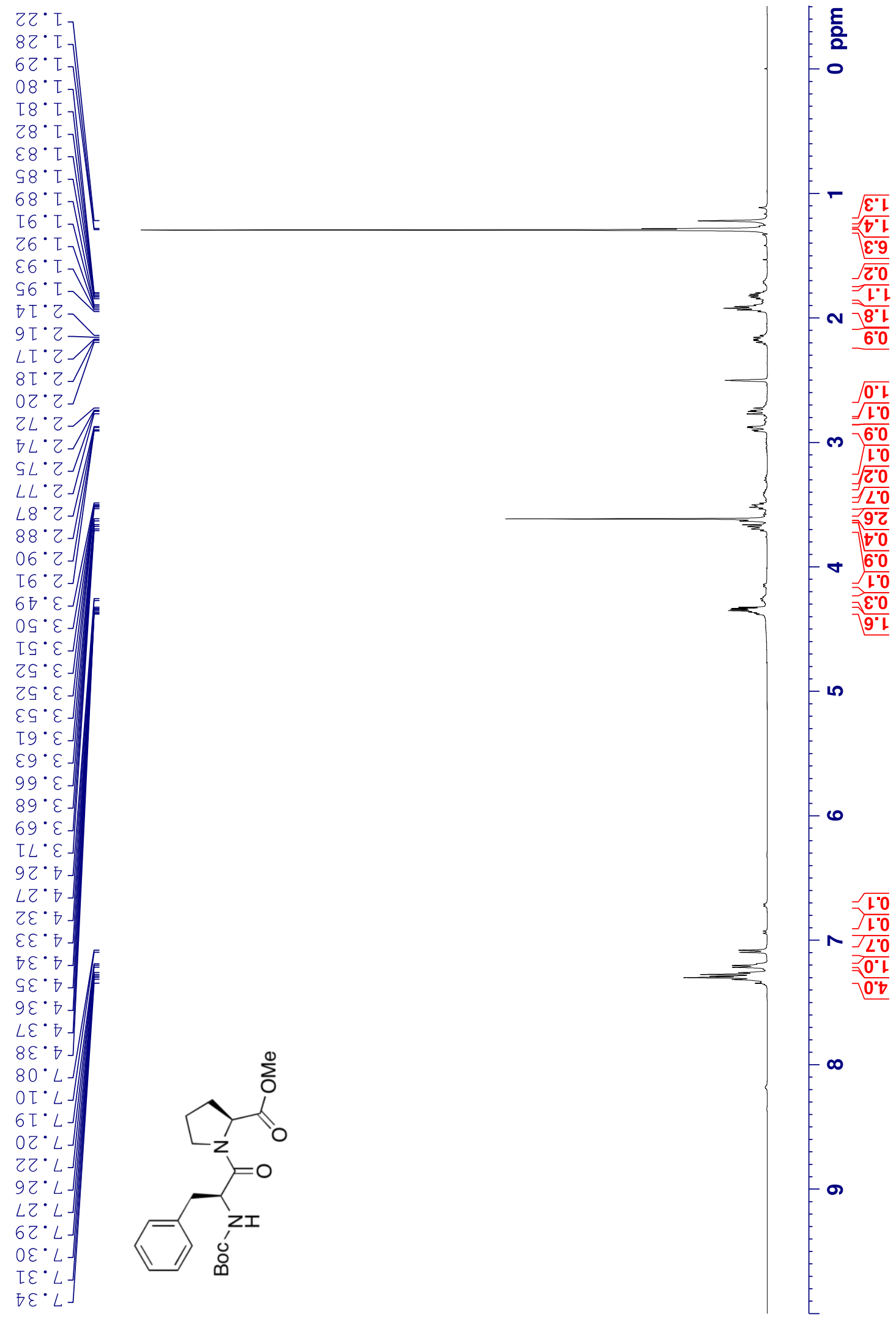


(S)-Methyl 1-((S)-2-((tert-butoxycarbonyl)amino)-3-phenylpropanoyl)pyrrolidine-2-carboxylate (BocL-Phe-L-Pro-OMe, KH_983) L,L-S10 - ${ }^{13}$ C NMR (126 MHz, DMSO-d , mixture of rotamers)
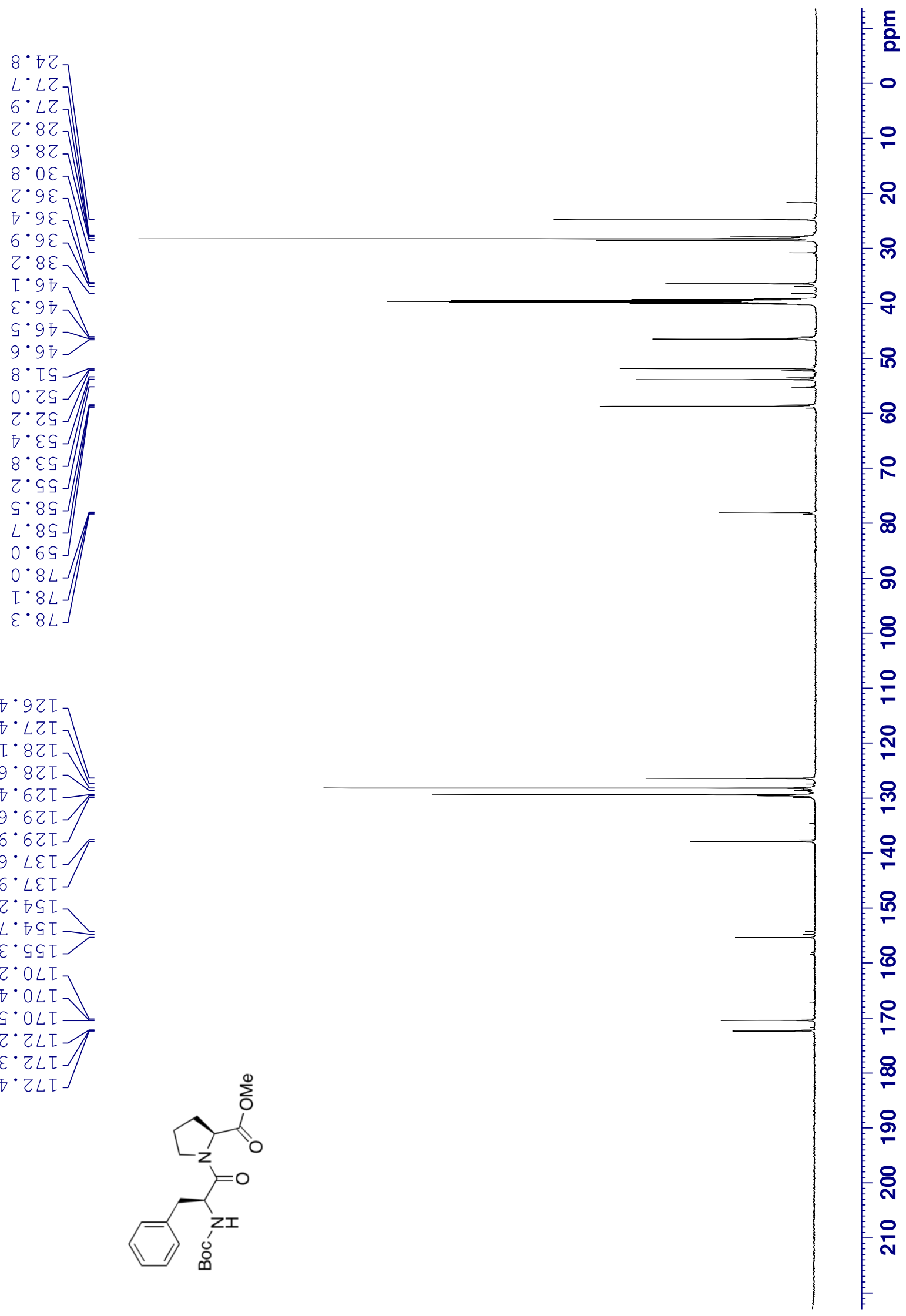
(S)-Methyl 1-((S)-2-((tert-butoxycarbonyl)amino)-3-phenylpropanoyl)pyrrolidine-2-carboxylate (BocL-Phe-L-Pro-OMe, KH_983) L,L-S10 - ${ }^{1} \mathbf{H}$ NMR (500 MHz, DMSO- $\boldsymbol{d}_{\boldsymbol{6}}, \mathbf{6 0}^{\circ} \mathbf{C}$, mixture of rotamers)
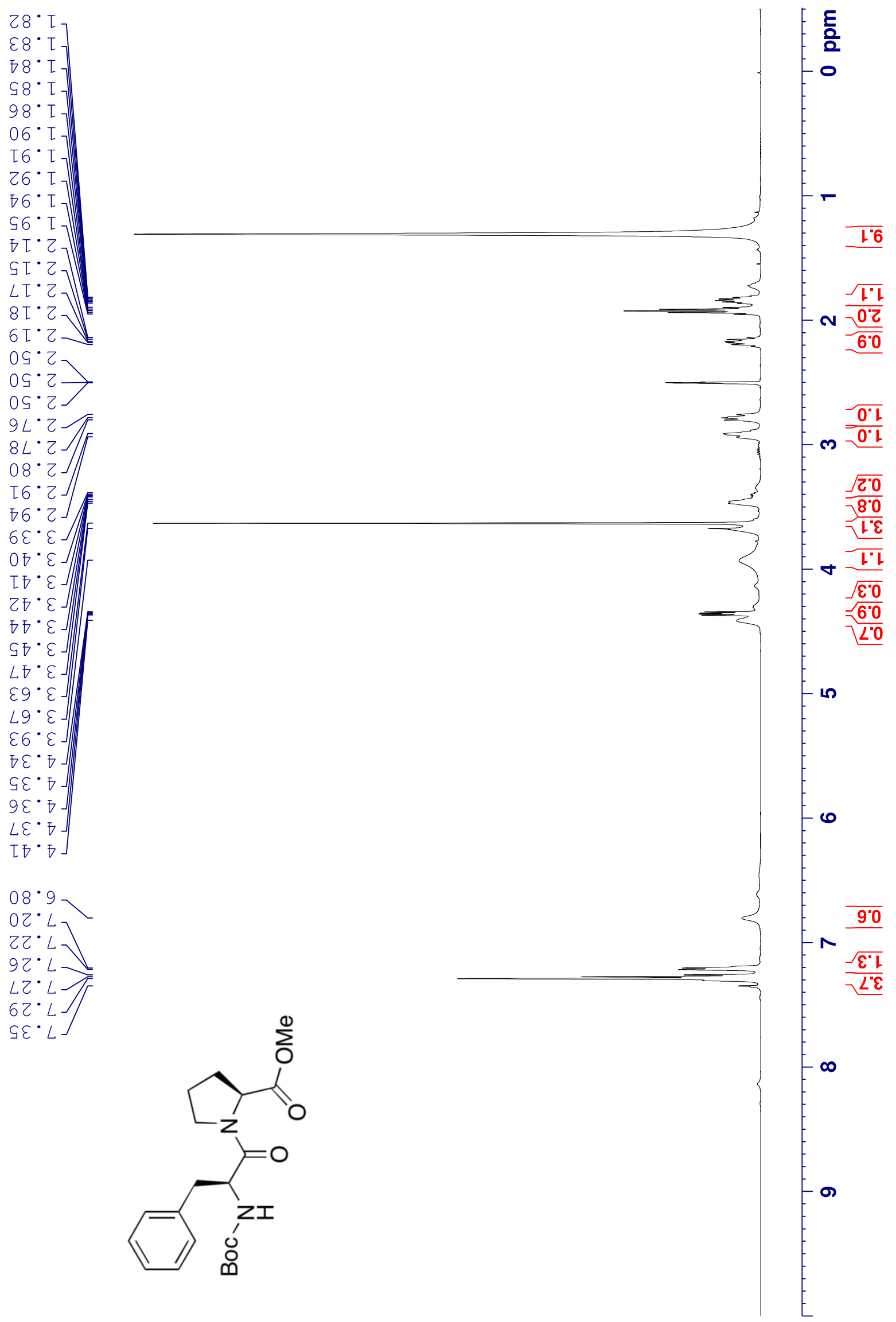
Methyl ((S)-2-((tert-butoxycarbonyl)amino)-3-(4-(((E)-prop-1-en-1-yl)oxy)phenyl)propanoyl)-Lphenylalanyl-L-prolinate (Boc-L-Tyr(All)-L-Phe-L-Pro-OMe, KH_985) L,L,L-S11 - ${ }^{\mathbf{1}}$ H NMR (500

\section{MHz, DMSO- $d_{6}$, mixture of rotamers)}

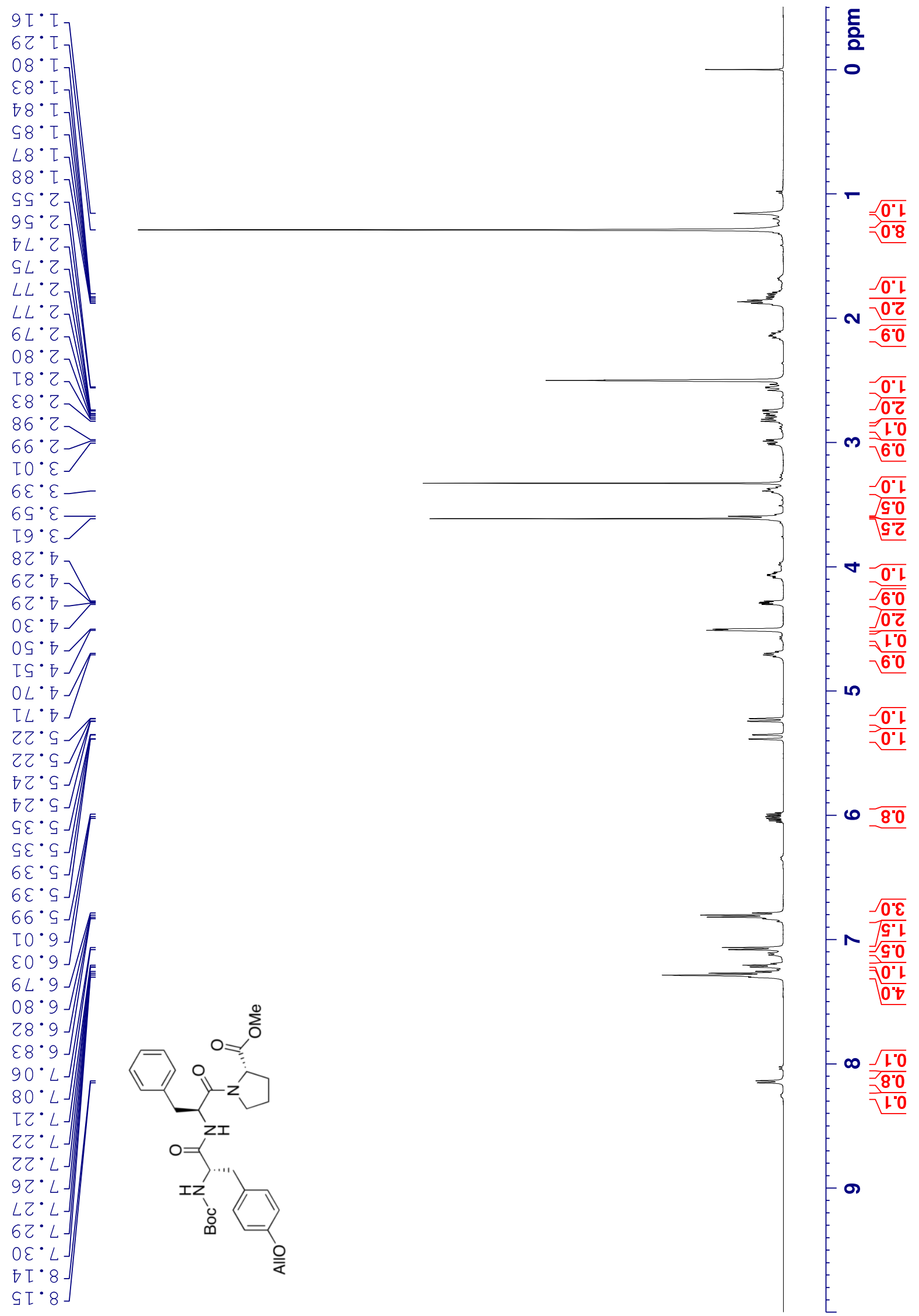


Methyl ((S)-2-((tert-butoxycarbonyl)amino)-3-(4-(((E)-prop-1-en-1-yl)oxy)phenyl)propanoyl)-Lphenylalanyl-L-prolinate (Boc-L-Tyr(All)-L-Phe-L-Pro-OMe, KH_985) L,L,L-S11 - ${ }^{13}$ C NMR (126 MHz, DMSO- $d_{6}$, mixture of rotamers)
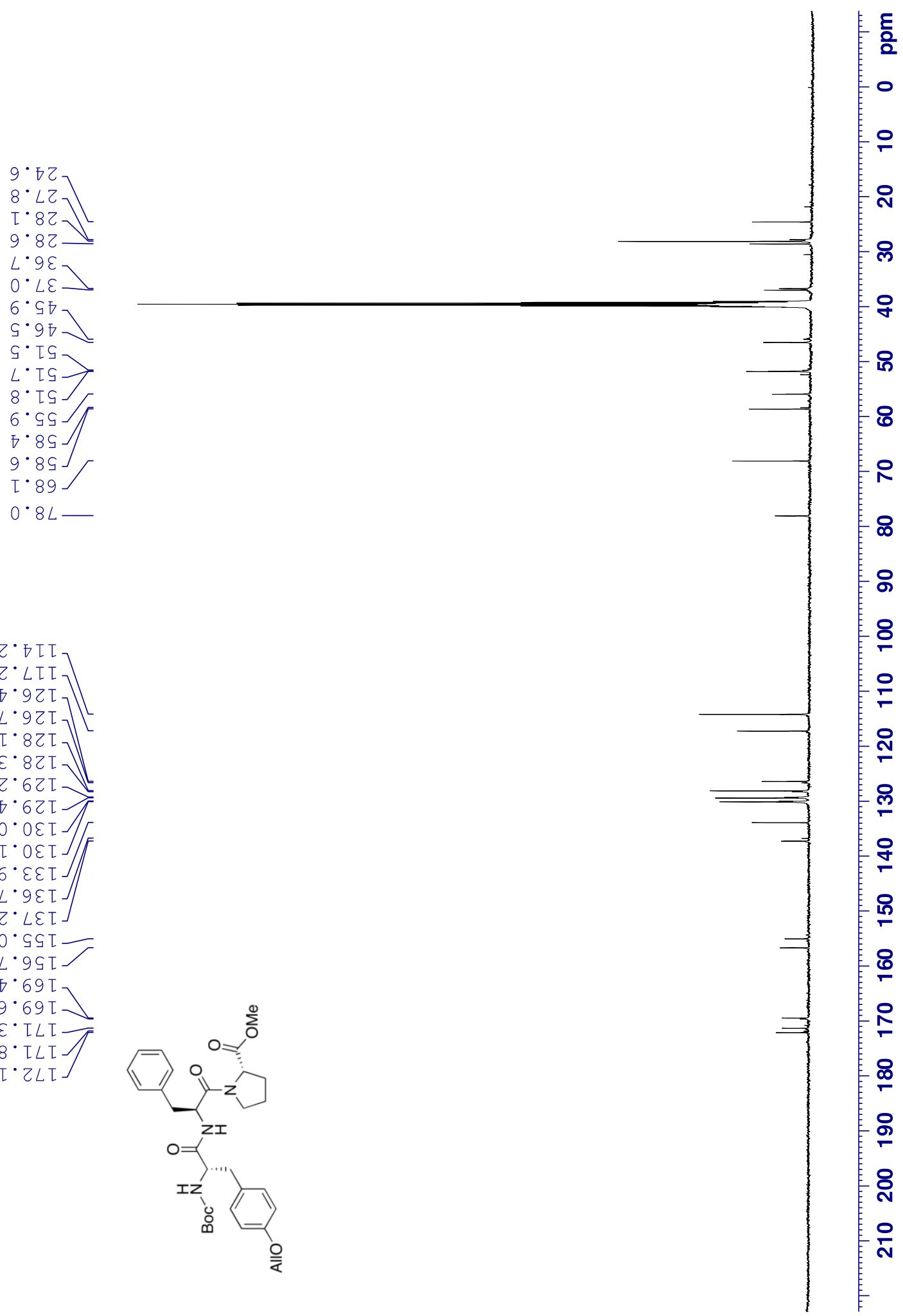
Methyl ((S)-2-((tert-butoxycarbonyl)amino)-3-(4-(((E)-prop-1-en-1-yl)oxy)phenyl)propanoyl)-Lphenylalanyl-L-prolinate (Boc-L-Tyr(All)-L-Phe-L-Pro-OMe, KH_985) L,L,L-S11 - ${ }^{\mathbf{1}}$ H NMR (500 MHz, DMSO- $d_{6}, 6^{\circ} \mathrm{C}$, mixture of rotamers)

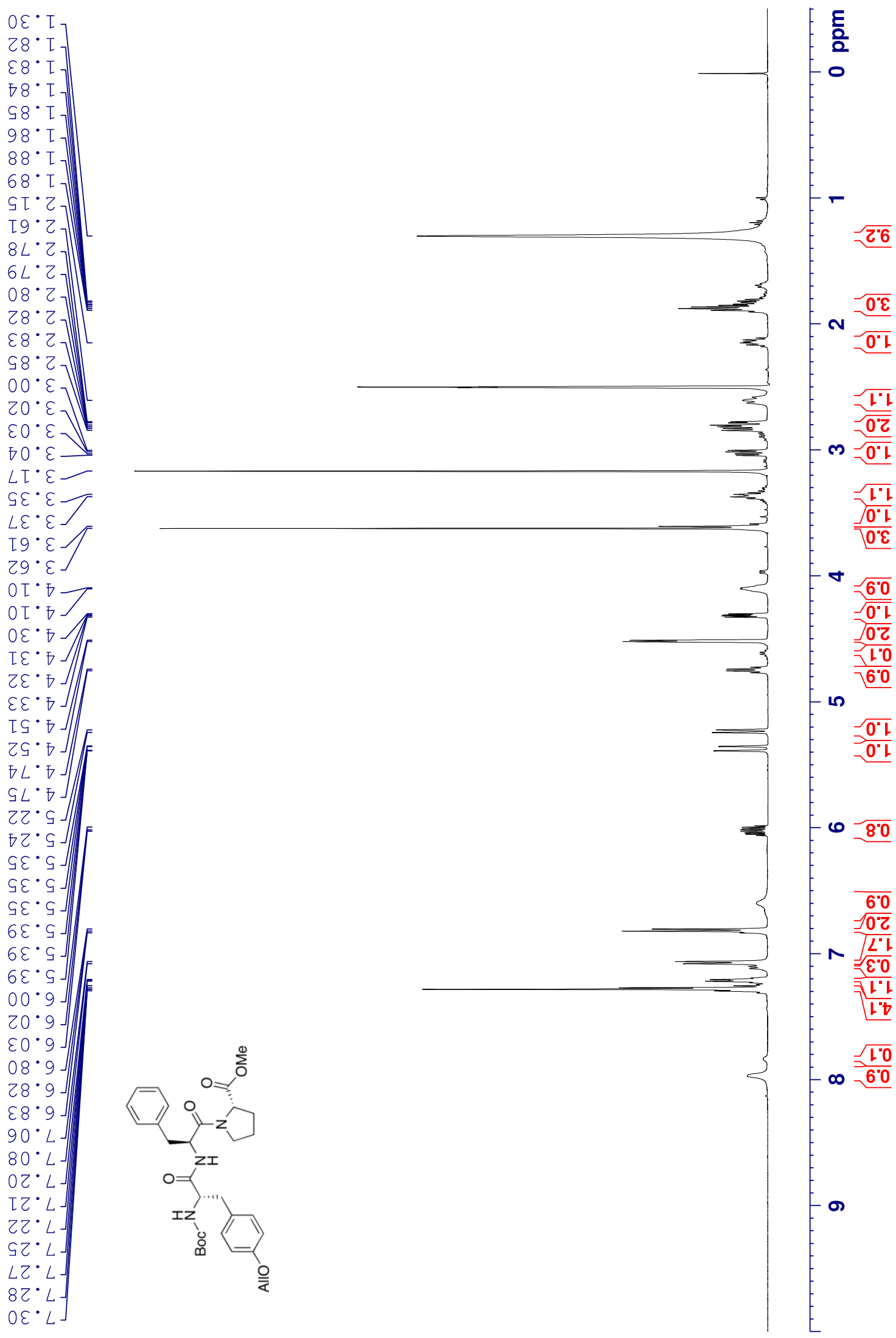


Methyl ((S)-2-((2S,3R)-2-((tert-butoxycarbonyl)amino)-3-methylpentanamido)-3-(4-(((E)-prop-1-en-1yl)oxy)phenyl)propanoyl)-L-phenylalanyl-L-prolinate

(Boc-L-Ile-L-Tyr(All)-L-Phe-L-Pro-OMe,

\section{KH_987) L,L,L,L-S12 - ${ }^{1} \mathrm{H}$ NMR (500 MHz, DMSO-d $\boldsymbol{d}_{6}$, mixture of rotamers)}
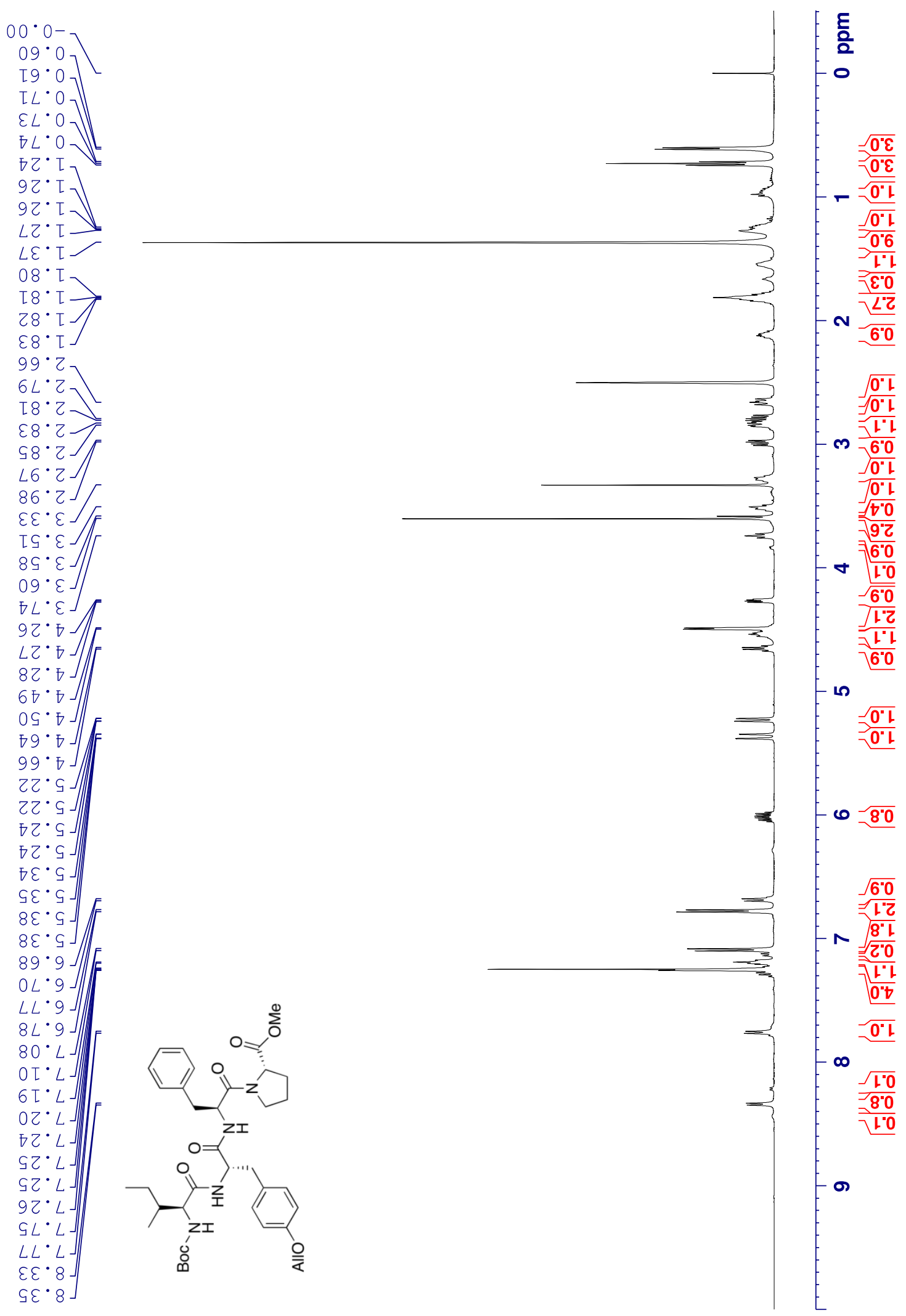
Methyl ((S)-2-((2S,3R)-2-((tert-butoxycarbonyl)amino)-3-methylpentanamido)-3-(4-(((E)-prop-1-en-1yl)oxy)phenyl)propanoyl)-L-phenylalanyl-L-prolinate (Boc-L-Ile-L-Tyr(All)-L-Phe-L-Pro-OMe, KH_987) L,L,L,L-S12 - ${ }^{13}$ C NMR (126 MHz, DMSO- $\boldsymbol{d}_{\boldsymbol{\sigma}}$, mixture of rotamers)
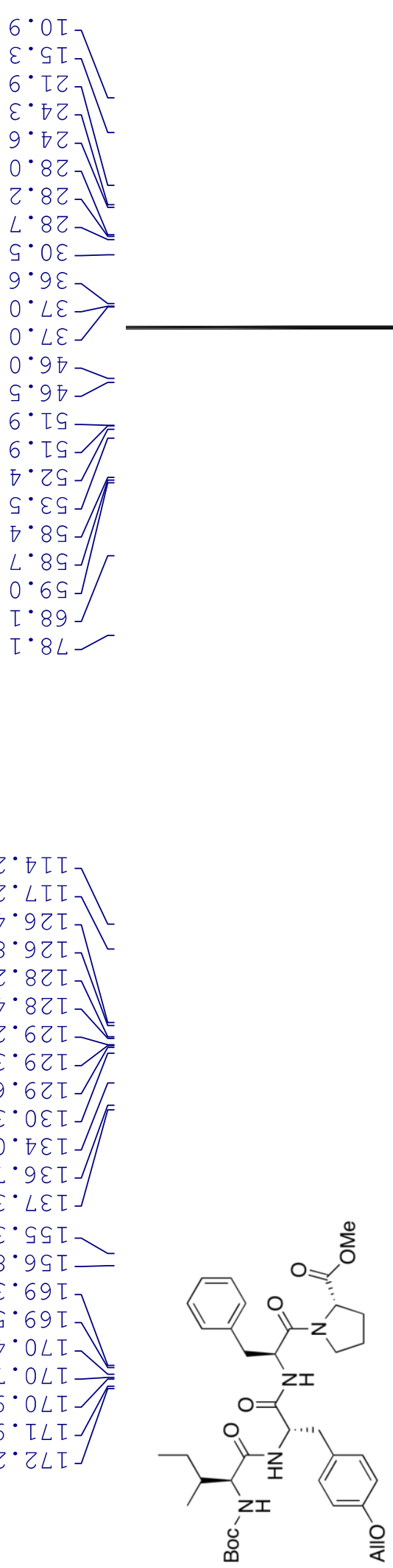

음

오

으

옴

10

8

옴

요

옹 
Methyl ((S)-2-((2S,3R)-2-((tert-butoxycarbonyl)amino)-3-methylpentanamido)-3-(4-(((E)-prop-1-en-1yl)oxy)phenyl)propanoyl)-L-phenylalanyl-L-prolinate (Boc-L-Ile-L-Tyr(All)-L-Phe-L-Pro-OMe, KH_987) L,L,L,L-S12 - ${ }^{1} \mathrm{H}$ NMR (500 MHz, DMSO- $d_{6}, \mathbf{6 0}^{\circ} \mathrm{C}$, mixture of rotamers)
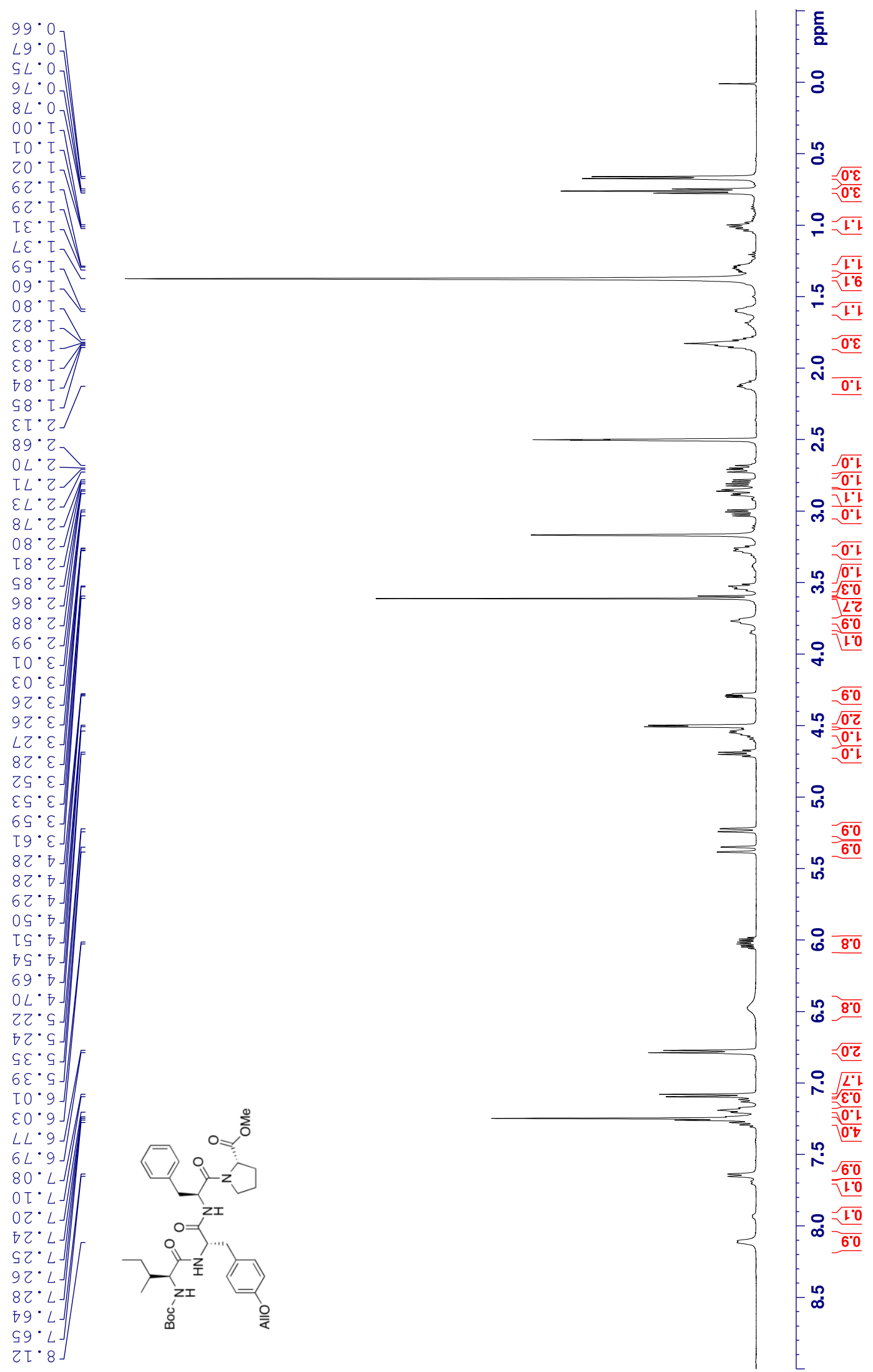
((S)-2-((2S,3R)-2-((tert-butoxycarbonyl)amino)-3-methylpentanamido)-3-(4-(((E)-prop-1-en-1yl)oxy)phenyl)propanoyl)-L-phenyl-alanyl-L-proline (Boc-L-Ile-L-Tyr(All)-L-Phe-L-Pro-OH, KH_988)

\section{L,L,L,L-S13 - ${ }^{1} \mathrm{H}$ NMR (500 MHz, DMSO- $d_{6}$, mixture of rotamers)}

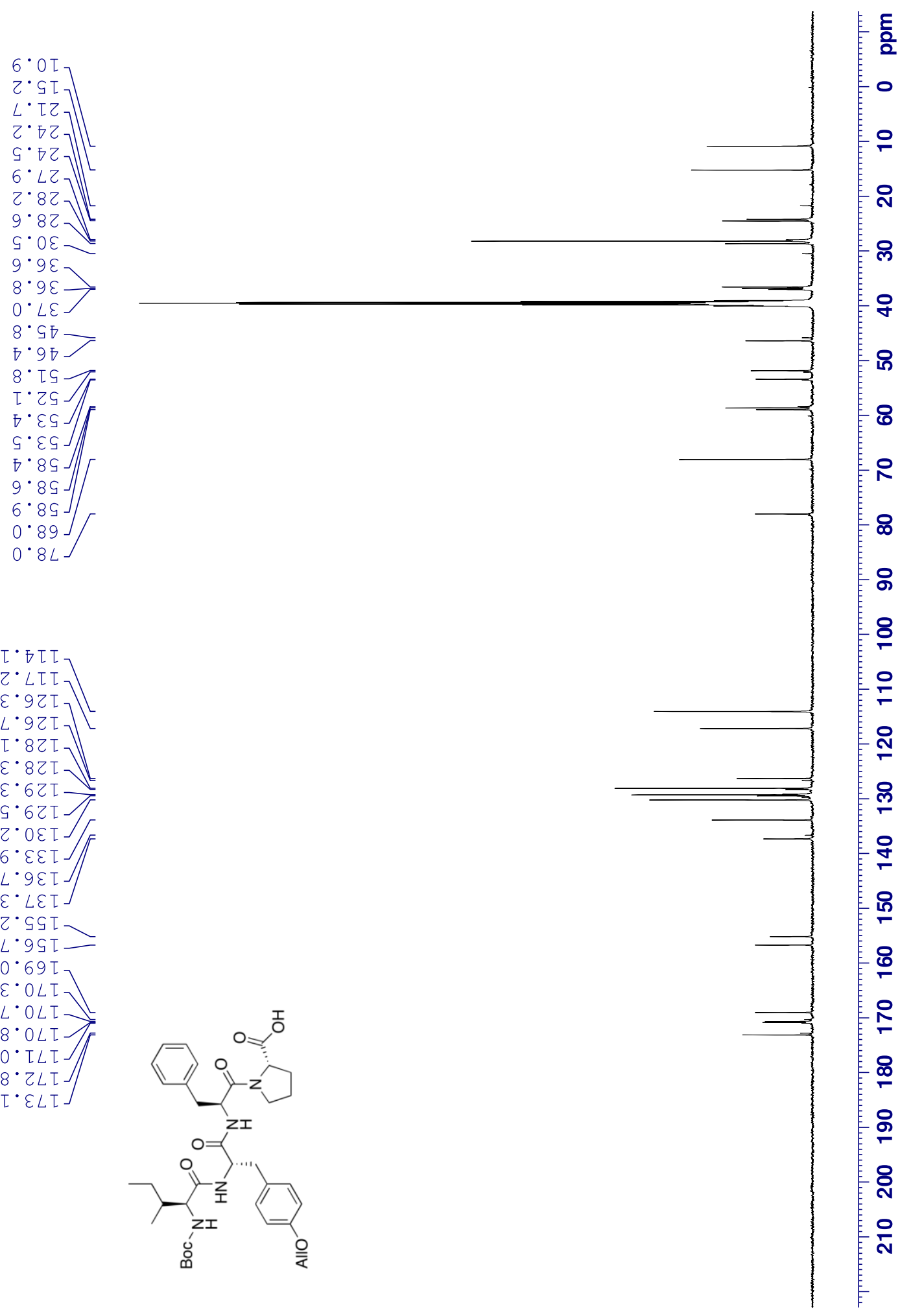


((S)-2-((2S,3R)-2-((tert-butoxycarbonyl)amino)-3-methylpentanamido)-3-(4-(((E)-prop-1-en-1yl)oxy)phenyl)propanoyl)-L-phenyl-alanyl-L-proline (Boc-L-Ile-L-Tyr(All)-L-Phe-L-Pro-OH, KH_988) L,L,L,L-S13 - ${ }^{13} \mathrm{C}$ NMR (126 MHz, DMSO- $d_{6}$, mixture of rotamers)

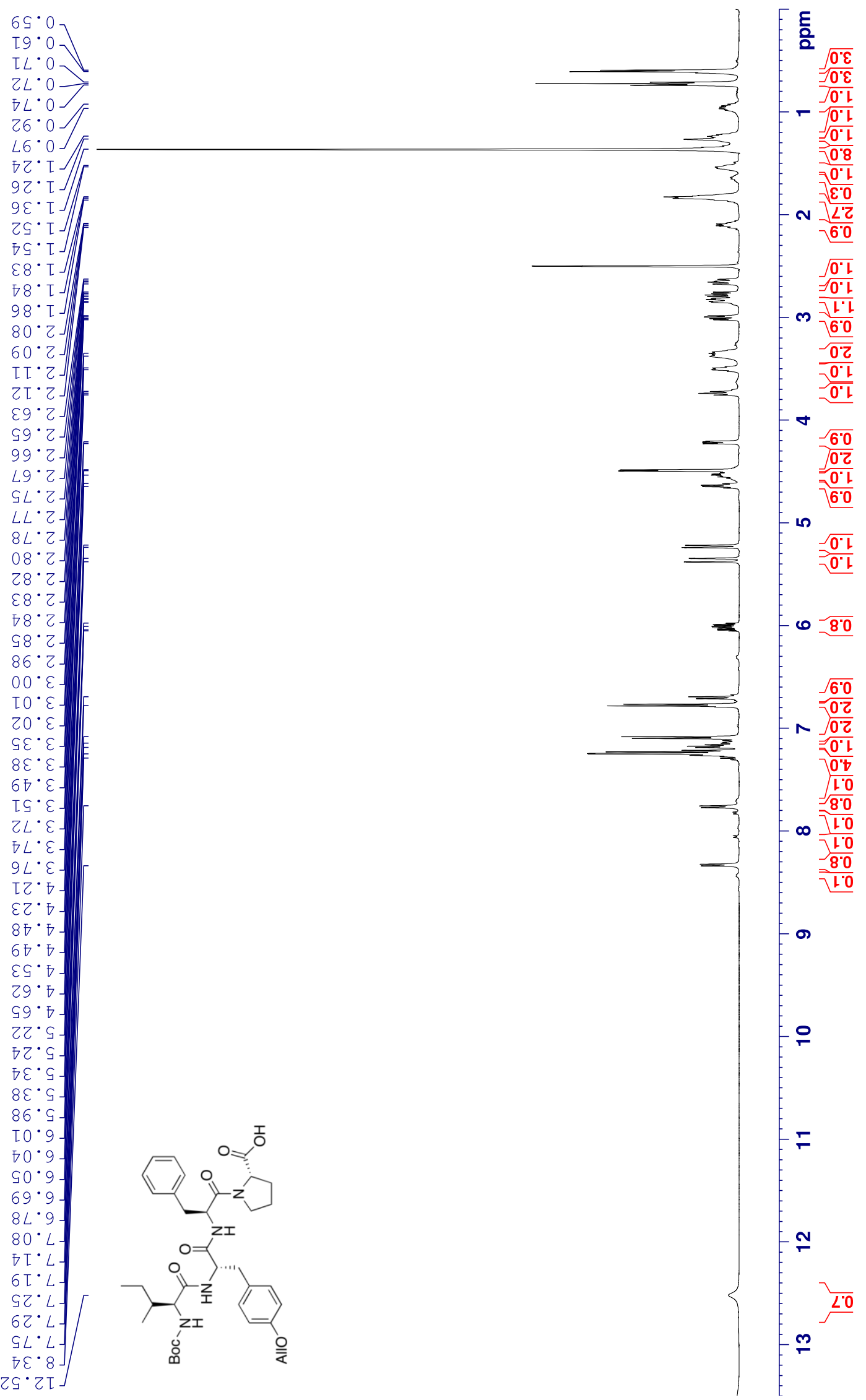


((S)-2-((2S,3R)-2-((tert-butoxycarbonyl)amino)-3-methylpentanamido)-3-(4-(((E)-prop-1-en-1yl)oxy)phenyl)propanoyl)-L-phenyl-alanyl-L-proline (Boc-L-Ile-L-Tyr(All)-L-Phe-L-Pro-OH, KH_988) L,L,L,L-S13 - ${ }^{1} \mathrm{H}$ NMR (500 MHz, DMSO-d ${ }_{6}, 6^{\circ} \mathrm{C}$, mixture of rotamers)
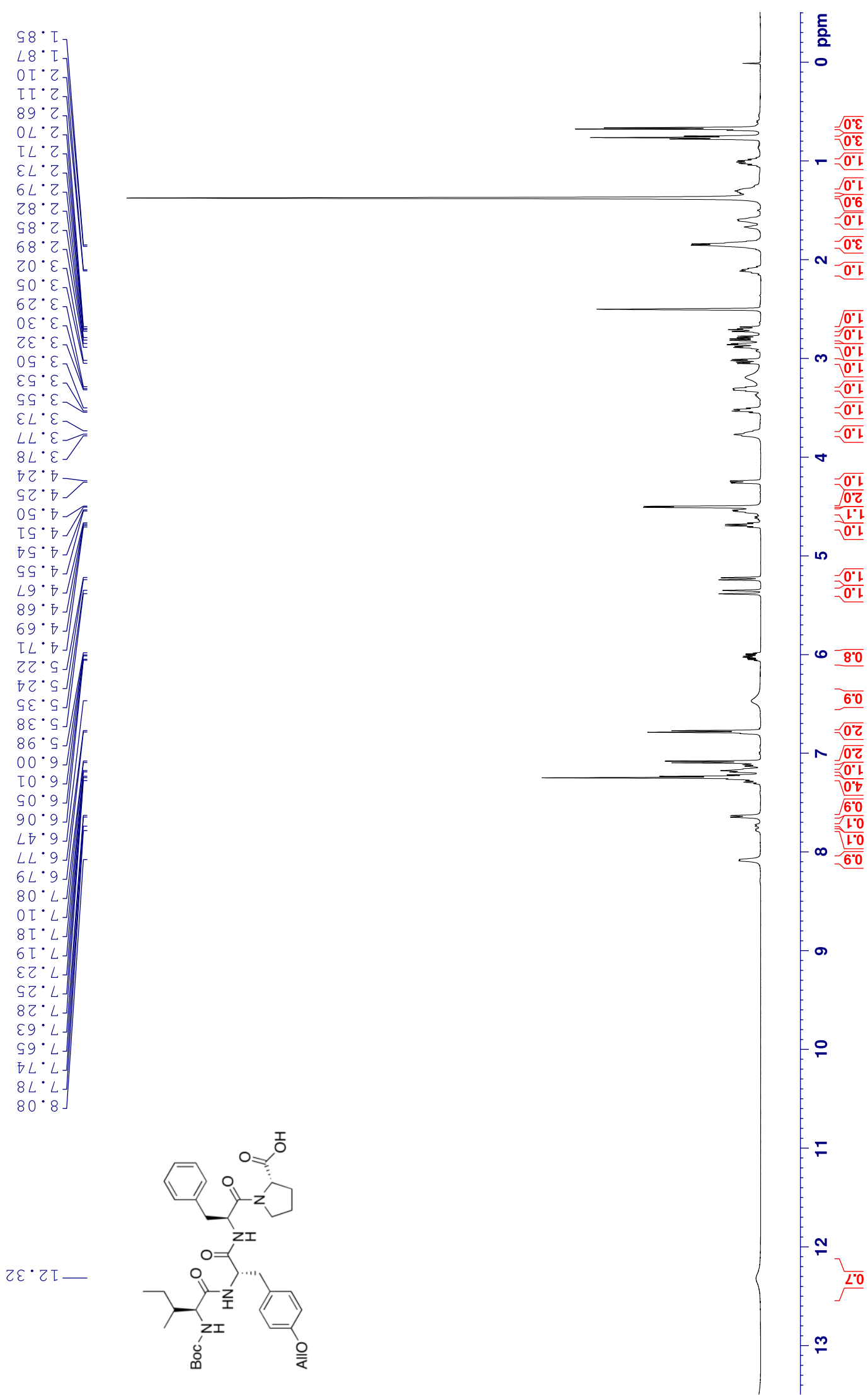
tert-Butyl 2-((2S)-2-(1-(tert-butoxycarbonyl)pyrrolidine-2-carboxamido)-3-

phenylpropanamido)nicotinate (Boc-Pro-Phe-NH- $t$ Bu-nic, KH_1214) L,L-S14 - ${ }^{\mathbf{1}}$ H NMR (500 MHz,

$\mathrm{CDCl}_{3}$,

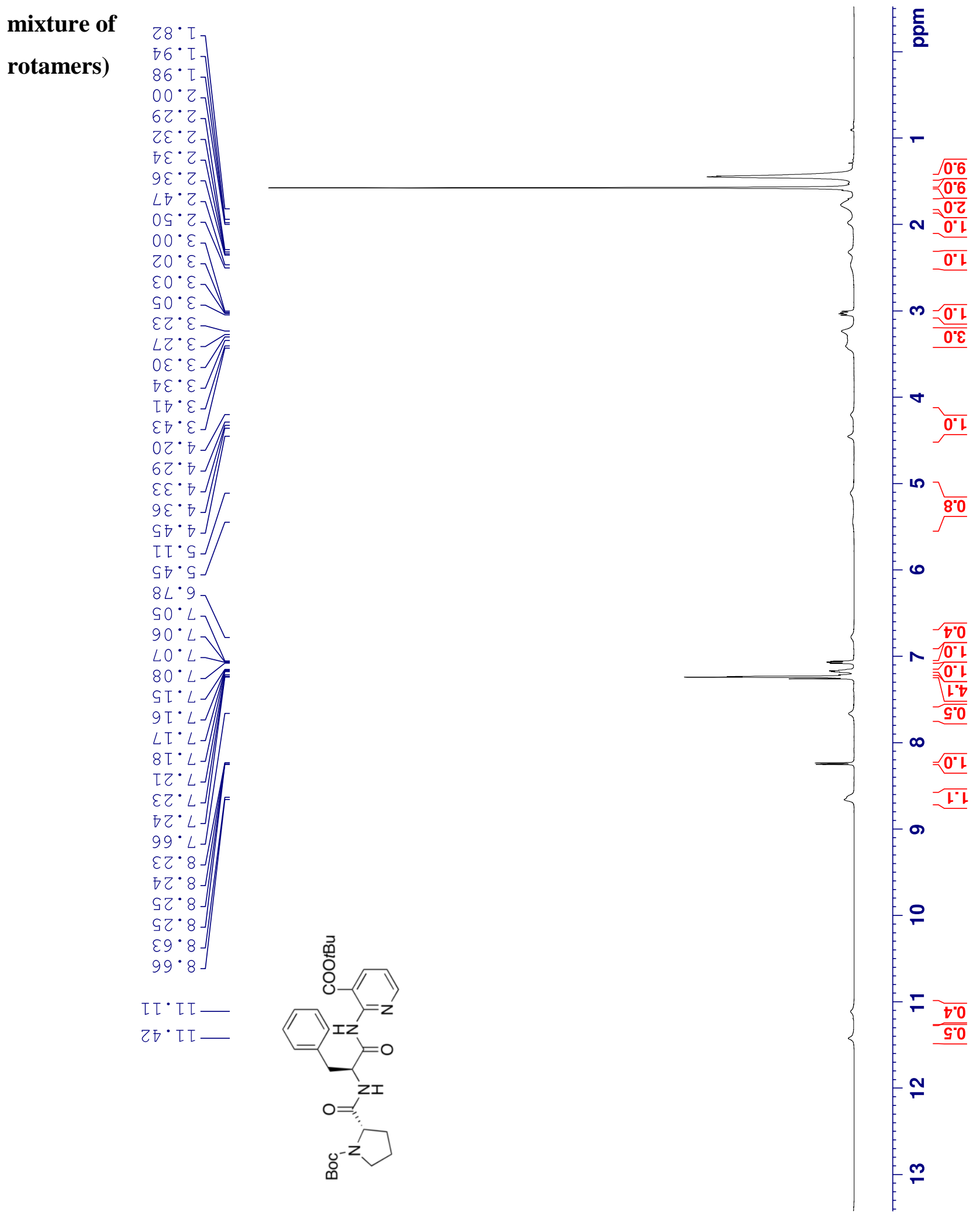


tert-Butyl 2-((2S)-2-(1-(tert-butoxycarbonyl)pyrrolidine-2-carboxamido)-3-

phenylpropanamido)nicotinate (Boc-Pro-Phe-NH-tBu-nic, KH_1214) L,L-S14 - ${ }^{\mathbf{1 3}}$ C NMR (500 MHz, $\mathrm{CDCl}_{3}$, mixture of rotamers)

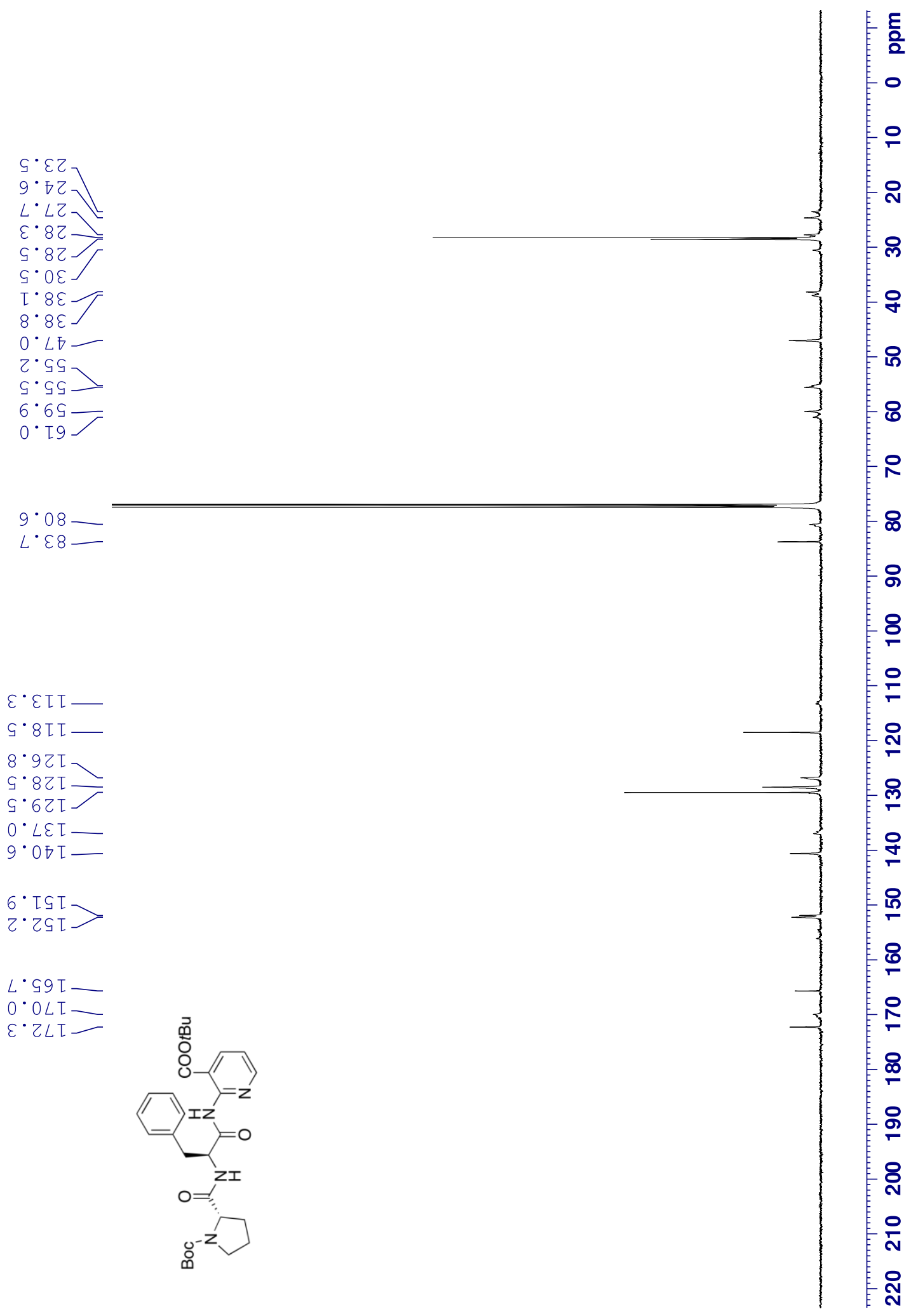


tert-Butyl 2-((2S)-2-(1-(tert-butoxycarbonyl)pyrrolidine-2-carboxamido)-3-

phenylpropanamido)nicotinate (Boc-Pro-Phe-NH- $t$ Bu-nic, KH_1214) L,L-S14 - ${ }^{\mathbf{1}}$ H NMR (500 MHz, DMSO- $\left.d_{6}, 8^{\circ} \mathrm{C}\right)$
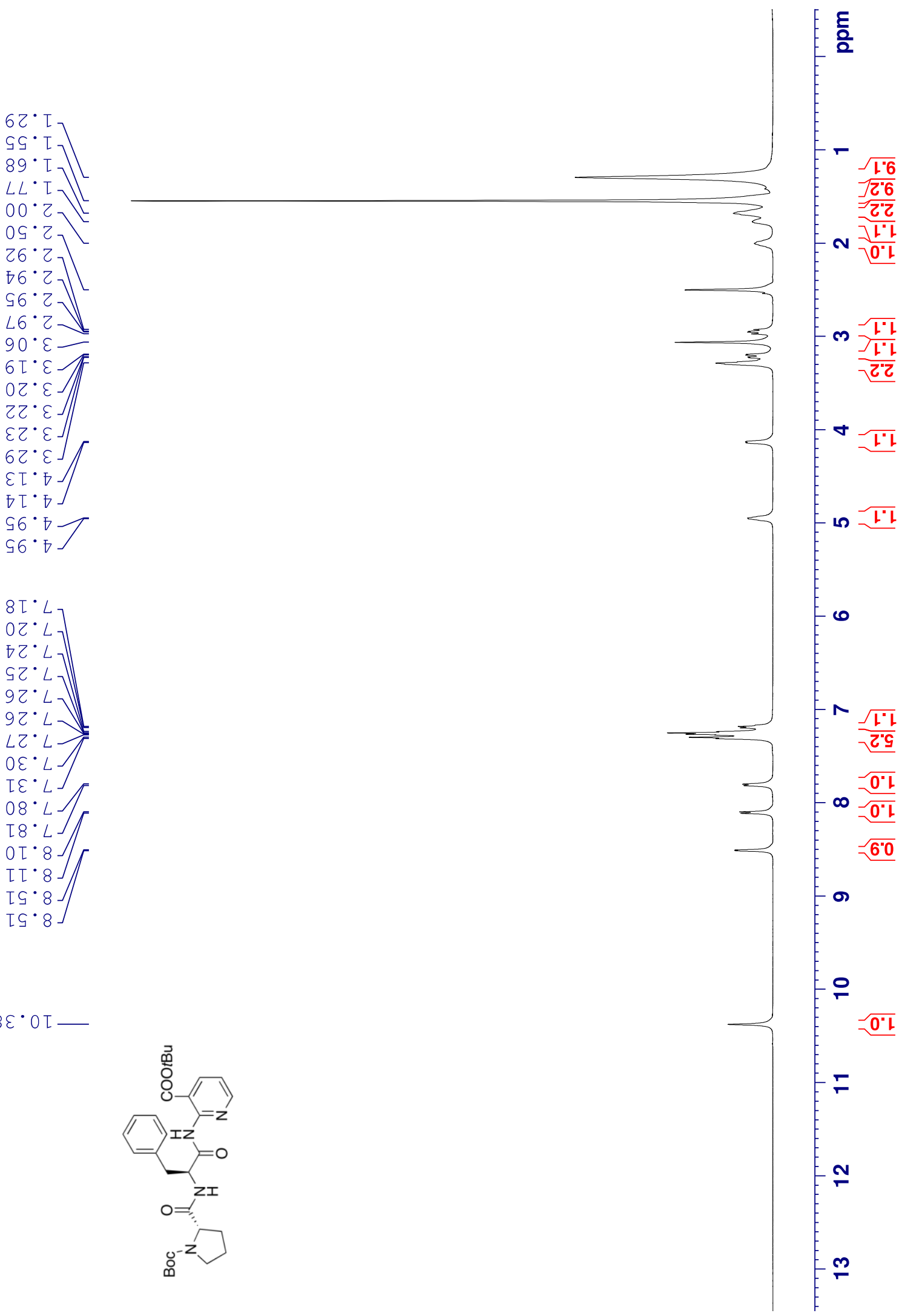
tert-Butyl 2-((S)-2-((S)-1-((2S,3S)-2-((tert-butoxycarbonyl)amino)-3-methylpentanoyl)pyrrolidine-2carboxamido)-3-phenylpropan-amido)nicotinate (Boc-L-Ile-L-Pro-L-Phe-NH-tBu-nic, KH_1039) L,L,LS15 - ${ }^{1}$ H NMR (500 MHz, DMSO-d $)^{\text {) }}$

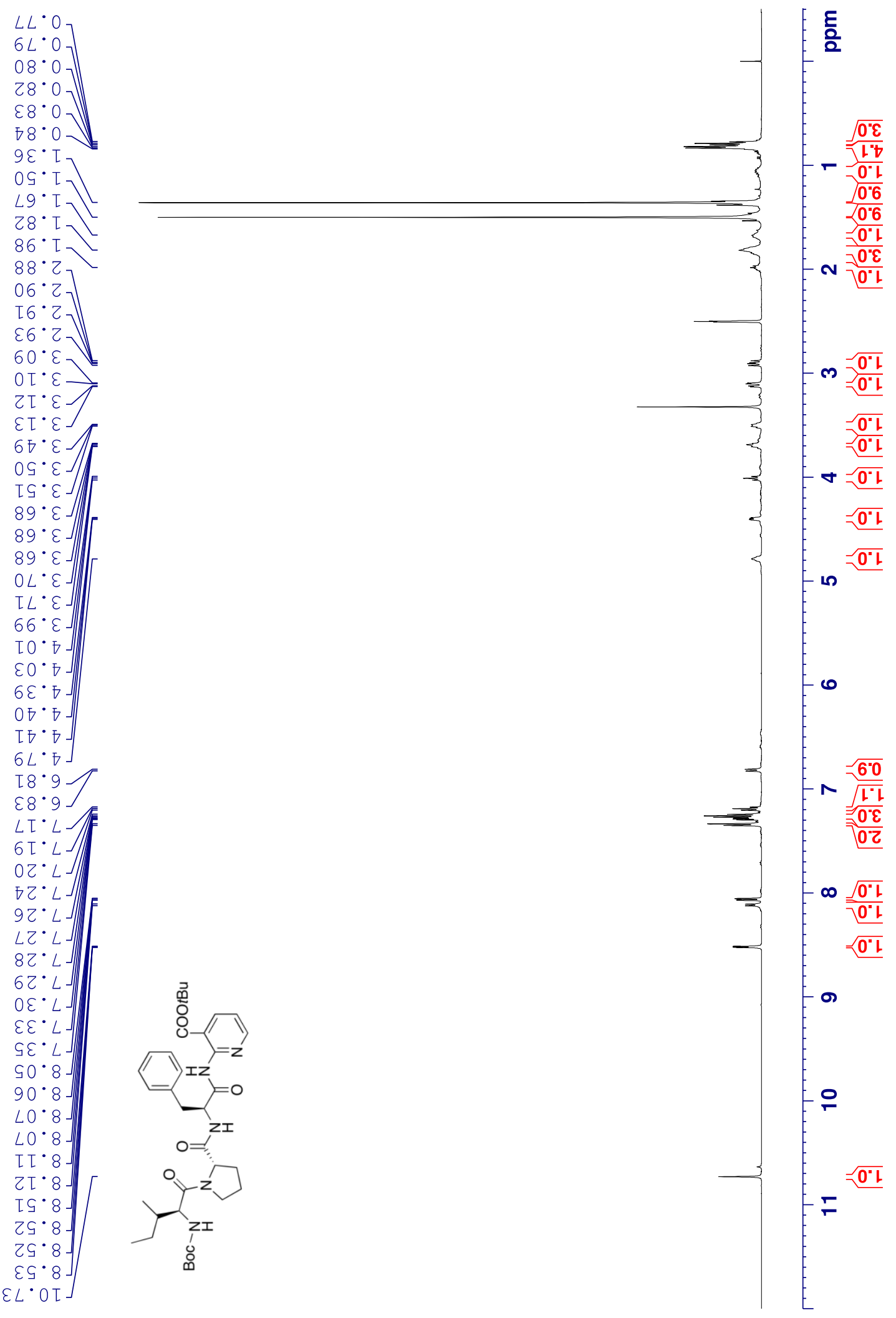


tert-Butyl 2-((S)-2-((S)-1-((2S,3S)-2-((tert-butoxycarbonyl)amino)-3-methylpentanoyl)pyrrolidine-2carboxamido)-3-phenylpropan-amido)nicotinate (Boc-L-Ile-L-Pro-L-Phe-NH-tBu-nic, KH_1039) L,L,LS15 - ${ }^{13}$ C NMR (126 MHz, DMSO-d 6$)$

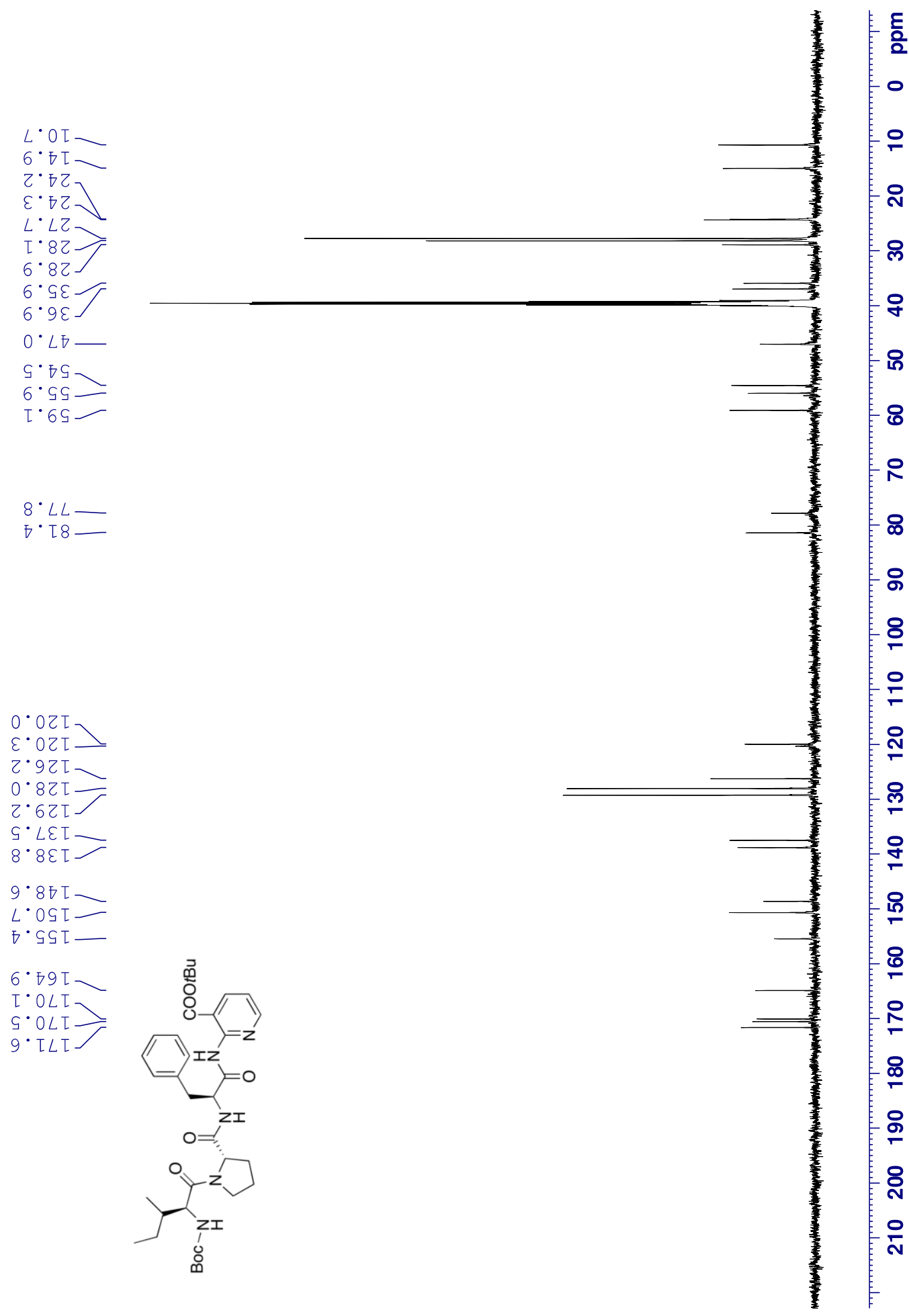


tert-Butyl 2-((S)-2-((S)-1-(((S)-2-((2S,3R)-2-((tert-butoxycarbonyl)amino)-3-methylpentanamido)-3-(4(((E)-prop-1-en-1-yl)oxy)phenyl) propanoyl)-L-phenylalanyl-L-prolyl-L-isoleucyl)pyrrolidine-2carboxamido)-3-phenylpropanamido)nicotinate (Boc-L-Ile-L-Tyr(All)-L-Phe-L-Pro-L-Ile-L-Pro-L-PheNH- $t$ Bu-nic, KH_1042) L,L,L,L,L,L,L-S16 - ${ }^{1}$ H NMR (500 MHz, DMSO-d $)^{\text {) }}$

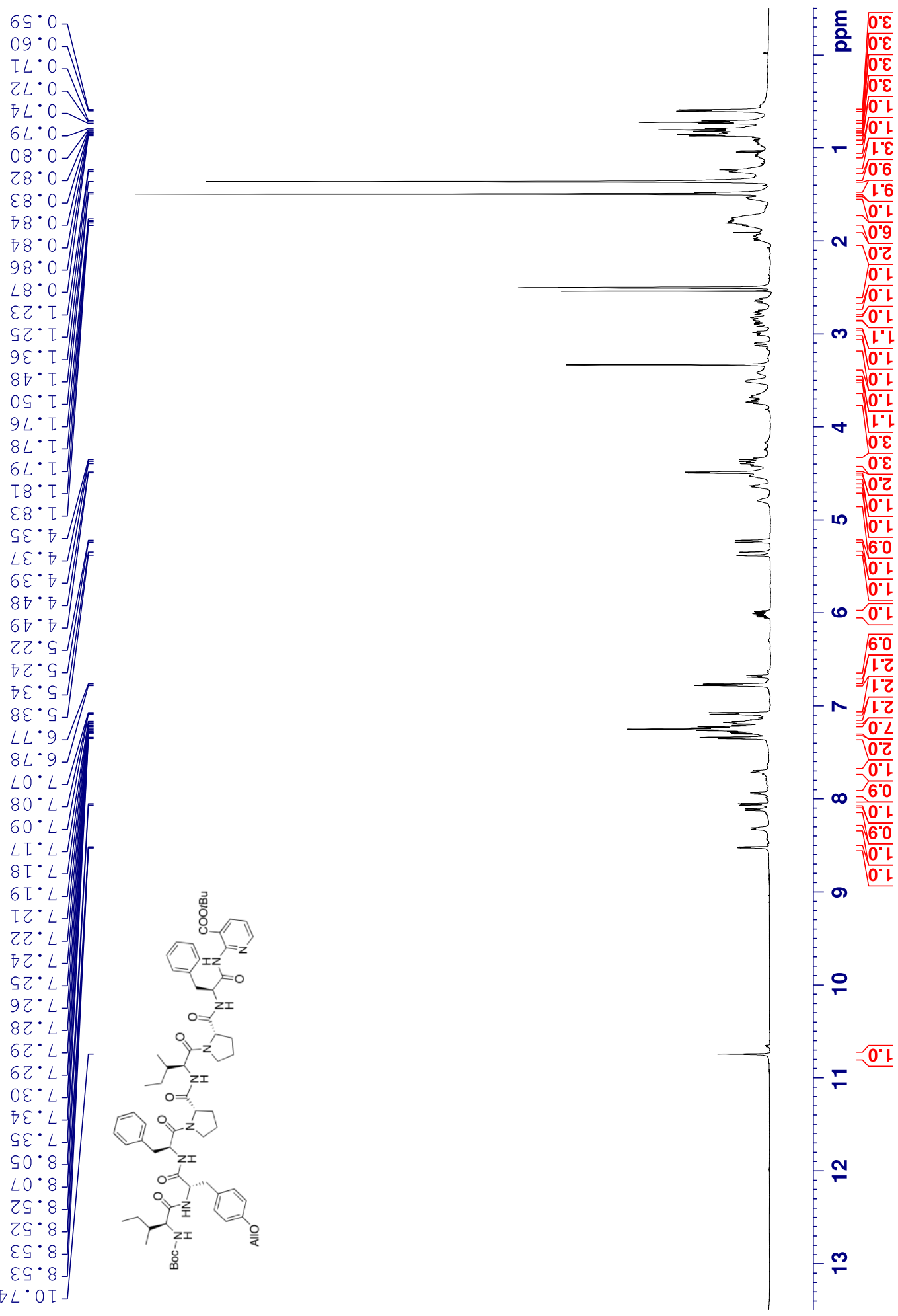


tert-Butyl 2-((S)-2-((S)-1-(((S)-2-((2S,3R)-2-((tert-butoxycarbonyl)amino)-3-methylpentanamido)-3-(4(((E)-prop-1-en-1-yl)oxy)phenyl) propanoyl)-L-phenylalanyl-L-prolyl-L-isoleucyl)pyrrolidine-2carboxamido)-3-phenylpropanamido)nicotinate (Boc-L-Ile-L-Tyr(All)-L-Phe-L-Pro-L-Ile-L-Pro-L-Phe$\mathrm{NH}-t \mathrm{Bu}-\mathrm{nic}$,

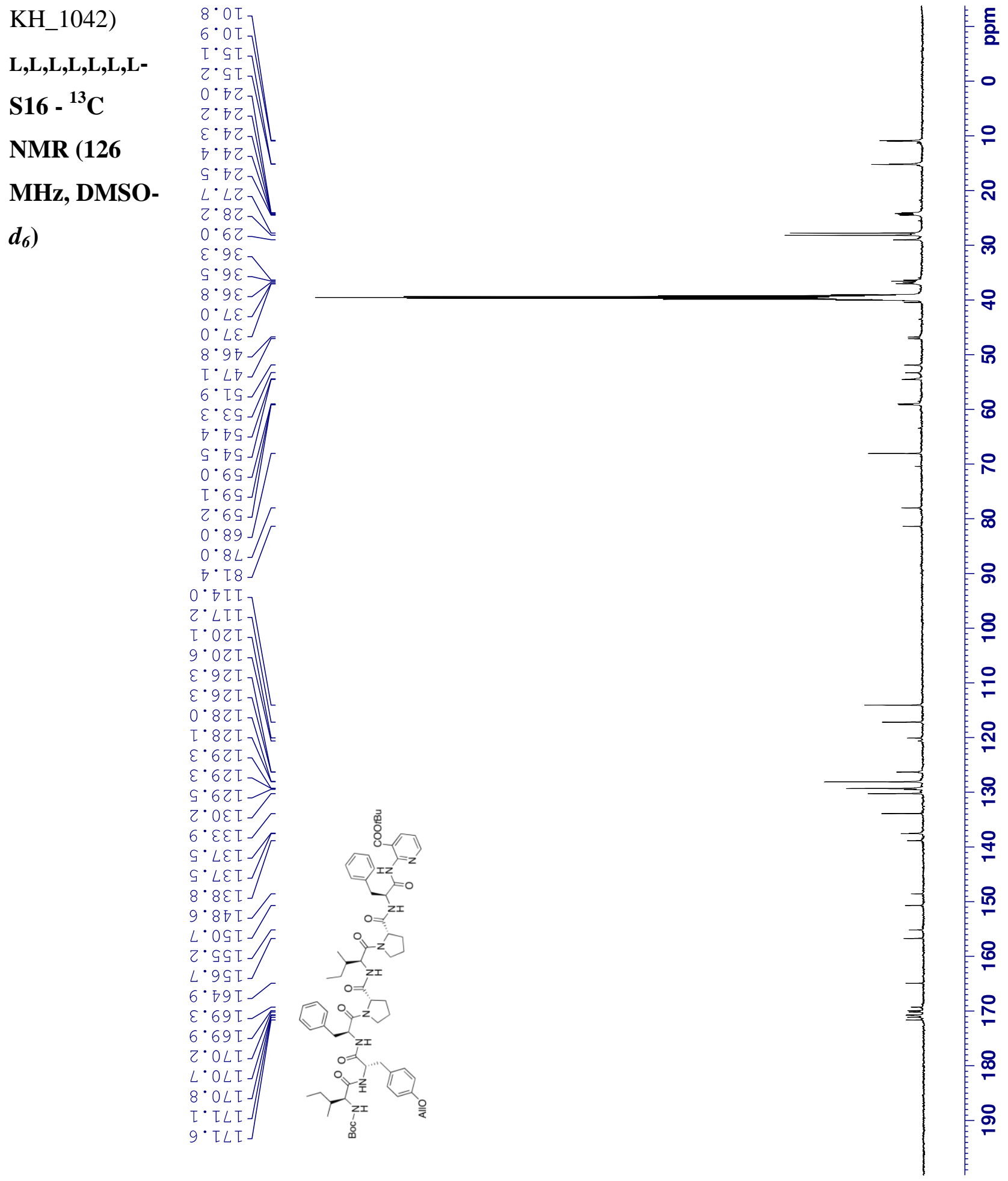


tert-Butyl 2-((S)-2-((S)-1-(((S)-2-((2S,3R)-2-amino-3-methylpentanamido)-3-(4-(((E)-prop-1-en-1yl)oxy)phenyl)propanoyl)-L-phenyl-alanyl-L-prolyl-L-isoleucyl)pyrrolidine-2-carboxamido)-3phenylpropanamido)nicotinate hydrochloride acid salt (H-L-Ile-L-Tyr(All)-L-Phe-L-Pro-L-Ile-L-Pro-LPhe-NH- $t$ Bu-nic.HCl, KH_1046) L,L,L,L,L,L,L-17.HCI - ${ }^{1}$ H NMR (500 MHz, DMSO-d 6 )

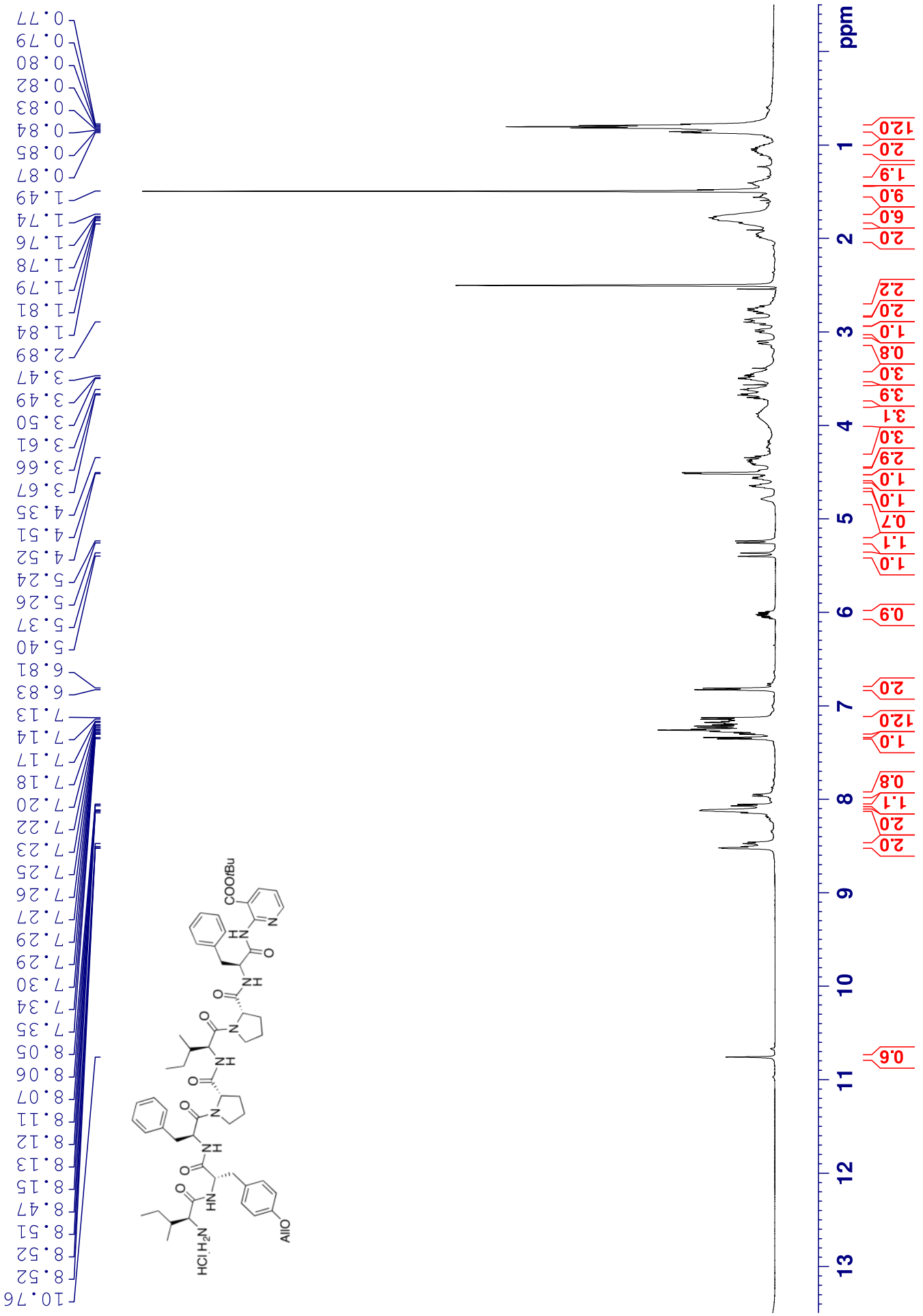


tert-Butyl 2-((S)-2-((S)-1-(((S)-2-((2S,3R)-2-amino-3-methylpentanamido)-3-(4-(((E)-prop-1-en-1yl)oxy)phenyl)propanoyl)-L-phenyl-alanyl-L-prolyl-L-isoleucyl)pyrrolidine-2-carboxamido)-3phenylpropanamido)nicotinate hydrochloride acid salt (H-L-Ile-L-Tyr(All)-L-Phe-L-Pro-L-Ile-L-Pro-LPhe-NH- $t$ Bu-nic.HCl, KH_1046) L,L,L,L,L,L,L-17.HCI - ${ }^{13}$ C NMR (126 MHz, DMSO-d 6 )

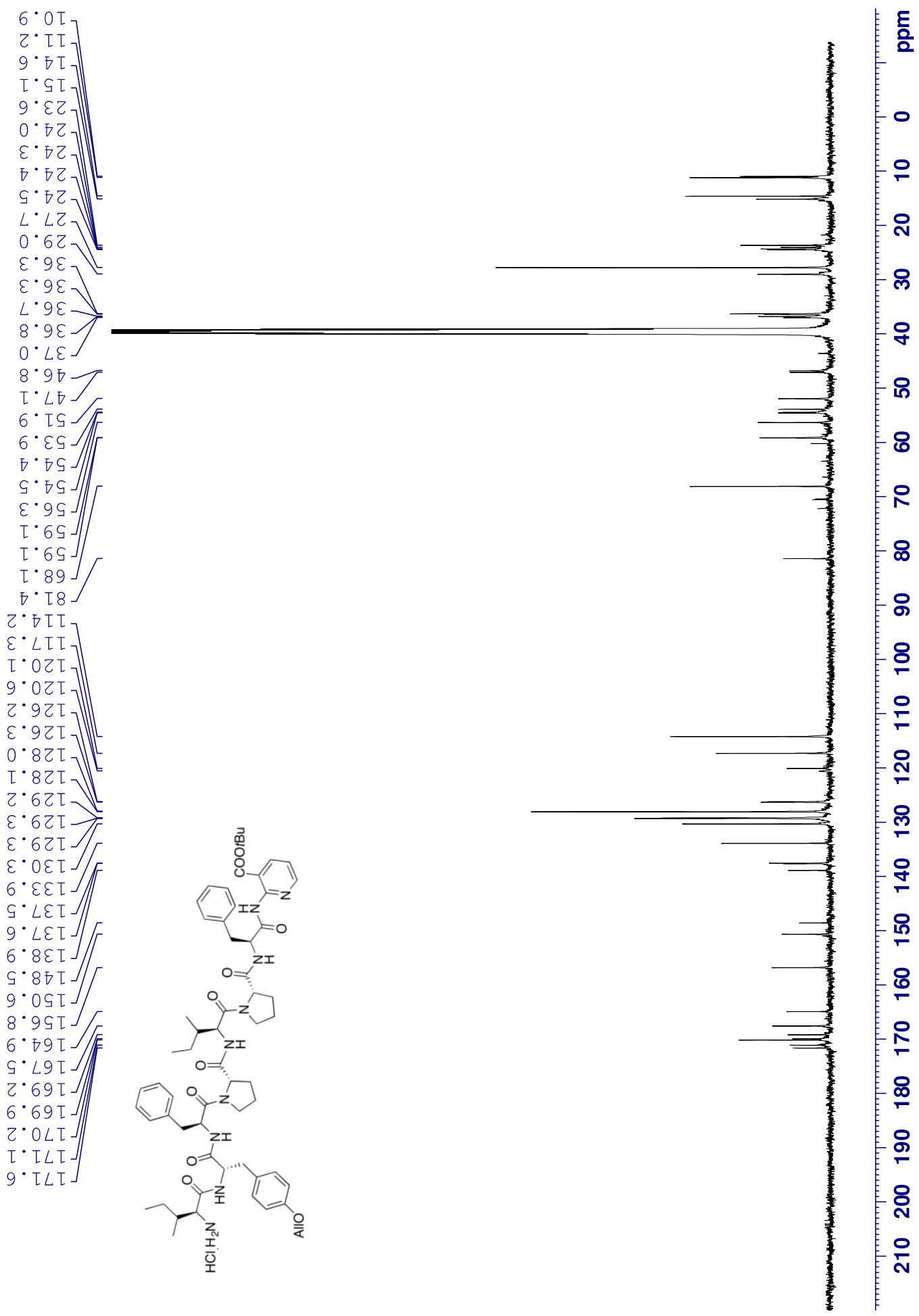


tert-Butyl 2-((S)-2-((S)-1-(((S)-2-((2S,3R)-2-amino-3-methylpentanamido)-3-(4-(((E)-prop-1-en-1yl)oxy)phenyl)propanoyl)-L-phenyl-alanyl-L-prolyl-L-isoleucyl)pyrrolidine-2-carboxamido)-3phenylpropanamido)nicotinate hydrochloride acid salt (H-L-Ile-L-Tyr(All)-L-Phe-L-Pro-L-Ile-L-Pro-LPhe-NH- $t$ Bu-nic.HCl, KH_1046) L,L,L,L,L,L,L-17.HCI - HSQC (DMSO-d $\boldsymbol{d}_{6}$ )

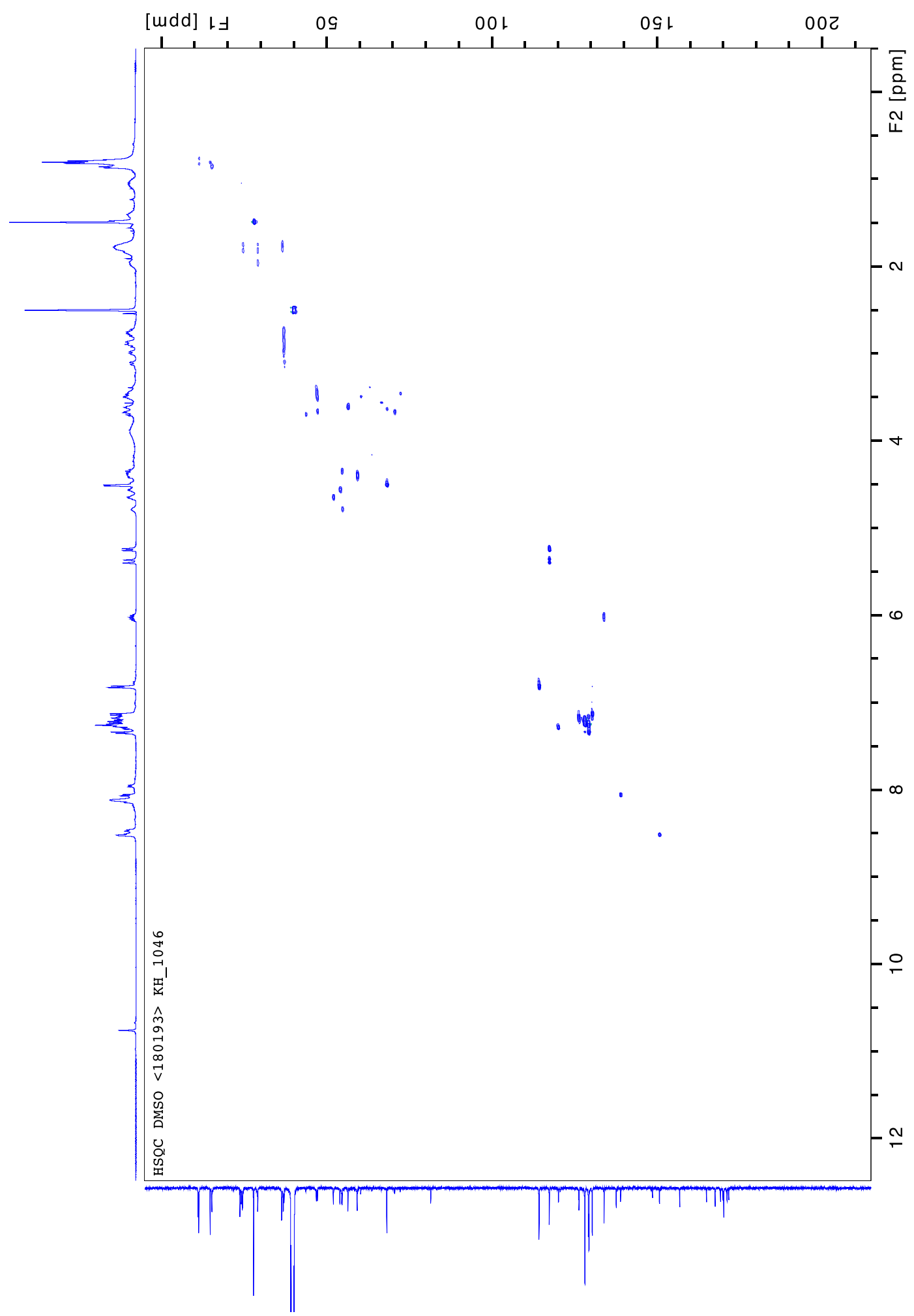


tert-Butyl 2-((S)-2-((S)-1-(((S)-2-((2S,3R)-2-amino-3-methylpentanamido)-3-(4-(((E)-prop-1-en-1yl)oxy)phenyl)propanoyl)-L-phenyl-alanyl-L-prolyl-L-isoleucyl)pyrrolidine-2-carboxamido)-3phenylpropanamido)nicotinate hydrochloride acid salt (H-L-Ile-L-Tyr(All)-L-Phe-L-Pro-L-Ile-L-Pro-L-

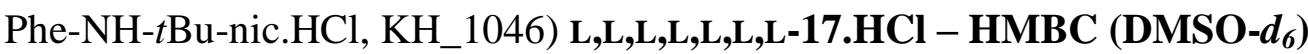

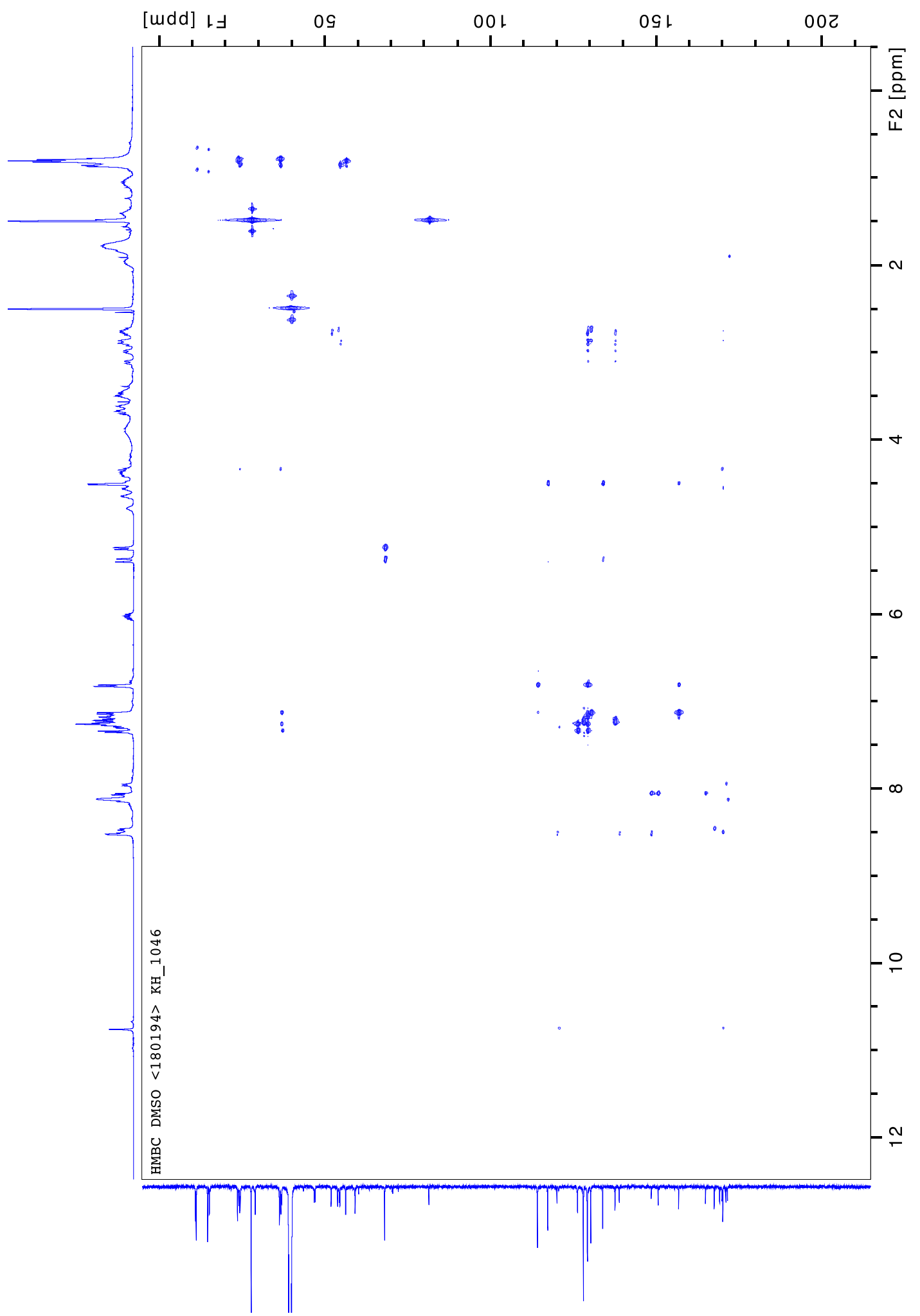


tert-Butyl 2-((S)-2-((S)-1-(((S)-2-((2S,3R)-2-amino-3-methylpentanamido)-3-(4-(((E)-prop-1-en-1yl)oxy)phenyl)propanoyl)-L-phenyl-alanyl-L-prolyl-L-isoleucyl)pyrrolidine-2-carboxamido)-3phenylpropanamido)nicotinate hydrochloride acid salt (H-L-Ile-L-Tyr(All)-L-Phe-L-Pro-L-Ile-L-Pro-LPhe-NH- $t$ Bu-nic.HCl, KH_1046) L,L,L,L,L,L,L-17.HCI - COSY (DMSO-d ) $^{-}$

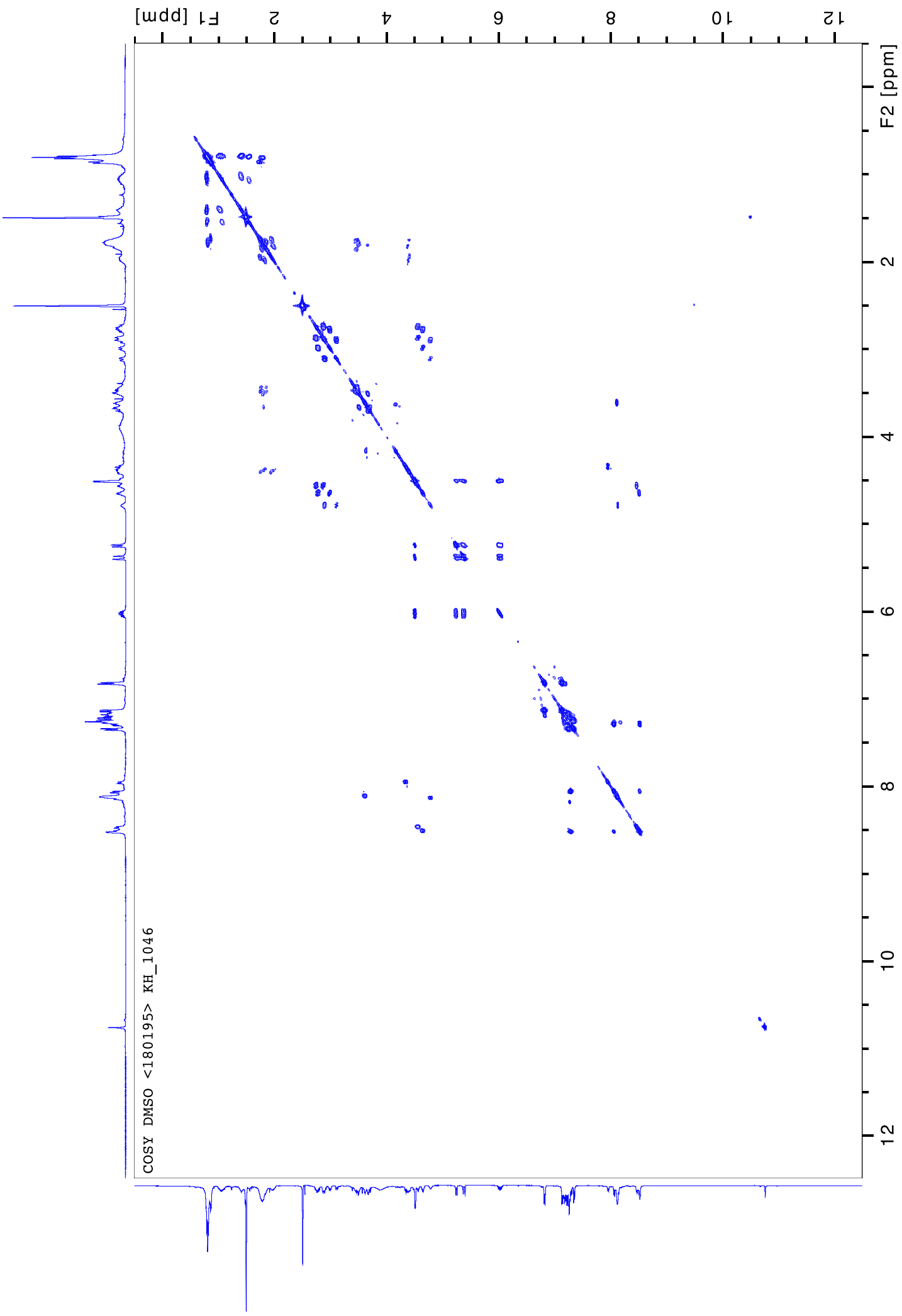


(6S,9S,12S,15S,17aS,23S,25aS)-6,15-dibenzyl-12,23-di((S)-sec-butyl)-9-(4-(((E)-prop-1-en-1yl)oxy)benzyl)octadecahydro-5H-dipyrrolo[1,2-a:1',2'-g][1,4,7,10,13,16,19]heptaazacyclohenicosine5,8,11,14,17,22,25-heptaone (c[-L-Ile-L-Tyr(All)-L-Phe-L-Pro-L-Ile-L-Pro-L-Phe-], KH_1236)

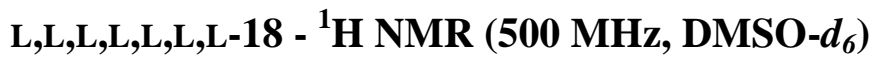
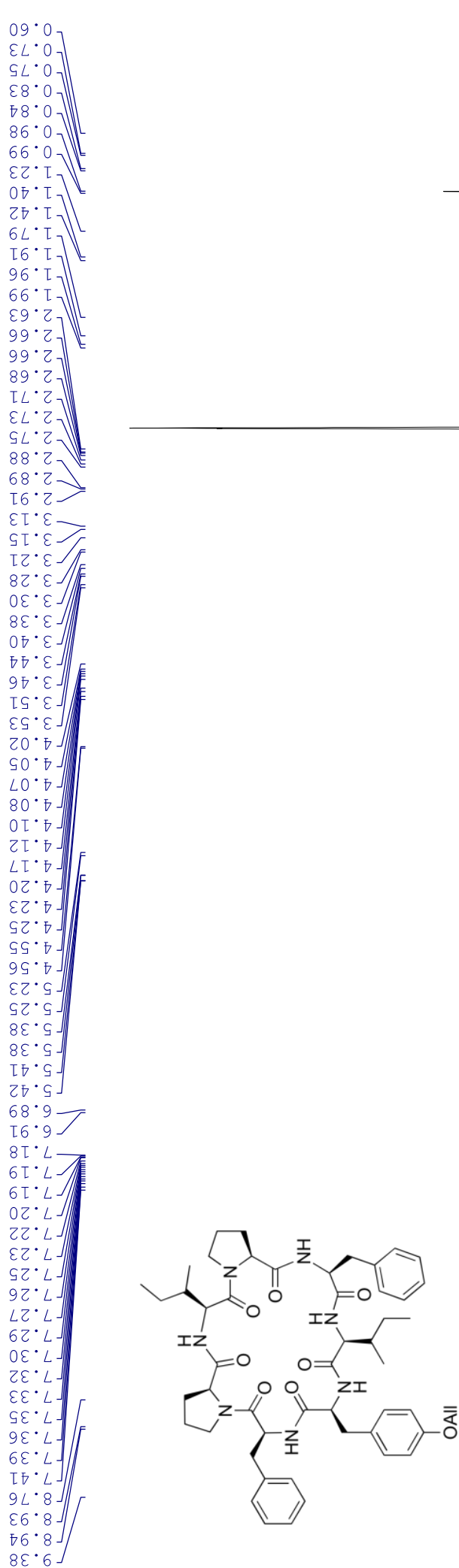

$\frac{\xi}{2}$

L 0.1
$\frac{109}{1.2}$
1.9

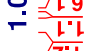

L $\frac{\sqrt{0.1}}{0.5}$

$-\frac{0^{\circ} 1}{6.0}$

i.

L

i $\sqrt{6^{\circ} .}$

$0 \frac{-60}{6.0}$

ले $\sqrt{60}$

$=\frac{60}{6.0}$

L

$0 \longdiv { 1 . 5 }$

× $\sqrt{\frac{10.1}{6.0}}$

on

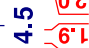

웅

in

$\stackrel{8}{80}$

边

$0 \frac{-6.6}{1 . t}$

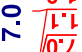

$\sqrt{1.1}$

$\stackrel{n}{\wedge}]$

$\circ$

$\stackrel{\infty}{\infty}$

$\therefore$

L 6 
$(6 S, 9 S, 12 S, 15 S, 17 \mathrm{a} S, 23 S, 25 \mathrm{a} S)-6,15$-dibenzyl-12,23-di((S)-sec-butyl)-9-(4-(((E)-prop-1-en-1yl)oxy)benzyl)octadecahydro-5H-dipyrrolo[1,2-a:1',2'-g][1,4,7,10,13,16,19]heptaazacyclohenicosine5,8,11,14,17,22,25-heptaone (c[-L-Ile-L-Tyr(All)-L-Phe-L-Pro-L-Ile-L-Pro-L-Phe-], KH_1236)

\section{L,L,L,L,L,L,L-18- ${ }^{13} \mathrm{C}$ NMR (126 MHz, DMSO-d $)$}

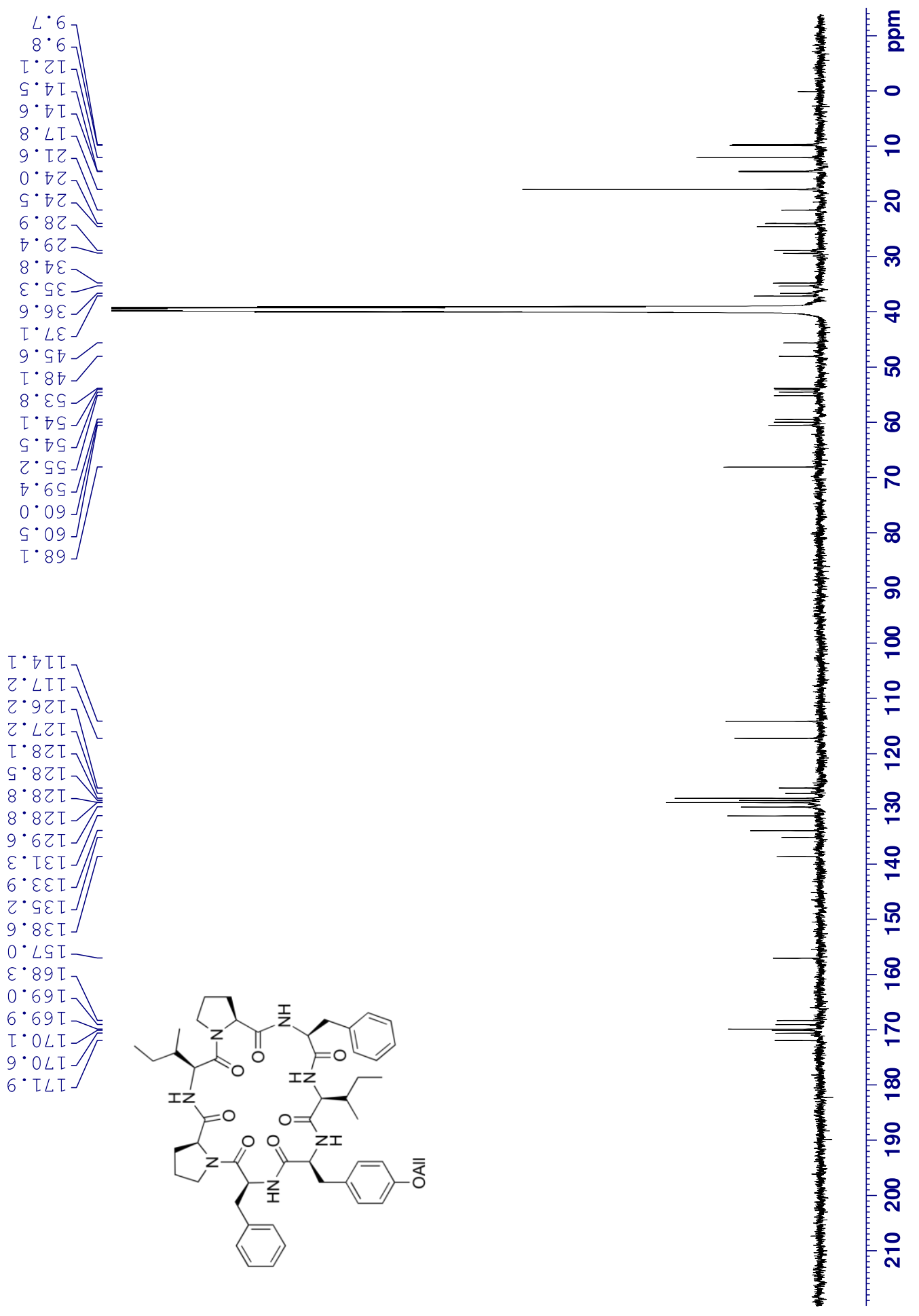


$(6 S, 9 S, 12 S, 15 S, 17 \mathrm{a} S, 23 S, 25 \mathrm{a} S)-6,15$-dibenzyl-12,23-di((S)-sec-butyl)-9-(4-(((E)-prop-1-en-1yl)oxy)benzyl)octadecahydro-5H-dipyrrolo[1,2-a:1',2'-g][1,4,7,10,13,16,19]heptaazacyclohenicosine5,8,11,14,17,22,25-heptaone (c[-L-Ile-L-Tyr(All)-L-Phe-L-Pro-L-Ile-L-Pro-L-Phe-], KH_1236)

\section{$L, L, L, L, L, L, L-18$ - HSQC (DMSO-d $d_{6}$ )}

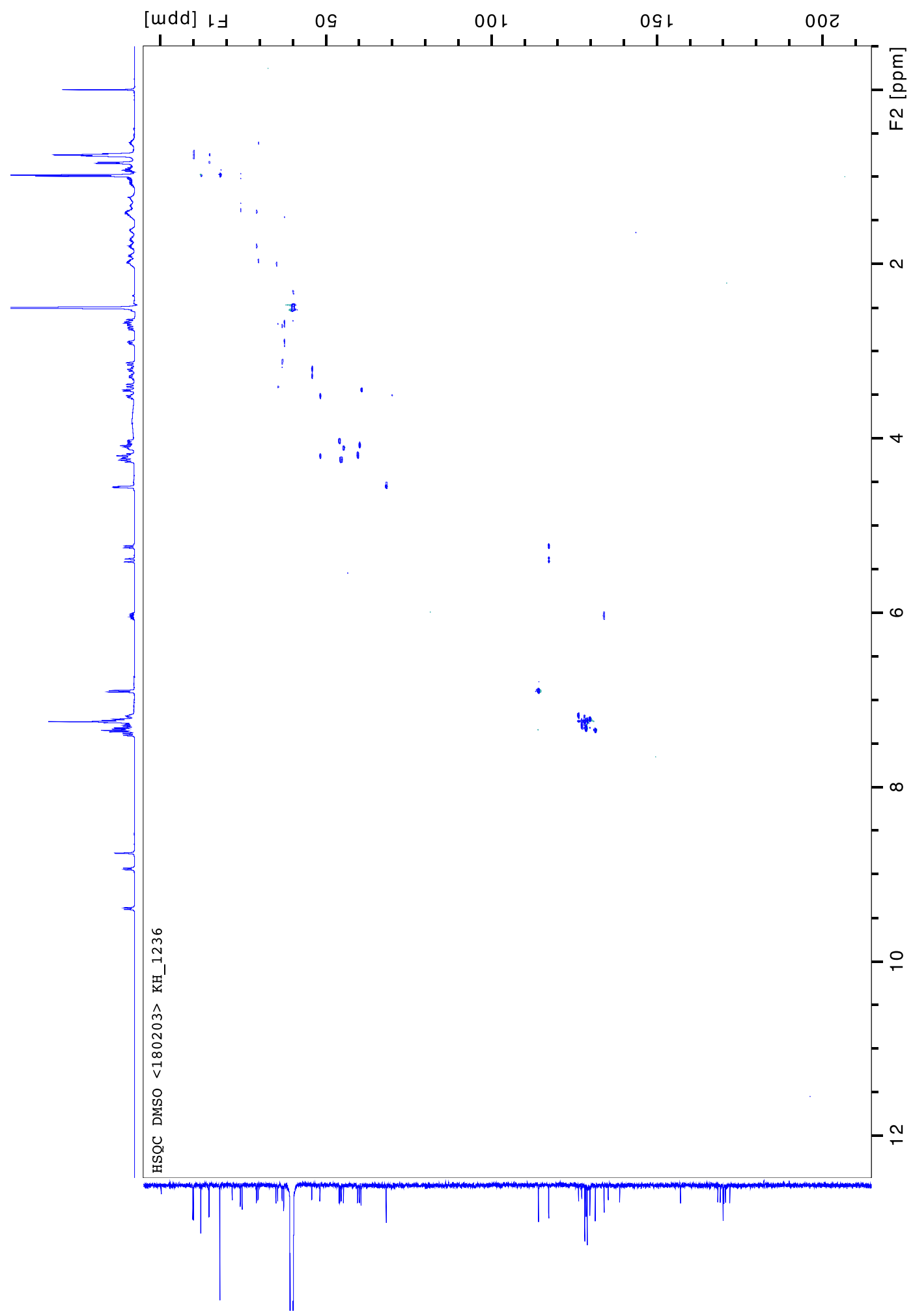


$(6 S, 9 S, 12 S, 15 S, 17 \mathrm{a} S, 23 S, 25 \mathrm{a} S)-6,15$-dibenzyl-12,23-di((S)-sec-butyl)-9-(4-(((E)-prop-1-en-1yl)oxy)benzyl)octadecahydro-5H-dipyrrolo[1,2-a:1',2'-g][1,4,7,10,13,16,19]heptaazacyclohenicosine5,8,11,14,17,22,25-heptaone (c[-L-Ile-L-Tyr(All)-L-Phe-L-Pro-L-Ile-L-Pro-L-Phe-], KH_1236)

\section{L,L,L,L,L,L,L-18 - HMBC (DMSO-d $\left.d_{6}\right)$}

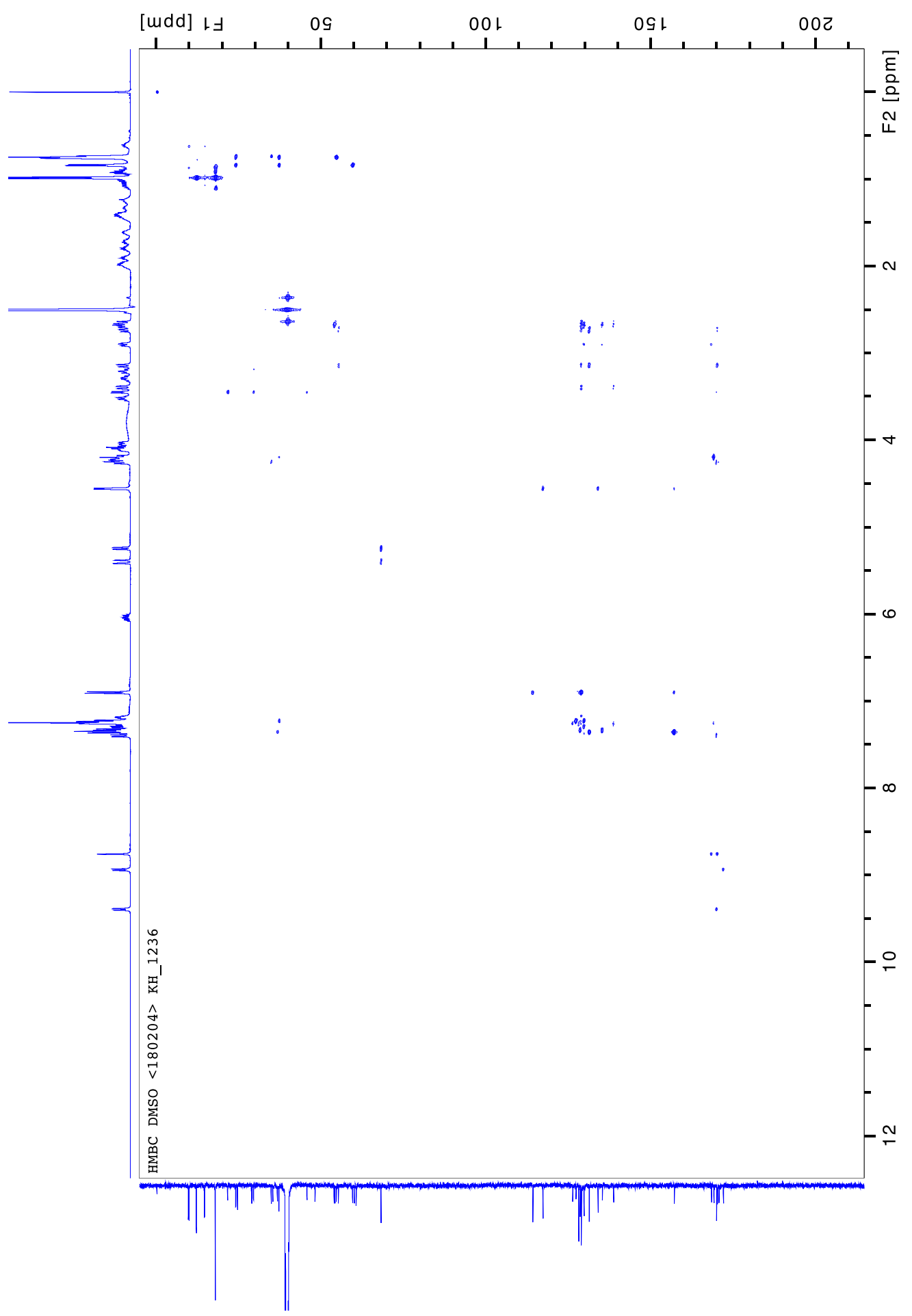


$(6 S, 9 S, 12 S, 15 S, 17 \mathrm{a} S, 23 S, 25 \mathrm{a} S)-6,15$-dibenzyl-12,23-di((S)-sec-butyl)-9-(4-(((E)-prop-1-en-1yl)oxy)benzyl)octadecahydro-5H-dipyrrolo[1,2-a:1',2'-g][1,4,7,10,13,16,19]heptaazacyclohenicosine5,8,11,14,17,22,25-heptaone (c[-L-Ile-L-Tyr(All)-L-Phe-L-Pro-L-Ile-L-Pro-L-Phe-], KH_1236)

\section{L,L,L,L,L,L,L-18- COSY (DMSO- $d_{6}$ )}

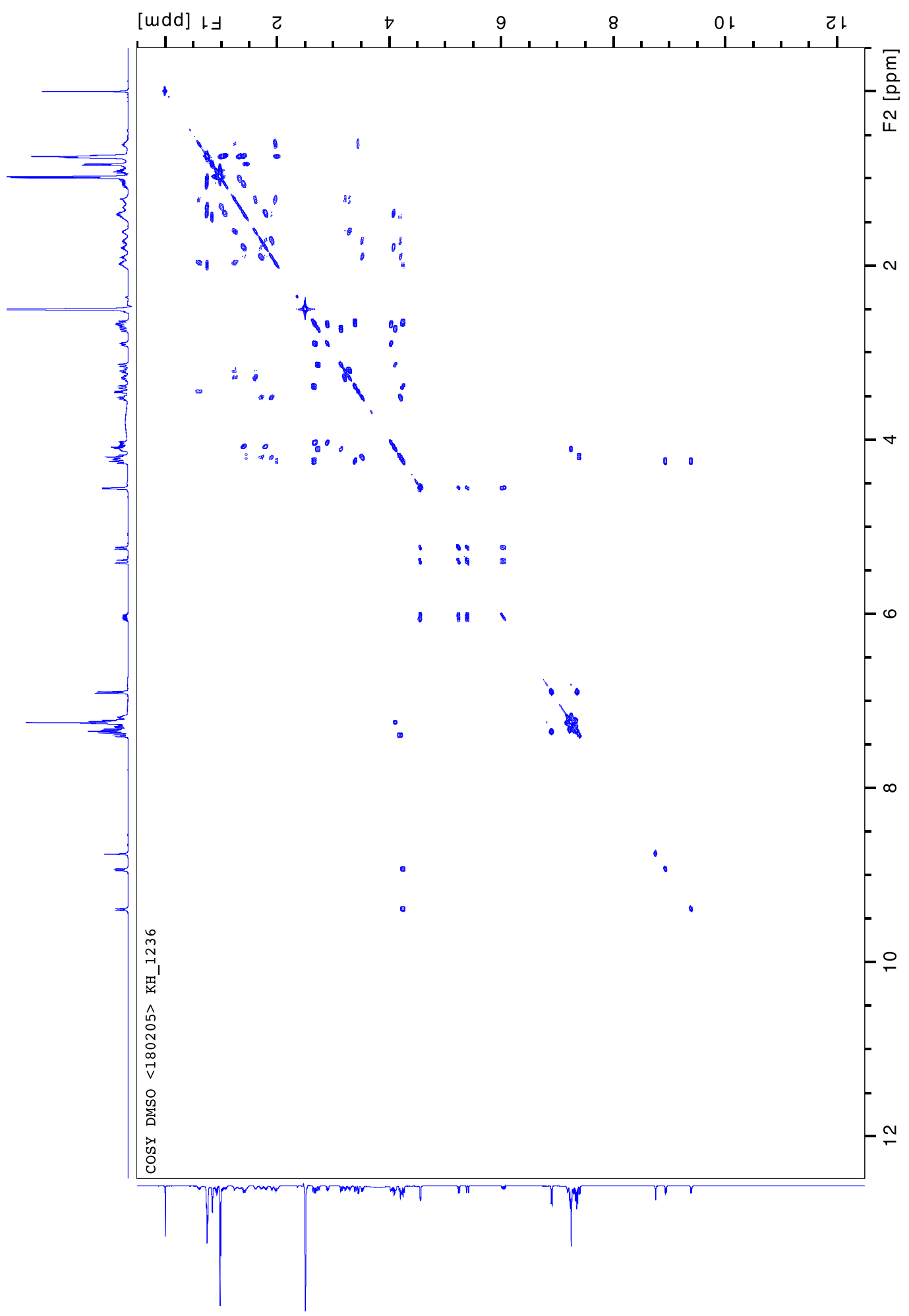


4-[methyl(phenyl)carbamoyl]benzoic acid (KH_808) S17 - ${ }^{\mathbf{1}} \mathbf{H}$ NMR (500 MHz, CDCl 3 )

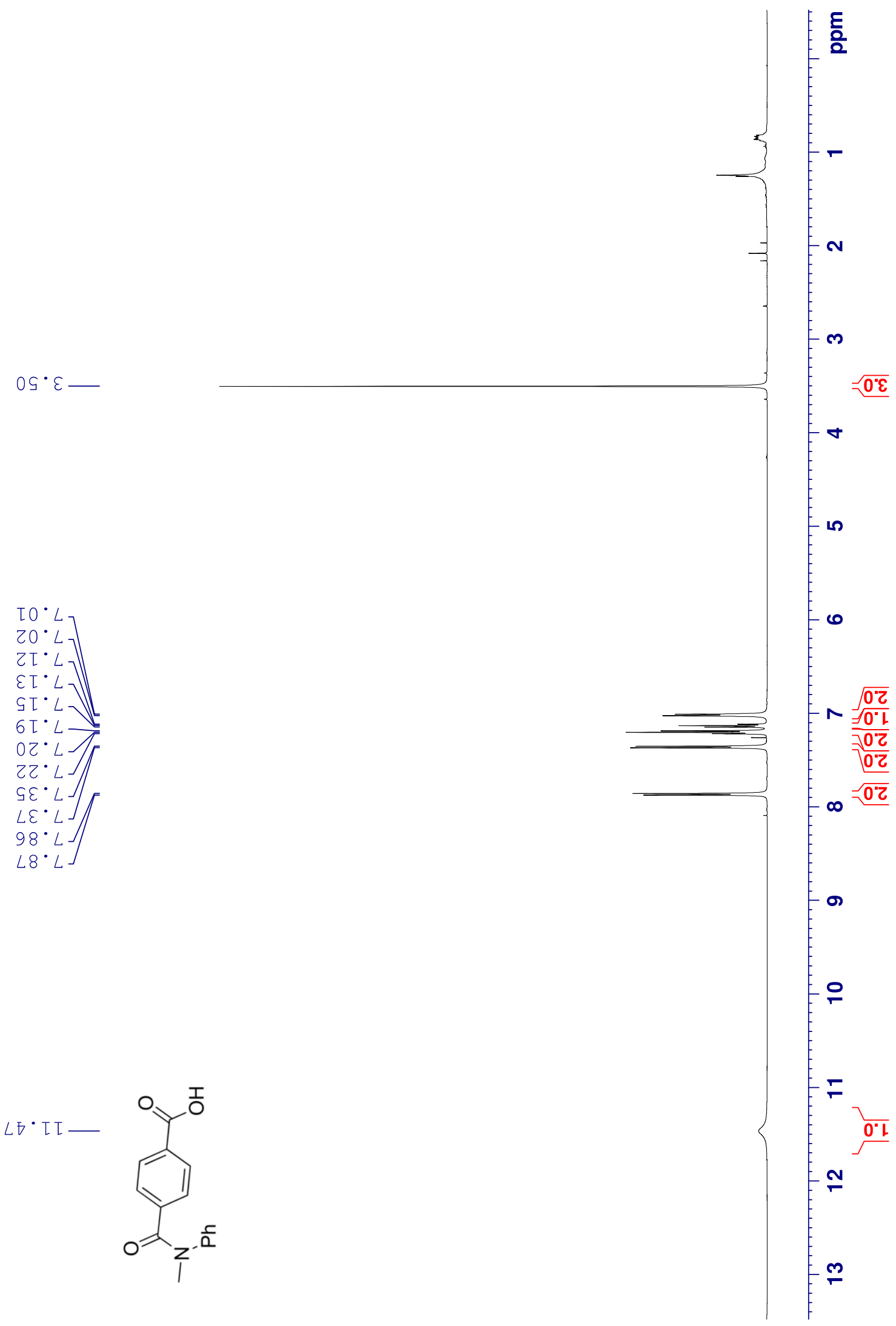


4-[methyl(phenyl)carbamoyl]benzoic acid (KH_808) S17 - ${ }^{13} \mathbf{C}$ NMR (126 MHz, CDCl $)$

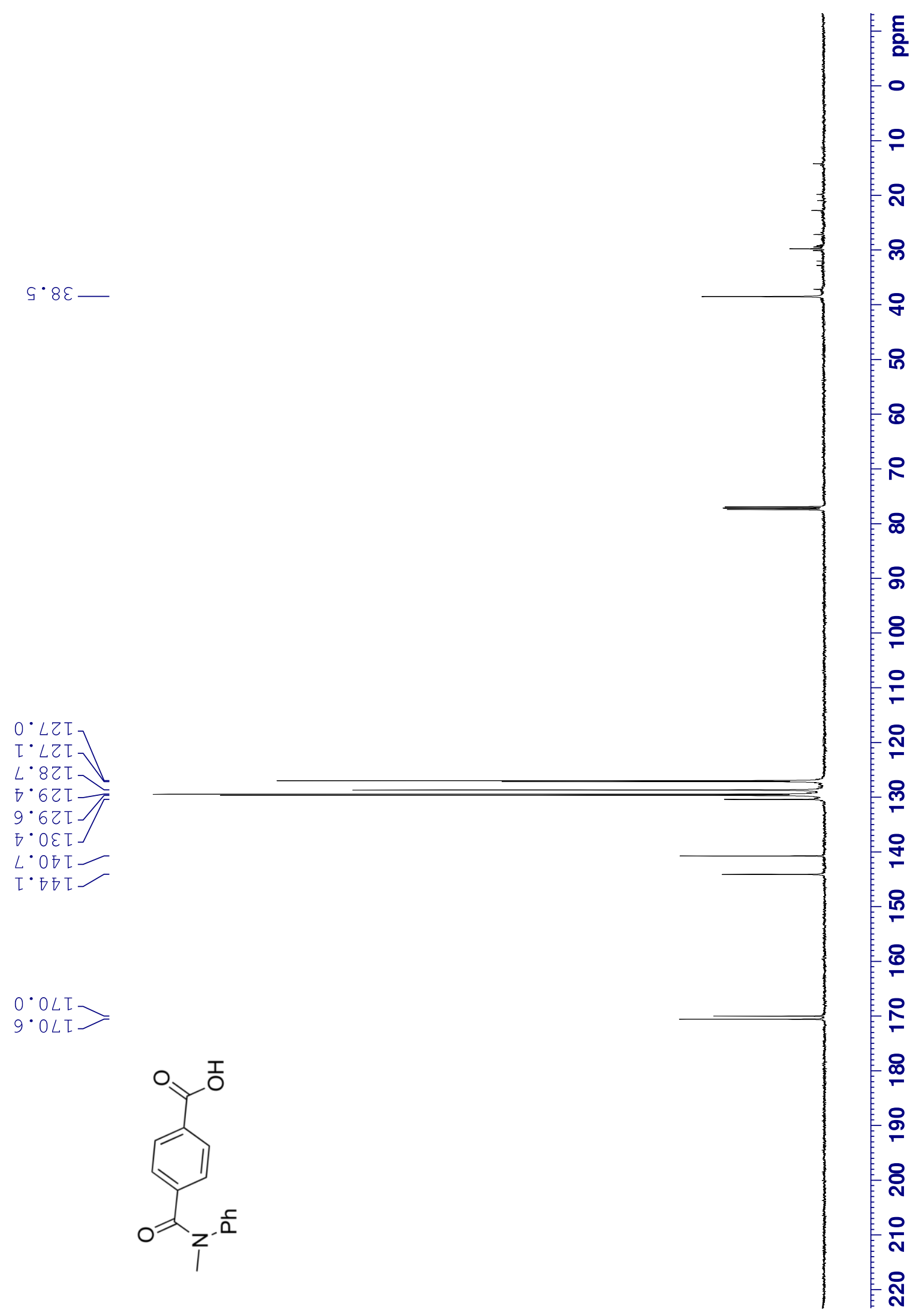


tert-Butyl 2-\{[(3S)-3-(\{[(3S)-6-amino-3-[\{tert-butoxycarbonyl)amino)-6-oxohex-1-en-2yl]amino \}pyridine-3-carboxylate (Boc-L-Gln-L-Phe-NH-tBu-nic, KH_905) L,L-S18 - ${ }^{\mathbf{1}}$ H NMR (500

\section{MHz, DMSO-d ${ }_{6}$, mixture of rotamers)}

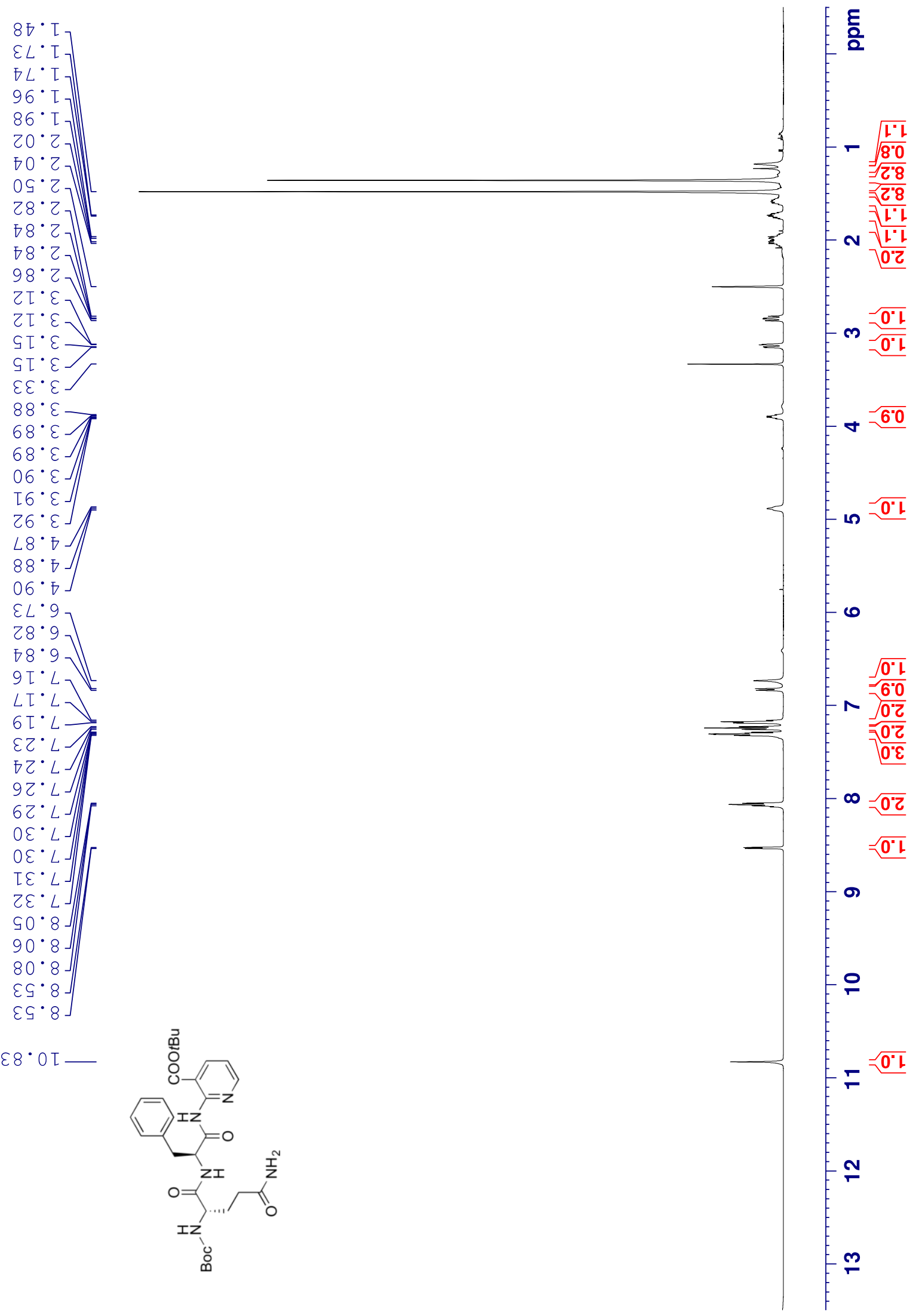


tert-Butyl 2-\{[(3S)-3-(\{[(3S)-6-amino-3-[\{tert-butoxycarbonyl)amino)-6-oxohex-1-en-2yl]amino pyridine-3-carboxylate (Boc-L-Gln-L-Phe-NH- $t$ Bu-nic, KH_905) L,L-S18 - ${ }^{\mathbf{1 3}}$ C NMR (126

\section{MHz, DMSO- $d_{6}$, mixture of rotamers)}
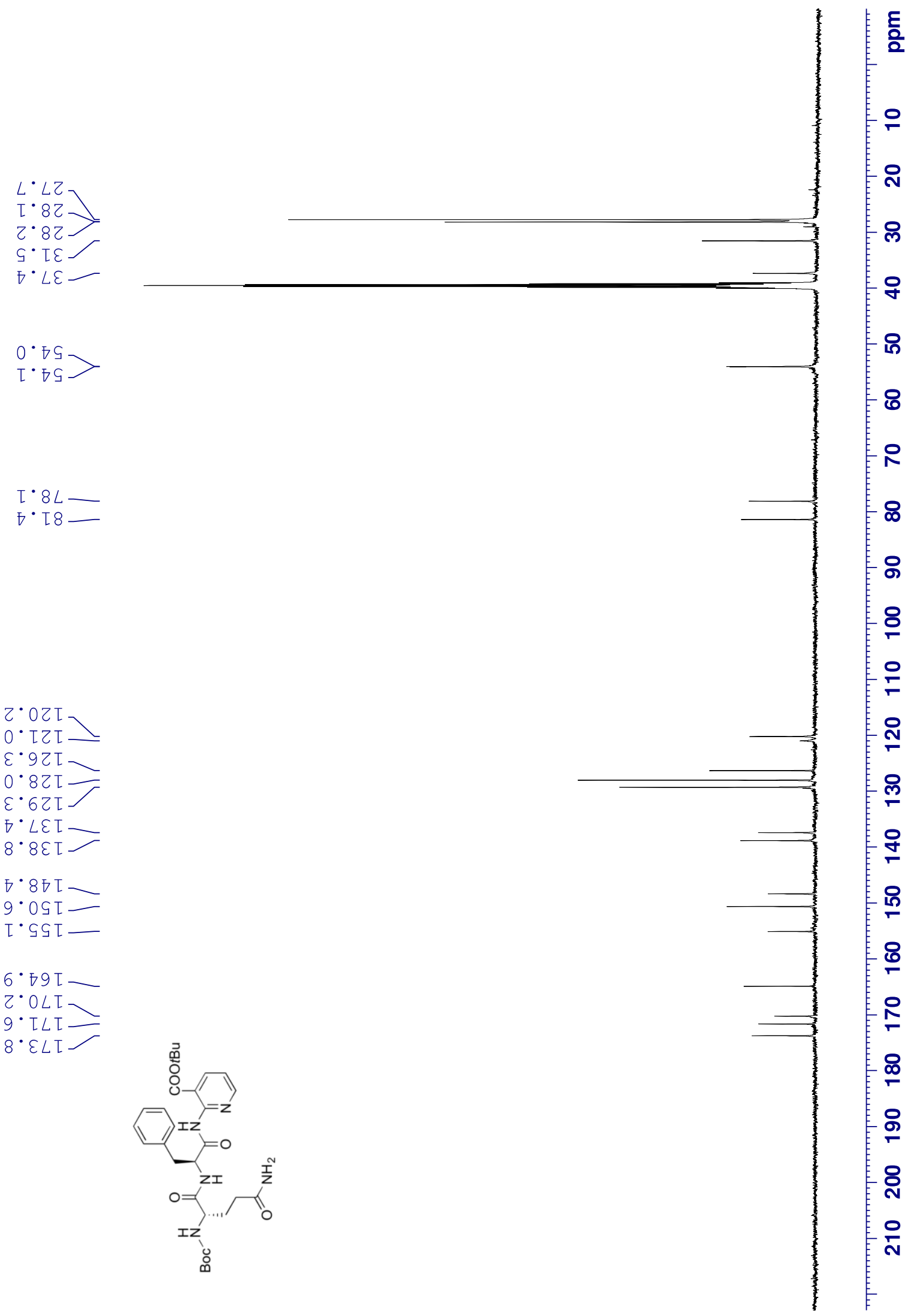
tert-Butyl 2-\{[(3S)-3-(\{[(3S)-6-amino-3-[\{tert-butoxycarbonyl)amino)-6-oxohex-1-en-2yl]amino \}pyridine-3-carboxylate (Boc-L-Gln-L-Phe-NH-tBu-nic, KH_905) L,L-S18 - ${ }^{\mathbf{1}}$ H NMR (500 MHz, DMSO-d $\left.{ }_{6}, 6^{\circ} \mathrm{C}\right)$
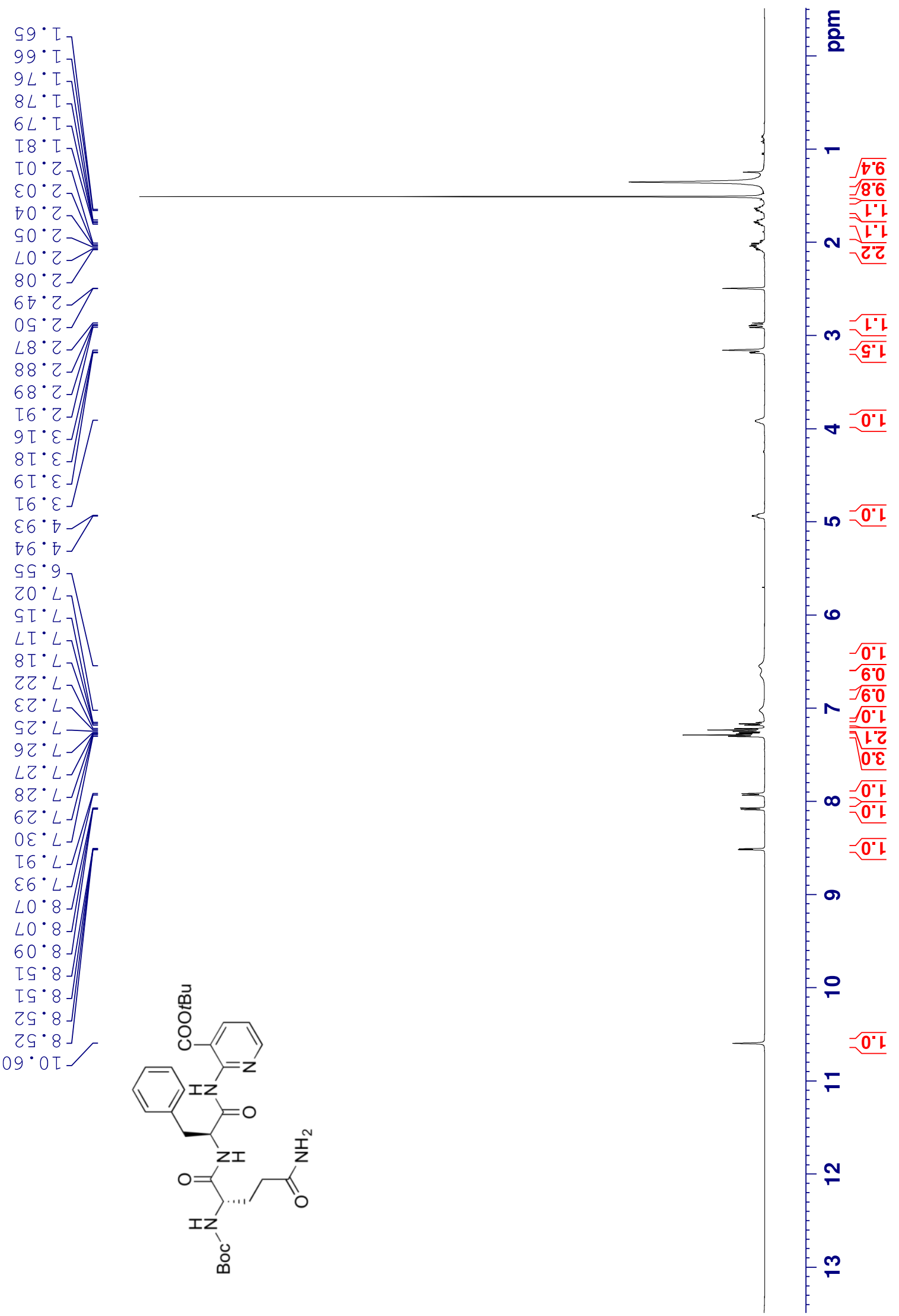
tert-Butyl 2-\{[(3S)-3-\{[(3S)-6-amino-3-\{4-[methyl(phenyl)carbamoyl]-benzamido $\}-6-o x o-h e x-1-e n-2-$ yl]amino \}-4-phenylbut-1-en-2yl] amino \} pyridine-3-carboxylate L,L-19 - ${ }^{\mathbf{1}}$ H NMR (500 MHz, DMSO$\left.d_{6}\right)$

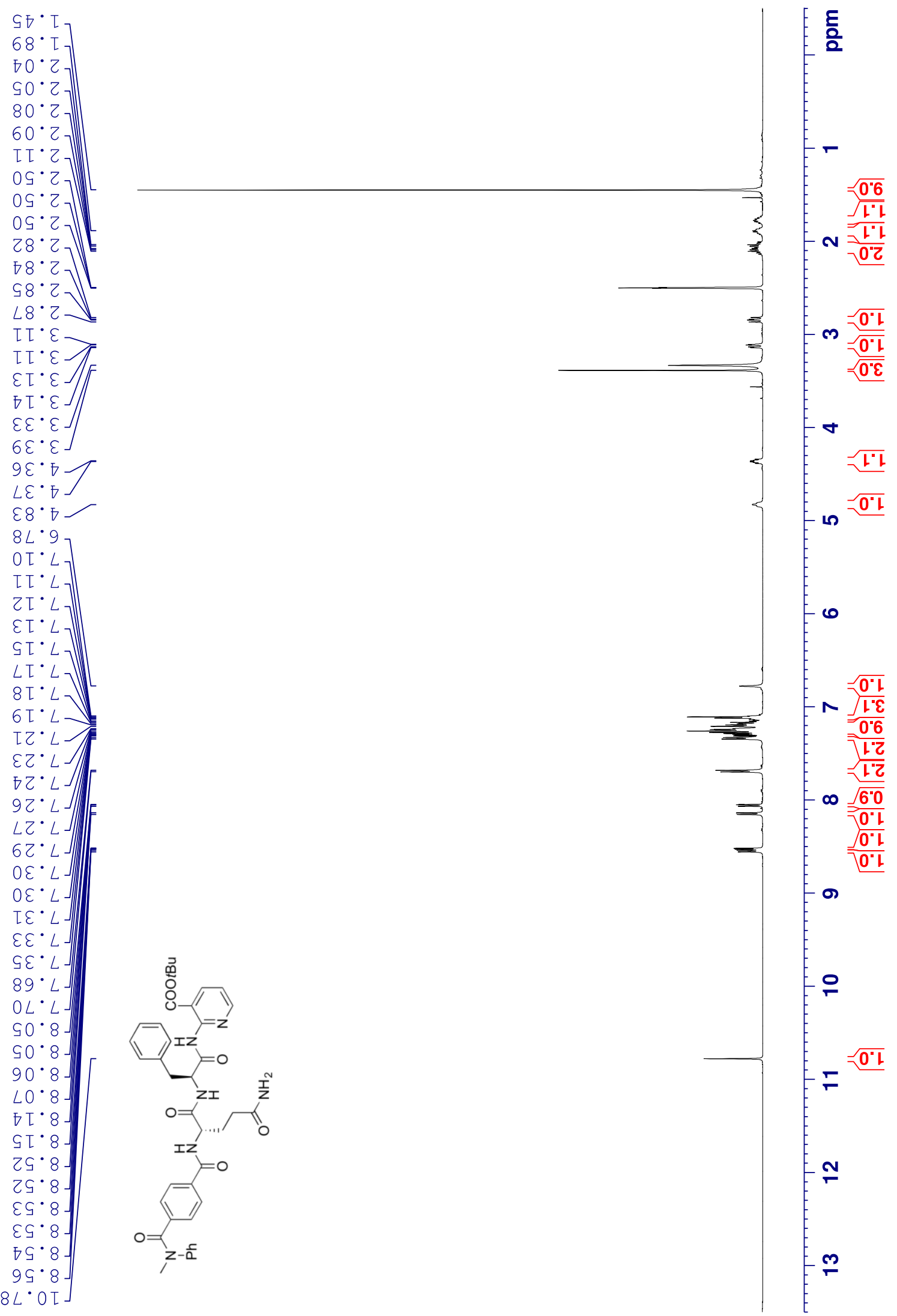


tert-Butyl 2-\{[(3S)-3-\{[(3S)-6-amino-3-\{4-[methyl(phenyl)carbamoyl]-benzamido $\}-6-0 x o-h e x-1-e n-2-$ yl]amino -4-phenylbut-1-en-2yl] amino\} pyridine-3-carboxylate L,L-19 - ${ }^{13}$ C NMR (126 MHz, DMSO- $d_{6}$ )

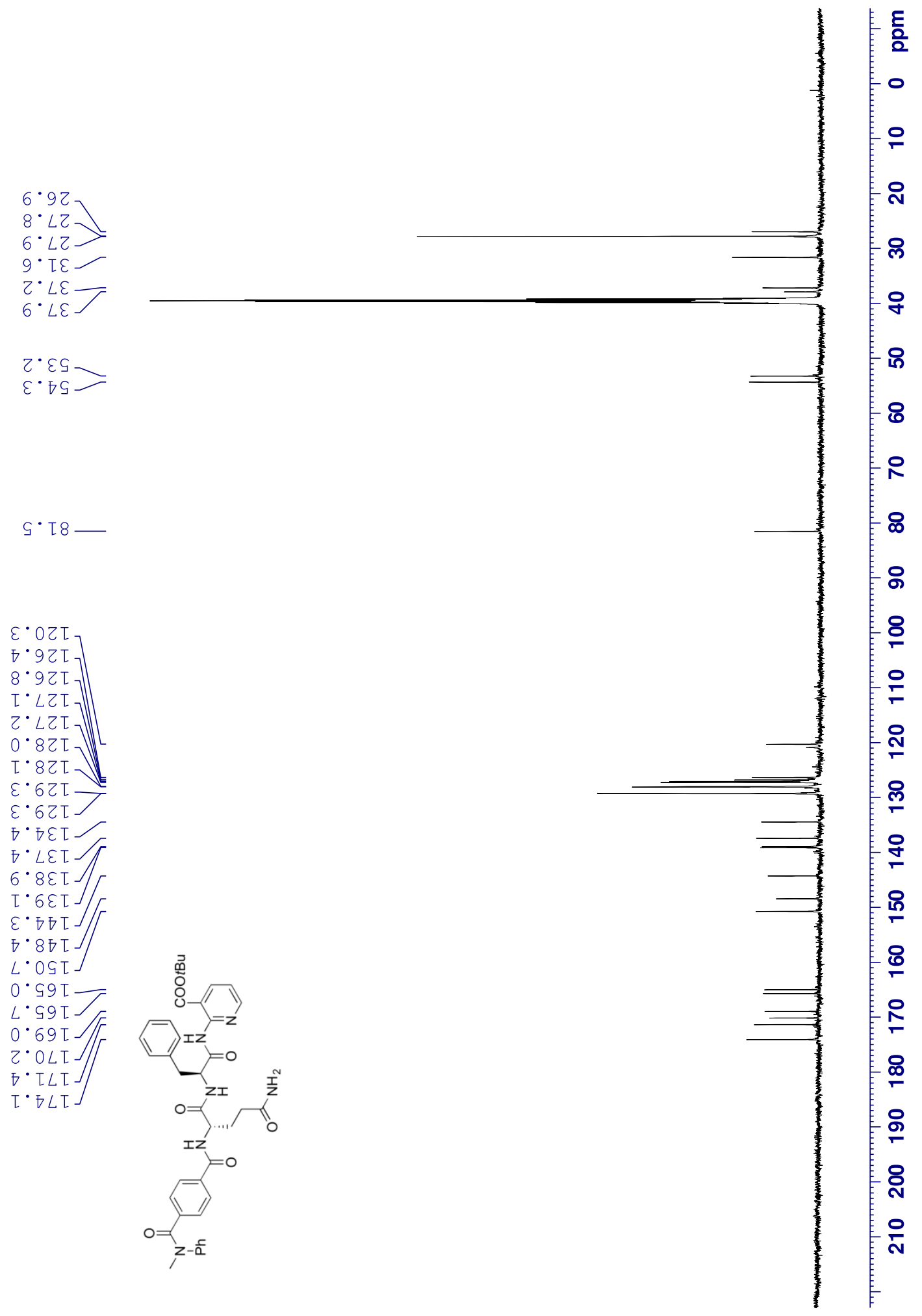


Methyl (4-(methyl(phenyl)carbamoyl)benzoyl)-L-glutaminyl-L-phenylalanyl-L-alaninate

(KH_1234) L,L,L-20 - ${ }^{1} \mathrm{H}$ NMR (500 MHz, DMSO- $d_{6}$, mixture of rotamers)

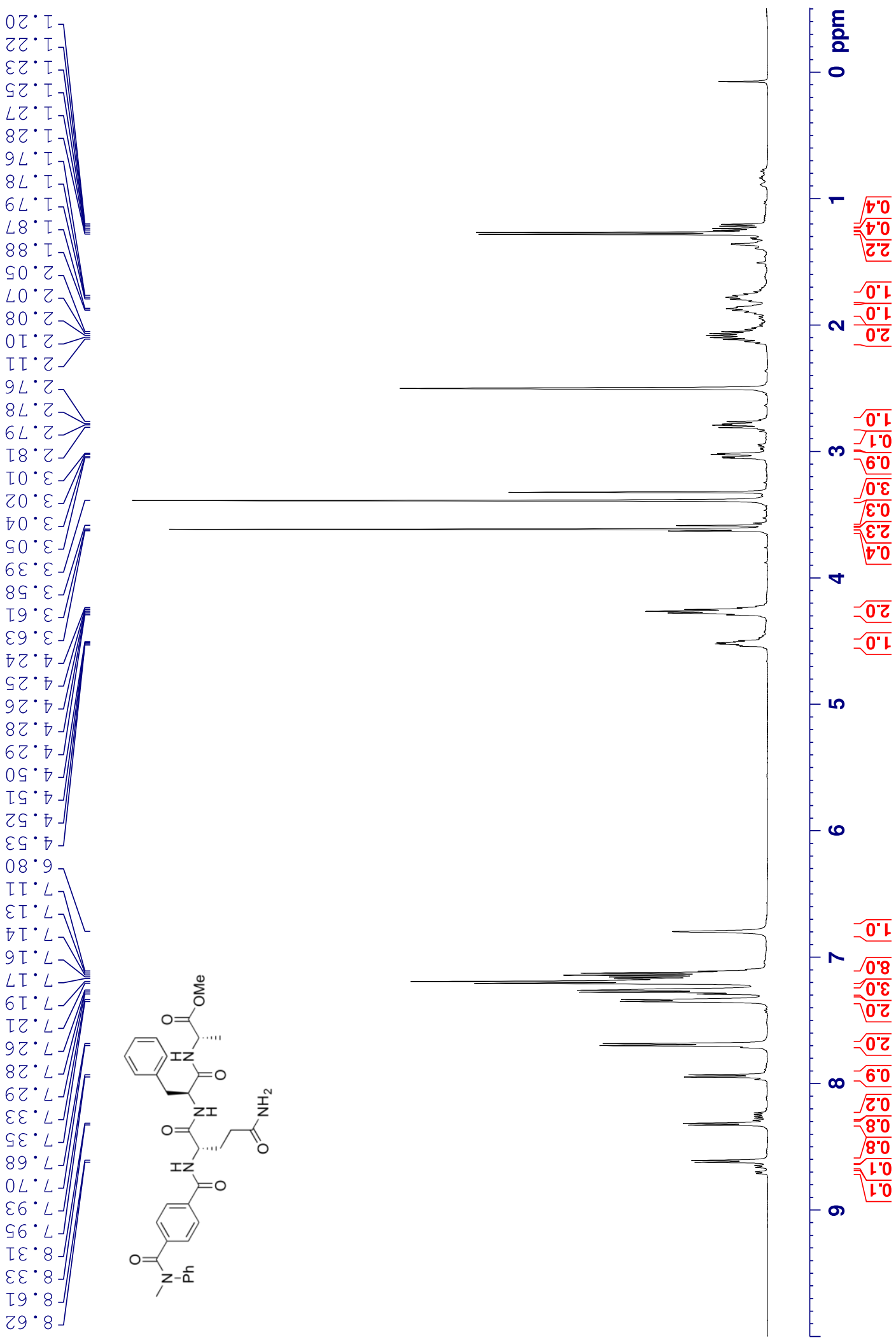


Methyl (4-(methyl(phenyl)carbamoyl)benzoyl)-L-glutaminyl-L-phenylalanyl-L-alaninate

(KH_1234)

L,L,L-20 - ${ }^{13} \mathrm{C}$ NMR (126 MHz, DMSO- $d_{6}$, mixture of rotamers)

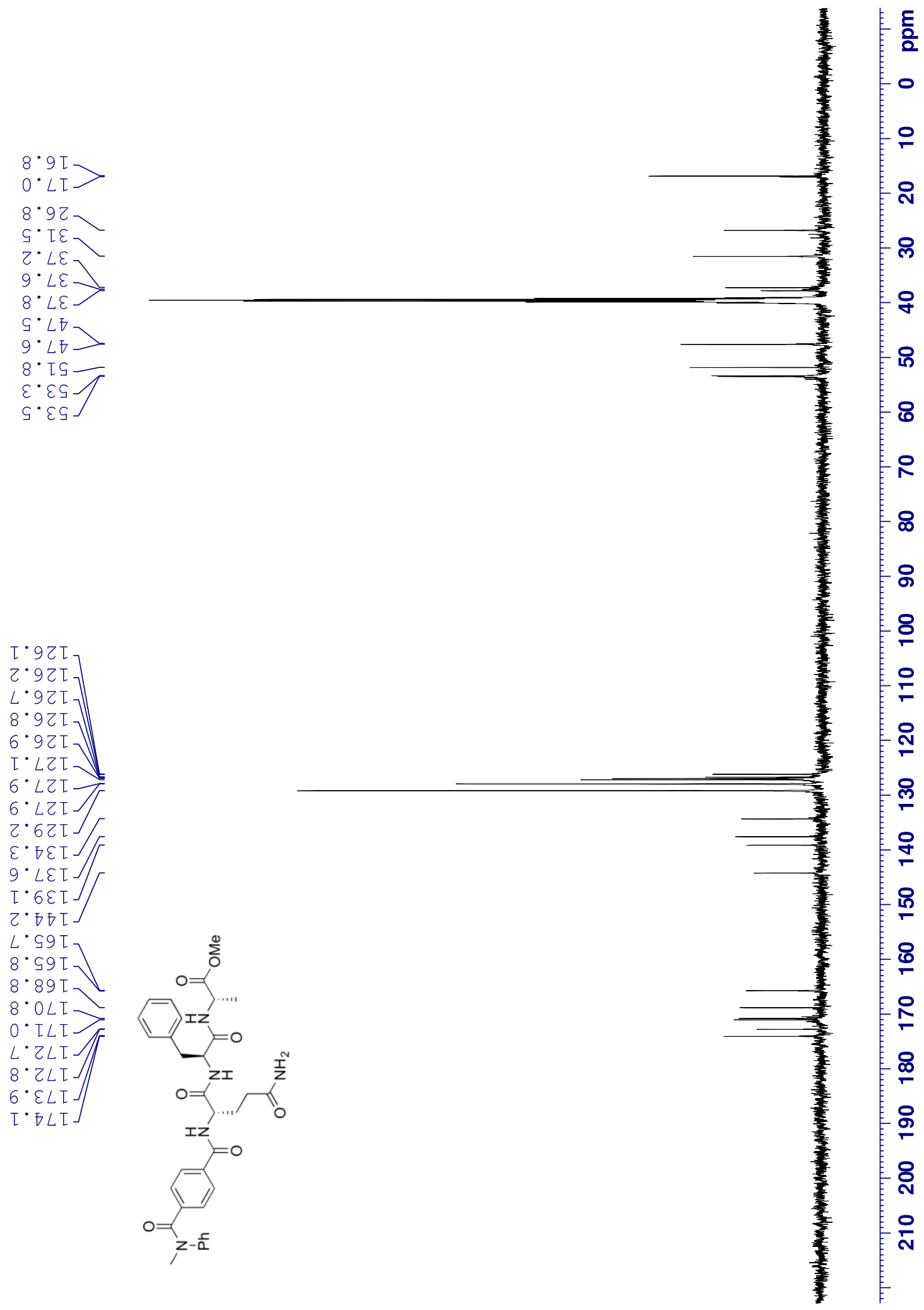


Methyl (4-(methyl(phenyl)carbamoyl)benzoyl)-L-glutaminyl-L-phenylalanyl-L-alaninate (KH_1234) L,L,L-20 - ${ }^{1} \mathrm{H}$ NMR (500 MHz, DMSO-d $\left.6,8^{\circ} \mathrm{C}\right)$

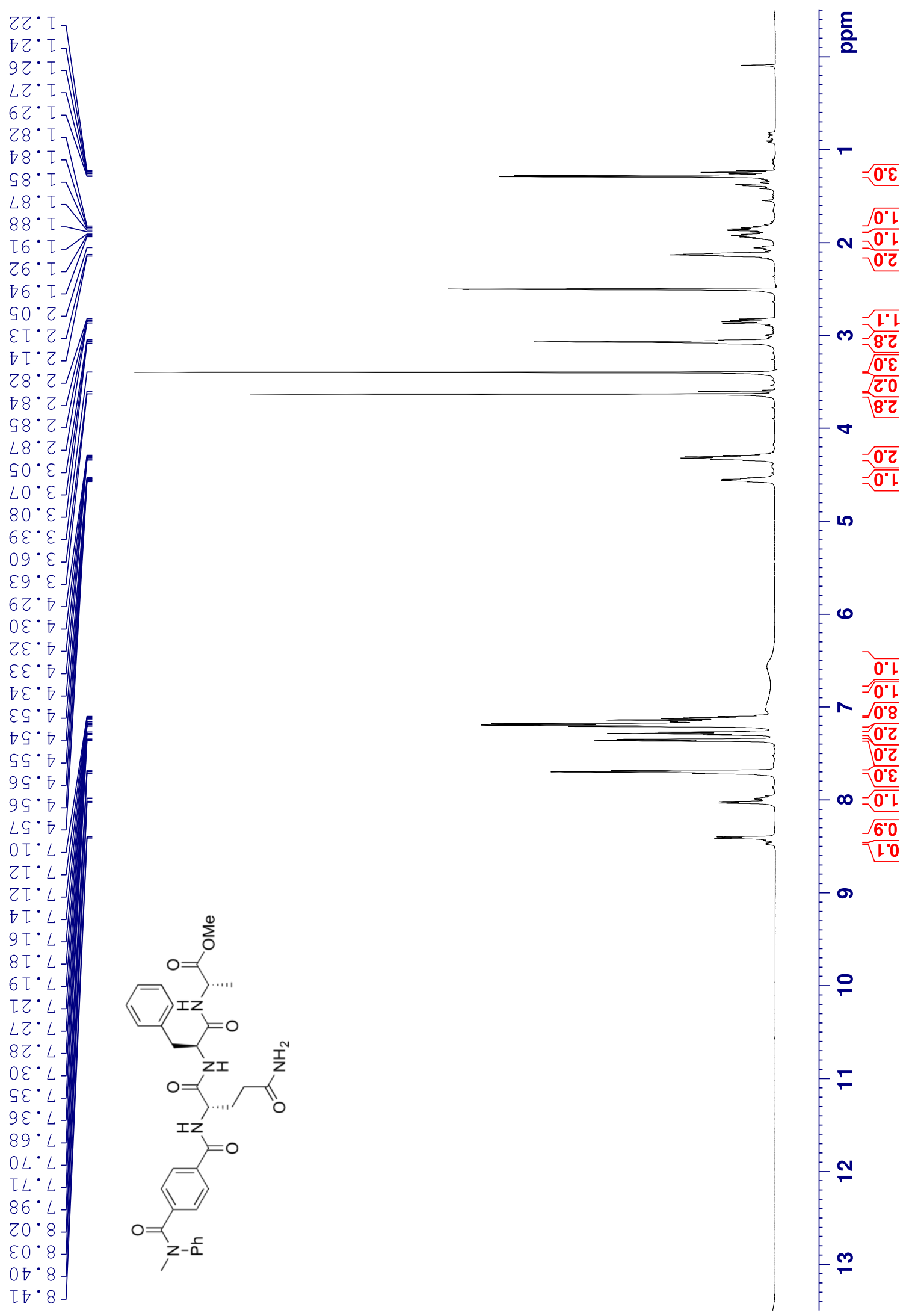


Methyl (4-(methyl(phenyl)carbamoyl)benzoyl)-L-glutaminyl-L-phenylalanyl-L-alaninate (KH_1234) L,L,L-20 - ${ }^{13} \mathrm{C}$ NMR (126 MHz, DMSO-d $\left.6,80^{\circ} \mathrm{C}\right)$

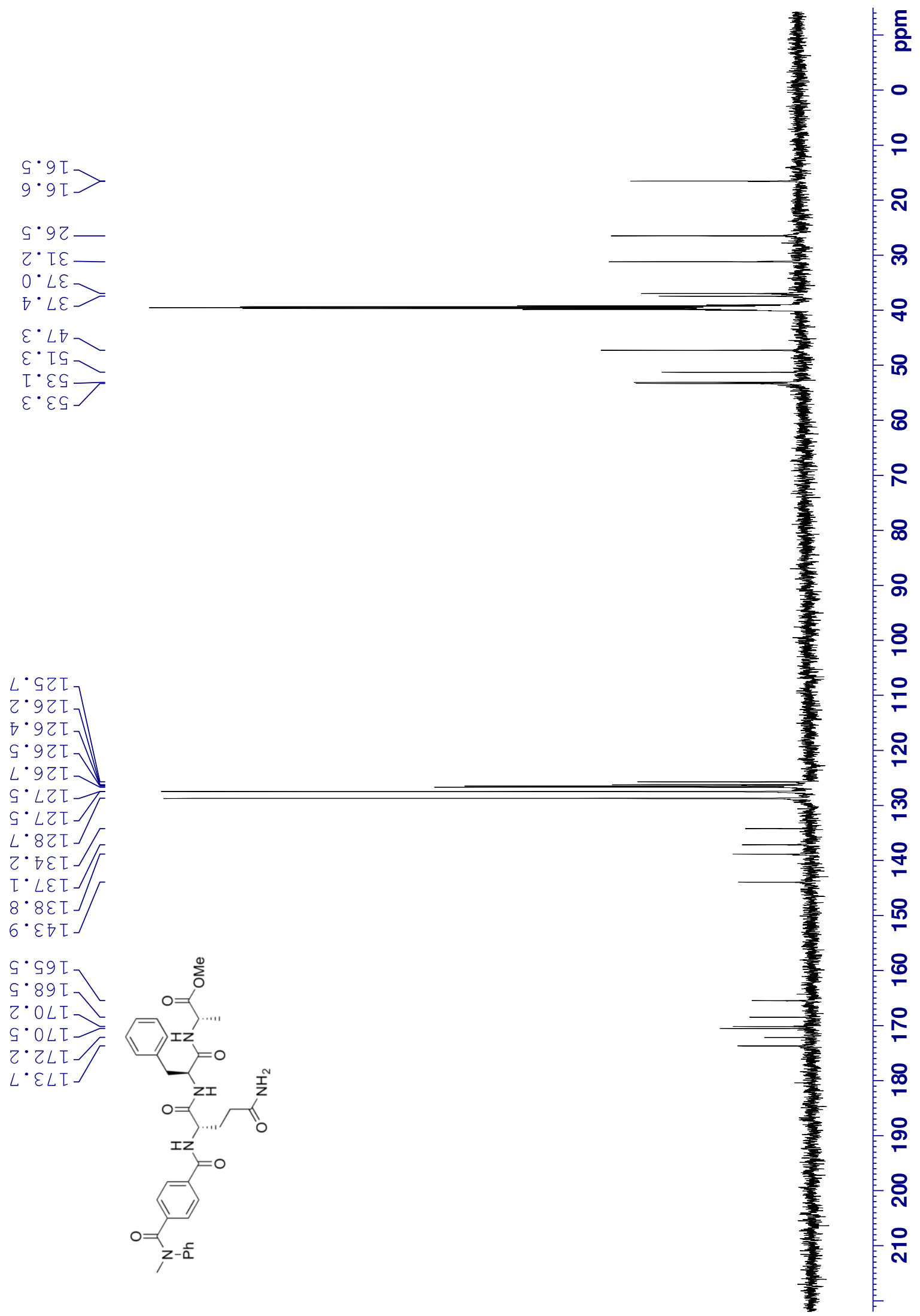




\section{References}

[1] Wybon, C. C. D.; Mensch, C.; Hollanders, K.; Gadais, C.; Herrebout, W. A.; Ballet, S.; Maes, B. U. W. Zn-Catalyzed tert-Butyl Nicotinate-Directed Amide Cleavage as a Biomimic of MetalloExopeptidase Activity. ACS Catal. 2018, 8, 203-218.

[2] Han, G.; Tamaki, M.; Hruby, V. J. Fast, Efficient and Selective Deprotection of the Tertbutoxycarbonyl (Boc) Group using HCL/dioxane (4 M). Chem. Biol. Drug Des. 2001, 58, 338341.

[3] Wright, S. W. Preparation of 2-, 4-, 5-, and 6-Aminonicotinic Acid Tert-butyl Esters. J. Heterocycl. Chem. 2012, 49, 442-445.

[4] Bruno, N. C.; Tudge, M. T.; Buchwald, S. L. Design and Preparation of New Palladium Precatalysts for C-C and C-N Cross-Coupling Reactions. Chem. Sci. 2013, 4, 916-920.

[5] Doroski, M. D.; Maderna, A.; O'donnell, C. J.; Subramanyam, C.; Vetelino, B.; Dushin, R. G; Strop, P.; Graziani, E. I. Cytotoxic Peptides and Antibody Drug Conjugates Thereof. 2012, WO2013072813A2.

[6] Declerck, V. Nun, P. Martinez, J. Lamaty, F. Solvent - Free Synthesis of Peptides. Angew. Chem. Int. Ed. 2009, 48, 9318-9321.

[7] Rydzewski, R. M.; Bryant, C. Oballa, R. Wesolowski, G. Rodan, S. B.; Bass, K. E.; Wong, D. H. Peptidic 1-Cyanopyrrolidines: Synthesis and SAR of a Series of Potent, Selective Cathepsin Inhibitors. Bioorg. Med. Chem. 2002, 10, 3277-3284.

[8] Pathak, S. K.; Nath, S. Gupta, R. K.; Rao, D. S.; Prasad, S. K.; Achalkumar, A. S. Effect of Regioisomerism on the Self-Assembly and Photophysical Behavior of 1,3,4-Thiadiazole-Based Polycatenars. J. Mater. Chem. C 2015, 3, 8166-8182.

[9] Greenberg A.; Venanzi, C. A. Structures and Energetics of Two Bridgehead Lactams and Their N- and O-Protonated Forms: an Ab Initio Molecular Orbital Study. J. Am. Chem. Soc., 1993, 115, 6951-6957.

[10] Szostak, R.; Meng, G.; Szostak, M. Resonance Destabilization in N-Acylanilines (Anilides): Electronically-Activated Planar Amides of Relevance in $\mathrm{N}-\mathrm{C}(\mathrm{O})$ Cross-Coupling. J. Org. Chem., 2017, 82, 6373-6378.

[11] PCmodel, version 10.0, 2020, Serena Soft, Bloomington, IN, USA.

[12] Gaussian 16, Revision A.03, M. J. Frisch, G. W. Trucks, H. B. Schlegel, G. E. Scuseria, M. A. Robb, J. R. Cheeseman, G. Scalmani, V. Barone, G. A. Petersson, H. Nakatsuji, X. Li, M. Caricato, A. V. Marenich, J. Bloino, B. G. Janesko, R. Gomperts, B. Mennucci, H. P. Hratchian, J. V. Ortiz, A. F. Izmaylov, J. L. Sonnenberg, D. Williams-Young, F. Ding, F. Lipparini, F. Egidi, J. Goings, B. Peng, A. 
Petrone, T. Henderson, D. Ranasinghe, V. G. Zakrzewski, J. Gao, N. Rega, G. Zheng, W. Liang, M. Hada, M. Ehara, K. Toyota, R. Fukuda, J. Hasegawa, M. Ishida, T. Nakajima, Y. Honda, O. Kitao, H. Nakai, T. Vreven, K. Throssell, J. A. Montgomery, Jr., J. E. Peralta, F. Ogliaro, M. J. Bearpark, J. J. Heyd, E. N. Brothers, K. N. Kudin, V. N. Staroverov, T. A. Keith, R. Kobayashi, J. Normand, K. Raghavachari, A. P. Rendell, J. C. Burant, S. S. Iyengar, J. Tomasi, M. Cossi, J. M. Millam, M. Klene, C. Adamo, R. Cammi, J. W. Ochterski, R. L. Martin, K. Morokuma, O. Farkas, J. B. Foresman, and D. J. Fox, Gaussian, Inc., Wallingford CT, 2016.

[13] NBO Version 3.1, E. D. Glendening, A. E. Reed, J. E. Carpenter, and F. Weinhold.

[14] Reed, A. E.; Curtiss, L. A.; Weinhold, F. Intermolecular Interactions from a Natural Bond Orbital, Donor-Acceptor Viewpoint. Chem. Rev., 1988, 88, 899-926. 